for

\title{
Copper-Catalyzed Asymmetric Cyanation of Alkenes via Carbonyl-Assisted Coupling of Alkyl-Substituted Carbon-Centered Radicals
}

Song Zhou, ${ }^{a}$ Guoyu Zhang, ${ }^{\mathrm{b}}$ Liang Fu, ${ }^{\mathrm{b}}$ Pinhong Chen, ${ }^{\mathrm{b}}$ Yibiao Li, ${ }^{* a}$ and Guosheng Liu ${ }^{* \mathrm{~b}}$

${ }^{a}$ School of Biotechnology and Health Science, Wuyi University, Jiangmen 529020, China. ${ }^{b}$ State

Key Laboratory of Organometallic Chemistry, Center for Excellence in Molecular Synthesis,

Shanghai Institute of Organic Chemistry, Chinese Academy of Sciences, 345 Lingling Road,

Shanghai 200032, China

E-mail:gliu@mail.sioc.ac.cn

\section{Table of Contents}

$\begin{array}{ll}\text { 1. General Considerations } & \text { S2 }\end{array}$

2. General Procedure for the Synthesis of Substrates $\quad$ S2

$\begin{array}{ll}\text { 3. Optimization of the Reaction Conditions } & \text { S12 }\end{array}$

4. General Procedure for the Asymmetric Cyanotrifluoromethylation $\quad$ S14

5. Scaled-up Reaction $\quad$ S15

$\begin{array}{lr}\text { 6. Product Transformations } & \text { S16 }\end{array}$

$\begin{array}{lr}\text { 7. Mechanistic Studies } & \text { S16 }\end{array}$

$\begin{array}{lr}\text { 8. Characterization of Products } & \text { S20 }\end{array}$

$\begin{array}{ll}\text { 9. Single Crystal X-Ray Diffraction Data (3g) } & \text { S44 }\end{array}$

10. NMR Spectra of Substrates and Products $\quad$ S53

$\begin{array}{lr}\text { 11. HPLC Analysis of Products } & \text { S276 }\end{array}$

$\begin{array}{lr}\text { 12. References } & \text { S328 }\end{array}$ 


\section{General Considerations}

All commercially available compounds were purchased from Aldrich, Alfa Aesar or Adamas. NMR spectra were recorded on Varian Inova 400 and Agilent 400 (400 MHz for ${ }^{1} \mathrm{H}$; $100 \mathrm{MHz}$ for ${ }^{13} \mathrm{C}$ ) spectrometer. The chemical shifts $(\delta)$ are given in parts per million relative to $\mathrm{CDCl}_{3}\left(7.26 \mathrm{ppm}\right.$ for $\left.{ }^{1} \mathrm{H}\right)$ or TMS $\left(0 \mathrm{ppm}\right.$ for $\left.{ }^{1} \mathrm{H}\right), d_{6}$-Acetone $(2.05$ ppm for $\left.{ }^{1} \mathrm{H}\right)$ and $\mathrm{CDCl}_{3}\left(77.0 \mathrm{ppm}\right.$ for $\left.{ }^{13} \mathrm{C}\right), d_{6}$-Acetone $\left(30.6 \mathrm{ppm}\right.$ for $\left.{ }^{13} \mathrm{C}\right)$. High performance liquid chromatography was performed on Waters 2487-600E, Waters ACQUITY UPC2, and Ultimate 3000 Series HPLC, using IC-3, IF-3, ID-3, IA, AD-H, OD-H, AY-3 chiral columns eluted with a mixture of hexane and isopropyl alcohol. Optical rotation was found on a Rudolph-Autopol I. High Resolution Mass spectral data were measured on Agilent Technologies 7250 GCQTOF spectrometer in EI mode. or on Agilent Technologies 6224 TOF LC MS spectrometer in ESI mode. Flash column chromatography was performed on silica gel (particle size 200-300 mesh, purchased from Canada) and eluted with EA/PE. Solvent was purified according to the procedure from the book named "Purification of Laboratory Chemicals". Methyl tert-butyl ether (MTBE) was deoxygenated with anhydrous $\mathrm{N}_{2}$ bubble for at least 30 min before used.

\section{General Procedure for the Synthesis of Substrates}

\subsection{General procedure for the preparation of $1 \mathrm{a}-1 \mathrm{k}$}

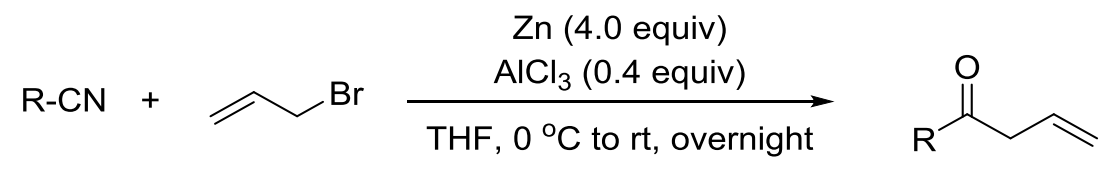

the reaction was quenched by $2 \mathrm{M} \mathrm{HCl}(5 \mathrm{~mL})$

Aluminum trichloride $(0.53 \mathrm{~g}, 4.0 \mathrm{mmol})$ was added at once to a solution of zinc powder $(2.6 \mathrm{~g}, 40.0 \mathrm{mmol})$, nitrile $(10.0 \mathrm{mmol})$ and allylic bromide $(1.8 \mathrm{~g}, 15.0 \mathrm{mmol})$ in anhydrous $\mathrm{THF}(50 \mathrm{~mL})$ at $0^{\circ} \mathrm{C}$ (ice-water bath). The reaction mixture was warmed up to room temperature and then stirred at room temperature overnight. After the reaction was completed (monitored by TLC), aqueous $2 \mathrm{M} \mathrm{HCl}(5 \mathrm{~mL})$ was added to the reaction mixture and stirred at room temperature for 5 minutes. The reaction mixture was passed through a short pad of silica gel and the organic solvent was 
removed directly under reduced pressure. Further purification is conducted on a flash chromatograph with ethyl acetate/hexane to afford products $\mathbf{1 a}, \mathbf{1 f}, \mathbf{1 h}, \mathbf{1 j}, \mathbf{1 k}{ }^{\mathrm{S} 1}$<smiles>C=CCC(=O)Cc1ccc(C(C)(C)C)cc1</smiles>

$1 \mathrm{a}(1.32 \mathrm{~g}, 61 \%)$, yellow liquid. ${ }^{1} \mathrm{H} \mathrm{NMR}\left(400 \mathrm{MHz}, \mathrm{CDCl}_{3}\right): \delta 7.36(\mathrm{~d}, J=8.0 \mathrm{~Hz}$, 2H), $7.14(\mathrm{~d}, J=8.0 \mathrm{~Hz}, 2 \mathrm{H}), 5.97-5.86(\mathrm{~m}, 1 \mathrm{H}), 5.20-5.10(\mathrm{~m}, 2 \mathrm{H}), 3.69$ (s, $2 \mathrm{H})$, $3.22(\mathrm{~d}, J=7.2 \mathrm{~Hz}, 2 \mathrm{H}), 1.32(\mathrm{~s}, 9 \mathrm{H}) .{ }^{13} \mathrm{C} \mathrm{NMR}\left(100 \mathrm{MHz}, \mathrm{CDCl}_{3}\right): \delta 206.5,149.9$, 130.8, 129.10, 125.7, 119.0, 49.0, 46.7, 34.4, 31.3. HRMS (EI) m/z: [M] ${ }^{+}$Calcd for $\mathrm{C}_{15} \mathrm{H}_{20} \mathrm{O} 216.1509$; Found 216.1506.<smiles>C=CCC(=O)Cc1ccc(F)cc1</smiles>

1f $(0.89 \mathrm{~g}, 50 \%)$, yellow liquid. ${ }^{1} \mathrm{H} \mathrm{NMR}\left(400 \mathrm{MHz}, \mathrm{CDCl}_{3}\right): \delta 7.17-7.13(\mathrm{~m}, 2 \mathrm{H})$, 7.01(t, $J=8.4 \mathrm{~Hz}, 2 \mathrm{H}), 5.95-5.84(\mathrm{~m}, 1 \mathrm{H}), 5.20-5.10(\mathrm{~m}, 2 \mathrm{H}), 3.70(\mathrm{~s}, 2 \mathrm{H}), 3.21$ (d, $J$ $=6.8 \mathrm{~Hz}, 2 \mathrm{H}) .{ }^{13} \mathrm{C} \mathrm{NMR}\left(100 \mathrm{MHz}, \mathrm{CDCl}_{3}\right): \delta 205.7,161.9(\mathrm{~d}, J=244.1 \mathrm{~Hz}), 130.9$ (d, $J=8.0 \mathrm{~Hz}), 130.2,129.5(\mathrm{~d}, J=2.5 \mathrm{~Hz}), 119.1,115.5(\mathrm{~d}, J=21.3 \mathrm{~Hz}), 48.3,46.8$. ${ }^{19} \mathrm{~F}$ NMR $\left(376 \mathrm{MHz}, \mathrm{CDCl}_{3}\right) \delta$-115.69- -115.76 (m). HRMS (EI) m/z: [M] ${ }^{+}$Calcd for $\mathrm{C}_{11} \mathrm{H}_{11} \mathrm{FO}$ 178.0788; Found 178.0788.<smiles>C=CCC(=O)Cc1ccc(C(F)(F)F)cc1</smiles>

1h $(1.14 \mathrm{~g}, 50 \%)$, yellow liquid. ${ }^{1} \mathrm{H}$ NMR (400 MHz, $\left.\mathrm{CDCl}_{3}\right): \delta 7.58$ (d, $J=8.4 \mathrm{~Hz}$, 2H), 7.31(t, $J=8.0 \mathrm{~Hz}, 2 \mathrm{H}), 5.96-5.86(\mathrm{~m}, 1 \mathrm{H}), 5.23-5.13(\mathrm{~m}, 2 \mathrm{H}), 3.80(\mathrm{~s}, 2 \mathrm{H}), 3.25$ $(\mathrm{d}, J=6.8 \mathrm{~Hz}, 2 \mathrm{H}) .{ }^{13} \mathrm{C} \mathrm{NMR}\left(100 \mathrm{MHz}, \mathrm{CDCl}_{3}\right): \delta 204.8,137.9,129.9,129.8,129.1$ $(\mathrm{q}, J=31.8 \mathrm{~Hz}), 125.4(\mathrm{q}, J=3.4 \mathrm{~Hz}), 124.1(\mathrm{q}, J=270.3 \mathrm{~Hz}), 119.2,48.5,47.1 .{ }^{19} \mathrm{~F}$ NMR (376 MHz, $\mathrm{CDCl}_{3}$ ) $\delta-62.56$ (s). HRMS (EI) m/z: [M] ${ }^{+}$Calcd for $\mathrm{C}_{12} \mathrm{H}_{13} \mathrm{~F}_{3} \mathrm{O}$ 228.0757; Found 228.0759.<smiles>C=CCC(=O)Cc1ccccc1C</smiles>

$\mathbf{1 j}(1.13 \mathrm{~g}, 65 \%)$, yellow liquid. ${ }^{1} \mathrm{H}$ NMR (400 MHz, $\left.\mathrm{CDCl}_{3}\right): \delta$ 7.22-7.14 (m, 4H), 6.01-5.9 (m, 1H), 5.23-5.13 (m, 2H), $3.76(\mathrm{~s}, 2 \mathrm{H}), 3.22(\mathrm{~d}, J=6.8 \mathrm{~Hz}, 2 \mathrm{H}), 2.27(\mathrm{~s}$, 
$3 \mathrm{H}) .{ }^{13} \mathrm{C} \mathrm{NMR}\left(100 \mathrm{MHz}, \mathrm{CDCl}_{3}\right): \delta 206.0,135.8,132.8,130.4,130.3,127.3,126.1$, 118.9, 47.6, 46.7, 19.6. HRMS (EI) m/z: [M] $]^{+}$Calcd for $\mathrm{C}_{12} \mathrm{H}_{14} \mathrm{O}$ 174.1039; Found 174.1038.<smiles>C=CCC(=O)Cc1cc(C)ccc1C</smiles>

1 k $(0.94 \mathrm{~g}, 50 \%)$, yellow liquid. ${ }^{1} \mathrm{H} \mathrm{NMR}\left(400 \mathrm{MHz}, \mathrm{CDCl}_{3}\right): \delta 7.08$ (d, $J=7.6 \mathrm{~Hz}$, $1 \mathrm{H}), 7.01(\mathrm{~d}, J=7.6 \mathrm{~Hz}, 1 \mathrm{H}), 6.95(\mathrm{~s}, 1 \mathrm{H}), 5.98-5.87(\mathrm{~m}, 1 \mathrm{H}), 5.21-5.10(\mathrm{~m}, 2 \mathrm{H})$, $3.71(\mathrm{~s}, 2 \mathrm{H}), 3.20(\mathrm{~d}, J=6.8 \mathrm{~Hz}, 2 \mathrm{H}), 2.31(\mathrm{~s}, 3 \mathrm{H}), 2.20(\mathrm{~s}, 3 \mathrm{H}) .{ }^{13} \mathrm{C} \mathrm{NMR}(100 \mathrm{MHz}$, $\left.\mathrm{CDCl}_{3}\right): \delta 206.3,135.7,133.6,132.6,131.1,130.5,130.4,128.1,118.9,47.7,46.7$, 20.9, 19.2. HRMS: (EI) $m / z:[\mathrm{M}]^{+}$Calcd for $\mathrm{C}_{13} \mathrm{H}_{16} \mathrm{O}$ 188.1196; Found 188.1188.

\subsection{General procedure for the preparation of 1l-1v}

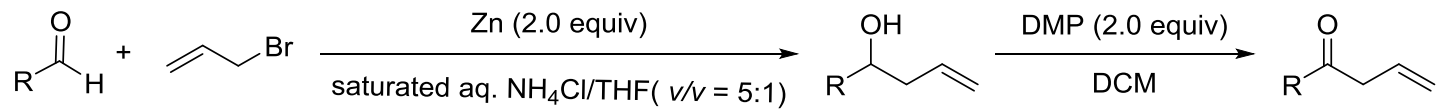

To a vigorously stirred emulsion of saturated aqueous $\mathrm{NH}_{4} \mathrm{Cl}(45 \mathrm{~mL})$ and $\mathrm{THF}(9 \mathrm{~mL})$ was added $\mathrm{Zn}(1.3 \mathrm{~g}, 20 \mathrm{mmol})$, aldehyde (10 mmol) and 3-bromopropene (2.4 g, 20 mmol). After stirring for $2 \mathrm{~h}$ at room temperature, the reaction was quenched by addition of water. The solvent was evaporated, and the remaining aqueous phase was extracted with EtOAc. The combined organic extracts were washed once with brine, dried over anhydrous $\mathrm{Na}_{2} \mathrm{SO}_{4}$, and concentrated under reduced pressure. The crude alcohol was directly used for next step without purification.

To a solution of DMP $(8.5 \mathrm{~g}, 20 \mathrm{mmol})$ in $\mathrm{CH}_{2} \mathrm{Cl}_{2}(20 \mathrm{~mL})$ at room temperature was added to a solution of the crude alcohol $(10 \mathrm{mmol})$ in $\mathrm{CH}_{2} \mathrm{Cl}_{2}(20 \mathrm{~mL})$. After $15 \mathrm{~min}$, the mixture was diluted with ether $(50 \mathrm{~mL})$ and washed once with $10 \%$ $\mathrm{Na}_{2} \mathrm{~S}_{2} \mathrm{O}_{3} /$ saturated aqueous $\mathrm{NaHCO}_{3}$ solution (1/1). The aqueous layer was extracted with ether. The combined organic extracts were washed once with brine, dried over anhydrous $\mathrm{MgSO}_{4}$, and concentrated under reduced pressure. The residue was purified by flash chromatography (10\% ethyl acetate/hexane) to afford products 11-1v. ${ }^{\text {S2 }}$ 
<smiles>C=CCC(=O)c1ccc(C(C)(C)C)cc1</smiles>

11 (1.65 g, 82\%), yellow liquid. ${ }^{1} \mathrm{H}$ NMR (400 MHz, $\left.\mathrm{CDCl}_{3}\right): \delta 7.91(\mathrm{~d}, J=8.8 \mathrm{~Hz}$, 2H), $7.48(\mathrm{~d}, J=8.8 \mathrm{~Hz}, 2 \mathrm{H}), 6.14-6.04(\mathrm{~m}, 1 \mathrm{H}), 5.24-5.19(\mathrm{~m}, 2 \mathrm{H}), 3.74(\mathrm{~d}, J=6.8$ $\mathrm{Hz}, 2 \mathrm{H}), 1.34$ (s, 9H). ${ }^{13} \mathrm{C}$ NMR $\left(100 \mathrm{MHz}, \mathrm{CDCl}_{3}\right): \delta 196.7,156.9,133.9,131.3$, $128.2,125.5,118.5,43.4,35.1,31.0$. These date are consistent with those reported in the literature. ${ }^{\mathrm{S} 3}$<smiles>C=CCC(=O)c1ccccc1</smiles>

$1 \mathrm{~m}(1.17 \mathrm{~g}, 80 \%)$, yellow liquid. ${ }^{1} \mathrm{H} \mathrm{NMR}\left(400 \mathrm{MHz}, \mathrm{CDCl}_{3}\right): \delta 7.95$ (d, $J=8.8 \mathrm{~Hz}$, 2H), $7.55(\mathrm{t}, J=7.2 \mathrm{~Hz}, 1 \mathrm{H}), 7.44(\mathrm{t}, J=7.2 \mathrm{~Hz}, 2 \mathrm{H}), 6.12-6.05(\mathrm{~m}, 1 \mathrm{H}), 5.24-5.18$ $(\mathrm{m}, 2 \mathrm{H}), 3.74(\mathrm{~d}, J=7.2 \mathrm{~Hz}, 2 \mathrm{H}) .{ }^{13} \mathrm{C} \mathrm{NMR}\left(100 \mathrm{MHz}, \mathrm{CDCl}_{3}\right): \delta$ 197.9, 136.4, 133.0, $131.0,128.5,128.1,118.6,43.3$. These data are consistent with those reported in the literature. $^{\mathrm{S} 3}$<smiles>C=CCC(=O)c1ccc(OC)cc1</smiles>

1n $(1.50 \mathrm{~g}, 85 \%)$, white solid. ${ }^{1} \mathrm{H} \mathrm{NMR}\left(400 \mathrm{MHz}, \mathrm{CDCl}_{3}\right): \delta 7.92(\mathrm{~d}, J=8.8 \mathrm{~Hz}, 2 \mathrm{H})$, $6.90(\mathrm{~d}, J=8.4 \mathrm{~Hz}, 2 \mathrm{H}), 6.11-6.01(\mathrm{~m}, 1 \mathrm{H}), 5.20-5.15(\mathrm{~m}, 2 \mathrm{H}), 3.83(\mathrm{~s}, 3 \mathrm{H}), 3.68(\mathrm{~d}, J$ $=7.2 \mathrm{~Hz}, 2 \mathrm{H}) .{ }^{13} \mathrm{C} \mathrm{NMR}\left(100 \mathrm{MHz}, \mathrm{CDCl}_{3}\right): \delta 196.6,163.4,131.4,130.5,129.5$, 118.4, 113.7, 55.41, 43.16. These data are consistent with those reported in the literature. $^{\mathrm{S3}}$<smiles>C=CCC(=O)c1ccc(OC(C)=O)cc1</smiles>

$10(1.73 \mathrm{~g}, 85 \%)$, yellow liquid. ${ }^{1} \mathrm{H}$ NMR (400 MHz, $\left.\mathrm{CDCl}_{3}\right): \delta 7.97(\mathrm{~d}, J=8.8 \mathrm{~Hz}$, 2H), $7.16(\mathrm{~d}, J=8.8 \mathrm{~Hz}, 2 \mathrm{H}), 6.09-6.00(\mathrm{~m}, 1 \mathrm{H}), 5.22-5.16(\mathrm{~m}, 2 \mathrm{H}), 3.71(\mathrm{~d}, J=6.4$ $\mathrm{Hz}, 2 \mathrm{H}), 2.28(\mathrm{~s}, 3 \mathrm{H}) .{ }^{13} \mathrm{C} \mathrm{NMR}\left(100 \mathrm{MHz}, \mathrm{CDCl}_{3}\right): \delta 196.6,168.7,154.2,133.9$, 
130.7, 129.7, 121.7, 118.7, 43.2, 21.0. HRMS (EI) m/z: [M] $]^{+}$Calcd for $\mathrm{C}_{12} \mathrm{H}_{12} \mathrm{O}_{3}$ 204.0781; Found 204.0779.<smiles>C=CCC(=O)c1ccc(-c2ccccc2)cc1</smiles>

$1 p(1.82 \mathrm{~g}, 82 \%)$, white solid. ${ }^{1} \mathrm{H}$ NMR $\left(400 \mathrm{MHz}, \mathrm{CDCl}_{3}\right): \delta 8.05(\mathrm{~d}, J=8.0 \mathrm{~Hz}, 2 \mathrm{H})$, $7.69(\mathrm{~d}, J=8.4 \mathrm{~Hz}, 2 \mathrm{H}), 7.63(\mathrm{~d}, J=8.0 \mathrm{~Hz}, 2 \mathrm{H}), 7.48(\mathrm{t}, J=7.4 \mathrm{~Hz}, 2 \mathrm{H}), 7.41(\mathrm{~d}, J$ $=7.2 \mathrm{~Hz}, 1 \mathrm{H}), 6.18-6.08(\mathrm{~m}, 1 \mathrm{H}), 5.28-5.23(\mathrm{~m}, 2 \mathrm{H}), 3.80(\mathrm{~d}, J=6.8 \mathrm{~Hz}, 2 \mathrm{H}) .{ }^{13} \mathrm{C}$ NMR $\left(100 \mathrm{MHz}, \mathrm{CDCl}_{3}\right): \delta 197.5,145.8,139.7,135.2,131.1,128.9,128.8,128.2$, $127.2,118.7,43.4$. These data are consistent with those reported in the literature. ${ }^{\mathrm{S} 4}$<smiles>C=CCC(=O)c1ccc(C)cc1</smiles>

$1 q(1.28 \mathrm{~g}, 80 \%)$, yellow liquid. ${ }^{1} \mathrm{H} \mathrm{NMR}\left(400 \mathrm{MHz}, \mathrm{CDCl}_{3}\right): \delta 7.83(\mathrm{~d}, J=8.0 \mathrm{~Hz}$, 2H), $7.21(\mathrm{~d}, J=8.0 \mathrm{~Hz}, 2 \mathrm{H}), 6.11-6.00(\mathrm{~m}, 1 \mathrm{H}), 5.19-5.14(\mathrm{~m}, 2 \mathrm{H}), 3.68(\mathrm{~d}, J=6.8$ $\mathrm{Hz}, 2 \mathrm{H}), 2.34$ (s, 3H). ${ }^{13} \mathrm{C} \mathrm{NMR}\left(100 \mathrm{MHz}, \mathrm{CDCl}_{3}\right): \delta$ 197.6, 143.9, 134.0, 131.2, $129.2,128.3,118.4,43.2,21.5$. These data are consistent with those reported in the literature. $^{\mathrm{S} 4}$<smiles>C=CCC(=O)c1ccc(C(=O)OC)cc1</smiles>

1r (1.67 g, 82\%), yellow liquid. ${ }^{1} \mathrm{H}$ NMR (400 MHz, $\left.\mathrm{CDCl}_{3}\right): \delta 8.10(\mathrm{~d}, J=8.4 \mathrm{~Hz}$, 2H), $8.00(\mathrm{~d}, J=8.4 \mathrm{~Hz}, 2 \mathrm{H}), 6.11-6.01(\mathrm{~m}, 1 \mathrm{H}), 5.25-5.19(\mathrm{~m}, 2 \mathrm{H}), 3.93(\mathrm{~s}, 3 \mathrm{H})$, $3.77(\mathrm{~d}, J=6.8 \mathrm{~Hz}, 2 \mathrm{H}) .{ }^{13} \mathrm{C} \mathrm{NMR}\left(100 \mathrm{MHz}, \mathrm{CDCl}_{3}\right): \delta 197.4,166.1,139.6,133.9$, $130.4,129.8,128.1,119.1,52.4,43.6$. These data are consistent with those reported in the literature. ${ }^{\mathrm{S} 5}$<smiles>C=CCC(=O)c1ccc(F)cc1</smiles>

1s $(1.31 \mathrm{~g}, 80 \%)$, yellow liquid. ${ }^{1} \mathrm{H}$ NMR (400 MHz, $\left.\mathrm{CDCl}_{3}\right): \delta 8.00-7.96(\mathrm{~m}, 2 \mathrm{H})$, 
$7.12(\mathrm{t}, J=8.6 \mathrm{~Hz}, 2 \mathrm{H}), 6.11-6.01(\mathrm{~m}, 1 \mathrm{H}), 5.24-5.18(\mathrm{~m}, 2 \mathrm{H}), 3.72(\mathrm{~d}, J=7.2 \mathrm{~Hz}$, 2H). ${ }^{13} \mathrm{C}$ NMR $\left(100 \mathrm{MHz}, \mathrm{CDCl}_{3}\right): \delta 196.2,165.6(\mathrm{~d}, J=252.8 \mathrm{~Hz}), 132.8(\mathrm{~d}, J=3.3$ Hz), $130.9(\mathrm{~d}, J=9.5 \mathrm{~Hz}), 130.8(\mathrm{~d}, J=9.2 \mathrm{~Hz}), 118.65,115.5(\mathrm{~d}, J=21.3 \mathrm{~Hz}), 43.1$. These data are consistent with those reported in the literature. ${ }^{\mathrm{S} 4}$<smiles>C=CCC(=O)c1ccc(Cl)cc1</smiles>

$1 t(1.10 \mathrm{~g}, 60 \%)$, white solid. ${ }^{1} \mathrm{H} \mathrm{NMR}\left(400 \mathrm{MHz}, \mathrm{CDCl}_{3}\right): \delta 7.89(\mathrm{~d}, J=8.8 \mathrm{~Hz}, 2 \mathrm{H})$, $7.41(\mathrm{~d}, J=8.4 \mathrm{~Hz}, 2 \mathrm{H}), 6.10-6.00(\mathrm{~m}, 1 \mathrm{H}), 5.24-5.17(\mathrm{~m}, 2 \mathrm{H}), 3.71(\mathrm{~d}, J=6.8 \mathrm{~Hz}$, 2H). ${ }^{13} \mathrm{C} \mathrm{NMR}\left(100 \mathrm{MHz}, \mathrm{CDCl}_{3}\right): \delta 196.6,139.5,134.7,130.6,129.6,128.8,118.9$, 43.3. These data are consistent with those reported in the literature. ${ }^{\mathrm{S}}$<smiles>C=CCC(=O)c1ccc(C(F)(F)F)cc1</smiles>

1u $(1.03 \mathrm{~g}, 48 \%)$, yellow liquid. ${ }^{1} \mathrm{H} \mathrm{NMR}\left(400 \mathrm{MHz}, \mathrm{CDCl}_{3}\right): \delta 8.07(\mathrm{~d}, J=8.8 \mathrm{~Hz}$, 2H), $7.74(\mathrm{~d}, J=8.4 \mathrm{~Hz}, 2 \mathrm{H}), 6.13-6.02(\mathrm{~m}, 1 \mathrm{H}), 5.29-5.21(\mathrm{~m}, 2 \mathrm{H}), 3.79(\mathrm{~d}, J=6.4$ $\mathrm{Hz}, 2 \mathrm{H}) .{ }^{13} \mathrm{C} \mathrm{NMR}\left(100 \mathrm{MHz}, \mathrm{CDCl}_{3}\right): \delta 196.9,139.1,134.4(\mathrm{q}, J=33.0 \mathrm{~Hz}), 130.3$, $128.5,125.6(\mathrm{q}, J=4.2 \mathrm{~Hz}), 123.5(\mathrm{q}, J=271.3 \mathrm{~Hz}), 119.2,43.6$. These data are consistent with those reported in the literature. ${ }^{\mathrm{S} 6}$<smiles>C=CCC(=O)c1ccc(C#N)cc1</smiles>

1v $(1.00 \mathrm{~g}, 58 \%)$, white solid. ${ }^{1} \mathrm{H} \mathrm{NMR}\left(400 \mathrm{MHz}, \mathrm{CDCl}_{3}\right): \delta 8.03(\mathrm{~d}, J=8.4 \mathrm{~Hz}, 2 \mathrm{H})$, $7.76(\mathrm{~d}, J=8.0 \mathrm{~Hz}, 2 \mathrm{H}), 6.09-6.98(\mathrm{~m}, 1 \mathrm{H}), 5.26-5.19(\mathrm{~m}, 2 \mathrm{H}), 3.76(\mathrm{~d}, J=6.4 \mathrm{~Hz}$, 2H). ${ }^{13} \mathrm{C} \mathrm{NMR}\left(100 \mathrm{MHz}, \mathrm{CDCl}_{3}\right): \delta 196.5,139.4,132.5,129.9,128.6,119.5,117.8$, $116.4,43.5$. These data are consistent with those reported in the literature. ${ }^{\mathrm{S} 7}$

2.3 General procedure for the preparation of $1 \mathrm{w}, 1 \mathrm{y}, 1 \mathrm{ac}, 1 \mathrm{ad}, 1 \mathrm{ag}$.<smiles>[R]NNC(=O)CC=C</smiles>

To a solution of corresponding amine $(10.0 \mathrm{mmoL})$ in $\mathrm{CH}_{2} \mathrm{Cl}_{2}(30 \mathrm{~mL})$ were added 
DCC (2.7 g, $13 \mathrm{mmoL})$, DMAP (0.16 g, $1.3 \mathrm{mmoL})$ and 3-butenoic acid (1.1 g, 13 $\mathrm{mmoL}$ ) at $0^{\circ} \mathrm{C}$. The reaction mixture was stirred for $10 \mathrm{~min}$ at $0{ }^{\circ} \mathrm{C}$, then for $24 \mathrm{~h}$ at room temperature. The solid was filtered off and washed with $\mathrm{CH}_{2} \mathrm{Cl}_{2}(25 \mathrm{~mL})$. The organic layer was hydrolyzed with saturated aqueous $\mathrm{NaHCO}_{3}$, the organic phase was separated and dried over $\mathrm{Na}_{2} \mathrm{SO}_{4}$ and concentrated under reduced pressure. The crude product was purified by silica gel column chromatography to afford to afford products 1w, 1y, 1ac, 1ad, 1ag. ${ }^{\text {s }}$<smiles>C=CCC(=O)Nc1ccccc1</smiles>

1w (1.42 g, 88\%), a light white solid. ${ }^{1} \mathrm{H}$ NMR (400 MHz, $\left.\mathrm{CDCl}_{3}\right): \delta 7.99$ (br, 1H), $7.52(\mathrm{~d}, J=8.0 \mathrm{~Hz}, 2 \mathrm{H}), 7.29(\mathrm{t}, J=8.0 \mathrm{~Hz}, 2 \mathrm{H}), 7.09$ (t, $J=7.4 \mathrm{~Hz}, 1 \mathrm{H}), 6.06-5.96$ $(\mathrm{m}, 1 \mathrm{H}), 5.29-5.24(\mathrm{~m}, 2 \mathrm{H}), 3.16(\mathrm{~d}, J=7.2 \mathrm{~Hz}, 2 \mathrm{H}) .{ }^{13} \mathrm{C} \mathrm{NMR}\left(100 \mathrm{MHz}, \mathrm{CDCl}_{3}\right): \delta$ $168.9,147.3,135.1,131.2,125.7,120.0,119.8,42.4$. These data are consistent with those reported in the literature. ${ }^{\mathrm{S} 8}$<smiles>C=CCC(=O)Nc1ccc(C(C)(C)C)cc1</smiles>

$1 \mathbf{y}\left(1.93\right.$ g, 89\%), light white solid, m.p. $62-64{ }^{\circ} \mathrm{C} .{ }^{1} \mathrm{H}$ NMR (400 MHz, $\left.\mathrm{CDCl}_{3}\right): \delta$ 7.77 (br, 1H), 7.44 (d, $J=8.8 \mathrm{~Hz}, 2 \mathrm{H}), 7.32$ (d, $J=8.8 \mathrm{~Hz}, 2 \mathrm{H}), 6.07-5.96$ (m, 1H), 5.30-5.25 (m, 2H), $3.15(\mathrm{~d}, J=6.8 \mathrm{~Hz}, 2 \mathrm{H}), 1.30(\mathrm{~s}, 9 \mathrm{H}) .{ }^{13} \mathrm{C}$ NMR (100 MHz, $\left.\mathrm{CDCl}_{3}\right): \delta 168.9,147.3,135.1,131.2,125.6,120.0,119.8,42.4,34.3,31.3 . \mathrm{HRMS}$ (ESI) m/z: [M+Na $]^{+}$Calcd for $\mathrm{C}_{14} \mathrm{H}_{19} \mathrm{NONa} 240.1359$; Found 240.1360.<smiles>C=CCC(=O)Nc1ccc(Br)cc1</smiles>

1ac (1.91 g, 80\%), light white solid, m.p. $61-63{ }^{\circ} \mathrm{C} .{ }^{1} \mathrm{H}$ NMR (400 MHz, $\left.\mathrm{CDCl}_{3}\right): \delta$ 8.31 (br, 1H), 7.41-7.35 (m, 4H), 6.02-5.92 (m, 1H), 5.26-5.21 (m, 2H), 3.14 (d, $J=$ $7.2 \mathrm{~Hz}, 2 \mathrm{H}) .{ }^{13} \mathrm{C} \mathrm{NMR}\left(100 \mathrm{MHz}, \mathrm{CDCl}_{3}\right): \delta 169.5,136.8,131.7,130.6,121.7,119.9$, 116.9, 42.2. HRMS (EI) m/z: [M] $]^{+}$Calcd for $\mathrm{C}_{10} \mathrm{H}_{10} \mathrm{BrNO} 238.9940$; Found 238.9941. 
$\overbrace{1}^{\mathrm{N}} \prod_{0}^{1 a d}$

$\operatorname{1ad}(1.85 \mathrm{~g}, 85 \%)$, yellow liquid. ${ }^{1} \mathrm{H} \mathrm{NMR}\left(400 \mathrm{MHz}, \mathrm{CDCl}_{3}\right): \delta$ 7.49-7.46 (m, $\left.1 \mathrm{H}\right)$, $7.38(\mathrm{~d}, J=7.6 \mathrm{~Hz}, 1 \mathrm{H}), 7.27-7.23(\mathrm{~m}, 2 \mathrm{H}), 7.14(\mathrm{~d}, J=8.0 \mathrm{~Hz}, 1 \mathrm{H}), 6.09-5.98(\mathrm{~m}$, 1H), 5.35-5.28 (m, 2H), $3.18(\mathrm{~d}, J=7.2 \mathrm{~Hz}, 2 \mathrm{H}), 1.30(\mathrm{~s}, 9 \mathrm{H}) .{ }^{13} \mathrm{C}$ NMR (100 MHz, $\left.\mathrm{CDCl}_{3}\right): \delta 168.8,152.2,137.4,131.1,128.6,121.5,120.4,117.2,117.0,42.7,34.7$, 31.2. HRMS (EI) m/z: [M] $]^{+}$Calcd for $\mathrm{C}_{14} \mathrm{H}_{19} \mathrm{NO} 217.1461$; Found 217.1460.<smiles>C=CCC(=O)NC(C)(C)c1ccccc1</smiles>

$1 \mathrm{ag}(1.63 \mathrm{~g}, 80 \%)$, light white solid, m.p. $60-62{ }^{\circ} \mathrm{C} .{ }^{1} \mathrm{H} \mathrm{NMR}\left(400 \mathrm{MHz}, \mathrm{CDCl}_{3}\right): \delta$ 7.38-7.31 (m, 4H), 7.26-7.21 (m, 1H), 6.01-5.91 (m, 1H), $5.83(\mathrm{br}, 1 \mathrm{H})$, 5.26-5.21 (m, 2H), $2.98(\mathrm{~d}, J=6.8 \mathrm{~Hz}, 2 \mathrm{H}), 1.70(\mathrm{~s}, 6 \mathrm{H}) .{ }^{13} \mathrm{C} \mathrm{NMR}\left(100 \mathrm{MHz}, \mathrm{CDCl}_{3}\right): \delta 169.4$, 146.7, 131.76, 128.4, 126.7, 124.6, 119.7, 55.9, 42.6, 29.0. HRMS (EI) m/z: $[M]^{+}$ Calcd for $\mathrm{C}_{13} \mathrm{H}_{17} \mathrm{NO} 203.1305$; Found 203.1306.

2.4 General procedure for the preparation of 1ah, 1aj, 1am, 1an, 1ao, 1ap, 1aq, 1ar, 1as

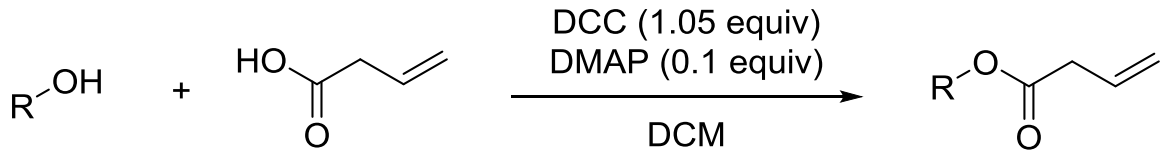

To a solution of the 3-butenoic acid $(0.86 \mathrm{~g}, 10 \mathrm{mmoL})$ and alcohols $(12.0 \mathrm{mmoL})$ in dichloromethane $(30 \mathrm{~mL})$ was added DCC $(2.2 \mathrm{~g}, 10.5 \mathrm{mmoL})$ followed by a catalytic amount of DMAP $(0.12 \mathrm{~g}, 1.0 \mathrm{mmoL})$. The mixture was stirred at room temperature overnight then concentrated under reduce pressure. The residue was triturated with $\mathrm{PE} / \mathrm{EA}=9 / 1$, filtered through a pad of celite then the filtrate was concentrated under reduce pressure. The desired product was obtained after purification by column chromatography on silica gel to afford products 1ah, 1aj, 1am, 1an, 1ao, 1ap, 1aq, 1ar, 1as. ${ }^{\text {S9 }}$<smiles>C=CCC(=O)Oc1ccccc1</smiles> 
1ah $(1.38 \mathrm{~g}, 85 \%)$, yellow liquid. ${ }^{1} \mathrm{H}$ NMR $\left(400 \mathrm{MHz}, \mathrm{CDCl}_{3}\right): \delta 7.39(\mathrm{t}, J=7.6 \mathrm{~Hz}$, 2H), $7.24(\mathrm{t}, J=6.8 \mathrm{~Hz}, 1 \mathrm{H}), 7.11(\mathrm{~d}, J=4.6 \mathrm{~Hz}, 2 \mathrm{H}), 6.11-6.00(\mathrm{~m}, 1 \mathrm{H}), 5.33-5.26$ $(\mathrm{m}, 2 \mathrm{H}), 3.36(\mathrm{~d}, J=7.2 \mathrm{~Hz}, 2 \mathrm{H}) .{ }^{13} \mathrm{C} \mathrm{NMR}\left(100 \mathrm{MHz}, \mathrm{CDCl}_{3}\right): \delta 169.9,150.6,129.3$, $125.8,121.4,119.11,39.0$. These data are consistent with those reported in the literature. $^{\mathrm{S} 9}$

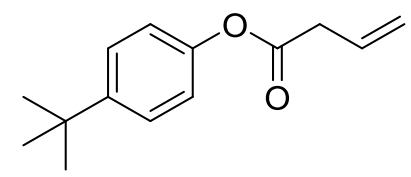

1aj

1aj (1.96 g, 90\%), yellow liquid. ${ }^{1} \mathrm{H}$ NMR (400 MHz, $\left.\mathrm{CDCl}_{3}\right): \delta 7.41(\mathrm{~d}, J=8.4 \mathrm{~Hz}$, 2H), $7.04(\mathrm{~d}, J=8.8 \mathrm{~Hz}, 2 \mathrm{H}), 6.11-6.01(\mathrm{~m}, 1 \mathrm{H}), 5.32-5.26(\mathrm{~m}, 2 \mathrm{H}), 3.35(\mathrm{~d}, J=6.8$ $\mathrm{Hz}, 2 \mathrm{H}), 1.34(\mathrm{~s}, 9 \mathrm{H}) .{ }^{13} \mathrm{C} \mathrm{NMR}\left(100 \mathrm{MHz}, \mathrm{CDCl}_{3}\right): \delta 170.0,148.6,148.2,129.7$, 126.2, 102.7, 119.0, 39.1, 34.4, 31.3. HRMS (EI) m/z: $[\mathrm{M}]^{+}$Calcd for $\mathrm{C}_{14} \mathrm{H}_{18} \mathrm{O}_{2}$ 218.1301; Found 218.1303.

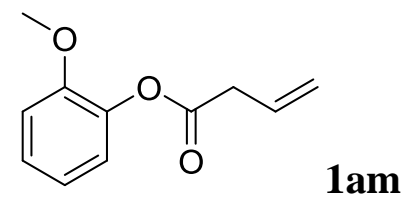

$\operatorname{1am}\left(1.50 \mathrm{~g}, 78 \%\right.$ ), yellow liquid. ${ }^{1} \mathrm{H} \mathrm{NMR}\left(400 \mathrm{MHz}, \mathrm{CDCl}_{3}\right): \delta 7.24-7.19(\mathrm{~m}, 1 \mathrm{H})$, 7.07-7.05 (m, 1H), 6.99-6.94 (m, 2H), 6.13-6.02 (m, 1H), 5.34-5.26 (m, 2H), $3.82(\mathrm{~s}$, $3 \mathrm{H}), 3.40(\mathrm{~d}, J=6.8 \mathrm{~Hz}, 2 \mathrm{H}) .{ }^{13} \mathrm{C} \mathrm{NMR}\left(100 \mathrm{MHz}, \mathrm{CDCl}_{3}\right): \delta 169.2,150.9,139.5$, 129.7, 126.7, 122.5, 120.5, 118.7, 112.2, 55.5, 38.4. HRMS (EI) m/z: [M] ${ }^{+}$Calcd for $\mathrm{C}_{11} \mathrm{H}_{12} \mathrm{O}_{3}$ 192.0781; Found 192.0778.

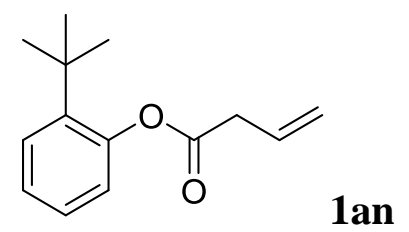

$\operatorname{1an}(1.75 \mathrm{~g}, 80 \%)$, yellow liquid. ${ }^{1} \mathrm{H}$ NMR (400 MHz, $\left.\mathrm{CDCl}_{3}\right): \delta 7.37(\mathrm{~d}, J=7.6 \mathrm{~Hz}$, 1H), 7.21-7.11 (m, 2H), $6.97(\mathrm{~d}, J=7.6 \mathrm{~Hz}, 1 \mathrm{H}), 6.10-5.99(\mathrm{~m}, 1 \mathrm{H}), 5.29-5.23(\mathrm{~m}$, $2 \mathrm{H}), 3.35(\mathrm{~d}, J=6.8 \mathrm{~Hz}, 2 \mathrm{H}), 1.34(\mathrm{~s}, 9 \mathrm{H}) .{ }^{13} \mathrm{C} \mathrm{NMR}\left(100 \mathrm{MHz}, \mathrm{CDCl}_{3}\right): \delta 169.8$, 149.1, 140.8, 129.4, 127.1, 126.8, 125.7, 123.8, 119.3, 39.6, 34.3, 30.1. HRMS (EI) $\mathrm{m} / \mathrm{z}:[\mathrm{M}]^{+}$Calcd for $\mathrm{C}_{14} \mathrm{H}_{18} \mathrm{O}_{2}$ 218.1301; Found 218.1294. 


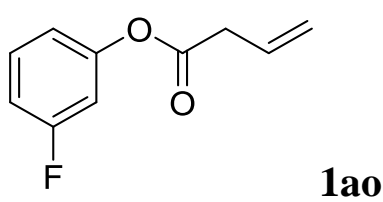

1 ao (1.44 g, 80\%), yellow liquid. ${ }^{1} \mathrm{H} \mathrm{NMR}\left(400 \mathrm{MHz}, \mathrm{CDCl}_{3}\right): \delta 7.29-7.23(\mathrm{~m}, 1 \mathrm{H})$, 6.91-6.84 (m, 3H), 6.03-5.93 (m, 1H), 5.25-5.20 (m, 2H), $3.28(\mathrm{~d}, J=6.8 \mathrm{~Hz}, 2 \mathrm{H})$; ${ }^{13} \mathrm{C} \mathrm{NMR}\left(100 \mathrm{MHz}, \mathrm{CDCl}_{3}\right): \delta 169.2,162.6(\mathrm{~d}, J=245.5 \mathrm{~Hz}), 151.3(\mathrm{~d}, J=10.8$ Hz), 130.0 (d, $J=9.3 \mathrm{~Hz}), 129.2,119.0,117.1(\mathrm{~d}, J=3.7 \mathrm{~Hz}), 112.6(\mathrm{~d}, J=20.5 \mathrm{~Hz})$, $109.4(\mathrm{~d}, J=24.4 \mathrm{~Hz}), 38.6 .{ }^{19} \mathrm{~F}$ NMR $\left(376 \mathrm{MHz}, \mathrm{CDCl}_{3}\right): \delta-111.10--111.04(\mathrm{~m})$. HRMS (EI) m/z: [M] ${ }^{+}$Calcd for $\mathrm{C}_{10} \mathrm{H}_{9} \mathrm{FO}_{2}$ 180.0581; Found 180.0575.<smiles>C=CCC(=O)Oc1c(Br)cccc1Br</smiles>

1ap (2.23 g, 70\%), yellow liquid. ${ }^{1} \mathrm{H}$ NMR (400 MHz, $\left.\mathrm{CDCl}_{3}\right): \delta 7.55-7.53(\mathrm{~m}, 2 \mathrm{H})$, 7.01-6.97 (m, 1H), 6.14-6.04 (m, 1H), 5.37-5.29 (m, 2H), 3.46 (d, $J=6.8 \mathrm{~Hz}, 2 \mathrm{H})$; ${ }^{13} \mathrm{C} \mathrm{NMR}\left(100 \mathrm{MHz}, \mathrm{CDCl}_{3}\right): \delta 167.6,146.0,132.2,128.8,128.1,119.6,117.6,38.5$. HRMS (EI) m/z: [M] Calcd for $\mathrm{C}_{10} \mathrm{H}_{8} \mathrm{Br}_{2} \mathrm{O}_{2}$ 317.8886; Found 317.8880.

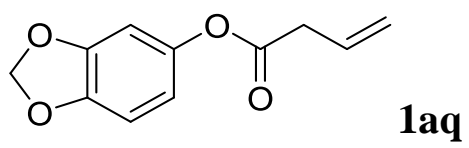

1aq $(1.55 \mathrm{~g}, 75 \%)$, yellow liquid. ${ }^{1} \mathrm{H} \mathrm{NMR}\left(400 \mathrm{MHz}, \mathrm{CDCl}_{3}\right): \delta 6.77(\mathrm{~d}, J=8.4 \mathrm{~Hz}$, $1 \mathrm{H}), 6.61(\mathrm{~d}, J=2.4 \mathrm{~Hz}, 1 \mathrm{H}), 6.54-6.51(\mathrm{~m}, 1 \mathrm{H}), 6.06-5.97(\mathrm{~m}, 1 \mathrm{H}), 5.97(\mathrm{~s}, 2 \mathrm{H})$, 5.29-5.24 (m, 2H), $3.31(\mathrm{~d}, J=6.8 \mathrm{~Hz}, 2 \mathrm{H}) .{ }^{13} \mathrm{C} \mathrm{NMR}\left(100 \mathrm{MHz}, \mathrm{CDCl}_{3}\right): \delta 170.2$, 147.9, 145.3, 144.9, 129.6, 119.2, 113.8, 107.9, 103.6, 101.7, 38.9. HRMS (EI) m/z: $[\mathrm{M}]^{+}$Calcd for $\mathrm{C}_{11} \mathrm{H}_{10} \mathrm{O}_{4}$ 206.0574; Found 206.0574.

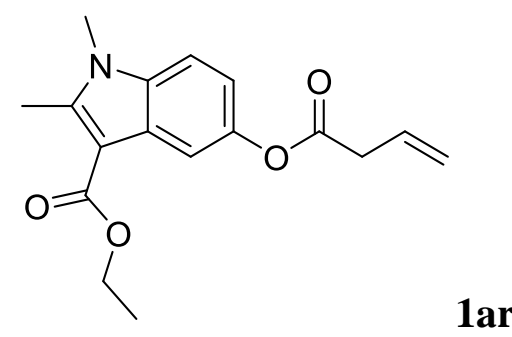

$\operatorname{1ar}(2.11 \mathrm{~g}, 70 \%)$, light white solid, m.p. $100-102{ }^{\circ} \mathrm{C} .{ }^{1} \mathrm{H}$ NMR $\left(400 \mathrm{MHz}, \mathrm{CDCl}_{3}\right): \delta$ $7.80(\mathrm{~d}, J=2.4 \mathrm{~Hz}, 1 \mathrm{H}), 7.26(\mathrm{~d}, J=8.8 \mathrm{~Hz}, 1 \mathrm{H}), 6.95(\mathrm{dd}, J=8.8,2.0 \mathrm{~Hz}, 1 \mathrm{H})$, 6.13-6.03 (m, 1H), 5.33-5.25 (m, 2H), 4.41-4.35 (m, 2H), 3.67 (s, 3H), 3.38 (d, $J=$ 
$6.8 \mathrm{~Hz}, 2 \mathrm{H}), 2.75(\mathrm{~s}, 3 \mathrm{H}), 1.43(\mathrm{t}, J=6.8 \mathrm{~Hz}, 3 \mathrm{H}) .{ }^{13} \mathrm{C} \mathrm{NMR}\left(100 \mathrm{MHz}, \mathrm{CDCl}_{3}\right): \delta$ $170.6,165.5$, 146.1 145.6, 134.0, 129.8, 126.8, 118.8, 115.6, 113.4, 109.2, 103.6, 59.2, 39.0, 29.3, 14.4, 11.6. HRMS (ESI) m/z: $[\mathrm{M}+\mathrm{Na}]^{+} \mathrm{Calcd}$ for $\mathrm{C}_{17} \mathrm{H}_{19} \mathrm{NO}_{4} \mathrm{Na}$ 324.1206; Found 324.1199.

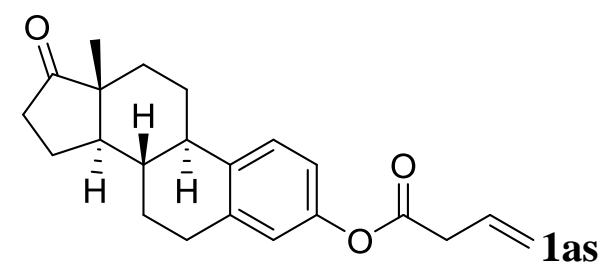

1as $(2.71 \mathrm{~g}, 80 \%)$, light white solid, m.p. $83-86{ }^{\circ} \mathrm{C} .{ }^{1} \mathrm{H}$ NMR (400 MHz, $\left.\mathrm{CDCl}_{3}\right): \delta$ $7.28(\mathrm{~d}, J=8.4 \mathrm{~Hz}, 1 \mathrm{H}), 6.86(\mathrm{dd}, J=8.8,2.0 \mathrm{~Hz}, 1 \mathrm{H}), 6.82(\mathrm{~s}, 1 \mathrm{H}), 6.06-5.99$ (m, 1H), 5.30-5.24 (m, 2H), $3.33(\mathrm{~d}, J=7.2 \mathrm{~Hz}, 2 \mathrm{H}), 2.90(\mathrm{t}, J=4.0 \mathrm{~Hz}, 2 \mathrm{H}), 2.54-1.94$ (m, 7H), 1.42-1.66 (m, 6H), 0.91 (s, 3H). $\left.{ }^{13} \mathrm{C} \mathrm{NMR} \mathrm{(100} \mathrm{MHz,} \mathrm{CDCl}_{3}\right): \delta 220.8$, 170.3, 148.5, 138.0, 137.4, 129.7, 126.4, 121.5, 119.1, 118.6, 50.4, 47.9, 44.1, 39.1, 37.9, 35.8, 31.5, 29.4, 26.3, 25.7, 21.6, 13.8. HRMS (ESI) m/z: $[\mathrm{M}+\mathrm{Na}]^{+}$Calcd for $\mathrm{C}_{22} \mathrm{H}_{26} \mathrm{O}_{3} \mathrm{Na}$ 361.1774; Found 361.1769.

\section{Optimization of the Reaction Conditions}

\subsection{Optimization of ligands}

Table S1. Optimization of ligands. ${ }^{a, b}$ 


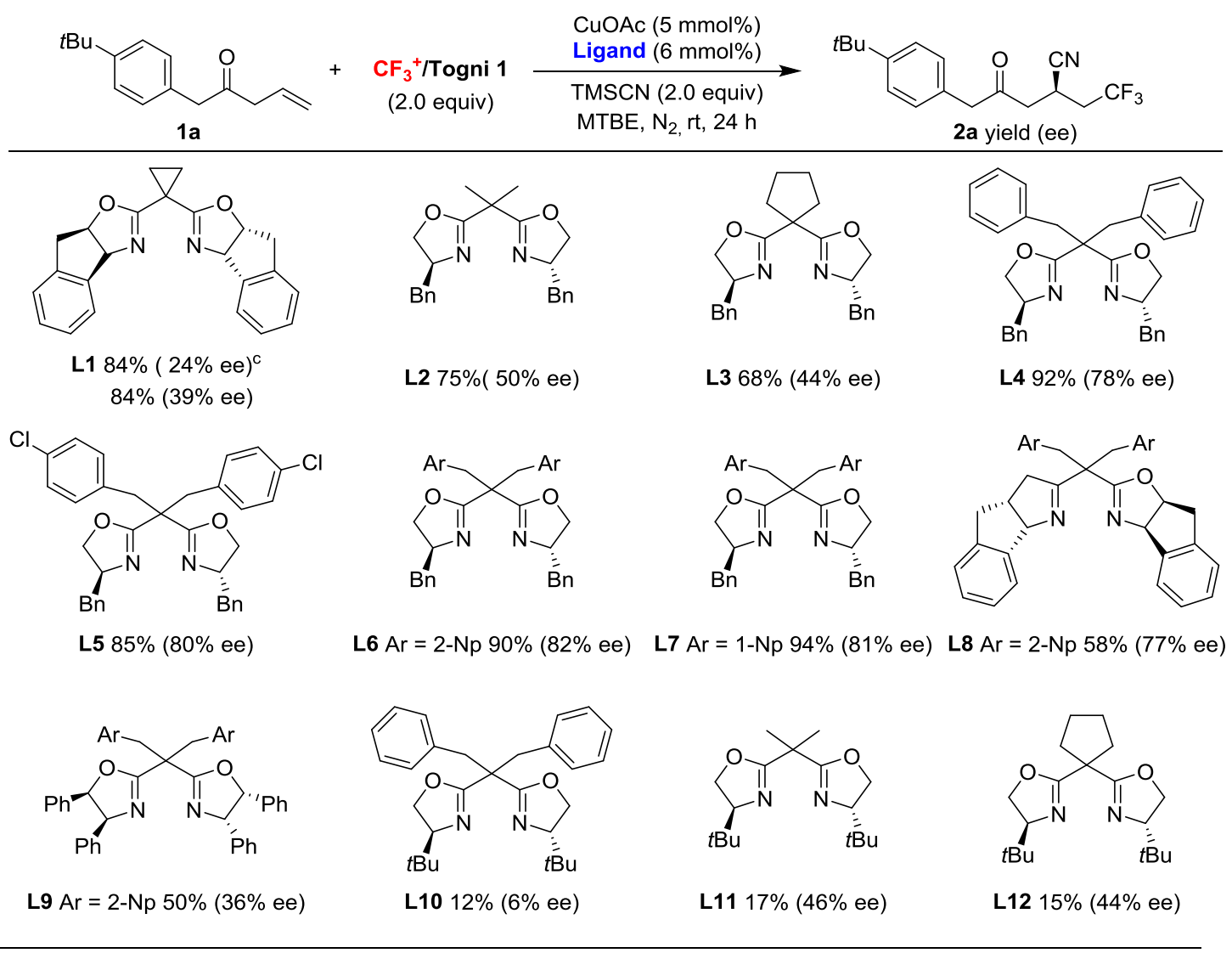

a All reactions were run on a $0.1 \mathrm{mmol}$ scale in MTBE $(1 \mathrm{~mL})$ at room temperature under a nitrogen atmosphere. ${ }^{b}$ Yields were determined by ${ }^{19} \mathrm{~F}$ NMR with $\mathrm{CF}_{3}$-DMAc as an internal standard and enantiomeric excess (ee) value was determined by HPLC on a chiral stationary phase. ${ }^{\mathrm{c}} \mathrm{CH}_{3} \mathrm{CN}$ as solvent

\subsection{Optimization of solvents}

Table S2. Solvent screening. ${ }^{a, b}$

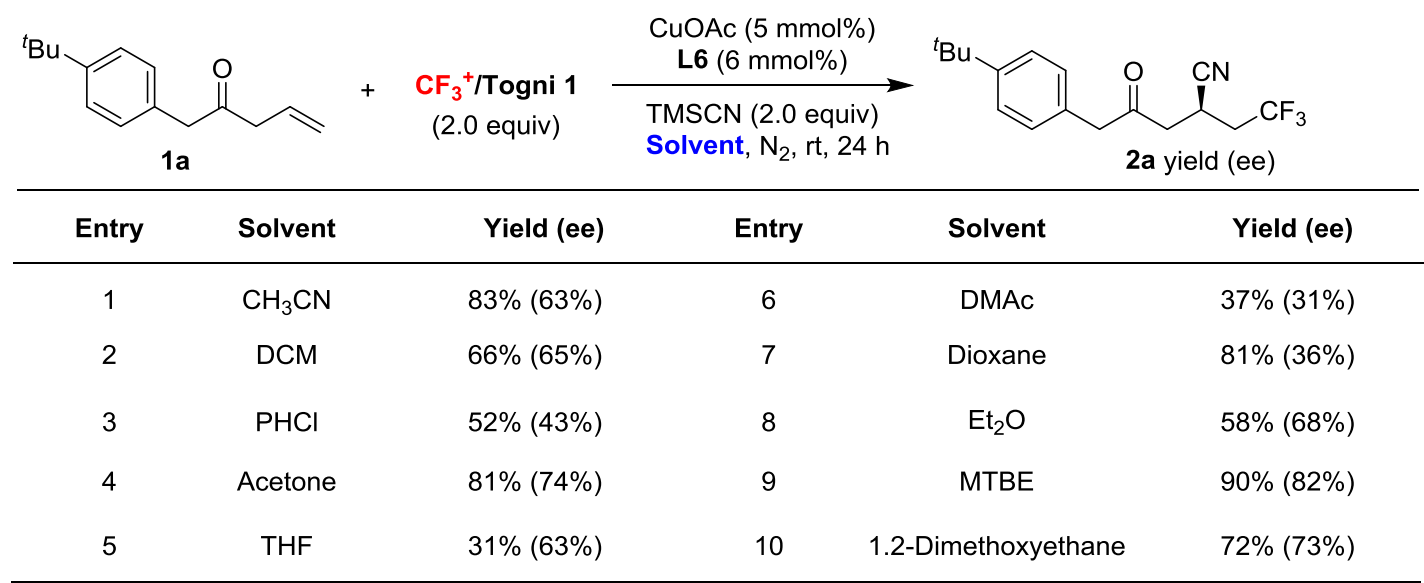

a All reactions were run on a $0.1 \mathrm{mmol}$ scale in solvent $(1 \mathrm{~mL})$ at room temperature under a nitrogen atmosphere. ${ }^{b}$ Yields were determined by ${ }^{19} \mathrm{~F}$ NMR with $\mathrm{CF}_{3}$-DMAc as an internal standard and enantiomeric excess (ee) value was determined by HPLC on a chiral stationary phase.

\subsection{Copper catalyst screening}


Table S3. Optimization of copper catalysts. ${ }^{a, b}$

\begin{tabular}{|c|c|c|c|c|c|}
\hline & $1 a$ & $\begin{array}{l}\mathrm{F}_{3}{ }^{+} / \text {Togni } 1 \\
(2.0 \text { equiv) }\end{array}$ & $\begin{array}{l}\text { Sat. (5 m } \\
\text { L6 (6 mr } \\
\text { ISCN (2. } \\
\text { BE, rt, }\end{array}$ & ${ }^{t} \mathrm{Bu}$ & $2 a$ yield $(e e)$ \\
\hline Entry & Cat. & Yield (ee) & Entry & Cat. & Yield (ee) \\
\hline 1 & $\mathrm{Cu}\left(\mathrm{CH}_{3} \mathrm{CN}\right)_{4} \mathrm{PF}_{6}$ & $79 \%(73 \%)$ & 6 & CuSCN & $80 \%(78 \%)$ \\
\hline 2 & $\mathrm{Cu}\left(\mathrm{CH}_{3} \mathrm{CN}\right)_{4} \mathrm{BF}_{4}$ & $86 \%(71 \%)$ & 7 & $\mathrm{CuCl}$ & $70 \%(75 \%)$ \\
\hline 3 & {$[\mathrm{Cu}(\mathrm{OTf})]_{2} \mathrm{PhH}$} & $90 \%(72 \%)$ & 8 & $\mathrm{CuBr}$ & $25 \%(47 \%)$ \\
\hline 4 & $\mathrm{Cu}(\mathrm{PPh})_{3} \mathrm{Br}$ & $78 \%(79 \%)$ & 9 & $\mathrm{Cu}(\mathrm{OTf})_{2}$ & $34 \%(71 \%)$ \\
\hline 5 & CuCN & $83 \%(81 \%)$ & 10 & CuOAc & $90 \%(82 \%)$ \\
\hline
\end{tabular}

${ }^{a}$ All reactions were run on a $0.1 \mathrm{mmol}$ scale in MTBE $(1 \mathrm{~mL})$ at room temperature under a nitrogen atmosphere. ${ }^{b}$ Yields were determined by ${ }^{19} \mathrm{~F}$ NMR with $\mathrm{CF}_{3}$-DMAc as an internal standard and enantiomeric excess (ee) value was determined by HPLC on a chiral stationary phase.

\subsection{Optimization of reaction temperature}

Table S4. Optimization of reaction temperature..$^{a, b}$

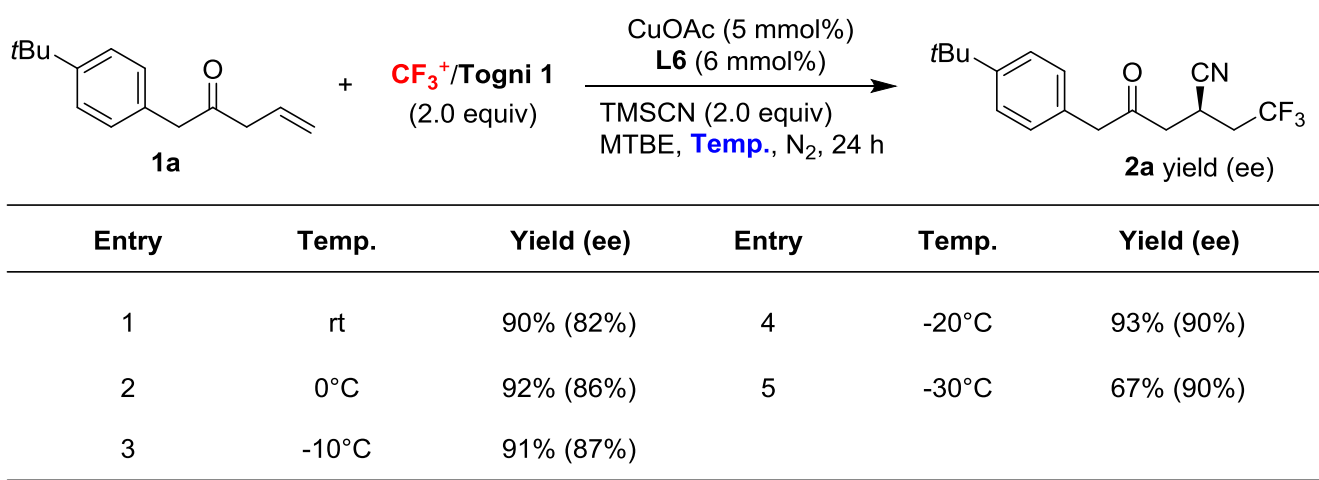

${ }^{a}$ All reactions were run on a $0.1 \mathrm{mmol}$ scale in MTBE $(1 \mathrm{~mL})$ under a nitrogen atmosphere. ${ }^{b}$ Yields were determined by ${ }^{19} \mathrm{~F} \mathrm{NMR}$ with $\mathrm{CF}_{3}$-DMAc as an internal standard and enantiomeric excess (ee) value was determined by HPLC on a chiral stationary phase.

\section{General Procedure for the Asymmetric Cyanotrifluoromethylation}

Condition A: In a dried sealed $10 \mathrm{~mL}$ Schlenk tube, a solution of ligand L6 (0.012 mmol, $6 \mathrm{~mol} \%, 7.4 \mathrm{mg})$ and CuOAc $(0.01 \mathrm{mmol}, 5 \mathrm{~mol} \%, 1.2 \mathrm{mg})$ in MTBE (2 mL) was stirred at $-20{ }^{\circ} \mathrm{C}$ under a $\mathrm{N}_{2}$ atmosphere for $30 \mathrm{~min}$. Then, alkene $(0.2 \mathrm{mmol}, 1.0$ equiv), TMSCN (0.4 mmol, 2.0 equiv, $52 \mu \mathrm{L}$ ) and Togni-1 ( 0.4 mmol, 2.0 equiv, 133 $\mathrm{mg}$ ) were added sequentially, then the mixture was stirred at $-20{ }^{\circ} \mathrm{C}$ for $24 \mathrm{~h}$. After the reaction was completed, solvent was evaporated under reduced pressure. The residue was purified by flash column chromatography on silica gel with gradient of petroleum 
ether/ethyl acetate to afford products.

Condition B: In a dried sealed $10 \mathrm{~mL}$ Schlenk tube, a solution of ligand L5 (0.012 mmol, $6 \mathrm{~mol} \%, 7.0 \mathrm{mg})$ and CuOAc $(0.01 \mathrm{mmol}, 5 \mathrm{~mol} \%, 1.2 \mathrm{mg})$ in MTBE $(2 \mathrm{~mL})$ was stirred at $-30{ }^{\circ} \mathrm{C}$ under a $\mathrm{N}_{2}$ atmosphere for $30 \mathrm{~min}$. Then, alkene $(0.2 \mathrm{mmol}, 1.0$ equiv), TMSCN (0.4 mmol, 2.0 equiv, $52 \mu \mathrm{L})$ and Togni-1 (0.4 mmol, 2.0 equiv, 133 mg) were added sequentially, and the tube was sealed with Teflon septum. then the mixture was stirred at $-30{ }^{\circ} \mathrm{C}$ for $72 \mathrm{~h}$. After the reaction was completed, solvent was evaporated under reduced pressure. The residue was purified by flash column chromatography on silica gel with gradient of petroleum ether/ethyl acetate to afford products.

Condition C: In a dried sealed $10 \mathrm{~mL}$ Schlenk tube, a solution of ligand L6 (0.012 mmol, $6 \mathrm{~mol} \%, 7.4 \mathrm{mg})$ and CuOAc $(0.01 \mathrm{mmol}, 5 \mathrm{~mol} \%, 1.2 \mathrm{mg})$ in MTBE (2 mL) was stirred at $-30{ }^{\circ} \mathrm{C}$ under a $\mathrm{N}_{2}$ atmosphere for $30 \mathrm{~min}$. Then, alkene $(0.2 \mathrm{mmol}, 1.0$ equiv), TMSCN (0.4 mmol, 2.0 equiv, $52 \mu \mathrm{L}$ ) and Togni-1 (0.4 mmol, 2.0 equiv, 133 mg) were added sequentially, then the mixture was stirred at $-30{ }^{\circ} \mathrm{C}$ for $72 \mathrm{~h}$. After the reaction was completed, solvent was evaporated under reduced pressure. The residue was purified by flash column chromatography on silica gel with gradient of petroleum ether/ethyl acetate to afford products.

\section{Scaled-up Reaction}
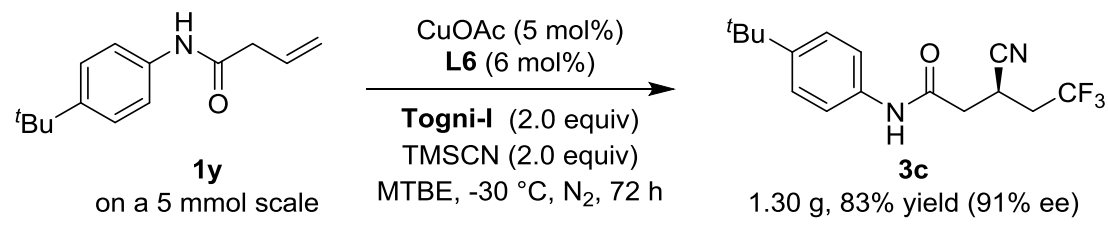

In a sealed $100 \mathrm{~mL}$ Schlenk tube, a solution of ligand L6 $(183.0 \mathrm{mg}, 0.3 \mathrm{mmol}, 6$ mol\%) and CuOAc (30.6 mg, $0.25 \mathrm{mmol}, 5 \mathrm{~mol} \%$ ) in degassed MTBE (50 mL) was stirred at $-30{ }^{\circ} \mathrm{C}$ under a $\mathrm{N}_{2}$ atmosphere for $30 \mathrm{~min}$. Then, $\mathbf{1 y}(1.09 \mathrm{~g}, 5 \mathrm{mmol}, 1.0$ equiv), TMSCN (0.99 g, $10 \mathrm{mmol}, 2.0$ equiv) and Togni-I (3.30 g, $10 \mathrm{mmol}, 2.0$ equiv) were added to the solution at $-30{ }^{\circ} \mathrm{C}$. After the reaction was completed, the reaction was quenched with water and extracted with EtOAc, the combined organic phase was washed with brine, dried over anhydrous $\mathrm{Na}_{2} \mathrm{SO}_{4}$ and concentrated under 
vacuum, and the residue was purified by flash column chromatography on silica gel with a gradient of PE/EtOAc (4:1) to afford the product $\mathbf{2 y}$ as a white solid (1.30 g, $83 \%$ yield, $91 \%$ ee).

\section{Product Transformations}
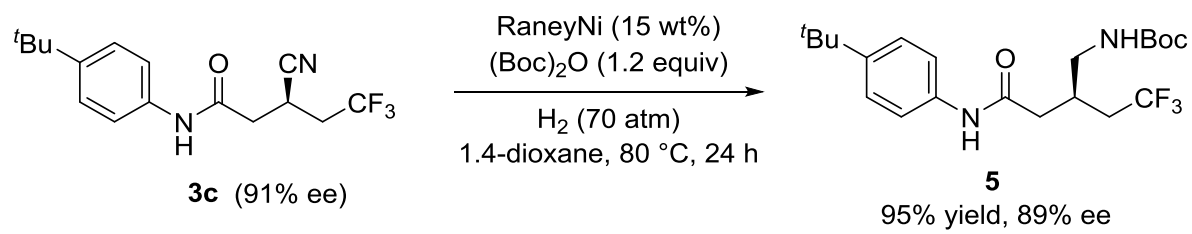

To a $4 \mathrm{~mL}$ glass vessel, the compound $3 \mathrm{c}(62.4 \mathrm{mg}, 0.2 \mathrm{mmol}, 1.0$ equiv, $91 \%$ ee), Raney $\mathrm{Ni}$ (6.4 mg, $15 \mathrm{wt} \%$ ) and $\mathrm{Boc}_{2} \mathrm{O}$ (87.3 mg, $0.4 \mathrm{mmol}, 2.0$ equiv) were dissolved in dioxane $(2 \mathrm{~mL})$. The glass vessel was placed in the autoclave, which was exchanged with $\mathrm{H}_{2}$ for three times and kept at 70 bar $\mathrm{H}_{2}$. The reaction was stirred at $80^{\circ} \mathrm{C}$ for $24 \mathrm{~h}$. After that, the mixture was filtered through a pad of celite with EtOAc. The filtrate was evaporated under reduced pressure and the residue was purified by flash column chromatography on silica gel to afford $\mathbf{5}$ as a light white solid (79.1 $\mathrm{mg}$, $95 \%$ yield, $89 \%$ ee), m.p. $45-48^{\circ} \mathrm{C}$.

$[\alpha]_{\mathrm{D}}^{25.3}=+8.800\left(\mathrm{c} 1.0, \mathrm{CHCl}_{3}\right) .{ }^{1} \mathrm{H} \mathrm{NMR}\left(400 \mathrm{MHz}, \mathrm{CDCl}_{3}\right): \delta 9.16(\mathrm{br}, 1 \mathrm{H}), 7.52(\mathrm{~d}$, $J=8.4 \mathrm{~Hz}, 2 \mathrm{H}), 7.34(\mathrm{~d}, J=8.0 \mathrm{~Hz}, 2 \mathrm{H}), 4.98(\mathrm{t}, J=5.6 \mathrm{~Hz}, 1 \mathrm{H}), 3.19-3.43(\mathrm{~m}, 2 \mathrm{H})$, 2.14-2.42 (m, 5H), 1.47 (s, 9H), $1.30(\mathrm{~s}, 9 \mathrm{H}) .{ }^{13} \mathrm{C} \mathrm{NMR}\left(100 \mathrm{MHz}, \mathrm{CDCl}_{3}\right): \delta 169.2$, 157.5, 147.1, 135.6, 126.7 (q, $J=276.1 \mathrm{~Hz}), 125.7,119.6,80.3,42.0,39.6,36.5$ (q, $J$ $=27.4 \mathrm{~Hz}), 34.3,32.2,31.3,28.3 .{ }^{19} \mathrm{~F}$ NMR $\left(376 \mathrm{MHz}, \mathrm{CDCl}_{3}\right): \delta-63.35(\mathrm{t}, J=10.53$ Hz). HRMS (ESI) m/z: $[\mathrm{M}+\mathrm{Na}]^{+}$Calcd for $\mathrm{C}_{21} \mathrm{H}_{31} \mathrm{~N}_{2} \mathrm{O}_{3} \mathrm{~F}_{3} \mathrm{Na}$ 439.2179; Found 439.2174 .

HPLC (IA, $0.46 * 25 \mathrm{~cm}, 5 \mu \mathrm{m}$, hexane/isopropanol = 95/5, flow $0.7 \mathrm{~mL} / \mathrm{min}$, detection at $214 \mathrm{~nm}$ ) retention time $=16.735 \mathrm{~min}$ (minor) and 19.137 min (major).

\section{Mechanistic Studies}

\subsection{Using TEMPO as a radical scavenger}
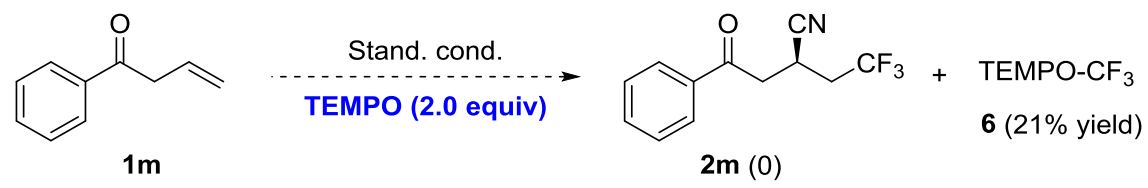
The reaction was conducted according to the condition A. In a dried sealed $10 \mathrm{~mL}$ Schlenk tube, a solution of $\mathbf{L 6}$ (6 mol \%, $3.7 \mathrm{mg}$ ) and CuOAc (5 mol \%, $0.6 \mathrm{mg}$ ) in MTBE was stirred under a $\mathrm{N}_{2}$ atmosphere for $30 \mathrm{~min}$. Then, $\mathbf{1 m}(0.1 \mathrm{mmol}, 1.0$ equiv $14.6 \mathrm{mg})$, TMSCN (0.2 mmol, 2.0 equiv, $26 \mu \mathrm{L})$, Togni-1 (0.2 mmol, 2.0 equiv, 66.5 $\mathrm{mg}$ ) and TEMPO (0.2 mmol, 2.0 equiv, $31.3 \mathrm{mg}$ ) were added. the reaction mixture was stirred at $-20{ }^{\circ} \mathrm{C}$ for $24 \mathrm{~h}$. The reaction mixture was analyzed by ${ }^{19} \mathrm{~F}-\mathrm{NMR}$, using $\mathrm{CF}_{3}$-DMAc as an internal standard. The formation of TEMPO- $\mathrm{CF}_{3} 6$ in $21 \%$ yield indicated the generation of highly reactive $\mathrm{CF}_{3}$ radical in our reaction. ${ }^{\mathrm{S} 10}$

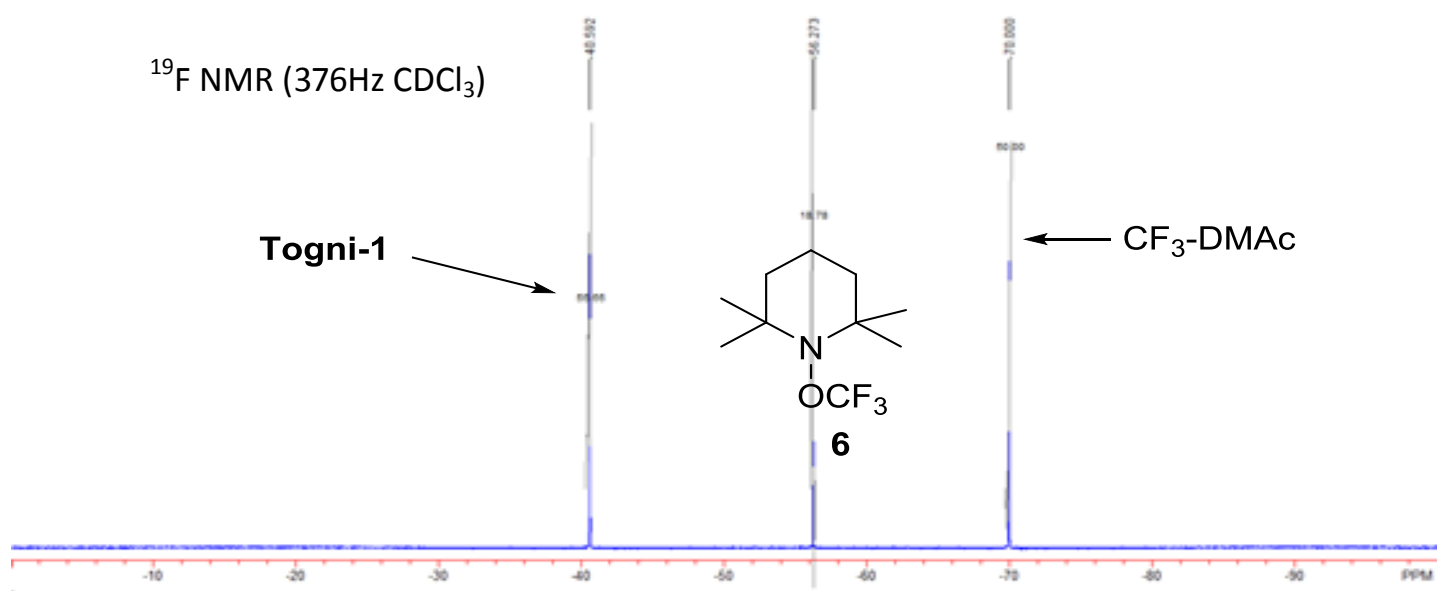

\subsection{Using $\mathrm{CBr}_{4}$ as a radical scavenger}

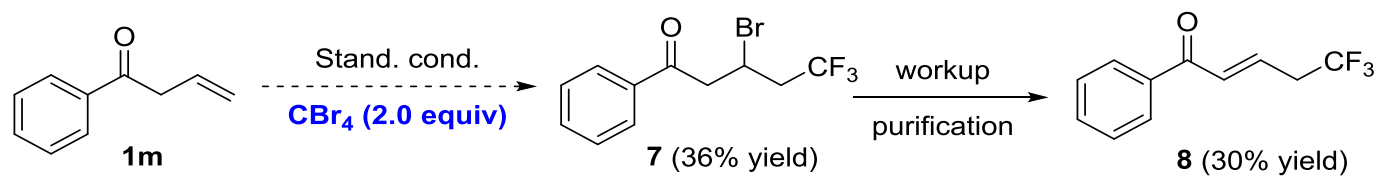

The reaction was conducted according to the condition A. In a dried sealed $10 \mathrm{~mL}$ Schlenk tube, a solution of $\mathbf{L 6}(6 \mathrm{~mol} \%, 3.7 \mathrm{mg})$ and CuOAc (5 mol \%, $0.6 \mathrm{mg}$ ) in MTBE was stirred under a $\mathrm{N}_{2}$ atmosphere at $-20{ }^{\circ} \mathrm{C}$ for $30 \mathrm{~min}$. Then, $\mathbf{1 m}(0.1 \mathrm{mmol}$, 1.0 equiv, $14.6 \mathrm{mg})$, TMSCN (0.2 mmol, 2.0 equiv, $26 \mu \mathrm{L})$, Togni-1 (0.2 mmol, 2.0 equiv, $66.5 \mathrm{mg})$ and $\mathrm{CBr}_{4}(0.2 \mathrm{mmol}, 2.0$ equiv, $66.3 \mathrm{mg})$ were added, the reaction mixture was stirred at $-20{ }^{\circ} \mathrm{C}$ for $24 \mathrm{~h}$. The reaction mixture was analyzed by ${ }^{1} \mathrm{H}-\mathrm{NMR}$ and ${ }^{19} \mathrm{~F}-\mathrm{NMR}$, using $\mathrm{CF}_{3}$-DMAc as an internal standard. The intermediate 7 was detected with ${ }^{1} \mathrm{H} /{ }^{19} \mathrm{~F}$ NMR spectroscopies of the crude mixture $\left[{ }^{19} \mathrm{~F}\right.$ NMR $(376 \mathrm{MHz}$, $\left.\left.\mathrm{CDCl}_{3}\right): \delta-64.07(\mathrm{t}, J=9.76 \mathrm{~Hz})\right]$ and HRMS $(\mathrm{M}-\mathrm{Br})$. However, in a silica gel 
column, the intermediate 7 was easily converted into alkene 8 by elimination $\left[{ }^{19} \mathrm{~F}\right.$ NMR $\left.\left(376 \mathrm{MHz}, \mathrm{CDCl}_{3}\right): \delta-65.80(\mathrm{t}, J=10.15 \mathrm{~Hz})\right]$. Although the bromination product 7 couldn't be isolated, this compound could also be confirmed in comparison to the ${ }^{1} \mathrm{H}$ NMR spectroscopy of 3-bromo-1-phenylbutan-1-one reported in the literature. ${ }^{\text {S11 }}$ The formation of $\mathbf{7}$ and $\mathbf{8}$ suggested the intermediacy of radical species generated by $\mathrm{CF}_{3}$ radical addition across alkene $\mathbf{1 m}$.

${ }^{1} \mathrm{H}$ NMR spectroscopy of the crude mixture:
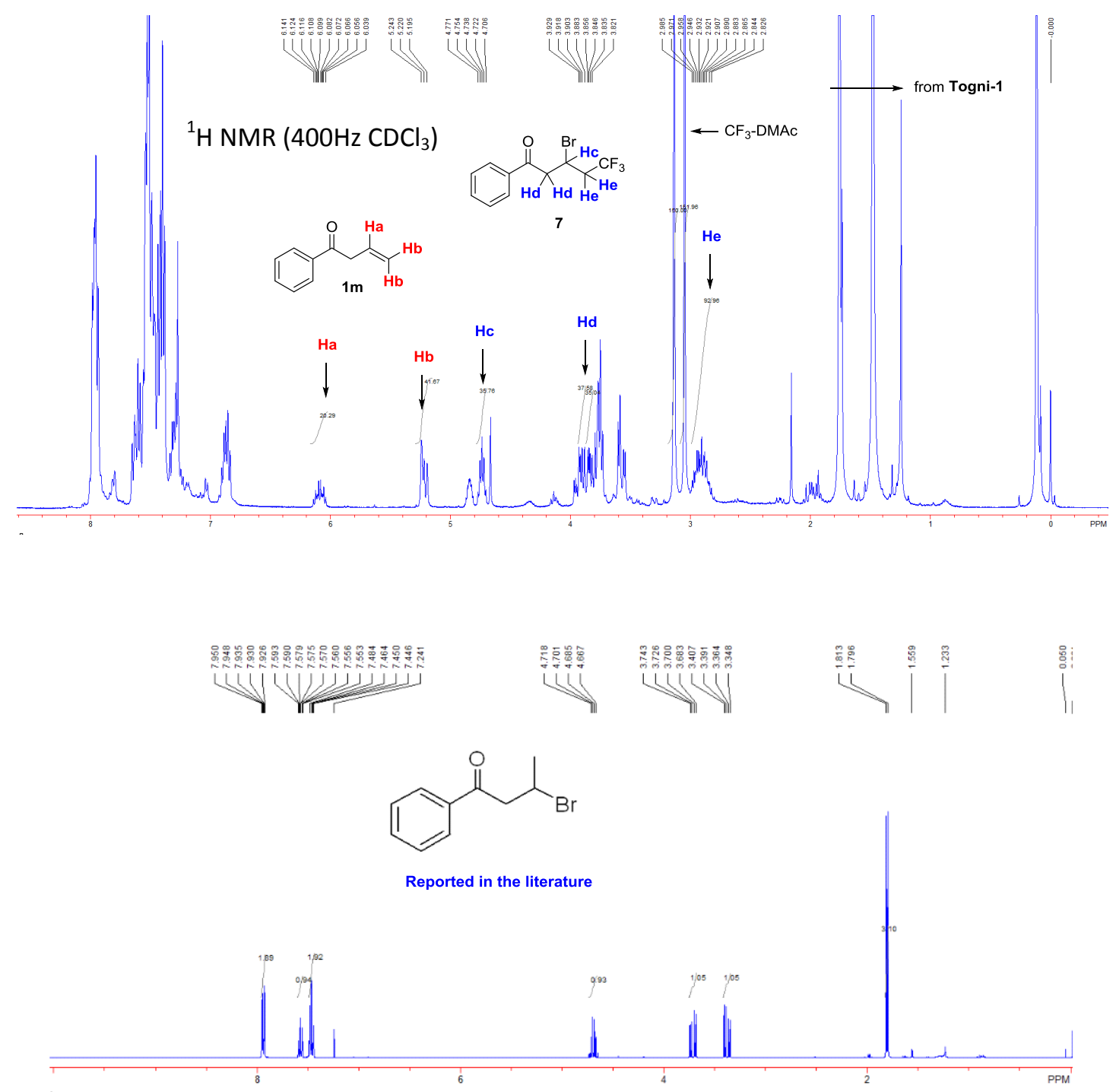
${ }^{19}$ F NMR spectroscopy of the crude mixture:
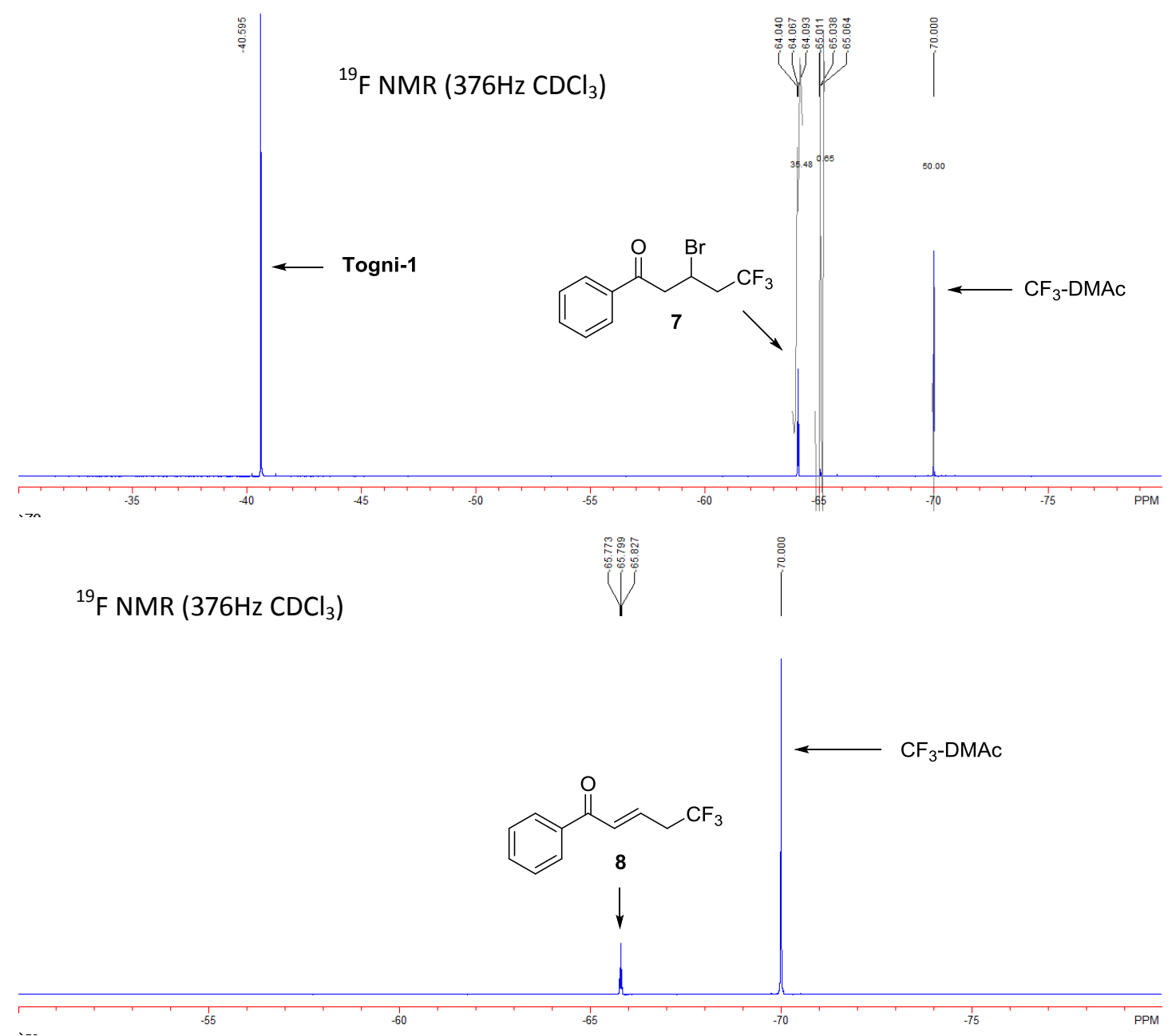<smiles>O=C(CC(Br)CC(F)(F)F)c1ccccc1</smiles>

HRMS (ESI) m/z: [M-Br] ${ }^{+}$Calcd for $\mathrm{C}_{11} \mathrm{H}_{10} \mathrm{~F}_{3} \mathrm{O}$ 215.0678; Found 215.0679.<smiles>O=C(/C=C/CC(F)(F)F)c1ccccc1</smiles>

${ }^{1} \mathrm{H}$ NMR (400 MHz, $\mathrm{CDCl}_{3}$ ): $\delta 7.94(\mathrm{~d}, J=7.2 \mathrm{~Hz}, 2 \mathrm{H}), 7.60(\mathrm{t}, J=7.2 \mathrm{~Hz}, 1 \mathrm{H}), 7.49$ (t, $J=7.6 \mathrm{~Hz}, 2 \mathrm{H}), 7.10$ (d, $J=15.6 \mathrm{~Hz}, 1 \mathrm{H}), 6.93-6.85(\mathrm{~m}, 1 \mathrm{H}), 3.16-3.07(\mathrm{~m}, 2 \mathrm{H})$. ${ }^{13} \mathrm{C}$ NMR (100 MHz, $\left.\mathrm{CDCl}_{3}\right): \delta 189.5,137.0,134.64(\mathrm{q}, J=3.5 \mathrm{~Hz}), 133.2,131.4$, 128.7, 128.6, $125.2(\mathrm{q}, J=275.6 \mathrm{~Hz}), 37.0(\mathrm{q}, J=30.4 \mathrm{~Hz}) .{ }^{19} \mathrm{~F}$ NMR $(376 \mathrm{MHz}$, $\left.\mathrm{CDCl}_{3}\right) \delta-65.29(\mathrm{t}, J=10.15 \mathrm{~Hz})$. HRMS (EI) m/z: $[\mathrm{M}]^{+}$Calcd for $\mathrm{C}_{11} \mathrm{H}_{9} \mathrm{~F}_{3} \mathrm{O} 214.06$; Found 214.0597. 


\section{Characterization of Products}<smiles>CC(C)(C)c1ccc(CC(=O)C[C@H](C#N)CC(F)(F)F)cc1</smiles>

The reaction was conducted on a $0.2 \mathrm{mmol}$ scale according to the condition $\mathbf{A}$. Column chromatography $(\mathrm{PE}: \mathrm{EA}=7: 1)$ yielded the desired product $2 \mathbf{a}(57.9 \mathrm{mg}$, $93 \%, 90 \%$ ee) as a light white solid, m.p. $43-46^{\circ} \mathrm{C}$.

$[\alpha]_{\mathrm{D}}^{25.3}=+213.27\left(\mathrm{c} 1.0, \mathrm{CHCl}_{3}\right) .{ }^{1} \mathrm{H}$ NMR $\left(400 \mathrm{MHz}, \mathrm{CDCl}_{3}\right): \delta 7.38(\mathrm{~d}, J=8.0 \mathrm{~Hz}$, 2H), $7.13(\mathrm{~d}, J=8.4 \mathrm{~Hz}, 2 \mathrm{H}), 3.70(\mathrm{~s}, 2 \mathrm{H}), 3.36-3.29(\mathrm{~m}, 1 \mathrm{H}), 2.98-2.81(\mathrm{~m}, 2 \mathrm{H})$, 2.54-2.30 (m, 2H), 1.32 (s, 9H). ${ }^{13} \mathrm{C}$ NMR (100 MHz, $\left.\mathrm{CDCl}_{3}\right): \delta 203.1,150.7,129.6$, 128.9, 126.1, 124.9 (q, $J=275.9 \mathrm{~Hz}), 119.1,49.4,42.2,35.0(\mathrm{q}, J=30 \mathrm{~Hz}), 34.5$, 31.2, 20.3 (q, $J=2.8 \mathrm{~Hz}) .{ }^{19} \mathrm{~F}$ NMR $\left(376 \mathrm{MHz}, \mathrm{CDCl}_{3}\right): \delta-64.72(\mathrm{t}, J=10.15 \mathrm{~Hz})$. HRMS (EI) m/z: [M] $]^{+}$Calcd for $\mathrm{C}_{17} \mathrm{H}_{20} \mathrm{~F}_{3} \mathrm{NO}$ 311.1492; Found 311.1492.

HPLC (IC-3, 0.46*15 cm, $3 \mu \mathrm{m}$, hexane/isopropanol = 95/5, flow $0.7 \mathrm{~mL} / \mathrm{min}$, detection at $214 \mathrm{~nm}$ ) retention time $=16.371 \mathrm{~min}$ (major) and $19.671 \mathrm{~min}$ (minor).<smiles>CC(C)(CC(C#N)CC(=O)Cc1ccccc1)C(F)(F)F</smiles>

The reaction was conducted on a $0.2 \mathrm{mmol}$ scale according to the conditions $\mathbf{A}$. Column chromatography (PE: EA = 7:1) yielded the desired product $2 \mathbf{b}(48.5 \mathrm{mg}$, $95 \%, 86 \%$ ee) as a yellow liquid.

$[\alpha]_{\mathrm{D}}{ }^{20.2}=-1.97\left(\mathrm{c} 0.5, \mathrm{CHCl}_{3}\right) .{ }^{1} \mathrm{H} \mathrm{NMR}\left(400 \mathrm{MHz} \mathrm{CDCl}_{3}\right): \delta 7.37(\mathrm{t}, J=6.8 \mathrm{~Hz}, 2 \mathrm{H})$, $7.31(\mathrm{t}, J=8.0 \mathrm{~Hz}, 1 \mathrm{H}), 7.20(\mathrm{~d}, J=7.6 \mathrm{~Hz}, 2 \mathrm{H}), 3.74(\mathrm{~s}, 2 \mathrm{H}), 3.35-3.29(\mathrm{~m}, 1 \mathrm{H})$, 2.98-2.80 (m, 2H), 2.54-2.29 (m, 2H). ${ }^{13} \mathrm{C}$ NMR (100 MHz, $\mathrm{CDCl}_{3}$ ): $\delta$ 202.8, 132.7, 129.3, 129.1, 127.7, 124.9 (q, $J=276.1 \mathrm{~Hz}), 119.1,49.9,42.2,35.0$ (q, $J=29.9 \mathrm{~Hz})$, $20.3(\mathrm{q}, J=2.7 \mathrm{~Hz}) .{ }^{19} \mathrm{~F}$ NMR $\left(376 \mathrm{MHz}, \mathrm{CDCl}_{3}\right): \delta-64.65(\mathrm{t}, J=10.15 \mathrm{~Hz})$. HRMS (EI) $\mathrm{m} / \mathrm{z}$ : $[\mathrm{M}]^{+}$Calcd for $\mathrm{C}_{13} \mathrm{H}_{12} \mathrm{~F}_{3} \mathrm{NO} 255.0866$; Found 255.0866.

HPLC (IC-3, 0.46*15 cm, $3 \mu \mathrm{m}$, hexane/isopropanol $=80 / 20$, flow $0.7 \mathrm{~mL} / \mathrm{min}$, detection at $214 \mathrm{~nm}$ ) retention time $=7.605 \mathrm{~min}$ (major) and $8.282 \mathrm{~min}$ (minor). 
<smiles>COc1ccc(CC(=O)CC(C#N)CC(F)(F)F)cc1</smiles>

The reaction was conducted on a $0.2 \mathrm{mmol}$ scale according to the conditions $\mathbf{A}$. Column chromatography (PE: EA=7:1) yielded the desired product $2 \mathrm{c}(45.6 \mathrm{mg}, 80 \%$, $87 \%$ ee) as a light white solid, m.p. $42-44{ }^{\circ} \mathrm{C}$.

$[\alpha]_{\mathrm{D}}^{24.6}=+1.36\left(\mathrm{c} 0.6, \mathrm{CHCl}_{3}\right) .{ }^{1} \mathrm{H}$ NMR $\left(400 \mathrm{MHz}, \mathrm{CDCl}_{3}\right): \delta 7.11(\mathrm{~d}, J=8.4 \mathrm{~Hz}$, $2 \mathrm{H}), 6.89(\mathrm{~d}, J=8.0 \mathrm{~Hz}, 2 \mathrm{H}), 3.81(\mathrm{~s}, 3 \mathrm{H}), 3.67(\mathrm{~s}, 2 \mathrm{H}), 3.36-3.29(\mathrm{~m}, 1 \mathrm{H}), 2.96-2.79$ (m, 2H), 2.54-2.29 (m, 2H). ${ }^{13} \mathrm{C}$ NMR (100 MHz, $\left.\mathrm{CDCl}_{3}\right): \delta 203.2,159.1,130.4$, 124.9 (q, $J=275.8 \mathrm{~Hz}), 124.6,119.1,114.5,55.3,49.1,42.1,35.1$ (q, $J=29.8 \mathrm{~Hz})$, $20.3(\mathrm{q}, J=2.9 \mathrm{~Hz}) .{ }^{19} \mathrm{~F}$ NMR $\left(376 \mathrm{MHz}, \mathrm{CDCl}_{3}\right): \delta-64.65(\mathrm{t}, J=9.78 \mathrm{~Hz})$. HRMS (EI) $\mathrm{m} / \mathrm{z}:[\mathrm{M}]^{+}$Calcd for $\mathrm{C}_{14} \mathrm{H}_{14} \mathrm{~F}_{3} \mathrm{NO}_{2}$ 285.0971; Found 285.0970.

HPLC (IC-3, 0.46*15 cm, $3 \mu \mathrm{m}$, hexane/isopropanol = 85/15, flow $0.7 \mathrm{~mL} / \mathrm{min}$, detection at $214 \mathrm{~nm}$ ) retention time $=15.688 \mathrm{~min}$ (minor) and $17.437 \mathrm{~min}$ (major).<smiles>Cc1ccc(CC(=O)CC(C#N)CC(F)(F)F)cc1</smiles>

The reaction was conducted on a $0.2 \mathrm{mmol}$ scale according to the conditions $\mathbf{A}$. Column chromatography $(\mathrm{PE}: \mathrm{EA}=7: 1)$ yielded the desired product $\mathbf{2 d}(43.1 \mathrm{mg}$, $80 \%, 86 \%$ ee) as a light white solid, m.p. $44-46^{\circ} \mathrm{C}$.

$[\alpha]_{\mathrm{D}}^{20.7}=-2.16\left(\mathrm{c} 0.5, \mathrm{CHCl}_{3}\right) .{ }^{1} \mathrm{H}$ NMR $\left(400 \mathrm{MHz}, \mathrm{CDCl}_{3}\right): \delta 7.17(\mathrm{~d}, J=8.0 \mathrm{~Hz}$, 2H), $7.08(\mathrm{~d}, J=8.0 \mathrm{~Hz}, 2 \mathrm{H}), 3.69(\mathrm{~s}, 2 \mathrm{H}), 3.35-3.28(\mathrm{~m}, 1 \mathrm{H}), 2.96-2.79(\mathrm{~m}, 2 \mathrm{H})$, 2.53-2.29 (m, 2H), 2.35 (s, 3H). ${ }^{13} \mathrm{C}$ NMR (100 MHz, $\left.\mathrm{CDCl}_{3}\right): \delta 203.0,137.4,129.8$, 129.6, 129.1, 124.9 (q, $J=275.6 \mathrm{~Hz}), 119.1,49.5,42.1,35.0$ (q, $J=29.8 \mathrm{~Hz}), 21.0$, $20.3(\mathrm{q}, J=3.2 \mathrm{~Hz}) .{ }^{19} \mathrm{~F}$ NMR $\left(376 \mathrm{MHz}, \mathrm{CDCl}_{3}\right): \delta-64.68(\mathrm{t}, J=9.78 \mathrm{~Hz})$. HRMS (EI) m/z: $[M]^{+}$Calcd for $\mathrm{C}_{14} \mathrm{H}_{14} \mathrm{~F}_{3} \mathrm{NO} 269.1022$; Found 269.1018 .

HPLC (IC-3, 0.46*15 cm, $3 \mu \mathrm{m}$, hexane/isopropanol $=80 / 20$, flow $0.7 \mathrm{~mL} / \mathrm{min}$, detection at $214 \mathrm{~nm}$ ) retention time $=7.553 \mathrm{~min}$ (major) and $8.217 \mathrm{~min}$ (minor).<smiles>N#CC(CC(=O)Cc1ccc(-c2ccccc2)cc1)CC(F)(F)F</smiles>

The reaction was conducted on a $0.2 \mathrm{mmol}$ scale according to the condition $\mathbf{A}$. 
Column chromatography $(\mathrm{PE}: \mathrm{EA}=7: 1)$ yielded the desired product $2 \mathrm{e}(56.3 \mathrm{mg}$, $85 \%, 85 \%$ ee) a light white solid, m.p. $80-81^{\circ} \mathrm{C}$.

$[\alpha]_{\mathrm{D}}^{22.1}=+11.06\left(\mathrm{c} 1.0, \mathrm{CHCl}_{3}\right) .{ }^{1} \mathrm{H}$ NMR $\left(400 \mathrm{MHz}, \mathrm{CDCl}_{3}\right): \delta 7.55-7.58(\mathrm{~m}, 4 \mathrm{H})$, $7.43(\mathrm{t}, J=8.0 \mathrm{~Hz}, 2 \mathrm{H}), 7.34(\mathrm{t}, J=7.2 \mathrm{~Hz}, 1 \mathrm{H}), 7.24(\mathrm{~s}, 2 \mathrm{H}), 3.76(\mathrm{~s}, 2 \mathrm{H}), 3.30-3.37$ $(\mathrm{m}, 1 \mathrm{H}), 2.83-3.0(\mathrm{~m}, 2 \mathrm{H}), 2.31-2.55(\mathrm{~m}, 2 \mathrm{H}) .{ }^{13} \mathrm{C} \mathrm{NMR}\left(100 \mathrm{MHz}, \mathrm{CDCl}_{3}\right) \delta 202.7$, 140.7, 140.4, 131.6, 129.7, 128.8, 127.8, 127.5, 127.0, 124.9 (q, $J=275.7$ Hz), 119.0, 49.5, 42.3, 35.1 (q, $J=29.7 \mathrm{~Hz}), 20.3(\mathrm{q}, J=3.1 \mathrm{~Hz}) .{ }^{19} \mathrm{~F} \mathrm{NMR}\left(376 \mathrm{MHz}, \mathrm{CDCl}_{3}\right) \delta$ $-64.61\left(\mathrm{t}, J=9.78 \mathrm{~Hz}\right.$ ). HRMS (EI) $\mathrm{m} / \mathrm{z}$ : $[\mathrm{M}]^{+}$Calcd for $\mathrm{C}_{19} \mathrm{H}_{16} \mathrm{~F}_{3} \mathrm{NO}$ 331.1179; Found 331.1173.

HPLC (IC-3, 0.46*15 cm, $3 \mu \mathrm{m}$, hexane/isopropanol = 95/5, flow $0.7 \mathrm{~mL} / \mathrm{min}$, detection at $214 \mathrm{~nm}$ ) retention time $=39.372 \mathrm{~min}$ (minor) and $41.085 \mathrm{~min}$ (major).<smiles>N#CC(CC(=O)Cc1ccc(F)cc1)CC(F)(F)F</smiles>

The reaction was conducted on a $0.2 \mathrm{mmol}$ scale according to the condition $\mathbf{A}$. Column chromatography $(\mathrm{PE}: \mathrm{EA}=7: 1)$ yielded the desired product $\mathbf{2 f}(47.0 \mathrm{mg}$, $86 \%, 81 \%$ ee) as a yellow liquid.

$[\alpha]_{\mathrm{D}}^{25.0}=+2.02\left(\mathrm{c} 0.9, \mathrm{CHCl}_{3}\right) .{ }^{1} \mathrm{H}$ NMR $\left(400 \mathrm{MHz}, \mathrm{CDCl}_{3}\right): \delta 7.19-7.14(\mathrm{~m}, 2 \mathrm{H})$, 7.08-7.02 (m, 2H), $3.72(\mathrm{~s}, 2 \mathrm{H}), 3.36-3.30(\mathrm{~m}, 1 \mathrm{H}), 2.99-2.82(\mathrm{~m}, 2 \mathrm{H}), 2.57-2.32(\mathrm{~m}$, 2H). ${ }^{13} \mathrm{C}$ NMR $\left(100 \mathrm{MHz}, \mathrm{CDCl}_{3}\right): \delta 202.6,162.2(\mathrm{~d}, J=244.9 \mathrm{~Hz}), 130.9(\mathrm{~d}, J=6.9$ $\mathrm{Hz}), 128.3(\mathrm{~d}, J=4.1 \mathrm{~Hz}), 124.9$ (q, $J=275.7 \mathrm{~Hz}), 119.1,115.1(\mathrm{~d}, J=21.4 \mathrm{~Hz}), 48.7$, 42.3, $35.0(\mathrm{q}, J=29.8 \mathrm{~Hz}), 20.3(\mathrm{q}, J=3.8 \mathrm{~Hz}) .{ }^{19} \mathrm{~F}$ NMR $\left(376 \mathrm{MHz}, \mathrm{CDCl}_{3}\right) \delta$ $-64.58(\mathrm{t}, J=10.53 \mathrm{~Hz}),-114.61--114.68(\mathrm{~m})$. HRMS (EI) $\mathrm{m} / \mathrm{z}:[\mathrm{M}]^{+}$Calcd for $\mathrm{C}_{13} \mathrm{H}_{11} \mathrm{~F}_{4} \mathrm{NO} 273.0771$; Found 273.0773.

HPLC (IC-3, 0.46*15 cm, $3 \mu \mathrm{m}$, hexane/isopropanol = 95/5, flow $0.7 \mathrm{~mL} / \mathrm{min}$, detection at $214 \mathrm{~nm}$ ) retention time $=20.935 \mathrm{~min}$ (minor) and $22.355 \mathrm{~min}$ (major).<smiles>N#CC(CC(=O)Cc1ccc(Cl)cc1)CC(F)(F)F</smiles>

The reaction was conducted on a $0.2 \mathrm{mmol}$ scale according to the condition $\mathbf{A}$. Column chromatography (PA: EA = 7:1) yielded the desired product $2 \mathrm{~g}(48.0 \mathrm{mg}$, $83 \%, 82 \%$ ee) as a light white solid, m.p. $41-43^{\circ} \mathrm{C}$. 
$[\alpha]_{\mathrm{D}}{ }^{25.5}=+3.53\left(\mathrm{c} 0.9, \mathrm{CHCl}_{3}\right) .{ }^{1} \mathrm{H} \mathrm{NMR}\left(400 \mathrm{MHz}, \mathrm{CDCl}_{3}\right): \delta 7.34(\mathrm{~d}, J=8.4 \mathrm{~Hz}$, $2 \mathrm{H}), 7.13(\mathrm{~d}, J=8.4 \mathrm{~Hz}, 2 \mathrm{H}), 3.72(\mathrm{~s}, 2 \mathrm{H}), 3.37-3.30(\mathrm{~m}, 1 \mathrm{H}), 2.99-2.82(\mathrm{~m}, 2 \mathrm{H})$, 2.58-2.33 (m, 2H). ${ }^{13} \mathrm{C}$ NMR (100 MHz, $\mathrm{CDCl}_{3}$ ): $\delta 202.2,133.7,131.0,130.7,129.2$, $124.9(\mathrm{q}, J=275.8 \mathrm{~Hz}), 118.9,48.9,42.4,35.0(\mathrm{q}, J=29.9 \mathrm{~Hz}), 20.3(\mathrm{q}, J=3.1 \mathrm{~Hz})$. ${ }^{19} \mathrm{~F}$ NMR $\left(376 \mathrm{MHz}, \mathrm{CDCl}_{3}\right): \delta-64.58(\mathrm{t}, J=10.53 \mathrm{~Hz})$. HRMS (EI) m/z: $[\mathrm{M}]^{+}$Calcd for $\mathrm{C}_{13} \mathrm{H}_{11} \mathrm{ClF}_{3} \mathrm{NO} 289.0476$; Found 289.0478.

HPLC (IC-3, 0.46*15 cm, $3 \mu \mathrm{m}$, hexane/isopropanol $=90 / 10$, flow $0.7 \mathrm{~mL} / \mathrm{min}$, detection at $214 \mathrm{~nm}$ ) retention time $=11.808 \mathrm{~min}$ (major) and $12.610 \mathrm{~min}$ (minor).<smiles>N#CC(CCCCCCCCCCCCCCC(=O)Cc1ccc(C(F)(F)F)cc1)CC(=O)c1ccccc1</smiles>

The reaction was conducted on a $0.2 \mathrm{mmol}$ scale according to the condition $\mathbf{A}$. Column chromatography (PA: EA $=7: 1)$ yielded the desired product $\mathbf{2 h}(56.2 \mathrm{mg}$, $87 \%, 81 \%$ ee) as a light white solid, m.p. $42-43^{\circ} \mathrm{C}$.

$[\alpha]_{\mathrm{D}}{ }^{25.6}=+5.67\left(\mathrm{c} 1.0, \mathrm{CHCl}_{3}\right) .{ }^{1} \mathrm{H} \mathrm{NMR}\left(400 \mathrm{MHz}, \mathrm{CDCl}_{3}\right): \delta 7.62(\mathrm{~d}, J=8.0 \mathrm{~Hz}$, $2 \mathrm{H}), 7.32(\mathrm{~d}, J=8.0 \mathrm{~Hz}, 2 \mathrm{H}), 3.82(\mathrm{~s}, 2 \mathrm{H}), 3.38-3.31(\mathrm{~m}, 1 \mathrm{H}), 3.03-2.85(\mathrm{~m}, 2 \mathrm{H})$, 2.60-2.35 (m, 2H). ${ }^{13} \mathrm{C}$ NMR (100 MHz, $\left.\mathrm{CDCl}_{3}\right): \delta 201.6,136.4,130.1$ (q, $J=33.2$ $\mathrm{Hz}), 129.8,125.9(\mathrm{q}, J=4.1 \mathrm{~Hz}), 124.9(\mathrm{q}, J=275.9 \mathrm{~Hz}), 123.9(\mathrm{q}, J=270.3 \mathrm{~Hz})$, 118.9, 48.2, 42.7, 35.0 (q, $J=29.8 \mathrm{~Hz}), 20.3(\mathrm{q}, J=3.2 \mathrm{~Hz}) .{ }^{19} \mathrm{~F}$ NMR $(376 \mathrm{MHz}$, $\left.\mathrm{CDCl}_{3}\right): \delta-64.58(\mathrm{t}, J=10.53 \mathrm{~Hz}),-62.68(\mathrm{~s})$. HRMS (EI) m/z: $[\mathrm{M}]^{+}$Calcd for $\mathrm{C}_{14} \mathrm{H}_{11} \mathrm{~F}_{6} \mathrm{NO} 323.0739$; Found 323.0742.

HPLC (AD-H, 0.46*25 cm, $5 \mu \mathrm{m}$, hexane/isopropanol $=90 / 10$, flow $0.7 \mathrm{~mL} / \mathrm{min}$, detection at $214 \mathrm{~nm}$ ) retention time $=12.815 \mathrm{~min}$ (major) and $13.683 \mathrm{~min}$ (minor).<smiles>Cc1ccccc1CC(=O)CC(C#N)CC([Mg])[As]</smiles>

The reaction was conducted on a $0.2 \mathrm{mmol}$ scale according to the condition $\mathbf{A}$. Column chromatography $(\mathrm{PE}: \mathrm{EA}=7: 1)$ yielded the desired product $2 \mathbf{i}(44.7 \mathrm{mg}$, $83 \%, 84 \%$ ee) as a yellow liquid.

$[\alpha]_{\mathrm{D}}^{21.8}=+4.94\left(\mathrm{c} 0.5, \mathrm{CHCl}_{3}\right) .{ }^{1} \mathrm{H}$ NMR $\left(400 \mathrm{MHz}, \mathrm{CDCl}_{3}\right): \delta$ 7.24-7.18 (m, 3H) $7.13(\mathrm{~d}, J=1.6 \mathrm{~Hz}, 1 \mathrm{H}), 3.75(\mathrm{~s}, 2 \mathrm{H}), 3.36-3.30(\mathrm{~m}, 1 \mathrm{H}), 2.93-2.76(\mathrm{~m}, 2 \mathrm{H})$, 2.56-2.31 (m, 2H), 2.25 (s, 3H). $\left.{ }^{13} \mathrm{C} \mathrm{NMR} \mathrm{(100} \mathrm{MHz,} \mathrm{CDCl}_{3}\right): \delta 202.9,136.8,131.6$, 
$130.8,130.3,128.0,126.6,124.9(\mathrm{~d}, J=275.7 \mathrm{~Hz}), 119.1,48.1,42.2,35.0(\mathrm{q}, J=$ $29.5 \mathrm{~Hz}), 20.3,19.6(\mathrm{q}, J=1.9 \mathrm{~Hz}) .{ }^{19} \mathrm{~F} \mathrm{NMR}\left(376 \mathrm{MHz}, \mathrm{CDCl}_{3}\right): \delta-64.62(\mathrm{t}, J=$ $9.02 \mathrm{~Hz}$ ). HRMS (EI) m/z: [M] ${ }^{+}$Calcd for $\mathrm{C}_{14} \mathrm{H}_{14} \mathrm{~F}_{3} \mathrm{NO} 269.1022$; Found 269.1023.

HPLC (IC-3, 0.46*15 cm, $3 \mu \mathrm{m}$, hexane/isopropanol $=90 / 10$, flow $0.7 \mathrm{~mL} / \mathrm{min}$, detection at $214 \mathrm{~nm}$ ) retention time $=10.800 \mathrm{~min}$ (major) and $12.975 \mathrm{~min}$ (minor).<smiles>Cc1ccc(C)c(CC(=O)CC(C#N)CC(F)(F)F)c1</smiles>

The reaction was conducted on a $0.2 \mathrm{mmol}$ scale according to the condition $\mathbf{A}$. Column chromatography $(\mathrm{PE}: \mathrm{EA}=7: 1)$ yielded the desired product $\mathbf{2 j}$ (47.0 $\mathrm{mg}$, $83 \%, 82 \%$ ee) as a light white solid, m.p. $43-45^{\circ} \mathrm{C}$.

$[\alpha]_{\mathrm{D}}{ }^{25.5}=-3.33\left(\mathrm{c} 1.0, \mathrm{CHCl}_{3}\right) .{ }^{1} \mathrm{H} \mathrm{NMR}\left(400 \mathrm{MHz}, \mathrm{CDCl}_{3}\right): \delta 7.10(\mathrm{~d}, J=7.6 \mathrm{~Hz}$, $1 \mathrm{H}), 7.04(\mathrm{~d}, J=8.0 \mathrm{~Hz}, 1 \mathrm{H}), 6.94(\mathrm{~s}, 1 \mathrm{H}), 3.71(\mathrm{~s}, 2 \mathrm{H}), 3.36-3.31(\mathrm{~m}, 1 \mathrm{H}), 2.93-2.76$ $(\mathrm{m}, 2 \mathrm{H}), 2.56-2.32(\mathrm{~m}, 2 \mathrm{H}), 2.32(\mathrm{~s}, 3 \mathrm{H}), 2.20(\mathrm{~s}, 3 \mathrm{H}) .{ }^{13} \mathrm{C} \mathrm{NMR}\left(100 \mathrm{MHz}, \mathrm{CDCl}_{3}\right)$ : $\delta 203.1,136.2,133.5,131.3,131.0,130.7,128.7,124.9$ (d, $J=275.9$ Hz), 119.1, 48.1, 42.1, 35.0 (q, $J=29.2 \mathrm{~Hz}), 20.8,20.3$ (q, $J=2.9 \mathrm{~Hz}), 19.1 .{ }^{19} \mathrm{~F}$ NMR (376 MHz, $\left.\mathrm{CDCl}_{3}\right): \delta-64.60\left(\mathrm{t}, J=9.78 \mathrm{~Hz}\right.$ ). HRMS (EI) $\mathrm{m} / \mathrm{z}:[\mathrm{M}]^{+}$Calcd for $\mathrm{C}_{15} \mathrm{H}_{16} \mathrm{~F}_{3} \mathrm{NO}$ 283.1179; Found 287.1177.

HPLC (IC-3, 0.46*15 cm, $3 \mu \mathrm{m}$, hexane/isopropanol = 90/10, flow $0.7 \mathrm{~mL} / \mathrm{min}$, detection at $214 \mathrm{~nm}$ ) retention time $=10.102 \mathrm{~min}$ (major) and $12.208 \mathrm{~min}$ (minor).

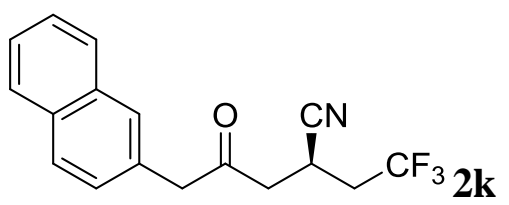

The reaction was conducted on a $0.2 \mathrm{mmol}$ scale according to the condition $\mathbf{B}$. Column chromatography (PE: EA = 7:1) yielded the desired product 2k $(39.7 \mathrm{mg}$, $65 \%, 86 \%$ ee) as a light white solid, m.p. $82-87^{\circ} \mathrm{C}$.

$[\alpha]_{\mathrm{D}}{ }^{21.5}=+3.28\left(\mathrm{c} 0.5, \mathrm{CHCl}_{3}\right) .{ }^{1} \mathrm{H} \mathrm{NMR}\left(400 \mathrm{MHz}, \mathrm{CDCl}_{3}\right): \delta$ 7.80-7.86 (m, 3H), $7.68(\mathrm{~s}, 1 \mathrm{H}), 7.48-7.53(\mathrm{~m}, 2 \mathrm{H}) 7.30(\mathrm{~d}, J=8.0 \mathrm{~Hz}, 1 \mathrm{H}), 3.90(\mathrm{~s}, 2 \mathrm{H}), 3.37-3.30(\mathrm{~m}$, 1H), 3.01-2.83 (m, 2H), 2.54-2.30 (m, 2H). ${ }^{13} \mathrm{C}$ NMR (100 MHz, $\left.\mathrm{CDCl}_{3}\right): \delta 202.8$, $133.5,132.6,130.2,129.0,128.3,127.8,127.6,126.9,126.6,126.3,124.9$ (d, $J=$ 
275.6 Hz), 119.0, 50.1, 42.4, 35.1 (q, $J=29.9 \mathrm{~Hz}), 20.3(\mathrm{q}, J=3.2 \mathrm{~Hz}) .{ }^{19} \mathrm{~F}$ NMR $\left(376 \mathrm{MHz}, \mathrm{CDCl}_{3}\right): \delta-64.66\left(\mathrm{t}, J=9.4 \mathrm{~Hz}\right.$ ). HRMS (EI) $\mathrm{m} / \mathrm{z}:[\mathrm{M}]^{+}$Calcd for $\mathrm{C}_{17} \mathrm{H}_{14} \mathrm{~F}_{3} \mathrm{NO}$ 305.1022; Found 305.1025.

HPLC (IC-3, 0.46*15 cm, $3 \mu \mathrm{m}$, hexane/isopropanol = 90/10, flow $0.7 \mathrm{~mL} / \mathrm{min}$, detection at $214 \mathrm{~nm}$ ) retention time $=20.755 \mathrm{~min}$ (minor) and $26.255 \mathrm{~min}$ (major).<smiles>CC(C)(C)c1ccc(C(=O)CC(C#N)CC(F)(F)F)cc1</smiles>

The reaction was conducted on a $0.2 \mathrm{mmol}$ scale according to the condition $\mathbf{A}$. Column chromatography $(\mathrm{PE}: \mathrm{EA}=10: 1)$ yielded the desired product $2 \mathrm{l}(53.5 \mathrm{mg}$, $90 \%, 80 \%$ ee) as a light white solid, m.p. $56-58^{\circ} \mathrm{C}$.

$[\alpha]_{\mathrm{D}}^{23.7}=-10.91\left(\mathrm{c} 1.0, \mathrm{CHCl}_{3}\right) .{ }^{1} \mathrm{H}$ NMR $\left(400 \mathrm{MHz}, \mathrm{CDCl}_{3}\right): \delta 7.84(\mathrm{~d}, J=8.4 \mathrm{~Hz}$, $2 \mathrm{H}), 7.52(\mathrm{~d}, J=8.4 \mathrm{~Hz}, 2 \mathrm{H}), 3.63-3.56(\mathrm{~m}, 1 \mathrm{H}), 3.52-3.36(\mathrm{~m}, 2 \mathrm{H}), 2.70-2.49(\mathrm{~m}$, 2H), 1.35 (s, 9H). ${ }^{13} \mathrm{C}$ NMR (100 MHz, $\left.\mathrm{CDCl}_{3}\right) \delta 193.7,158.3,132.8,125.1$ (q, $J=$ $275.9 \mathrm{~Hz}), 128.0,125.9,119.5,39.6,35.4(\mathrm{q}, J=28.9 \mathrm{~Hz}), 35.3,31.0,20.6$ (q, $J=3.1$ Hz). ${ }^{19} \mathrm{~F} \mathrm{NMR}\left(376 \mathrm{MHz}, \mathrm{CDCl}_{3}\right): \delta-64.54(\mathrm{t}, J=9.75 \mathrm{~Hz}) . \mathrm{HRMS}(\mathrm{EI}) \mathrm{m} / \mathrm{z}:[\mathrm{M}]^{+}$ Calcd for $\mathrm{C}_{16} \mathrm{H}_{18} \mathrm{~F}_{3} \mathrm{NO} 297.1335$; Found 297.1335.

HPLC (OD-H, 0.46*25 cm, $5 \mu \mathrm{m}$, hexane/isopropanol = 90/10, flow $0.7 \mathrm{~mL} / \mathrm{min}$, detection at $214 \mathrm{~nm}$ ) retention time $=32.553 \mathrm{~min}$ (minor) and $48.083 \mathrm{~min}$ (major).<smiles>N#C[C@H](CC(=O)c1ccccc1)CC(F)(F)F</smiles>

The reaction was conducted on a $0.2 \mathrm{mmol}$ scale according to the condition $\mathbf{A}$. Column chromatography (PE: EA = 10:1) yielded the desired product $\mathbf{2 m}(41.0 \mathrm{mg}$, $85 \%, 80 \%$ ee) as a light white solid, m.p. $55-56^{\circ} \mathrm{C}$.

$[\alpha]_{\mathrm{D}}^{25.1}=-7.84\left(\mathrm{c} 1.0, \mathrm{CHCl}_{3}\right) .{ }^{1} \mathrm{H} \mathrm{NMR}\left(400 \mathrm{MHz}, \mathrm{CDCl}_{3}\right): \delta 7.94(\mathrm{~d}, J=7.6 \mathrm{~Hz}$, 2H), $7.63(\mathrm{t}, J=7.6 \mathrm{~Hz}, 1 \mathrm{H}), 7.50(\mathrm{t}, J=8.0 \mathrm{~Hz}, 2 \mathrm{H}), 3.62-3.38(\mathrm{~m}, 3 \mathrm{H}), 2.71-2.49$ (m, 2H). ${ }^{13} \mathrm{C}$ NMR (100 MHz, $\left.\mathrm{CDCl}_{3}\right): \delta 194.1,135.3,134.2,125.1$ (q, $J=275.8 \mathrm{~Hz}$ ), 129.0, 128.0, 119.5, 39.7, $35.4(\mathrm{q}, J=30.0 \mathrm{~Hz}), 20.6(\mathrm{q}, J=3.2 \mathrm{~Hz}) .{ }^{19} \mathrm{~F}$ NMR $(376$ $\mathrm{MHz}, \mathrm{CDCl}_{3}$ ): $\delta-64.53\left(\mathrm{t}, J=9.02 \mathrm{~Hz}\right.$ ). HRMS (EI) m/z: $[\mathrm{M}]^{+}$Calcd for $\mathrm{C}_{12} \mathrm{H}_{10} \mathrm{~F}_{3} \mathrm{NO}$ 
241.0709; Found 241.0710.

HPLC (AD-H, 0.46*25 cm, $5 \mu \mathrm{m}$, hexane/isopropanol = 90/10, flow $0.7 \mathrm{~mL} / \mathrm{min}$, detection at $214 \mathrm{~nm}$ ) retention time $=23.228 \mathrm{~min}$ (major) and $24.788 \mathrm{~min}$ (minor).<smiles>COc1ccc(C(=O)C[C@H](C#N)CC(F)(F)F)cc1</smiles>

The reaction was conducted on a $0.2 \mathrm{mmol}$ scale according to the condition A. Column chromatography (PE: EA = 10:1) yielded the desired product 2n (48.3 mg, $89 \%, 81 \%$ ee) as a light white solid, m.p. $55-56{ }^{\circ} \mathrm{C}$.

$[\alpha]_{\mathrm{D}}{ }^{23.3}=-11.73\left(\mathrm{c} 1.0, \mathrm{CHCl}_{3}\right) .{ }^{1} \mathrm{H}$ NMR $\left(400 \mathrm{MHz}, \mathrm{CDCl}_{3}\right): \delta 7.92(\mathrm{~d}, J=8.8 \mathrm{~Hz}$, 2H), $6.96(\mathrm{~d}, J=8.8 \mathrm{~Hz}, 2 \mathrm{H}), 3.89(\mathrm{~s}, 3 \mathrm{H}), 3.61-3.54(\mathrm{~m}, 1 \mathrm{H}), 3.49-3.32(\mathrm{~m}, 2 \mathrm{H})$, 2.69-2.49 (m, 2H). ${ }^{13} \mathrm{C}$ NMR $\left(100 \mathrm{MHz}, \mathrm{CDCl}_{3}\right): \delta 192.5,164.3,130.4,125.1$ (q, $J=$ $275.7 \mathrm{~Hz}), 129.3,119.6,114.1,55.6,39.3,35.4(\mathrm{q}, J=30.1 \mathrm{~Hz}), 20.7$ (q, $J=3.2 \mathrm{~Hz})$. ${ }^{19} \mathrm{~F} \mathrm{NMR}\left(376 \mathrm{MHz}, \mathrm{CDCl}_{3}\right): \delta-64.56(\mathrm{t}, J=10.50 \mathrm{~Hz})$. HRMS (EI) m/z: [M] Calcd for $\mathrm{C}_{13} \mathrm{H}_{12} \mathrm{~F}_{3} \mathrm{NO}_{2}$ 271.0815; Found 271.0815.

HPLC (AY-3, 0.46*15 cm, $3 \mu \mathrm{m}$, hexane/isopropanol = 90/10, flow $0.7 \mathrm{~mL} / \mathrm{min}$, detection at $214 \mathrm{~nm}$ ) retention time $=33.172 \mathrm{~min}$ (major) and $39.813 \mathrm{~min}$ (minor).<smiles>CC(=O)Oc1ccc(C(=O)C[C@H](C#N)CC(F)(F)F)cc1</smiles>

The reaction was conducted on a $0.2 \mathrm{mmol}$ scale according to the condition $\mathbf{A}$. Column chromatography $(\mathrm{PE}: \mathrm{EA}=10: 1)$ yielded the desired product $20(53.8 \mathrm{mg}$, $90 \%, 84 \%$ ee) as a light white solid, m.p. $56-57^{\circ} \mathrm{C}$.

$[\alpha]_{\mathrm{D}}{ }^{24.6}=-8.56\left(\mathrm{c} 1.0, \mathrm{CHCl}_{3}\right) .{ }^{1} \mathrm{H} \mathrm{NMR}\left(400 \mathrm{MHz}, \mathrm{CDCl}_{3}\right): \delta 7.97(\mathrm{~d}, J=8.8 \mathrm{~Hz}$, $2 \mathrm{H}), 7.24(\mathrm{~d}, J=8.8 \mathrm{~Hz}, 2 \mathrm{H}), 3.62-3.35(\mathrm{~m}, 3 \mathrm{H}), 2.68-2.49(\mathrm{~m}, 2 \mathrm{H}), 2.33(\mathrm{~s}, 3 \mathrm{H}) .{ }^{13} \mathrm{C}$ NMR (100 MHz, $\left.\mathrm{CDCl}_{3}\right): \delta 192.9,168.7,155.2,132.9,129.7,125.1$ (q, $\left.J=275.9 \mathrm{~Hz}\right)$, 122.2, 119.4, 39.7, $35.4(\mathrm{q}, J=30.0 \mathrm{~Hz}), 21.1,20.6(\mathrm{q}, J=3.2 \mathrm{~Hz}) .{ }^{19} \mathrm{~F}$ NMR $(376$ $\left.\mathrm{MHz}, \mathrm{CDCl}_{3}\right): \delta-64.53\left(\mathrm{t}, J=10.15 \mathrm{~Hz}\right.$ ). HRMS (EI) $\mathrm{m} / \mathrm{z}:[\mathrm{M}]^{+}$Calcd for $\mathrm{C}_{14} \mathrm{H}_{12} \mathrm{~F}_{3} \mathrm{NO}_{3}$ 299.0764; Found 299.0758.

HPLC (IC-3, 0.46*15 cm, $3 \mu \mathrm{m}$, hexane/isopropanol = 70/30, flow $0.7 \mathrm{~mL} / \mathrm{min}$, 
detection at $214 \mathrm{~nm}$ ) retention time $=13.028 \mathrm{~min}$ (minor) and $22.583 \mathrm{~min}$ (major).<smiles>N#CC(CC(=O)c1ccc(-c2ccccc2)cc1)CC(F)(F)F</smiles>

The reaction was conducted on a $0.2 \mathrm{mmol}$ scale according to the condition $\mathbf{A}$. Column chromatography (PE: EA $=10: 1)$ yielded the desired product $\mathbf{2 p}(57.1 \mathrm{mg}$, $90 \%, 80 \%$ ee) as a light white solid, m.p. $115-118^{\circ} \mathrm{C}$.

$[\alpha]_{\mathrm{D}}{ }^{24.3}=-12.53\left(\mathrm{c} 1.0, \mathrm{CHCl}_{3}\right) .{ }^{1} \mathrm{H}$ NMR $\left(400 \mathrm{MHz}, \mathrm{CDCl}_{3}\right): \delta 8.02(\mathrm{~d}, J=8.8 \mathrm{~Hz}$, 2H), 7.57 (d, $J=8.4 \mathrm{~Hz}, 2 \mathrm{H}), 7.63(\mathrm{~d}, J=7.2 \mathrm{~Hz}, 2 \mathrm{H}), 7.49$ (t, $J=6.8 \mathrm{~Hz}, 2 \mathrm{H}), 7.43$ $(\mathrm{d}, J=7.2 \mathrm{~Hz}, 1 \mathrm{H}), 3.65-3.41(\mathrm{~m}, 3 \mathrm{H}), 2.73-2.52(\mathrm{~m}, 2 \mathrm{H}) .{ }^{13} \mathrm{C}$ NMR $(100 \mathrm{MHz}$, $\left.\mathrm{CDCl}_{3}\right): \delta 193.7,146.9,139.4,134.0,129.0,128.6,128.6,127.5,127.2,125.1(\mathrm{q}, J=$ $275.7 \mathrm{~Hz}), 119.5,39.7,35.4(\mathrm{q}, J=29.7 \mathrm{~Hz}), 20.6(\mathrm{q}, J=3.0 \mathrm{~Hz}) .{ }^{19} \mathrm{~F}$ NMR $(376$ $\left.\mathrm{MHz}, \mathrm{CDCl}_{3}\right): \delta-64.47(\mathrm{t}, J=9.78 \mathrm{~Hz})$. HRMS (EI) $\mathrm{m} / \mathrm{z}$ : $[\mathrm{M}]^{+}$Calcd for $\mathrm{C}_{18} \mathrm{H}_{14} \mathrm{~F}_{3} \mathrm{NO}$ 317.1022; Found 317.1021.

HPLC (IC-3, 0.46*15 cm, $3 \mu \mathrm{m}$, hexane/isopropanol = 90/10, flow $0.7 \mathrm{~mL} / \mathrm{min}$, detection at $214 \mathrm{~nm}$ ) retention time $=25.177 \mathrm{~min}$ (minor) and $30.442 \mathrm{~min}$ (major).<smiles>Cc1ccc(C(=O)C[C@H](C#N)CC(F)(F)F)cc1</smiles>

The reaction was conducted on a $0.2 \mathrm{mmol}$ scale according to the condition $\mathbf{A}$. Column chromatography $(\mathrm{PE}: \mathrm{EA}=10: 1)$ yielded the desired product $\mathbf{2 q}(34.2 \mathrm{mg}$, $67 \%, 78 \%$ ee) as a light white solid, m.p. $60-63^{\circ} \mathrm{C}$.

$[\alpha]_{\mathrm{D}}^{22.3}=-6.87\left(\mathrm{c} 1.0, \mathrm{CHCl}_{3}\right) .{ }^{1} \mathrm{H} \mathrm{NMR}\left(400 \mathrm{MHz}, \mathrm{CDCl}_{3}\right): \delta 7.84(\mathrm{~d}, J=8.0 \mathrm{~Hz}$, 2H), $7.30(\mathrm{~d}, J=8.4 \mathrm{~Hz}, 2 \mathrm{H}), 3.60-3.55(\mathrm{~m}, 1 \mathrm{H}), 3.52-3.35(\mathrm{~m}, 2 \mathrm{H}), 2.70-2.49$ (m, 2H), 2.43 (s, 9H). $\left.{ }^{13} \mathrm{C} \mathrm{NMR} \mathrm{(100} \mathrm{MHz,} \mathrm{CDCl}_{3}\right): \delta$ 193.7, 145.3, 132.9, 129.6, 128.1, $125.1(\mathrm{q}, J=275.8 \mathrm{~Hz}), 119.5,39.6,35.4(\mathrm{q}, J=30.2 \mathrm{~Hz}), 20.7,20.59(\mathrm{q}, J=3.0 \mathrm{~Hz})$. ${ }^{19} \mathrm{~F}$ NMR $\left(376 \mathrm{MHz}, \mathrm{CDCl}_{3}\right): \delta-64.53(\mathrm{t}, J=10.13 \mathrm{~Hz})$. HRMS (EI) m/z: [M] ${ }^{+}$Calcd for $\mathrm{C}_{13} \mathrm{H}_{12} \mathrm{~F}_{3} \mathrm{NO} 255.0866$; Found 255.0865.

HPLC (AD-H, 0.46*25 cm, $5 \mu \mathrm{m}$, hexane/isopropanol = 95/5, flow $0.7 \mathrm{~mL} / \mathrm{min}$, detection at $214 \mathrm{~nm}$ ) retention time $=27.445 \mathrm{~min}$ (major) and $29.550 \mathrm{~min}$ (minor). 
<smiles>COC(=O)c1ccc(C(=O)C[C@H](C#N)CC(F)(F)F)cc1</smiles>

The reaction was conducted on a $0.2 \mathrm{mmol}$ scale according to the condition $\mathbf{A}$. Column chromatography (PE: $\mathrm{EA}=10: 1)$ yielded the desired product 2 r $(55.6 \mathrm{mg}$, $93 \%, 75 \%$ ee) as a light white solid, m.p. $55-56^{\circ} \mathrm{C}$.

$[\alpha]_{\mathrm{D}}{ }^{24.5}=-5.80\left(\mathrm{c} 1.0, \mathrm{CHCl}_{3}\right) .{ }^{1} \mathrm{H} \mathrm{NMR}\left(400 \mathrm{MHz}, \mathrm{CDCl}_{3}\right): \delta 8.14(\mathrm{~d}, J=8.4 \mathrm{~Hz}$, $2 \mathrm{H}), 7.98(\mathrm{~d}, J=8.4 \mathrm{~Hz}, 2 \mathrm{H}), 3.95(\mathrm{~s}, 3 \mathrm{H}), 3.61-3.40(\mathrm{~m}, 3 \mathrm{H}), 2.73-2.50(\mathrm{~m}, 2 \mathrm{H}) .{ }^{13} \mathrm{C}$ NMR (100 MHz, $\left.\mathrm{CDCl}_{3}\right): \delta 193.8,165.8,138.3,134.8,130.0,127.9,125.0$ (q, $J=$ $275.4 \mathrm{~Hz}), 119.2,52.6,40.0,35.3(\mathrm{q}, J=29.8 \mathrm{~Hz}), 20.6(\mathrm{q}, J=3.3 \mathrm{~Hz}) .{ }^{19} \mathrm{~F}$ NMR $\left(376 \mathrm{MHz}, \mathrm{CDCl}_{3}\right): \delta-64.53\left(\mathrm{t}, J=10.15 \mathrm{~Hz}\right.$ ). HRMS (EI) $\mathrm{m} / \mathrm{z}:[\mathrm{M}]^{+}$Calcd for $\mathrm{C}_{14} \mathrm{H}_{12} \mathrm{~F}_{3} \mathrm{NO}_{3}$ 299.0764; Found 299.0754.

HPLC (OD-H, 0.46*25 cm, $5 \mu \mathrm{m}$, hexane/isopropanol = 70/30, flow $0.7 \mathrm{~mL} / \mathrm{min}$, detection at $214 \mathrm{~nm}$ ) retention time $=25.822 \mathrm{~min}$ (major) and $31.263 \mathrm{~min}$ (minor).<smiles>N#C[C@H](CC(=O)c1ccc(F)cc1)CC(F)(F)F</smiles>

The reaction was conducted on a $0.2 \mathrm{mmol}$ scale according to the condition $\mathbf{A}$. Column chromatography $(\mathrm{PE}: \mathrm{EA}=10: 1)$ yielded the desired product $2 \mathrm{~s}(41.4 \mathrm{mg}$, $80 \%, 71 \%$ ee) as a yellow liquid.

$[\alpha]_{\mathrm{D}}^{23.2}=-7.22\left(\mathrm{c} 1.0, \mathrm{CHCl}_{3}\right) .{ }^{1} \mathrm{H}$ NMR $\left(400 \mathrm{MHz}, \mathrm{CDCl}_{3}\right): \delta 8.01-7.96(\mathrm{~m}, 2 \mathrm{H})$, 7.21-7.15 (m, 2H), 3.62-3.55 (m, 1H), 3.53-3.35 (m, 2H), 2.72-2.50 (m, 2H). ${ }^{13} \mathrm{C}$ NMR (100 MHz, $\left.\mathrm{CDCl}_{3}\right): \delta$ 192.5, $166.5(\mathrm{~d}, J=275.1 \mathrm{~Hz}), 131.8(\mathrm{~d}, J=2.9 \mathrm{~Hz})$, $130.8(\mathrm{~d}, J=10.4 \mathrm{~Hz}), 125.1(\mathrm{q}, J=275.7 \mathrm{~Hz}), 119.3,116.2(\mathrm{~d}, J=21.7 \mathrm{~Hz}), 39.6$, $35.4(\mathrm{q}, J=29.8 \mathrm{~Hz}), 20.6(\mathrm{q}, J=3.9 \mathrm{~Hz}) .{ }^{19} \mathrm{~F} \mathrm{NMR}\left(376 \mathrm{MHz}, \mathrm{CDCl}_{3}\right): \delta-64.5(\mathrm{t}, J$ $=9.78 \mathrm{~Hz}$ ), $-102.70,-102.77(\mathrm{~m})$. HRMS (EI) m/z: $[\mathrm{M}]^{+}$Calcd for $\mathrm{C}_{12} \mathrm{H}_{9} \mathrm{~F}_{4} \mathrm{NO}$ 259.0615; Found 259.0616.

HPLC (IC-3, 0.46*15 cm, $3 \mu \mathrm{m}$, hexane/isopropanol = 90/10, flow $0.7 \mathrm{~mL} / \mathrm{min}$, detection at $214 \mathrm{~nm}$ ) retention time $=12.543 \mathrm{~min}$ (major) and $14.717 \mathrm{~min}$ (minor). 
<smiles>N#C[C@H](CC(=O)c1ccc(Cl)cc1)CC(F)(F)F</smiles>

The reaction was conducted on a $0.2 \mathrm{mmol}$ scale according to the condition $\mathbf{A}$. Column chromatography $(\mathrm{PE}: \mathrm{EA}=10: 1)$ yielded the desired product $2 \mathrm{t}(51.2 \mathrm{mg}$, $93 \%, 76 \%$ ee) as a light white solid, m.p. $42-45^{\circ} \mathrm{C}$.

$[\alpha]_{\mathrm{D}}{ }^{23.8}=-13.01\left(\mathrm{c} 1.0, \mathrm{CHCl}_{3}\right) .{ }^{1} \mathrm{H}$ NMR $\left(400 \mathrm{MHz}, \mathrm{CDCl}_{3}\right): \delta 7.88(\mathrm{~d}, J=8.4 \mathrm{~Hz}$, 2H), $7.47(\mathrm{~d}, J=8.0 \mathrm{~Hz}, 2 \mathrm{H}), 3.60-3.54(\mathrm{~m}, 1 \mathrm{H}), 3.52-3.34(\mathrm{~m}, 2 \mathrm{H}), 2.72-2.49(\mathrm{~m}$, 2H). ${ }^{13} \mathrm{C}$ NMR $\left(100 \mathrm{MHz}, \mathrm{CDCl}_{3}\right): \delta 193.0,140.9,133.6,129.4,129.3,125.1$ (q, $J=$ $275.7 \mathrm{~Hz}), 119.3,39.4,35.3(\mathrm{q}, J=29.9 \mathrm{~Hz}), 20.6(\mathrm{q}, J=3.1 \mathrm{~Hz}) .{ }^{19} \mathrm{~F}$ NMR $(376$ $\left.\mathrm{MHz}, \mathrm{CDCl}_{3}\right): \delta-64.51\left(\mathrm{t}, J=10.53 \mathrm{~Hz}\right.$ ). HRMS (EI) $\mathrm{m} / \mathrm{z}:[\mathrm{M}]^{+}$Calcd for $\mathrm{C}_{12} \mathrm{H}_{9} \mathrm{ClF}_{3} \mathrm{NO} 275.0319$; Found 275.0320.

HPLC (OD-H, 0.46*25 cm, $5 \mu \mathrm{m}$, hexane/isopropanol = 80/20, flow $0.7 \mathrm{~mL} / \mathrm{min}$, detection at $214 \mathrm{~nm}$ ) retention time $=22.642 \mathrm{~min}$ (major) and $30.833 \mathrm{~min}$ (minor).<smiles>N#CC(CC(=O)c1ccc(C(F)(F)F)cc1)CC(F)(F)F</smiles>

The reaction was conducted on a $0.2 \mathrm{mmol}$ scale according to the conditions $\mathbf{A}$. Column chromatography (PE: EA = 10:1) yielded the desired product $\mathbf{2 u}(47.6 \mathrm{mg}$, $77 \%, 75 \%$ ee) as a light white solid. m.p. $50-52{ }^{\circ} \mathrm{C}$.

$[\alpha]_{\mathrm{D}}{ }^{21.9}-2.95\left(\mathrm{c} 1.0, \mathrm{CHCl}_{3}\right) .{ }^{1} \mathrm{H} \mathrm{NMR}\left(400 \mathrm{MHz}, \mathrm{CDCl}_{3}\right) \delta 8.06(\mathrm{~d}, J=8.0 \mathrm{~Hz}, 2 \mathrm{H})$, $7.78(\mathrm{~d}, J=8.4 \mathrm{~Hz}, 2 \mathrm{H}), 3.63-3.41(\mathrm{~m}, 3 \mathrm{H}), 2.75-2.51(\mathrm{~m}, 2 \mathrm{H}) .{ }^{13} \mathrm{C}$ NMR $(100 \mathrm{MHz}$, $\left.\mathrm{CDCl}_{3}\right) \delta 193.4,137.9,135.5(\mathrm{q}, J=33.1 \mathrm{~Hz}), 126.5(\mathrm{q}, J=275.8 \mathrm{~Hz}), 127.6(\mathrm{q}, J=$ $4.0 \mathrm{~Hz}), 123.3(\mathrm{q}, J=271.5 \mathrm{~Hz}), 119.1,40.0,35.3(\mathrm{q}, J=29.9 \mathrm{~Hz}), 20.6(\mathrm{q}, J=3.9$ $\mathrm{Hz}) .{ }^{19} \mathrm{~F}$ NMR $\left(376 \mathrm{MHz}, \mathrm{CDCl}_{3}\right) \delta-63.31(\mathrm{~s}),-64.46(\mathrm{t}, J=10.53 \mathrm{~Hz})$. HRMS (EI) $\mathrm{m} / \mathrm{z}:[\mathrm{M}]^{+}$Calcd for $\mathrm{C}_{13} \mathrm{H}_{9} \mathrm{~F}_{6} \mathrm{NO}$ 309.0583; Found 309.0585.

HPLC (AD-H, 0.46*25 cm, $5 \mu \mathrm{m}$, hexane/isopropanol = 95/5, flow $0.7 \mathrm{~mL} / \mathrm{min}$, detection at $214 \mathrm{~nm}$ ) retention time $=42.713 \mathrm{~min}$ (major) and $44.978 \mathrm{~min}$ (minor). 
<smiles>N#Cc1ccc(C(=O)C[C@@H](C#N)CC(F)(F)F)cc1</smiles>

The reaction was conducted on a $0.2 \mathrm{mmol}$ scale according to the condition $\mathbf{A}$. Column chromatography $(\mathrm{PE}: \mathrm{EA}=10: 1)$ yielded the desired product $\mathbf{2 v}(45.2 \mathrm{mg}$, $85 \%, 70 \%$ ee) as a light white solid, m.p. $67-70^{\circ} \mathrm{C}$.

$[\alpha]_{\mathrm{D}}{ }^{24.9}=-5.56\left(\mathrm{c} 1.0, \mathrm{CHCl}_{3}\right) .{ }^{1} \mathrm{H} \mathrm{NMR}\left(400 \mathrm{MHz}, \mathrm{CDCl}_{3}\right): \delta 8.04(\mathrm{~d}, J=8.0 \mathrm{~Hz}$, 2H), $7.81(\mathrm{~d}, J=8.8 \mathrm{~Hz}, 2 \mathrm{H}), 3.60-3.39(\mathrm{~m}, 3 \mathrm{H}), 2.75-2.50(\mathrm{~m}, 2 \mathrm{H}) .{ }^{13} \mathrm{C}$ NMR $(100$ $\left.\mathrm{MHz}, \mathrm{CDCl}_{3}\right): \delta 193.2,138.1,132.8,128.4,125.0(\mathrm{q}, J=275.7 \mathrm{~Hz}), 119.0,117.5$, 117.4, 39.9, 35.2 (q, $J=29.8 \mathrm{~Hz}), 20.6$ (q, $J=3.9 \mathrm{~Hz}) .{ }^{19} \mathrm{~F} \mathrm{NMR}\left(376 \mathrm{MHz}, \mathrm{CDCl}_{3}\right)$ : $\delta$-64.44 (t, $J=9.78 \mathrm{~Hz}$ ). HRMS (EI) $\mathrm{m} / \mathrm{z}$ : $[\mathrm{M}]^{+}$Calcd for $\mathrm{C}_{13} \mathrm{H}_{9} \mathrm{~F}_{3} \mathrm{~N}_{2} \mathrm{O}$ 266.0661; Found 266.0660.

HPLC (IC-3, 0.46*15 cm, $3 \mu \mathrm{m}$, hexane/isopropanol = 80/20, flow $0.7 \mathrm{~mL} / \mathrm{min}$, detection at $214 \mathrm{~nm}$ ) retention time $=16.952 \mathrm{~min}$ (major) and $21.642 \mathrm{~min}$ (minor).<smiles>N#CC(CC(=O)Nc1ccccc1)CC(F)(F)F</smiles>

The reaction was conducted on a $0.2 \mathrm{mmol}$ scale according to the condition $\mathbf{A}$. Column chromatography $(\mathrm{PE}: \mathrm{EA}=4: 1)$ yielded the desired product 3a $(46.1 \mathrm{mg}$, $90 \%, 85 \%$ ee) as a light white solid, m.p. $128-130{ }^{\circ} \mathrm{C}$.

$[\alpha]_{\mathrm{D}}{ }^{24.7}=+3.42\left(\mathrm{c} 0.80\right.$, Acetone). ${ }^{1} \mathrm{H}$ NMR $\left(400 \mathrm{MHz}, d_{6}\right.$-Acetone): $\delta 9.42(\mathrm{br}, 1 \mathrm{H})$, $7.64(\mathrm{~d}, J=8.4 \mathrm{~Hz}, 2 \mathrm{H}), 7.31(\mathrm{t}, J=7.6 \mathrm{~Hz}, 2 \mathrm{H}), 7.08(\mathrm{t}, J=7.2 \mathrm{~Hz}, 1 \mathrm{H}), 3.61-3.54$ (m, 1H), 3.05-2.73 (m, 4H). ${ }^{13} \mathrm{C}$ NMR (100 MHz, $d_{6}$-Acetone): $\delta$ 168.3, 140.5, 130.4, $127.7(\mathrm{q}, J=275.2 \mathrm{~Hz}), 125.4,121.2,121.0,39.2,36.5(\mathrm{q}, J=29.1 \mathrm{~Hz}), 23.2(\mathrm{q}, J=$ $3.5 \mathrm{~Hz}) .{ }^{19} \mathrm{~F}$ NMR $\left(376 \mathrm{MHz}, d_{6}\right.$-Acetone): $\delta-64.04(\mathrm{t}, J=10.53 \mathrm{~Hz})$. HRMS (EI) $\mathrm{m} / \mathrm{z}$ : $[\mathrm{M}]^{+}$Calcd for $\mathrm{C}_{12} \mathrm{H}_{11} \mathrm{~F}_{3} \mathrm{~N}_{2} \mathrm{O}$ 256.0818; Found 256.0817.

HPLC (AD-H, 0.46*25 cm, $5 \mu \mathrm{m}$, hexane/isopropanol $=90 / 10$, flow $0.7 \mathrm{~mL} / \mathrm{min}$, detection at $214 \mathrm{~nm}$ ) retention time $=17.700 \mathrm{~min}$ (major) and $20.235 \mathrm{~min}$ (minor).<smiles>COc1ccc(NC(=O)CC(C#N)CC(F)(F)F)cc1</smiles> 
The reaction was conducted on a $0.2 \mathrm{mmol}$ scale according to the condition $\mathbf{A}$. Column chromatography $(\mathrm{PE}: \mathrm{EA}=4: 1)$ yielded the desired product $\mathbf{3 b}(49.2 \mathrm{mg}$, $86 \%, 84 \%$ ee) as a light white solid, m.p. $125-128{ }^{\circ} \mathrm{C}$.

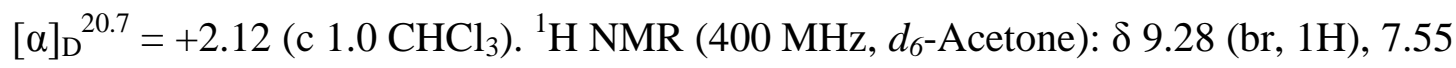
$(\mathrm{d}, J=9.2 \mathrm{~Hz}, 2 \mathrm{H}), 6.88(\mathrm{~d}, J=9.2 \mathrm{~Hz}, 2 \mathrm{H}), 3.77(\mathrm{~s}, 3 \mathrm{H}) 3.60-3.53(\mathrm{~m}, 1 \mathrm{H})$, 3.00-2.73 (m, 4H). ${ }^{13} \mathrm{C}$ NMR (100 MHz, $d_{6}$-Acetone): $\delta$ 167.8, 157.8, 133.6, 127.8 (q, $J=274.5 \mathrm{~Hz}), 122.6,121.3,114.5,56.4,39.1,36.5(\mathrm{q}, J=29.1 \mathrm{~Hz}), 23.3(\mathrm{q}, J=3.3$

Hz). ${ }^{19} \mathrm{~F}$ NMR (376 MHz, $d_{6}$-Acetone): $\delta-60.25$ (t, $\left.J=10.50 \mathrm{~Hz}\right)$. HRMS (EI) m/z: $[\mathrm{M}]^{+}$Calcd for $\mathrm{C}_{13} \mathrm{H}_{13} \mathrm{~F}_{3} \mathrm{~N}_{2} \mathrm{O}_{2}$ 286.0924; Found 286.0921.

HPLC (ID-3, 0.46*15 cm, $3 \mu \mathrm{m}$, hexane/isopropanol $=90 / 10$, flow $0.7 \mathrm{~mL} / \mathrm{min}$, detection at $214 \mathrm{~nm}$ ) retention time $=21.482 \mathrm{~min}$ (major) and $25.568 \mathrm{~min}$ (minor).<smiles>CC(F)(F)CC(C#N)CC(=O)Nc1ccc(C(C)(C)C)cc1</smiles>

The reaction was conducted on a $0.2 \mathrm{mmol}$ scale according to the condition $\mathbf{C}$. Column chromatography $(\mathrm{PE}: \mathrm{EA}=4: 1)$ yielded the desired product $3 \mathbf{c}(46.8 \mathrm{mg}$, $75 \%, 91 \%$ ee) as a light white solid, m.p. $126-127^{\circ} \mathrm{C}$.

$[\alpha]_{\mathrm{D}}{ }^{24.4}=0.34$ (c 1.0, Acetone). ${ }^{1} \mathrm{H}$ NMR (400 MHz, $d_{6}$-Acetone): $\delta 9.32$ (br, 1H), $7.52(\mathrm{~d}, J=8.0 \mathrm{~Hz}, 2 \mathrm{H}), 7.35(\mathrm{~d}, J=8.4 \mathrm{~Hz}, 2 \mathrm{H}), 3.61-3.54(\mathrm{~m}, 1 \mathrm{H}), 3.03-2.72(\mathrm{~m}$, 4H), 1.29 (s, 9H). ${ }^{13} \mathrm{C} \mathrm{NMR} \mathrm{(100} \mathrm{MHz,} d_{6}$-Acetone): $\delta$ 168.1, 148.1, 138.0, 127.7 (q, $J=274.8 \mathrm{~Hz}), 127.1,121.2,120.8,39.2,36.5(\mathrm{q}, J=29.1 \mathrm{~Hz}), 35.6,32.4,23.3(\mathrm{q}, J=$ $3.4 \mathrm{~Hz}) .{ }^{19} \mathrm{~F}$ NMR $\left(376 \mathrm{MHz}, d_{6}\right.$-Acetone): $\delta-60.22(\mathrm{t}, J=10.53 \mathrm{~Hz})$. HRMS (EI) $\mathrm{m} / \mathrm{z}$ : $[\mathrm{M}]^{+}$Calcd for $\mathrm{C}_{16} \mathrm{H}_{19} \mathrm{~F}_{3} \mathrm{~N}_{2} \mathrm{O}$ 312.1444; Found 312.1444.

HPLC (AD-H, 0.46*25 cm, $5 \mu \mathrm{m}$, hexane/isopropanol = 90/10, flow $0.7 \mathrm{~mL} / \mathrm{min}$, detection at $214 \mathrm{~nm}$ ) retention time $=35.315 \mathrm{~min}$ (major) and $46.665 \mathrm{~min}$ (minor).<smiles>Cc1ccc(NC(=O)CC(C#N)CC(F)(F)F)cc1</smiles>

The reaction was conducted on a $0.2 \mathrm{mmol}$ scale according to the condition $\mathbf{A}$. Column chromatography $(\mathrm{PE}: \mathrm{EA}=4: 1)$ yielded the desired product 3d $(48.6 \mathrm{mg}$, 
$90 \%, 84 \%$ ee) as a light white solid, m.p. $148-150{ }^{\circ} \mathrm{C}$.

$[\alpha]_{\mathrm{D}}{ }^{24.4}=+1.4817$ (c 0.5, Acetone). ${ }^{1} \mathrm{H}$ NMR (400 MHz, $d_{6}$-Acetone): $\delta 9.29$ (br, $\left.1 \mathrm{H}\right)$, $7.56(\mathrm{~d}, J=8.4 \mathrm{~Hz}, 2 \mathrm{H}), 7.12(\mathrm{~d}, J=8.4 \mathrm{~Hz}, 2 \mathrm{H}), 3.53-3.60(\mathrm{~m}, 1 \mathrm{H}), 2.72-3.02(\mathrm{~m}$, 4H), $2.27(\mathrm{~s}, 3 \mathrm{H}) .{ }^{13} \mathrm{C}$ NMR (100 MHz, $d_{6}$-Acetone): $\delta 168.1,138.0,134.7,130.8$, $127.7(\mathrm{q}, J=274.0 \mathrm{~Hz}), 121.2,121.0,39.2,36.5(\mathrm{q}, J=29.1 \mathrm{~Hz}), 23.2(\mathrm{q}, J=2.9 \mathrm{~Hz})$, 21.6. ${ }^{19}$ F NMR (376 MHz, $d_{6}$-Acetone): $\delta-60.24(\mathrm{t}, J=10.90 \mathrm{~Hz}) . \mathrm{HRMS}(\mathrm{EI}) \mathrm{m} / \mathrm{z}$ : $[\mathrm{M}]^{+}$Calcd for $\mathrm{C}_{13} \mathrm{H}_{13} \mathrm{~F}_{3} \mathrm{~N}_{2} \mathrm{O}$ 270.0974; Found 271.0971.

HPLC (AD-H, 0.46*25 cm, $5 \mu \mathrm{m}$, hexane/isopropanol = 90/10, flow $0.7 \mathrm{~mL} / \mathrm{min}$, detection at $214 \mathrm{~nm}$ ) retention time $=32.747 \mathrm{~min}$ (major) and $36.327 \mathrm{~min}$ (minor).<smiles>N#CC(CC(=O)Nc1ccc(F)cc1)CC(F)(F)F</smiles>

The reaction was conducted on a $0.2 \mathrm{mmol}$ scale according to the condition $\mathbf{C}$. Column chromatography $(\mathrm{PE}: \mathrm{EA}=4: 1)$ yielded the desired product $3 \mathrm{e}(41.7 \mathrm{mg}$, $76 \%, 83 \%$ ee) as a light white solid, m.p. $116-119^{\circ} \mathrm{C}$.

$[\alpha]_{\mathrm{D}}^{25.1}=-13.61\left(\mathrm{c} 0.50, \mathrm{CHCl}_{3}\right) .{ }^{1} \mathrm{H}$ NMR $\left(400 \mathrm{MHz}, d_{6}\right.$-Acetone): $\delta 9.42(\mathrm{br}, 1 \mathrm{H})$, $7.64(\mathrm{dd}, J=8.8,5.2 \mathrm{~Hz}, 2 \mathrm{H}), 7.31(\mathrm{t}, J=8.8 \mathrm{~Hz}, 2 \mathrm{H}), 3.61-3.54(\mathrm{~m}, 1 \mathrm{H}), 3.04-2.73$ (m, 4H). ${ }^{13} \mathrm{C}$ NMR (100 MHz, $d_{6}$-Acetone): $\delta$ 168.3, $160.6(\mathrm{~d}, J=240.3 \mathrm{~Hz}), 136.8(\mathrm{~d}$, $J=2.3 \mathrm{~Hz}), 127.7(\mathrm{q}, J=275.1 \mathrm{~Hz}), 122.8(\mathrm{~d}, J=7.7 \mathrm{~Hz}), 121.2,116.8(\mathrm{~d}, J=21.9$ $\mathrm{Hz}), 39.1,36.5(\mathrm{q}, J=23.7 \mathrm{~Hz}), 23.2(\mathrm{q}, J=3.5 \mathrm{~Hz}) .{ }^{19} \mathrm{~F}$ NMR $(376 \mathrm{MHz}$, $d_{6}$-Acetone): $\delta-60.25(\mathrm{t}, J=10.15 \mathrm{~Hz}),-115.33--115.42(\mathrm{~m}) . \operatorname{HRMS}(\mathrm{EI}) \mathrm{m} / \mathrm{z}:[\mathrm{M}]^{+}$ Calcd for $\mathrm{C}_{12} \mathrm{H}_{10} \mathrm{~F}_{4} \mathrm{~N}_{2} \mathrm{O} 274.0724$; Found 274.0724.

HPLC (AD-H, 0.46*25 cm, $5 \mu \mathrm{m}$, hexane/isopropanol $=90 / 10$, flow $0.7 \mathrm{~mL} / \mathrm{min}$, detection at $214 \mathrm{~nm}$ ) retention time $=16.115 \mathrm{~min}$ (minor) and $17.542 \mathrm{~min}$ (major).<smiles>N#CC(CC(=O)Nc1ccc(Cl)cc1)CC(F)(F)F</smiles>

The reaction was conducted on a $0.2 \mathrm{mmol}$ scale according to the condition $\mathbf{C}$. Column chromatography (PE: EA = 4:1) yielded the desired product $3 f$ (44.1 mg, $76 \%, 81 \%$ ee) as a light white solid, m.p. $132-134{ }^{\circ} \mathrm{C}$. 
$[\alpha]_{\mathrm{D}}{ }^{24.5}=+3.59$ (c 0.5, acetone). ${ }^{1} \mathrm{H}$ NMR $\left(400 \mathrm{MHz}, d_{6}\right.$-Acetone): $\delta 9.53(\mathrm{br}, 1 \mathrm{H})$, $7.67(\mathrm{~d}, J=8.8 \mathrm{~Hz}, 2 \mathrm{H}), 7.48(\mathrm{~d}, J=8.8 \mathrm{~Hz}, 2 \mathrm{H}), 3.61-3.54(\mathrm{~m}, 1 \mathrm{H}), 3.06-2.73(\mathrm{~m}$, 4H). ${ }^{13} \mathrm{C}$ NMR (100 MHz, $d_{6}$-Acetone): $\delta 168.5,139.4,130.4,129.7,127.7$ (q, $J=$ $275.2 \mathrm{~Hz}), 122.5,121.2,39.2,36.5(\mathrm{q}, J=29.1 \mathrm{~Hz}), 23.2(\mathrm{q}, J=3.9 \mathrm{~Hz}) .{ }^{19} \mathrm{~F}$ NMR (376 MHz, $d_{6}$-Acetone): $\delta-64.37(\mathrm{t}, J=9.78 \mathrm{~Hz})$. HRMS (EI) m/z: $[\mathrm{M}]^{+}$Calcd for $\mathrm{C}_{12} \mathrm{H}_{10} \mathrm{ClF}_{3} \mathrm{~N}_{2} \mathrm{O} 290.0428$; Found 290.0427.

HPLC (AD-H, 0.46*25 cm, $5 \mu \mathrm{m}$, hexane/isopropanol $=90 / 10$, flow $0.7 \mathrm{~mL} / \mathrm{min}$, detection at $214 \mathrm{~nm}$ ) retention time $=24.740 \mathrm{~min}$ (major) and $27.425 \mathrm{~min}$ (minor).<smiles>N#CC(CC(=O)Nc1ccc(Br)cc1)CC(F)(F)F</smiles>

The reaction was conducted on a $0.2 \mathrm{mmol}$ scale according to the condition $\mathbf{C}$. Column chromatography $(\mathrm{PE}: \mathrm{EA}=4: 1)$ yielded the desired product $3 \mathrm{~g}(53.4 \mathrm{mg}$, $80 \%, 80 \%$ ee) as a light white solid, m.p. $125-126^{\circ} \mathrm{C}$.

$[\alpha]_{\mathrm{D}}{ }^{24.7}=+6.25$ (c 0.8 , Acetone). ${ }^{1} \mathrm{H}$ NMR $\left(400 \mathrm{MHz}, d_{6}\right.$-Acetone): $\delta 9.55$ (br, $\left.1 \mathrm{H}\right)$ $7.62(\mathrm{~d}, J=8.8 \mathrm{~Hz}, 2 \mathrm{H}), 7.34(\mathrm{~d}, J=8.8 \mathrm{~Hz}, 2 \mathrm{H}), 3.60-3.53(\mathrm{~m}, 1 \mathrm{H}), 3.06-2.73(\mathrm{~m}$, 4H). ${ }^{13} \mathrm{C}$ NMR (100 MHz, $d_{6}$-Acetone): $\delta 168.6,139.8,133.3,127.7(\mathrm{q}, J=274.8 \mathrm{~Hz})$, 122.8, 121.2, 117.3, 39.2, $36.4(\mathrm{q}, J=29.1 \mathrm{~Hz}), 23.1(\mathrm{q}, J=3.2 \mathrm{~Hz}) .{ }^{19} \mathrm{~F}$ NMR $(376$ MHz, $d_{6}$-Acetone): $\delta-60.26\left(\mathrm{t}, J=10.53 \mathrm{~Hz}\right.$ ). HRMS (EI) $\mathrm{m} / \mathrm{z}$ : $[\mathrm{M}]^{+}$Calcd for $\mathrm{C}_{12} \mathrm{H}_{10} \mathrm{BrF}_{3} \mathrm{~N}_{2} \mathrm{O}$ 333.9923; Found 333.9926.

HPLC (AD-H, 0.46*25 cm, $5 \mu \mathrm{m}$, hexane/isopropanol $=90 / 10$, flow $0.7 \mathrm{~mL} / \mathrm{min}$, detection at $214 \mathrm{~nm}$ ) retention time $=25.137 \mathrm{~min}$ (major) and $28.012 \mathrm{~min}$ (minor).<smiles>CC(CC(=O)Nc1cccc(NC(=O)CC(C)(C)C)c1)CC(C)(C)CC(C)CC(F)(C#N)C(F)(F)c1cccc(NC(=O)CC(C)(C)C)c1</smiles>

$3 h$

The reaction was conducted on a $0.2 \mathrm{mmol}$ scale according to the condition $\mathbf{C}$. Column chromatography $(\mathrm{PE}$ : EA = 4:1) yielded the desired product $\mathbf{3 h}(43.7 \mathrm{mg}$, $70 \%, 82 \%$ ee) as a light white solid, m.p. $129-130^{\circ} \mathrm{C}$.

$[\alpha]_{\mathrm{D}}{ }^{23.1}=-7.74$ (c 0.5, Acetone). ${ }^{1} \mathrm{H}$ NMR $\left(400 \mathrm{MHz}, \mathrm{CDCl}_{3}\right): \delta 7.91(\mathrm{br}, 1 \mathrm{H}), 7.47(\mathrm{~s}$, $1 \mathrm{H}), 7.34(\mathrm{~d}, J=8.0 \mathrm{~Hz}, 1 \mathrm{H}), 7.23(\mathrm{t}, J=8.0 \mathrm{~Hz}, 1 \mathrm{H}), 7.17(\mathrm{~d}, J=7.6 \mathrm{~Hz}, 1 \mathrm{H})$, 
3.51-3.44 (m, 1H), 2.84-2.69 (m, 2H), 2.63-2.45 (m, 2H), $1.28(\mathrm{~s}, 9 \mathrm{H}) .{ }^{13} \mathrm{C}$ NMR (100 $\left.\mathrm{MHz}, \mathrm{CDCl}_{3}\right): \delta 165.8,152.4,136.8,128.7,125.0(\mathrm{q}, J=275.9 \mathrm{~Hz}), 122.2,119.4$, 117.5, 117.4, 37.8, $35.2(\mathrm{q}, J=29.6 \mathrm{~Hz}), 31.1,21.8(\mathrm{q}, J=2.6 \mathrm{~Hz}) .{ }^{19} \mathrm{~F}$ NMR $(376$ $\left.\mathrm{MHz}, \mathrm{CDCl}_{3}\right): \delta-64.50\left(\mathrm{t}, J=9.78 \mathrm{~Hz}\right.$ ). HRMS (EI) m/z: $[\mathrm{M}]^{+}$Calcd for $\mathrm{C}_{16} \mathrm{H}_{19} \mathrm{~F}_{3} \mathrm{~N}_{2} \mathrm{O}$ 312.1444; Found 312.1451.

HPLC (AD-H, 0.46*25 cm, $5 \mu \mathrm{m}$, hexane/isopropanol $=90 / 10$, flow $0.7 \mathrm{~mL} / \mathrm{min}$, detection at $214 \mathrm{~nm}$ ) retention time $=10.277 \mathrm{~min}$ (major) and $12.460 \mathrm{~min}$ (minor).<smiles>COc1ccccc1NC(=O)CC(C#N)CC(F)(F)F</smiles>

The reaction was conducted on a $0.2 \mathrm{mmol}$ scale according to the condition $\mathbf{A}$. Column chromatography $(\mathrm{PE}: \mathrm{EA}=4: 1)$ yielded the desired product $3 \mathbf{i}(45.8 \mathrm{mg}, 80 \%$, $72 \%$ ee) as a light white solid, m.p. $127-130^{\circ} \mathrm{C}$.

$[\alpha]_{\mathrm{D}}{ }^{25.4}=-5.62\left(\mathrm{c} 1.0, \mathrm{CHCl}_{3}\right) .{ }^{1} \mathrm{H} \mathrm{NMR}\left(400 \mathrm{MHz}, \mathrm{CDCl}_{3}\right): \delta 8.29(\mathrm{~d}, J=6.8 \mathrm{~Hz}, 1 \mathrm{H})$, 7.86 (br, 1H), 7.08 (t, $J=8.0 \mathrm{~Hz}, 1 \mathrm{H}), 6.96(\mathrm{t}, J=7.6 \mathrm{~Hz}, 1 \mathrm{H}), 6.89$ (d, $J=8.0 \mathrm{~Hz}$, $1 \mathrm{H}), 3.88(\mathrm{~s}, 3 \mathrm{H}), 3.56-3.49(\mathrm{~m}, 1 \mathrm{H}), 2.94-2.74(\mathrm{~m}, 2 \mathrm{H}), 2.64-2.55(\mathrm{~m}, 2 \mathrm{H}) .{ }^{13} \mathrm{C}$ NMR $\left(100 \mathrm{MHz}, \mathrm{CDCl}_{3}\right): \delta 165.2,147.8,126.6,125.0(\mathrm{q}, J=275.7 \mathrm{~Hz}), 124.5,121.0$, 120.0, 119.1, 110.0, 55.6, 38.2, $35.3(\mathrm{q}, J=29.8 \mathrm{~Hz}), 21.7(\mathrm{q}, J=3.0 \mathrm{~Hz}) .{ }^{19} \mathrm{~F} \mathrm{NMR}$ $\left(376 \mathrm{MHz}, \mathrm{CDCl}_{3}\right): \delta-64.49(\mathrm{t}, J=9.4 \mathrm{~Hz})$. HRMS (EI) $\mathrm{m} / \mathrm{z}:[\mathrm{M}]^{+}$Calcd for $\mathrm{C}_{13} \mathrm{H}_{13} \mathrm{~F}_{3} \mathrm{~N}_{2} \mathrm{O}_{2}$ 286.0924; Found 286.0929.

HPLC (AD-H, 0.46*25 cm, $5 \mu \mathrm{m}$, hexane/isopropanol = 90/10, flow $0.7 \mathrm{~mL} / \mathrm{min}$, detection at $214 \mathrm{~nm}$ ) retention time $=19.527 \mathrm{~min}$ (major) and $23.568 \mathrm{~min}$ (minor).<smiles>CCN(C(=O)CC(C#N)CC(F)(F)F)c1ccccc1</smiles>

The reaction was conducted on a $0.2 \mathrm{mmol}$ scale according to the condition $\mathbf{A}$. Column chromatography $(\mathrm{PE}: \mathrm{EA}=4: 1)$ yielded the desired product $\mathbf{3 j}(28.4 \mathrm{mg}$, $50 \%, 59 \%$ ee) as a yellow liquid.

$[\alpha]_{\mathrm{D}}{ }^{23.9}=-7.8556(\mathrm{c} 0.5$, Acetone $) .{ }^{1} \mathrm{H}$ NMR $\left(400 \mathrm{MHz}, \mathrm{CDCl}_{3}\right): \delta 7.49-7.40(\mathrm{~m}, 3 \mathrm{H})$, $7.15(\mathrm{~d}, J=8.0 \mathrm{~Hz}, 2 \mathrm{H}), 3.84-3.7(\mathrm{~m}, 2 \mathrm{H}), 3.46-3.39(\mathrm{~m}, 1 \mathrm{H}), 2.53-2.33(\mathrm{~m}, 4 \mathrm{H})$, 
$1.13(\mathrm{t}, J=7.2 \mathrm{~Hz}, 3 \mathrm{H}) .{ }^{13} \mathrm{C} \mathrm{NMR}\left(100 \mathrm{MHz}, \mathrm{CDCl}_{3}\right): \delta 166.8,140.9,130.2,128.8$, $128.3,125.1(\mathrm{q}, J=275.9 \mathrm{~Hz}), 119.5,44.4,36.0,35.4(\mathrm{q}, J=29.7 \mathrm{~Hz}), 21.9(\mathrm{q}, J=$ 2.7 Hz), 21.9. ${ }^{19} \mathrm{~F}$ NMR (376 MHz, $\left.\mathrm{CDCl}_{3}\right): \delta-64.63(\mathrm{t}, J=10.15 \mathrm{~Hz})$. HRMS (EI) $\mathrm{m} / \mathrm{z}$ : $[\mathrm{M}]^{+}$Calcd for $\mathrm{C}_{14} \mathrm{H}_{15} \mathrm{~F}_{3} \mathrm{~N}_{2} \mathrm{O}$ 284.1131; Found 284.1130.

HPLC (IF-3, 0.46*25 cm, $3 \mu \mathrm{m}$, hexane/isopropanol = 95/5, flow $0.7 \mathrm{~mL} / \mathrm{min}$, detection at $214 \mathrm{~nm}$ ) retention time $=12.830 \mathrm{~min}$ (minor) and $16.538 \mathrm{~min}$ (major).<smiles>CC(C)(C[C@H](C#N)CC(=O)NC(C)(F)c1ccccc1)c1ccccc1</smiles>

The reaction was conducted on a $0.2 \mathrm{mmol}$ scale according to the condition A. Column chromatography $(\mathrm{PE}: \mathrm{EA}=4: 1)$ yielded the desired product $3 \mathrm{k}(50.7 \mathrm{mg}$, $85 \%, 85 \%$ ee) as a light white solid, m.p. $125-127^{\circ} \mathrm{C}$.

$[\alpha]_{\mathrm{D}}{ }^{23.5}=-10.37\left(\mathrm{c} 0.5\right.$, Acetone). ${ }^{1} \mathrm{H}$ NMR $\left(400 \mathrm{MHz}, \mathrm{CDCl}_{3}\right): \delta 7.39-7.32(\mathrm{~m}, 4 \mathrm{H})$, 7.26-7.23 (m, 1H), 6.12 (br, 1H), 3.39-3.32 (m, 1H), 2.61-2.41 (m, 4H), 1.69 (s, 6H). ${ }^{13} \mathrm{C} \mathrm{NMR}\left(100 \mathrm{MHz}, \mathrm{CDCl}_{3}\right): \delta 166.2,146.1,128.5,126.9,125.0(\mathrm{q}, J=275.8 \mathrm{~Hz})$, 124.6, 119.3, 56.4, 37.7, 35.1 (q, $J=29.7 \mathrm{~Hz}), 28.9,28.8,21.9$ (q, $J=3.1 \mathrm{~Hz}) .{ }^{19} \mathrm{~F}$ NMR (376 MHz, $\left.\mathrm{CDCl}_{3}\right): \delta-64.38(\mathrm{t}, J=10.15 \mathrm{~Hz})$. HRMS (EI) m/z: [M] ${ }^{+}$Calcd for $\mathrm{C}_{15} \mathrm{H}_{17} \mathrm{~F}_{3} \mathrm{~N}_{2} \mathrm{O} 298.1287$; Found 298.1297.

HPLC (AD-H, 0.46*25 cm, $5 \mu \mathrm{m}$, hexane/isopropanol = 90/10, flow $0.7 \mathrm{~mL} / \mathrm{min}$, detection at $214 \mathrm{~nm}$ ) retention time $=9.717 \mathrm{~min}$ (major) and $10.025 \mathrm{~min}$ (minor).<smiles>N#CC(CC(=O)Oc1ccccc1)CC(F)(F)F</smiles>

The reaction was conducted on a $0.2 \mathrm{mmol}$ scale according to the condition $\mathbf{A}$. Column chromatography $(\mathrm{PE}: \mathrm{EA}=10: 1)$ yielded the desired product $4 \mathbf{a}(45.2 \mathrm{mg}$, $88 \%, 85 \%$ ee) as a light white solid, m.p. $40-42{ }^{\circ} \mathrm{C}$.

$[\alpha]_{\mathrm{D}}^{25.6}=+2.13\left(\mathrm{c} 1.0, \mathrm{CHCl}_{3}\right) .{ }^{1} \mathrm{H}$ NMR $\left(400 \mathrm{MHz}, \mathrm{CDCl}_{3}\right): \delta 7.41(\mathrm{t}, J=8.4 \mathrm{~Hz}$, 2H), $7.28(\mathrm{~d}, J=7.2 \mathrm{~Hz}, 1 \mathrm{H}), 7.11(\mathrm{~d}, J=7.6 \mathrm{~Hz}, 2 \mathrm{H}), 3.46-3.39(\mathrm{~m}, 1 \mathrm{H}), 3.10-2.96$ (m, 2H), 2.76-2.51 (m, 2H). ${ }^{13} \mathrm{C}$ NMR (100 MHz, $\left.\mathrm{CDCl}_{3}\right): \delta$ 167.5, 150.0, 129.6, $126.5,124.9$ (q, $J=274.8 \mathrm{~Hz}), 121.2,118.5,35.5,35.3(\mathrm{q}, J=30.1 \mathrm{~Hz}), 20.8(\mathrm{q}, J=$ $3.1 \mathrm{~Hz}) .{ }^{19} \mathrm{~F}$ NMR $\left(376 \mathrm{MHz}, \mathrm{CDCl}_{3}\right): \delta-64.61(\mathrm{t}, J=9.78 \mathrm{~Hz})$. HRMS (EI) m/z: 
$[\mathrm{M}]^{+}$Calcd for $\mathrm{C}_{12} \mathrm{H}_{10} \mathrm{~F}_{3} \mathrm{NO}_{2}$ 257.0658; Found 257.0657.

HPLC (AD-H, 0.46*25 cm, $5 \mu \mathrm{m}$, hexane/isopropanol = 90/10, flow $0.7 \mathrm{~mL} / \mathrm{min}$, detection at $214 \mathrm{~nm}$ ) retention time $=11.162 \mathrm{~min}$ (major) and $11.833 \mathrm{~min}$ (minor).<smiles>COc1ccc(OC(=O)CC(C#N)C[13CH3])cc1</smiles>

The reaction was conducted on a $0.2 \mathrm{mmol}$ scale according to the condition $\mathbf{A}$. Column chromatography $(\mathrm{PE}: \mathrm{EA}=10: 1)$ yielded the desired product $\mathbf{4 b}(54.5 \mathrm{mg}$, $95 \%, 86 \%$ ee) as a light white solid, m.p. $40-43^{\circ} \mathrm{C}$.

$[\alpha]_{\mathrm{D}}{ }^{25.9}=+18.37\left(\mathrm{c} 0.5, \mathrm{CHCl}_{3}\right) .{ }^{1} \mathrm{H} \mathrm{NMR}\left(400 \mathrm{MHz}, \mathrm{CDCl}_{3}\right): \delta 7.03(\mathrm{~d}, J=9.2 \mathrm{~Hz}$, 2H), $6.90(\mathrm{~d}, J=8.8 \mathrm{~Hz}, 2 \mathrm{H}), 3.81(\mathrm{~s}, 3 \mathrm{H}), 3.47-3.40(\mathrm{~m}, 1 \mathrm{H}), 3.10-2.96(\mathrm{~m}, 2 \mathrm{H})$, 2.77-2.52 (m, 2H). ${ }^{13} \mathrm{C} \mathrm{NMR}\left(100 \mathrm{MHz}, \mathrm{CDCl}_{3}\right): \delta 167.8,157.7,143.5,124.9$ (q, $J=$ $274.6 \mathrm{~Hz}), 122.0,118.4,114.6,55.6,35.7,35.4(\mathrm{q}, J=29.5 \mathrm{~Hz}), 20.8(\mathrm{q}, J=3.0 \mathrm{~Hz})$. ${ }^{19} \mathrm{~F} \mathrm{NMR}\left(376 \mathrm{MHz}, \mathrm{CDCl}_{3}\right): \delta-64.58(\mathrm{t}, J=10.15 \mathrm{~Hz})$. HRMS (EI) m/z: [M] ${ }^{+}$Calcd for $\mathrm{C}_{13} \mathrm{H}_{12} \mathrm{~F}_{3} \mathrm{NO}_{3} 287.0764$; Found 287.0765.

HPLC (IC-3, 0.46*15 cm, $3 \mu \mathrm{m}$, hexane/isopropanol = 95/5, flow $0.7 \mathrm{~mL} / \mathrm{min}$, detection at $214 \mathrm{~nm}$ ) retention time $=27.493 \mathrm{~min}$ (major) and $35.078 \mathrm{~min}$ (minor).<smiles>CC(C)(C)c1ccc(OC(=O)C[C@H](C#N)CC(F)(F)F)cc1</smiles>

The reaction was conducted on a $0.2 \mathrm{mmol}$ scale according to the condition $\mathbf{B}$. Column chromatography (PE: $\mathrm{EA}=10: 1)$ yielded the desired product $4 \mathrm{c}(52.0 \mathrm{mg}$, $83 \%, 91 \%$ ee) as a light white solid, m.p. $42-44^{\circ} \mathrm{C}$.

$[\alpha]_{\mathrm{D}}{ }^{25.9}=+11.19\left(\mathrm{c} 1.0, \mathrm{CHCl}_{3}\right) .{ }^{1} \mathrm{H} \mathrm{NMR}\left(400 \mathrm{MHz}, \mathrm{CDCl}_{3}\right): \delta 7.41(\mathrm{~d}, J=8.8 \mathrm{~Hz}$, 2H), $7.03(\mathrm{~d}, J=8.8 \mathrm{~Hz}, 2 \mathrm{H}), 3.47-3.40(\mathrm{~m}, 1 \mathrm{H}), 3.11-2.97(\mathrm{~m}, 2 \mathrm{H}), 2.77-2.53(\mathrm{~m}$, 2H), 1.32 (s, 9H). ${ }^{13} \mathrm{C}$ NMR (100 MHz, $\left.\mathrm{CDCl}_{3}\right): \delta 167.7,149.4,147.6,126.5,124.9$ $(\mathrm{q}, J=276.1 \mathrm{~Hz}), 120.5,118.5,35.7,35.3(\mathrm{q}, J=29.7 \mathrm{~Hz}), 34.5,31.3,21.8(\mathrm{q}, J=3.0$ Hz). ${ }^{19} \mathrm{~F} \mathrm{NMR}\left(376 \mathrm{MHz}, \mathrm{CDCl}_{3}\right): \delta-64.59$ (t, $\left.J=9.02 \mathrm{~Hz}\right)$. HRMS (EI) m/z: $[\mathrm{M}]^{+}$ Calcd for $\mathrm{C}_{16} \mathrm{H}_{18} \mathrm{~F}_{3} \mathrm{NO}_{2}$ 313.1284; Found 313.1284.

HPLC (AD-H, 0.46*25 cm, $5 \mu \mathrm{m}$, hexane/isopropanol = 95/5, flow $0.7 \mathrm{~mL} / \mathrm{min}$, detection at $214 \mathrm{~nm}$ ) retention time $=14.235 \mathrm{~min}$ (major) and $15.563 \mathrm{~min}$ (minor). 
<smiles>N#CC(C[18F])CC(=O)Oc1ccc(F)cc1</smiles>

The reaction was conducted on a $0.2 \mathrm{mmol}$ scale according to the condition $\mathbf{A}$. Column chromatography (PE: EA = 10:1) yielded the desired product 4d $(49.5 \mathrm{mg}$, $90 \%, 79 \%$ ee) as a light white solid, m.p. $41-43{ }^{\circ} \mathrm{C}$.

$[\alpha]_{\mathrm{D}}^{25.9}=+8.13\left(\mathrm{c} 1.0, \mathrm{CHCl}_{3}\right) .{ }^{1} \mathrm{H}$ NMR $\left(400 \mathrm{MHz}, \mathrm{CDCl}_{3}\right): \delta 7.08(\mathrm{~d}, J=6.4 \mathrm{~Hz}$, $4 \mathrm{H}), 3.46-3.39(\mathrm{~m}, 1 \mathrm{H}), 3.09-2.96(\mathrm{~m}, 2 \mathrm{H}), 2.76-2.51(\mathrm{~m}, 2 \mathrm{H}) .{ }^{13} \mathrm{C}$ NMR $(100 \mathrm{MHz}$, $\left.\mathrm{CDCl}_{3}\right): \delta 167.5,160.5(\mathrm{~d}, J=244.5 \mathrm{~Hz}), 145.8(\mathrm{~d}, J=2.7 \mathrm{~Hz}), 124.8(\mathrm{q}, J=276.00$ $\mathrm{Hz}), 122.7$ (d, $J=8.6 \mathrm{~Hz}), 118.4,116.3$ (d, $J=23.7 \mathrm{~Hz}), 35.6,35.2$ (q, $J=30.7 \mathrm{~Hz})$,

$21.7(\mathrm{q}, J=3.5 \mathrm{~Hz}) .{ }^{19} \mathrm{~F}$ NMR $\left(376 \mathrm{MHz}, \mathrm{CDCl}_{3}\right): \delta-64.60(\mathrm{t}, J=9.78 \mathrm{~Hz})$, 115.94-115.88 (m). HRMS (EI) m/z: [M] $]^{+}$Calcd for $\mathrm{C}_{12} \mathrm{H}_{9} \mathrm{~F}_{4} \mathrm{NO}_{2}$ 275.0564; Found 275.0563.

HPLC (IC-3, 0.46*15 cm, $3 \mu \mathrm{m}$, hexane/isopropanol = 95/5, flow $0.7 \mathrm{~mL} / \mathrm{min}$, detection at $214 \mathrm{~nm}$ ) retention time $=12.983 \mathrm{~min}$ (major) and $15.200 \mathrm{~min}$ (minor).<smiles>N#CC(CC(=O)Oc1ccc(Cl)cc1)C[13C](F)(F)F</smiles>

The reaction was conducted on a $0.2 \mathrm{mmol}$ scale according to the condition $\mathbf{B}$. Column chromatography $(\mathrm{PE}: \mathrm{EA}=10: 1)$ yielded the desired product $4 \mathrm{e}(51.2 \mathrm{mg}$, $88 \%, 81 \%$ ee) as a light white solid, m.p. $41-42^{\circ} \mathrm{C}$.

$[\alpha]_{\mathrm{D}}{ }^{25.9}=+22.61\left(\mathrm{c} 1.0, \mathrm{CHCl}_{3}\right) .{ }^{1} \mathrm{H} \mathrm{NMR}\left(400 \mathrm{MHz}, \mathrm{CDCl}_{3}\right): \delta 7.37(\mathrm{~d}, J=8.8 \mathrm{~Hz}$, $2 \mathrm{H}), 7.07(\mathrm{~d}, J=8.8 \mathrm{~Hz}, 2 \mathrm{H}) 3.47-3.40(\mathrm{~m}, 1 \mathrm{H}), 3.11-2.98(\mathrm{~m}, 2 \mathrm{H}), 2.77-2.52(\mathrm{~m}, 2 \mathrm{H})$. ${ }^{13} \mathrm{C}$ NMR (100 MHz, $\left.\mathrm{CDCl}_{3}\right): \delta 167.3,148.4,131.9,129.7,124.8(\mathrm{q}, J=275.9 \mathrm{~Hz})$, 122.6, 118.3, 35.6, $35.3(\mathrm{q}, J=30.1 \mathrm{~Hz}), 21.8(\mathrm{q}, J=3.2 \mathrm{~Hz}) .{ }^{19} \mathrm{~F}$ NMR $(376 \mathrm{MHz}$, $\left.\mathrm{CDCl}_{3}\right): \delta-64.54(\mathrm{t}, J=8.65 \mathrm{~Hz})$. HRMS (EI) m/z: $[\mathrm{M}]^{+}$Calcd for $\mathrm{C}_{12} \mathrm{H}_{9} \mathrm{ClF}_{3} \mathrm{NO}_{2}$ 291.0268; Found 291.0268.

HPLC (IC-3, 0.46*15 cm, $3 \mu \mathrm{m}$, hexane/isopropanol = 95/5, flow $0.7 \mathrm{~mL} / \mathrm{min}$, detection at $214 \mathrm{~nm}$ ) retention time $=13.792 \mathrm{~min}$ (major) and $16.375 \mathrm{~min}$ (minor).<smiles>COc1ccccc1OC(=O)CC(C#N)CC(F)(F)F</smiles> 
The reaction was conducted on a $0.2 \mathrm{mmol}$ scale according to the condition $\mathbf{A}$. Column chromatography $(\mathrm{PE}: \mathrm{EA}=10: 1)$ yielded the desired product $\mathbf{4 f}(43.6 \mathrm{mg}$, $76 \%, 86 \%$ ee) as a yellow liquid.

$[\alpha]_{\mathrm{D}}^{25.9}=+8.85\left(\mathrm{c} 1.0, \mathrm{CHCl}_{3}\right) .{ }^{1} \mathrm{H}$ NMR $\left(400 \mathrm{MHz}, \mathrm{CDCl}_{3}\right): \delta 7.24(\mathrm{~d}, J=8.0 \mathrm{~Hz}$, 1H), $7.06(\mathrm{~d}, J=7.6 \mathrm{~Hz}, 1 \mathrm{H}), 7.00-6.95(\mathrm{~m}, 2 \mathrm{H}), 3.83(\mathrm{~s}, 3 \mathrm{H}), 3.49-3.43(\mathrm{~m}, 1 \mathrm{H})$, 3.14-2.97 (m, 2H), 2.77-2.59 (m, 2H). ${ }^{13} \mathrm{C} \mathrm{NMR}\left(100 \mathrm{MHz}, \mathrm{CDCl}_{3}\right): \delta$ 166.9, 150.6, $139.0,127.5,124.9$ (q, $J=275.9 \mathrm{~Hz}), 122.4,120.9,118.4,112.4,55.7,35.6,35.3$ (q, $J$ $=30.2 \mathrm{~Hz}), 21.9(\mathrm{q}, J=3.4 \mathrm{~Hz}) .{ }^{19} \mathrm{~F}$ NMR $\left(376 \mathrm{MHz}, \mathrm{CDCl}_{3}\right): \delta-64.71(\mathrm{t}, J=9.02$ Hz). HRMS (EI) m/z: [M] $]^{+}$Calcd for $\mathrm{C}_{13} \mathrm{H}_{12} \mathrm{~F}_{3} \mathrm{NO}_{3}$ 287.0764; Found 287.0763.

HPLC (IC-3, 0.46*15 cm, $3 \mu \mathrm{m}$, hexane/isopropanol = 95/5, flow $0.7 \mathrm{~mL} / \mathrm{min}$, detection at $214 \mathrm{~nm}$ ) retention time $=18.193 \mathrm{~min}$ (major) and $21.235 \mathrm{~min}$ (minor).<smiles>CC(C)(C)c1ccccc1OC(=O)C[C@H](C#N)CC(F)(F)F</smiles>

The reaction was conducted on a $0.2 \mathrm{mmol}$ scale according to the condition $\mathbf{A}$. Column chromatography $(\mathrm{PE}: \mathrm{EA}=10: 1)$ yielded the desired product $\mathbf{4 g}(59.5 \mathrm{mg}$, $95 \%, 78 \%$ ee) as a yellow liquid.

$[\alpha]_{\mathrm{D}}^{25.9}=+9.15\left(\mathrm{c} 0.6, \mathrm{CHCl}_{3}\right) .{ }^{1} \mathrm{H}$ NMR $\left(400 \mathrm{MHz}, \mathrm{CDCl}_{3}\right): \delta 7.42(\mathrm{~d}, J=8.0 \mathrm{~Hz}$, $1 \mathrm{H}), 6.19-7.25(\mathrm{~m}, 2 \mathrm{H}), 6.99(\mathrm{~d}, J=7.6 \mathrm{~Hz}, 1 \mathrm{H}) 3.50-3.43(\mathrm{~m}, 1 \mathrm{H}), 3.16-3.01(\mathrm{~m}$, 2H), 2.78-2.55 (m, 2H), $1.35(\mathrm{~s}, 9 \mathrm{H}) .{ }^{13} \mathrm{C} \mathrm{NMR}\left(100 \mathrm{MHz}, \mathrm{CDCl}_{3}\right): \delta 167.8,148.5$, $140.8,127.5,127.1,126.4,126.3,124.9$ (q, $J=275.5 \mathrm{~Hz}), 118.4,36.1,35.2$ (q, $J=$ $29.6 \mathrm{~Hz}), 34.4,30.2,21.7$ (q, $J=3.0 \mathrm{~Hz}) .{ }^{19} \mathrm{~F} \mathrm{NMR}\left(376 \mathrm{MHz}, \mathrm{CDCl}_{3}\right) \delta-64.49(\mathrm{t}, J$ $=10.15 \mathrm{~Hz}$ ). HRMS (EI) m/z: $[\mathrm{M}]^{+}$Calcd for $\mathrm{C}_{16} \mathrm{H}_{18} \mathrm{~F}_{3} \mathrm{NO}_{2}$ 313.1284; Found 313.1282 .

HPLC (IC-3, 0.46*15 cm, $3 \mu \mathrm{m}$, hexane/isopropanol = 95/5, flow $0.7 \mathrm{~mL} / \mathrm{min}$, detection at $214 \mathrm{~nm}$ ) retention time $=12.830 \mathrm{~min}$ (major) and $14.518 \mathrm{~min}$ (minor).<smiles>N#C[C@H](CC(=O)Oc1cccc(F)c1)CC(F)(F)F</smiles>

The reaction was conducted on a $0.2 \mathrm{mmol}$ scale according to the condition $\mathbf{B}$. 
Column chromatography $(\mathrm{PE}: \mathrm{EA}=10: 1)$ yielded the desired product $\mathbf{4 h}(49.5 \mathrm{mg}$, $90 \%, 80 \%$ ee) as a light white solid, m.p. $39-41^{\circ} \mathrm{C}$.

$[\alpha]_{\mathrm{D}}^{25.4}=+10.00\left(\mathrm{c} 1.0, \mathrm{CHCl}_{3}\right) .{ }^{1} \mathrm{H}$ NMR $\left(400 \mathrm{MHz}, \mathrm{CDCl}_{3}\right): \delta 7.39-7.34(\mathrm{~m}, 1 \mathrm{H})$, 7.02-6.88 (m, 3H), 3.46-3.39 (m, 1H), 3.10-2.97 (m, 2H), 2.76-2.51 (m, 2H). ${ }^{13} \mathrm{C}$ NMR (100 MHz, $\left.\mathrm{CDCl}_{3}\right): \delta 167.1,162.8(\mathrm{~d}, J=247.1 \mathrm{~Hz}), 150.6(\mathrm{~d}, J=10.6 \mathrm{~Hz})$, $130.4(\mathrm{~d}, J=9.3 \mathrm{~Hz}), 124.8(\mathrm{q}, J=276.4 \mathrm{~Hz}), 118.4,117.0(\mathrm{~d}, J=3.2 \mathrm{~Hz}), 113.5$ (d, $J$ $=20.8 \mathrm{~Hz}), 109.4(\mathrm{~d}, J=24.6 \mathrm{~Hz}), 35.6,35.2(\mathrm{q}, J=30.0 \mathrm{~Hz}), 21.7(\mathrm{q}, J=3.2 \mathrm{~Hz})$.

${ }^{19} \mathrm{~F}$ NMR (376 MHz, $\left.\mathrm{CDCl}_{3}\right): \delta-64.6(\mathrm{t}, J=10.15 \mathrm{~Hz}),-110.47--110.404(\mathrm{~m})$. HRMS (EI) m/z: [M] $]^{+}$Calcd for $\mathrm{C}_{12} \mathrm{H}_{9} \mathrm{~F}_{4} \mathrm{NO}_{2}$ 275.0564; Found 271.056.

HPLC (IC-3, 0.46*15 cm, $3 \mu \mathrm{m}$, hexane/isopropanol = 95/5, flow $0.7 \mathrm{~mL} / \mathrm{min}$, detection at $214 \mathrm{~nm}$ ) retention time $=13.148 \mathrm{~min}$ (major) and $14.758 \mathrm{~min}$ (minor).<smiles>N#C[C@H](CC(=O)Oc1c(Br)cccc1Br)CC(F)(F)F</smiles>

The reaction was conducted on a $0.2 \mathrm{mmol}$ scale according to the condition $\mathbf{A}$. Column chromatography $(\mathrm{PE}: \mathrm{EA}=10: 1)$ yielded the desired product $4 \mathbf{i}(66.1 \mathrm{mg}$, $80 \%, 81 \%$ ee) as a light white solid, m.p. $41-44^{\circ} \mathrm{C}$.

$[\alpha]_{\mathrm{D}}^{25.9}=+8.32\left(\mathrm{c} 1.0, \mathrm{CHCl}_{3}\right) .{ }^{1} \mathrm{H}$ NMR $\left(400 \mathrm{MHz}, \mathrm{CDCl}_{3}\right): \delta 7.59(\mathrm{~d}, J=8.0 \mathrm{~Hz}$, 2H), $7.07(\mathrm{t}, J=8.4 \mathrm{~Hz}, 1 \mathrm{H}), 3.53-3.45(\mathrm{~m}, 1 \mathrm{H}), 3.27-3.10(\mathrm{~m}, 2 \mathrm{H}), 2.80-2.59(\mathrm{~m}$, $2 \mathrm{H}) .{ }^{13} \mathrm{C} \mathrm{NMR}\left(100 \mathrm{MHz}, \mathrm{CDCl}_{3}\right): \delta 165.5,145.5,132.5,128.8,124.8$ (q, $J=276.3$ $\mathrm{Hz}), 118.1,117.3,35.3,35.3(\mathrm{q}, J=29.6 \mathrm{~Hz}), 21.6(\mathrm{q}, J=3.3 \mathrm{~Hz}) .{ }^{19} \mathrm{~F}$ NMR $(376$ $\left.\mathrm{MHz}, \mathrm{CDCl}_{3}\right): \delta-64.49\left(\mathrm{t}, J=10.53 \mathrm{~Hz}\right.$ ). HRMS (EI) $\mathrm{m} / \mathrm{z}:[\mathrm{M}]^{+}$Calcd for $\mathrm{C}_{12} \mathrm{H}_{8} \mathrm{BrF}_{3} \mathrm{NO}_{2} 412.8868$; Found 412.8864 .

HPLC (IC-3, 0.46*15 cm, $3 \mu \mathrm{m}$, hexane/isopropanol = 95/5, flow $0.7 \mathrm{~mL} / \mathrm{min}$, detection at $214 \mathrm{~nm}$ ) retention time $=12.927$ min (major) and $14.593 \mathrm{~min}$ (minor).<smiles>[14CH3]C[C@H](C#N)CC(=O)Oc1ccc2c(c1)OCO2</smiles>

The reaction was conducted on a $0.2 \mathrm{mmol}$ scale according to the condition $\mathbf{A}$. Column chromatography $(\mathrm{PE}: \mathrm{EA}=4: 1)$ yielded the desired product $\mathbf{4 j}(54.2 \mathrm{mg}$, $90 \%, 81 \%$ ee) as a light white solid, m.p. $43-45^{\circ} \mathrm{C}$. 
$[\alpha]_{\mathrm{D}}{ }^{19.8}=+13.26\left(\mathrm{c} 1.0, \mathrm{CHCl}_{3}\right) .{ }^{1} \mathrm{H} \mathrm{NMR}\left(400 \mathrm{MHz}, \mathrm{CDCl}_{3}\right): \delta 6.78(\mathrm{~d}, J=8.4 \mathrm{~Hz}$, $1 \mathrm{H}), 6.62(\mathrm{~d}, J=2.4 \mathrm{~Hz}, 1 \mathrm{H}), 6.55(\mathrm{dd}, J=8.4,2.0 \mathrm{~Hz}, 1 \mathrm{H}), 5.99$ (s, 2H) 3.43-3.38 (m, 1H), 3.03-2.94 (m, 2H), 2.73-2.53 (m, 2H). $\left.{ }^{13} \mathrm{C} \mathrm{NMR} \mathrm{(100} \mathrm{MHz,} \mathrm{CDCl}_{3}\right): \delta 167.7$, 148.1, 145.8, 144.2, 124.9 (q, $J=275.6$ Hz), 118.4, 113.6, 108.0, 103.3, 101.9, 35.6, $35.3(\mathrm{q}, J=29.4 \mathrm{~Hz}), 21.8(\mathrm{q}, J=3.6 \mathrm{~Hz}) .{ }^{19} \mathrm{~F} \mathrm{NMR}\left(376 \mathrm{MHz}, \mathrm{CDCl}_{3}\right): \delta-64.59(\mathrm{t}$, $J=10.53 \mathrm{~Hz}$ ). HRMS (EI) m/z: [M] $]^{+}$Calcd for $\mathrm{C}_{13} \mathrm{H}_{10} \mathrm{~F}_{3} \mathrm{NO}_{4}$ 301.0556; Found 301.0554 .

HPLC (IC-3, 0.46*15 cm, $3 \mu \mathrm{m}$, hexane/isopropanol = 90/10, flow $0.7 \mathrm{~mL} / \mathrm{min}$, detection at $214 \mathrm{~nm}$ ) retention time $=23.868 \mathrm{~min}$ (major) and $28.350 \mathrm{~min}$ (minor).

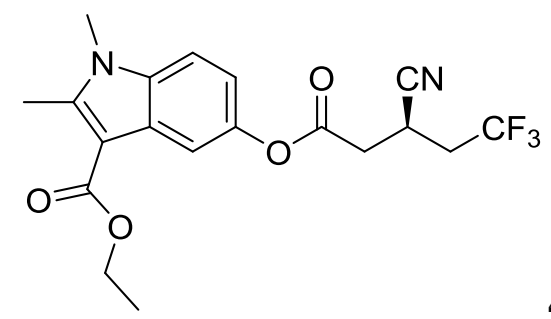

The reaction was conducted on a $0.2 \mathrm{mmol}$ scale according to the condition $\mathbf{A}$. Column chromatography $(\mathrm{PE}: \mathrm{EA}=4: 1)$ yielded the desired product $\mathbf{4 k}(39.6 \mathrm{mg}$, $50 \%, 81 \%$ ee) as a light white solid, m.p. $88-90{ }^{\circ} \mathrm{C}$.

$[\alpha]_{\mathrm{D}}^{20.3}=+5.24\left(\mathrm{c} 0.5, \mathrm{CHCl}_{3}\right) .{ }^{1} \mathrm{H}$ NMR $\left(400 \mathrm{MHz} \mathrm{CDCl}_{3}\right): \delta 7.82(\mathrm{~d}, J=2.0 \mathrm{~Hz}$, $1 \mathrm{H}), 7.25(\mathrm{~d}, J=8.8 \mathrm{~Hz}, 1 \mathrm{H}), 6.95(\mathrm{dd}, J=8.8,2.0 \mathrm{~Hz}, 1 \mathrm{H}), 4.38(\mathrm{q}, J=7.2 \mathrm{~Hz}, 2 \mathrm{H})$, $3.66(\mathrm{~s}, 3 \mathrm{H}), 3.49-3.42(\mathrm{~m}, 1 \mathrm{H}), 3.13-2.98(\mathrm{~m}, 2 \mathrm{H}), 2.76(\mathrm{~s}, 3 \mathrm{H}), 2.74-2.53(\mathrm{~m}, 2 \mathrm{H})$, $1.43(\mathrm{t}, J=7.2 \mathrm{~Hz}, 3 \mathrm{H}) .{ }^{13} \mathrm{C} \mathrm{NMR}\left(100 \mathrm{MHz}, \mathrm{CDCl}_{3}\right): \delta 168.1,165.7,146.6,145.2$, 134.6, 127.1, 124.9 (q, $J=275.6 \mathrm{~Hz}), 118.5,115.5,113.6,109.6,104.4,59.5,35.8$, $35.4(\mathrm{q}, J=29.5 \mathrm{~Hz}), 29.8,21.9(\mathrm{q}, J=3.0 \mathrm{~Hz}), 14.6,12.0 .{ }^{19} \mathrm{~F}$ NMR $(376 \mathrm{MHz}$, $\mathrm{CDCl}_{3}$ ): $\delta-64.60\left(\mathrm{t}, J=9.02 \mathrm{~Hz}\right.$ ). HRMS (EI) m/z: $[\mathrm{M}]^{+}$Calcd for $\mathrm{C}_{19} \mathrm{H}_{19} \mathrm{~F}_{3} \mathrm{~N}_{2} \mathrm{O}_{4}$ 396.1291; Found 396.1292.

HPLC (ID-3, 0.46*15 cm, $3 \mu \mathrm{m}$, hexane/isopropanol $=70 / 30$, flow $0.7 \mathrm{~mL} / \mathrm{min}$, detection at $214 \mathrm{~nm}$ ) retention time $=22.568 \mathrm{~min}$ (major) and $36.548 \mathrm{~min}$ (minor).

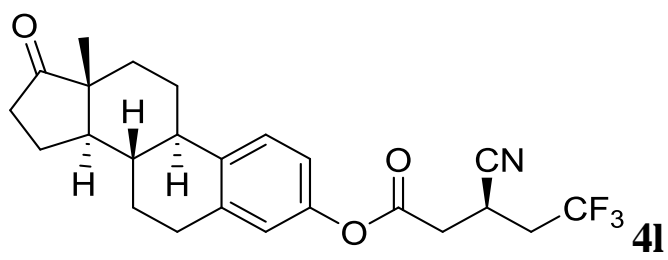


The reaction was conducted on a $0.2 \mathrm{mmol}$ scale according to the condition $\mathbf{A}$. Column chromatography (PE: EA=4:1) yielded the desired product $\mathbf{4 l}(62.4 \mathrm{mg}, 72 \%$, $81 \%$ de) as a light white solid, m.p. $106-109^{\circ} \mathrm{C}$.

$[\alpha]_{\mathrm{D}}^{25.0}=+103.54\left(\mathrm{c} 1.0, \mathrm{CHCl}_{3}\right) .{ }^{1} \mathrm{H}$ NMR $\left(400 \mathrm{MHz}, \mathrm{CDCl}_{3}\right): \delta 7.31(\mathrm{~d}, J=8.4 \mathrm{~Hz}$, $1 \mathrm{H}), 6.87(\mathrm{~d}, J=8.4 \mathrm{~Hz}, 1 \mathrm{H}), 6.84(\mathrm{~s}, 1 \mathrm{H}), 3.47-3.40(\mathrm{~m}, 1 \mathrm{H}), 3.10-2.90(\mathrm{~m}, 4 \mathrm{H})$, 2.76-1.95 (m, 9H), 1.68-1.40 (m, 6H), $\left.0.91(\mathrm{~s}, 3 \mathrm{H}) .{ }^{13} \mathrm{C} \mathrm{NMR} \mathrm{(100} \mathrm{MHz,} \mathrm{CDCl}_{3}\right): \delta$ 202.6, 167.7, 147.9, 138.3, 138.1, 126.6, 124.9 (q, $J=275.8$ Hz), 121.2, 118.4, 118.30, 50.4, 47.9, 44.1, 37.9, 35.8, 35.7, $35.4(\mathrm{q}, J=29.8 \mathrm{~Hz}), 31.5,29.4,26.2,25.7,21.8(\mathrm{q}$, $J=2.0 \mathrm{~Hz}), 21.5,13.8 .{ }^{19} \mathrm{~F} \mathrm{NMR}\left(376 \mathrm{MHz}, \mathrm{CDCl}_{3}\right): \delta-64.58(\mathrm{t}, J=9.40 \mathrm{~Hz})$. HRMS (EI) m/z: [M] $]^{+}$Calcd for $\mathrm{C}_{24} \mathrm{H}_{26} \mathrm{~F}_{3} \mathrm{NO}_{3}$ 433.1859; Found 433.1863.

HPLC (IF-3, 0.46*15 cm, $3 \mu \mathrm{m}$, hexane/isopropanol = 80/20, flow $0.7 \mathrm{~mL} / \mathrm{min}$, detection at $214 \mathrm{~nm}$ ) retention time $=15.398 \mathrm{~min}$ (major) and $26.180 \mathrm{~min}$ (minor).<smiles>N#CC(CCC(=O)c1ccccc1)CC(F)(F)F</smiles>

The reaction was conducted on a $0.2 \mathrm{mmol}$ scale according to the condition $\mathbf{A}$. Column chromatography (PE: EA = 10:1) yielded the desired product $\mathbf{2 w}(45.9 \mathrm{mg}$, $90 \%, 59 \%$ ee) as a light white solid, m.p. $40-43{ }^{\circ} \mathrm{C}$.

$[\alpha]_{\mathrm{D}}{ }^{24.8}=+20.81\left(\mathrm{c} 1.0, \mathrm{CHCl}_{3}\right) .{ }^{1} \mathrm{H}$ NMR $\left(400 \mathrm{MHz}, \mathrm{CDCl}_{3}\right): \delta 7.97(\mathrm{~d}, J=7.6 \mathrm{~Hz}$, 2H), $7.61(\mathrm{t}, J=7.6 \mathrm{~Hz}, 1 \mathrm{H}), 7.49(\mathrm{t}, J=7.2 \mathrm{~Hz}, 2 \mathrm{H}), 3.28(\mathrm{t}, J=6.8 \mathrm{~Hz}, 2 \mathrm{H})$ 3.11-3.19 (m, 1H) 2.37-2.66 (m, 2H), 1.99-2.31 (m, 2H). ${ }^{13} \mathrm{C}$ NMR (100 MHz, $\left.\mathrm{CDCl}_{3}\right): \delta 197.6,136.2,133.7,125.0(\mathrm{q}, J=275.6 \mathrm{~Hz}), 128.8,128.0,119.4,36.7$ (q, $J$ $=21.9 \mathrm{~Hz}), 35.0,26.3,25.1(\mathrm{q}, J=3.2 \mathrm{~Hz}) .{ }^{19} \mathrm{~F} \mathrm{NMR}\left(376 \mathrm{MHz}, \mathrm{CDCl}_{3}\right): \delta-64.79(\mathrm{t}$, $J=10.15 \mathrm{~Hz}$ ). HRMS (EI) m/z: $[\mathrm{M}]^{+}$Calcd for $\mathrm{C}_{13} \mathrm{H}_{12} \mathrm{~F}_{3} \mathrm{NO}$ 255.0866; Found 255.0865 .

HPLC (IC-3, 0.46*15 cm, $3 \mu \mathrm{m}$, hexane/isopropanol = 90/10, flow $0.7 \mathrm{~mL} / \mathrm{min}$, detection at $214 \mathrm{~nm}$ ) retention time $=11.265 \mathrm{~min}$ (major) and $12.232 \mathrm{~min}$ (minor).<smiles>N#C[C@H](CCCC(=O)c1ccccc1)CC(F)(F)F</smiles> 
The reaction was conducted on a $0.2 \mathrm{mmol}$ scale according to the condition $\mathbf{A}$. Column chromatography $(\mathrm{PE}: \mathrm{EA}=10: 1)$ yielded the desired product $\mathbf{2 x}(47.4 \mathrm{mg}$, $88 \%, 53 \%$ ee) as a light white solid, m.p. $38-40{ }^{\circ} \mathrm{C}$.

$[\alpha]_{\mathrm{D}}{ }^{24.1}=+3.66\left(\mathrm{c} 1.0, \mathrm{CHCl}_{3}\right) .{ }^{1} \mathrm{H}$ NMR $\left(400 \mathrm{MHz}, \mathrm{CDCl}_{3}\right): \delta 7.95(\mathrm{~d}, J=7.6 \mathrm{~Hz}$, 2H), $7.58(\mathrm{t}, J=6.8 \mathrm{~Hz}, 1 \mathrm{H}), 7.47(\mathrm{t}, J=7.6 \mathrm{~Hz}, 2 \mathrm{H}), 3.07(\mathrm{t}, J=6.8 \mathrm{~Hz}, 2 \mathrm{H})$, 2.97-2.90 (m, 1H), 2.62-2.32 (m, 2H), 2.08-1.73 (m, 4H). ${ }^{13} \mathrm{C}$ NMR (100 MHz, $\left.\mathrm{CDCl}_{3}\right): \delta 198.7,136.5,133.3,128.7,127.9,125.1(\mathrm{q}, J=276.1 \mathrm{~Hz}), 119.5,37.1,36.3$ $(\mathrm{q}, J=29.9 \mathrm{~Hz}), 31.5,25.6(\mathrm{q}, J=3.2 \mathrm{~Hz}), 20.1 .{ }^{19} \mathrm{~F}$ NMR $\left(376 \mathrm{MHz}, \mathrm{CDCl}_{3}\right): \delta$ -64.91 (t, $J=9.78 \mathrm{~Hz}$ ). HRMS (EI) m/z: $[\mathrm{M}]^{+}$Calcd for $\mathrm{C}_{14} \mathrm{H}_{14} \mathrm{~F}_{3} \mathrm{NO}$ 269.1022; Found 269.1024.

HPLC (AD-H, 0.46*25 cm, $5 \mu \mathrm{m}$, hexane/isopropanol = 90/10, flow $0.7 \mathrm{~mL} / \mathrm{min}$, detection at $214 \mathrm{~nm}$ ) retention time $=20.332 \mathrm{~min}$ (minor) and $22.417 \mathrm{~min}$ (major).<smiles>N#CC(CCCCC(=O)c1ccccc1)CC(F)(F)F</smiles>

The reaction was conducted on a $0.2 \mathrm{mmol}$ scale according to the condition $\mathbf{A}$. Column chromatography (PE: EA = 10:1) yielded the desired product $\mathbf{2 y}(48.1 \mathrm{mg}$, $85 \%, 17 \%$ ee) as a light white solid, m.p. $45-48^{\circ} \mathrm{C}$.

$[\alpha]_{\mathrm{D}}{ }^{25.1}=+2.04\left(\mathrm{c} 1.0, \mathrm{CHCl}_{3}\right) .{ }^{1} \mathrm{H} \mathrm{NMR}\left(400 \mathrm{MHz}, \mathrm{CDCl}_{3}\right): \delta 7.95(\mathrm{~d}, J=7.2 \mathrm{~Hz}$, 2H), $7.57(\mathrm{t}, J=7.2 \mathrm{~Hz}, 1 \mathrm{H}), 7.47(\mathrm{t}, J=7.6 \mathrm{~Hz}, 2 \mathrm{H}), 3.02(\mathrm{t}, J=7.2 \mathrm{~Hz}, 2 \mathrm{H})$, 2.94-2.87 (m, 1H), 2.60-2.28 (m, 2H), 1.89-1.51 (m, 6H). ${ }^{13} \mathrm{C}$ NMR (100 MHz, $\left.\mathrm{CDCl}_{3}\right): \delta 199.5,136.8,133.1,128.6,128.0,125.1(\mathrm{q}, J=275.4 \mathrm{~Hz}), 119.6,37.9,36.4$ $(\mathrm{q}, J=30.2 \mathrm{~Hz}), 32.0,26.4,25.5(\mathrm{q}, J=2.8 \mathrm{~Hz}), 23.2 .{ }^{19} \mathrm{~F} \mathrm{NMR}\left(376 \mathrm{MHz}, \mathrm{CDCl}_{3}\right): \delta$ $-64.93(\mathrm{t}, J=10.15 \mathrm{~Hz})$. HRMS (ESI) $\mathrm{m} / \mathrm{z}$ : $[\mathrm{M}+\mathrm{Na}]^{+}$Calcd for $\mathrm{C}_{15} \mathrm{H}_{16} \mathrm{NOF}_{3} \mathrm{Na}$ 306.1076; Found 306.1078.

HPLC (IC-3, 0.46*15 cm, $3 \mu \mathrm{m}$, hexane/isopropanol = 90/10, flow $0.7 \mathrm{~mL} / \mathrm{min}$, detection at $214 \mathrm{~nm}$ ) retention time $=25.905 \mathrm{~min}$ (major) and $28.588 \mathrm{~min}$ (minor).<smiles>N#C[C@H](CCc1ccccc1)CC(F)(F)F</smiles> 
The reaction was conducted on a $0.2 \mathrm{mmol}$ scale according to the condition $\mathbf{A}$. Column chromatography $(\mathrm{PE}: \mathrm{EA}=10: 1)$ yielded the desired product 9 (40.9 mg, $90 \%, 11 \%$ ee) as yellow liquid.

$[\alpha]_{\mathrm{D}}{ }^{18.8}=+2.77\left(\mathrm{c} 0.5, \mathrm{CHCl}_{3}\right) .{ }^{1} \mathrm{H} \mathrm{NMR}\left(400 \mathrm{MHz}, \mathrm{CDCl}_{3}\right): \delta 7.33(\mathrm{t}, J=7.6 \mathrm{~Hz}$, 2H), 7.27-7.19 (m, 3H), 2.98-2.91 (m, 1H), 2.87-2.75 (m, 2H), 2.61-2.27 (m, 2H), 1.94-2.09 (m, 2H). ${ }^{13} \mathrm{C}$ NMR (100 MHz, $\left.\mathrm{CDCl}_{3}\right): \delta 139.1,128.8,128.4,126.8,125.0$ $(\mathrm{q}, J=275.7 \mathrm{~Hz}), 119.4,36.4(\mathrm{q}, J=30.2 \mathrm{~Hz}), 33.7,32.8,24.9$ (q, $J=2.9 \mathrm{~Hz}) .{ }^{19} \mathrm{~F}$ NMR (376 MHz, $\left.\mathrm{CDCl}_{3}\right): \delta-64.77\left(\mathrm{t}, J=9.02 \mathrm{~Hz}\right.$ ). HRMS (EI) m/z: [M] ${ }^{+}$Calcd for $\mathrm{C}_{12} \mathrm{H}_{12} \mathrm{~F}_{3} \mathrm{~N}$ 227.0916; Found 227.0920.

HPLC (IA, $0.46^{*} 25 \mathrm{~cm}, 5 \mu \mathrm{m}$, hexane/isopropanol $=99 / 1$, flow $0.7 \mathrm{~mL} / \mathrm{min}$, detection at $214 \mathrm{~nm}$ ) retention time $=17.178 \mathrm{~min}$ (minor) and $21.433 \mathrm{~min}$ (major).<smiles>N#C[C@H](CC(F)F)CS(=O)(=O)c1ccccc1</smiles>

The reaction was conducted on a $0.2 \mathrm{mmol}$ scale according to the condition $\mathbf{A}$. Column chromatography $(\mathrm{PE}: \mathrm{EA}=4: 1)$ yielded the desired product $10(30.5 \mathrm{mg}$, $55 \%, 50 \%$ ee) as a light white solid, m.p. $66-68^{\circ} \mathrm{C}$.

$[\alpha]_{\mathrm{D}}^{22.2}=+7.16\left(\mathrm{c} 0.5, \mathrm{CHCl}_{3}\right) .{ }^{1} \mathrm{H}$ NMR $\left(400 \mathrm{MHz} \mathrm{CDCl}_{3}\right): \delta 7.96(\mathrm{~d}, J=7.6 \mathrm{~Hz}$, 2H), $7.76(\mathrm{t}, J=7.2 \mathrm{~Hz}, 1 \mathrm{H}), 7.65(\mathrm{~d}, J=8.0 \mathrm{~Hz}, 2 \mathrm{H}), 3.54-3.34(\mathrm{~m}, 3 \mathrm{H}), 2.91-2.61$ $(\mathrm{m}, 2 \mathrm{H}) .{ }^{13} \mathrm{C} \mathrm{NMR}\left(100 \mathrm{MHz}, \mathrm{CDCl}_{3}\right): \delta 137.8,135.0,129.9,128.3,124.5(\mathrm{q}, J=$ $276.1 \mathrm{~Hz}), 116.7,55.8,35.5(\mathrm{q}, J=30 \mathrm{~Hz}), 20.9(\mathrm{q}, J=2.9 \mathrm{~Hz}) .{ }^{19} \mathrm{~F}$ NMR $(376 \mathrm{MHz}$, $\left.\mathrm{CDCl}_{3}\right): \delta-64.50\left(\mathrm{t}, J=9.4 \mathrm{~Hz}\right.$ ). HRMS (EI) m/z: $[\mathrm{M}]^{+}$Calcd for $\mathrm{C}_{11} \mathrm{H}_{10} \mathrm{~F}_{3} \mathrm{NO}_{2} \mathrm{~S}$ 277.0379; Found 277.0375.

HPLC (IC-3, 0.46*15 cm, $3 \mu \mathrm{m}$, hexane/isopropanol = 80/20, flow $0.7 \mathrm{~mL} / \mathrm{min}$, detection at $214 \mathrm{~nm}$ ) retention time $=21.588 \mathrm{~min}$ (major) and $23.250 \mathrm{~min}$ (minor).<smiles>N#C[C@H](CC(F)F)CS(=O)(=O)Oc1ccccc1</smiles>

The reaction was conducted on a $0.2 \mathrm{mmol}$ scale according to the condition $\mathbf{A}$. Column chromatography $(\mathrm{PE}: \mathrm{EA}=4: 1)$ yielded the desired product $11(41.0 \mathrm{mg}$, $70 \%, 29 \%$ ee) as a light white solid, m.p. $70-71^{\circ} \mathrm{C}$. 
$[\alpha]_{\mathrm{D}}{ }^{24.7}=+0.57\left(\mathrm{c} 0.7, \mathrm{CHCl}_{3}\right) .{ }^{1} \mathrm{H}$ NMR $\left(400 \mathrm{MHz}, \mathrm{CDCl}_{3}\right): \delta 7.46(\mathrm{t}, J=8.0 \mathrm{~Hz}$, 2H), $7.38(\mathrm{t}, J=7.6 \mathrm{~Hz}, 1 \mathrm{H}), 7.32(\mathrm{~d}, J=8.0 \mathrm{~Hz}, 2 \mathrm{H}) 3.74-3.51(\mathrm{~m}, 3 \mathrm{H}), 2.86-2.68$ (m, 2H). ${ }^{13} \mathrm{C}$ NMR (100 MHz, $\left.\mathrm{CDCl}_{3}\right): \delta 148.5,130.3,128.0,124.5$ (q, $\left.J=275.9 \mathrm{~Hz}\right)$, 121.8, 116.5, 50.2, $35.4(\mathrm{q}, J=30.5 \mathrm{~Hz}), 22.0(\mathrm{q}, J=3.4 \mathrm{~Hz}) .{ }^{19} \mathrm{~F}$ NMR $(376 \mathrm{MHz}$, $\mathrm{CDCl}_{3}$ ): $\delta-64.37\left(\mathrm{t}, J=9.4 \mathrm{~Hz}\right.$ ). HRMS (EI) m/z: $[\mathrm{M}]^{+}$Calcd for $\mathrm{C}_{11} \mathrm{H}_{10} \mathrm{~F}_{3} \mathrm{NO}_{3} \mathrm{~S}$ 293.0328; Found 293.0325.

HPLC (OD-H, 0.46*25 cm, $5 \mu \mathrm{m}$, hexane/isopropanol = 80/20, flow $0.7 \mathrm{~mL} / \mathrm{min}$, detection at $214 \mathrm{~nm}$ ) retention time $=8.710 \mathrm{~min}$ (minor) and $10.356 \mathrm{~min}$ (major).

\section{Single Crystal X-Ray Diffraction Data (3g)}

ORTEP of $3 g$ (CCDC 2011013)<smiles>N#CC(CC(=O)Nc1ccc(Br)cc1)CC(F)(F)F</smiles>

$3 g$

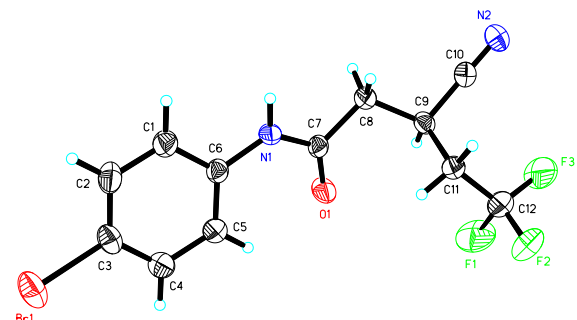

drawn at 30\% ellipsoid contour levels

A crystal of $\mathbf{3 g}$ was obtained by recrystallization from PE/EA and was analyzed on a Bruker APEX-II CCD at $293 \mathrm{~K}$. 
Table 1. Crystal data and structure refinement for mo_d8v19667_0m.

Identification code

Empirical formula

Formula weight

Temperature

Wavelength

Crystal system

Space group

Unit cell dimensions

Volume

Z

Density (calculated)

Absorption coefficient

$\mathrm{F}(000)$

Crystal size

Theta range for data collection

Index ranges

Reflections collected

Independent reflections

Completeness to theta $=25.242^{\circ}$

Absorption correction

Max. and min. transmission

Refinement method

Data / restraints / parameters

Goodness-of-fit on $\mathrm{F}^{2}$

Final R indices [I $>2 \operatorname{sigma}(\mathrm{I})]$

$\mathrm{R}$ indices (all data)

Absolute structure parameter

Extinction coefficient

Largest diff. peak and hole mo_d8v19667_0m

C12 H10 Br F3 N2 O

335.13

293(2) K

$0.71073 \AA$

Orthorhombic

P 212121

$\begin{array}{ll}\mathrm{a}=5.1828(6) \AA & \alpha=90^{\circ} . \\ \mathrm{b}=10.8817(11) \AA & \beta=90^{\circ} . \\ \mathrm{c}=23.519(3) \AA & \gamma=90^{\circ} .\end{array}$

1326.4(3) $\AA^{3}$

4

$1.678 \mathrm{Mg} / \mathrm{m}^{3}$

$3.127 \mathrm{~mm}^{-1}$

664

$0.170 \times 0.140 \times 0.120 \mathrm{~mm}^{3}$

2.550 to $25.999^{\circ}$.

$-6<=\mathrm{h}<=6,-11<=\mathrm{k}<=13,-23<=\mathrm{l}<=29$

6276

$2586[\mathrm{R}(\mathrm{int})=0.0356]$

$99.4 \%$

Semi-empirical from equivalents

0.7456 and 0.3389

Full-matrix least-squares on $\mathrm{F}^{2}$

$2586 / 0 / 172$

1.022

$\mathrm{R} 1=0.0347, \mathrm{wR} 2=0.0768$

$\mathrm{R} 1=0.0508, \mathrm{wR} 2=0.0824$

$0.054(10)$

$\mathrm{n} / \mathrm{a}$

0.337 and -0.273 e. $\AA^{-3}$ 
Table 2. Atomic coordinates (x 10 $)$ and equivalent isotropic displacement parameters $\left(\AA^{2} \times 10^{3}\right)$ for mo_d8v19667_0m. U(eq) is defined as one third of the trace of the orthogonalized Uij tensor.

\begin{tabular}{|c|c|c|c|c|}
\hline & $\mathrm{x}$ & $\mathrm{y}$ & $\mathrm{z}$ & $\mathrm{U}(\mathrm{eq})$ \\
\hline $\operatorname{Br}(1)$ & $11527(1)$ & $-710(1)$ & $5196(1)$ & $84(1)$ \\
\hline $\mathrm{F}(1)$ & $4952(8)$ & $7197(3)$ & $3412(2)$ & $118(1)$ \\
\hline $\mathrm{F}(2)$ & $1773(8)$ & $7434(3)$ & $3954(2)$ & $112(1)$ \\
\hline $\mathrm{F}(3)$ & $1288(11)$ & $7550(3)$ & $3052(2)$ & $151(2)$ \\
\hline $\mathrm{O}(1)$ & $6884(5)$ & $3920(3)$ & $3390(1)$ & $62(1)$ \\
\hline $\mathrm{N}(1)$ & $4846(6)$ & 2101(3) & $3524(2)$ & $49(1)$ \\
\hline $\mathrm{N}(2)$ & $-861(8)$ & $5565(4)$ & $2118(2)$ & $69(1)$ \\
\hline $\mathrm{C}(1)$ & $6067(9)$ & $250(4)$ & $4001(2)$ & $59(1)$ \\
\hline$C(2)$ & $7541(9)$ & $-406(4)$ & $4378(2)$ & $64(1)$ \\
\hline $\mathrm{C}(3)$ & $9489(8)$ & $180(4)$ & $4660(2)$ & $56(1)$ \\
\hline $\mathrm{C}(4)$ & $9993(10)$ & 1393(4) & $4577(2)$ & $59(1)$ \\
\hline$C(5)$ & $8489(9)$ & 2061(4) & $4194(2)$ & $54(1)$ \\
\hline$C(6)$ & $6520(8)$ & $1486(3)$ & $3907(2)$ & $45(1)$ \\
\hline$C(7)$ & $5073(7)$ & $3229(4)$ & $3302(2)$ & $43(1)$ \\
\hline $\mathrm{C}(8)$ & $2832(8)$ & $3600(3)$ & $2922(2)$ & $49(1)$ \\
\hline $\mathrm{C}(9)$ & $2635(7)$ & $5003(4)$ & $2871(2)$ & $45(1)$ \\
\hline$C(10)$ & $679(8)$ & $5318(4)$ & $2439(2)$ & $52(1)$ \\
\hline $\mathrm{C}(11)$ & 1946(8) & $5604(3)$ & $3442(2)$ & $50(1)$ \\
\hline $\mathrm{C}(12)$ & $2423(11)$ & $6951(5)$ & $3459(3)$ & $76(2)$ \\
\hline
\end{tabular}


Table 3. Bond lengths $[\AA]$ and angles $\left[{ }^{\circ}\right]$ for mo_d8v19667_0m.

\begin{tabular}{|c|c|}
\hline $\operatorname{Br}(1)-C(3)$ & $1.909(4)$ \\
\hline $\mathrm{F}(1)-\mathrm{C}(12)$ & $1.342(7)$ \\
\hline $\mathrm{F}(2)-\mathrm{C}(12)$ & $1.321(7)$ \\
\hline $\mathrm{F}(3)-\mathrm{C}(12)$ & $1.299(6)$ \\
\hline $\mathrm{O}(1)-\mathrm{C}(7)$ & $1.220(5)$ \\
\hline $\mathrm{N}(1)-\mathrm{C}(7)$ & $1.339(5)$ \\
\hline $\mathrm{N}(1)-\mathrm{C}(6)$ & $1.419(5)$ \\
\hline $\mathrm{N}(1)-\mathrm{H}(1)$ & 0.8600 \\
\hline $\mathrm{N}(2)-\mathrm{C}(10)$ & $1.131(5)$ \\
\hline$C(1)-C(2)$ & $1.371(6)$ \\
\hline$C(1)-C(6)$ & $1.383(6)$ \\
\hline $\mathrm{C}(1)-\mathrm{H}(1 \mathrm{~A})$ & 0.9300 \\
\hline $\mathrm{C}(2)-\mathrm{C}(3)$ & $1.366(6)$ \\
\hline $\mathrm{C}(2)-\mathrm{H}(2)$ & 0.9300 \\
\hline $\mathrm{C}(3)-\mathrm{C}(4)$ & $1.359(6)$ \\
\hline$C(4)-C(5)$ & $1.396(6)$ \\
\hline $\mathrm{C}(4)-\mathrm{H}(4)$ & 0.9300 \\
\hline$C(5)-C(6)$ & $1.374(6)$ \\
\hline $\mathrm{C}(5)-\mathrm{H}(5)$ & 0.9300 \\
\hline$C(7)-C(8)$ & $1.521(5)$ \\
\hline $\mathrm{C}(8)-\mathrm{C}(9)$ & $1.535(5)$ \\
\hline $\mathrm{C}(8)-\mathrm{H}(8 \mathrm{~A})$ & 0.9700 \\
\hline $\mathrm{C}(8)-\mathrm{H}(8 \mathrm{~B})$ & 0.9700 \\
\hline $\mathrm{C}(9)-\mathrm{C}(10)$ & $1.476(6)$ \\
\hline $\mathrm{C}(9)-\mathrm{C}(11)$ & $1.537(6)$ \\
\hline $\mathrm{C}(9)-\mathrm{H}(9)$ & 0.9800 \\
\hline $\mathrm{C}(11)-\mathrm{C}(12)$ & $1.487(6)$ \\
\hline $\mathrm{C}(11)-\mathrm{H}(11 \mathrm{~A})$ & 0.9700 \\
\hline $\mathrm{C}(11)-\mathrm{H}(11 \mathrm{~B})$ & 0.9700 \\
\hline $\mathrm{C}(7)-\mathrm{N}(1)-\mathrm{C}(6)$ & $128.7(3)$ \\
\hline $\mathrm{C}(7)-\mathrm{N}(1)-\mathrm{H}(1)$ & 115.6 \\
\hline $\mathrm{C}(6)-\mathrm{N}(1)-\mathrm{H}(1)$ & 115.6 \\
\hline$C(2)-C(1)-C(6)$ & $121.0(5)$ \\
\hline $\mathrm{C}(2)-\mathrm{C}(1)-\mathrm{H}(1 \mathrm{~A})$ & 119.5 \\
\hline $\mathrm{C}(6)-\mathrm{C}(1)-\mathrm{H}(1 \mathrm{~A})$ & 119.5 \\
\hline
\end{tabular}




\begin{tabular}{|c|c|}
\hline$C(3)-C(2)-C(1)$ & $118.8(4)$ \\
\hline $\mathrm{C}(3)-\mathrm{C}(2)-\mathrm{H}(2)$ & 120.6 \\
\hline $\mathrm{C}(1)-\mathrm{C}(2)-\mathrm{H}(2)$ & 120.6 \\
\hline $\mathrm{C}(4)-\mathrm{C}(3)-\mathrm{C}(2)$ & $121.7(4)$ \\
\hline $\mathrm{C}(4)-\mathrm{C}(3)-\mathrm{Br}(1)$ & $118.7(4)$ \\
\hline $\mathrm{C}(2)-\mathrm{C}(3)-\mathrm{Br}(1)$ & $119.5(3)$ \\
\hline$C(3)-C(4)-C(5)$ & $119.4(5)$ \\
\hline $\mathrm{C}(3)-\mathrm{C}(4)-\mathrm{H}(4)$ & 120.3 \\
\hline $\mathrm{C}(5)-\mathrm{C}(4)-\mathrm{H}(4)$ & 120.3 \\
\hline$C(6)-C(5)-C(4)$ & $119.7(4)$ \\
\hline $\mathrm{C}(6)-\mathrm{C}(5)-\mathrm{H}(5)$ & 120.2 \\
\hline $\mathrm{C}(4)-\mathrm{C}(5)-\mathrm{H}(5)$ & 120.2 \\
\hline$C(5)-C(6)-C(1)$ & $119.3(4)$ \\
\hline$C(5)-C(6)-N(1)$ & $123.5(3)$ \\
\hline $\mathrm{C}(1)-\mathrm{C}(6)-\mathrm{N}(1)$ & $117.2(4)$ \\
\hline $\mathrm{O}(1)-\mathrm{C}(7)-\mathrm{N}(1)$ & $124.5(4)$ \\
\hline $\mathrm{O}(1)-\mathrm{C}(7)-\mathrm{C}(8)$ & $121.6(3)$ \\
\hline $\mathrm{N}(1)-\mathrm{C}(7)-\mathrm{C}(8)$ & $113.9(3)$ \\
\hline $\mathrm{C}(7)-\mathrm{C}(8)-\mathrm{C}(9)$ & 111.1(3) \\
\hline $\mathrm{C}(7)-\mathrm{C}(8)-\mathrm{H}(8 \mathrm{~A})$ & 109.4 \\
\hline $\mathrm{C}(9)-\mathrm{C}(8)-\mathrm{H}(8 \mathrm{~A})$ & 109.4 \\
\hline $\mathrm{C}(7)-\mathrm{C}(8)-\mathrm{H}(8 \mathrm{~B})$ & 109.4 \\
\hline $\mathrm{C}(9)-\mathrm{C}(8)-\mathrm{H}(8 \mathrm{~B})$ & 109.4 \\
\hline $\mathrm{H}(8 \mathrm{~A})-\mathrm{C}(8)-\mathrm{H}(8 \mathrm{~B})$ & 108.0 \\
\hline $\mathrm{C}(10)-\mathrm{C}(9)-\mathrm{C}(8)$ & 109.3(3) \\
\hline $\mathrm{C}(10)-\mathrm{C}(9)-\mathrm{C}(11)$ & $110.1(3)$ \\
\hline $\mathrm{C}(8)-\mathrm{C}(9)-\mathrm{C}(11)$ & $111.7(4)$ \\
\hline $\mathrm{C}(10)-\mathrm{C}(9)-\mathrm{H}(9)$ & 108.5 \\
\hline $\mathrm{C}(8)-\mathrm{C}(9)-\mathrm{H}(9)$ & 108.5 \\
\hline $\mathrm{C}(11)-\mathrm{C}(9)-\mathrm{H}(9)$ & 108.5 \\
\hline $\mathrm{N}(2)-\mathrm{C}(10)-\mathrm{C}(9)$ & $178.3(5)$ \\
\hline $\mathrm{C}(12)-\mathrm{C}(11)-\mathrm{C}(9)$ & $113.8(4)$ \\
\hline $\mathrm{C}(12)-\mathrm{C}(11)-\mathrm{H}(11 \mathrm{~A})$ & 108.8 \\
\hline $\mathrm{C}(9)-\mathrm{C}(11)-\mathrm{H}(11 \mathrm{~A})$ & 108.8 \\
\hline $\mathrm{C}(12)-\mathrm{C}(11)-\mathrm{H}(11 \mathrm{~B})$ & 108.8 \\
\hline $\mathrm{C}(9)-\mathrm{C}(11)-\mathrm{H}(11 \mathrm{~B})$ & 108.8 \\
\hline $\mathrm{H}(11 \mathrm{~A})-\mathrm{C}(11)-\mathrm{H}(11 \mathrm{~B})$ & 107.7 \\
\hline $\mathrm{F}(3)-\mathrm{C}(12)-\mathrm{F}(2)$ & $109.5(5)$ \\
\hline
\end{tabular}




$\begin{array}{ll}F(3)-C(12)-F(1) & 106.3(6) \\ F(2)-C(12)-F(1) & 104.0(5) \\ F(3)-C(12)-C(11) & 113.6(5) \\ F(2)-C(12)-C(11) & 112.0(5) \\ F(1)-C(12)-C(11) & 110.9(4)\end{array}$

Symmetry transformations used to generate equivalent atoms: 
Table 4. Anisotropic displacement parameters $\left(\AA^{2} \mathrm{x} 10^{3}\right)$ for mo_d8v19667_0m. The anisotropic displacement factor exponent takes the form: $\quad-2 \pi^{2}\left[\mathrm{~h}^{2} \mathrm{a}^{* 2} \mathrm{U}^{11}+\ldots \quad+2 \mathrm{hk} \mathrm{a*} \mathrm{b}^{*} \mathrm{U}^{12}\right]$

\begin{tabular}{|c|c|c|c|c|c|c|}
\hline & $\mathrm{U}^{11}$ & $\mathrm{U}^{22}$ & $\mathrm{U}^{33}$ & $\mathrm{U}^{23}$ & $\mathrm{U}^{13}$ & $\mathrm{U}^{12}$ \\
\hline $\operatorname{Br}(1)$ & $91(1)$ & $99(1)$ & $62(1)$ & $24(1)$ & $-2(1)$ & $26(1)$ \\
\hline $\mathrm{F}(1)$ & $119(3)$ & $90(2)$ & $144(3)$ & $-3(3)$ & $-6(3)$ & $-52(2)$ \\
\hline $\mathrm{F}(2)$ & $134(3)$ & $74(2)$ & $128(3)$ & $-46(2)$ & $-14(3)$ & $13(2)$ \\
\hline $\mathrm{F}(3)$ & $249(5)$ & $47(2)$ & $157(3)$ & $4(2)$ & $-113(4)$ & $22(3)$ \\
\hline $\mathrm{O}(1)$ & $40(2)$ & $53(2)$ & $92(2)$ & $14(2)$ & $-14(2)$ & $-10(1)$ \\
\hline $\mathrm{N}(1)$ & $40(2)$ & $40(2)$ & 68(2) & $1(2)$ & $-15(2)$ & $-6(2)$ \\
\hline $\mathrm{N}(2)$ & $71(3)$ & $67(2)$ & 70(3) & $6(2)$ & $-19(2)$ & $13(2)$ \\
\hline $\mathrm{C}(1)$ & $52(3)$ & $47(2)$ & $79(3)$ & $4(2)$ & $-9(2)$ & $-3(2)$ \\
\hline $\mathrm{C}(2)$ & $65(3)$ & $46(2)$ & $80(3)$ & $11(2)$ & $0(3)$ & $6(2)$ \\
\hline$C(3)$ & $55(3)$ & $66(3)$ & $47(3)$ & $12(2)$ & $3(2)$ & $12(2)$ \\
\hline $\mathrm{C}(4)$ & $60(3)$ & $61(3)$ & $56(3)$ & $-1(2)$ & $-4(2)$ & $2(2)$ \\
\hline$C(5)$ & $56(2)$ & $49(2)$ & $57(3)$ & $0(2)$ & $-6(3)$ & $-2(2)$ \\
\hline$C(6)$ & $39(2)$ & $42(2)$ & $54(2)$ & $-2(2)$ & $1(2)$ & $4(2)$ \\
\hline $\mathrm{C}(7)$ & $34(2)$ & $40(2)$ & $55(3)$ & $-4(2)$ & $1(2)$ & $5(2)$ \\
\hline $\mathrm{C}(8)$ & $43(2)$ & $45(2)$ & $61(3)$ & $-2(2)$ & $-7(2)$ & $1(2)$ \\
\hline $\mathrm{C}(9)$ & $34(2)$ & $46(2)$ & $55(3)$ & $3(2)$ & $-3(2)$ & $1(2)$ \\
\hline$C(10)$ & $54(3)$ & $47(2)$ & $55(3)$ & $2(2)$ & $1(2)$ & $5(2)$ \\
\hline $\mathrm{C}(11)$ & $47(2)$ & $43(2)$ & $60(3)$ & $-4(2)$ & $-6(2)$ & $0(2)$ \\
\hline$C(12)$ & $85(4)$ & $49(3)$ & $94(5)$ & $-10(3)$ & $-23(3)$ & 1(3) \\
\hline
\end{tabular}


Table 5. Hydrogen coordinates $\left(\mathrm{x} 10^{4}\right)$ and isotropic displacement parameters $\left(\AA^{2} \times 10^{3}\right)$ for mo_d8v19667_0m.

\begin{tabular}{|c|c|c|c|c|}
\hline & $\mathrm{x}$ & $\mathrm{y}$ & $\mathrm{z}$ & $\mathrm{U}(\mathrm{eq})$ \\
\hline $\mathrm{H}(1)$ & 3505 & 1694 & 3420 & 59 \\
\hline $\mathrm{H}(1 \mathrm{~A})$ & 4741 & -141 & 3806 & 71 \\
\hline $\mathrm{H}(2)$ & 7220 & -1236 & 4441 & 76 \\
\hline $\mathrm{H}(4)$ & 11328 & 1775 & 4774 & 71 \\
\hline $\mathrm{H}(5)$ & 8817 & 2891 & 4133 & 65 \\
\hline $\mathrm{H}(8 \mathrm{~A})$ & 1237 & 3277 & 3077 & 59 \\
\hline $\mathrm{H}(8 \mathrm{~B})$ & 3072 & 3246 & 2547 & 59 \\
\hline $\mathrm{H}(9)$ & 4309 & 5323 & 2745 & 54 \\
\hline $\mathrm{H}(11 \mathrm{~A})$ & 137 & 5454 & 3522 & 60 \\
\hline $\mathrm{H}(11 \mathrm{~B})$ & 2947 & 5215 & 3741 & 60 \\
\hline
\end{tabular}


Table 6. Torsion angles $\left[{ }^{\circ}\right]$ for mo_d8v19667_0m.

\begin{tabular}{|c|c|}
\hline$C(6)-C(1)-C(2)-C(3)$ & $-0.3(7)$ \\
\hline $\mathrm{C}(1)-\mathrm{C}(2)-\mathrm{C}(3)-\mathrm{C}(4)$ & $0.2(7)$ \\
\hline $\mathrm{C}(1)-\mathrm{C}(2)-\mathrm{C}(3)-\mathrm{Br}(1)$ & $178.9(4)$ \\
\hline $\mathrm{C}(2)-\mathrm{C}(3)-\mathrm{C}(4)-\mathrm{C}(5)$ & $-0.1(7)$ \\
\hline $\mathrm{Br}(1)-\mathrm{C}(3)-\mathrm{C}(4)-\mathrm{C}(5)$ & $-178.9(3)$ \\
\hline$C(3)-C(4)-C(5)-C(6)$ & $0.1(7)$ \\
\hline$C(4)-C(5)-C(6)-C(1)$ & $-0.2(7)$ \\
\hline $\mathrm{C}(4)-\mathrm{C}(5)-\mathrm{C}(6)-\mathrm{N}(1)$ & $177.9(4)$ \\
\hline$C(2)-C(1)-C(6)-C(5)$ & $0.3(7)$ \\
\hline$C(2)-C(1)-C(6)-N(1)$ & $-178.0(4)$ \\
\hline $\mathrm{C}(7)-\mathrm{N}(1)-\mathrm{C}(6)-\mathrm{C}(5)$ & $11.8(7)$ \\
\hline $\mathrm{C}(7)-\mathrm{N}(1)-\mathrm{C}(6)-\mathrm{C}(1)$ & $-170.0(4)$ \\
\hline $\mathrm{C}(6)-\mathrm{N}(1)-\mathrm{C}(7)-\mathrm{O}(1)$ & $2.2(7)$ \\
\hline $\mathrm{C}(6)-\mathrm{N}(1)-\mathrm{C}(7)-\mathrm{C}(8)$ & $-177.8(4)$ \\
\hline $\mathrm{O}(1)-\mathrm{C}(7)-\mathrm{C}(8)-\mathrm{C}(9)$ & $-21.9(6)$ \\
\hline $\mathrm{N}(1)-\mathrm{C}(7)-\mathrm{C}(8)-\mathrm{C}(9)$ & $158.0(4)$ \\
\hline$C(7)-C(8)-C(9)-C(10)$ & $172.3(3)$ \\
\hline$C(7)-C(8)-C(9)-C(11)$ & $-65.6(4)$ \\
\hline $\mathrm{C}(10)-\mathrm{C}(9)-\mathrm{C}(11)-\mathrm{C}(12)$ & $-74.1(4)$ \\
\hline$C(8)-C(9)-C(11)-C(12)$ & $164.2(4)$ \\
\hline $\mathrm{C}(9)-\mathrm{C}(11)-\mathrm{C}(12)-\mathrm{F}(3)$ & $54.5(6)$ \\
\hline$C(9)-C(11)-C(12)-F(2)$ & $179.1(4)$ \\
\hline $\mathrm{C}(9)-\mathrm{C}(11)-\mathrm{C}(12)-\mathrm{F}(1)$ & $-65.2(6)$ \\
\hline
\end{tabular}

Symmetry transformations used to generate equivalent atoms: 
Table 7. Hydrogen bonds for mo_d8v19667_0m [ $\AA$ and $\left.{ }^{\circ}\right]$.

\begin{tabular}{lllll}
\hline $\mathrm{D}-\mathrm{H} \ldots \mathrm{A}$ & $\mathrm{d}(\mathrm{D}-\mathrm{H})$ & $\mathrm{d}(\mathrm{H} \ldots \mathrm{A})$ & $\mathrm{d}(\mathrm{D} \ldots \mathrm{A})$ & $<(\mathrm{DHA})$ \\
\hline $\mathrm{C}(11)-\mathrm{H}(11 \mathrm{~B}) \ldots \mathrm{O}(1)$ & 0.97 & 2.61 & $3.151(5)$ & 115.2 \\
$\mathrm{C}(11)-\mathrm{H}(11 \mathrm{~A}) \ldots \mathrm{O}(1) \# 1$ & 0.97 & 2.39 & $3.202(5)$ & 140.7 \\
$\mathrm{C}(8)-\mathrm{H}(8 \mathrm{~A}) \ldots \mathrm{O}(1) \# 1$ & 0.97 & 2.47 & $3.292(5)$ & 142.0 \\
$\mathrm{C}(5)-\mathrm{H}(5) \ldots \mathrm{O}(1)$ & 0.93 & 2.30 & $2.890(5)$ & 120.6 \\
$\mathrm{~N}(1)-\mathrm{H}(1) \ldots \mathrm{N}(2) \# 2$ & 0.86 & 2.23 & $3.055(5)$ & 160.2 \\
$\mathrm{C}(11)-\mathrm{H}(11 \mathrm{~B}) \ldots \mathrm{O}(1)$ & 0.97 & 2.61 & $3.151(5)$ & 115.2 \\
$\mathrm{C}(11)-\mathrm{H}(11 \mathrm{~A}) \ldots \mathrm{O}(1) \# 1$ & 0.97 & 2.39 & $3.202(5)$ & 140.7 \\
$\mathrm{C}(8)-\mathrm{H}(8 \mathrm{~A}) \ldots \mathrm{O}(1) \# 1$ & 0.97 & 2.47 & $3.292(5)$ & 142.0 \\
$\mathrm{C}(5)-\mathrm{H}(5) \ldots \mathrm{O}(1)$ & 0.93 & 2.30 & $2.890(5)$ & 120.6 \\
$\mathrm{~N}(1)-\mathrm{H}(1) \ldots \mathrm{N}(2) \# 2$ & 0.86 & 2.23 & $3.055(5)$ & 160.2 \\
$\mathrm{~N}(1)-\mathrm{H}(1) \ldots \mathrm{N}(2) \# 2$ & 0.86 & 2.23 & $3.055(5)$ & 160.2 \\
$\mathrm{C}(5)-\mathrm{H}(5) \ldots \mathrm{O}(1)$ & 0.93 & 2.30 & $2.890(5)$ & 120.6 \\
$\mathrm{C}(8)-\mathrm{H}(8 \mathrm{~A}) \ldots \mathrm{O}(1) \# 1$ & 0.97 & 2.47 & $3.292(5)$ & 142.0 \\
$\mathrm{C}(11)-\mathrm{H}(11 \mathrm{~A}) \ldots \mathrm{O}(1) \# 1$ & 0.97 & 0.97 & $3.202(5)$ & 140.7 \\
$\mathrm{C}(11)-\mathrm{H}(11 \mathrm{~B}) \ldots \mathrm{O}(1)$ & & $3.151(5)$ & 115.2 \\
\hline & & & \\
\hline
\end{tabular}

Symmetry transformations used to generate equivalent atoms:

\#1 x-1,y,z \#2 -x,y-1/2,-z+1/2 


\section{${ }^{1} \mathrm{H}$ NMR (400 MHz $\left.\mathrm{CDCl}_{3}\right)$}

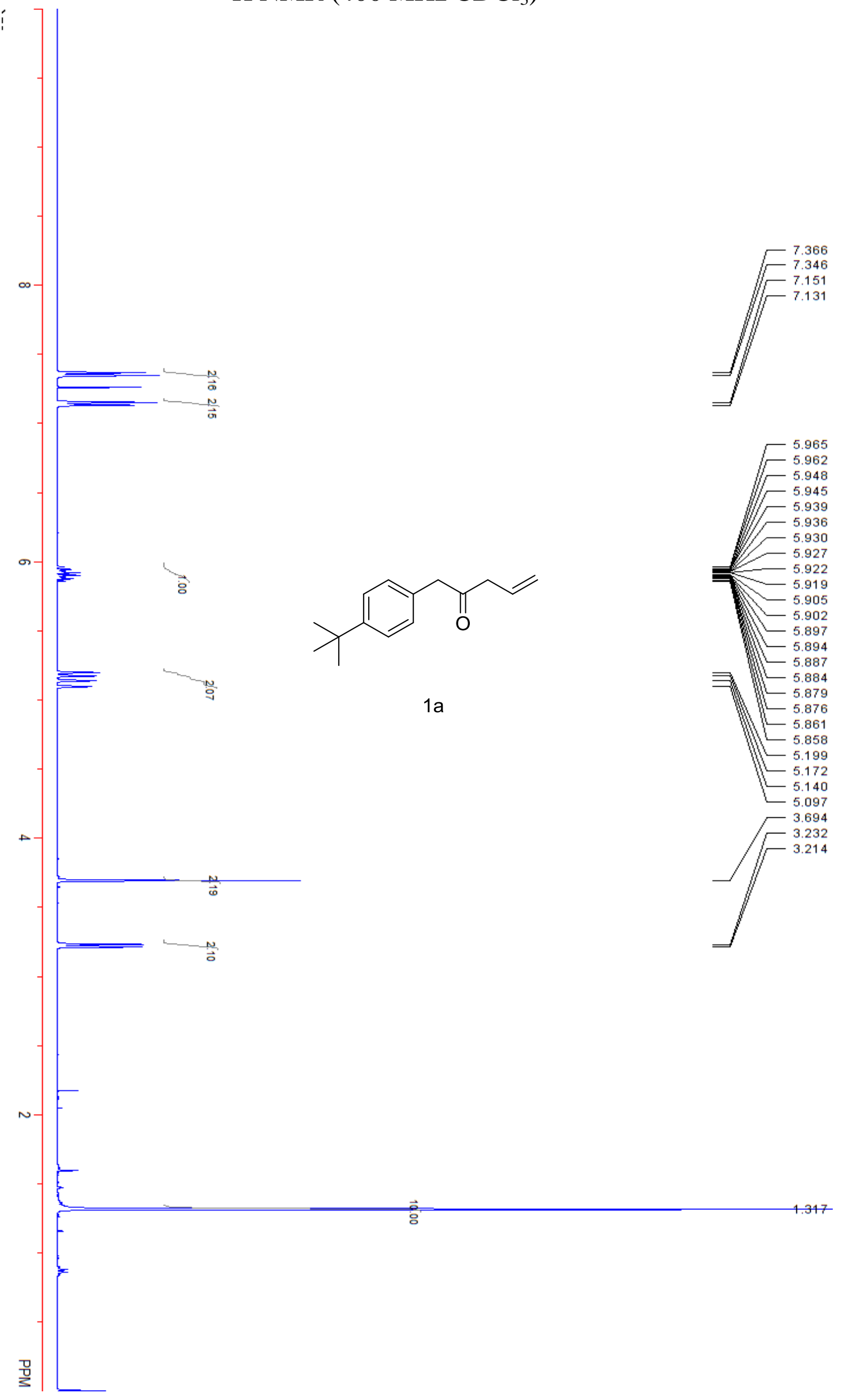




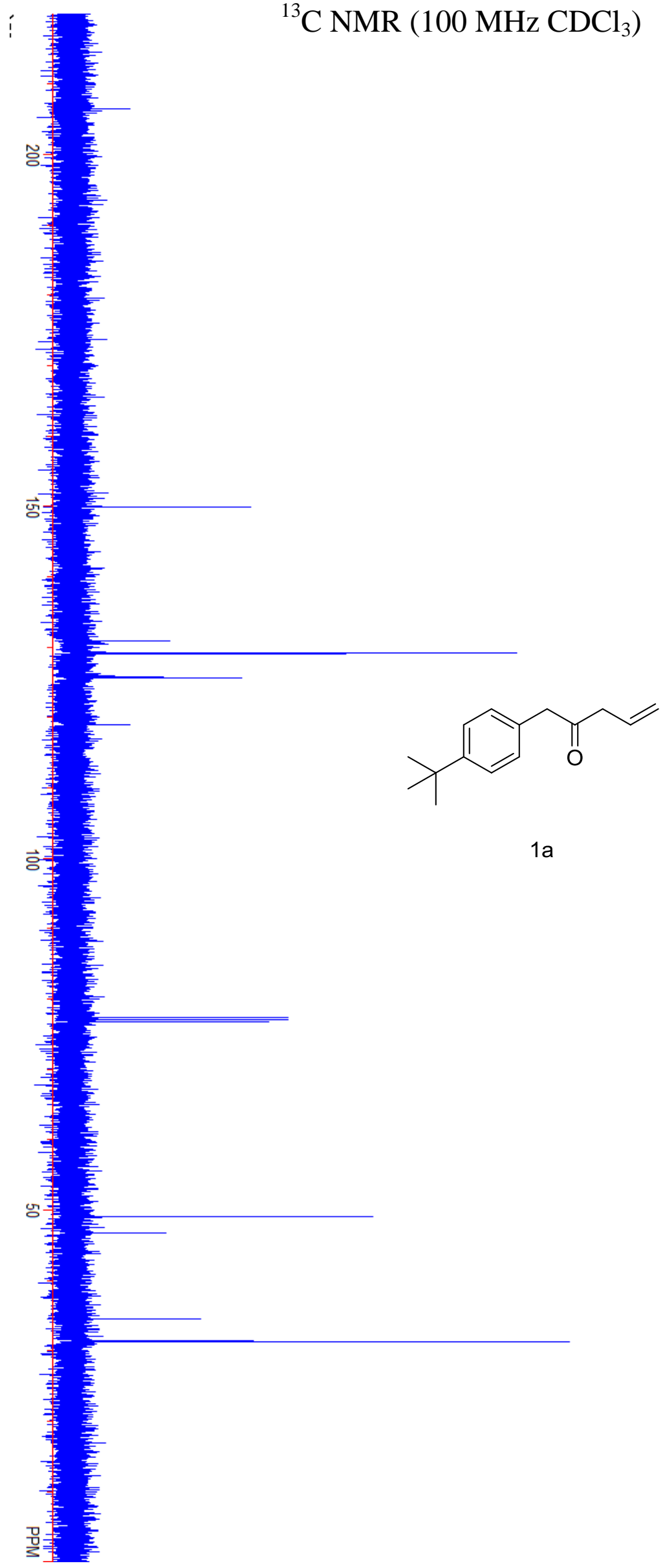

206.456

149.935

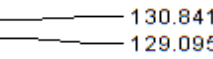

125.667

118.948

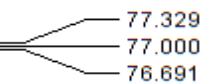

$-76.691$

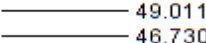

46.73

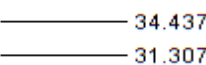



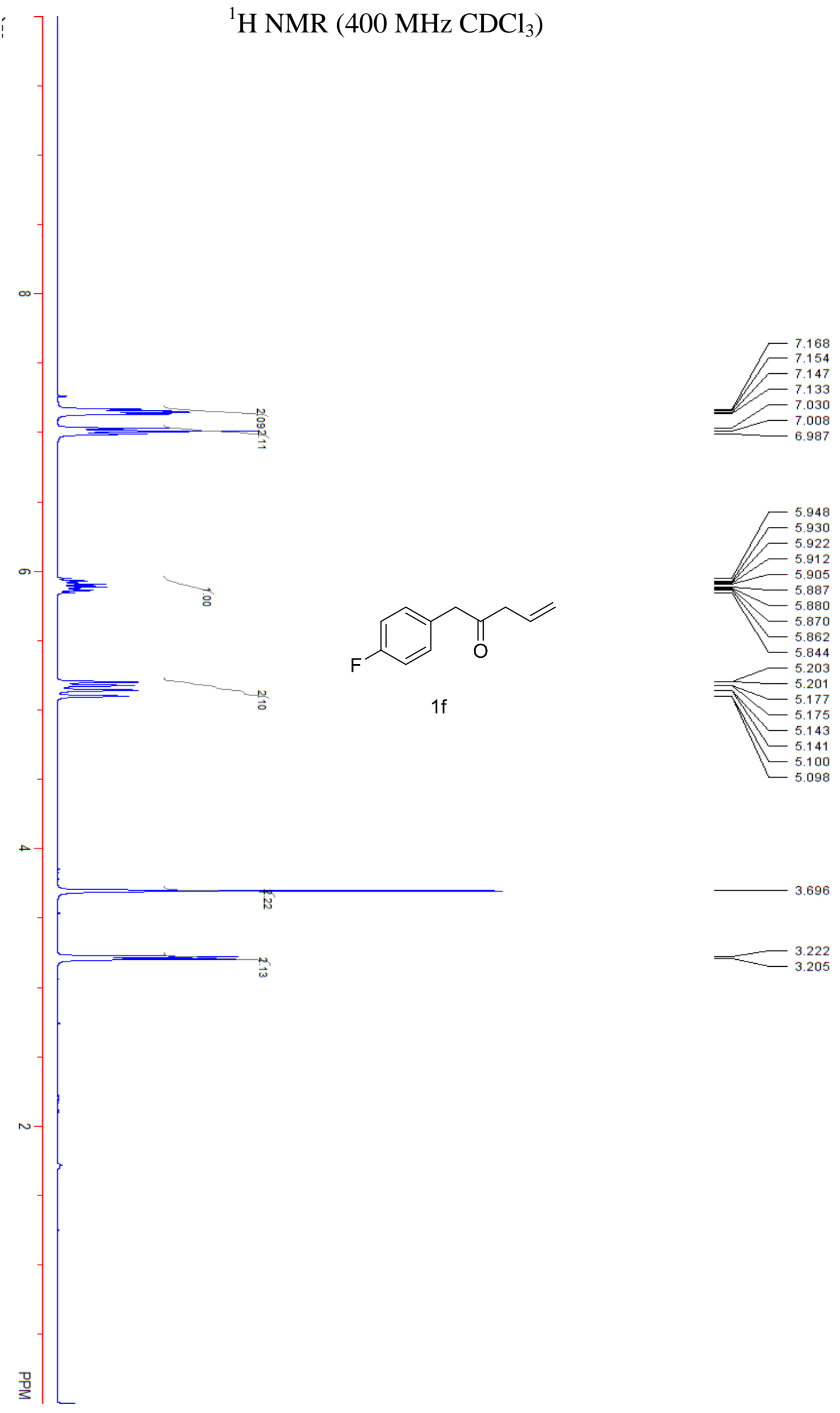


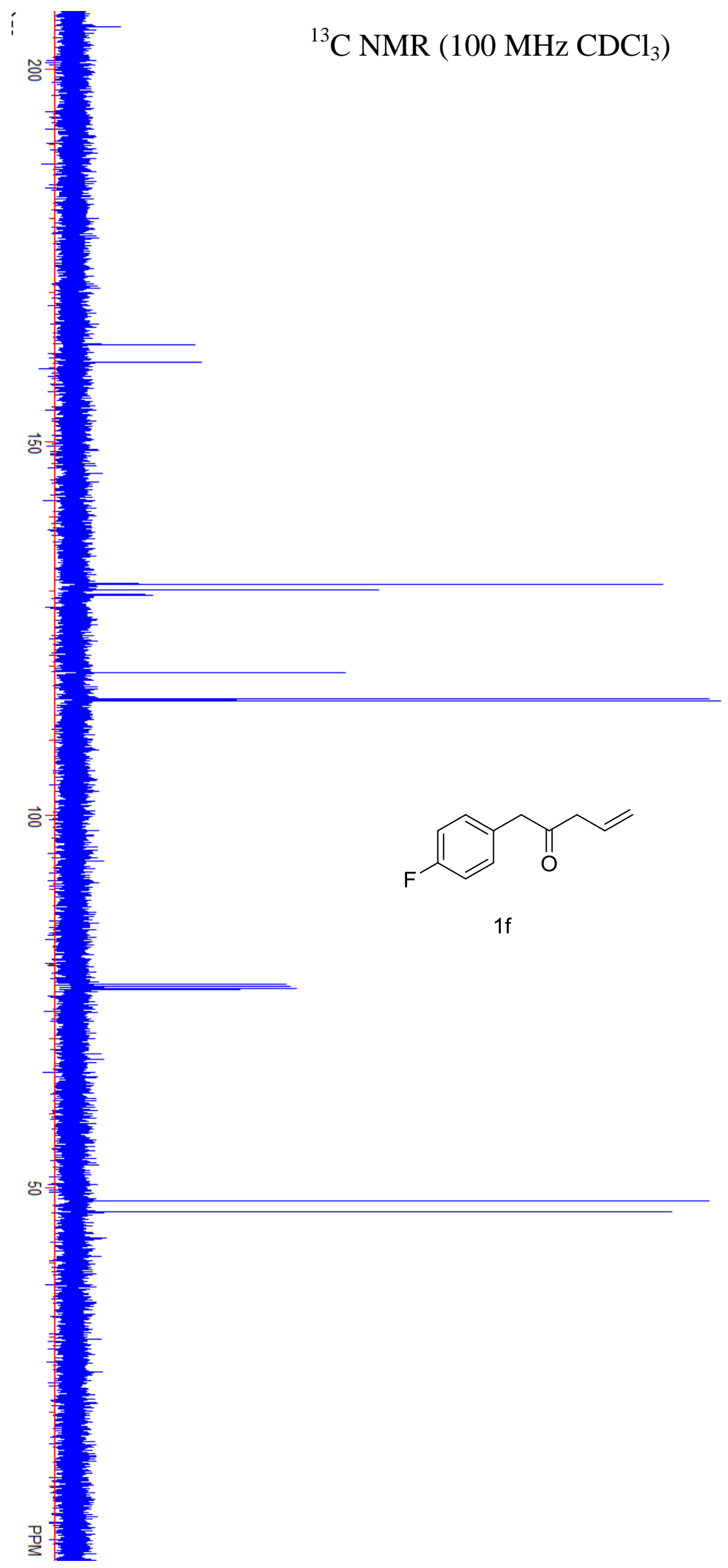

$-205.661$

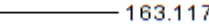

160.676

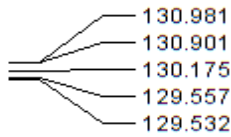

119.067

115.576

115.363
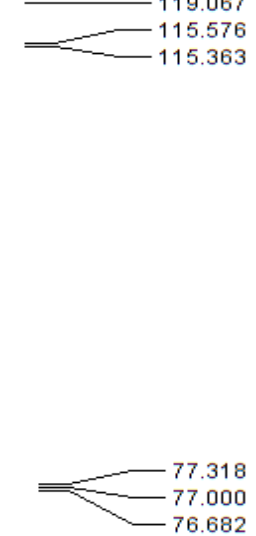

76.682 

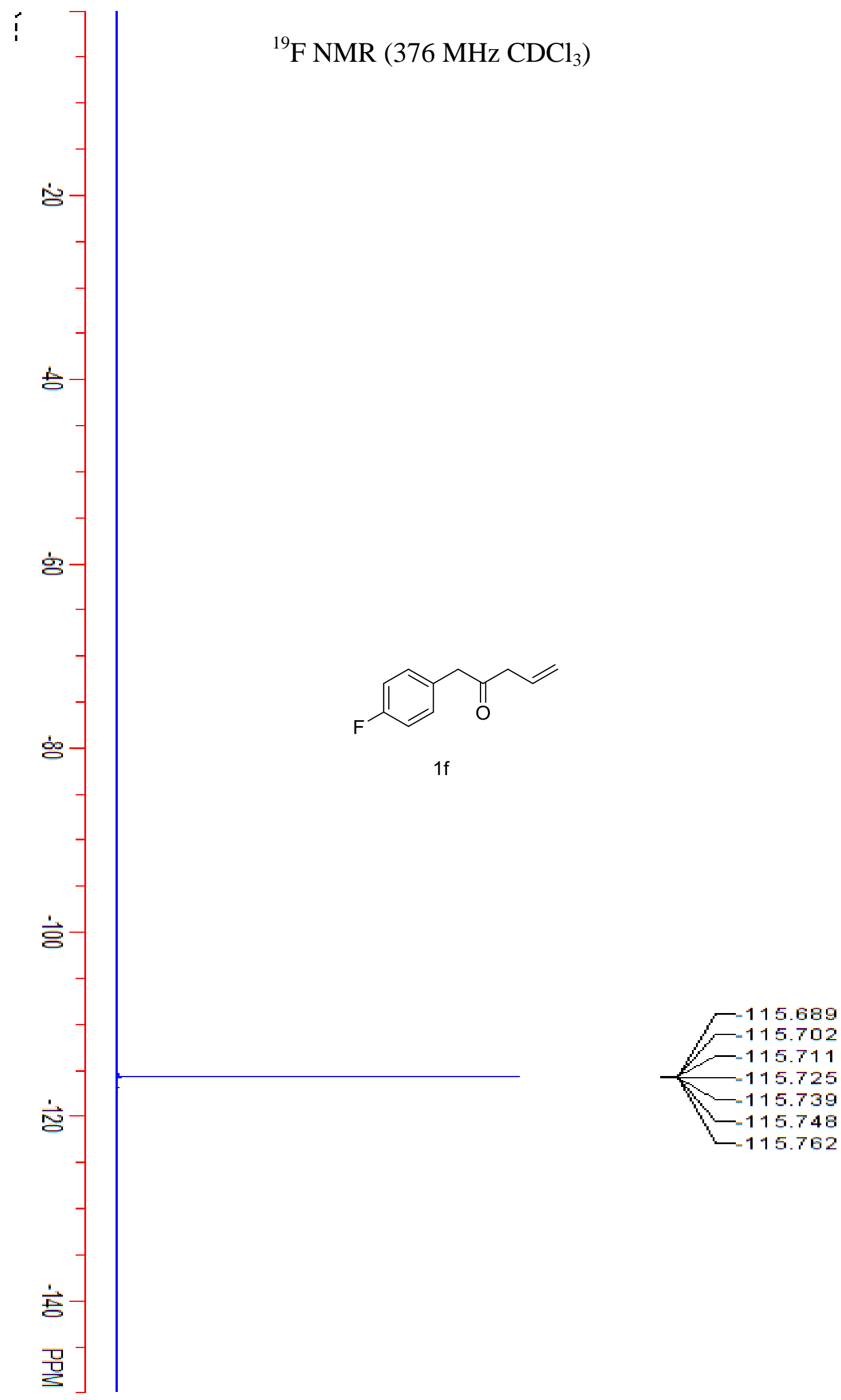


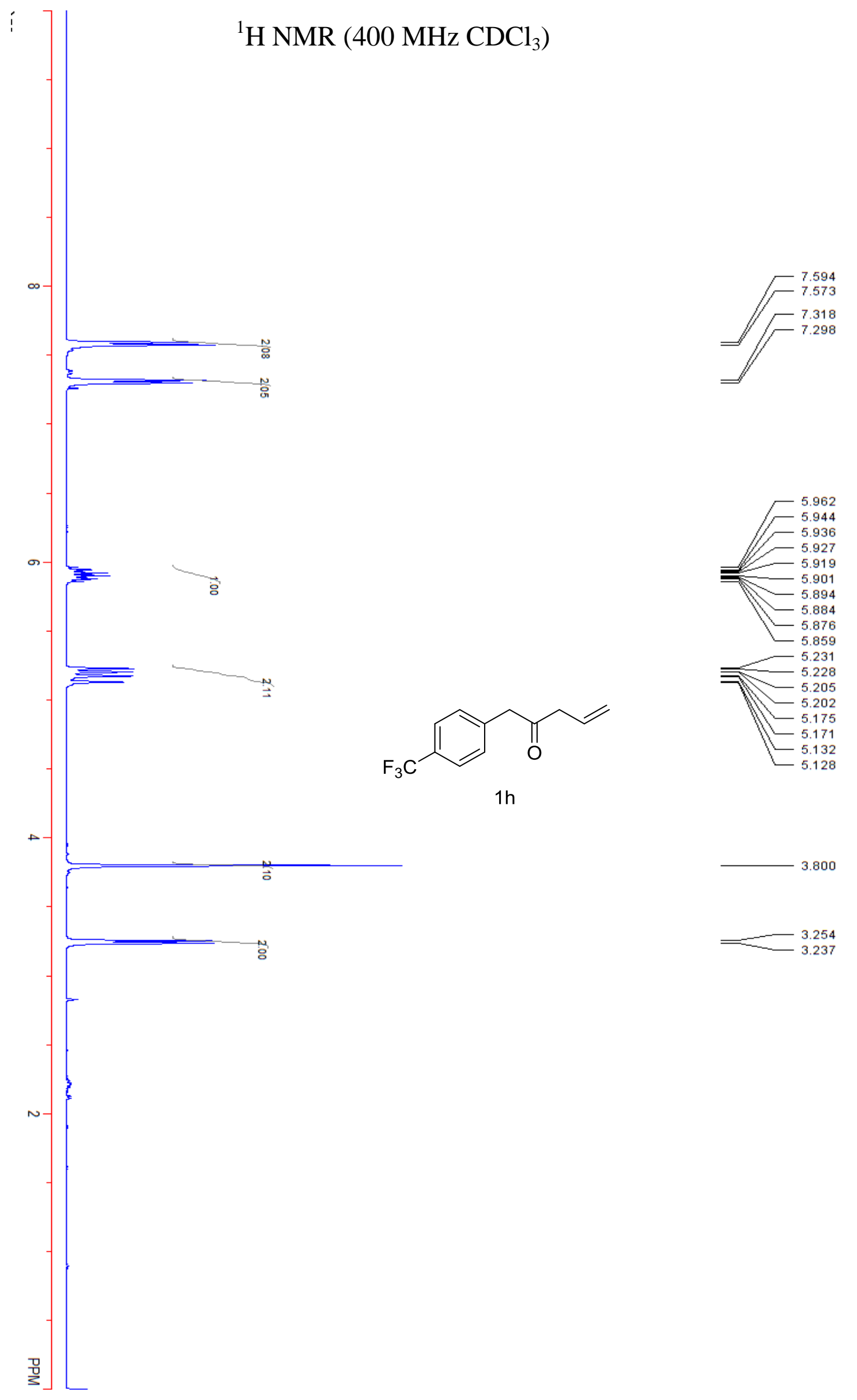




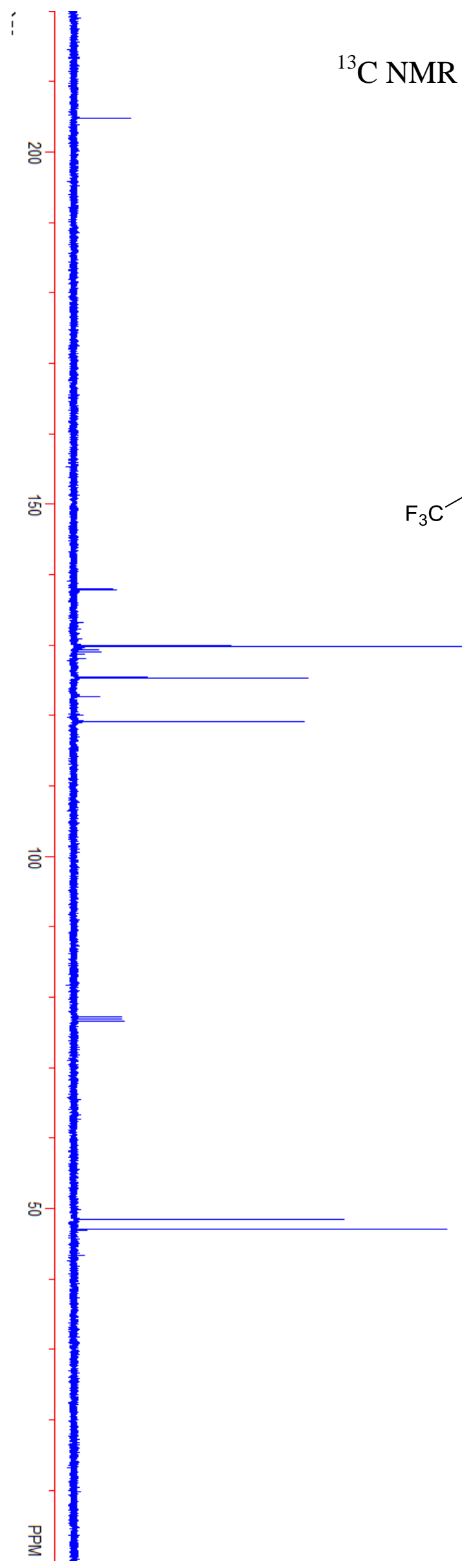

204.746<smiles>C=CCC(=O)Cc1ccc(C)cc1</smiles>
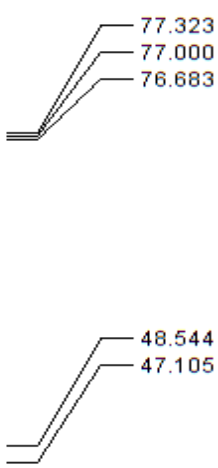


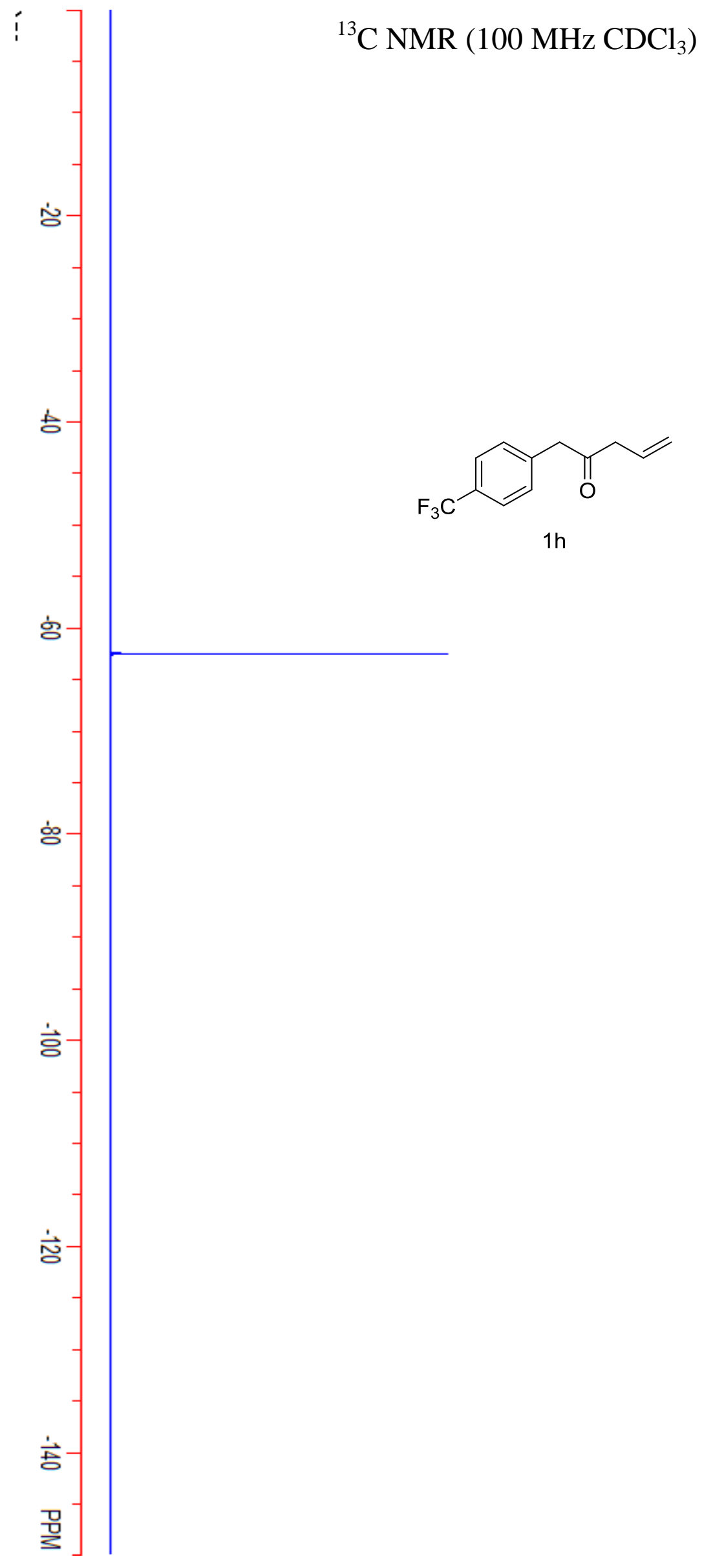

$-62.556$ 

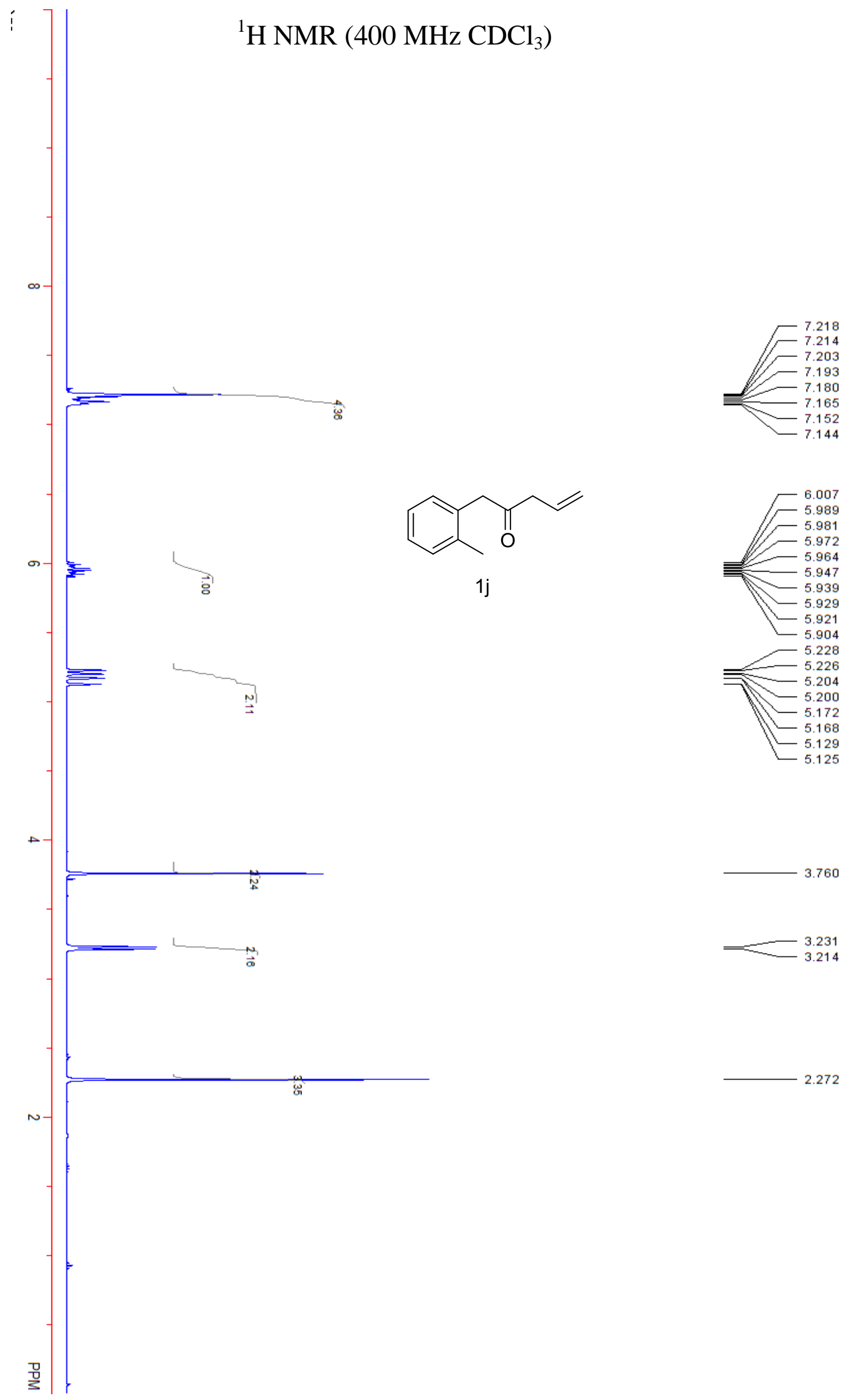

3.760

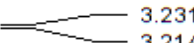

. 214

272 


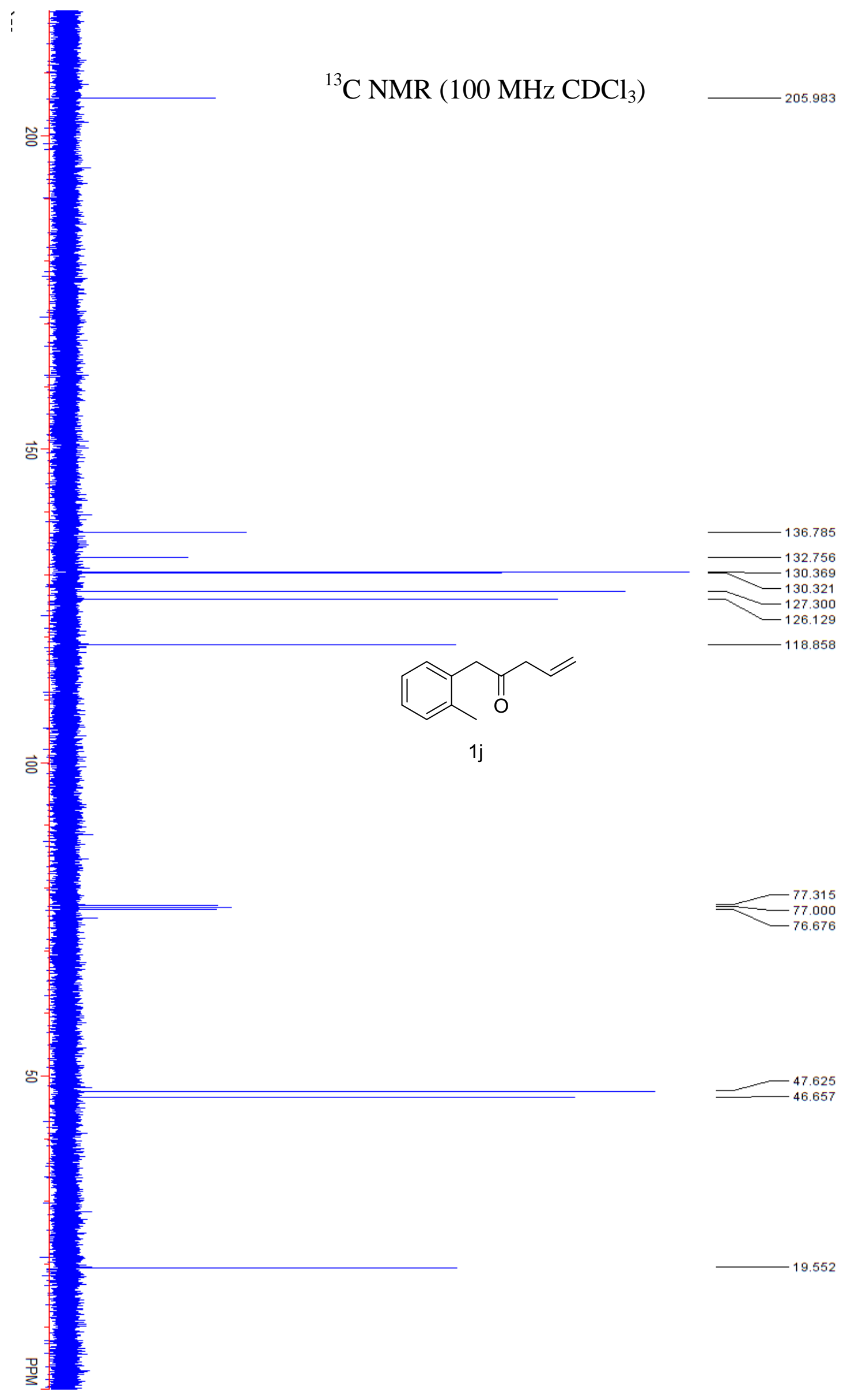



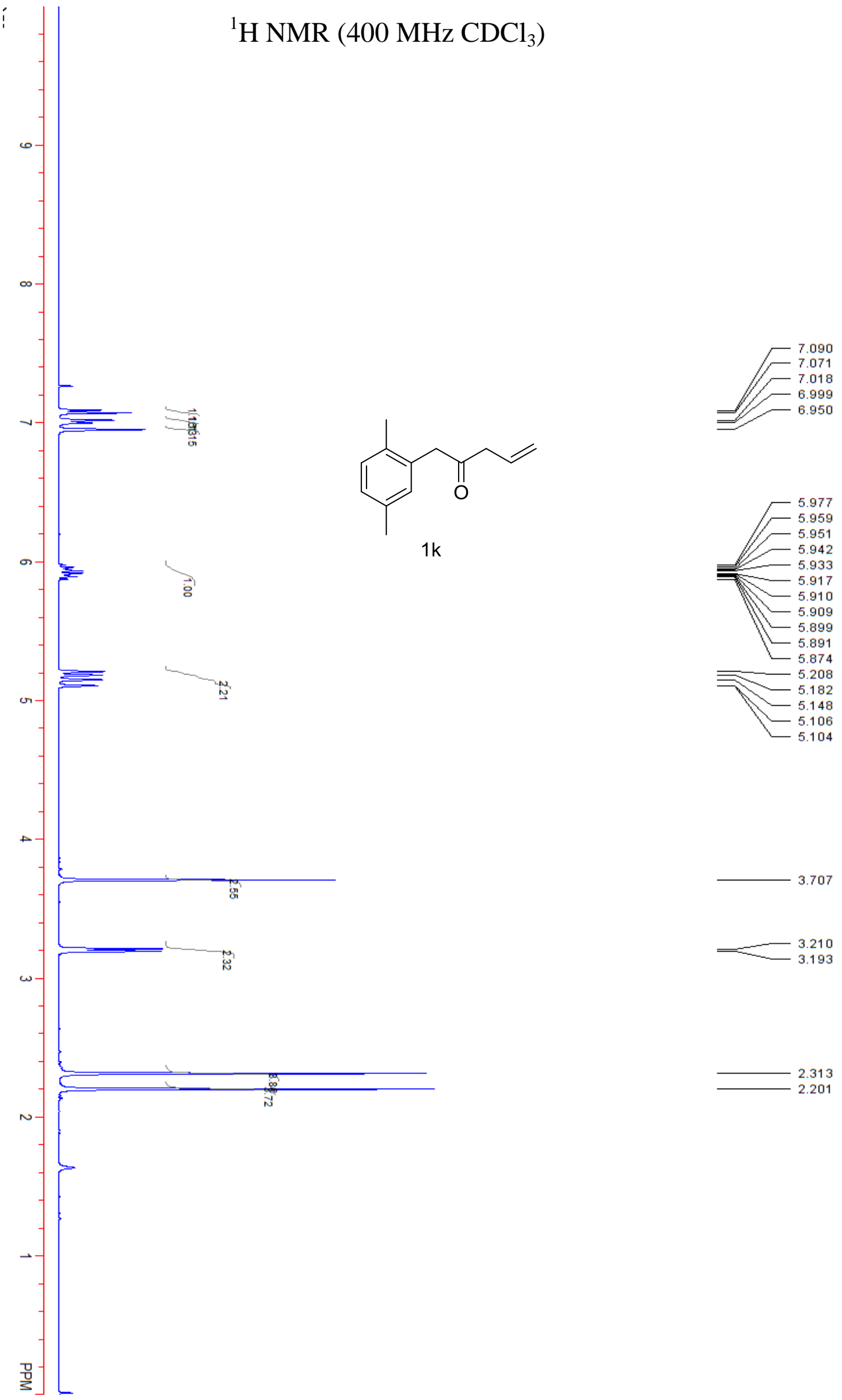


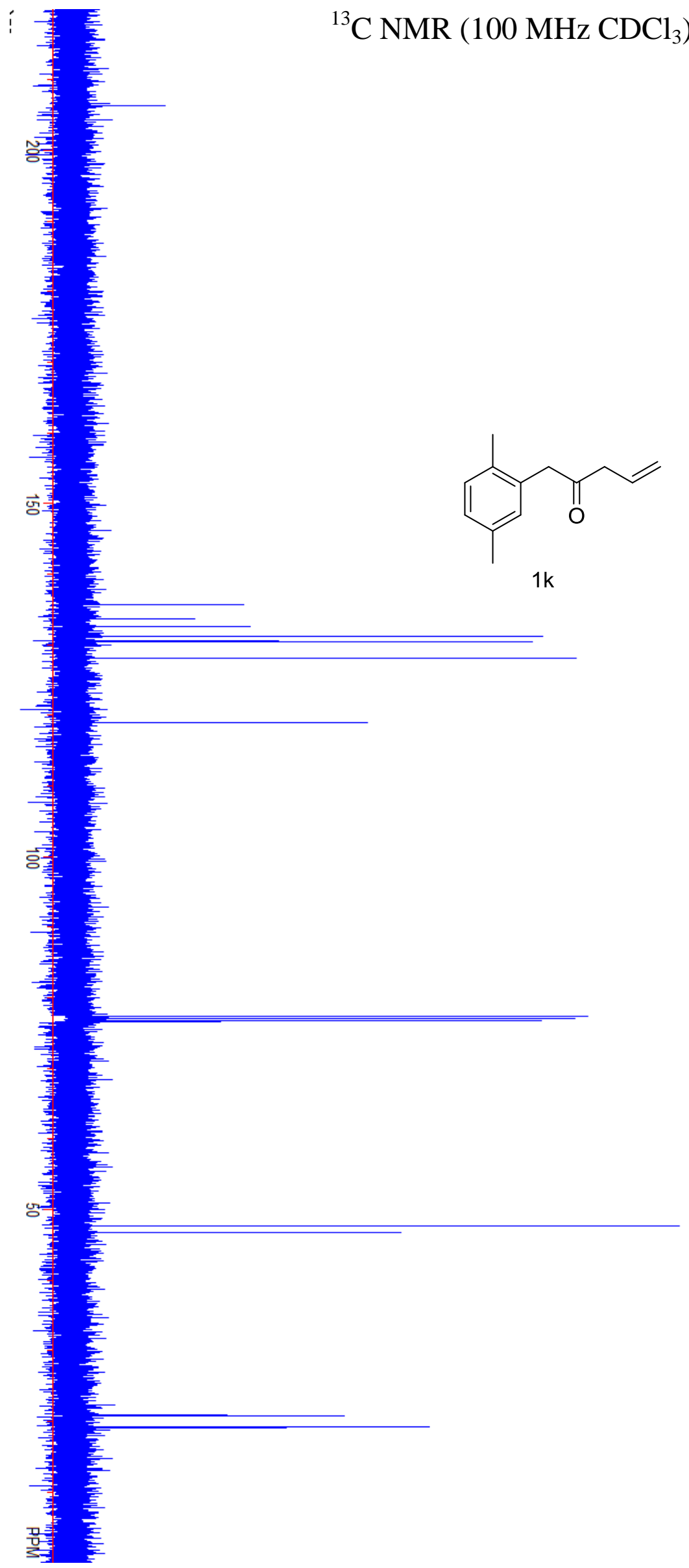

206.290

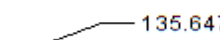

133.633 132.583

=-131.109

$\longrightarrow-130.482$

130.352

128.068

118.917

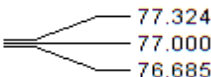

$-76.685$

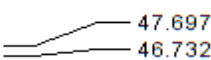

$-46.732$

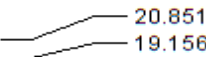


${ }^{1} \mathrm{H}$ NMR (400 MHz $\left.\mathrm{CDCl}_{3}\right)$

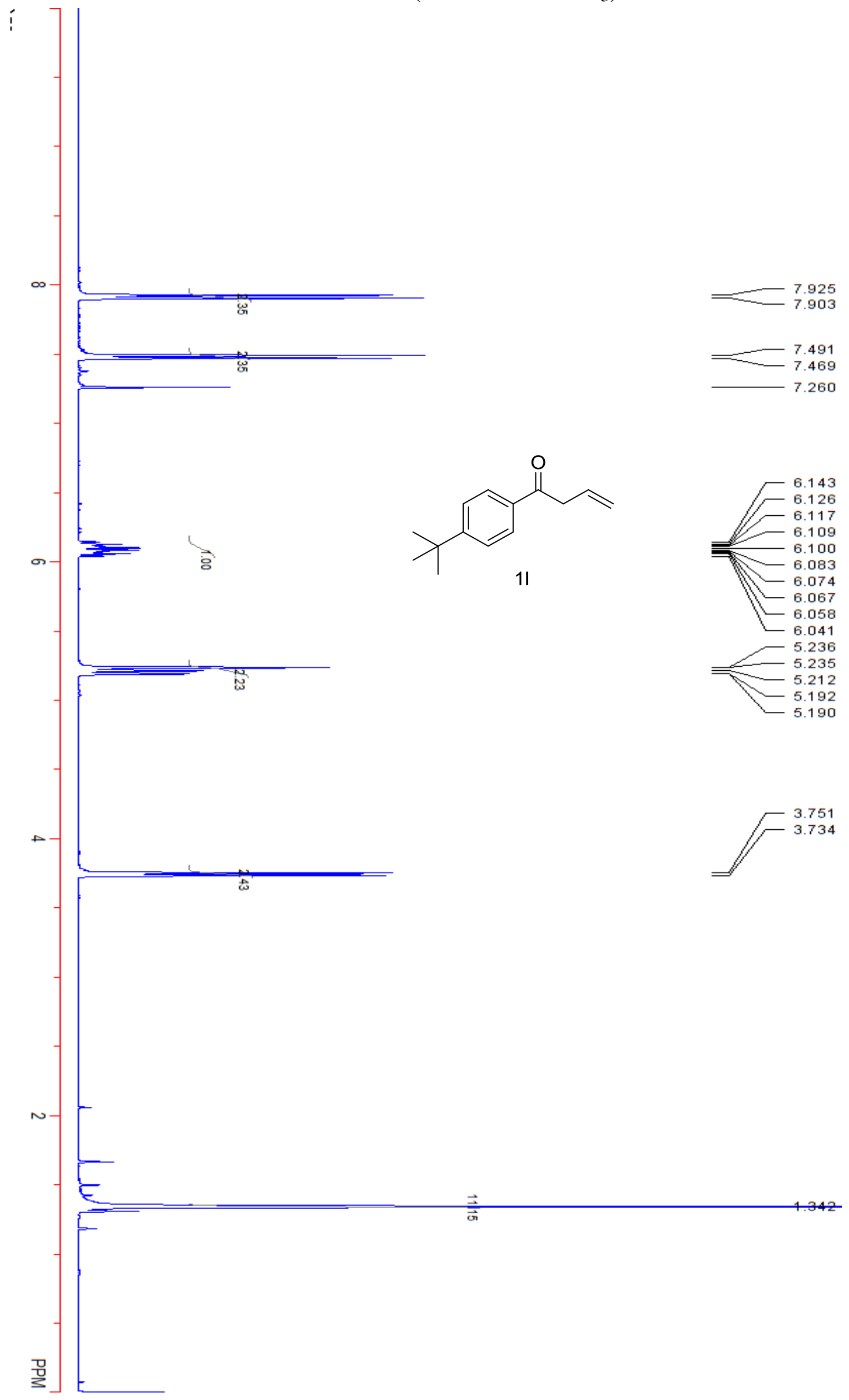




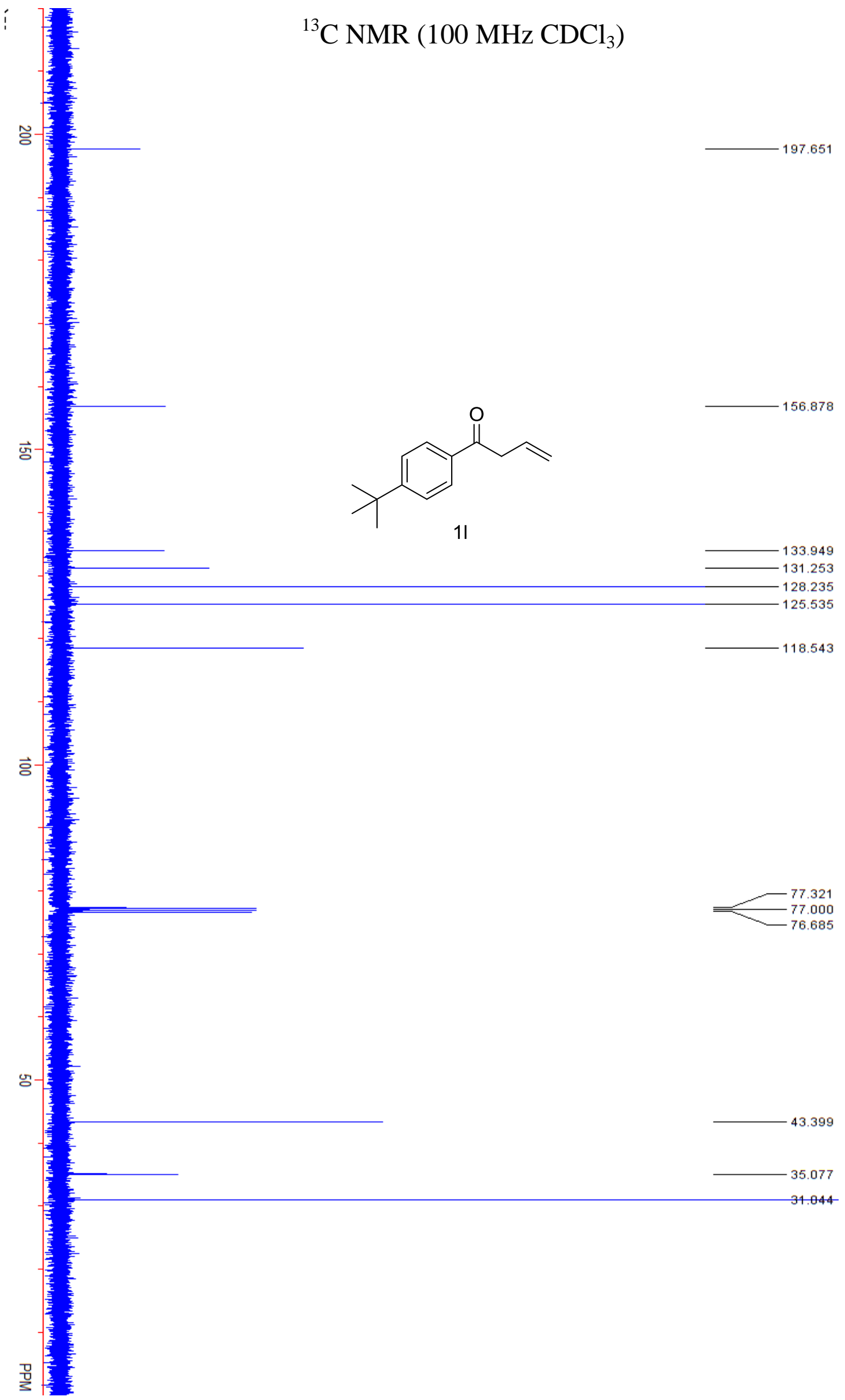



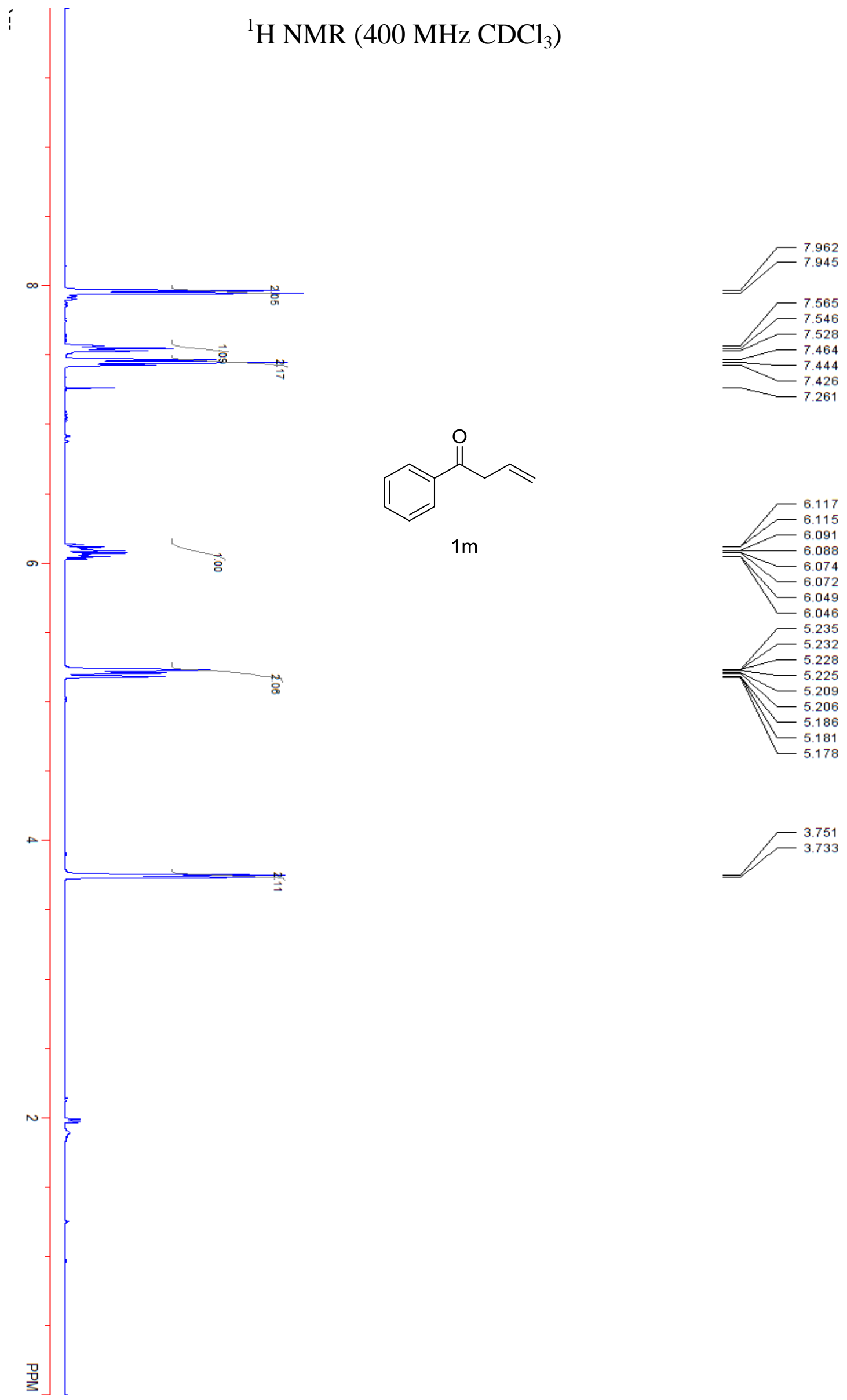


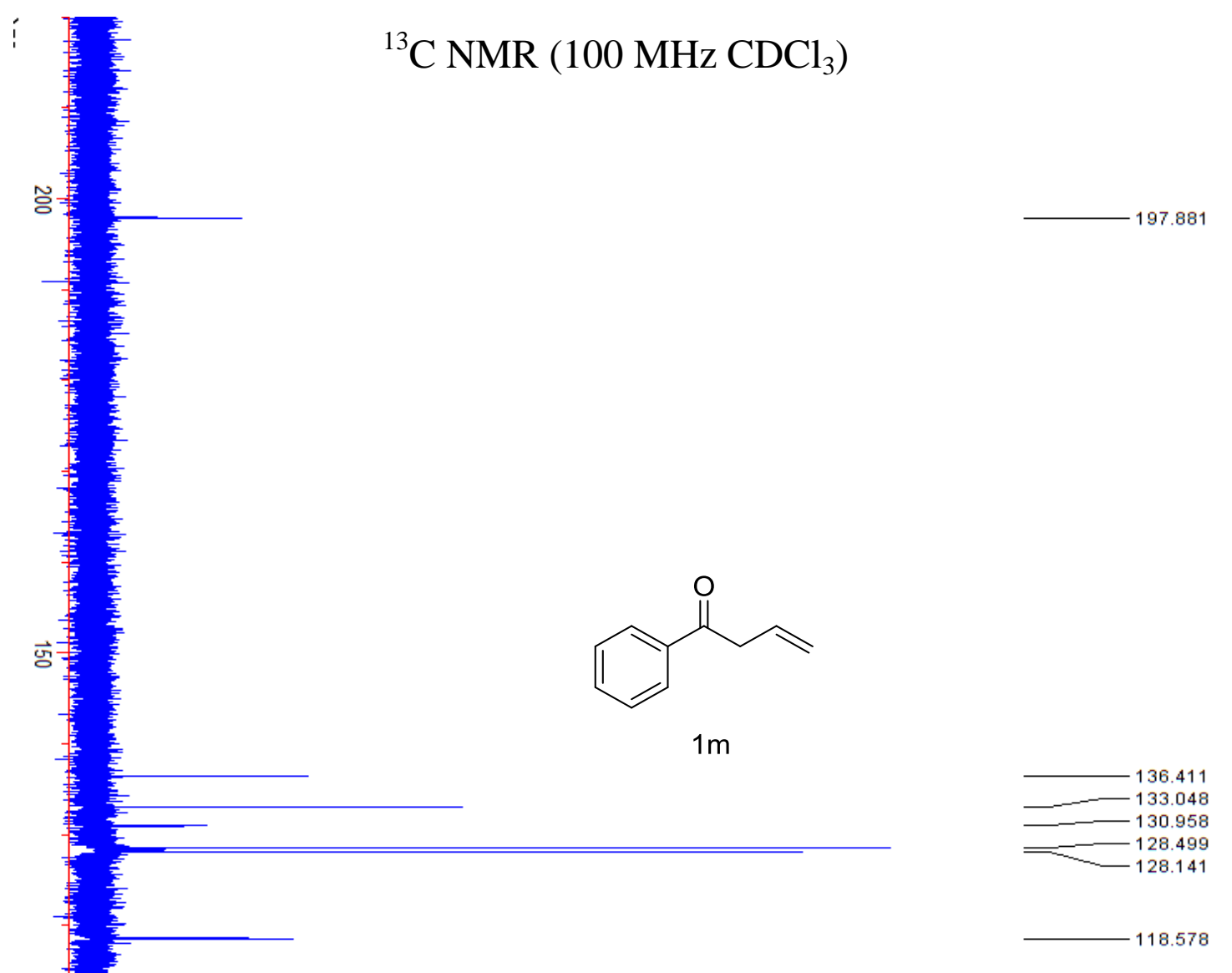

S69 


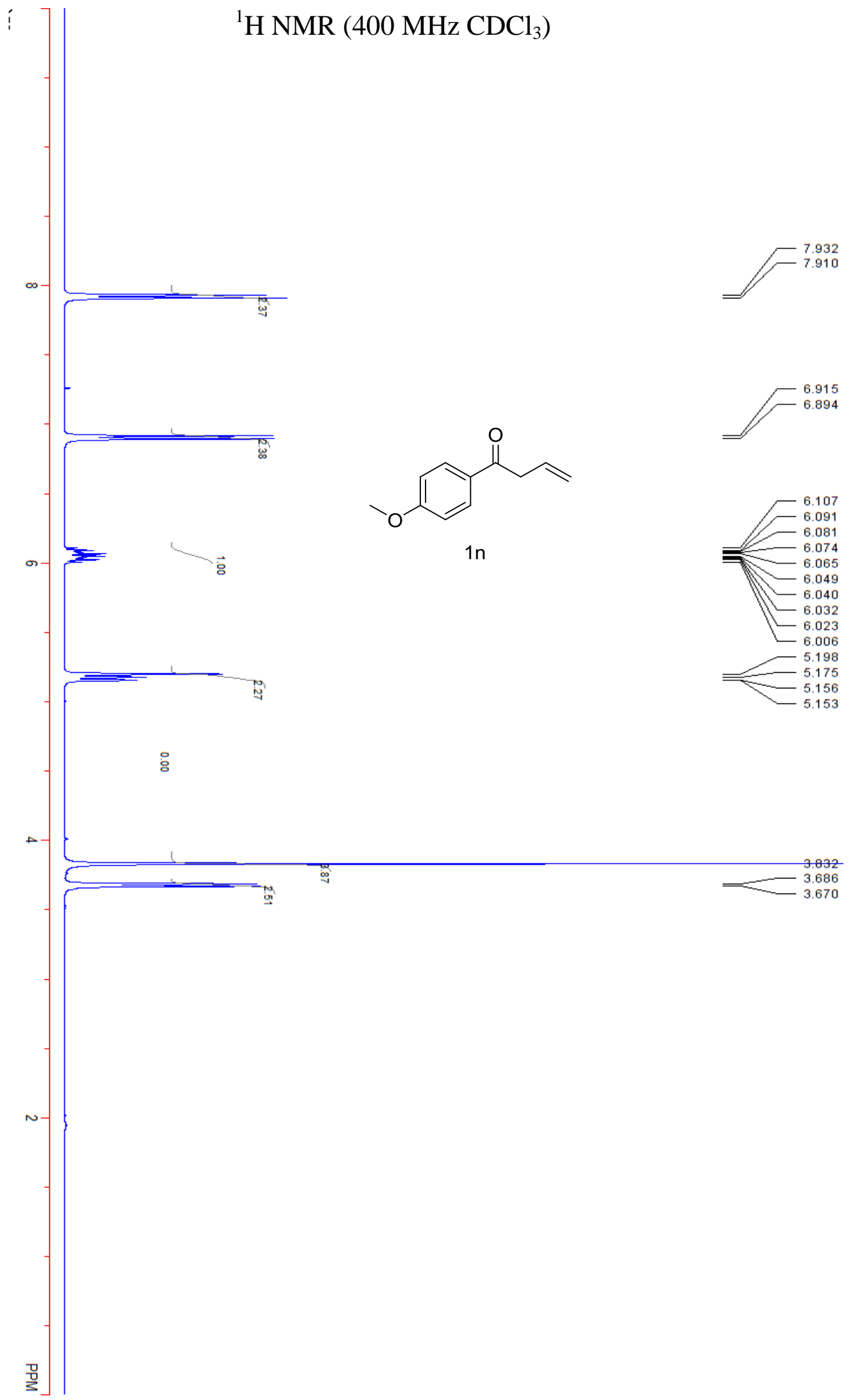



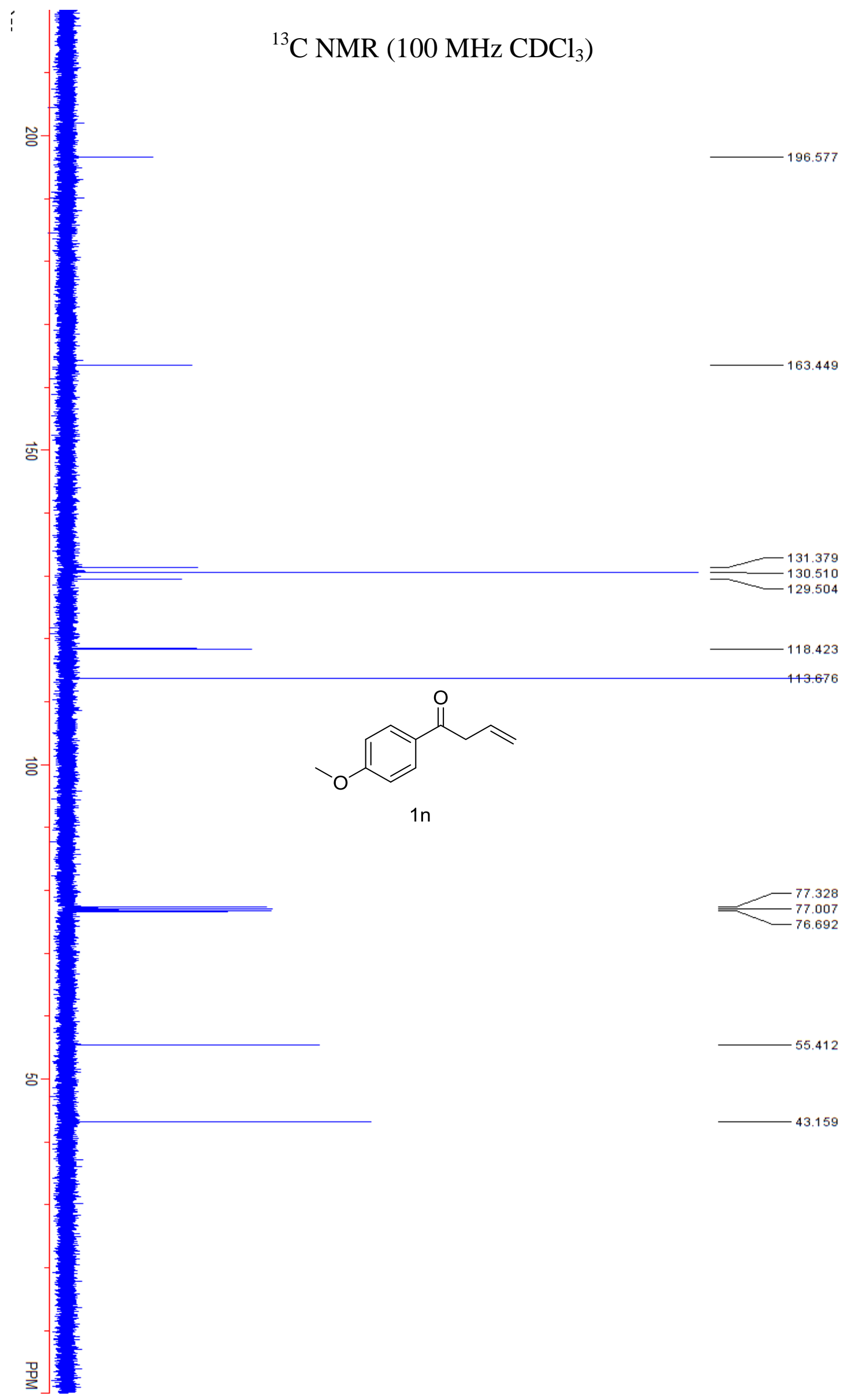

113.676<smiles>C=CCC(=O)c1ccc(OC)cc1</smiles>

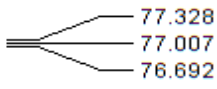

55.412

43.159 

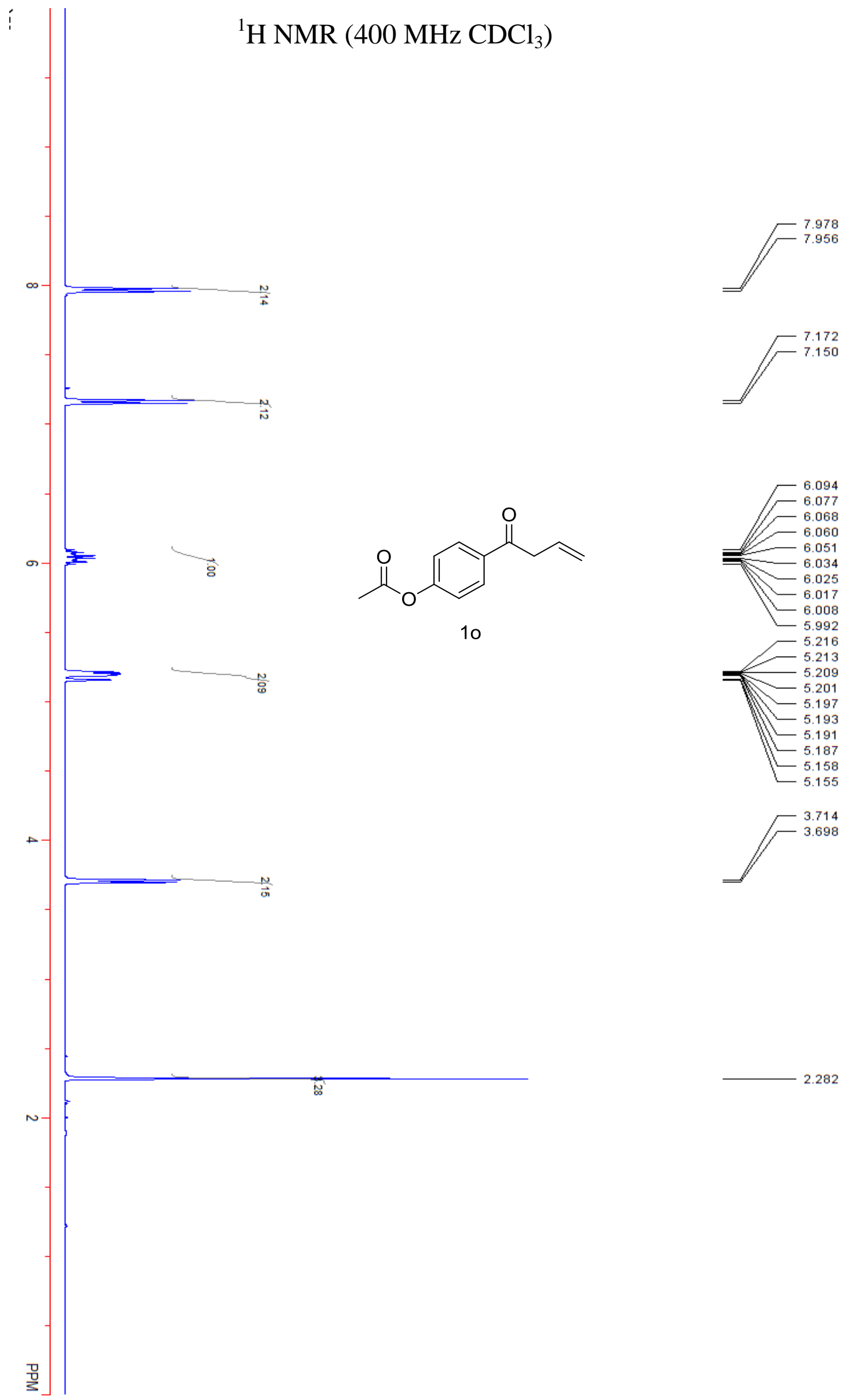

2.282 

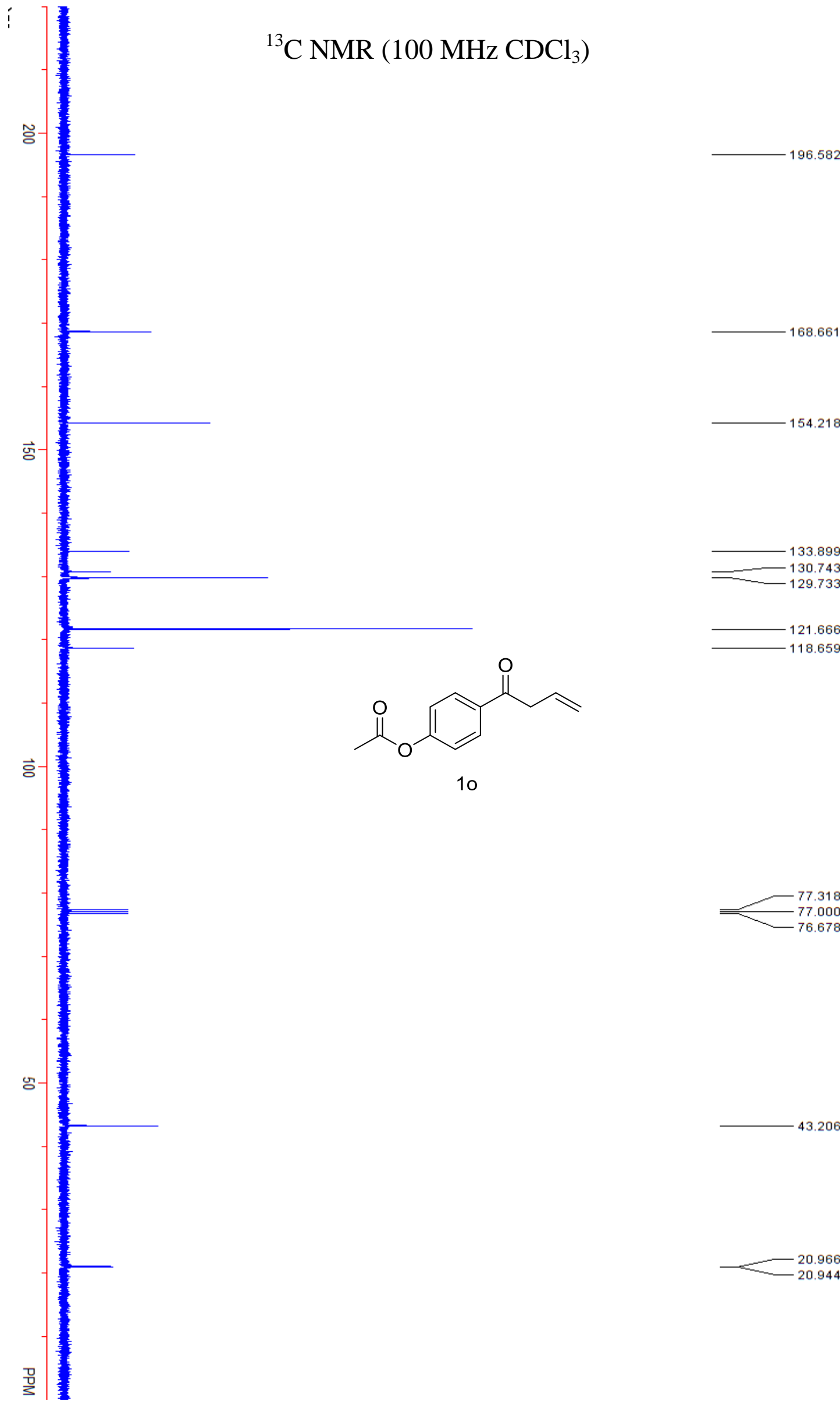
129.733

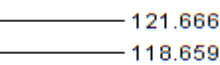

21.666
118.659
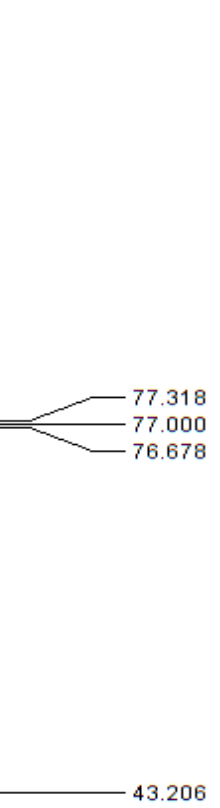

43.206

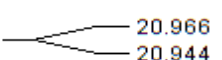



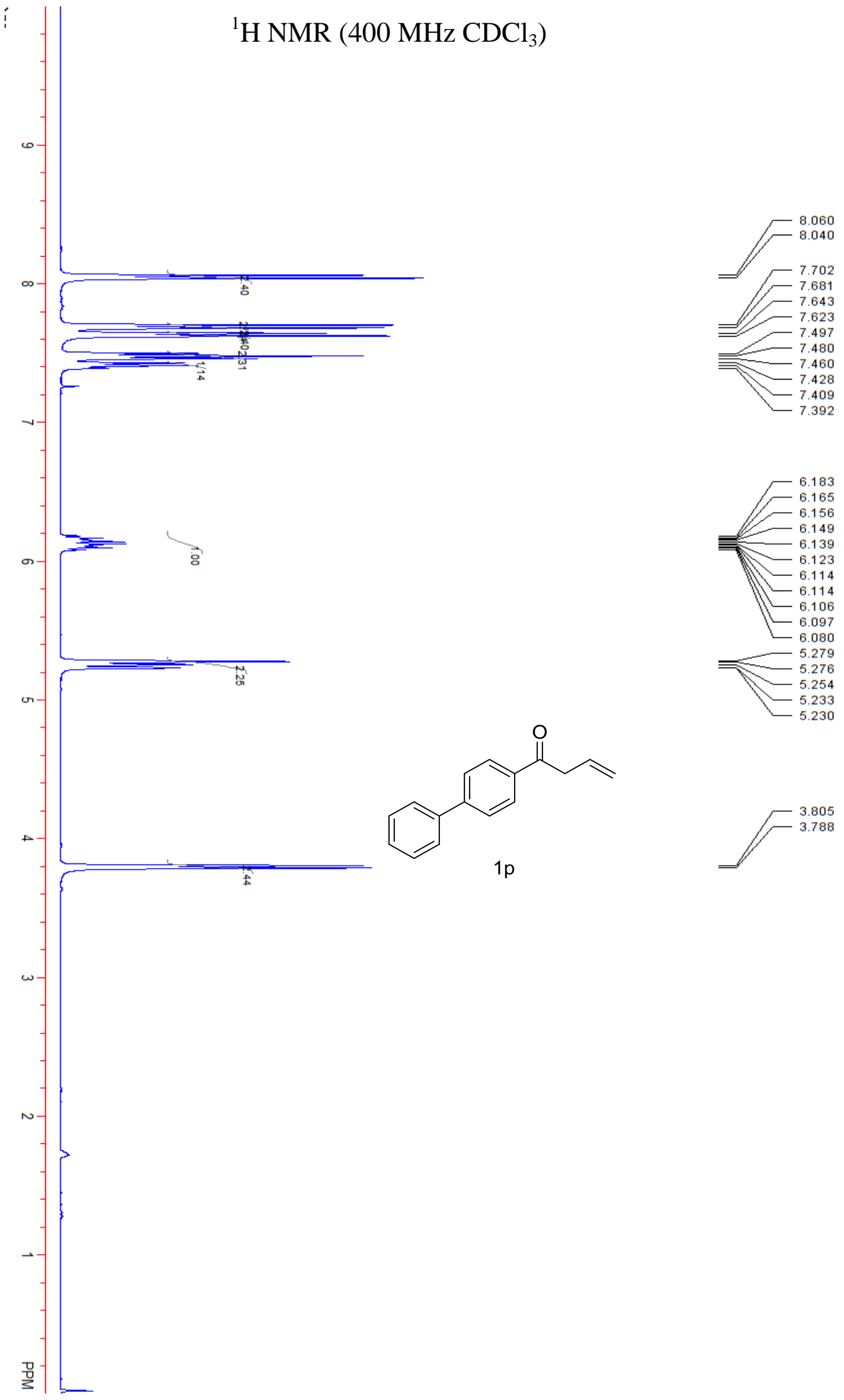


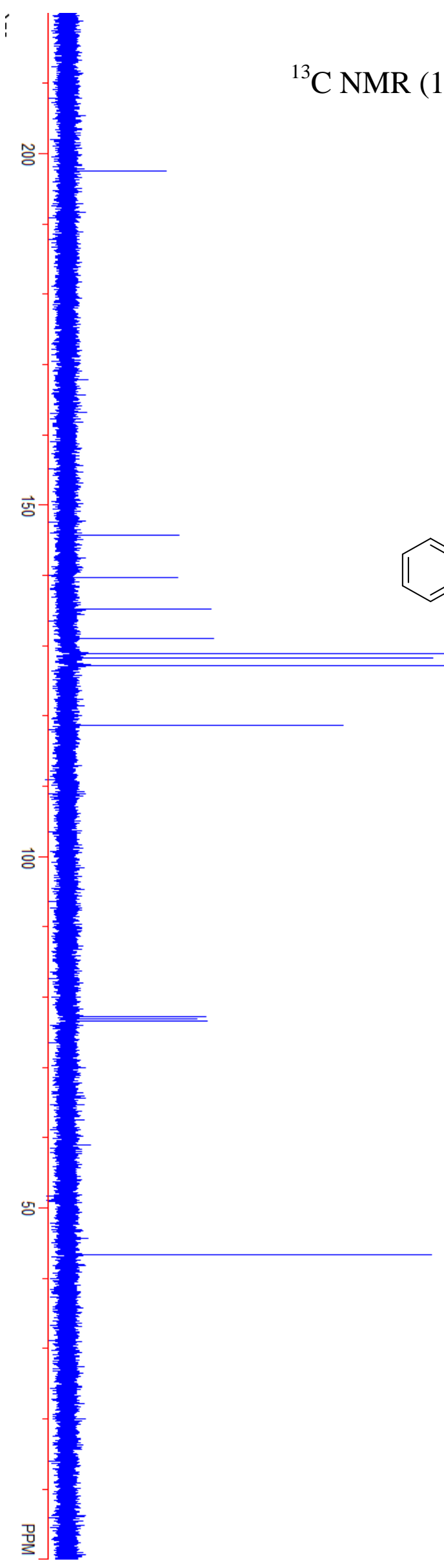

$-197.535$

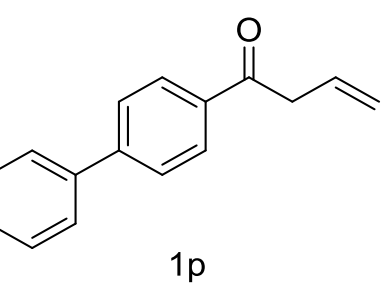

135.169

131.052

128.897 128.823 127.191

118.690

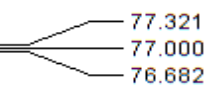

76.682 

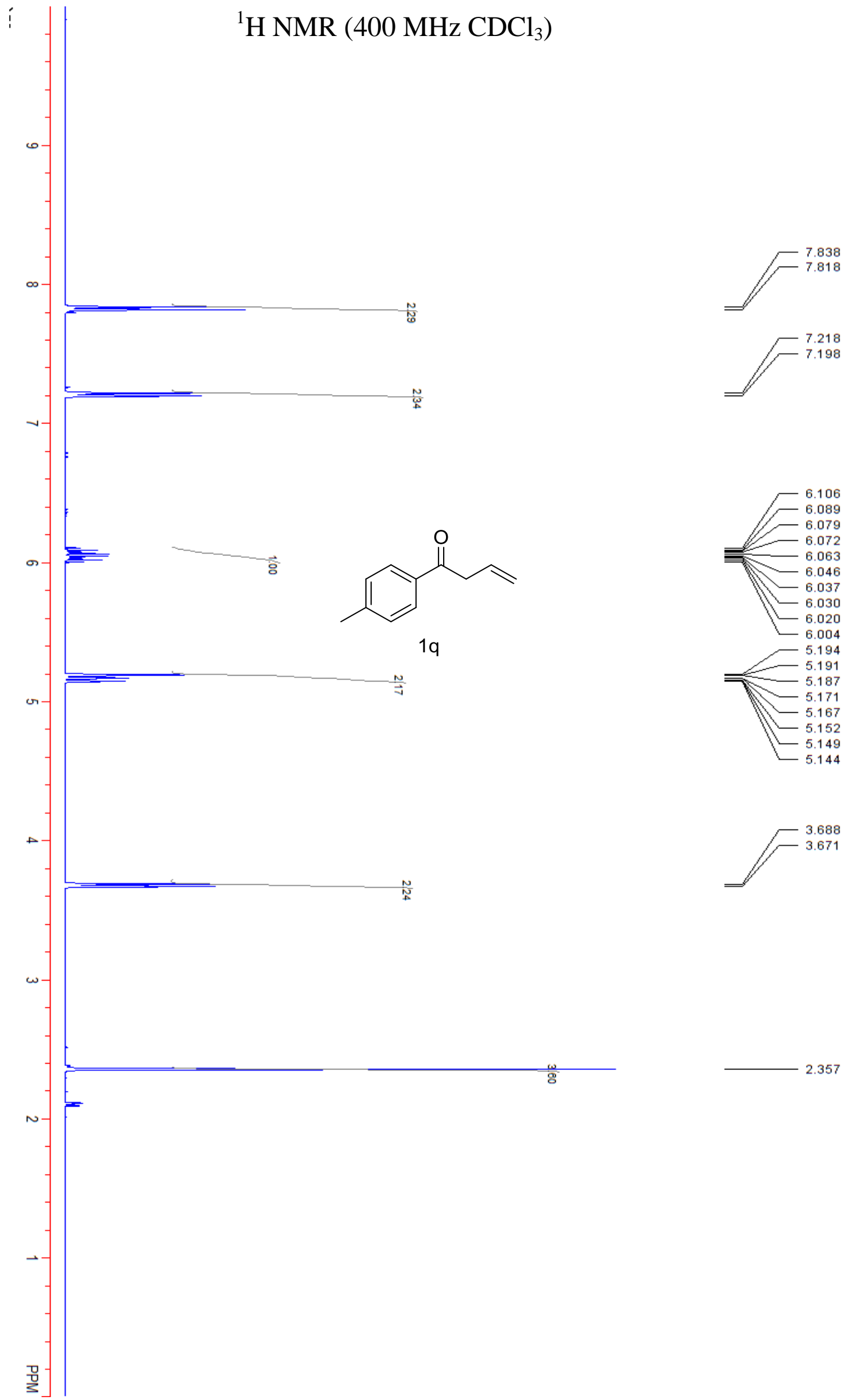


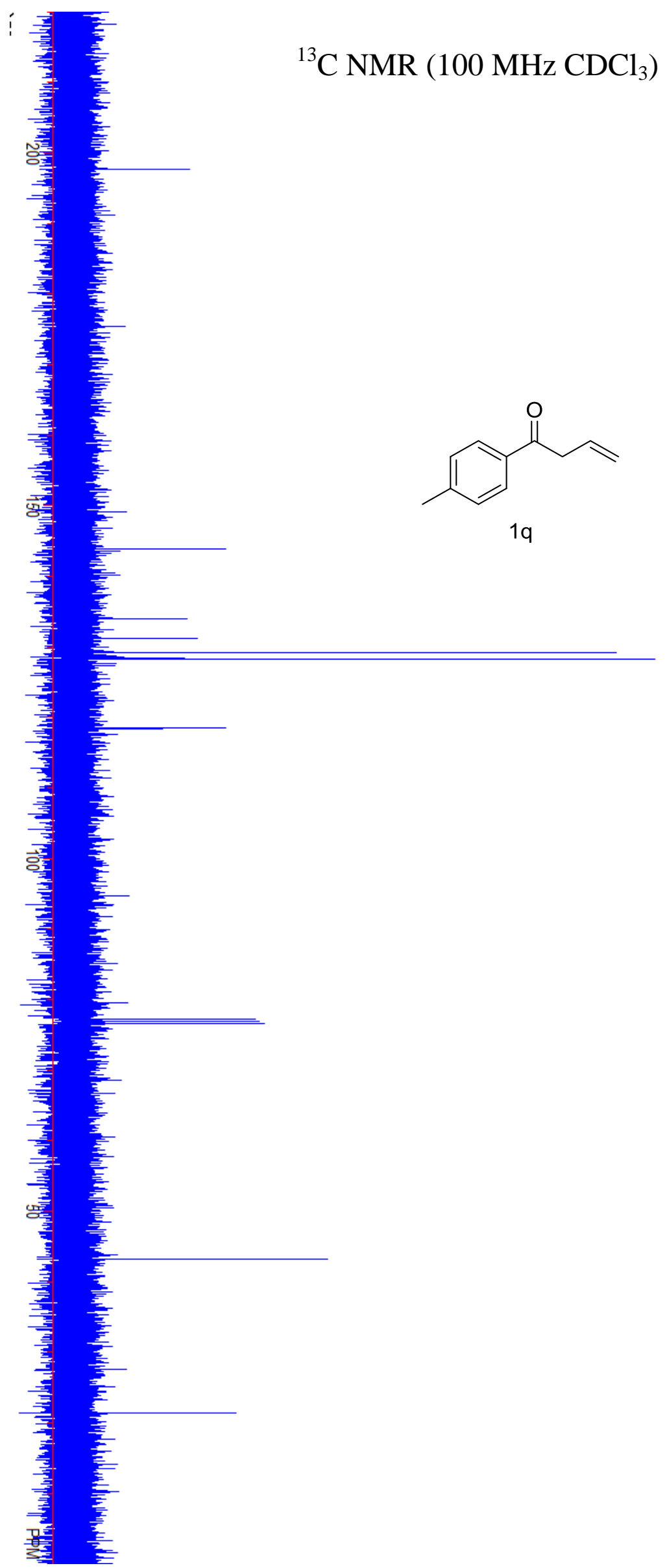

197.639
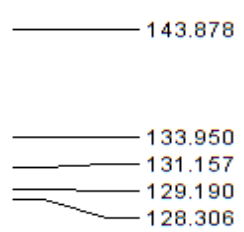

118.444

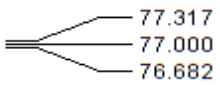

43.249 


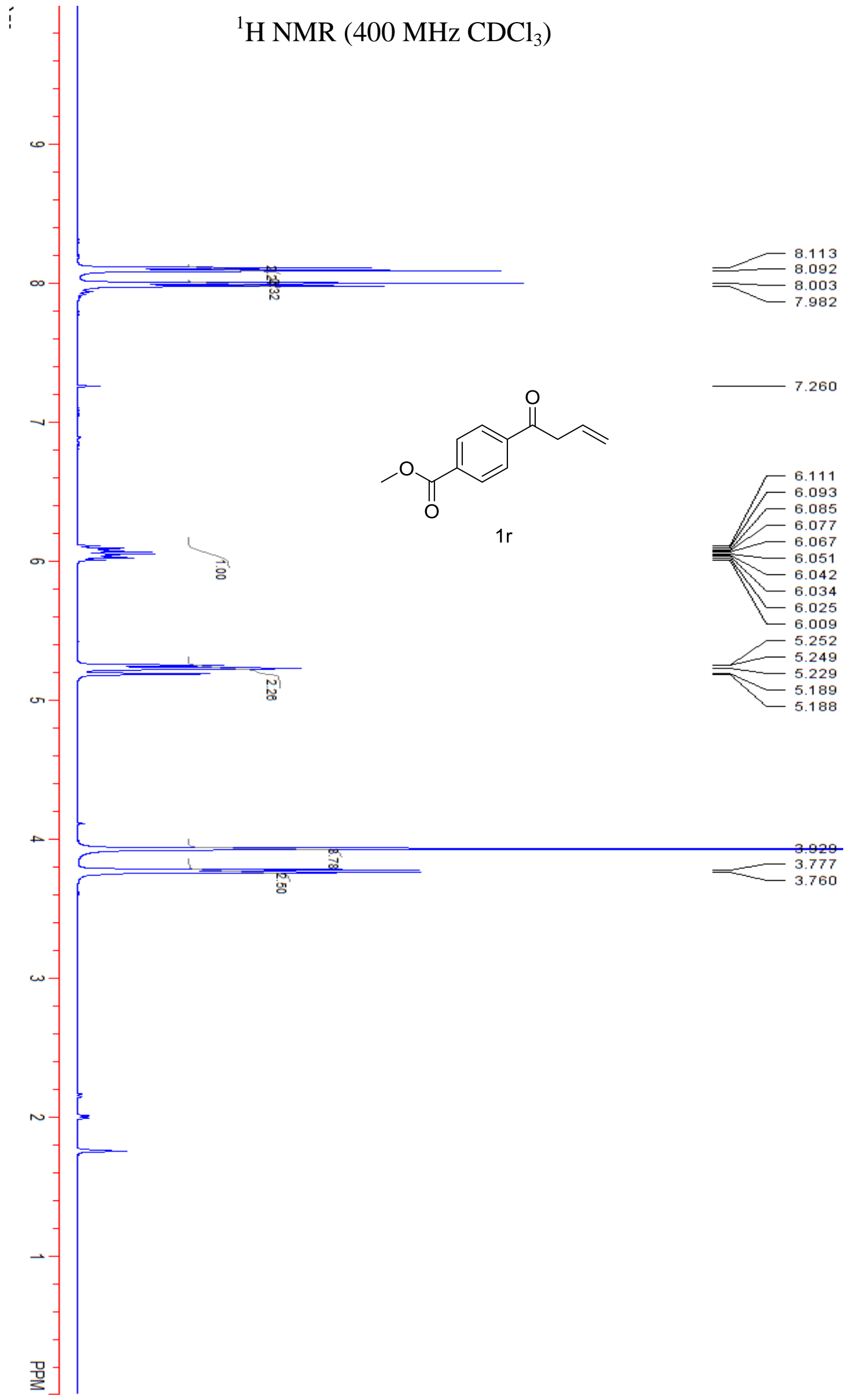




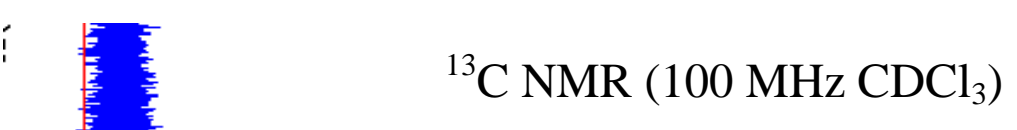

○。

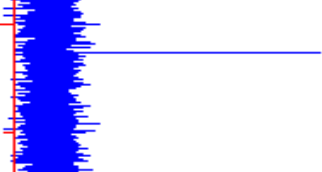

$-197.433$

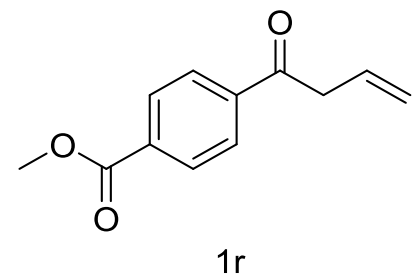

166.105

जु

$1 r$

133.867

130.433

128.104

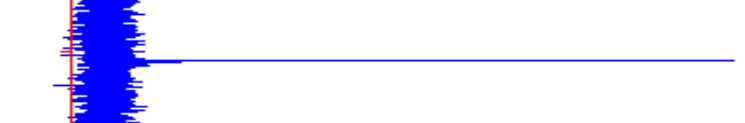

119.066

흥

8
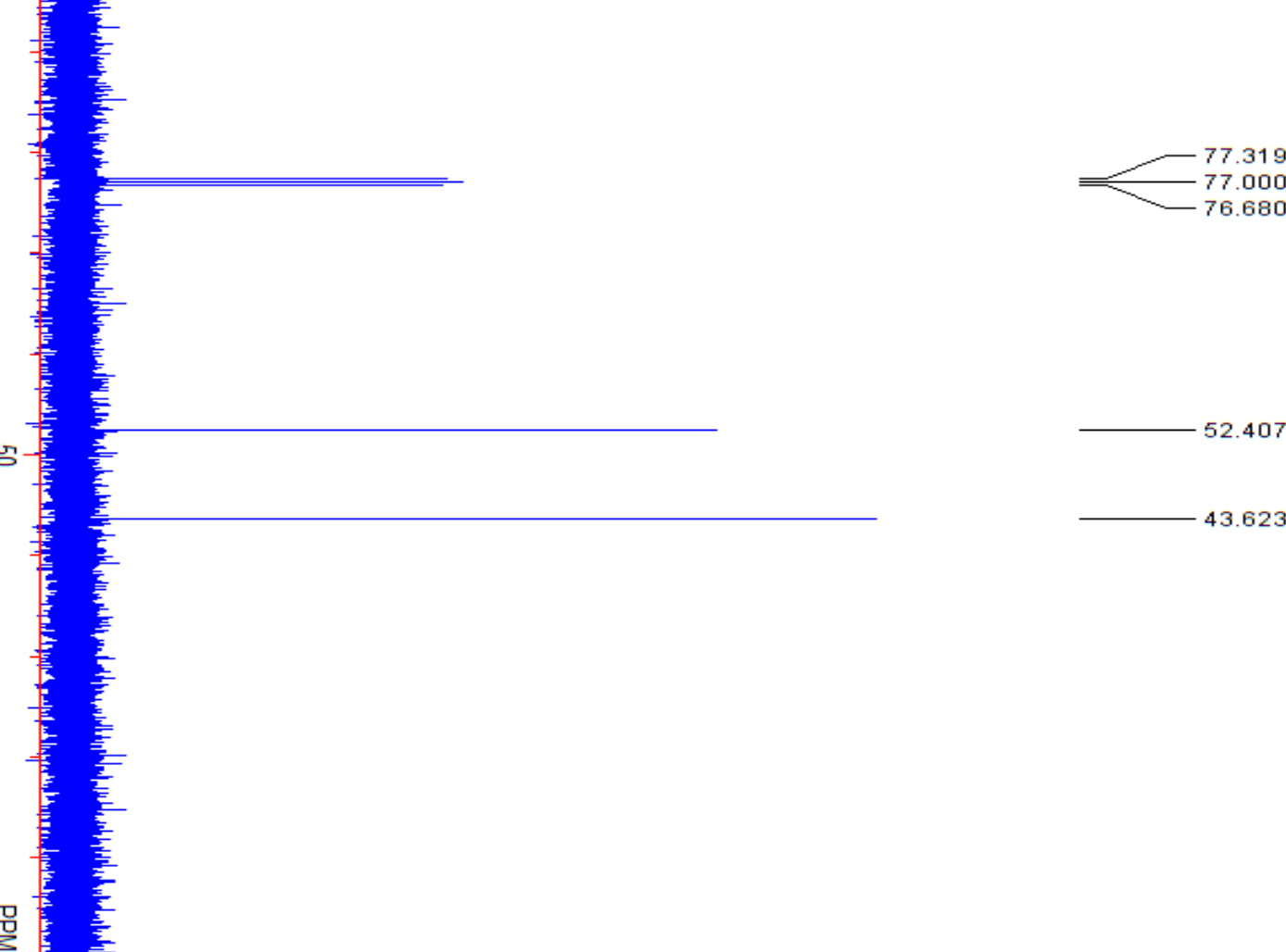

77.000
76.680

52.407

43.623 


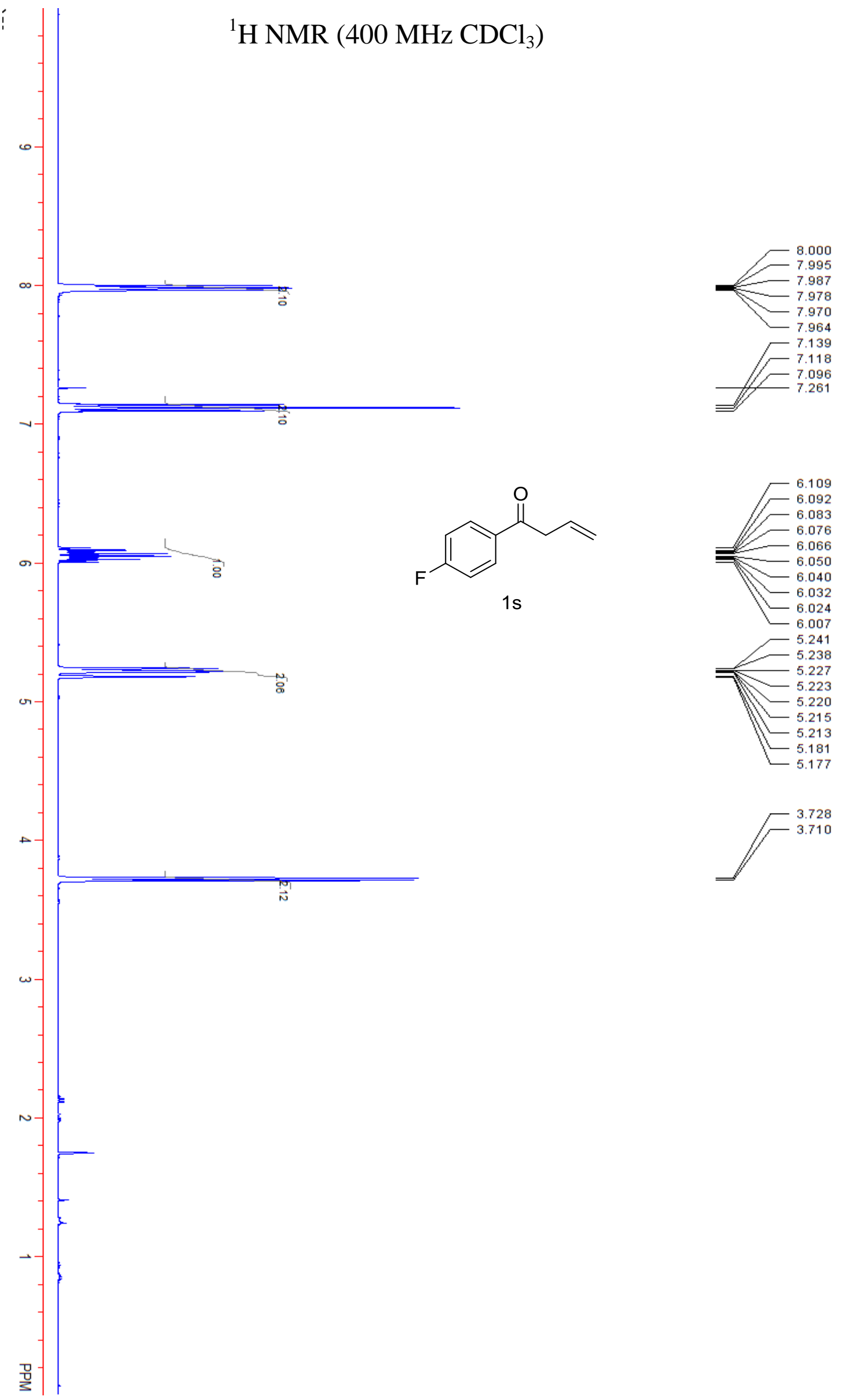




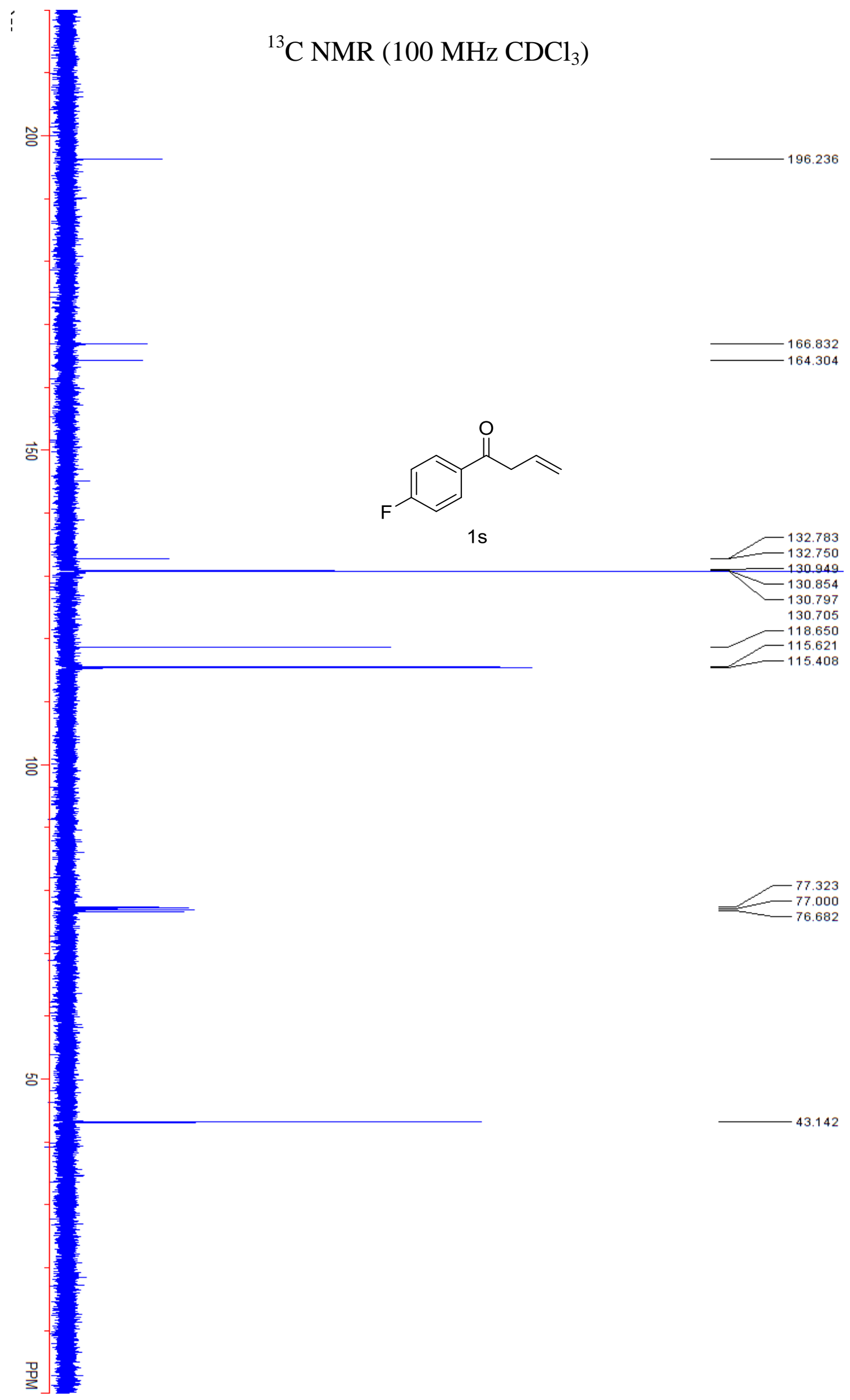




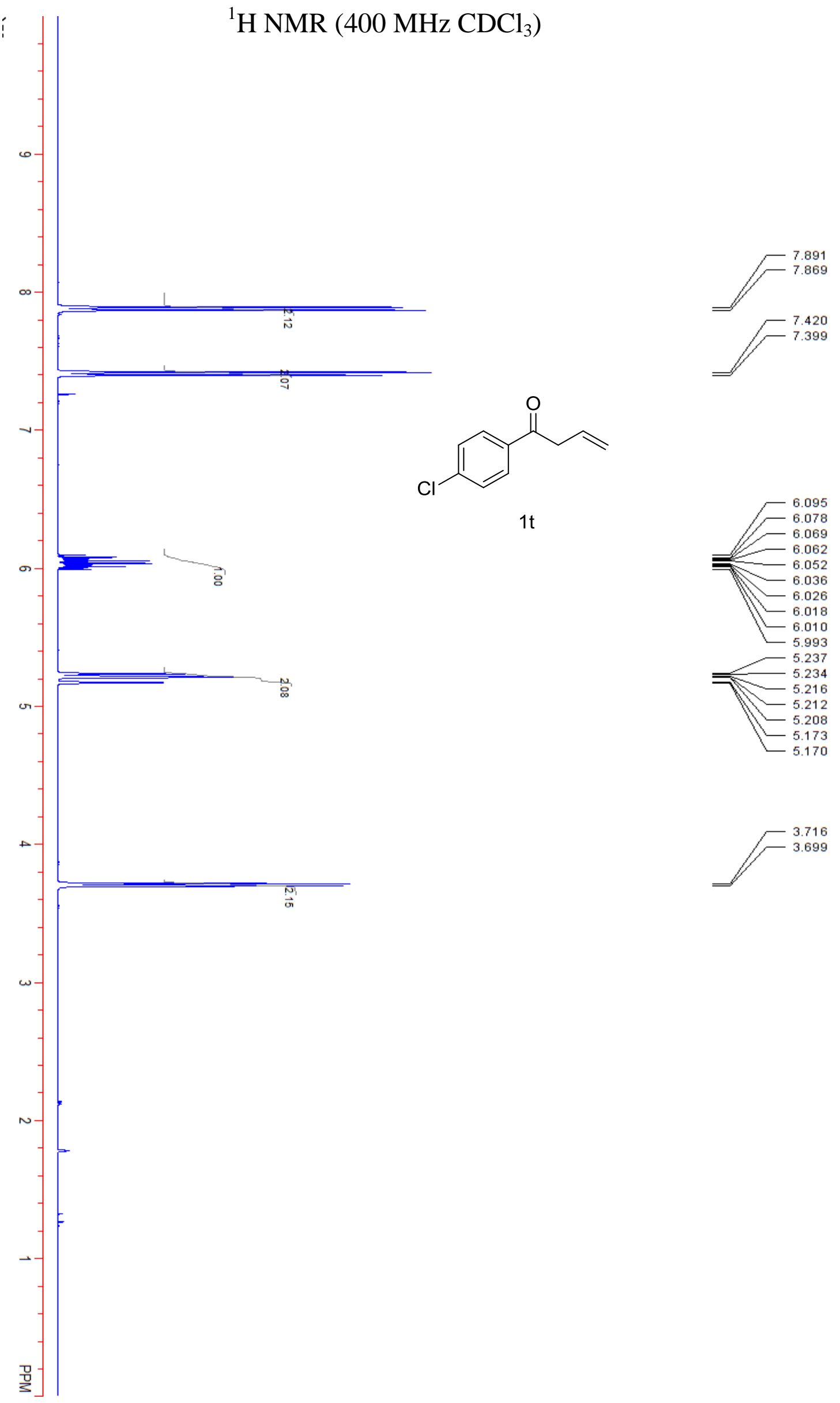




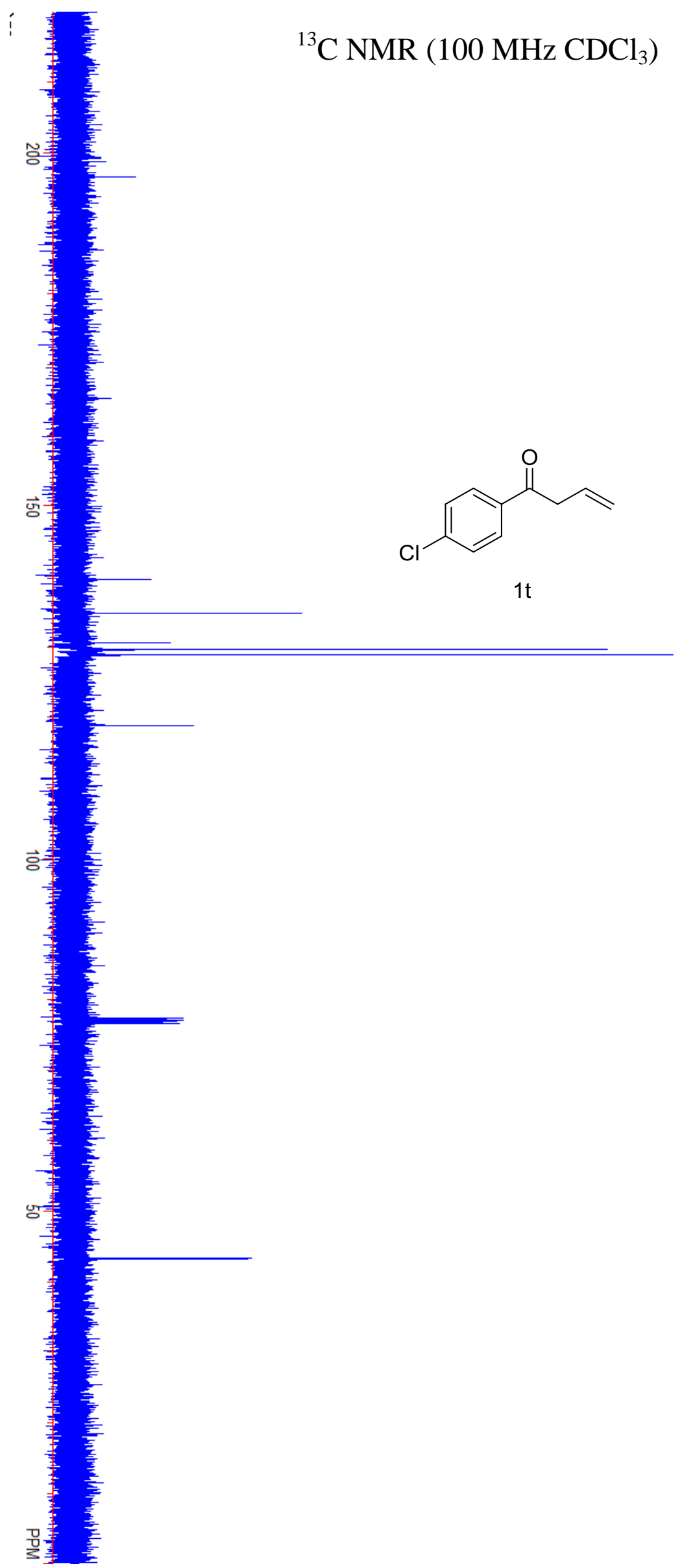

$\longrightarrow 196.615$

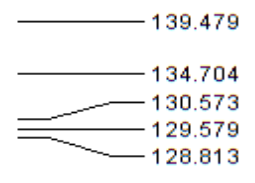

118.860

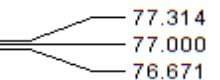

$-76.67$

43.252 


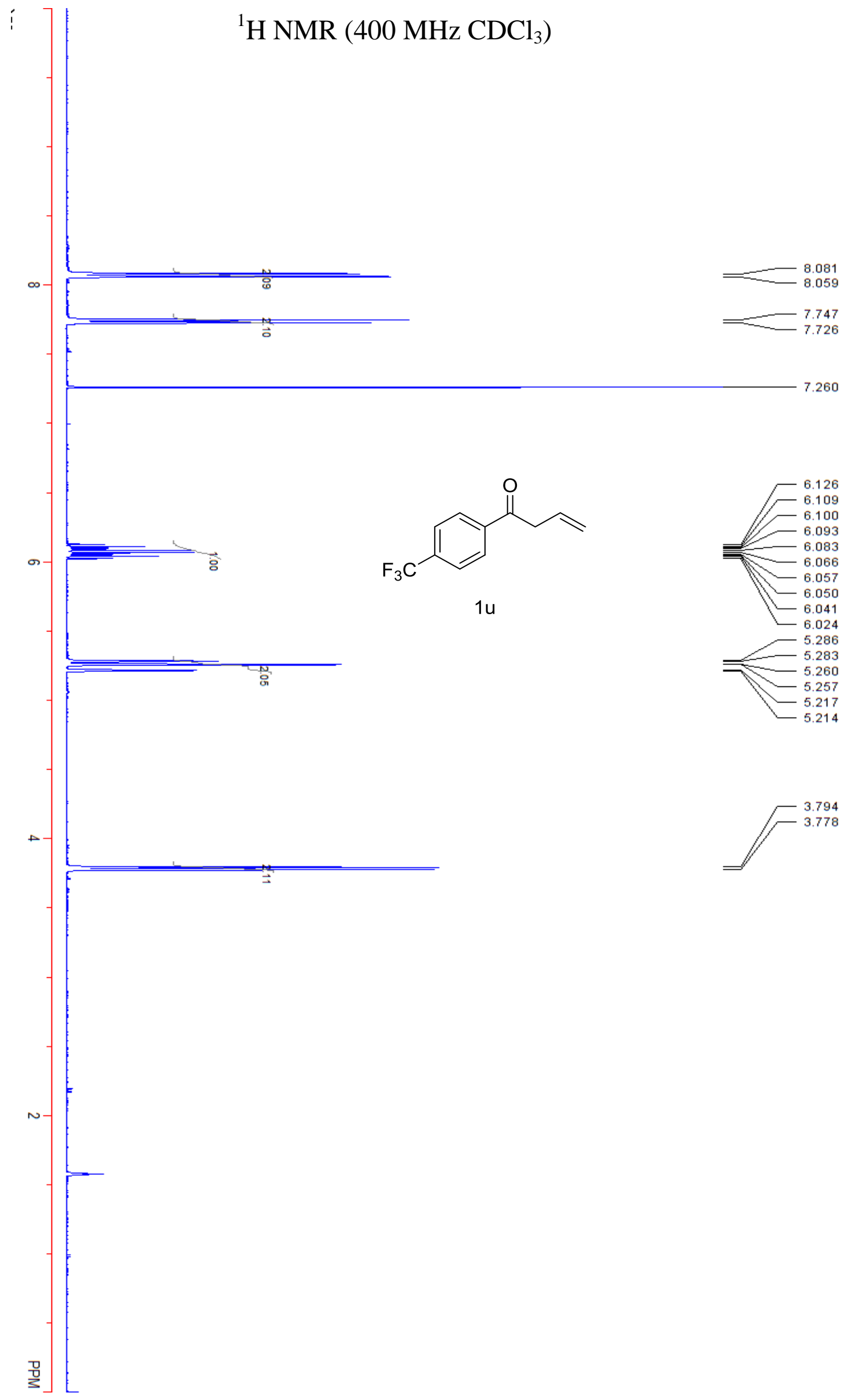


$\left.{ }^{13} \mathrm{C} \mathrm{NMR}(100 \mathrm{MHz} \mathrm{CDCl})_{3}\right)$

$-196.903$
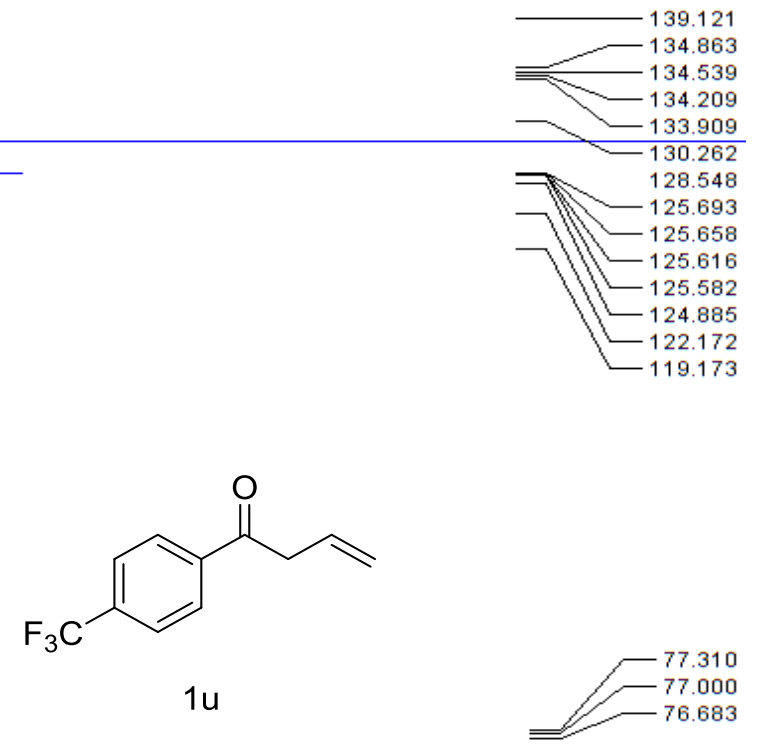

S85 


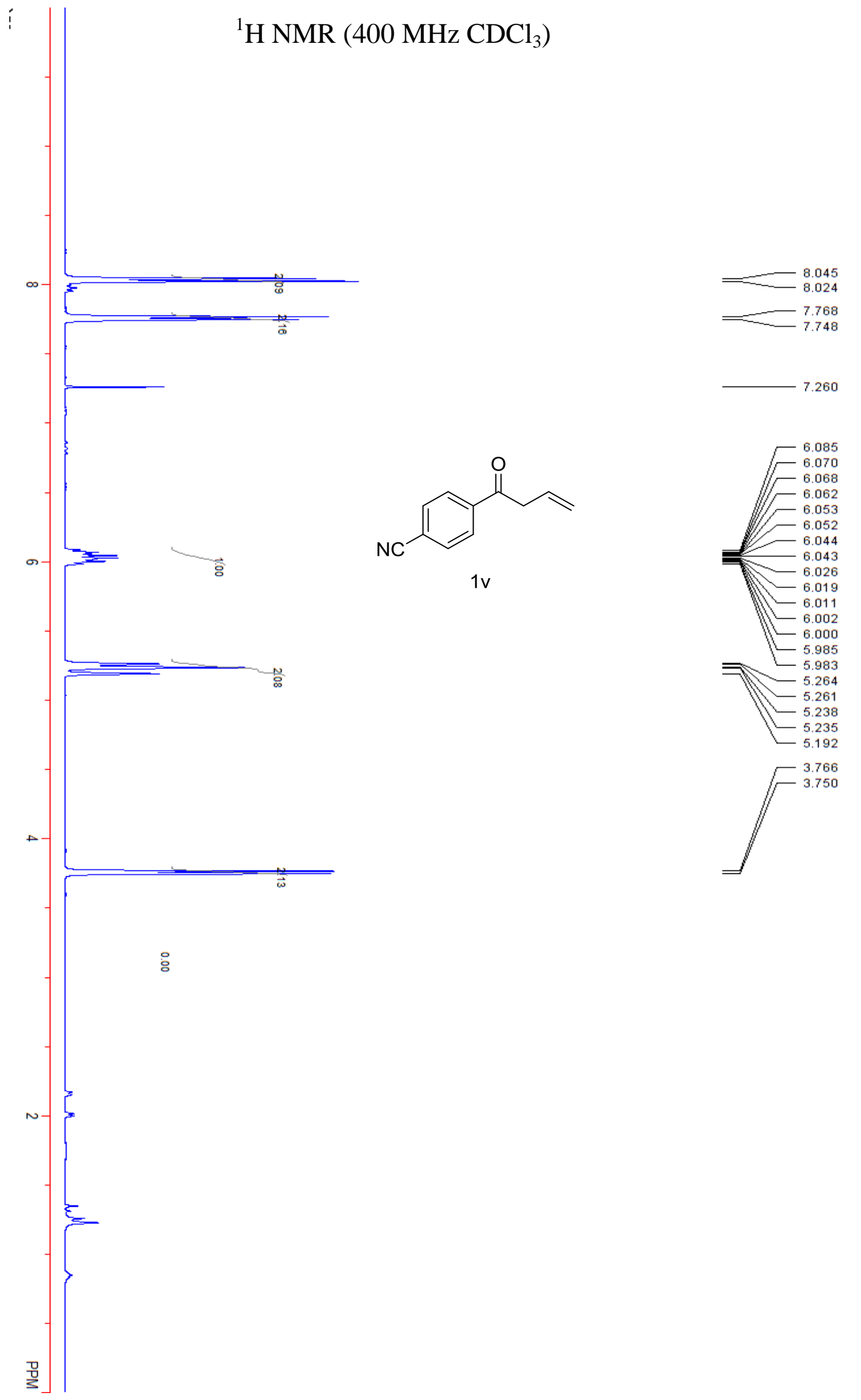




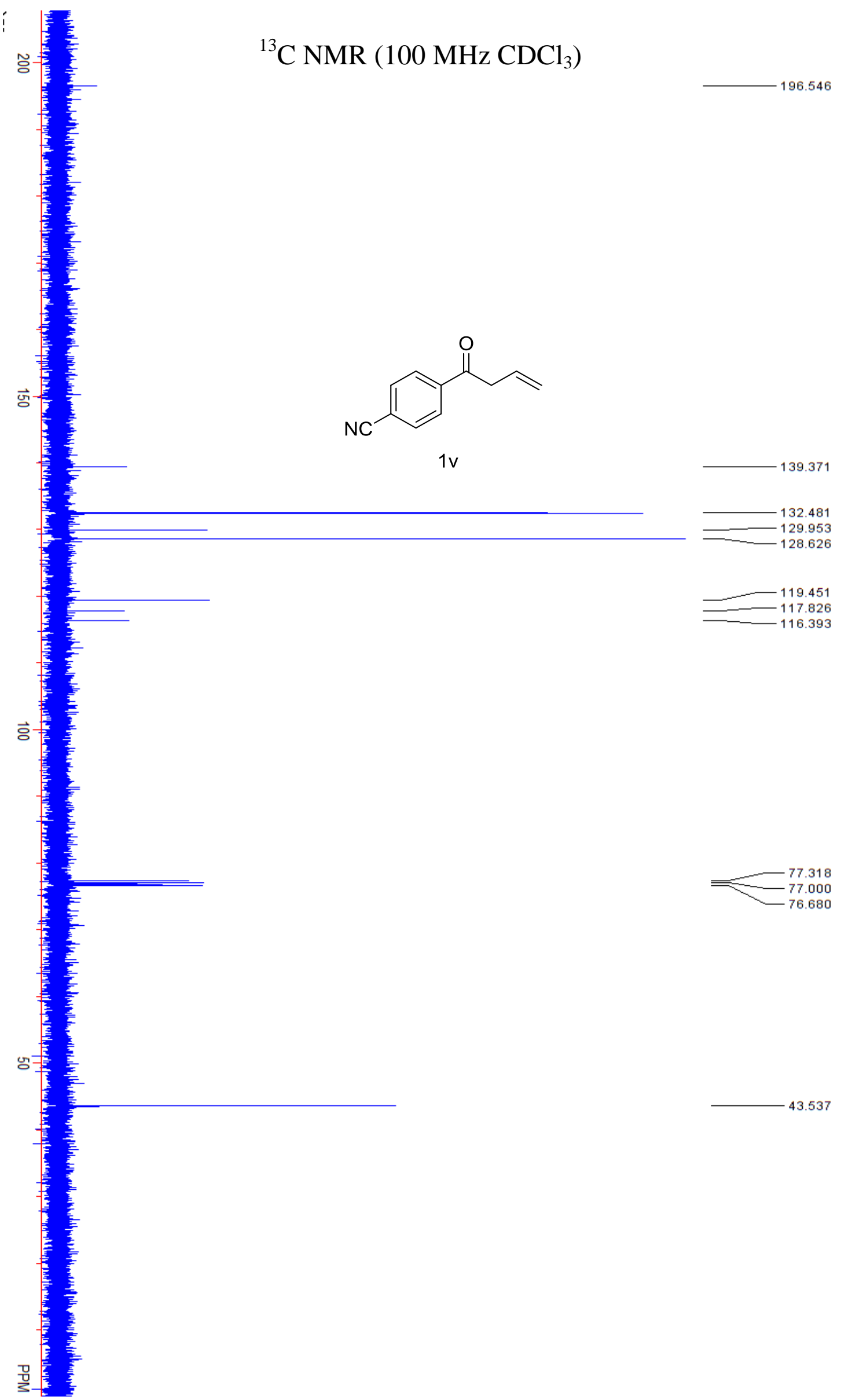




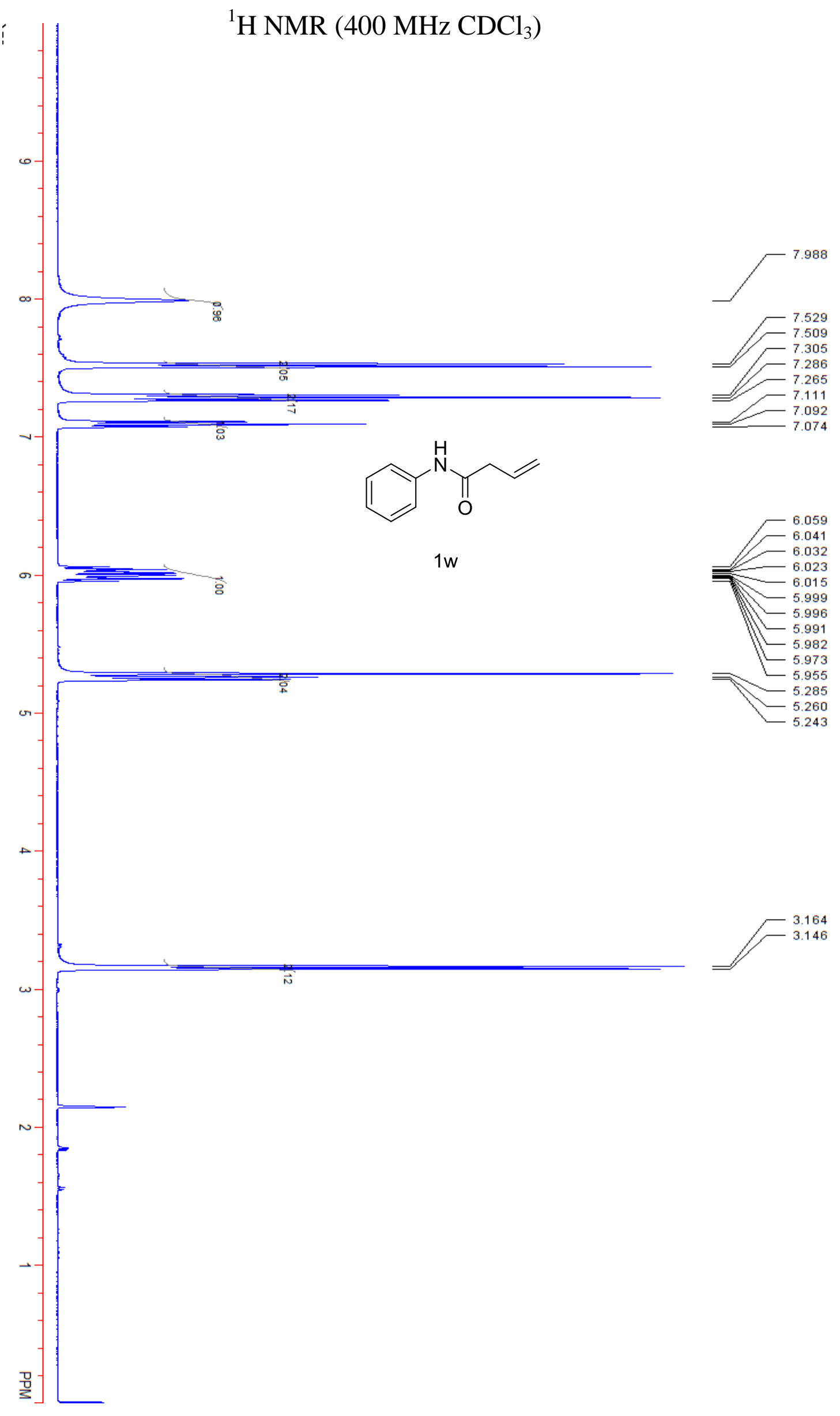



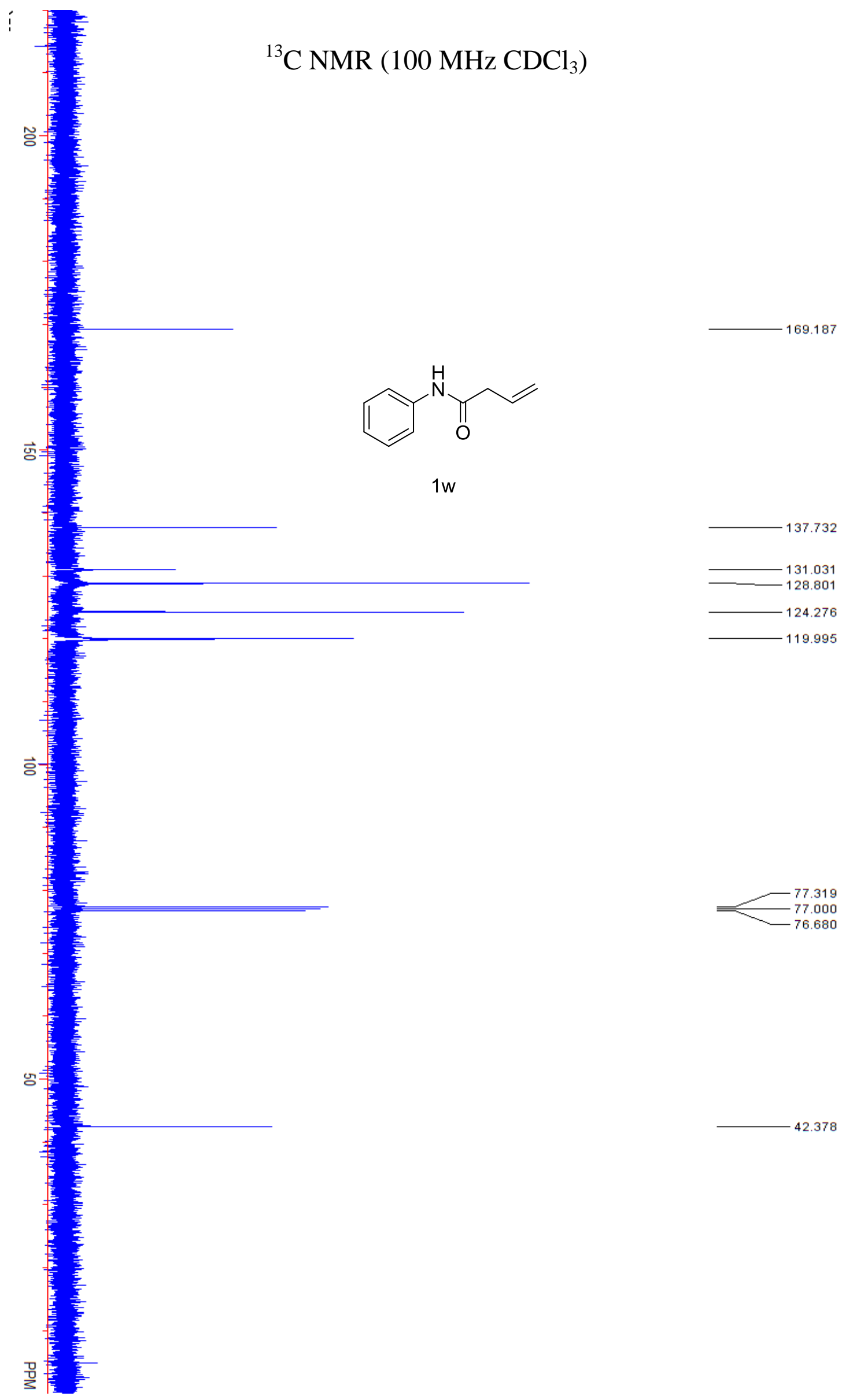


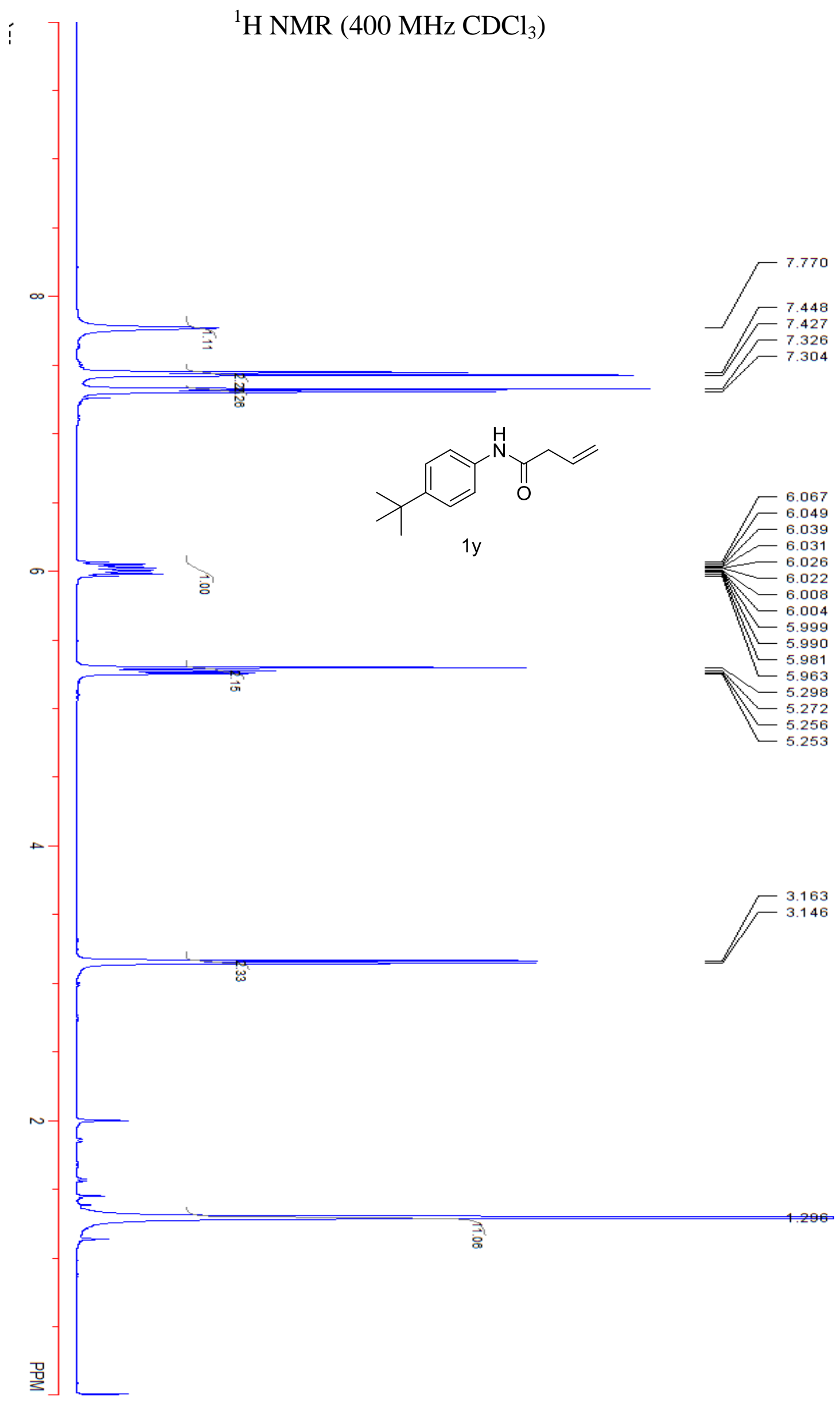



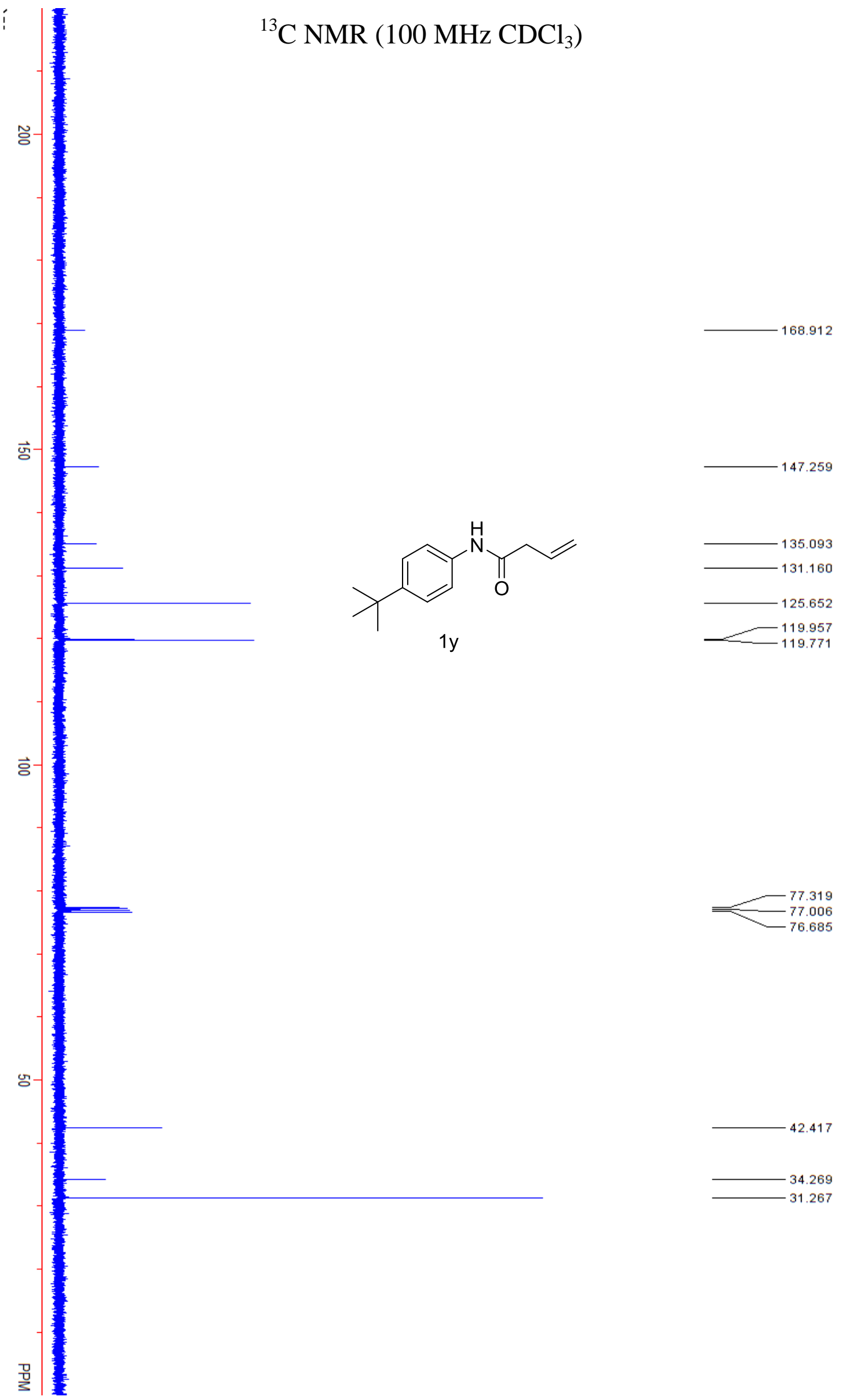

76.685

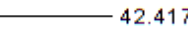

$-34.269$ 
${ }^{1} \mathrm{H}$ NMR $\left(400 \mathrm{MHz} \mathrm{CDCl}_{3}\right)$
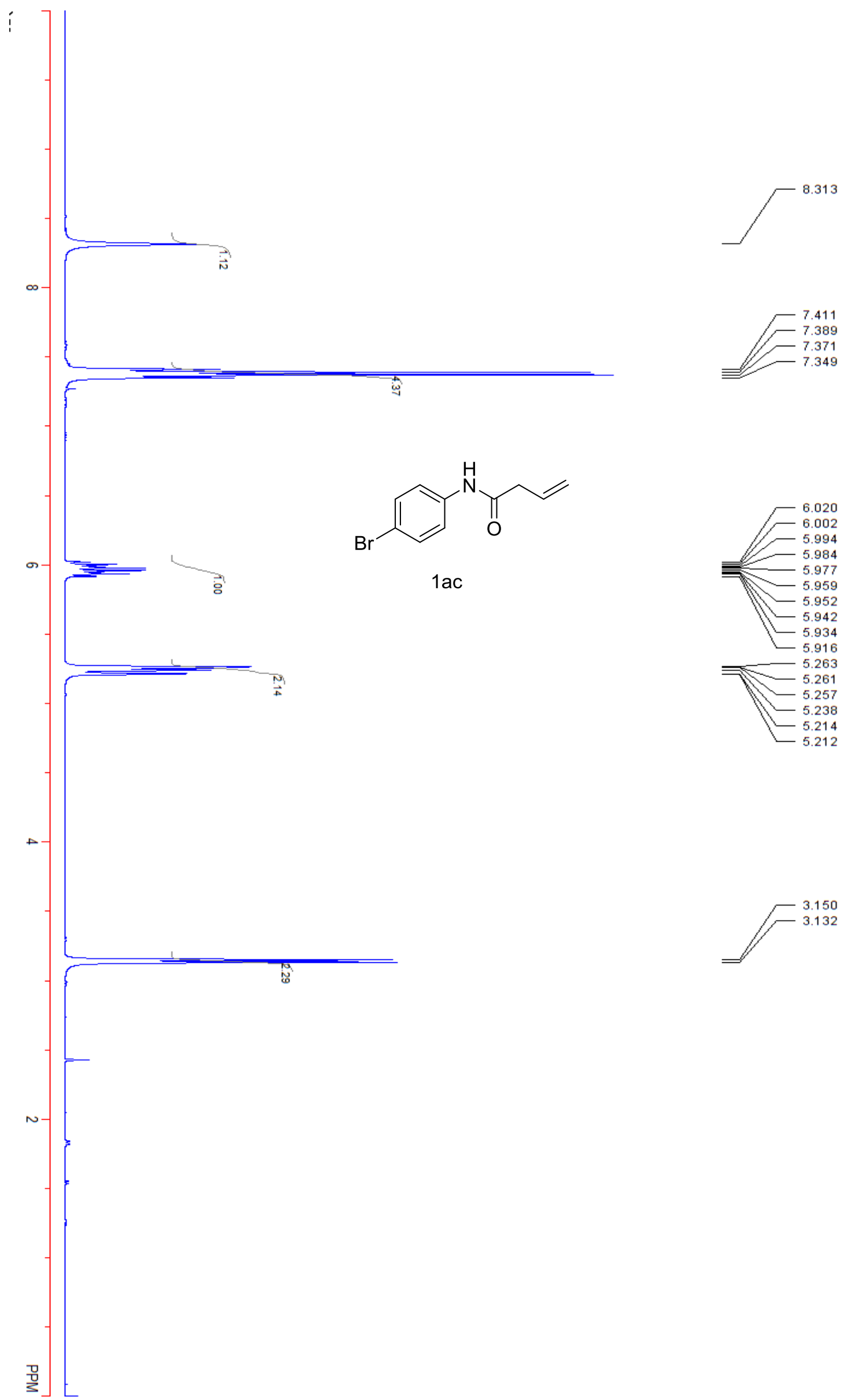


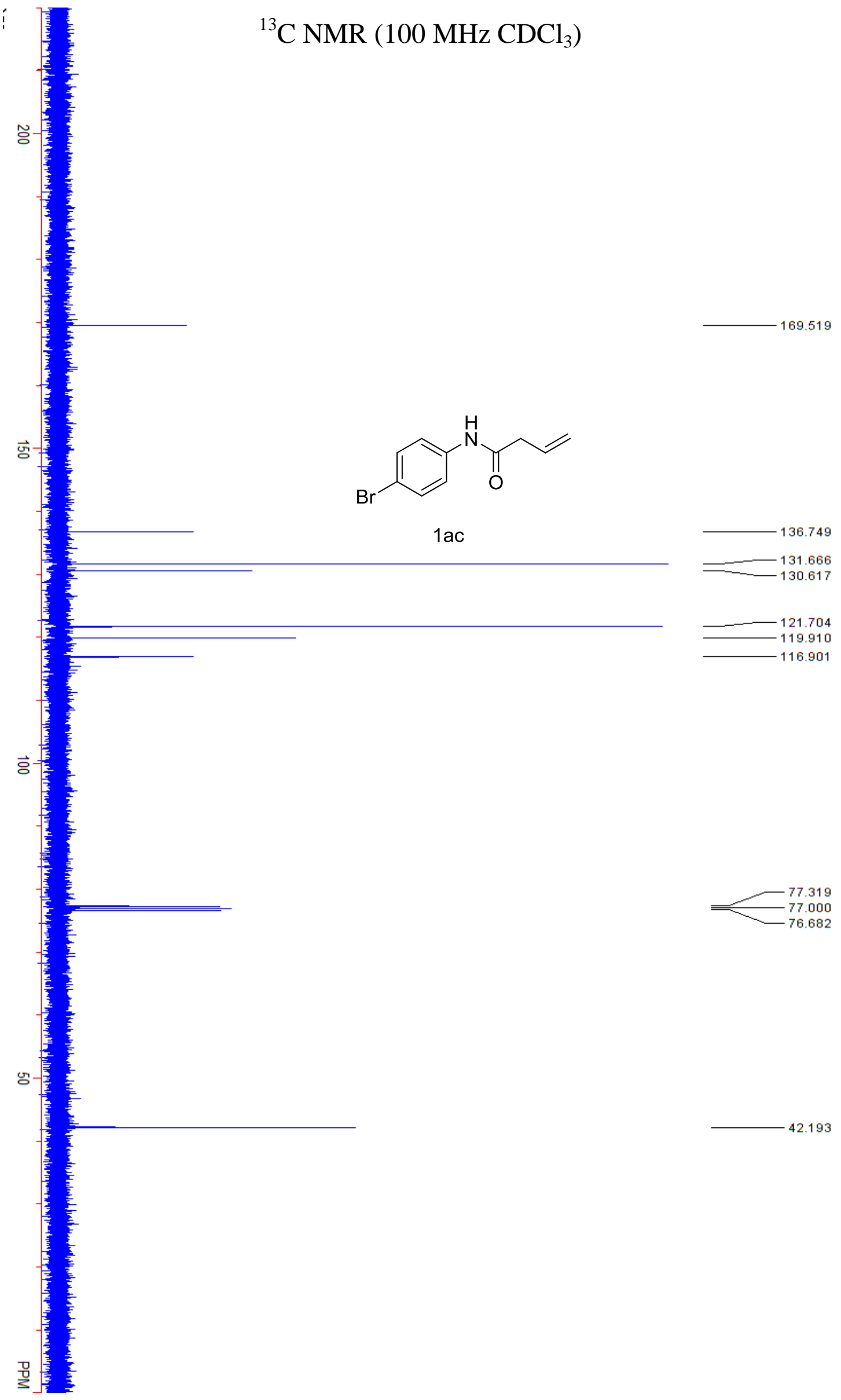




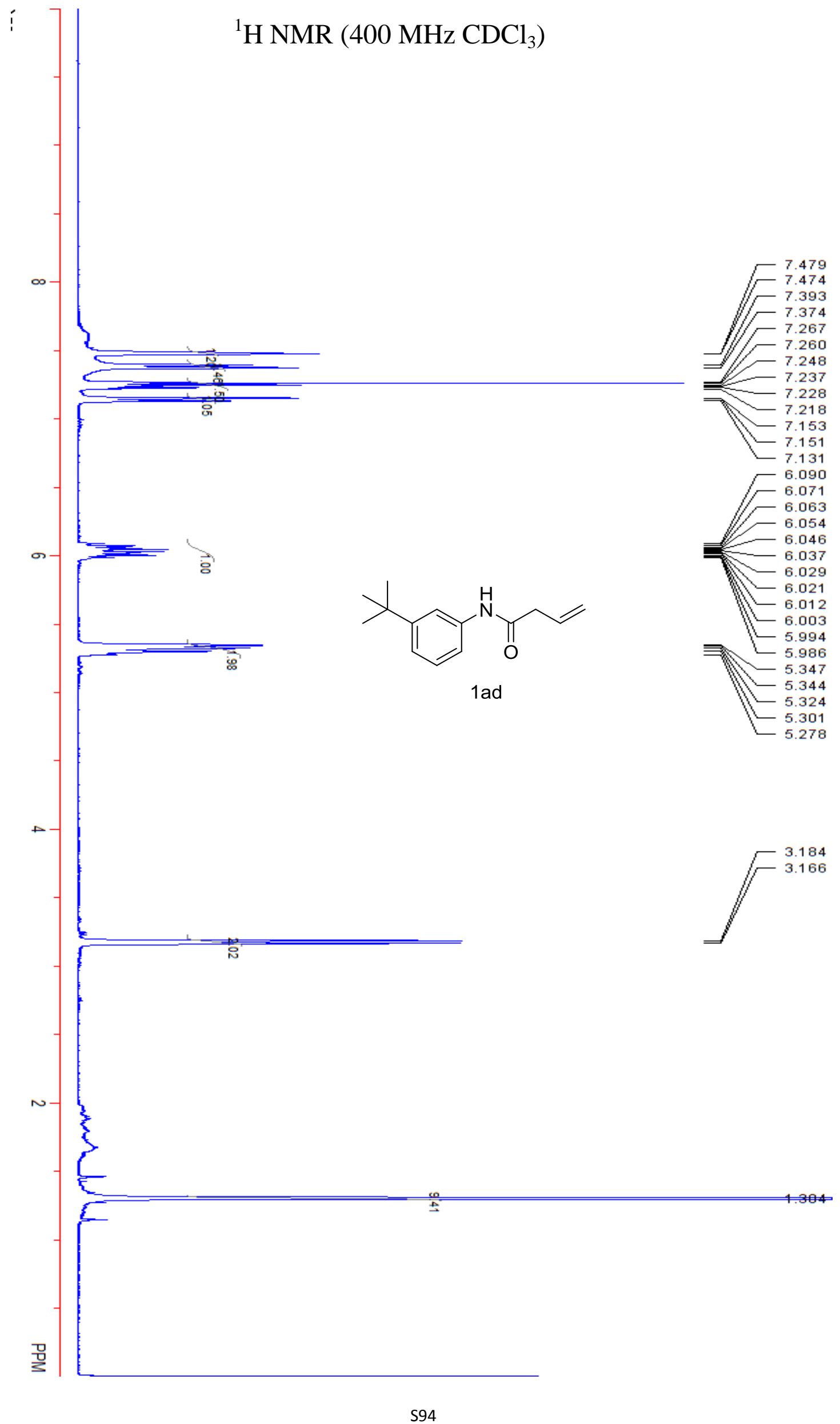




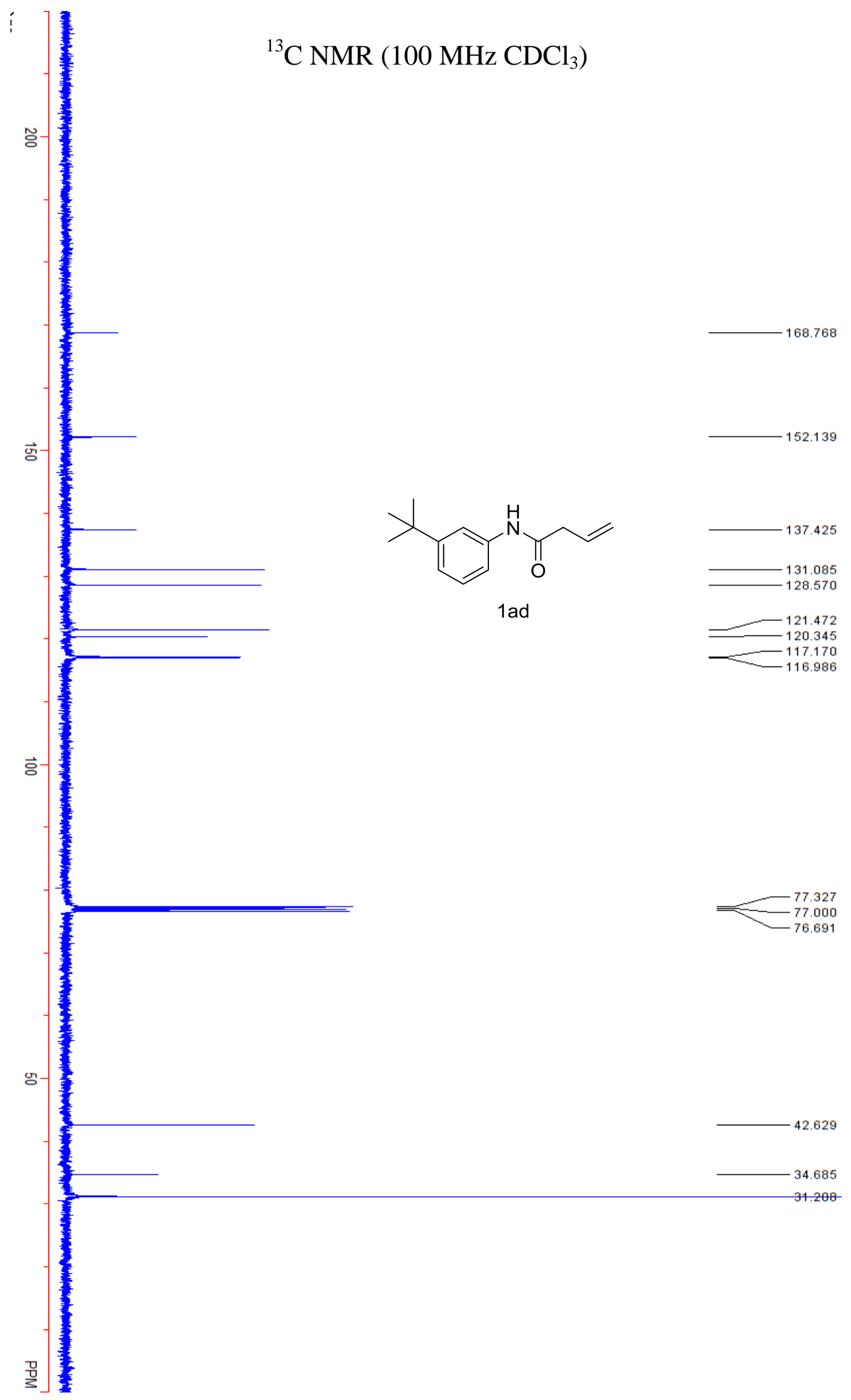




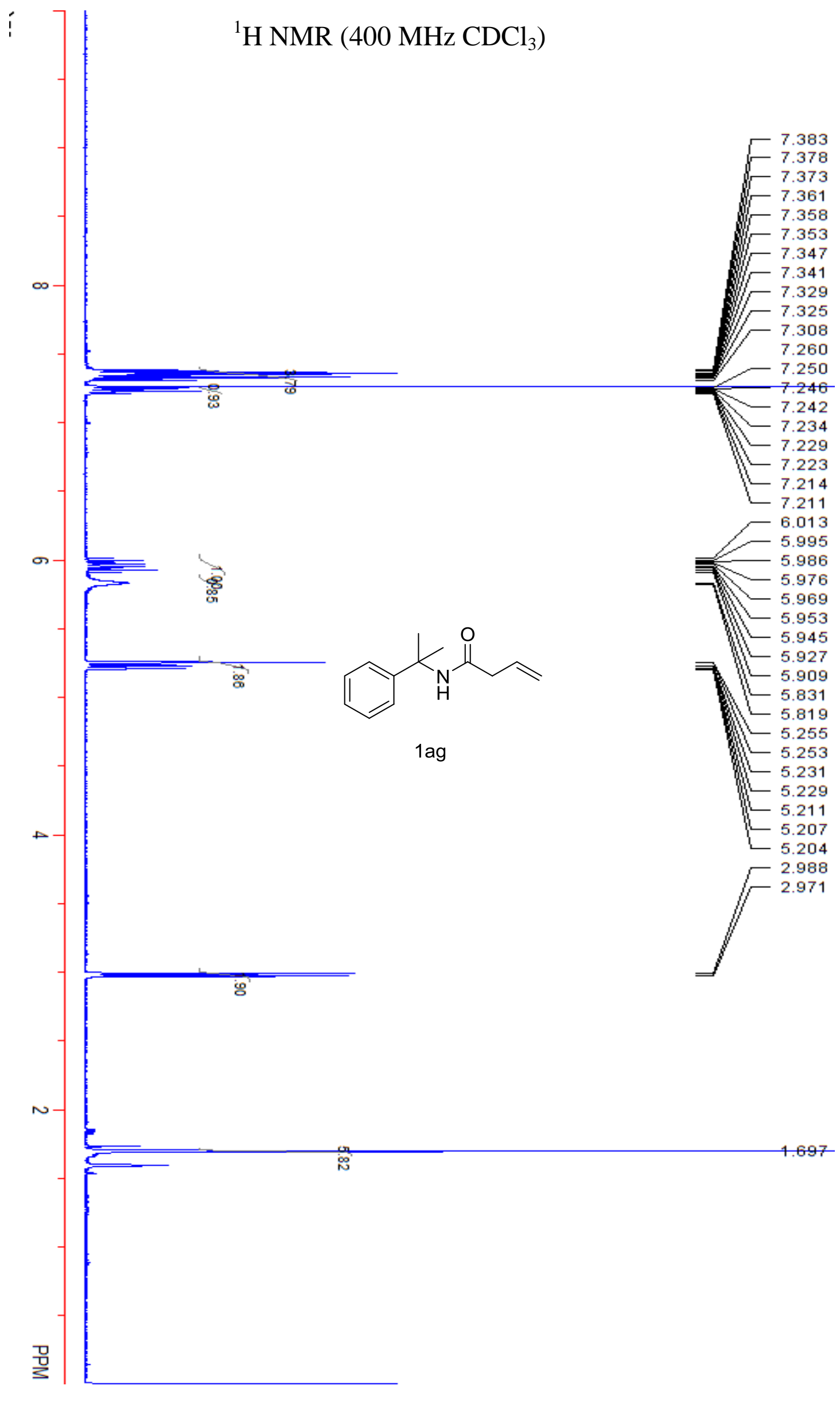




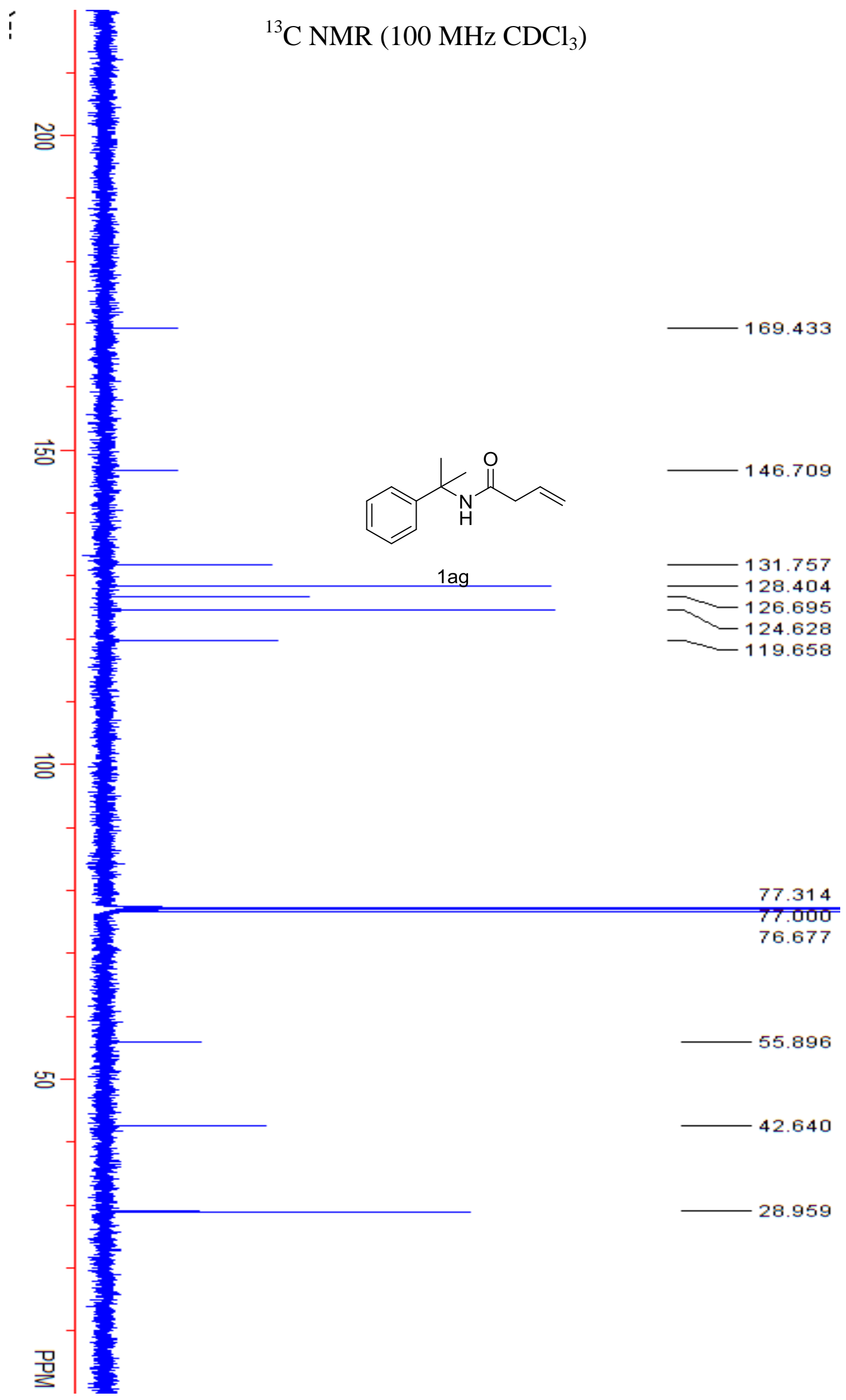




\section{${ }^{1} \mathrm{H}$ NMR (400 MHz $\mathrm{CDCl}_{3}$ )}
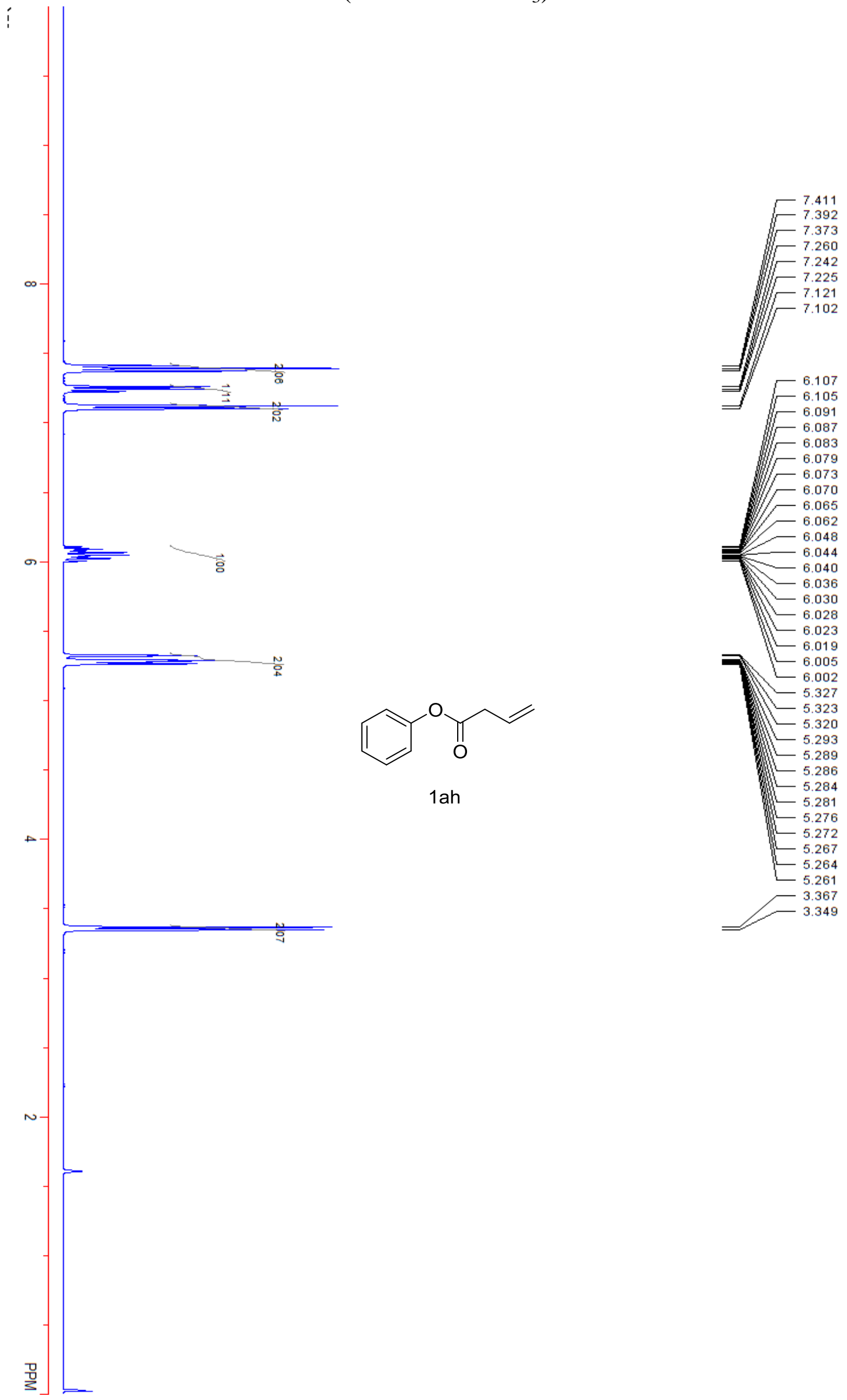
<smiles>C=CCC(=O)Oc1ccccc1</smiles>

$-169.887$

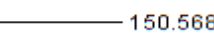

\begin{tabular}{r} 
\\
\hline \\
129.345 \\
-125.791 \\
\hline \\
121.419 \\
119.112
\end{tabular}

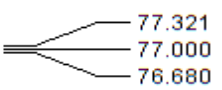

76.680

$-39.040$ 


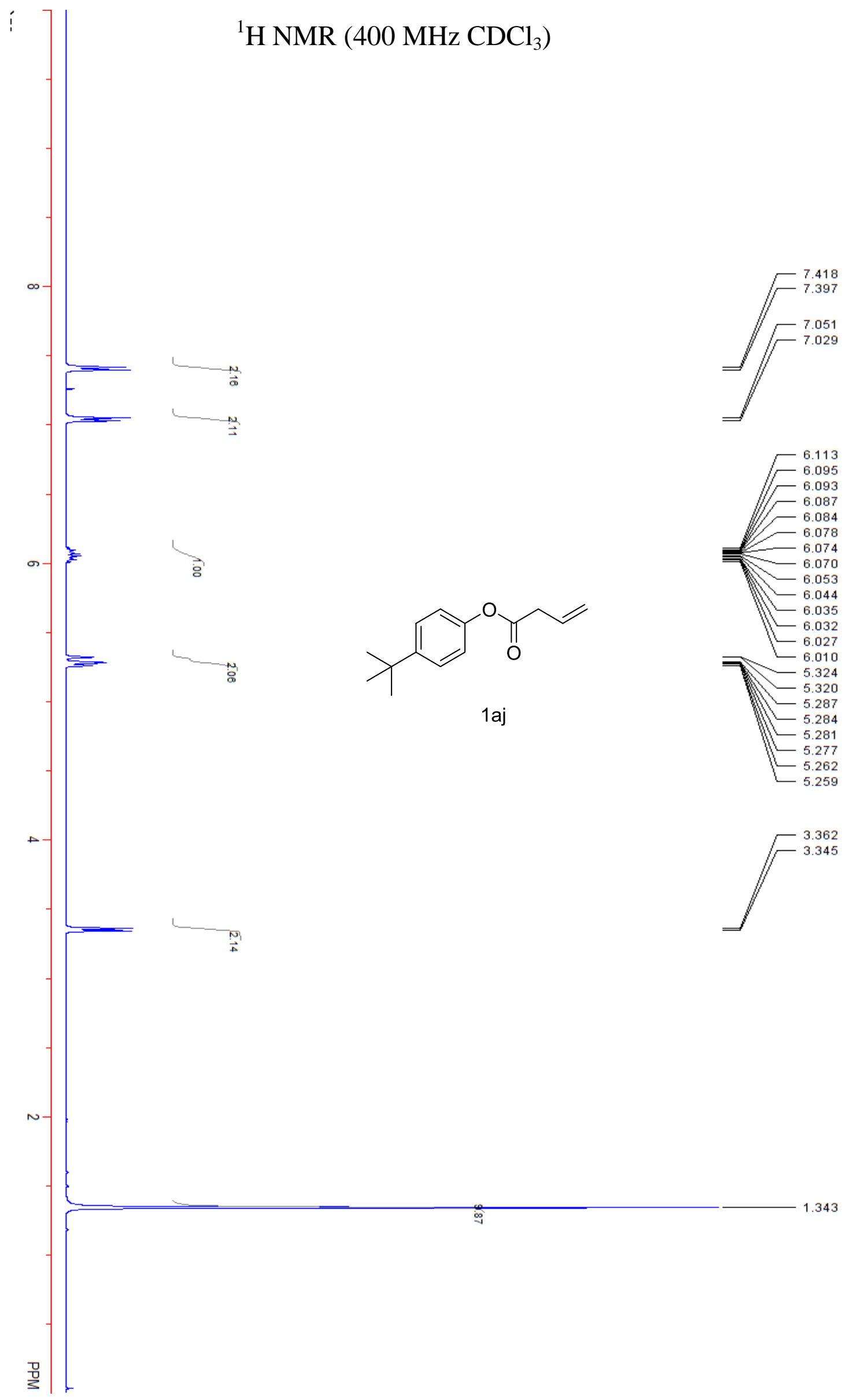




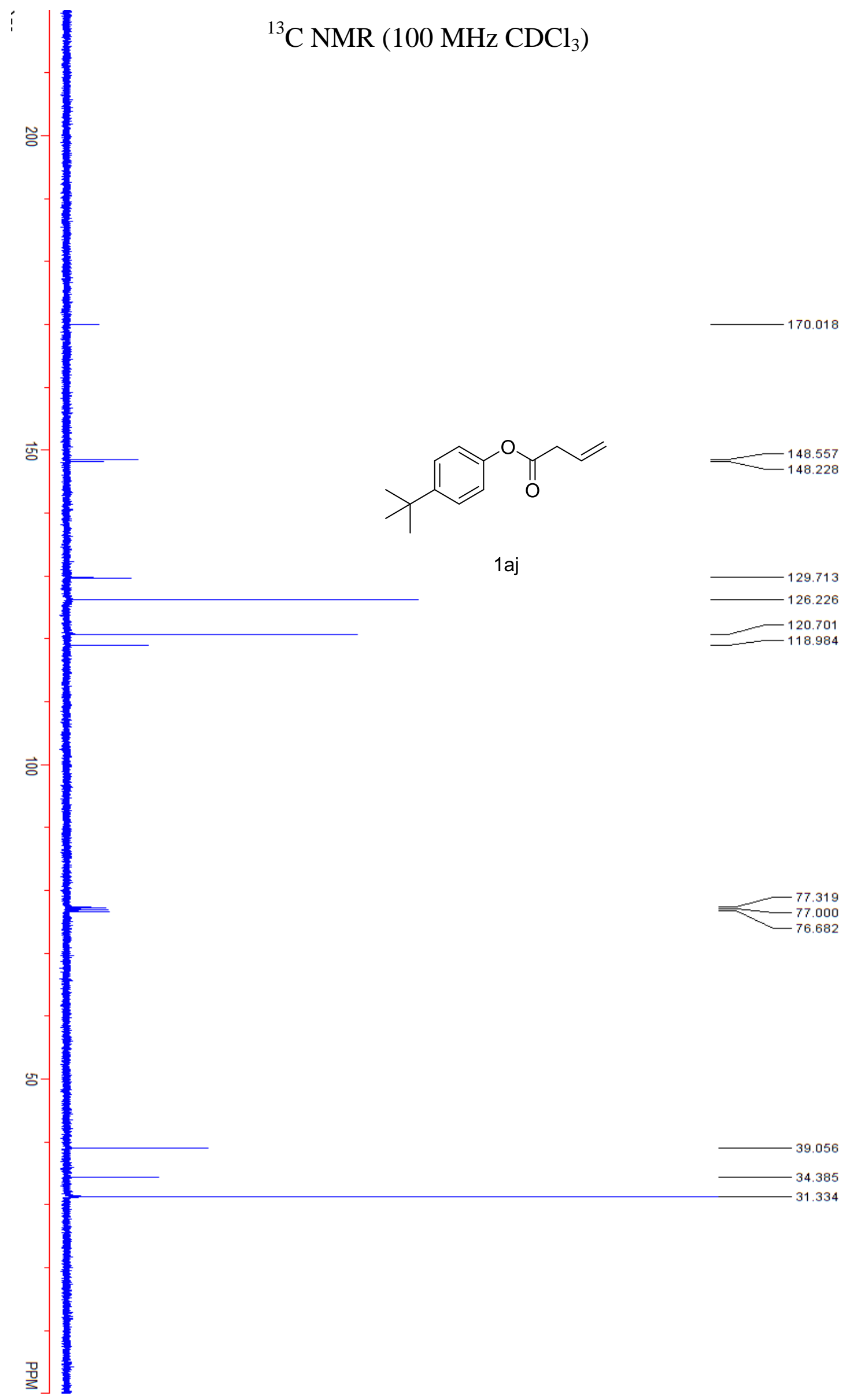



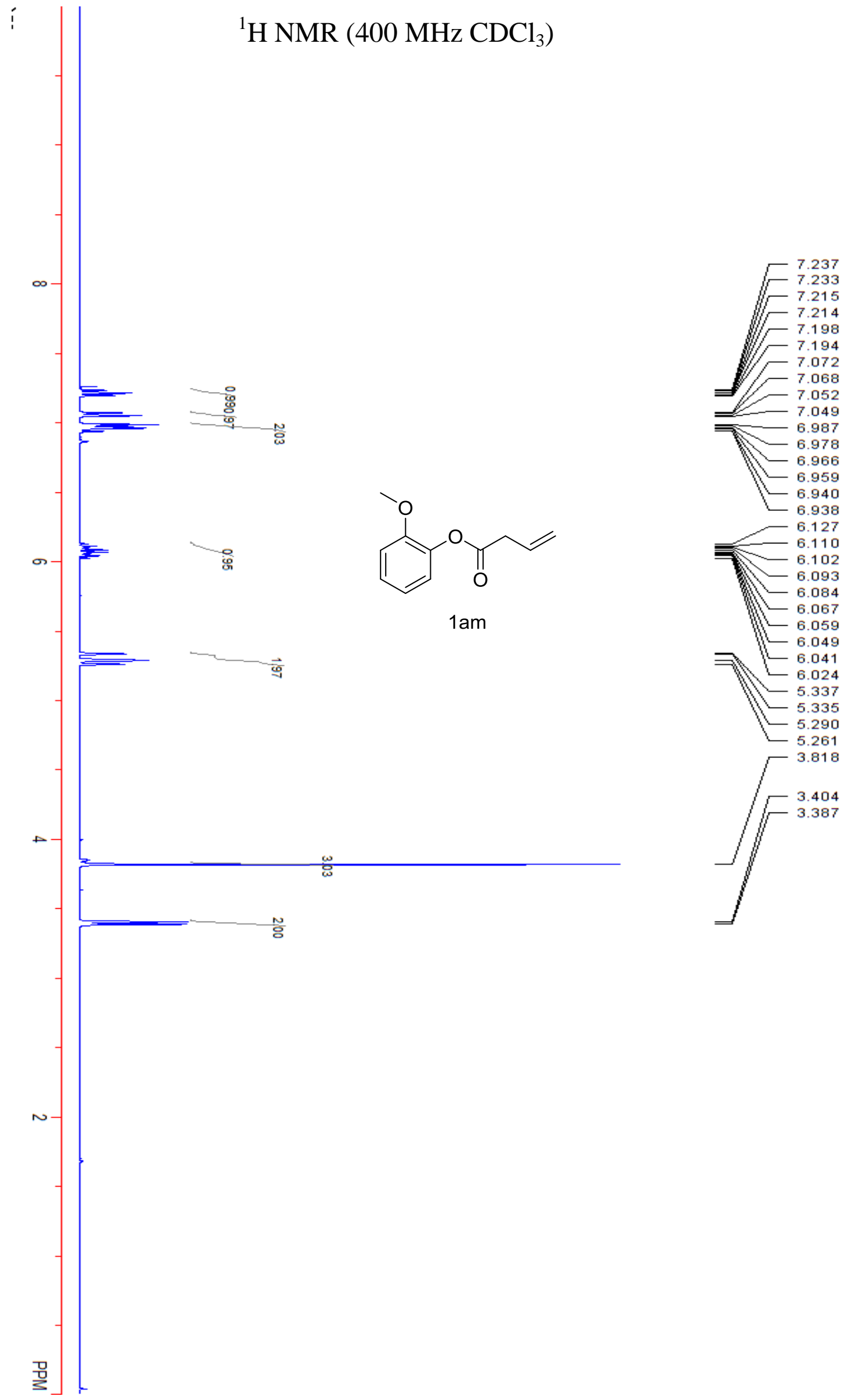

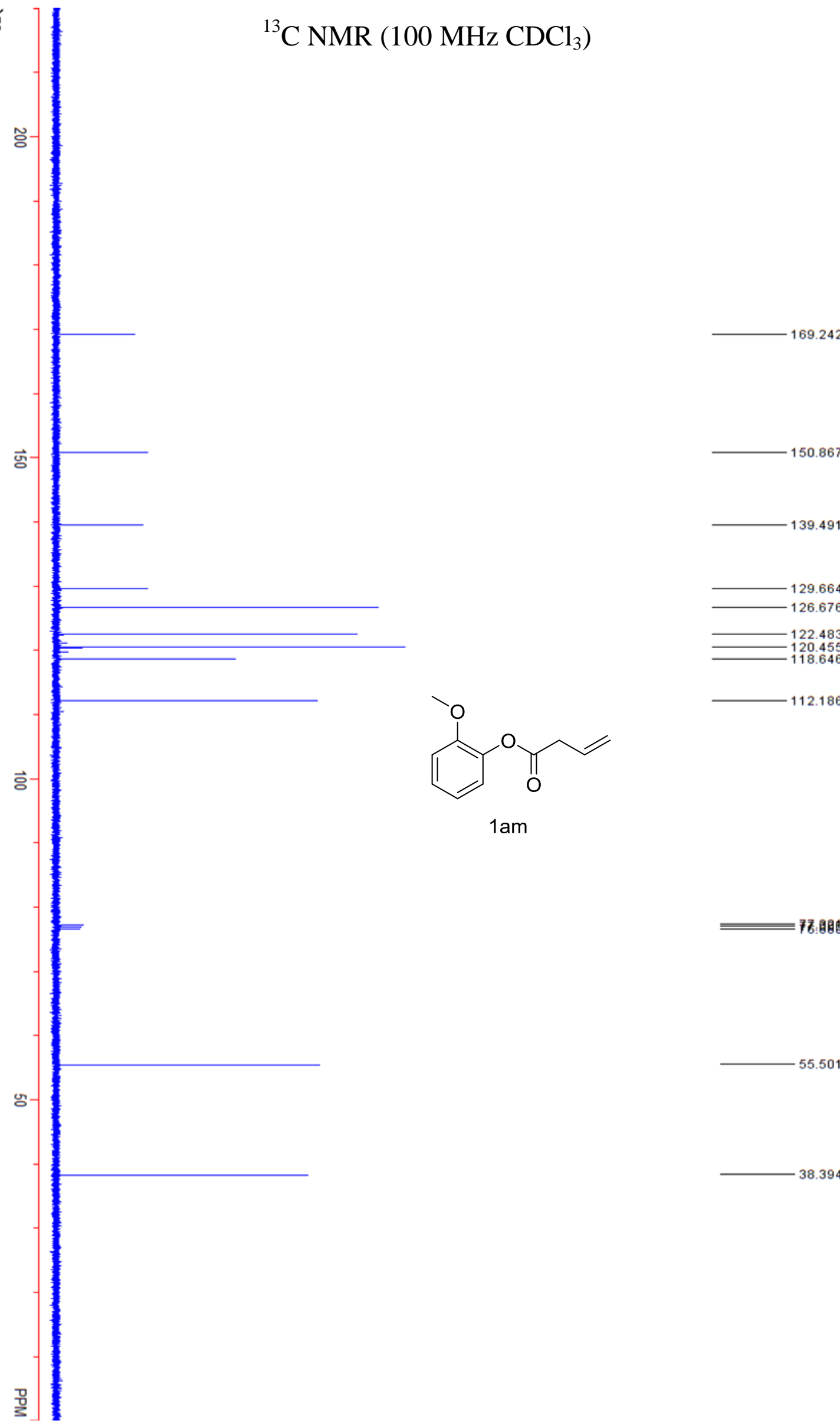

169.242

150.867

139.491

$-129.664$

$-126.676$

22.483 118646<smiles>C=CCC(=O)Oc1ccccc1OC</smiles>

112.186 


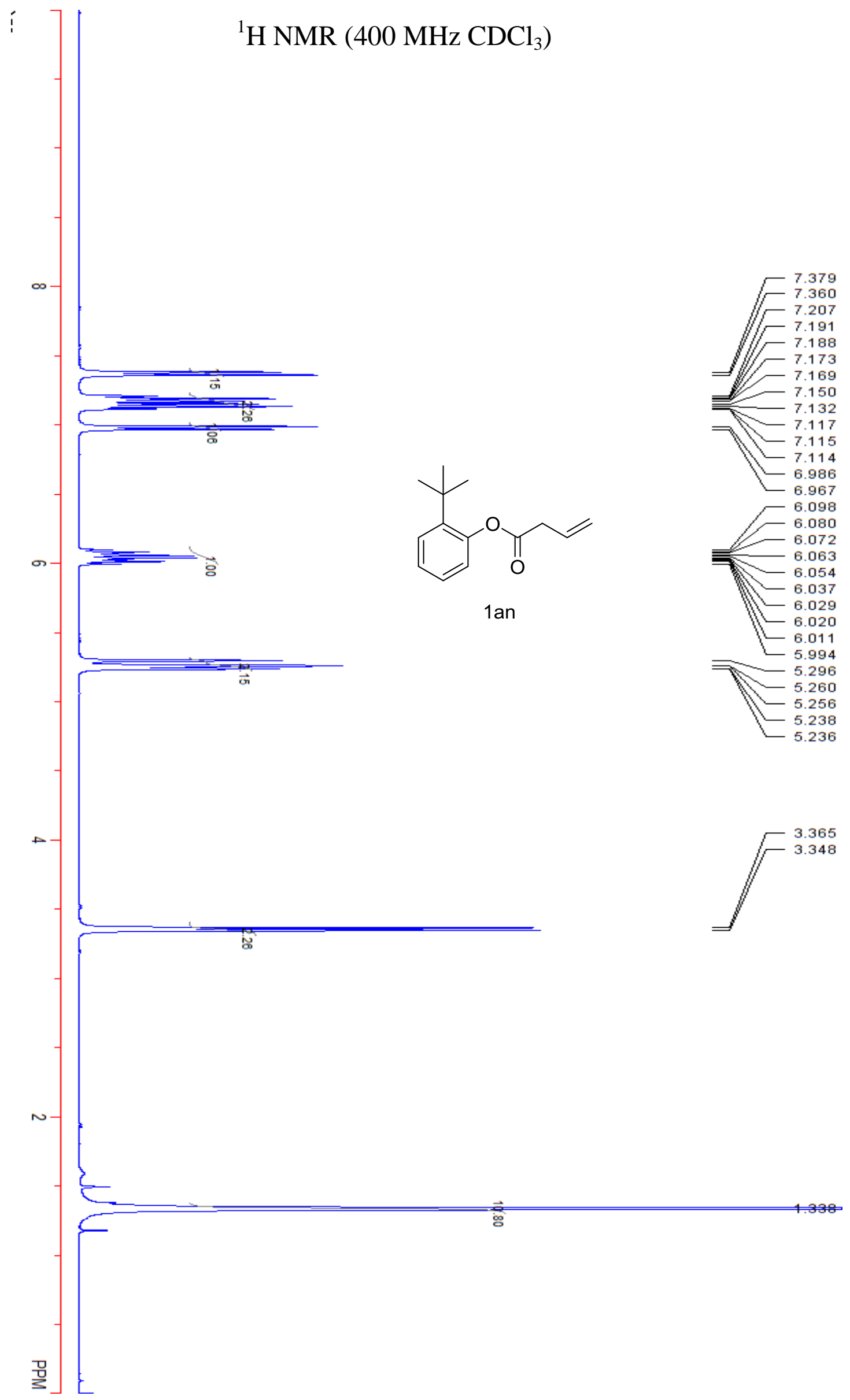




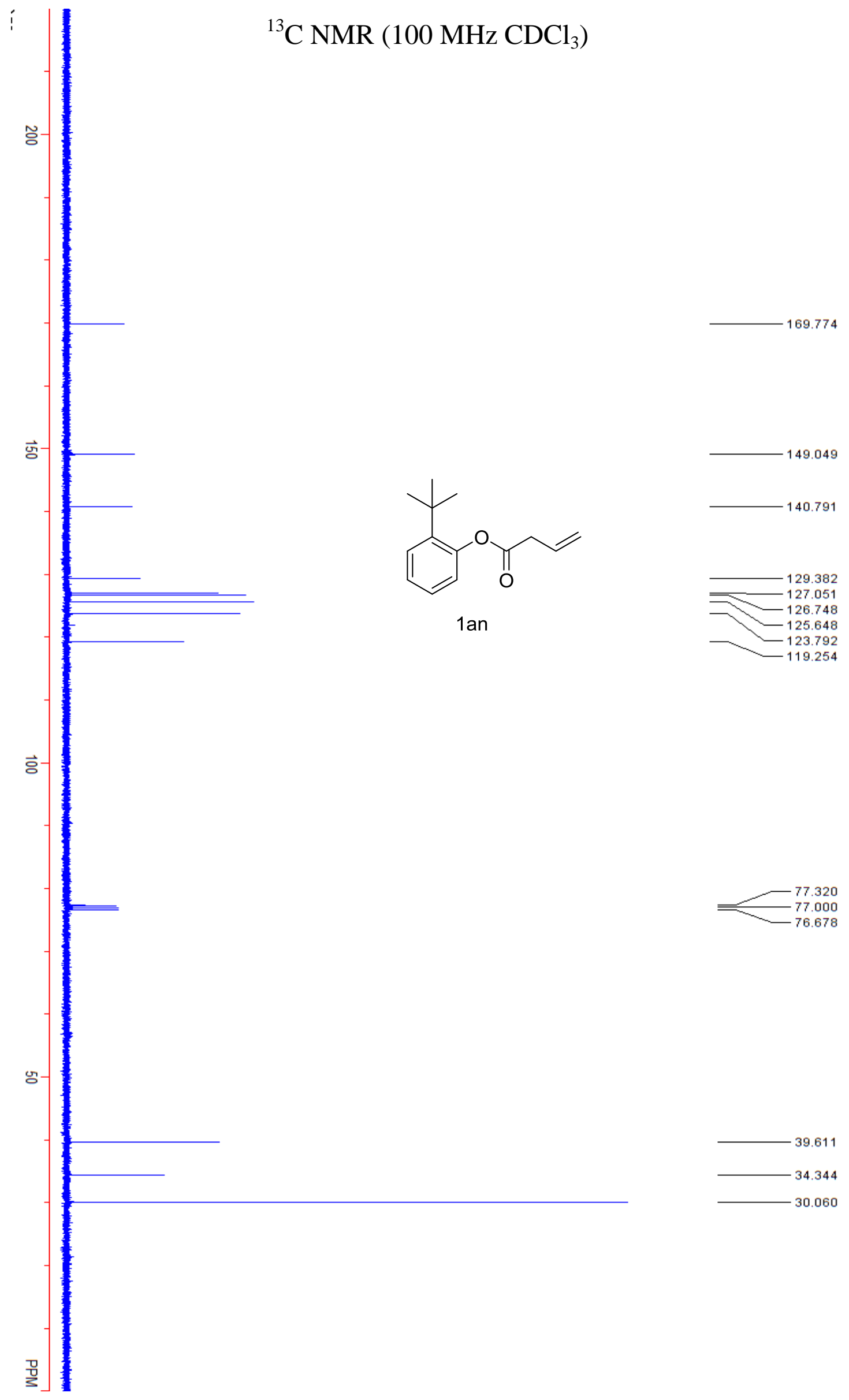



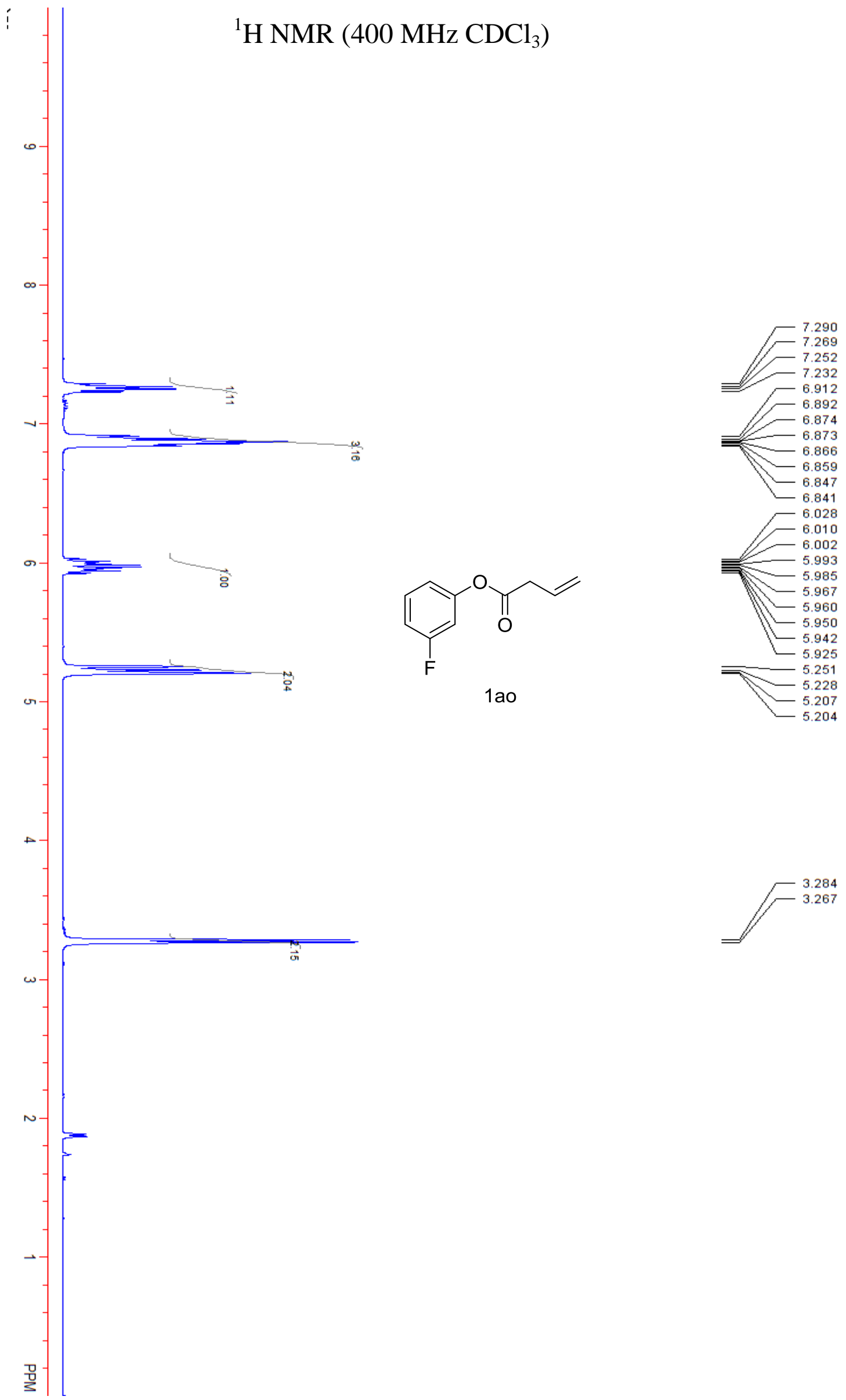


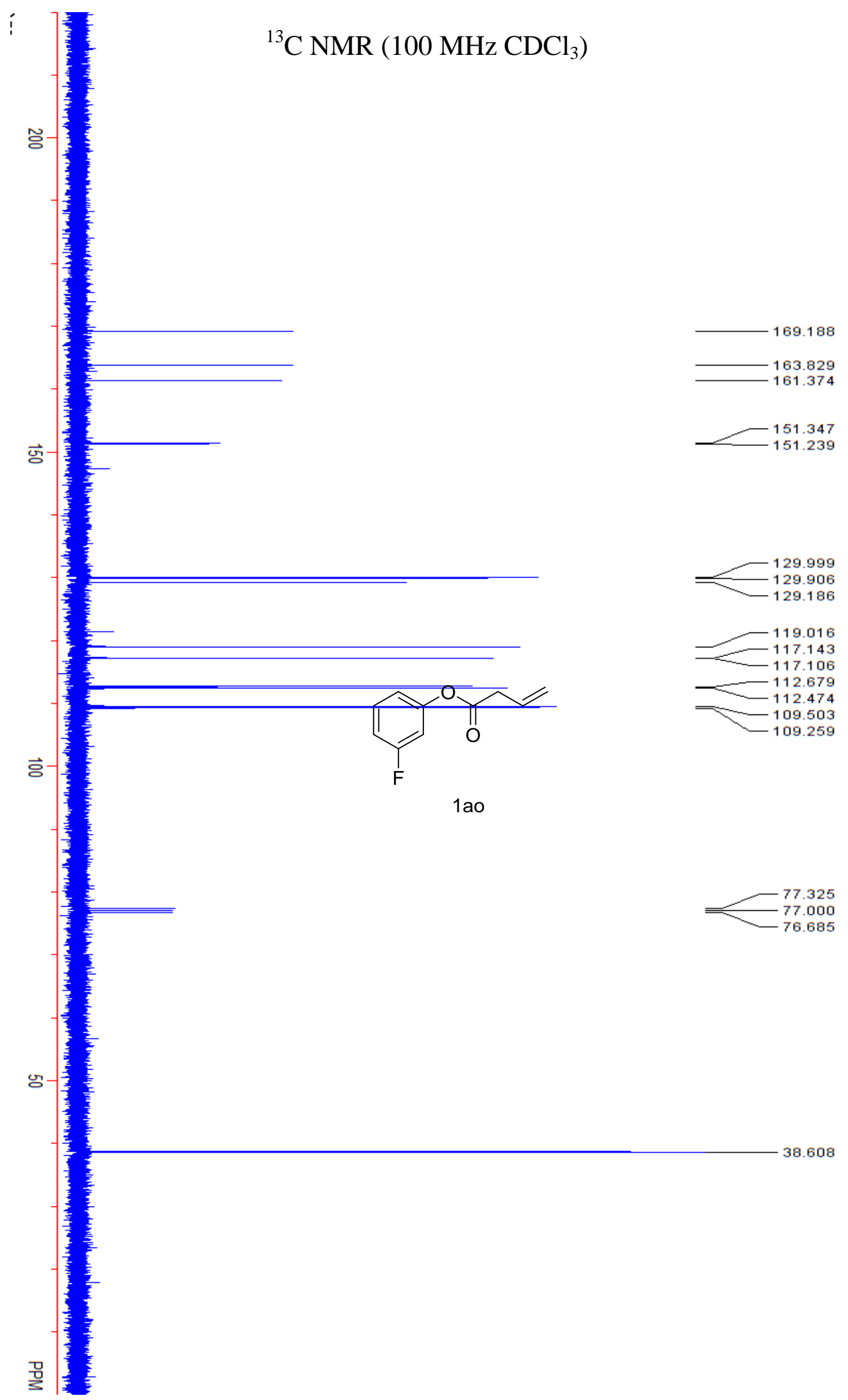



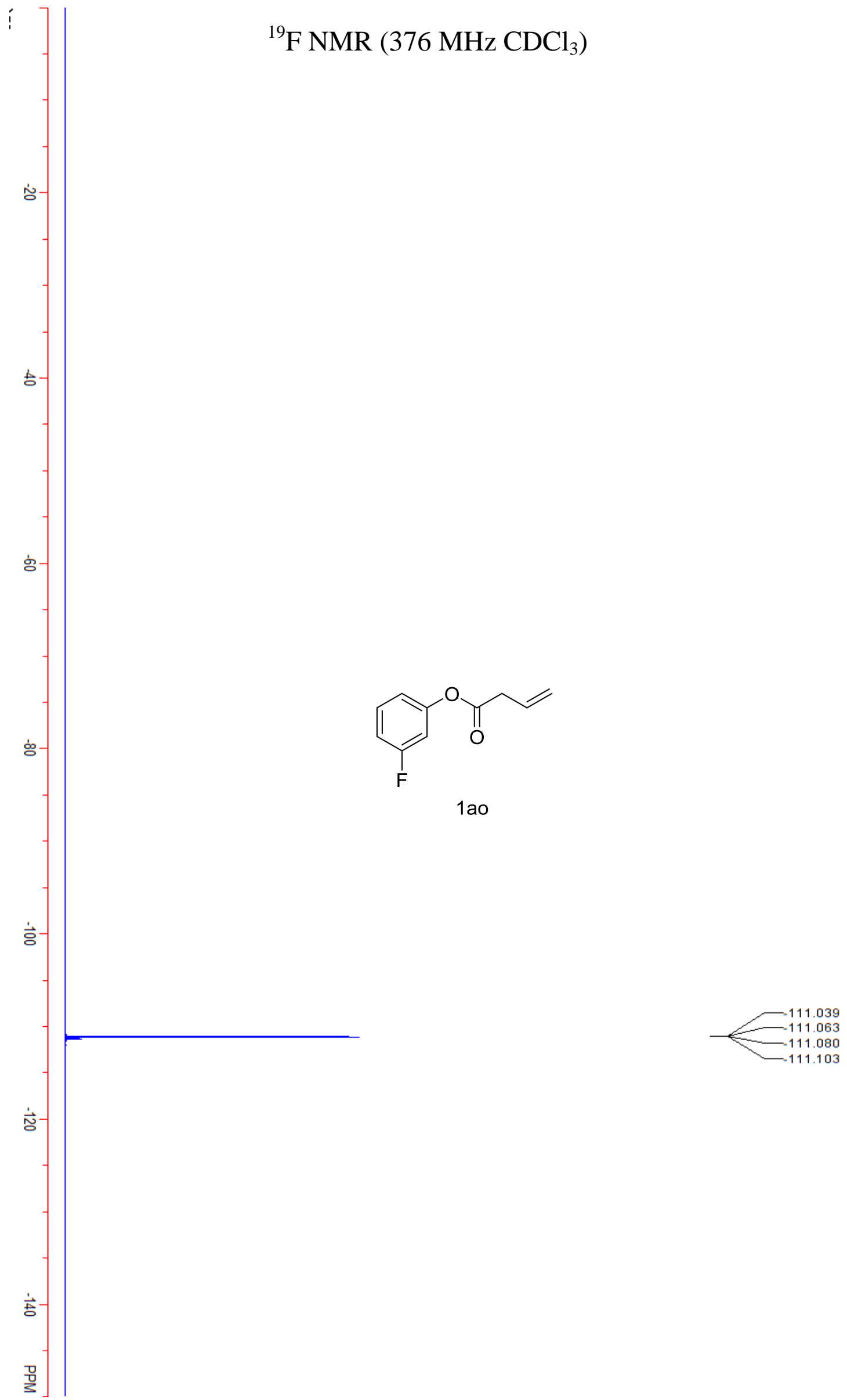

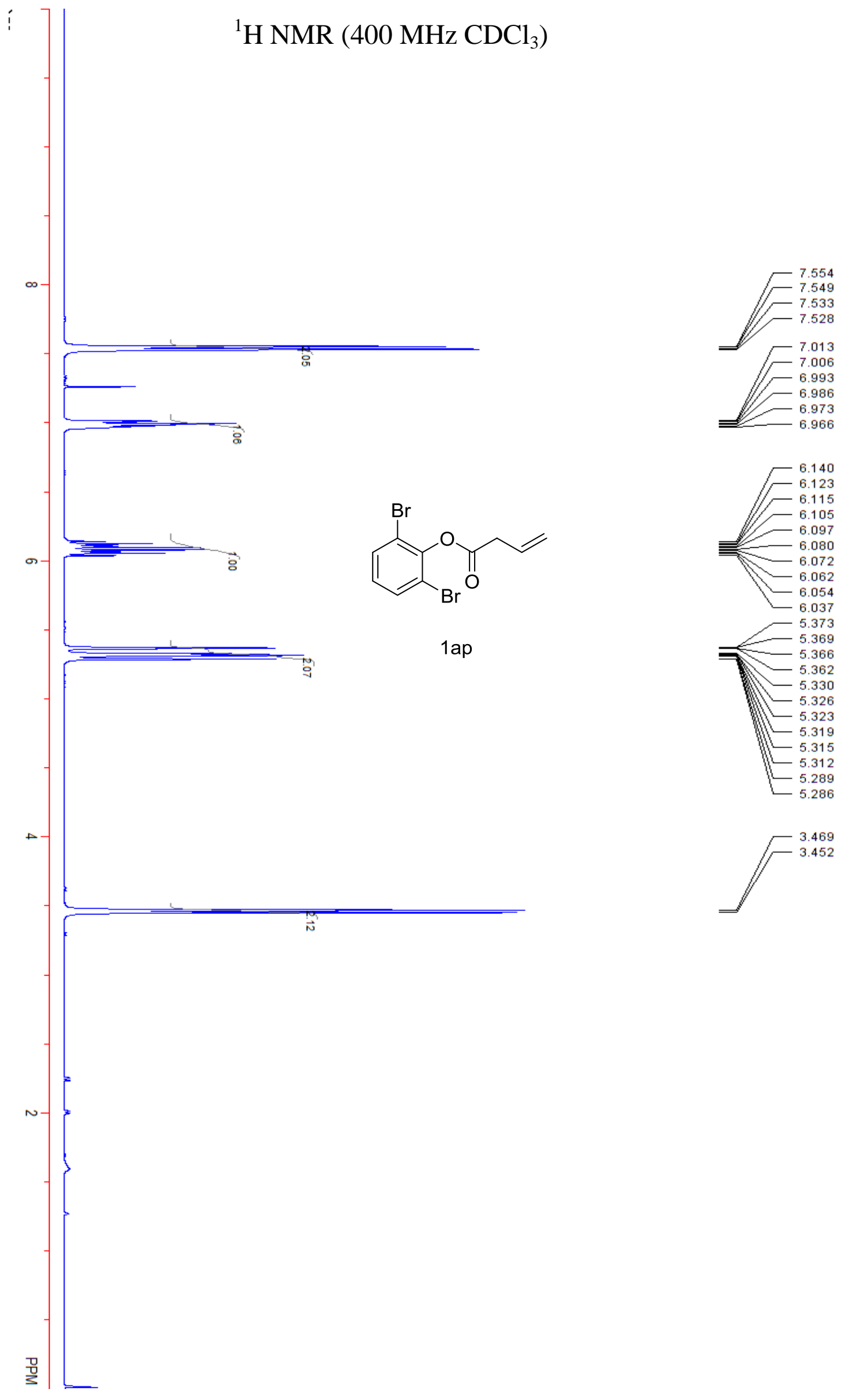


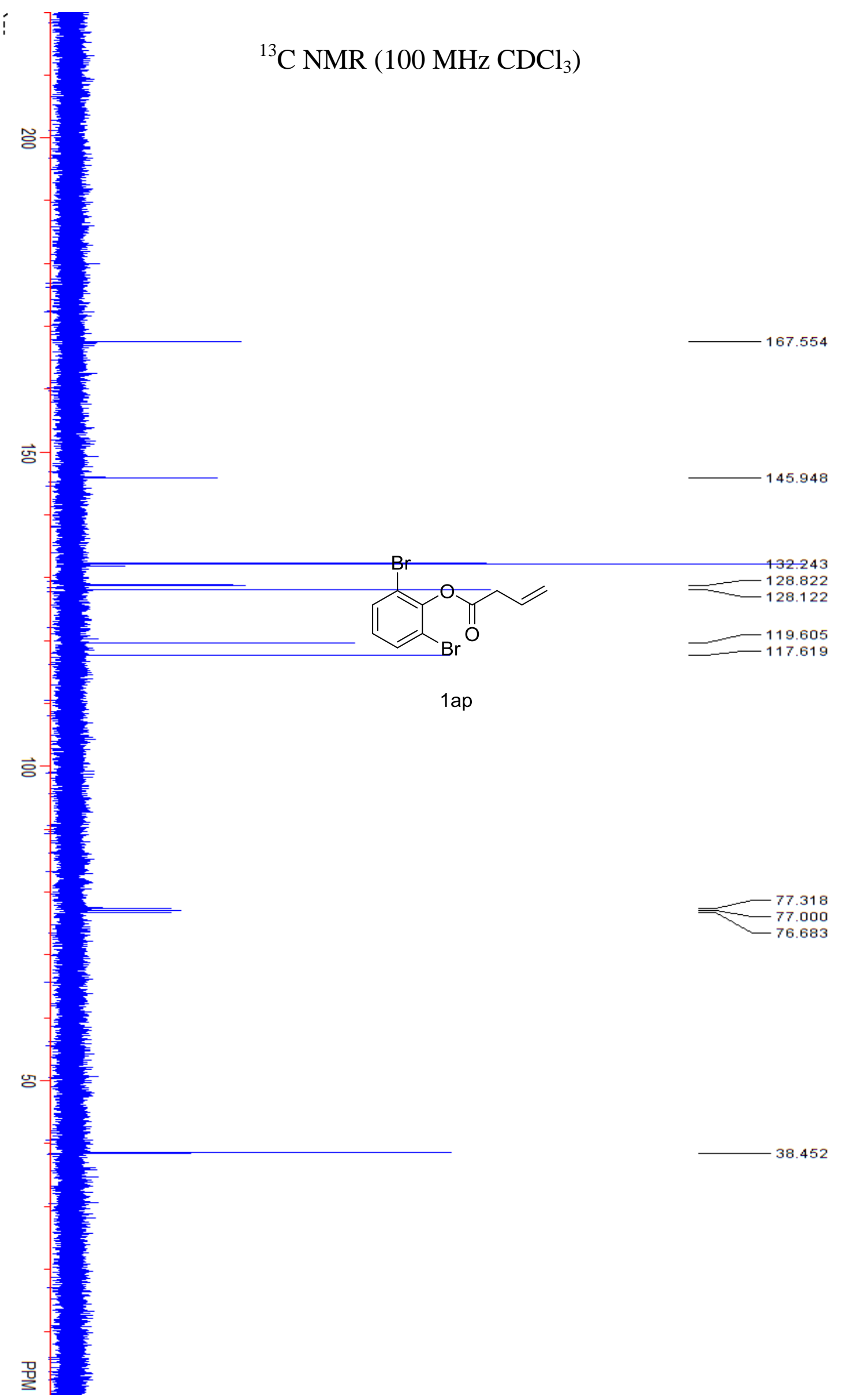



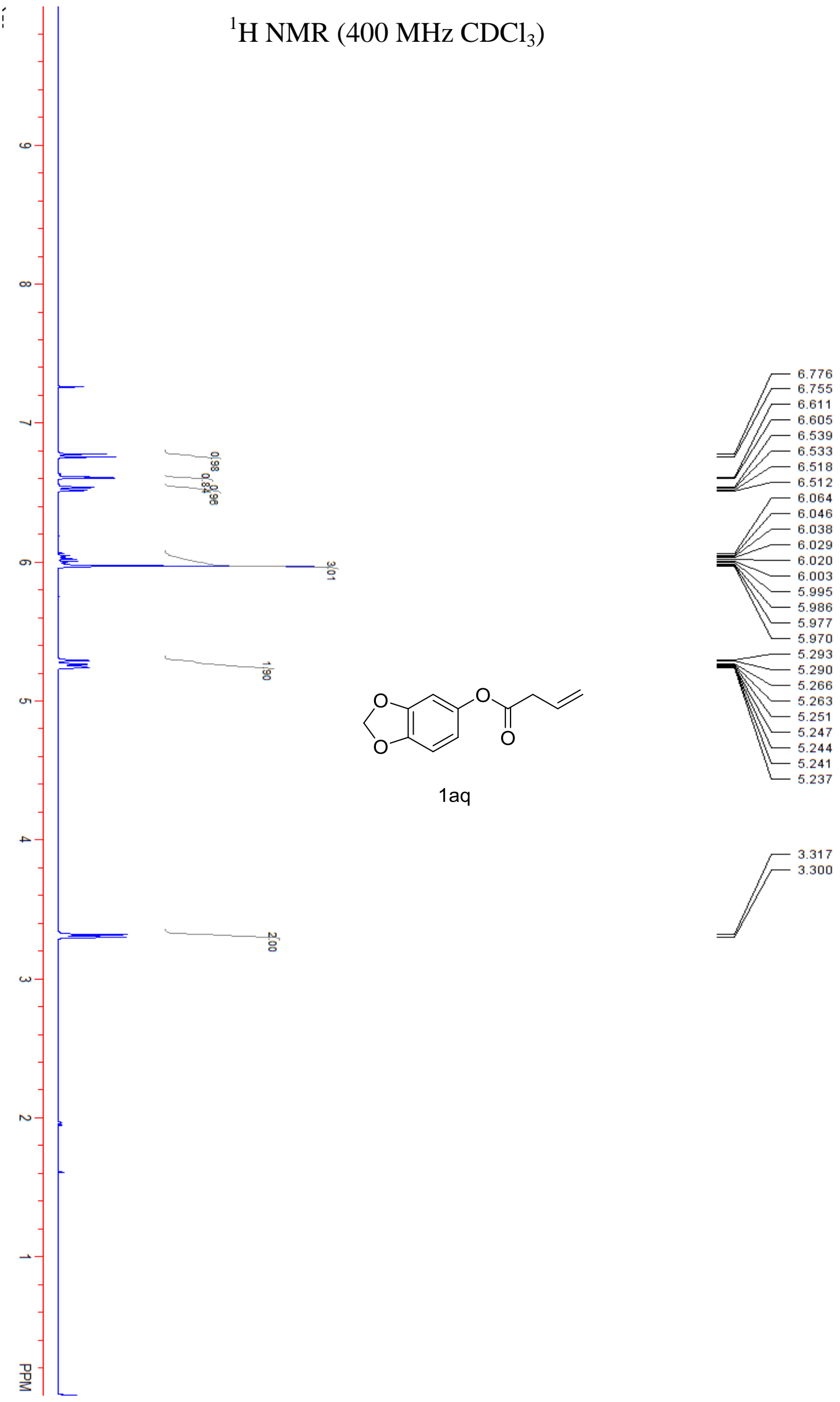

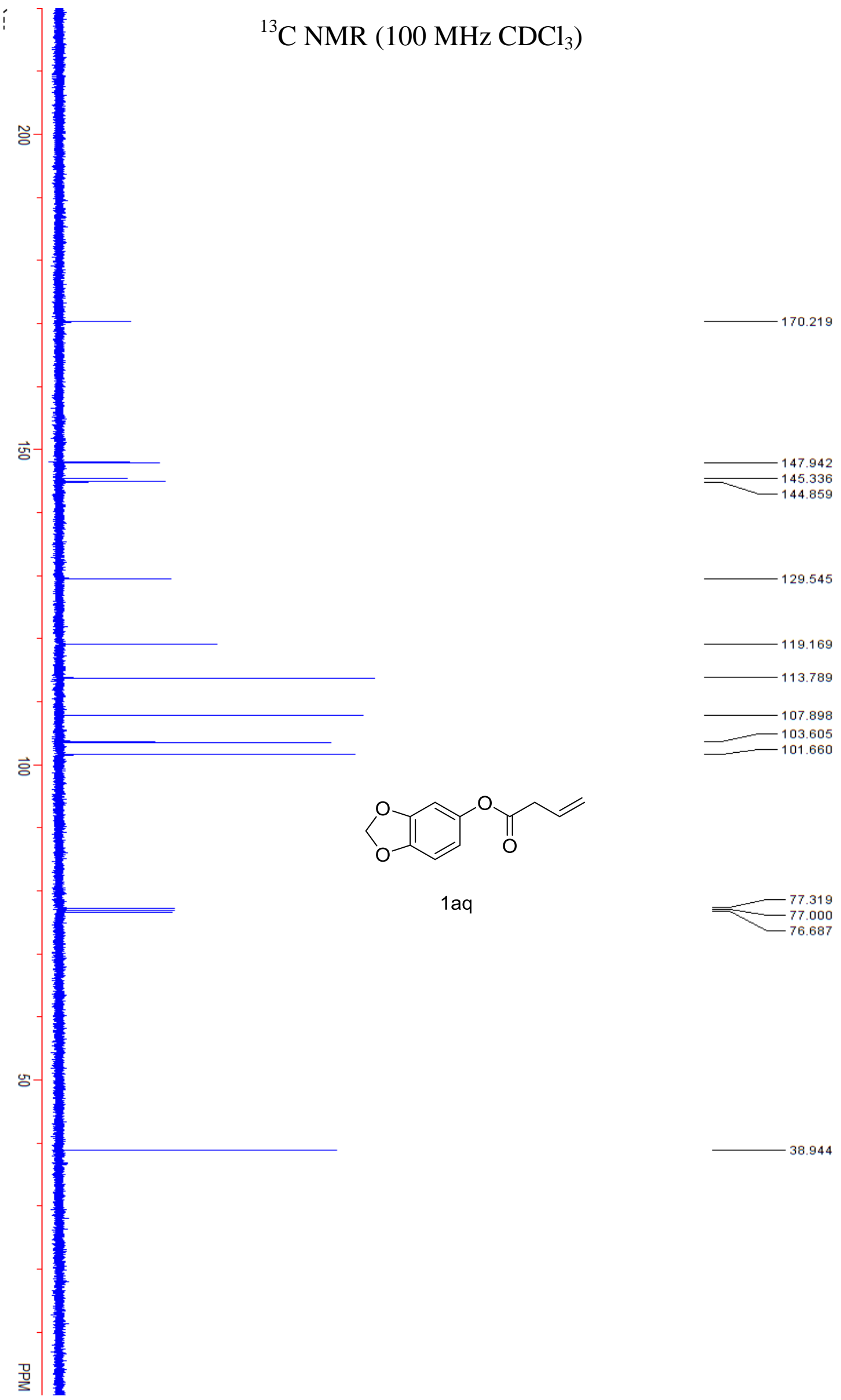


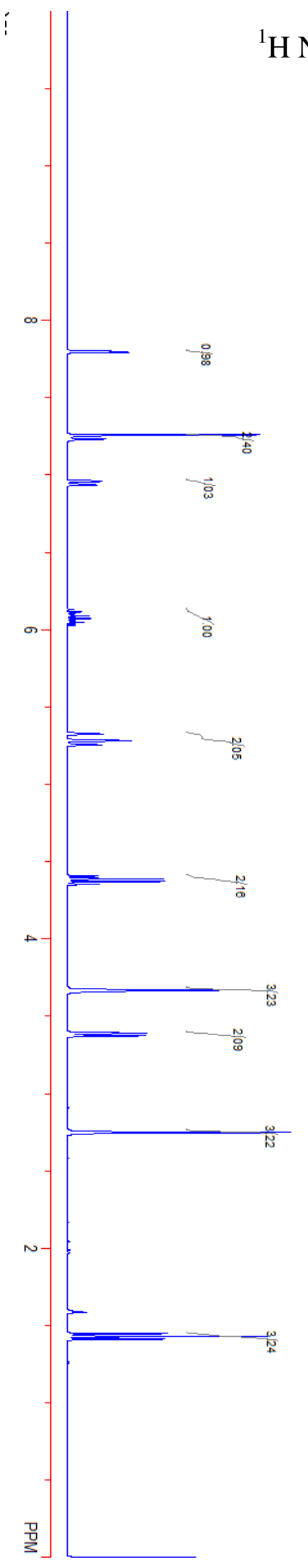

H NMR (400 MHz $\mathrm{CDCl}_{3}$ )
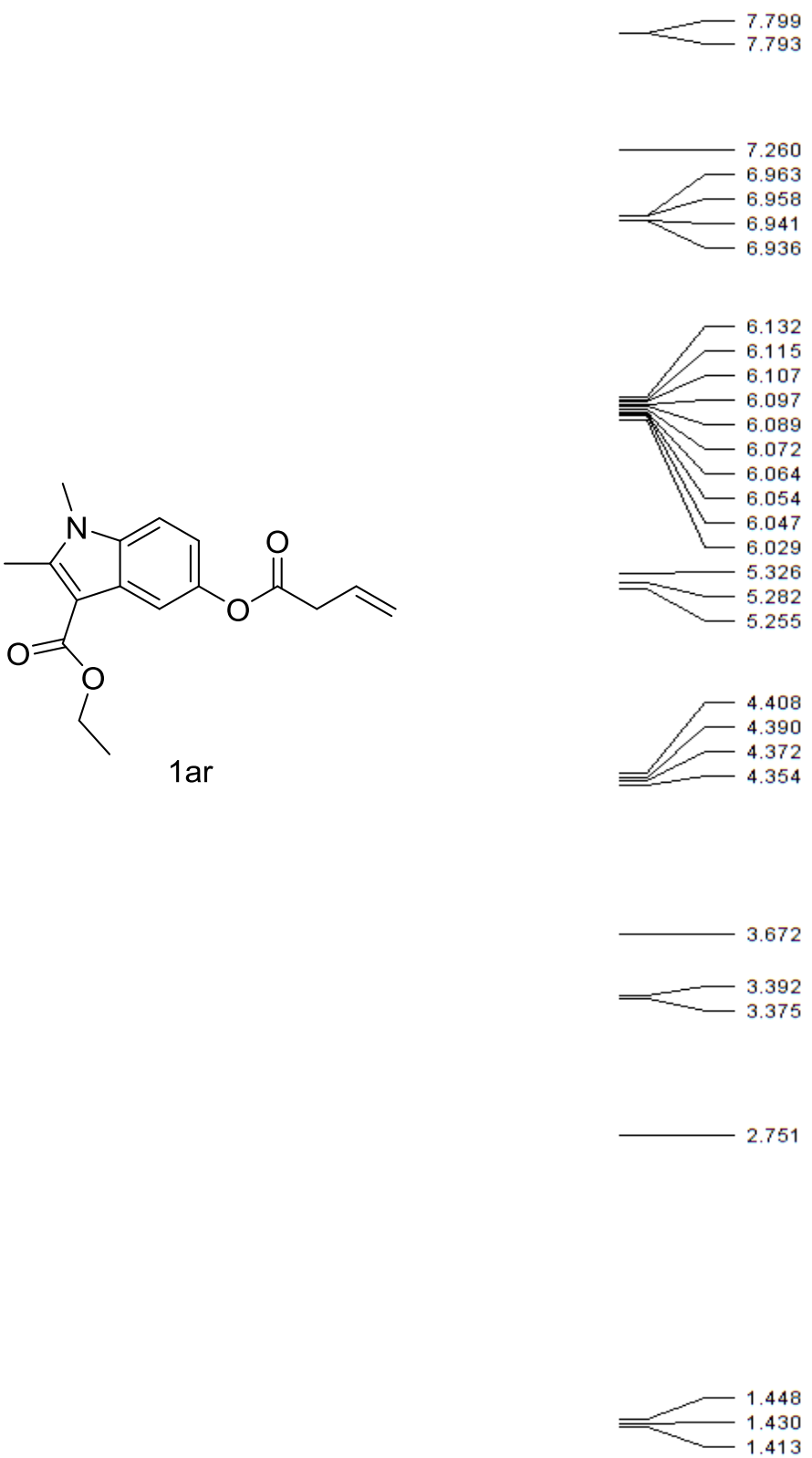


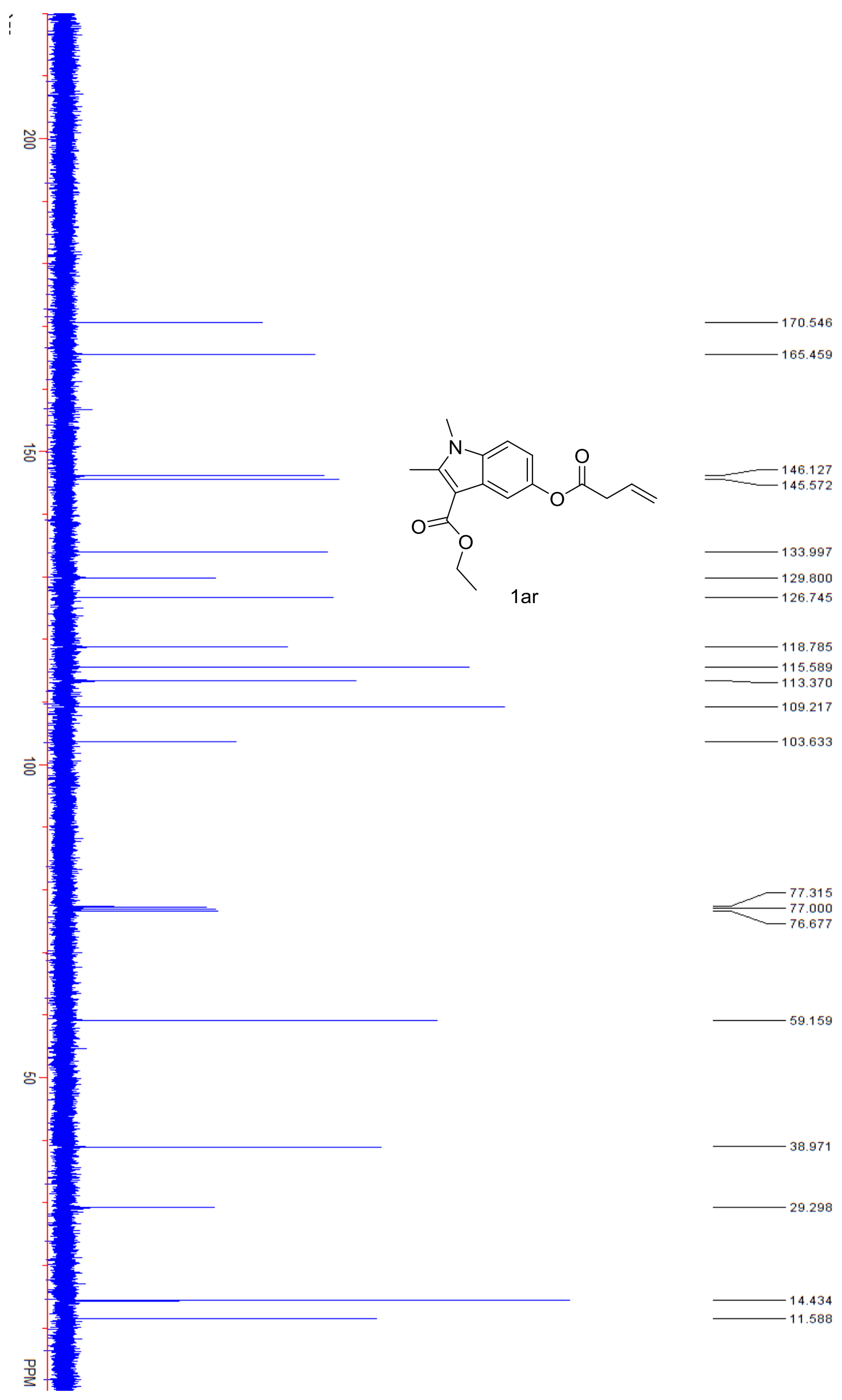




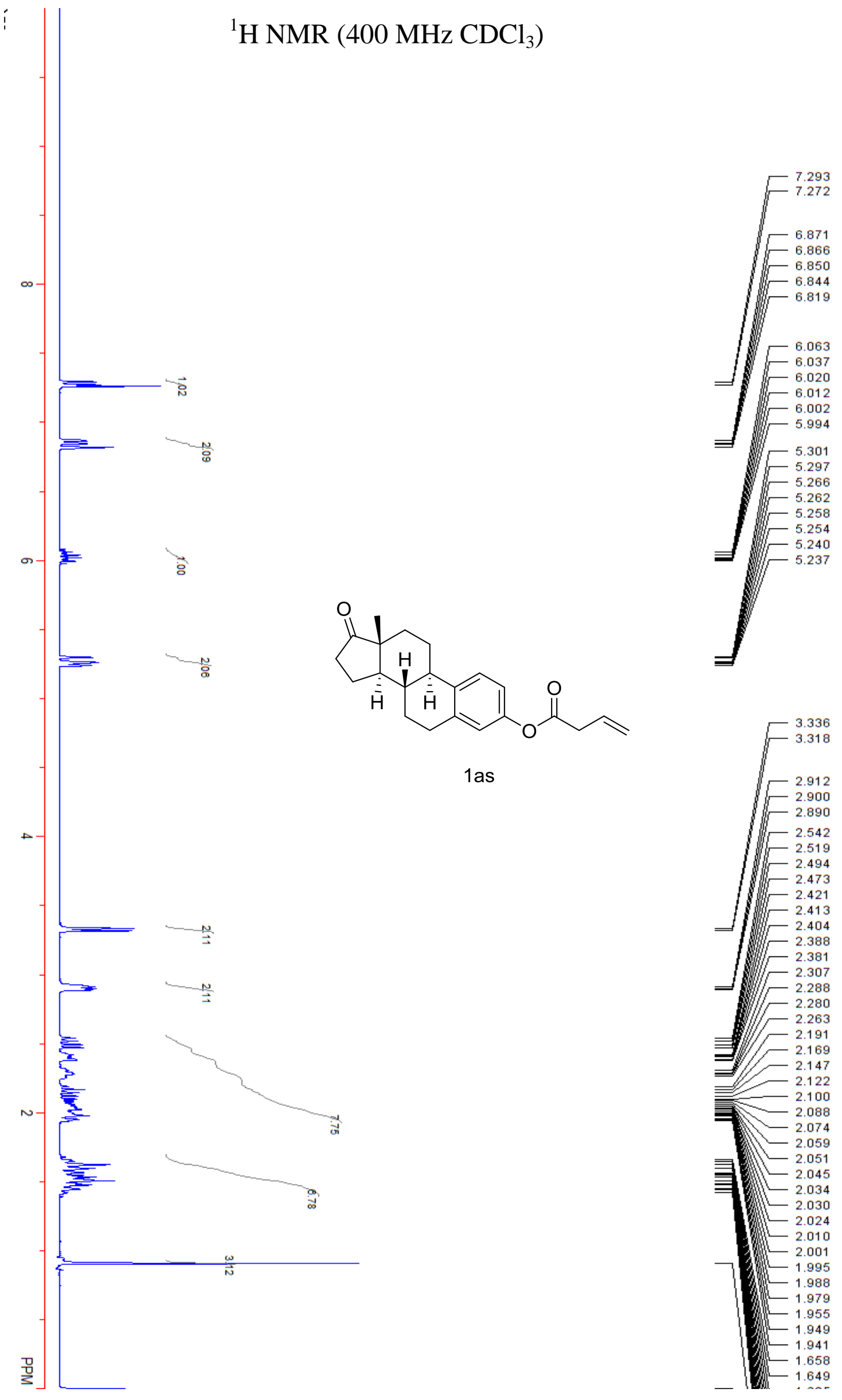




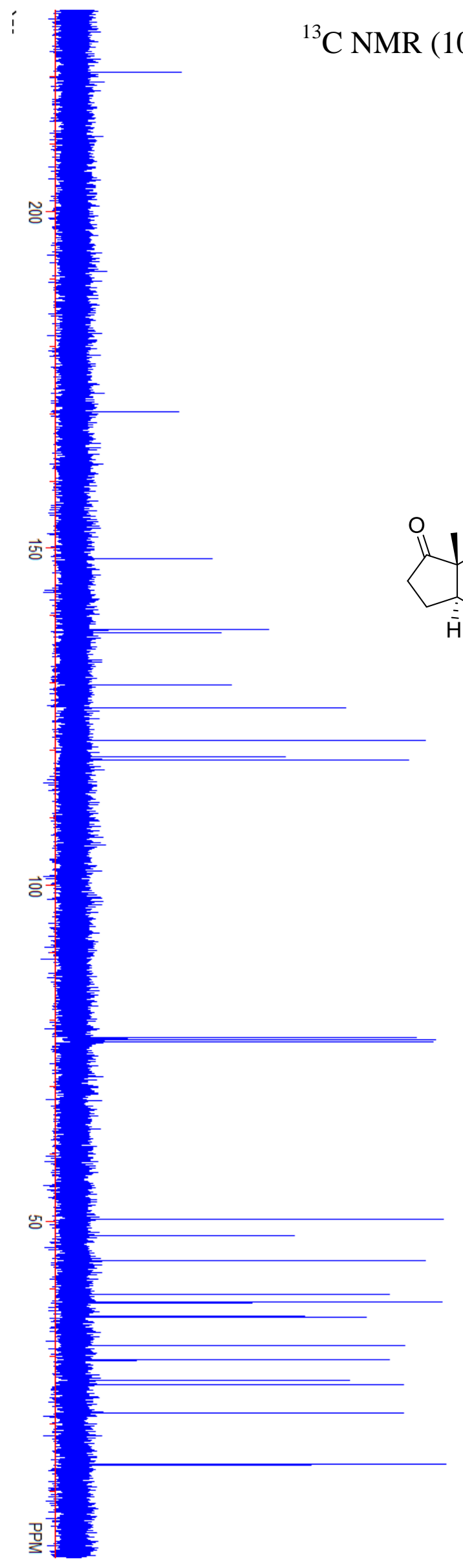

$-220.774$

170.254

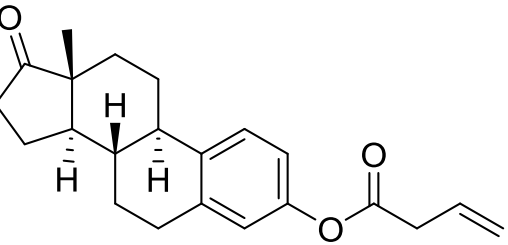

148.461
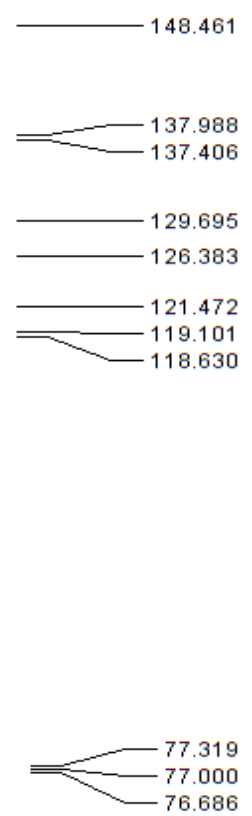

76.686

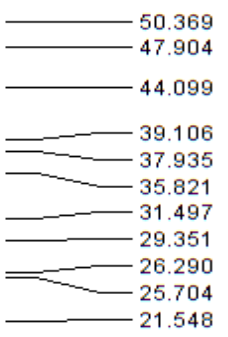

13.788 


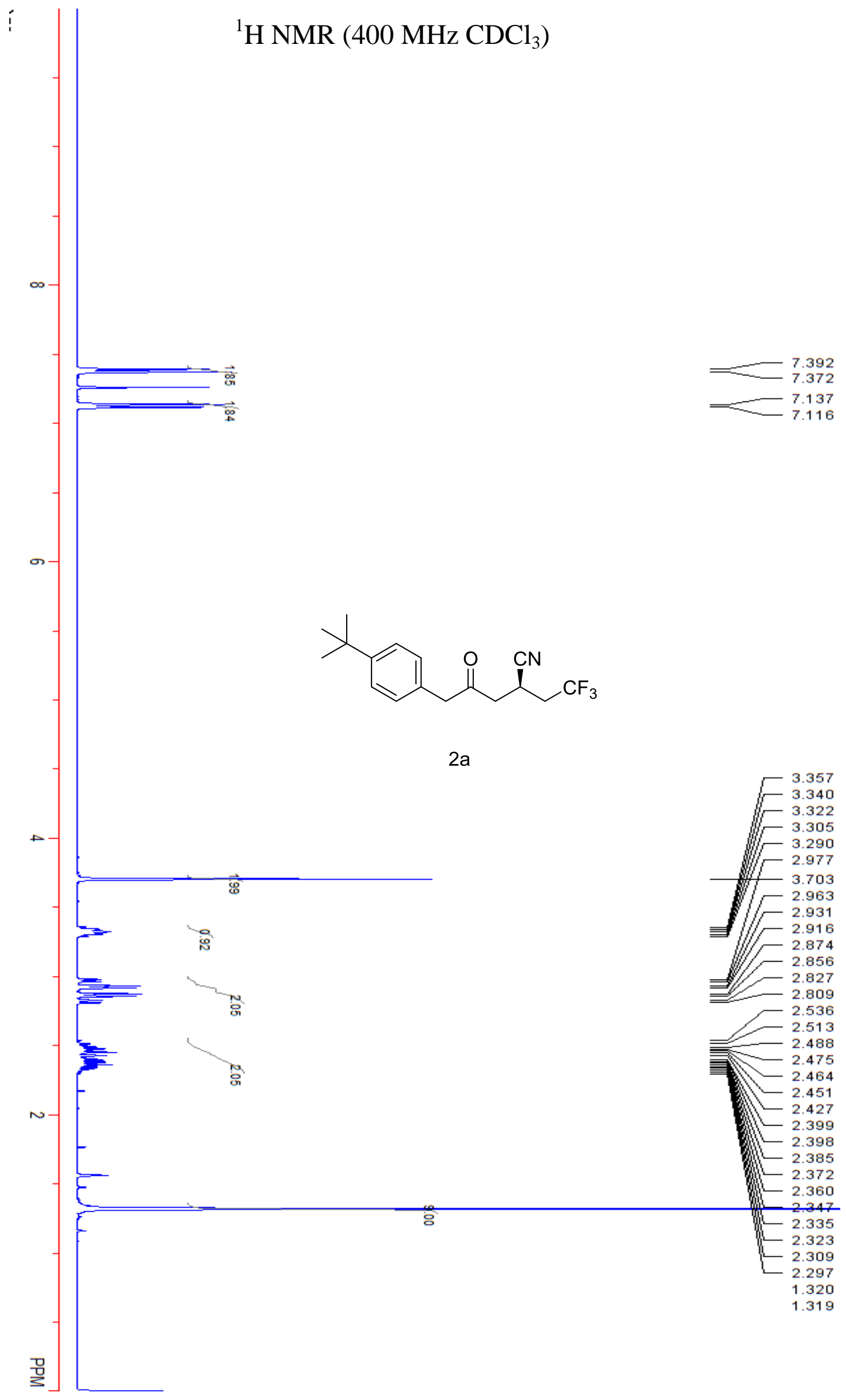




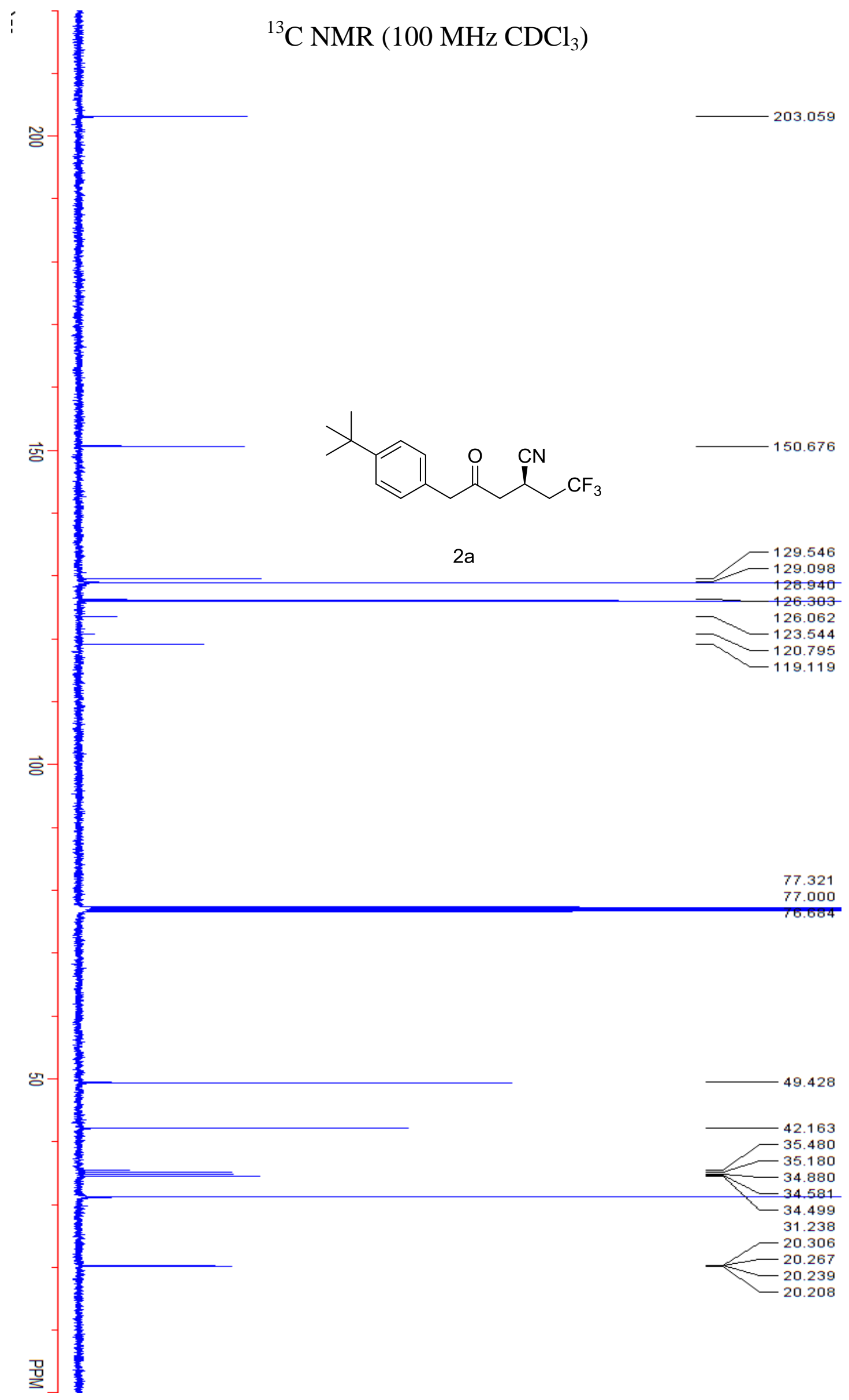




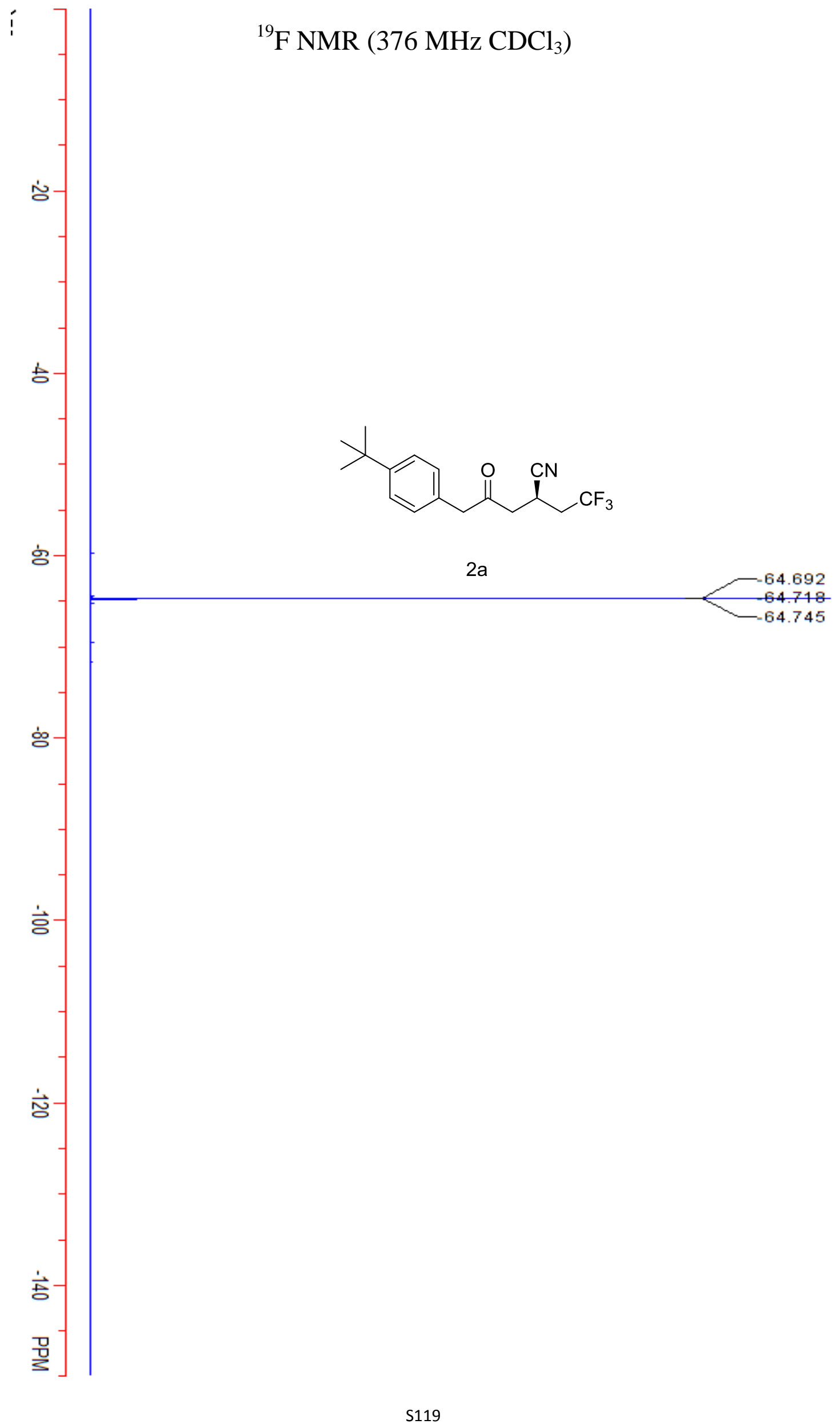




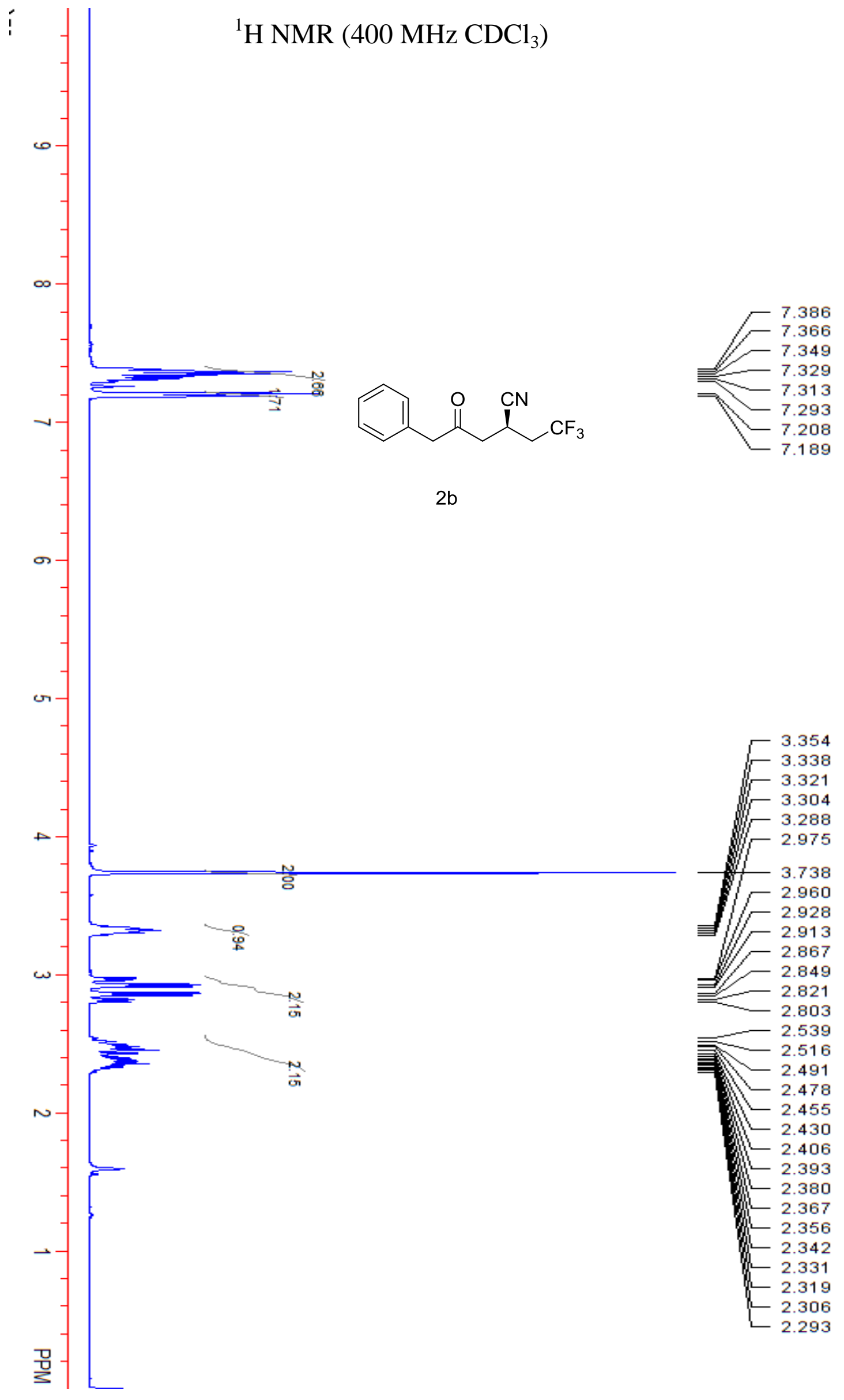




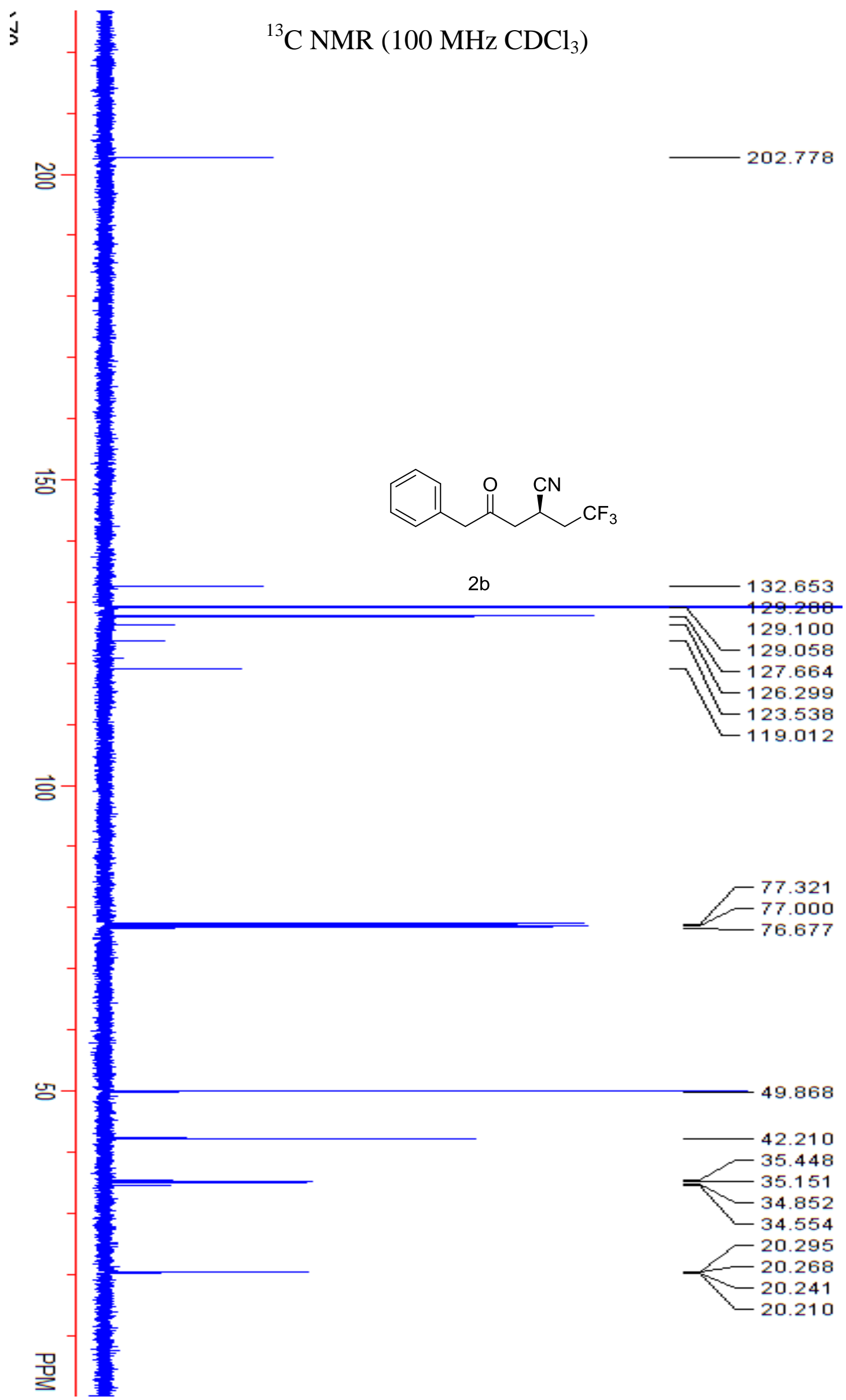


${ }^{19} \mathrm{~F}$ NMR (376 MHz $\mathrm{CDCl}_{3}$ )

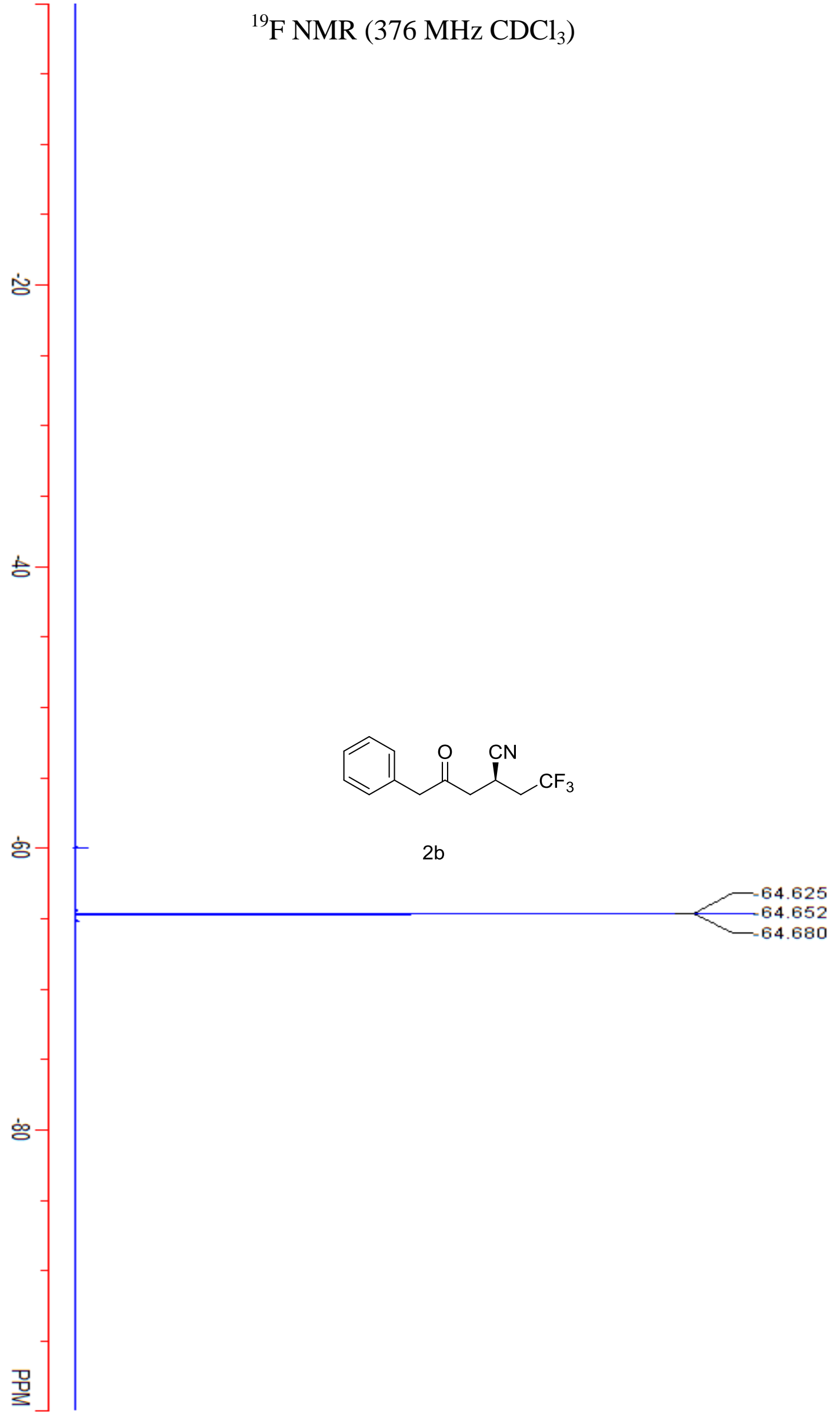




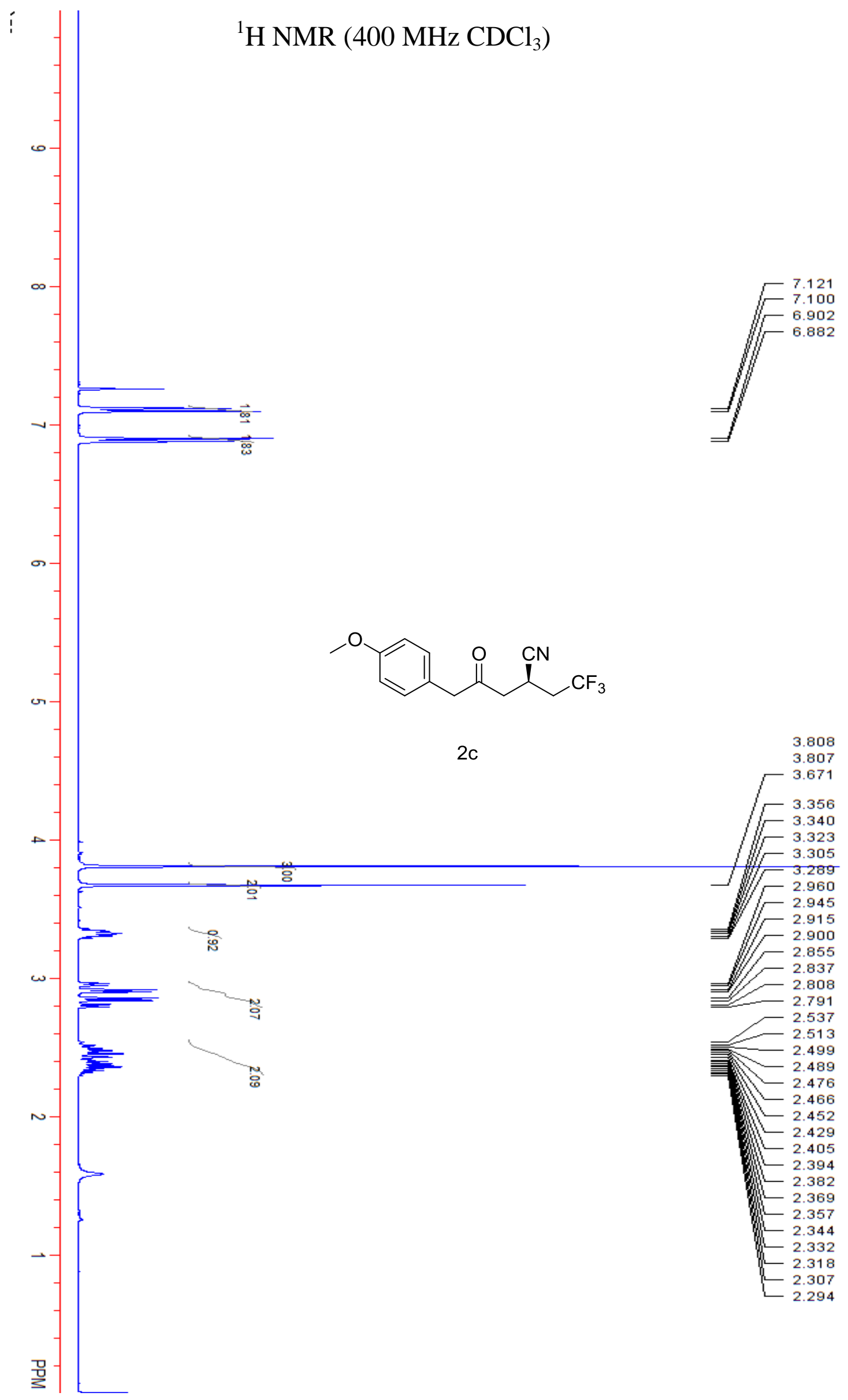




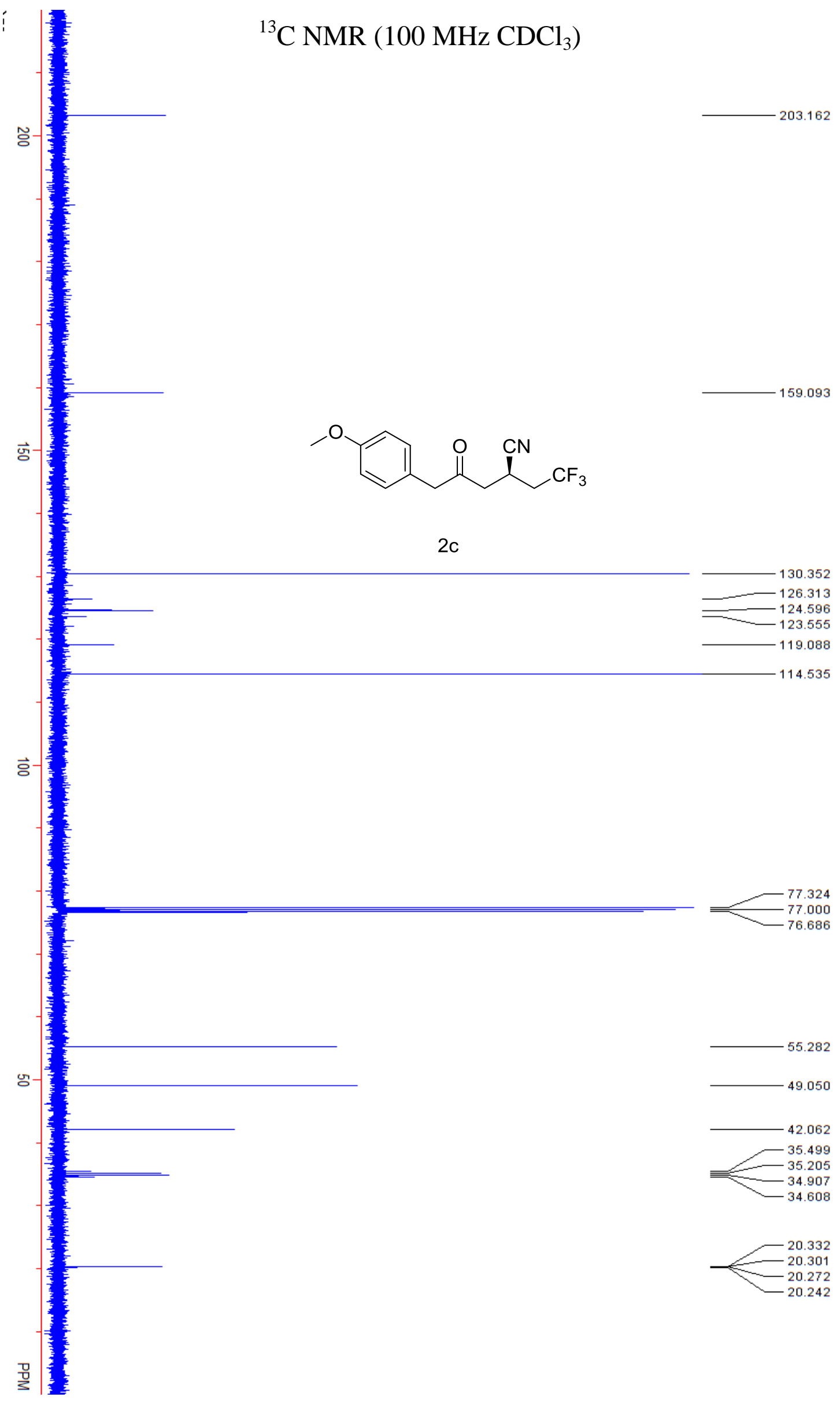




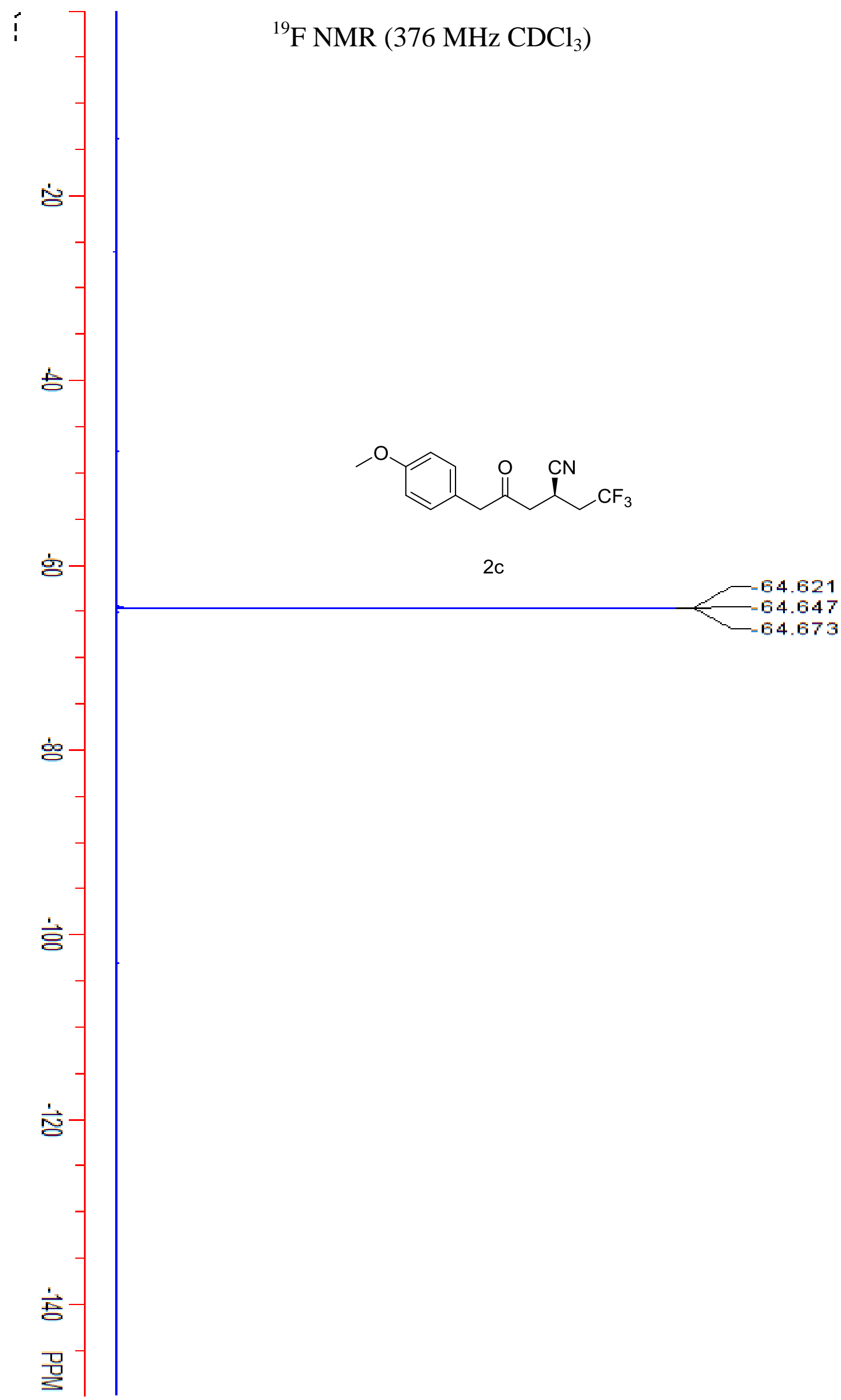




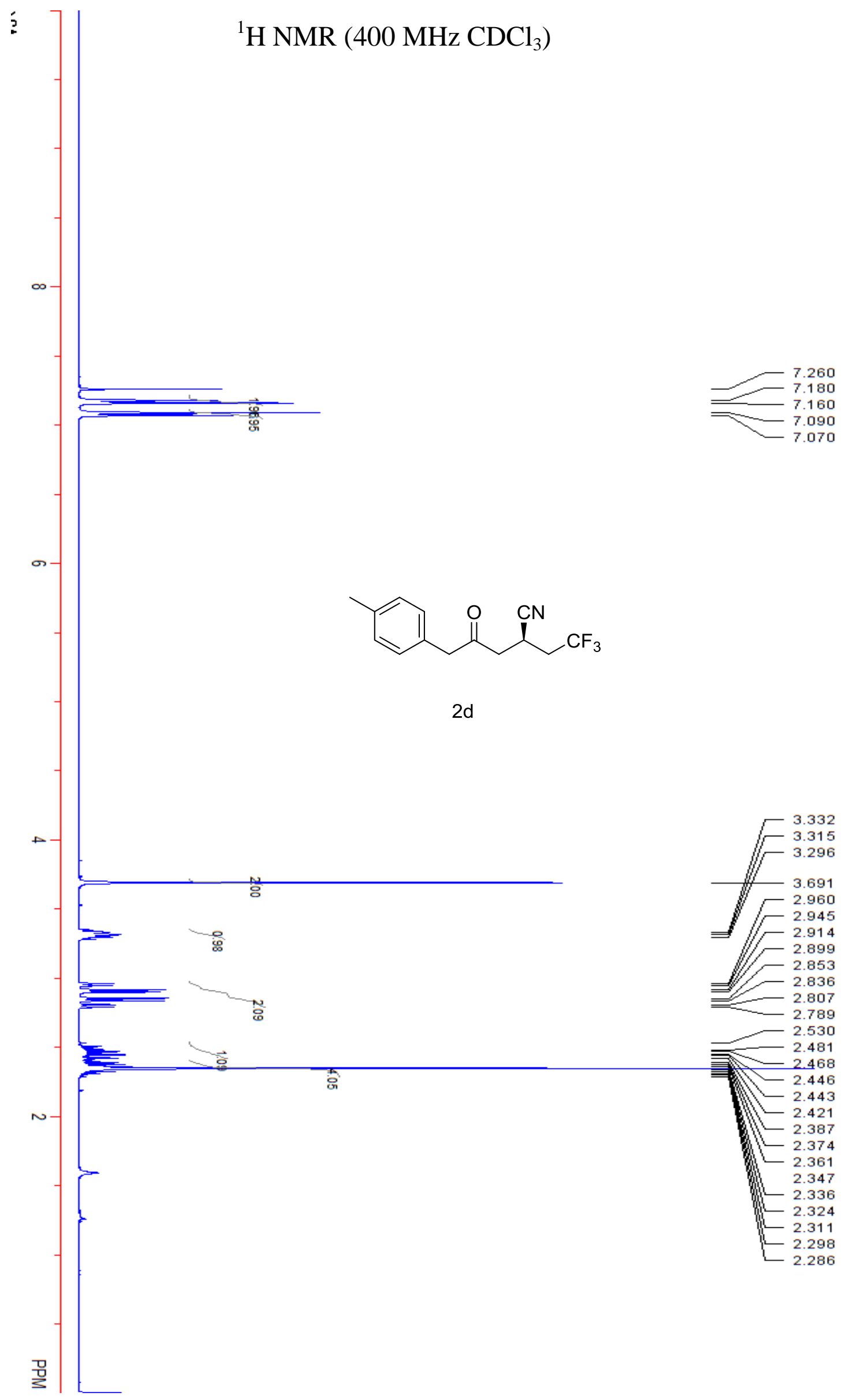




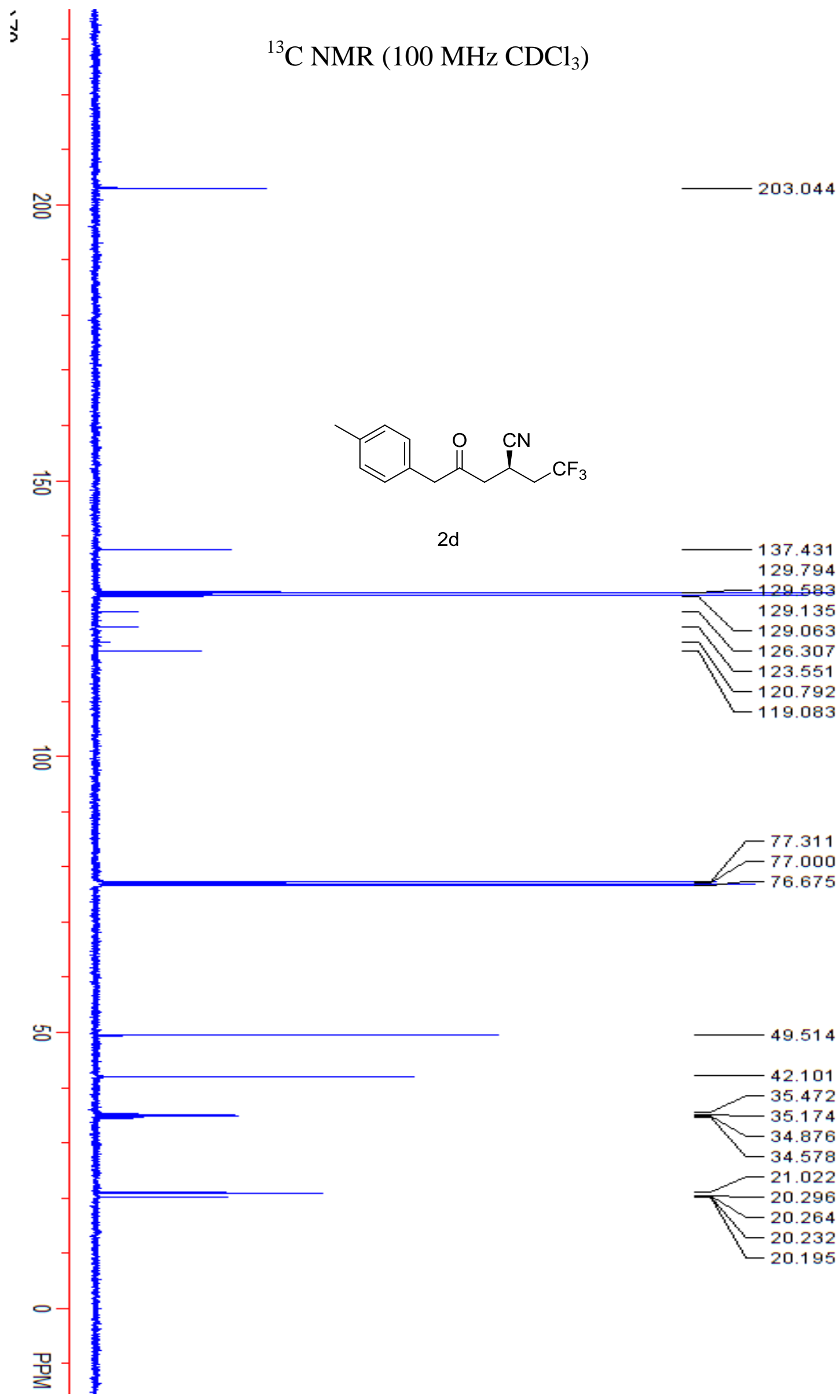




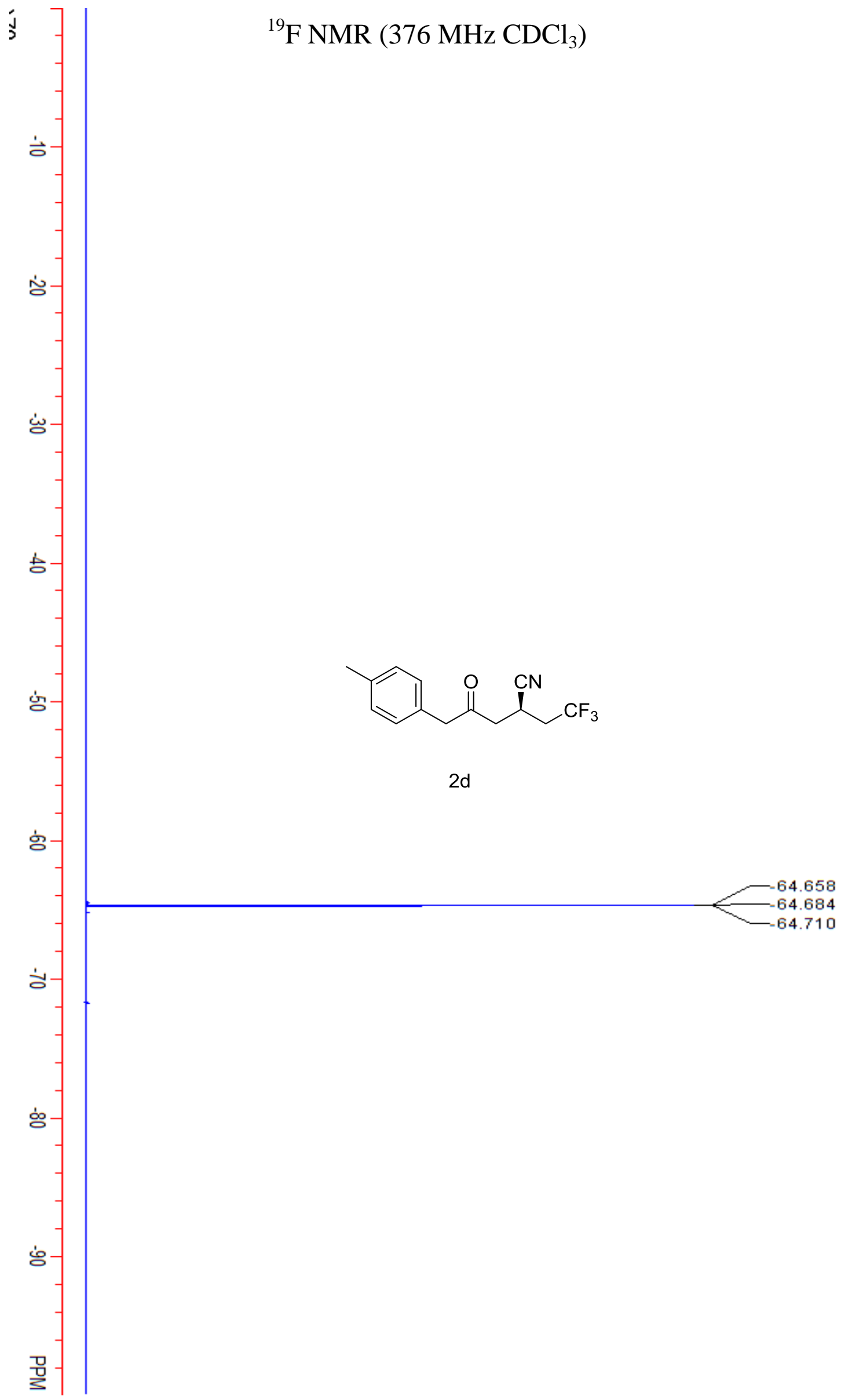




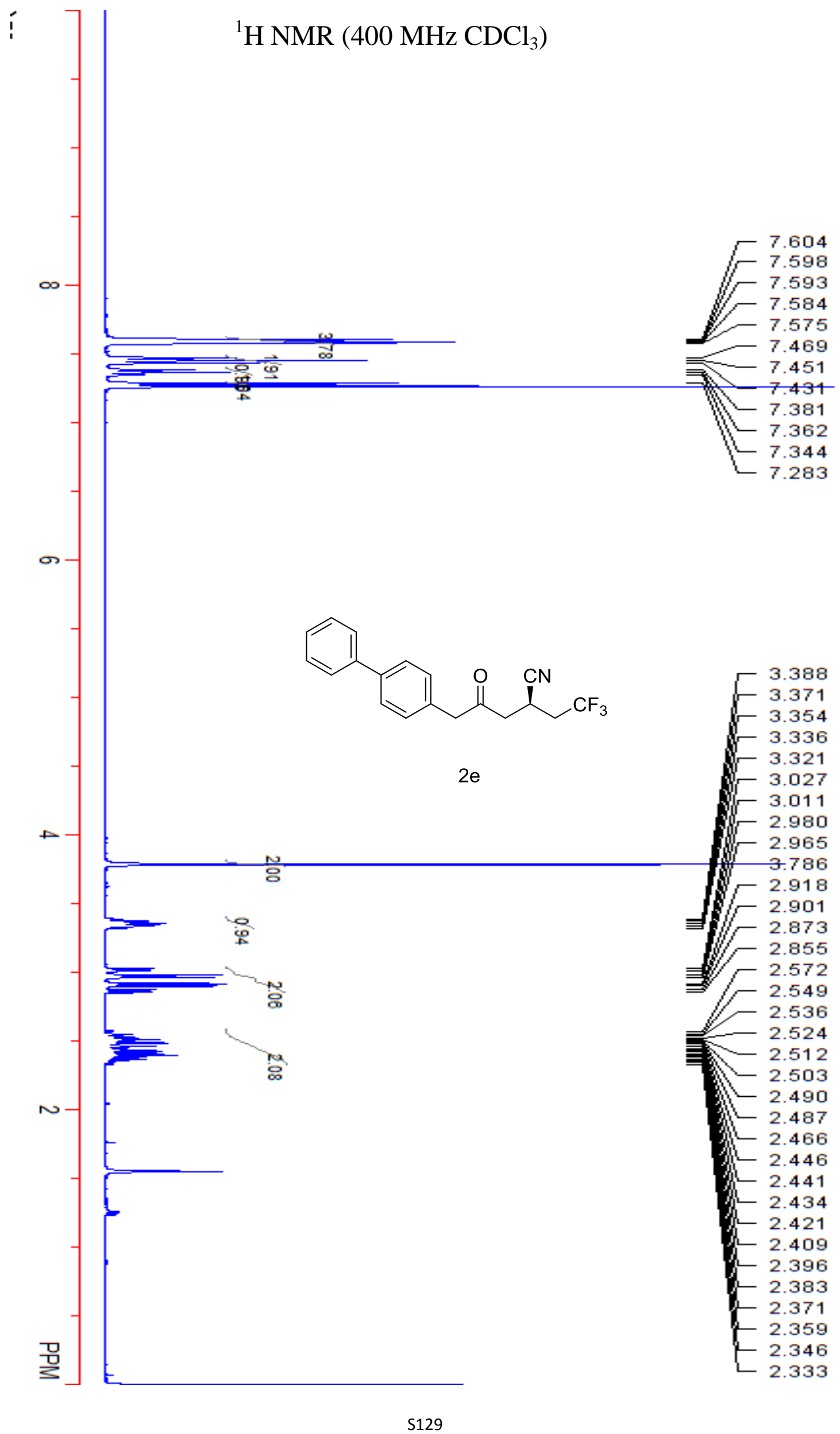




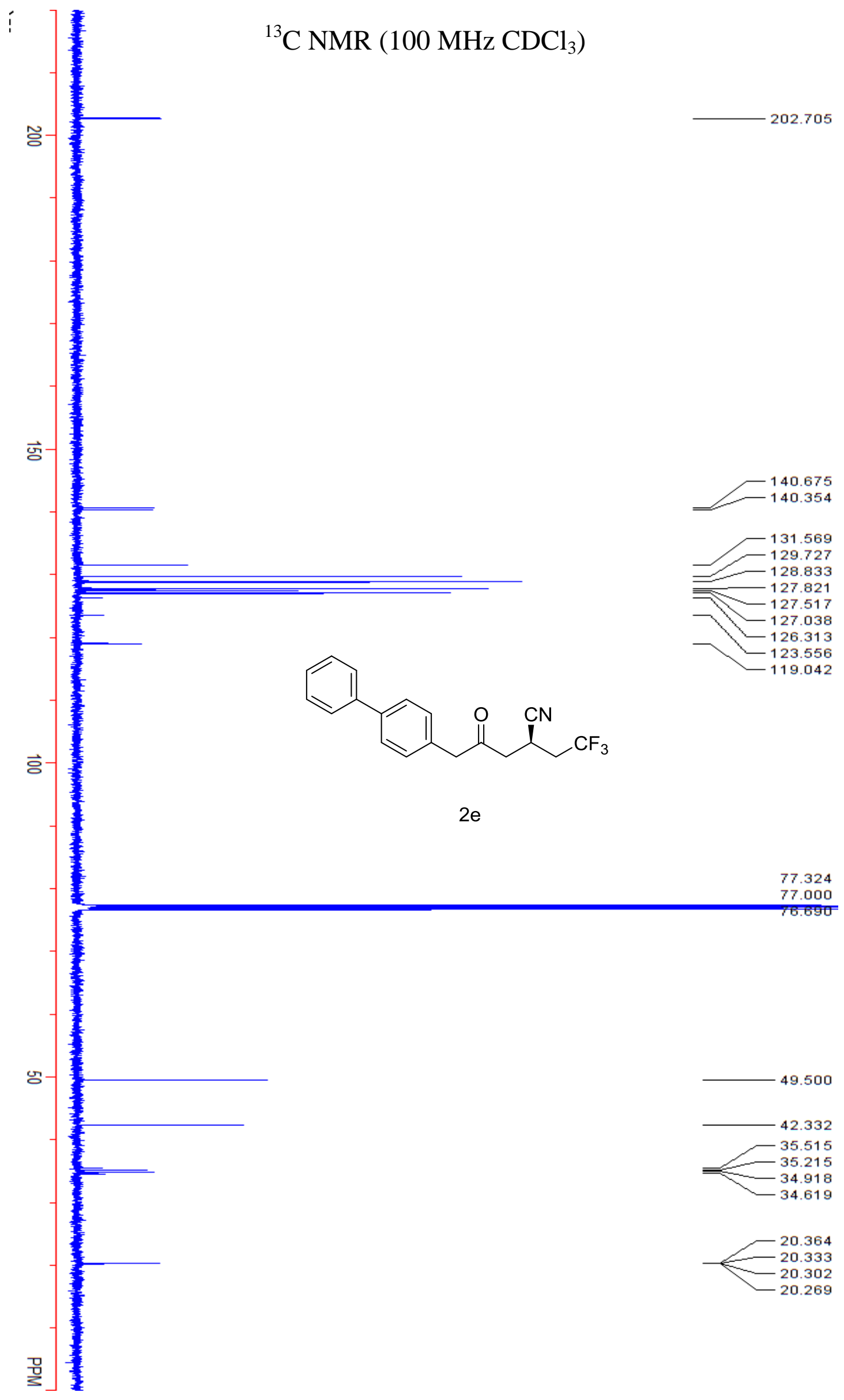




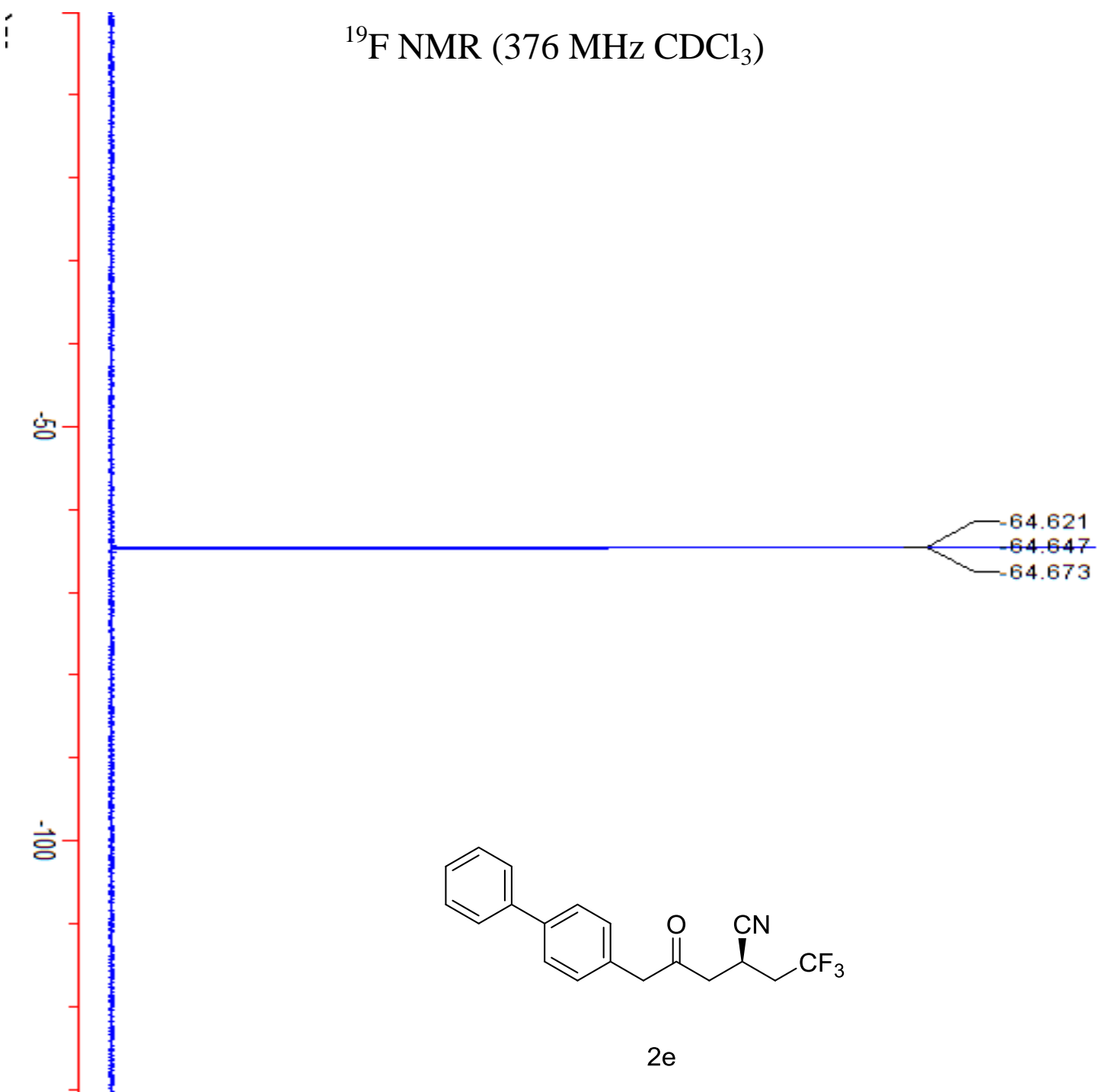

S131 


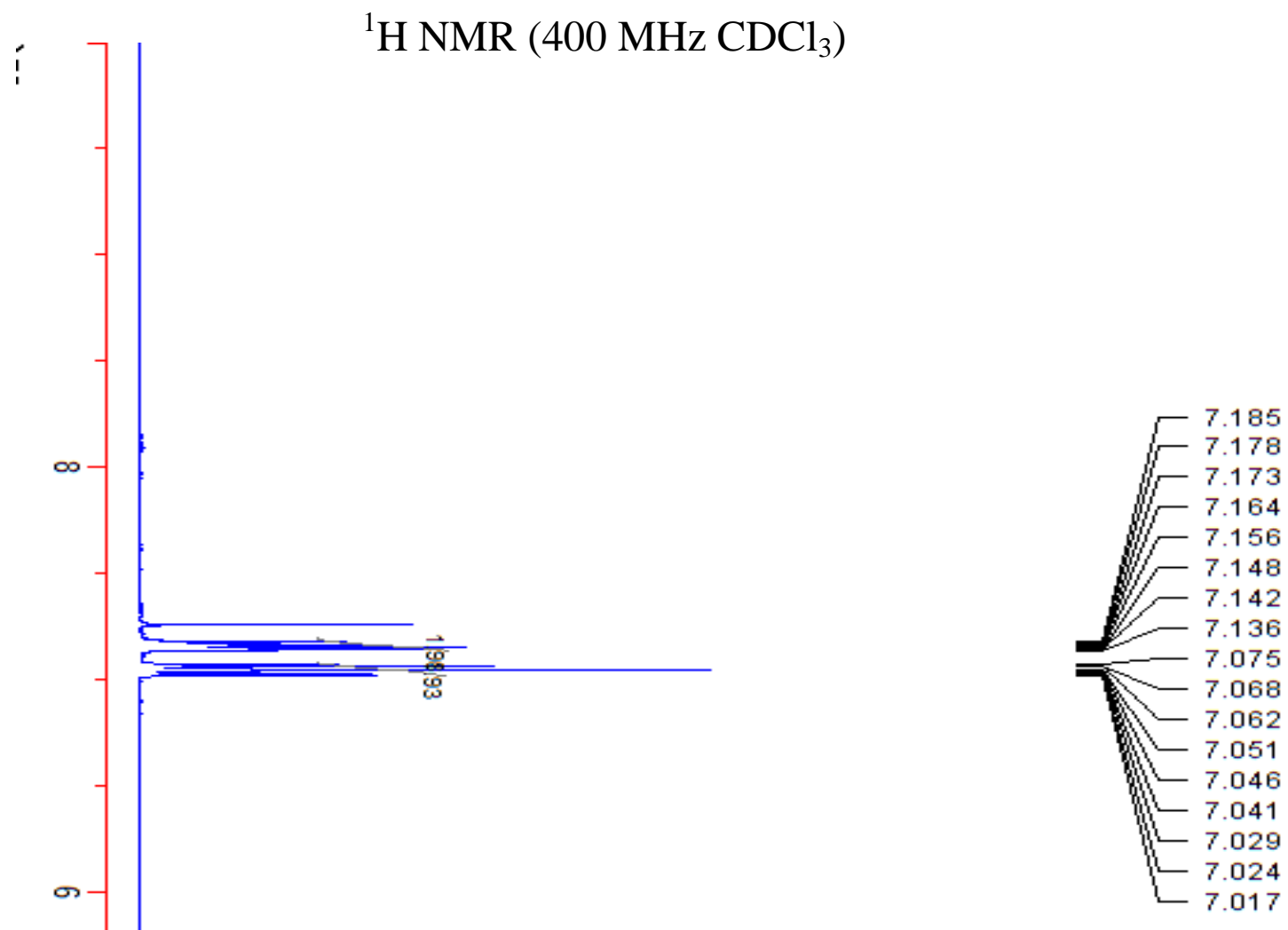<smiles>N#CC(CC(=O)Cc1ccc(F)cc1)CC(F)(F)F</smiles>

$-3.363$

- 3.347

3.331

$2 f$

- 3.329

$-3.312$

3.295

2.991

2.975

$\rightarrow-\left\{\begin{array}{l} \\ -2.945 \\ 2.929\end{array}\right.$

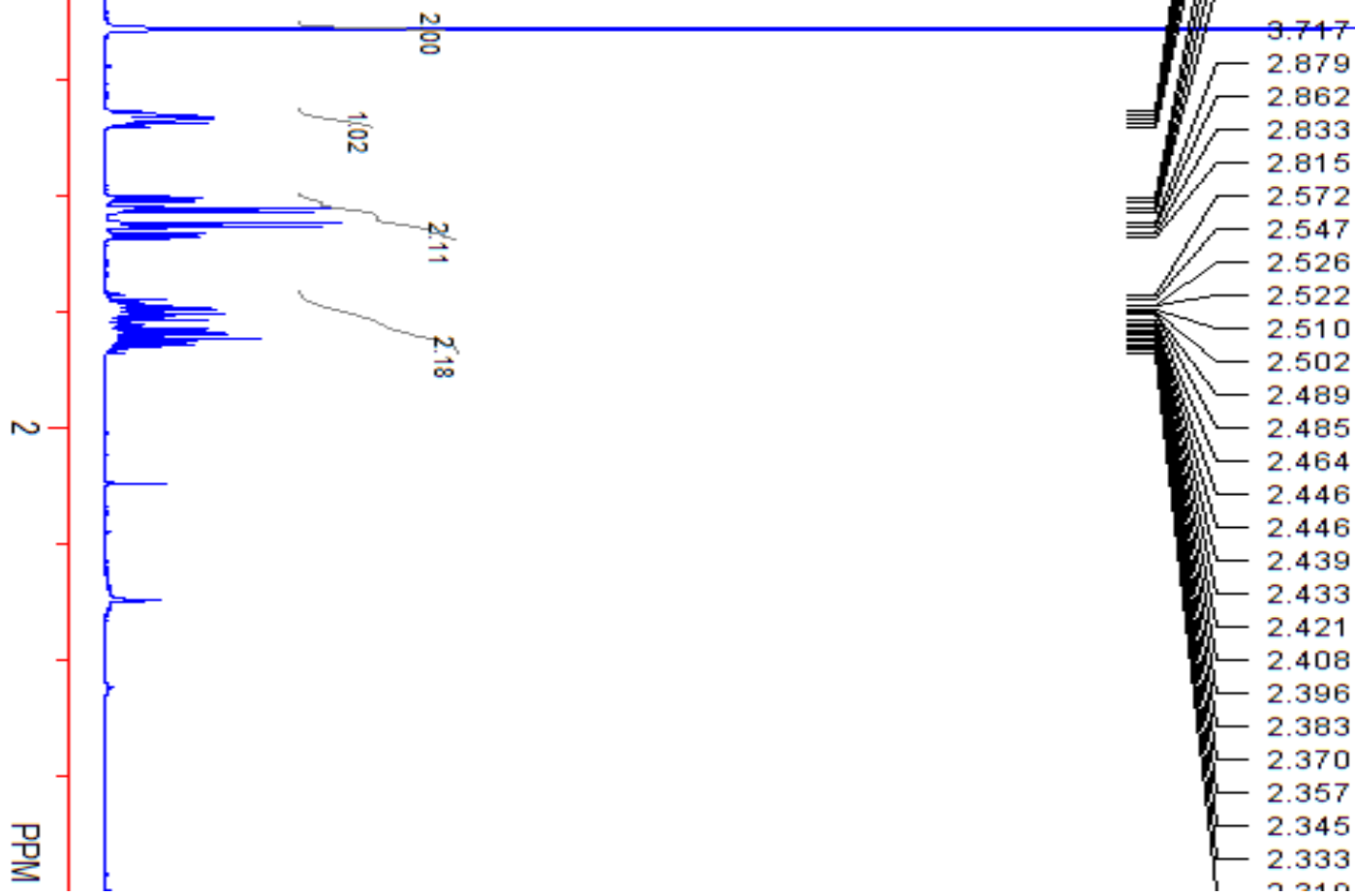




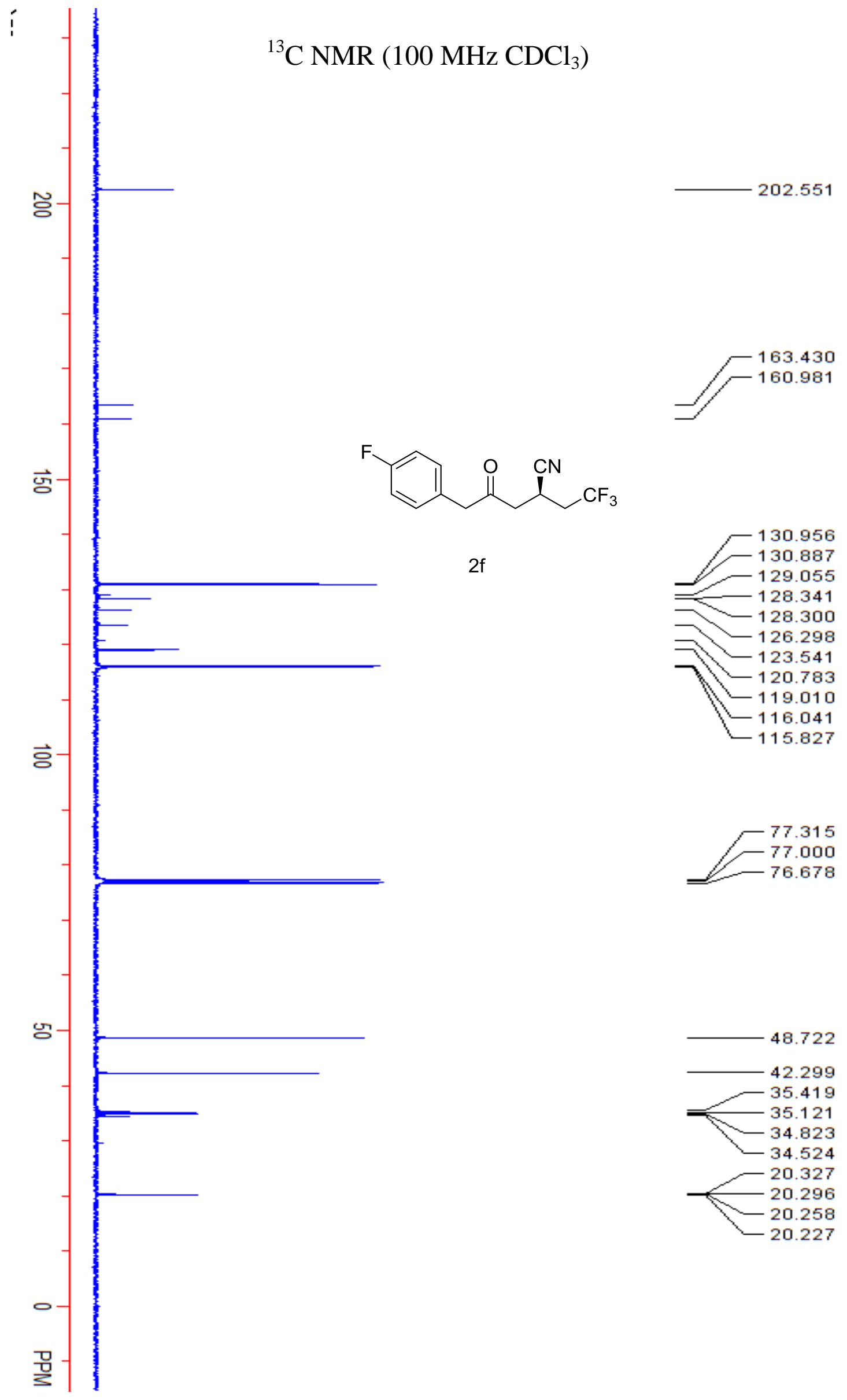




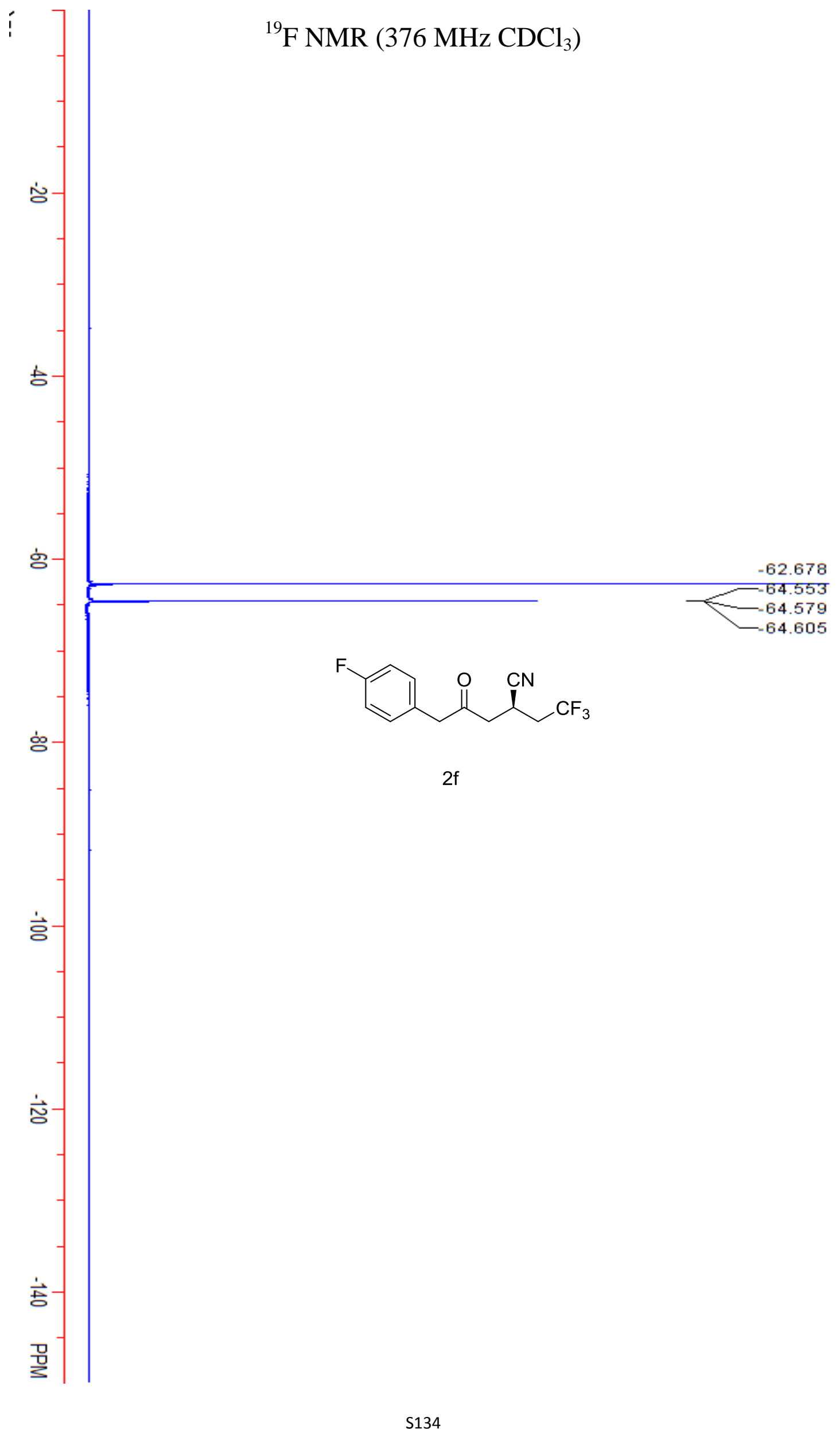




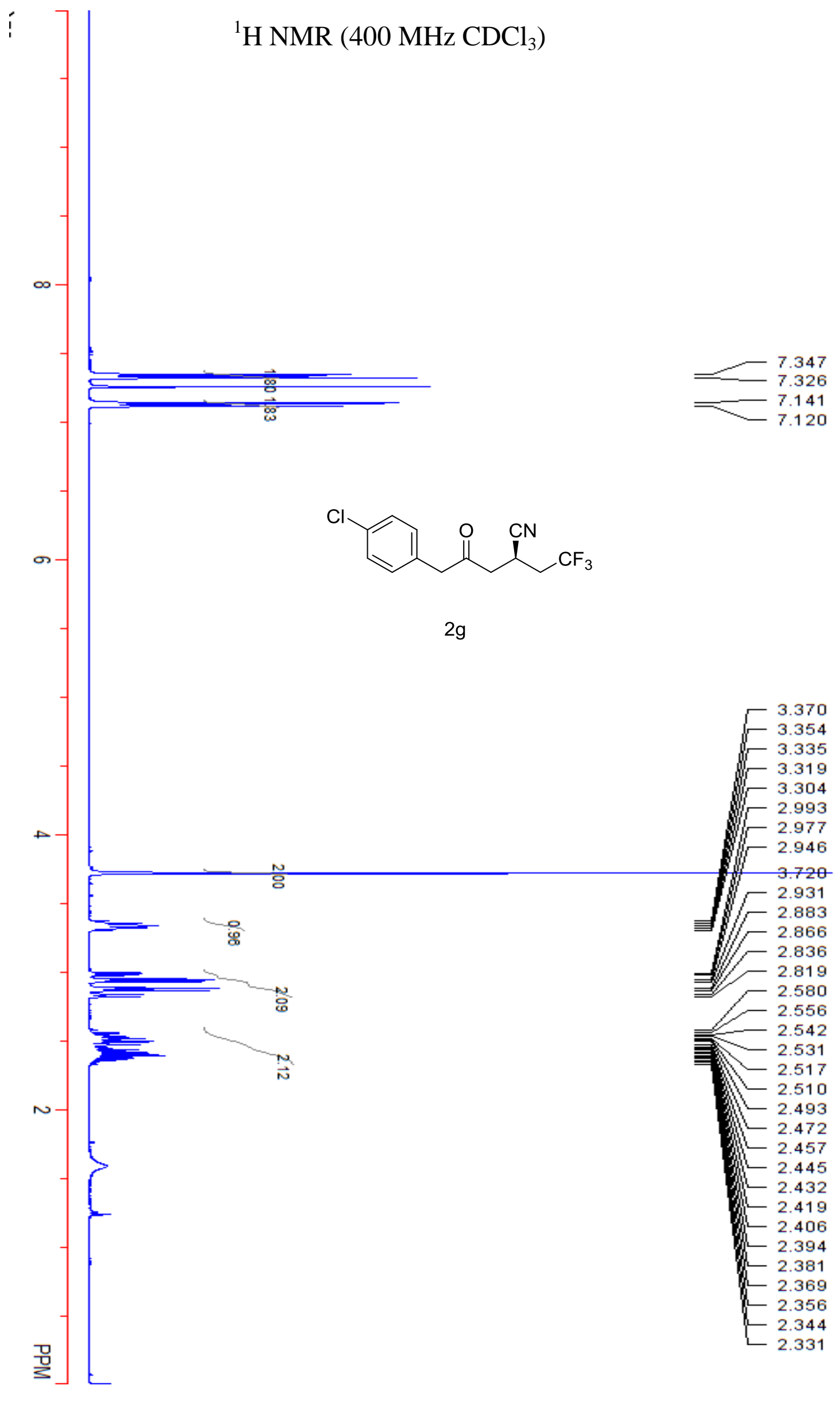




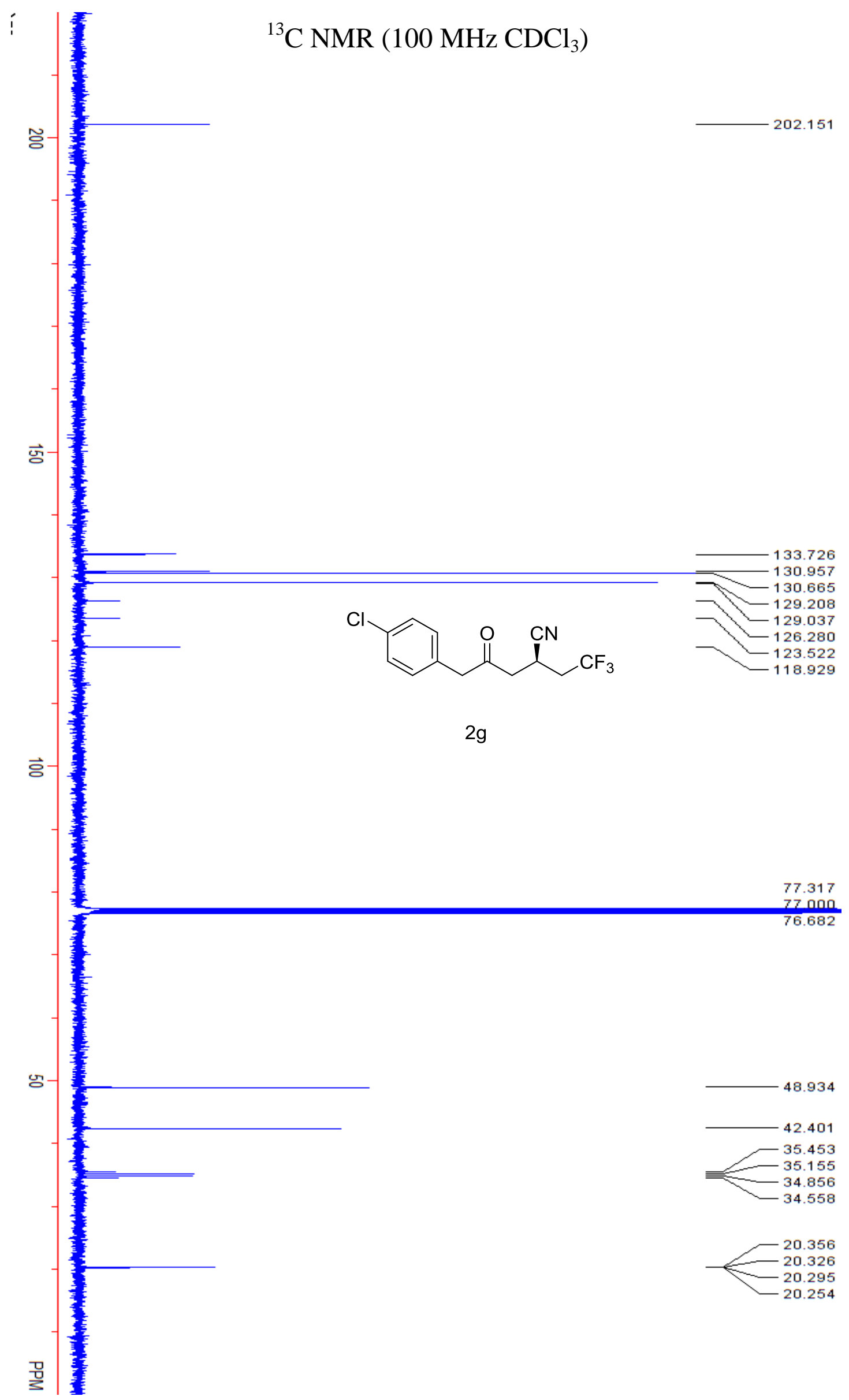




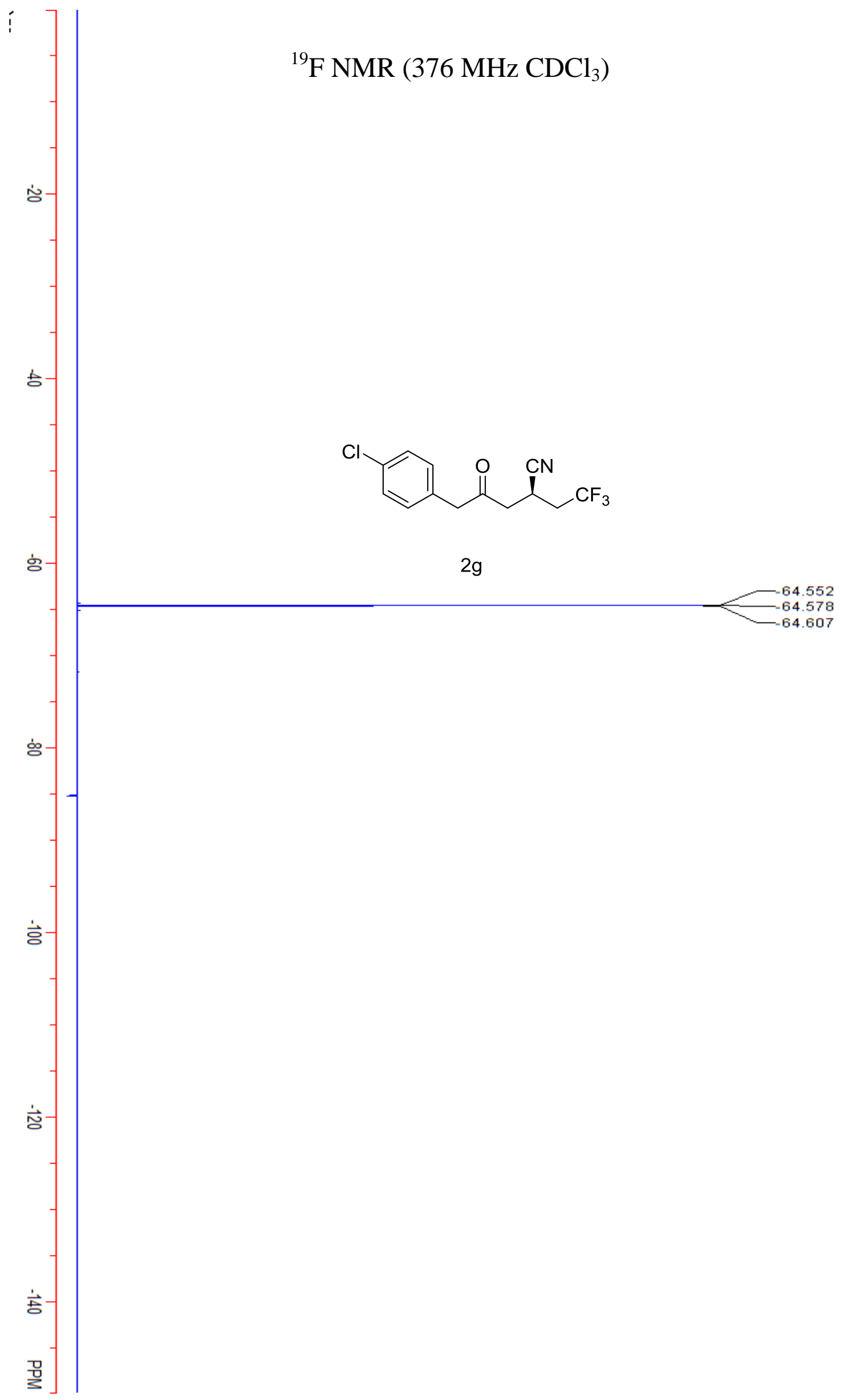




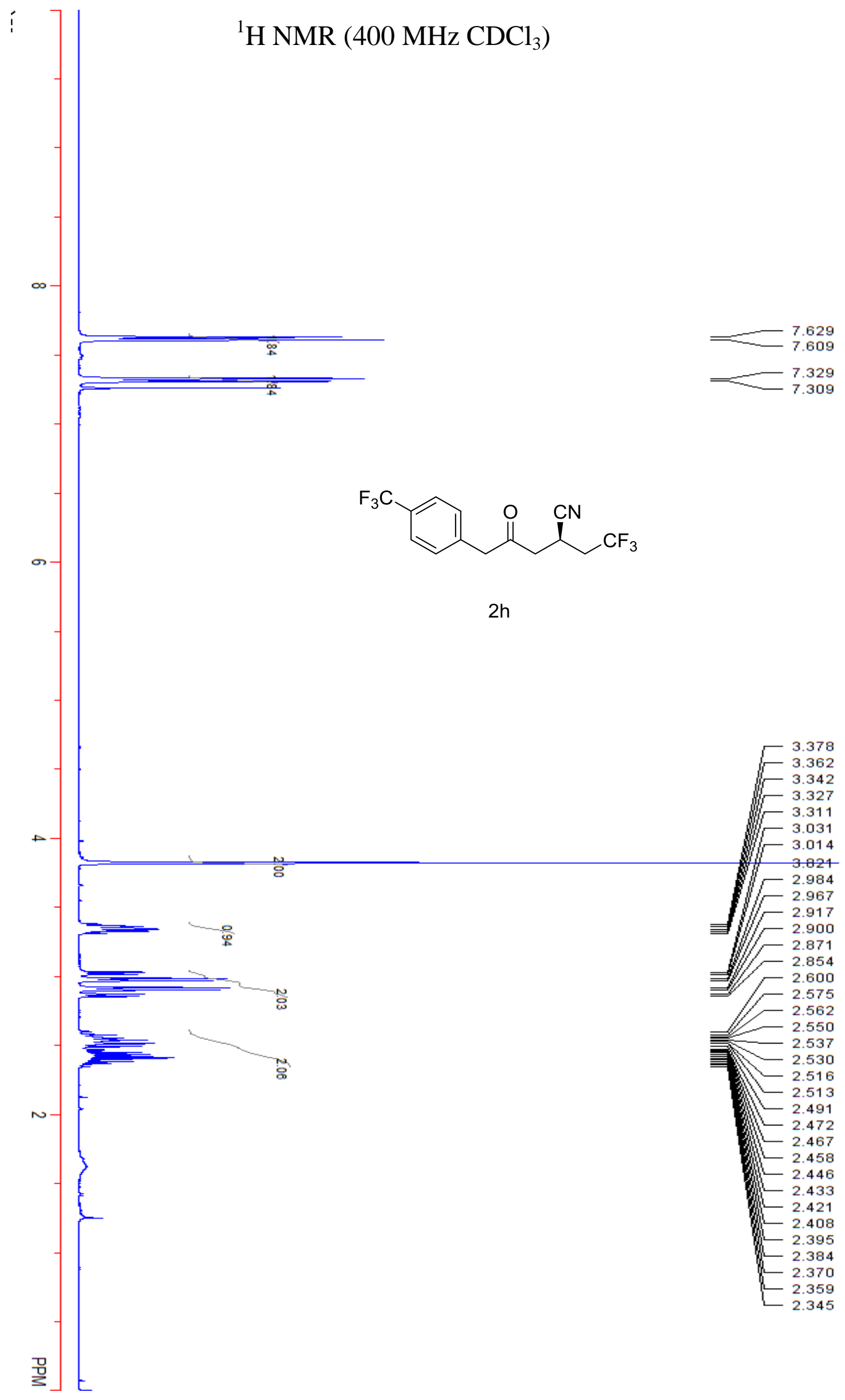




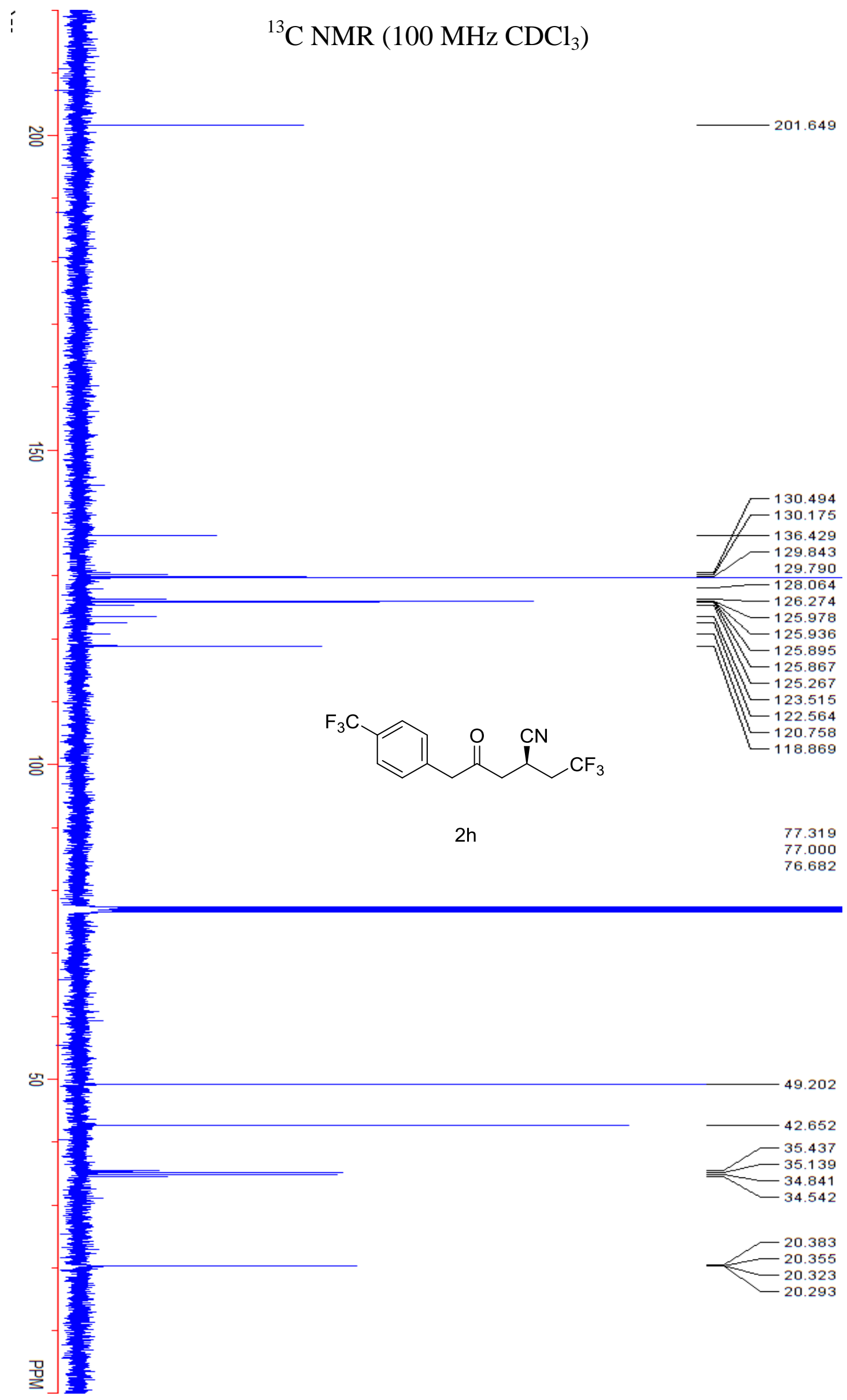




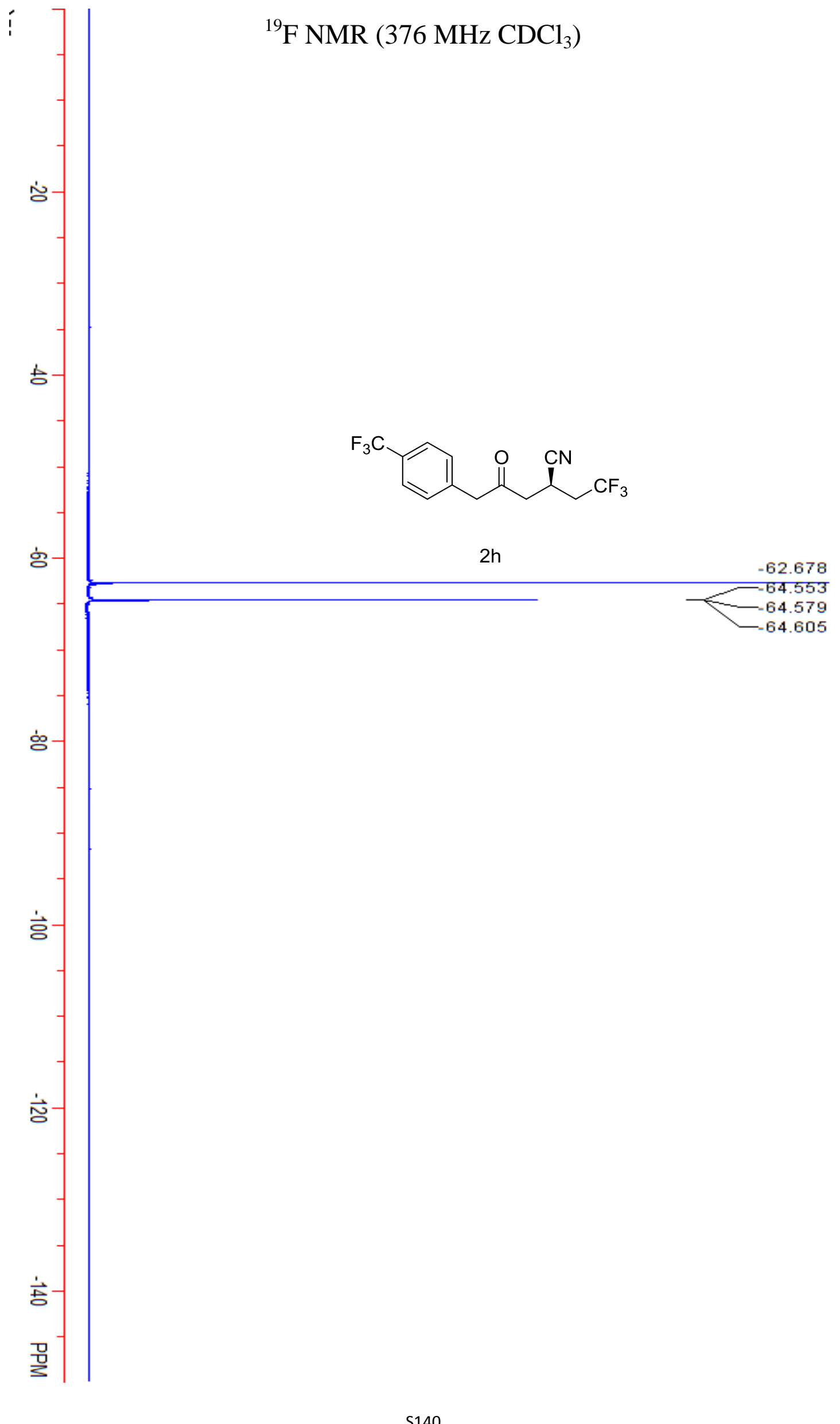




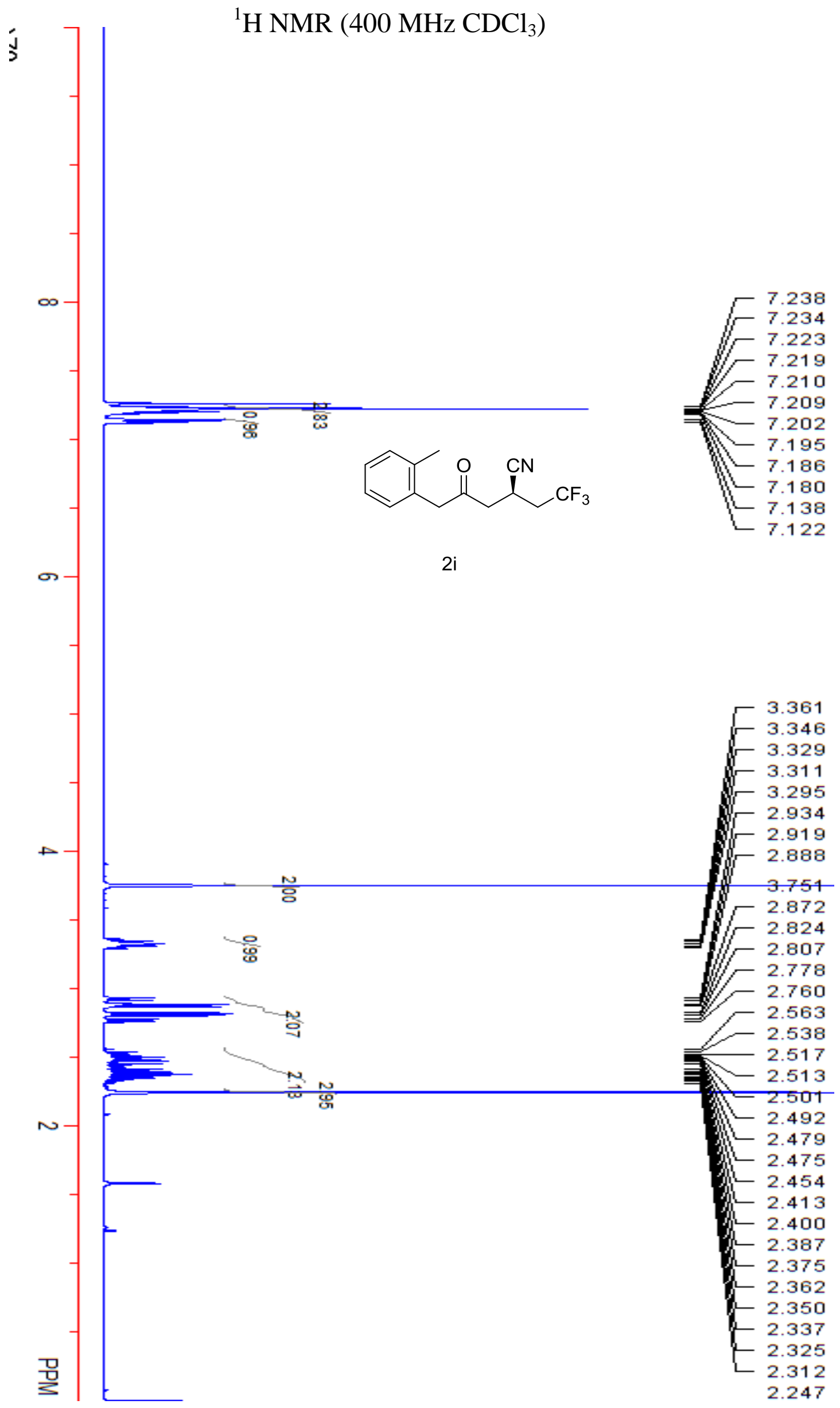




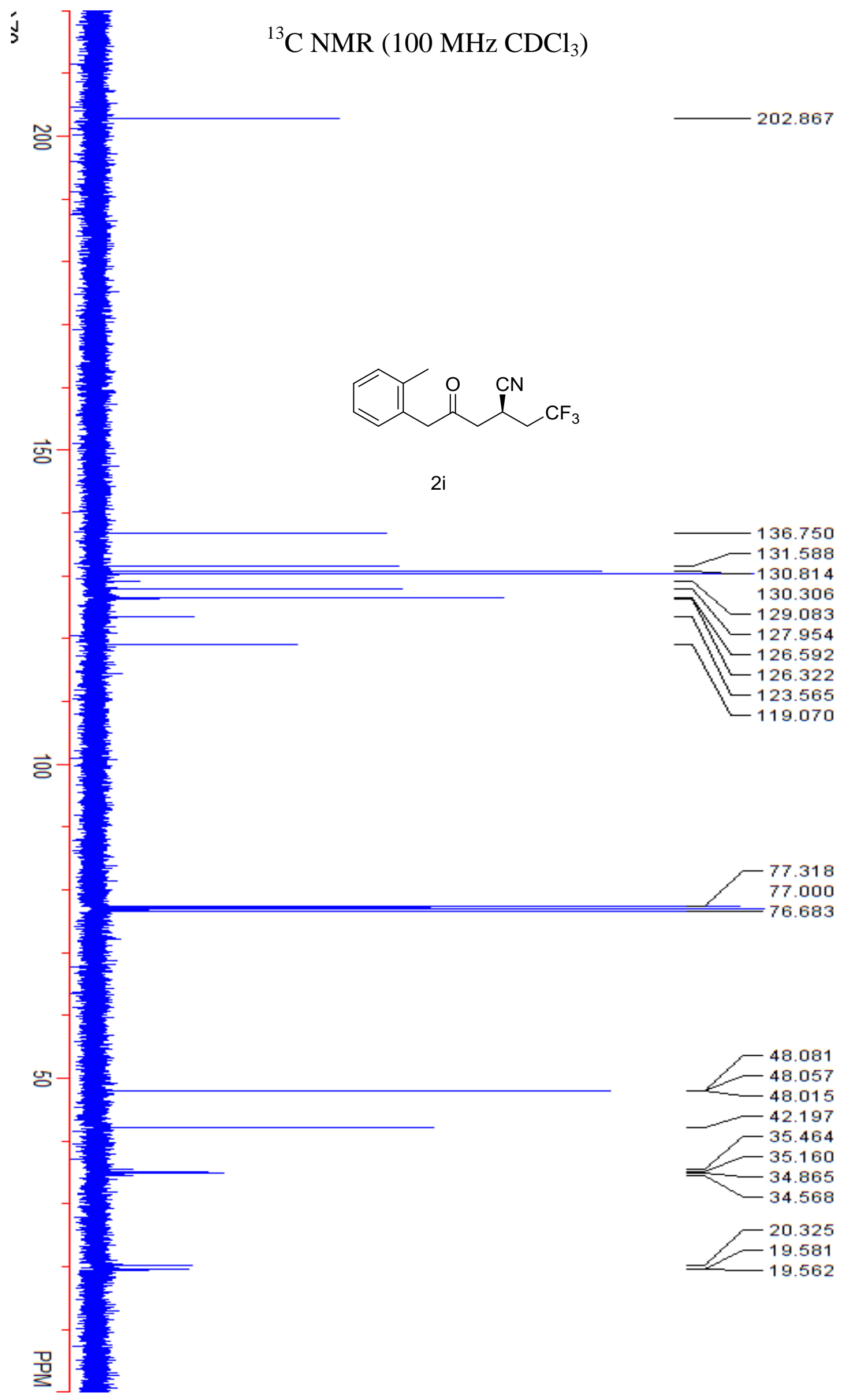


${ }^{19} \mathrm{~F}$ NMR (376 MHz $\mathrm{CDCl}_{3}$ )

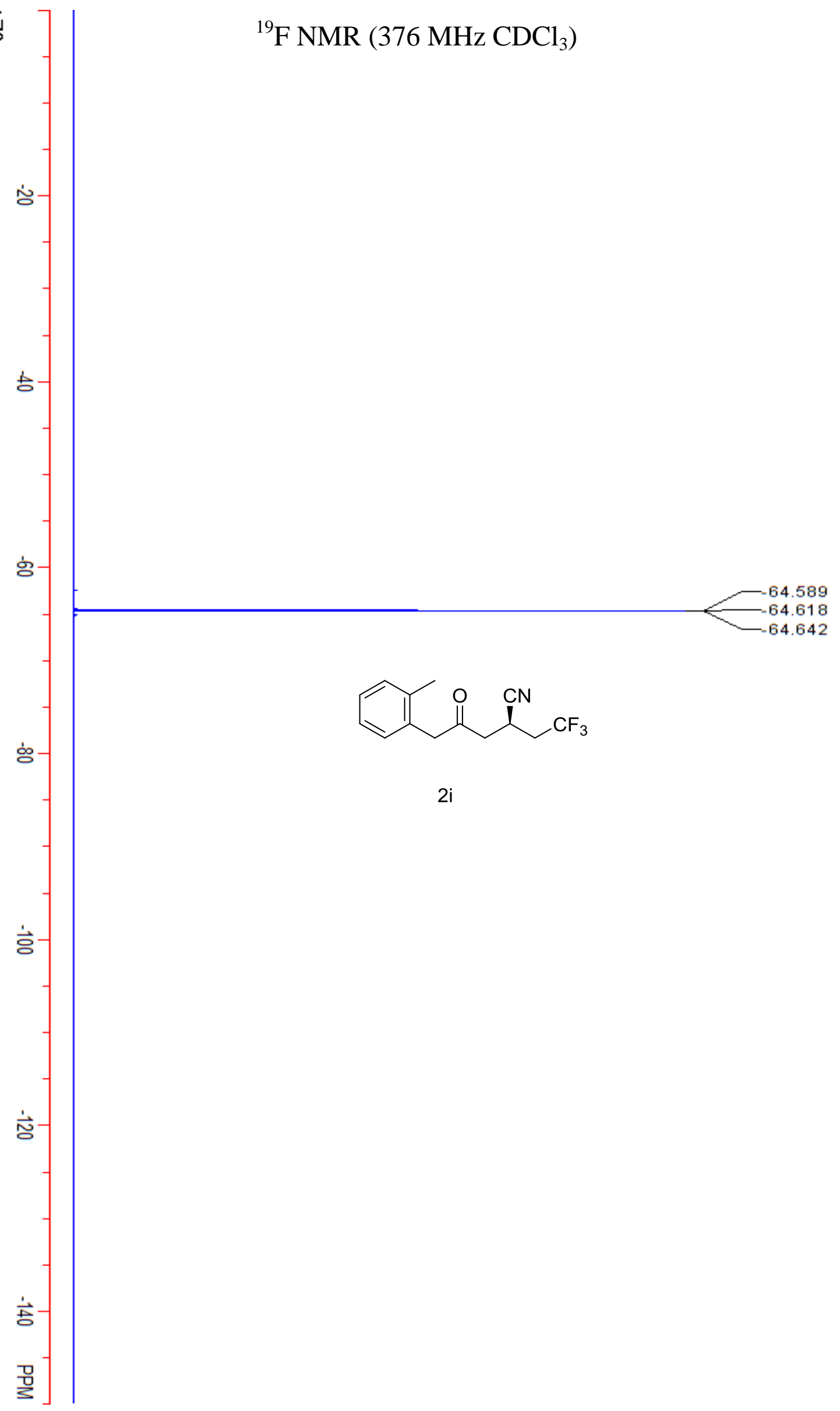




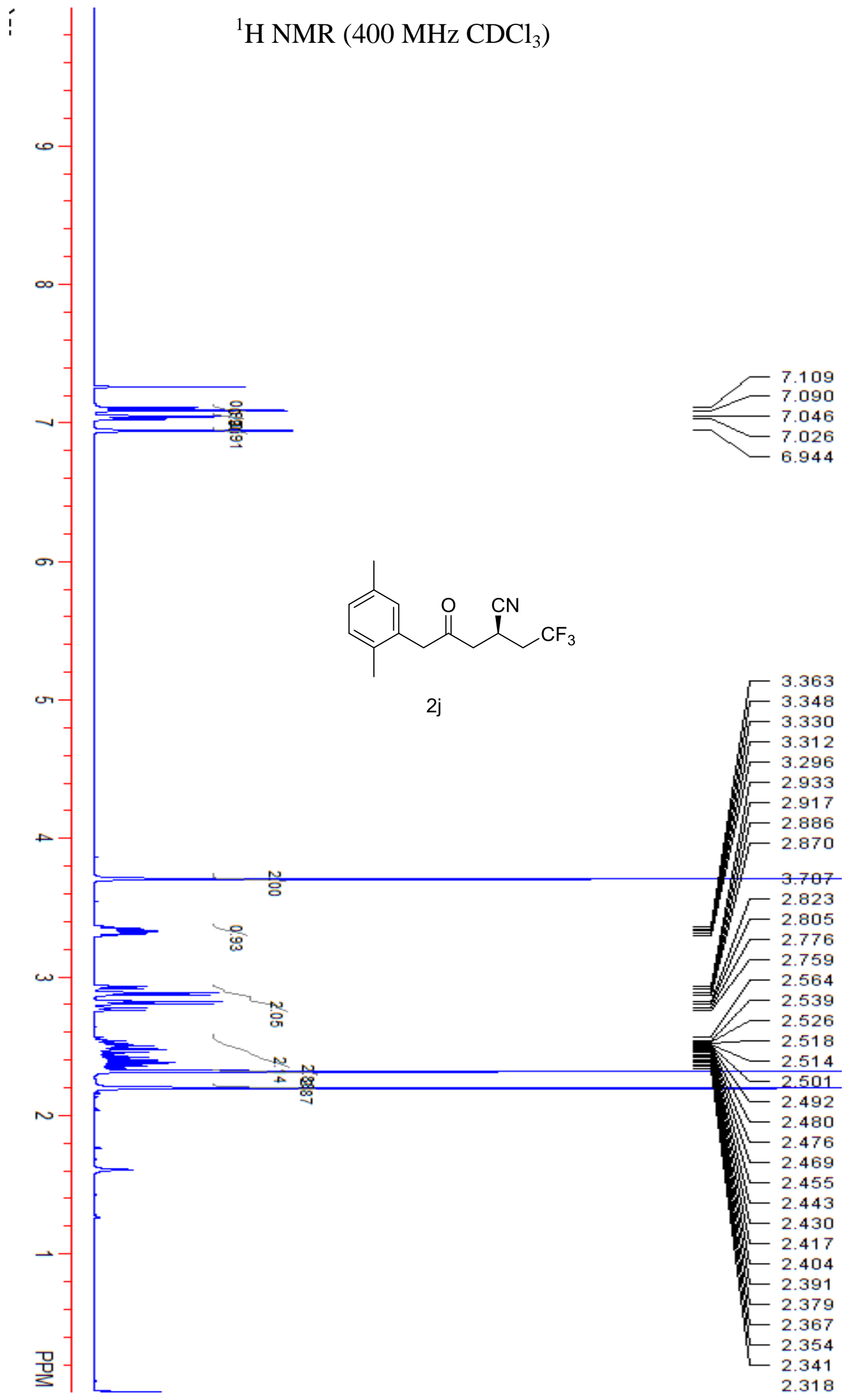




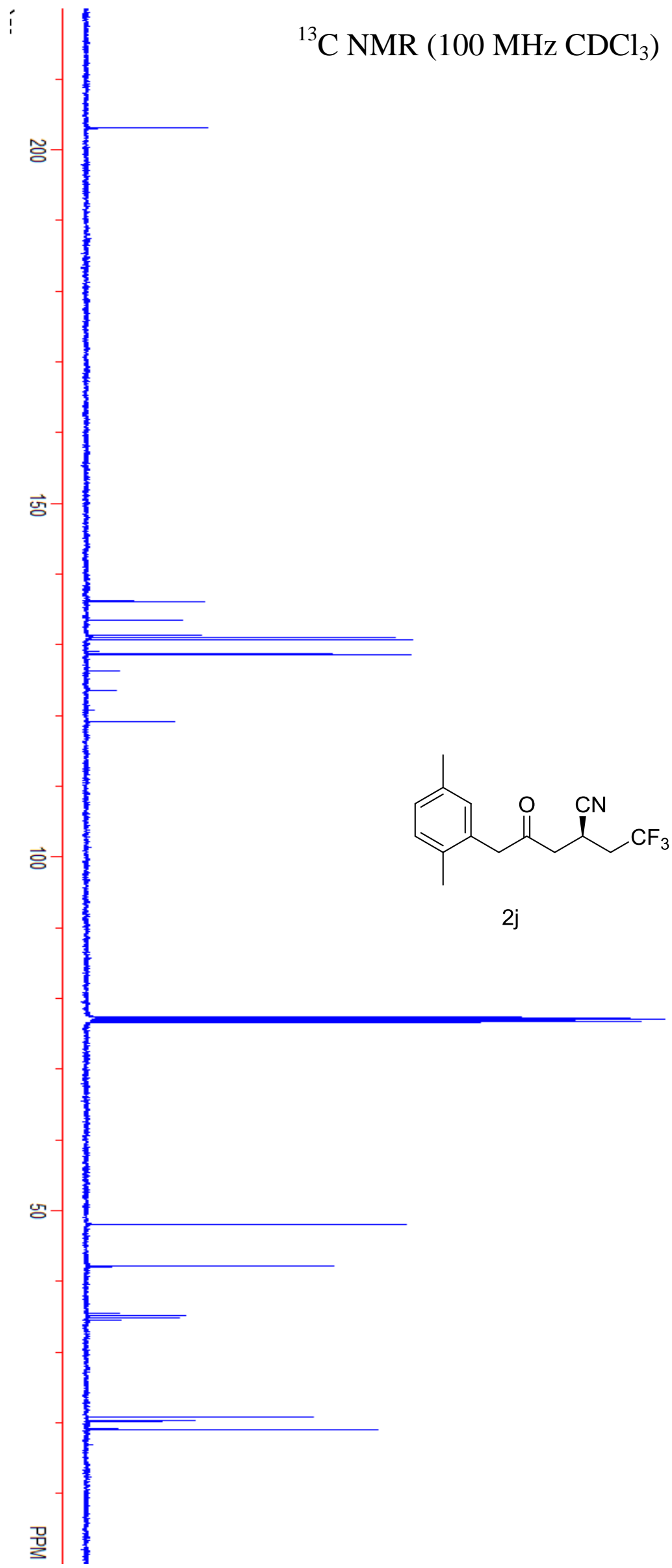

203.046
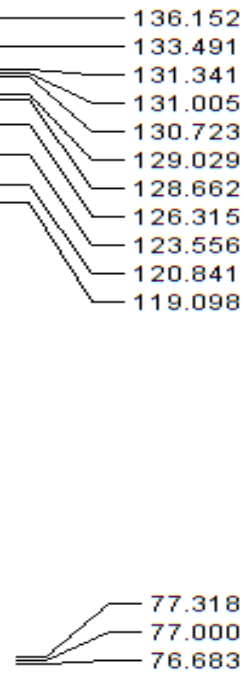


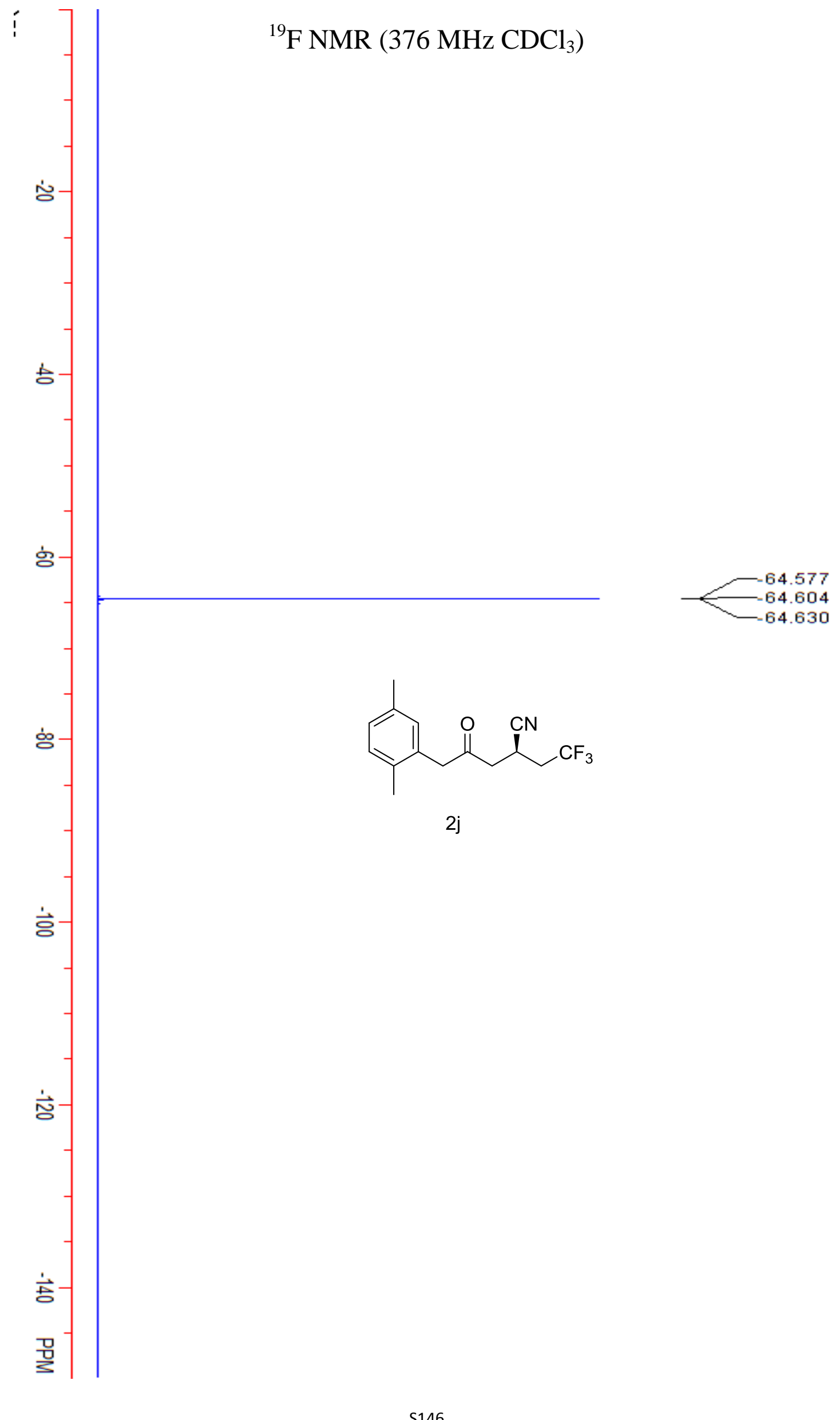




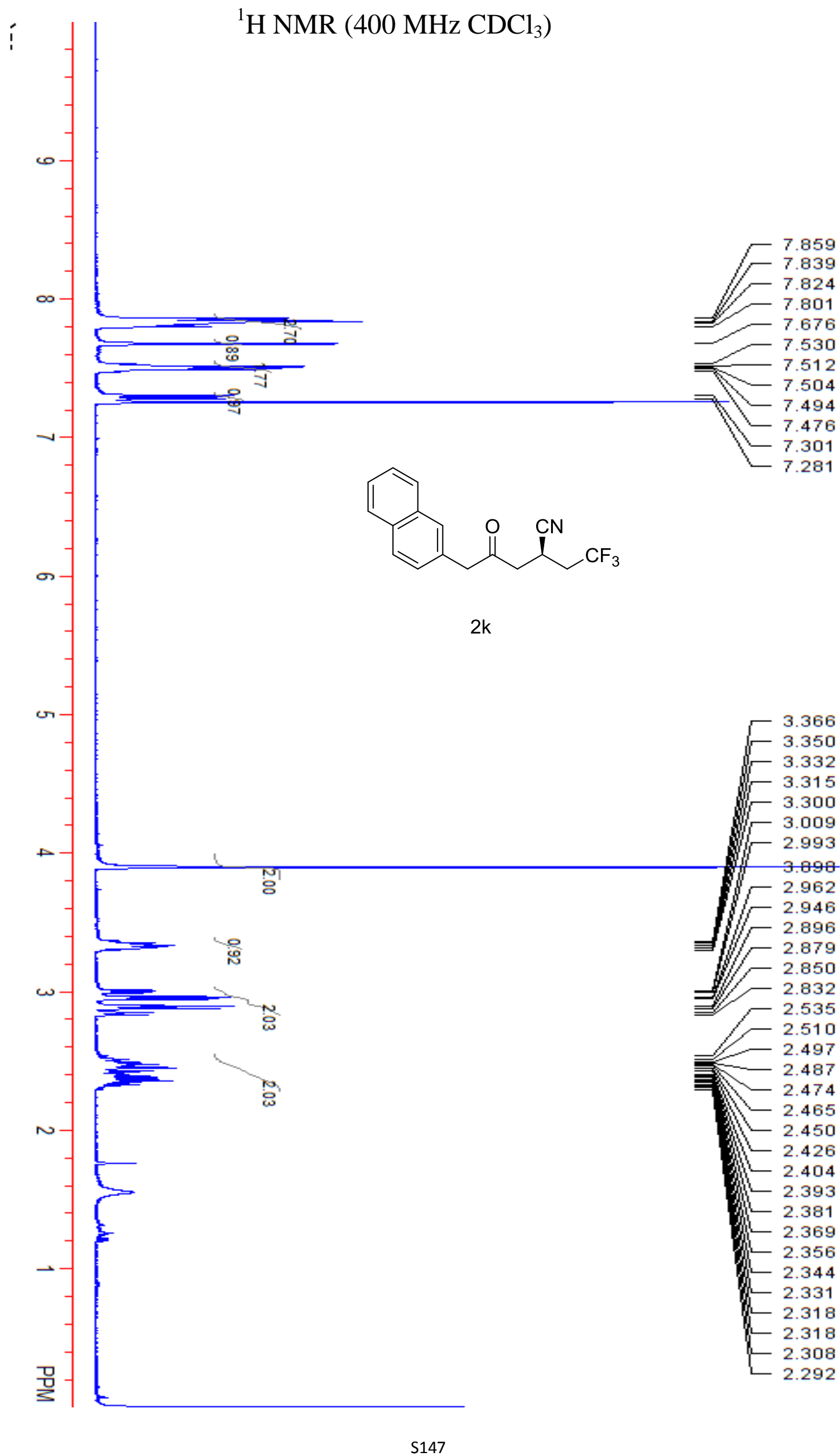




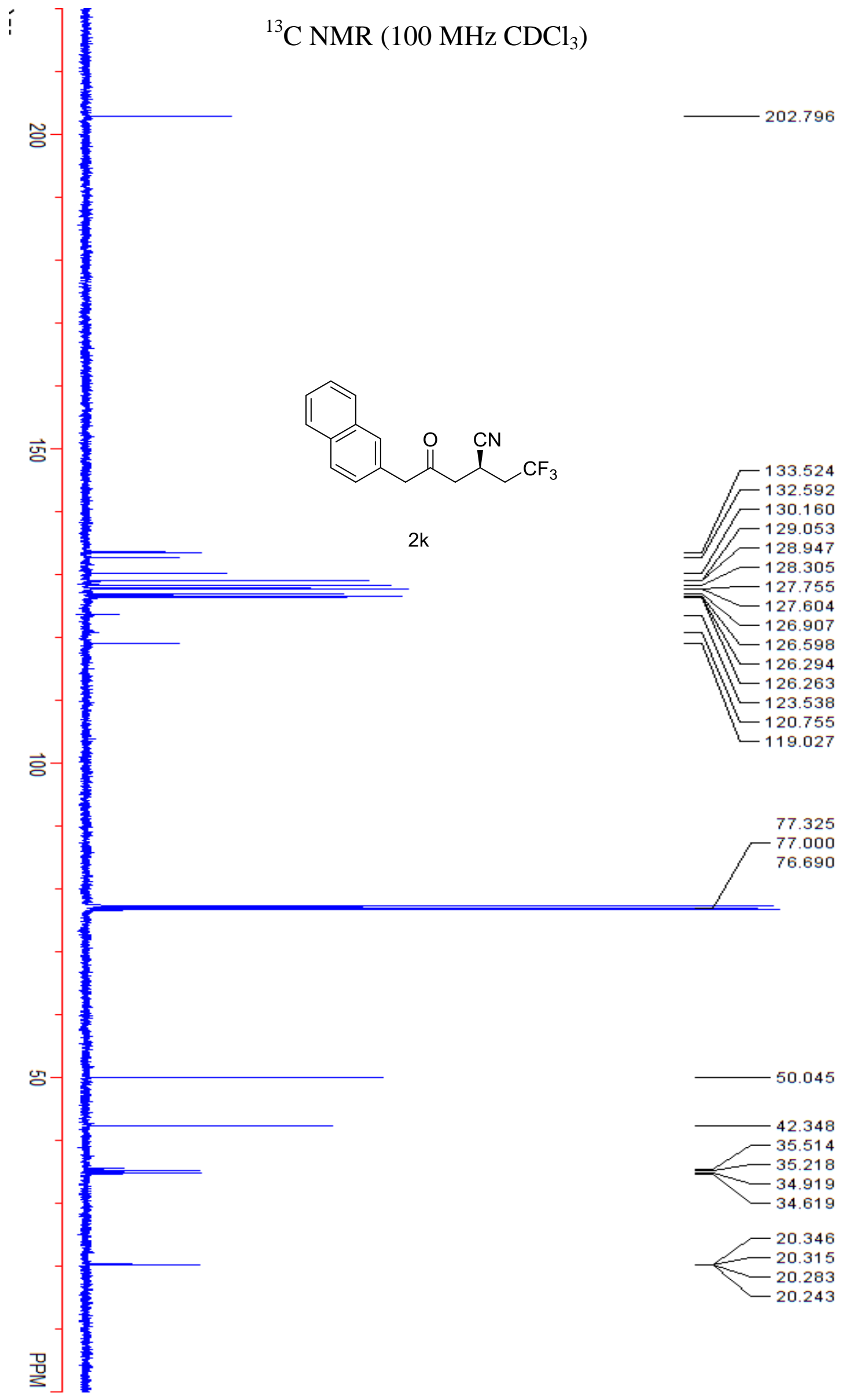




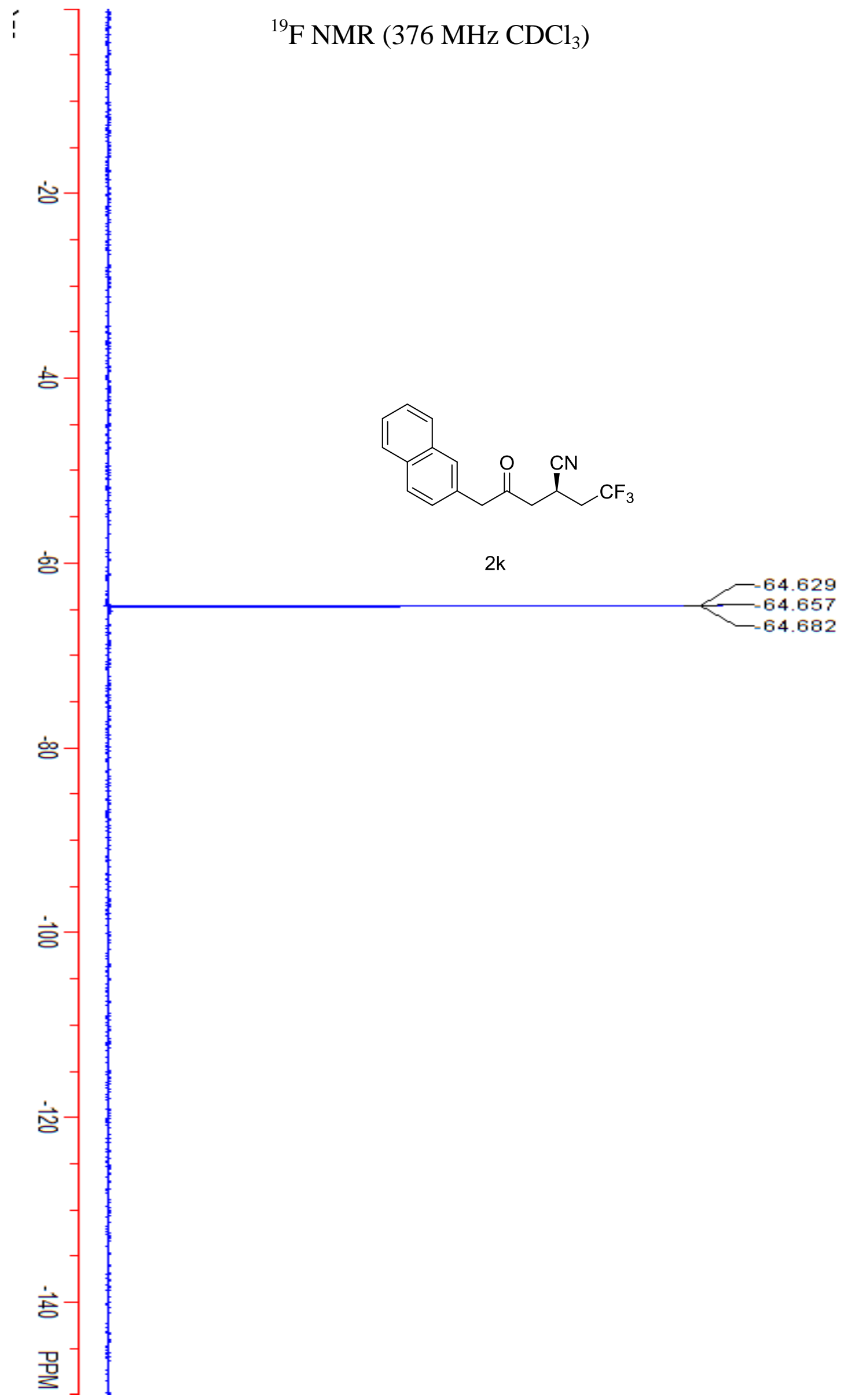









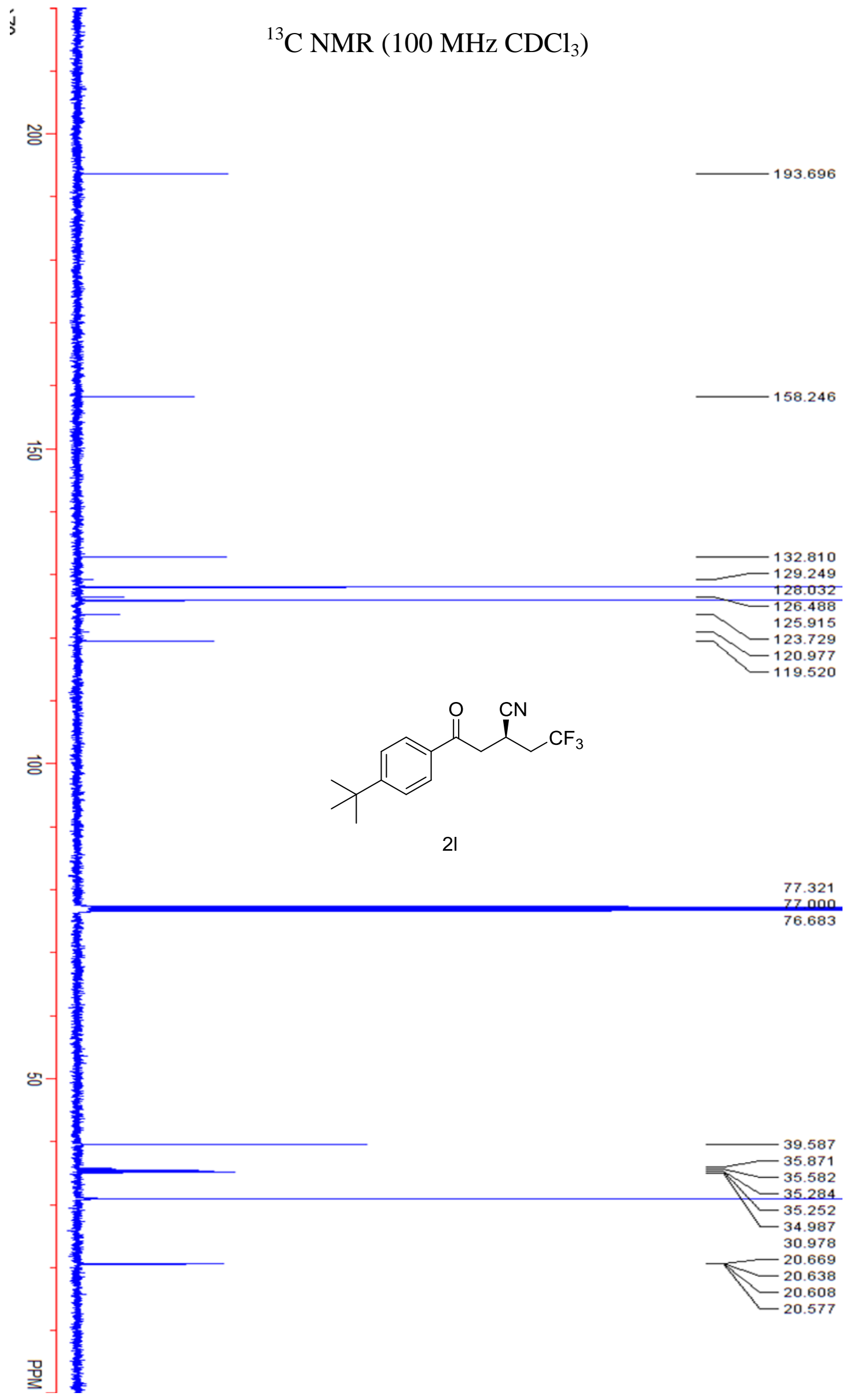




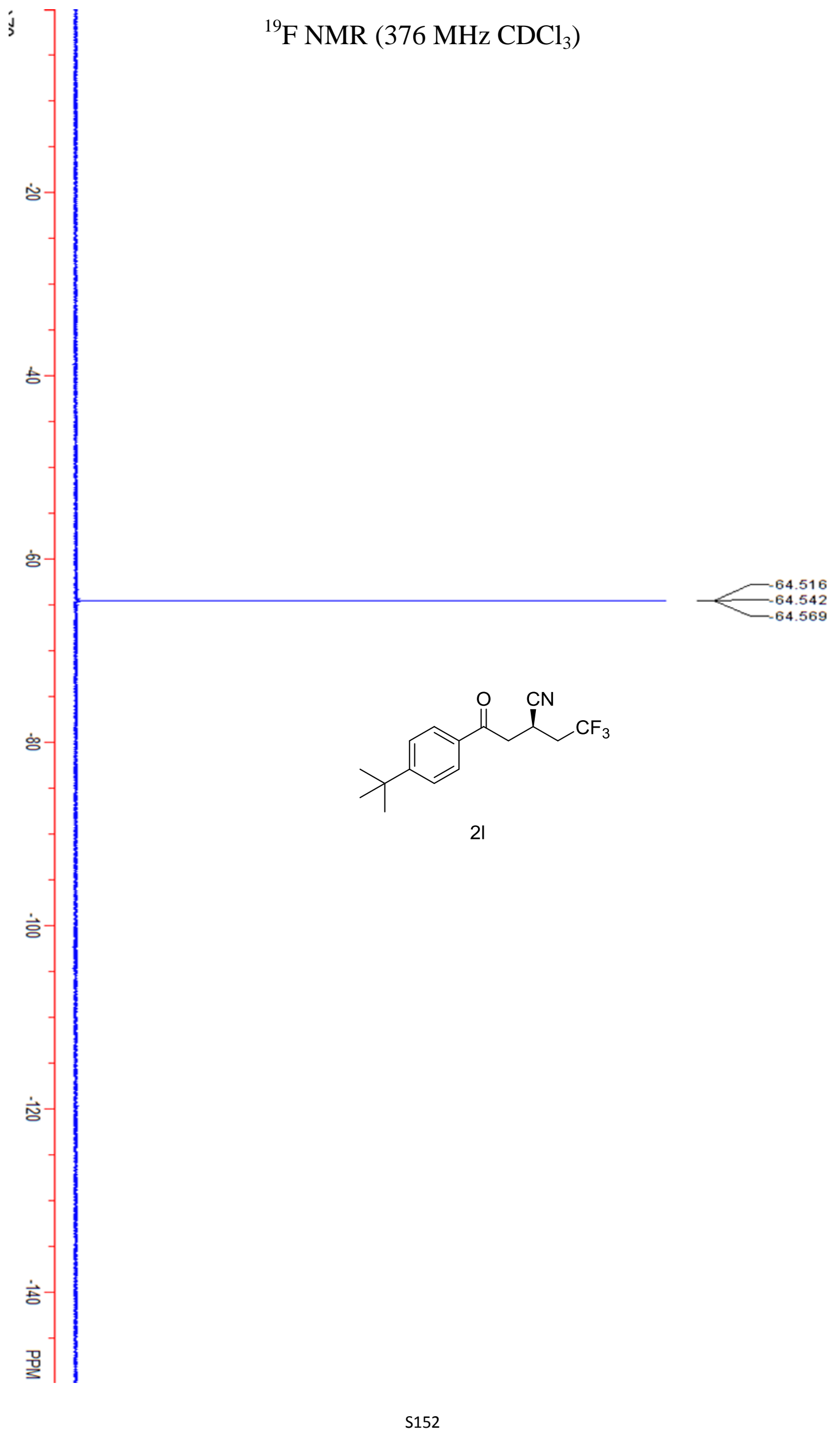




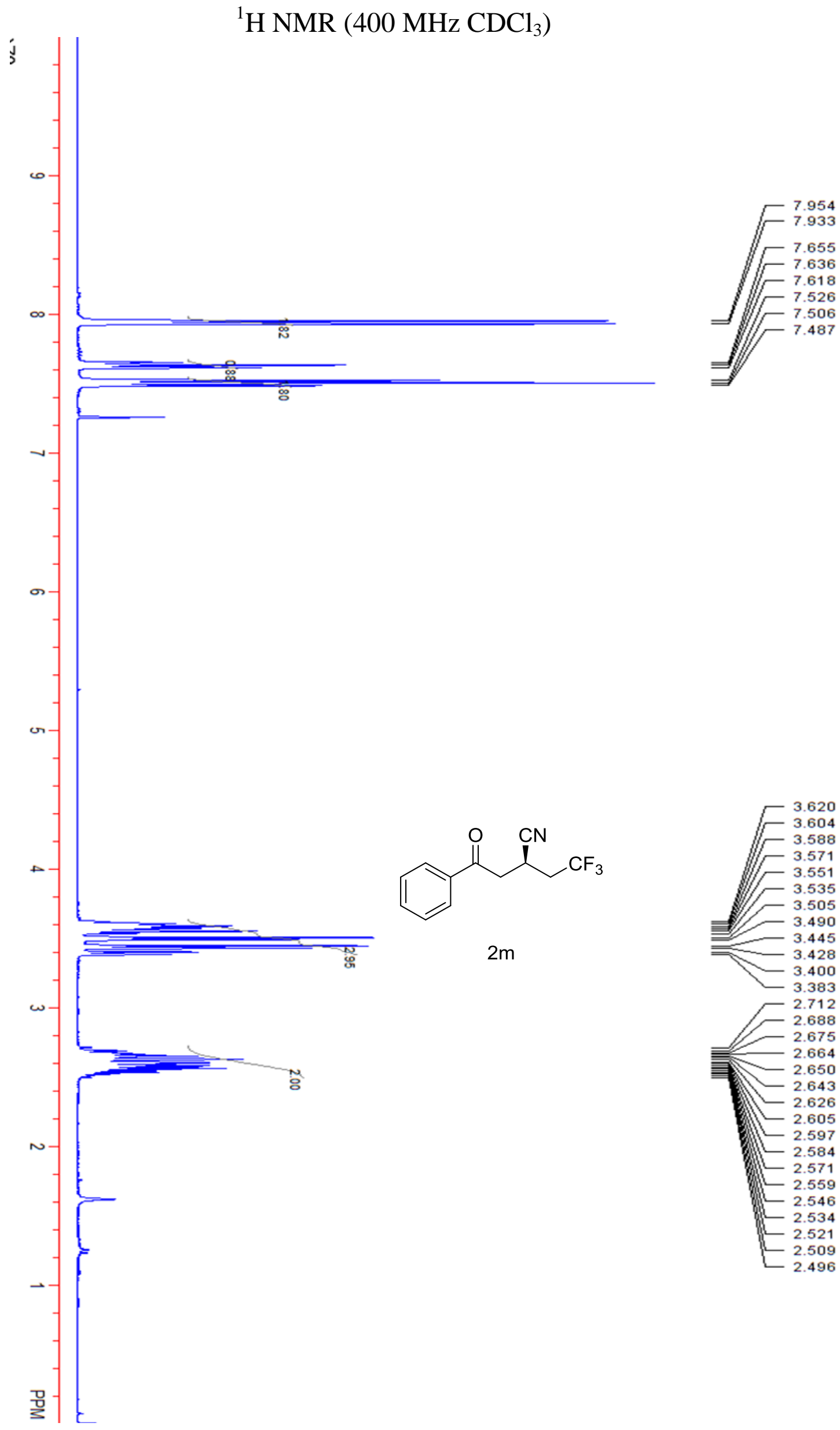




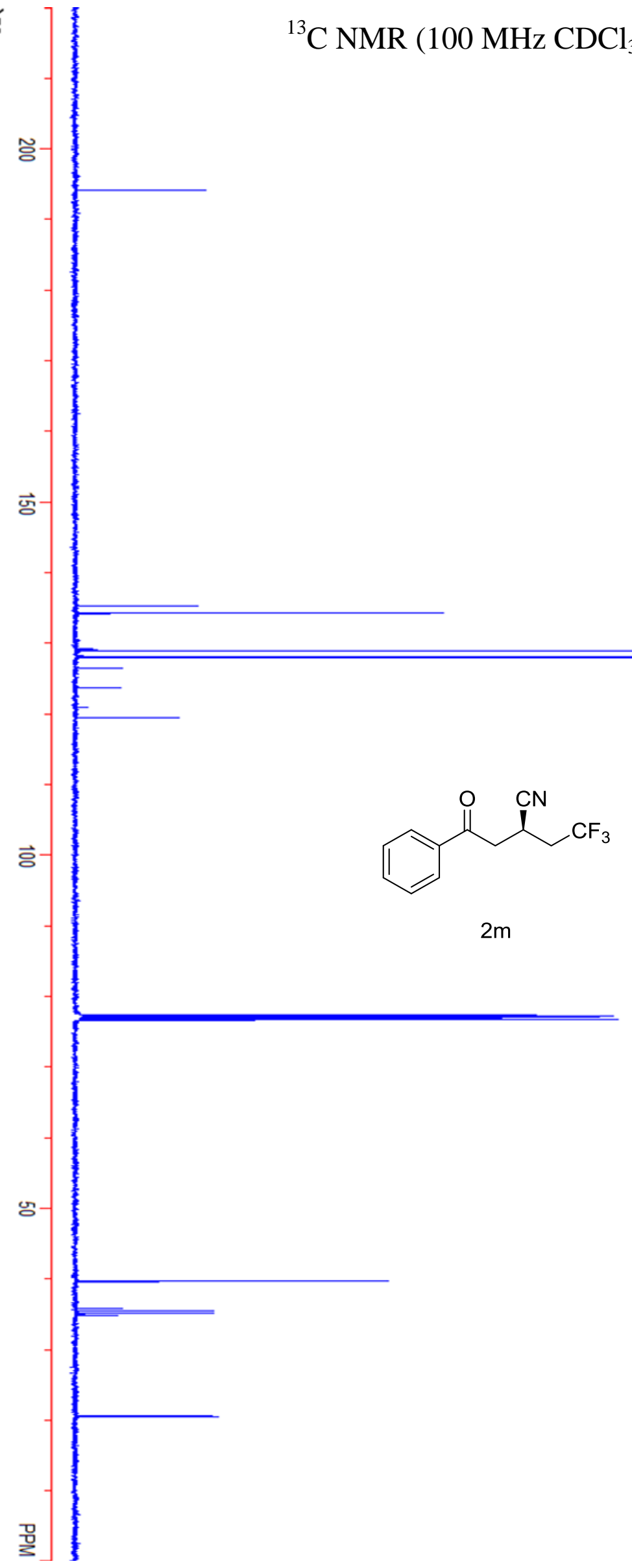

194.130

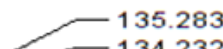

134.232

129.217 128.951 $-128.021$

$-126.461$

$-123.703$

-120.945
-119.453

$2 m$

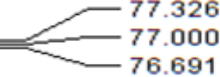




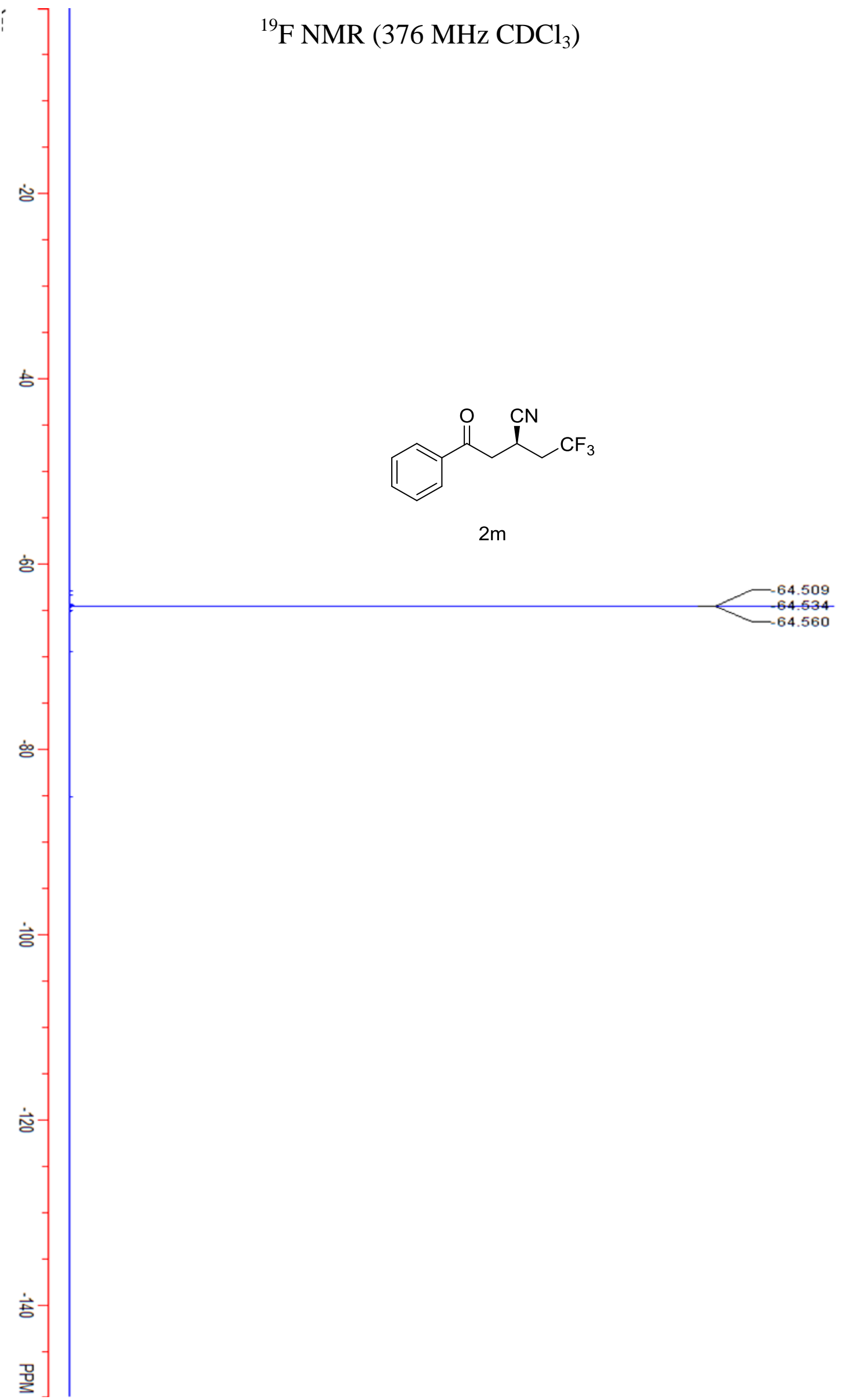




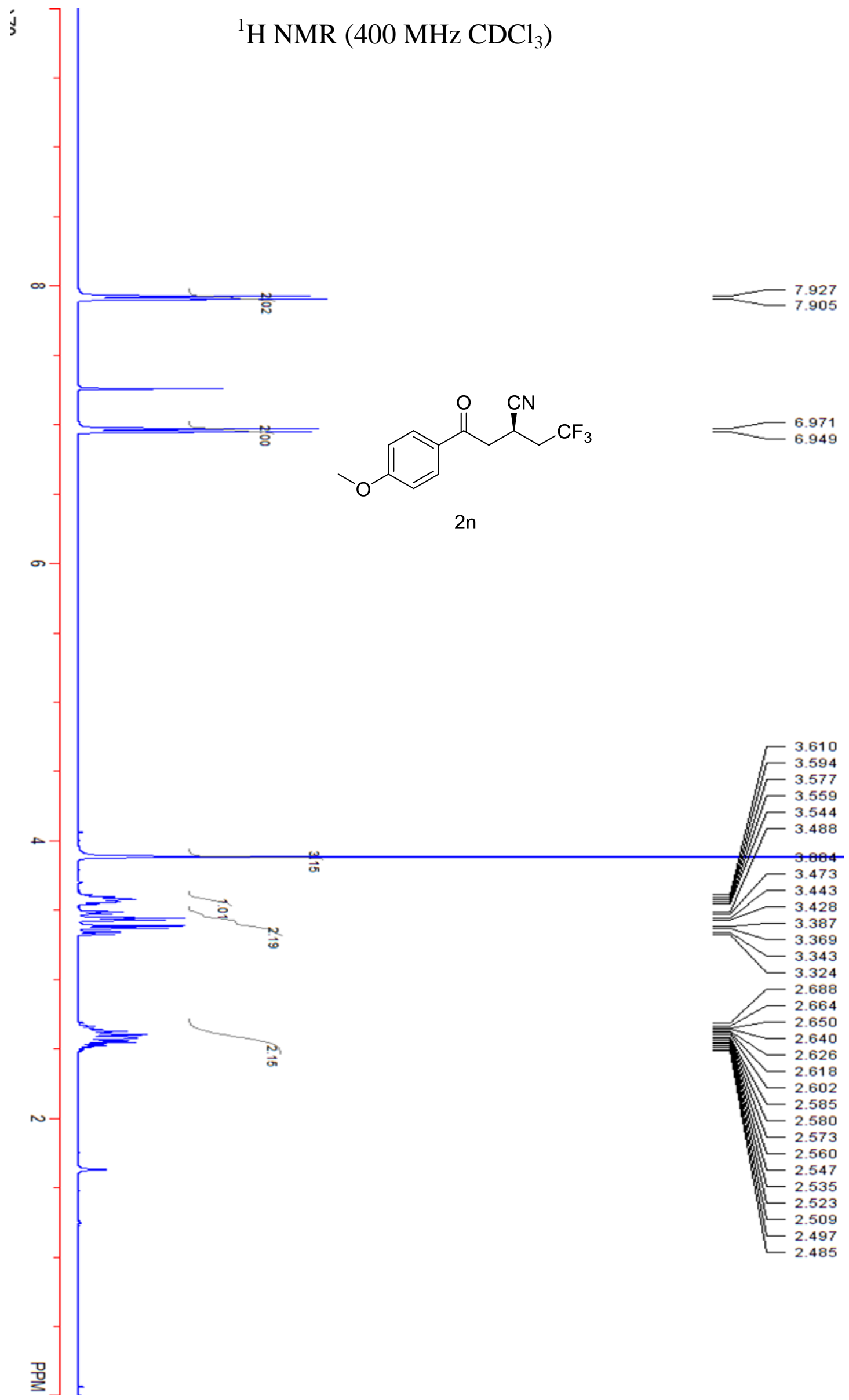




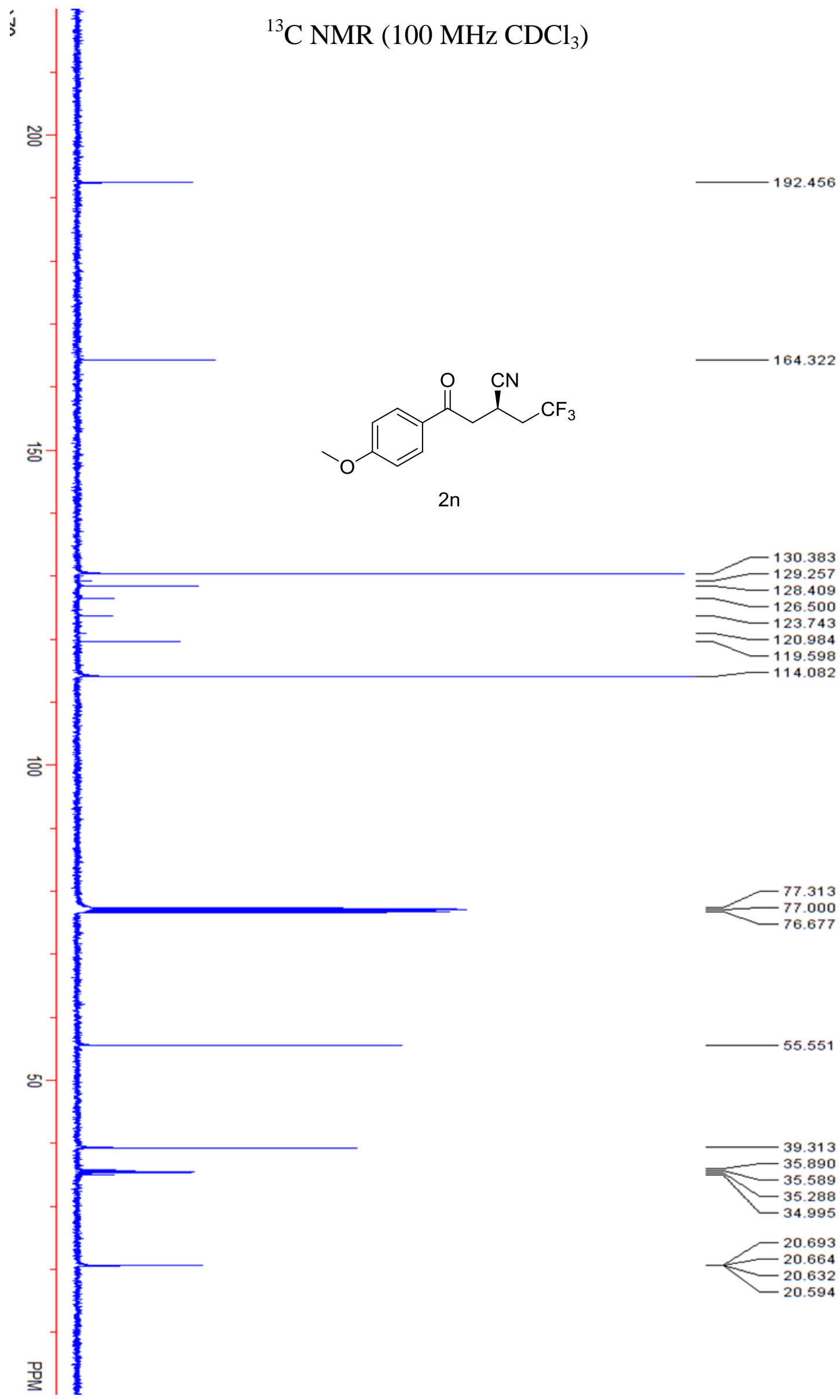




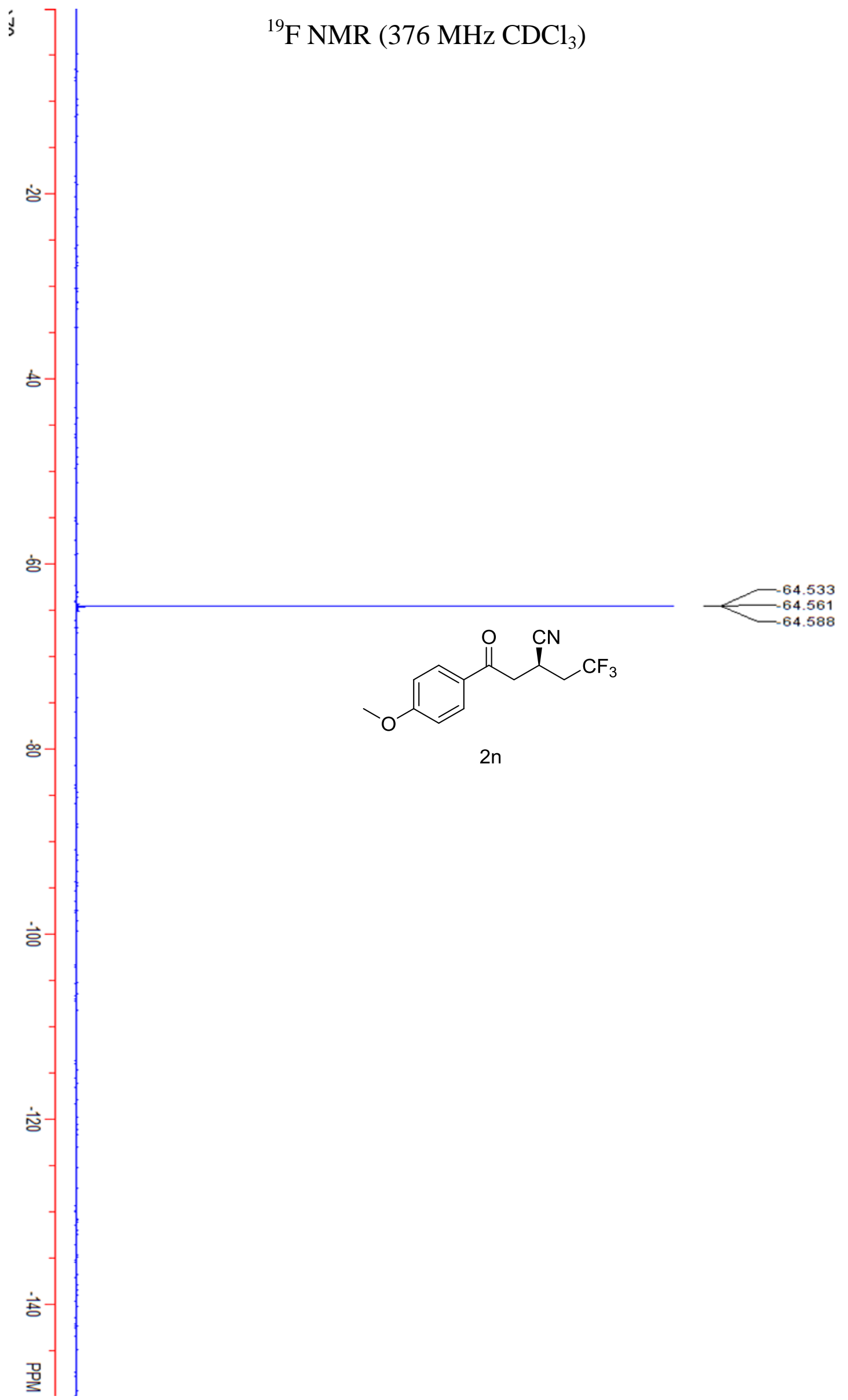




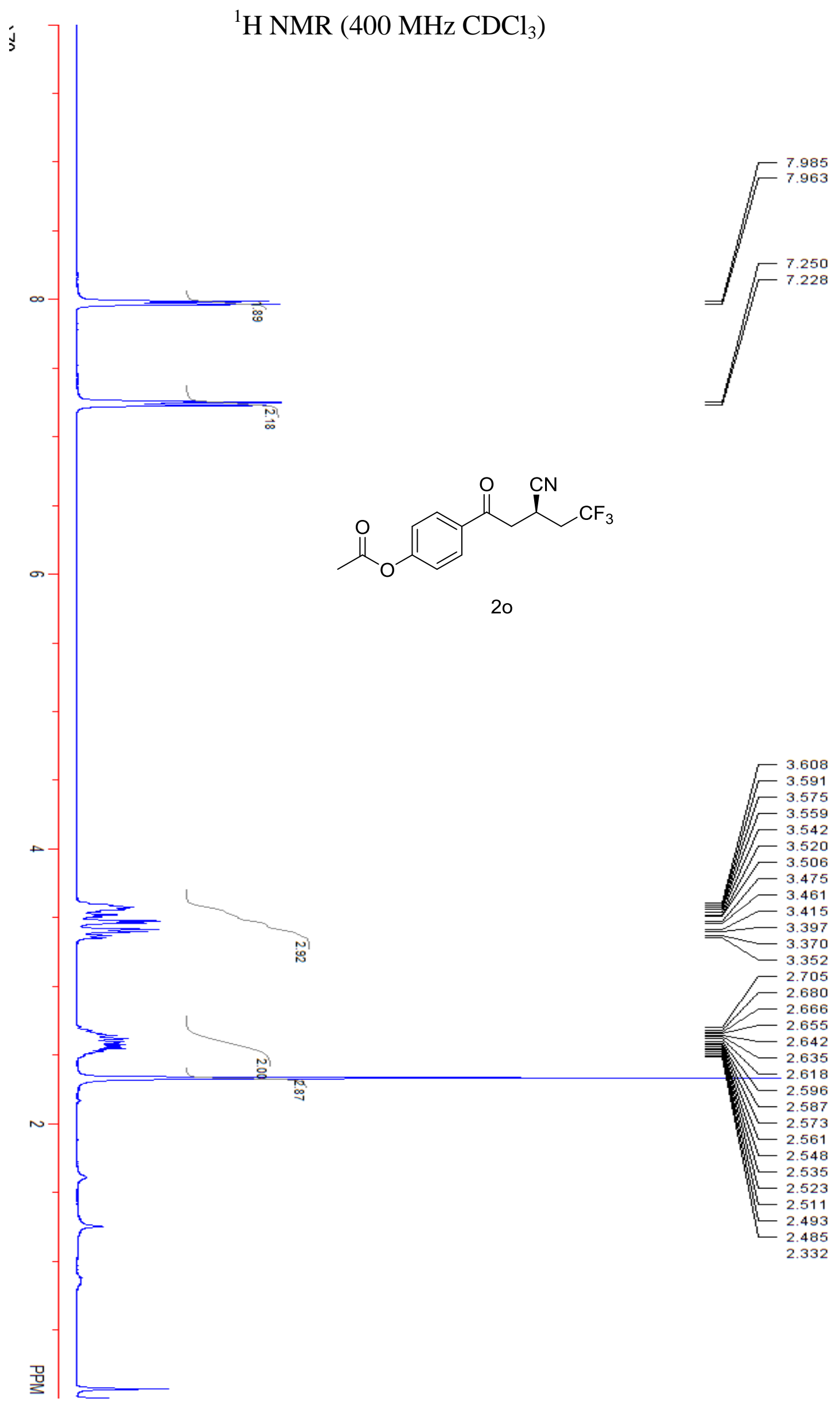




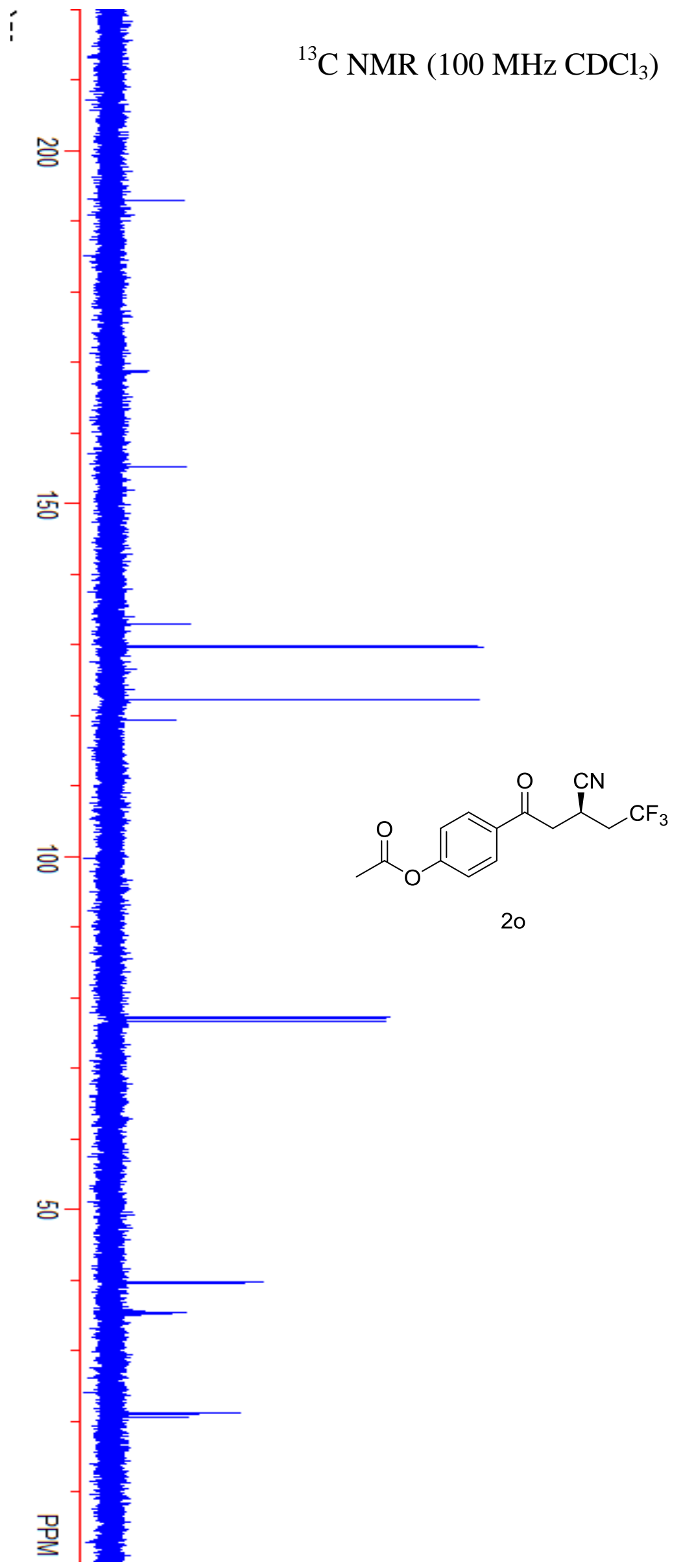

192.889

168.663

155.158
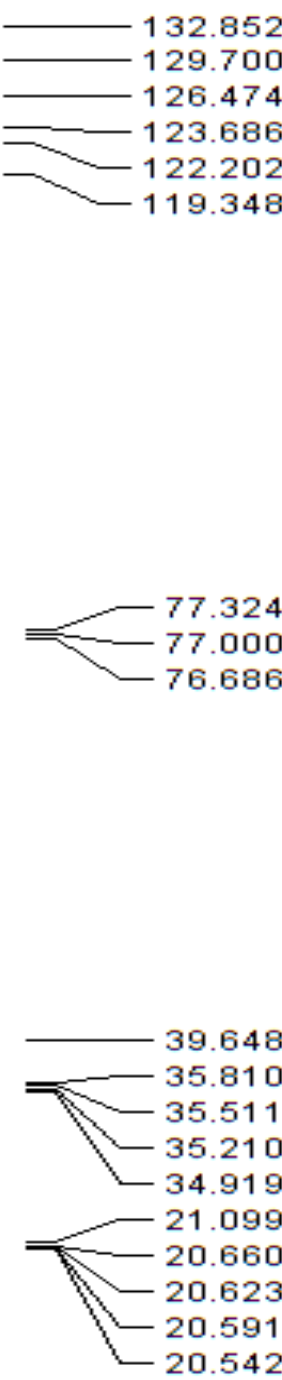


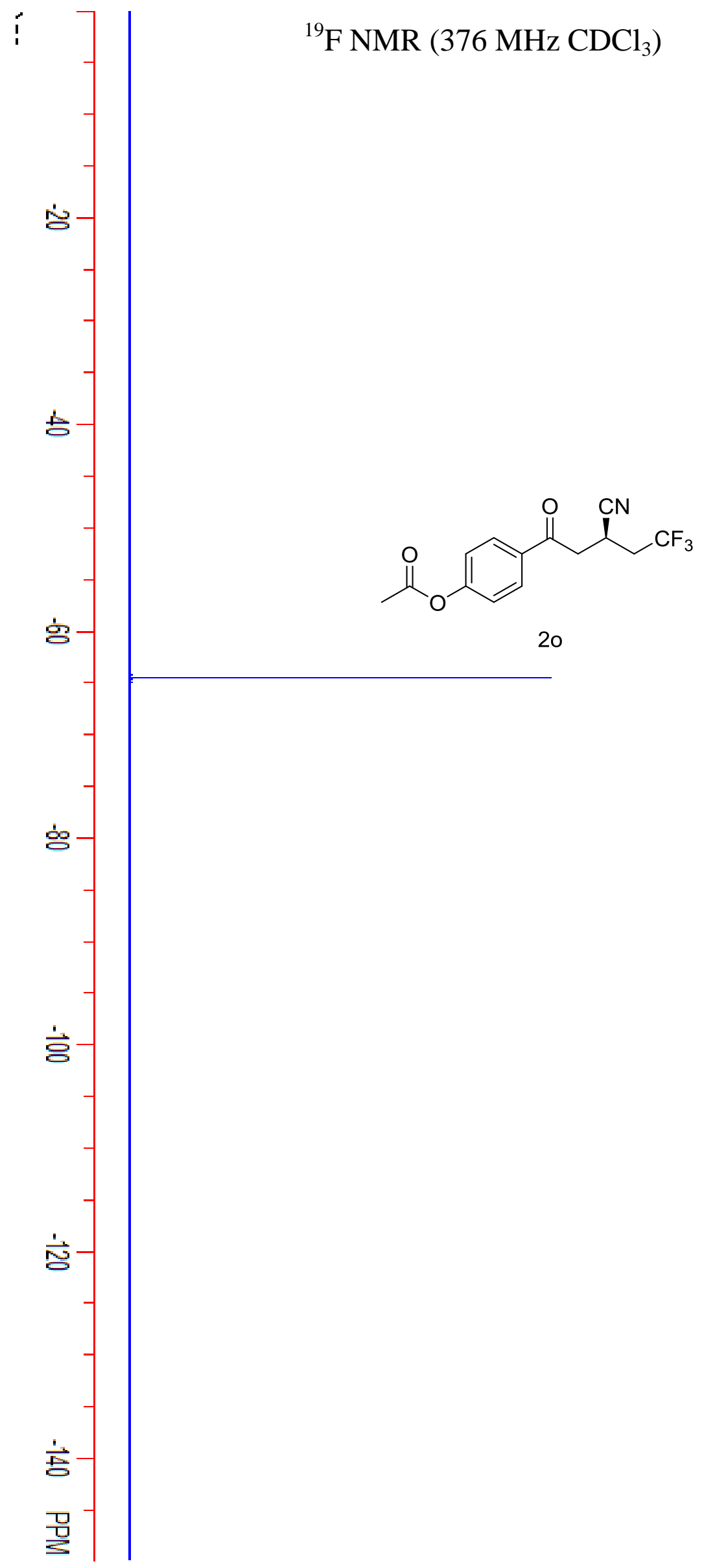




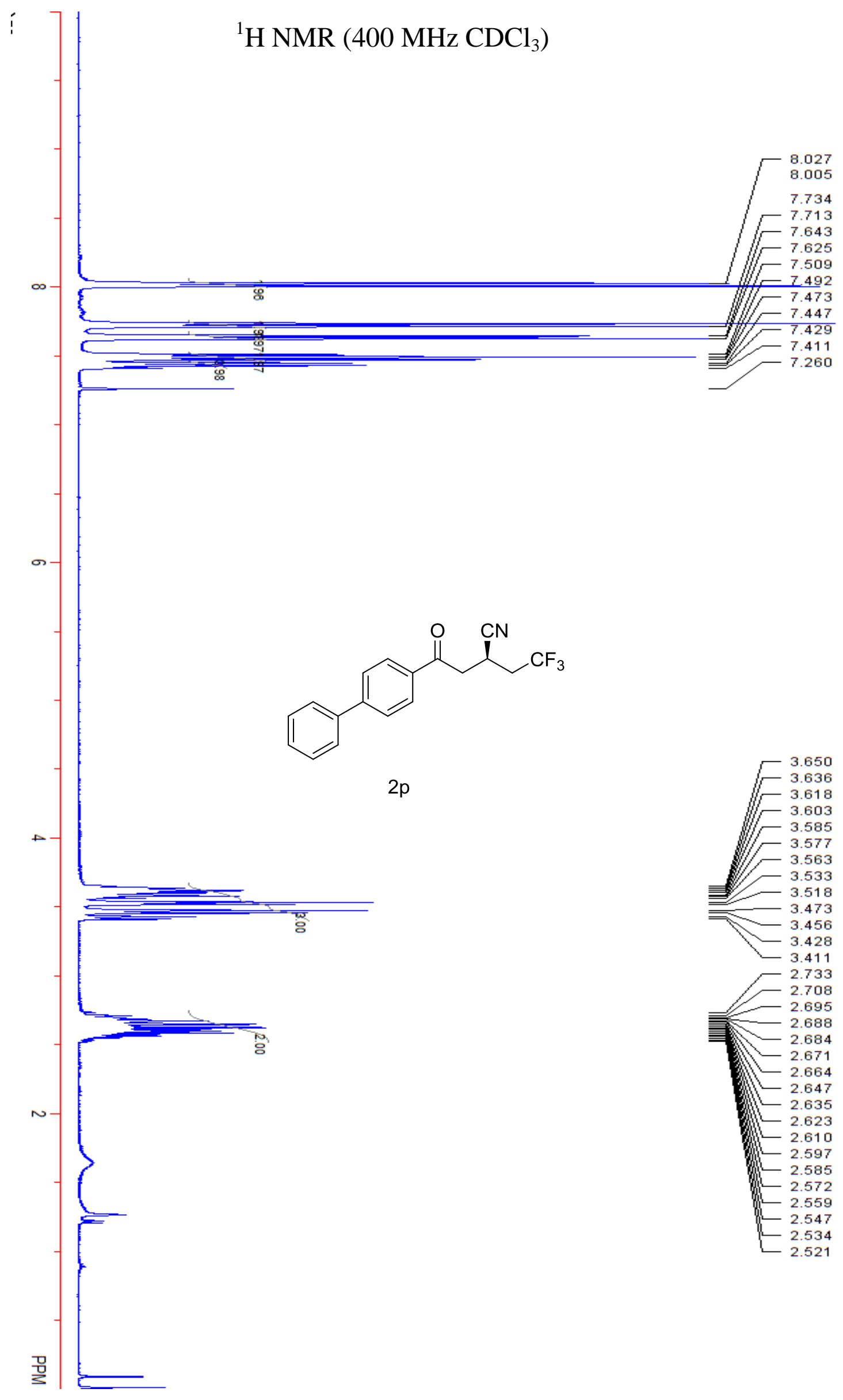




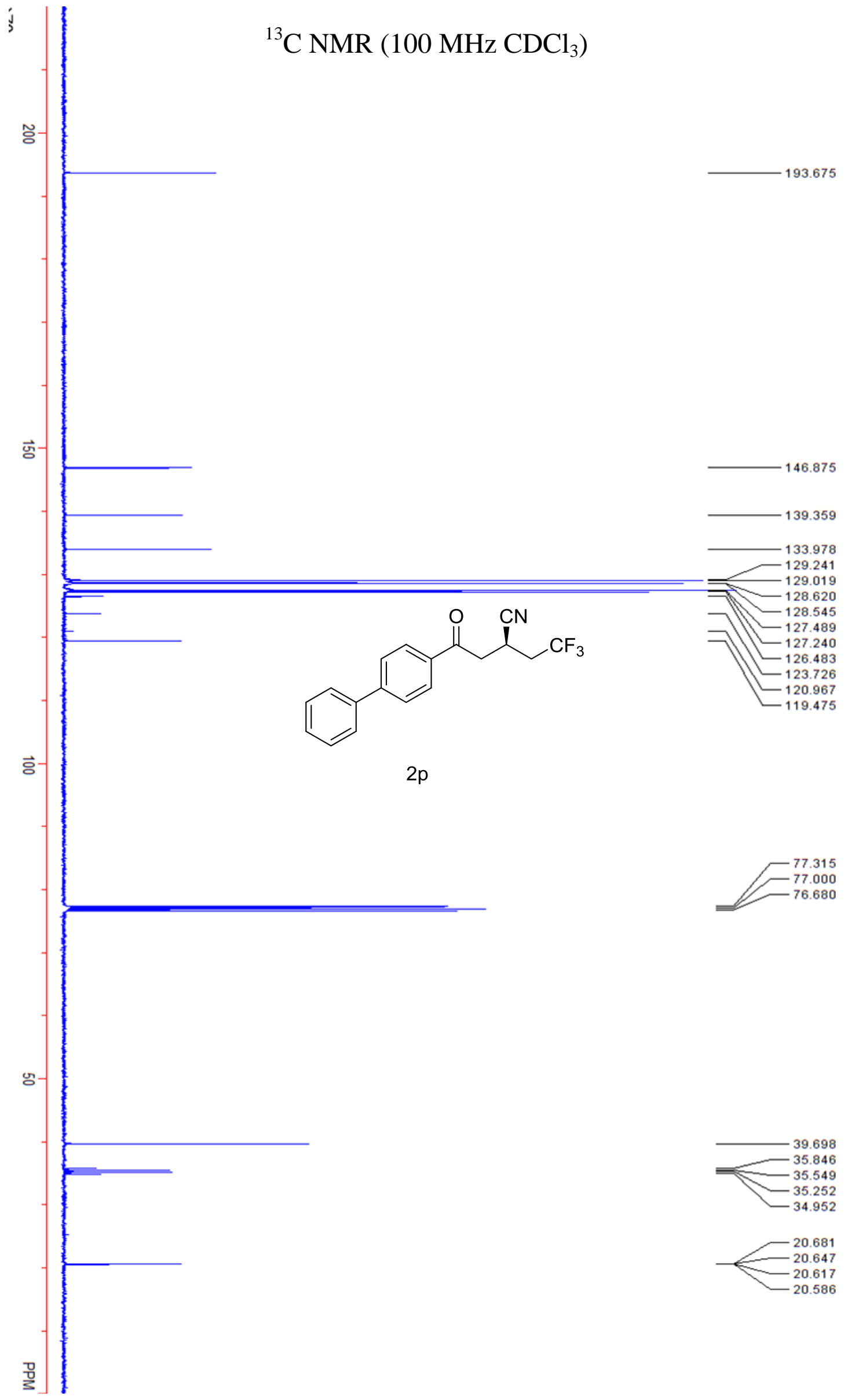



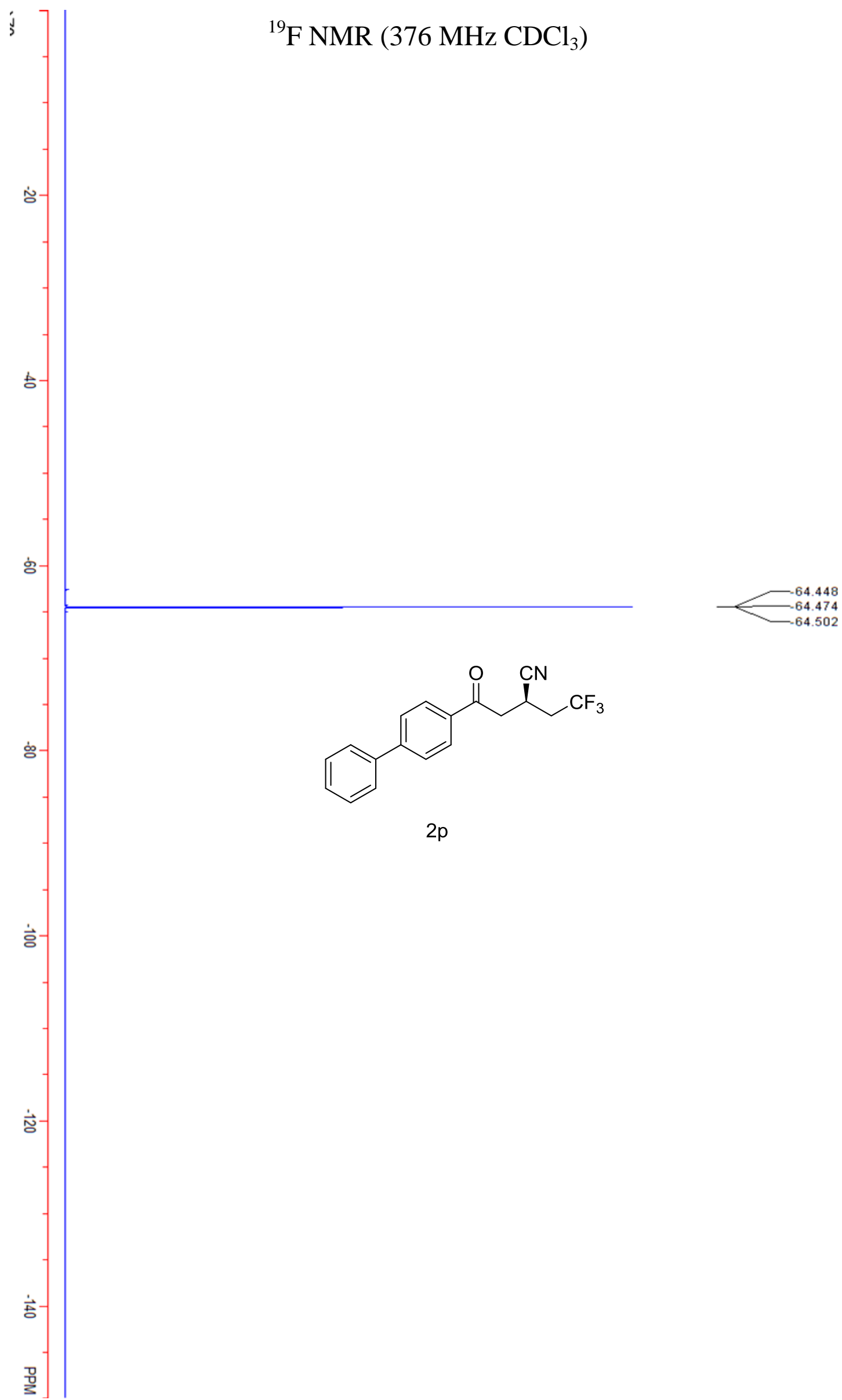


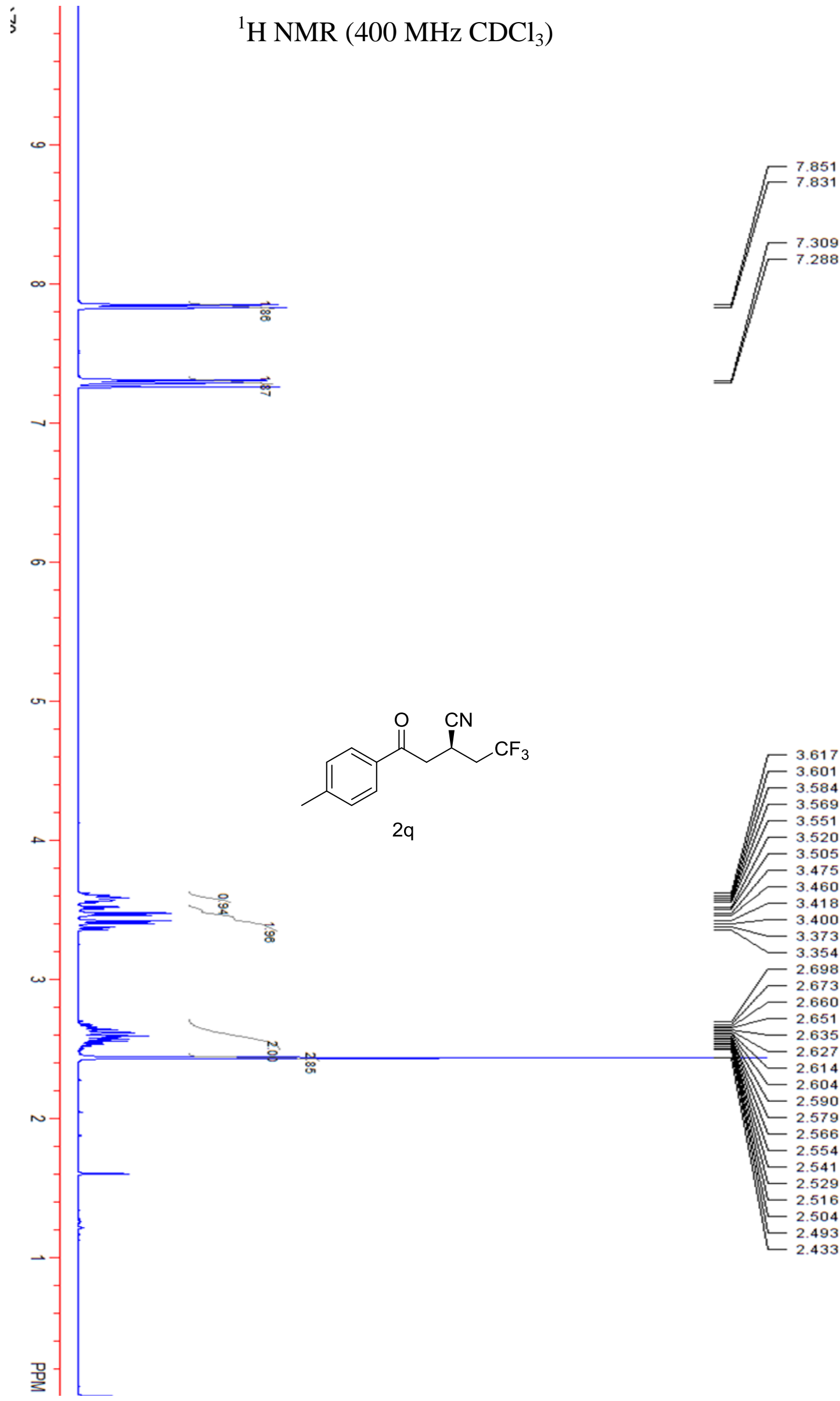




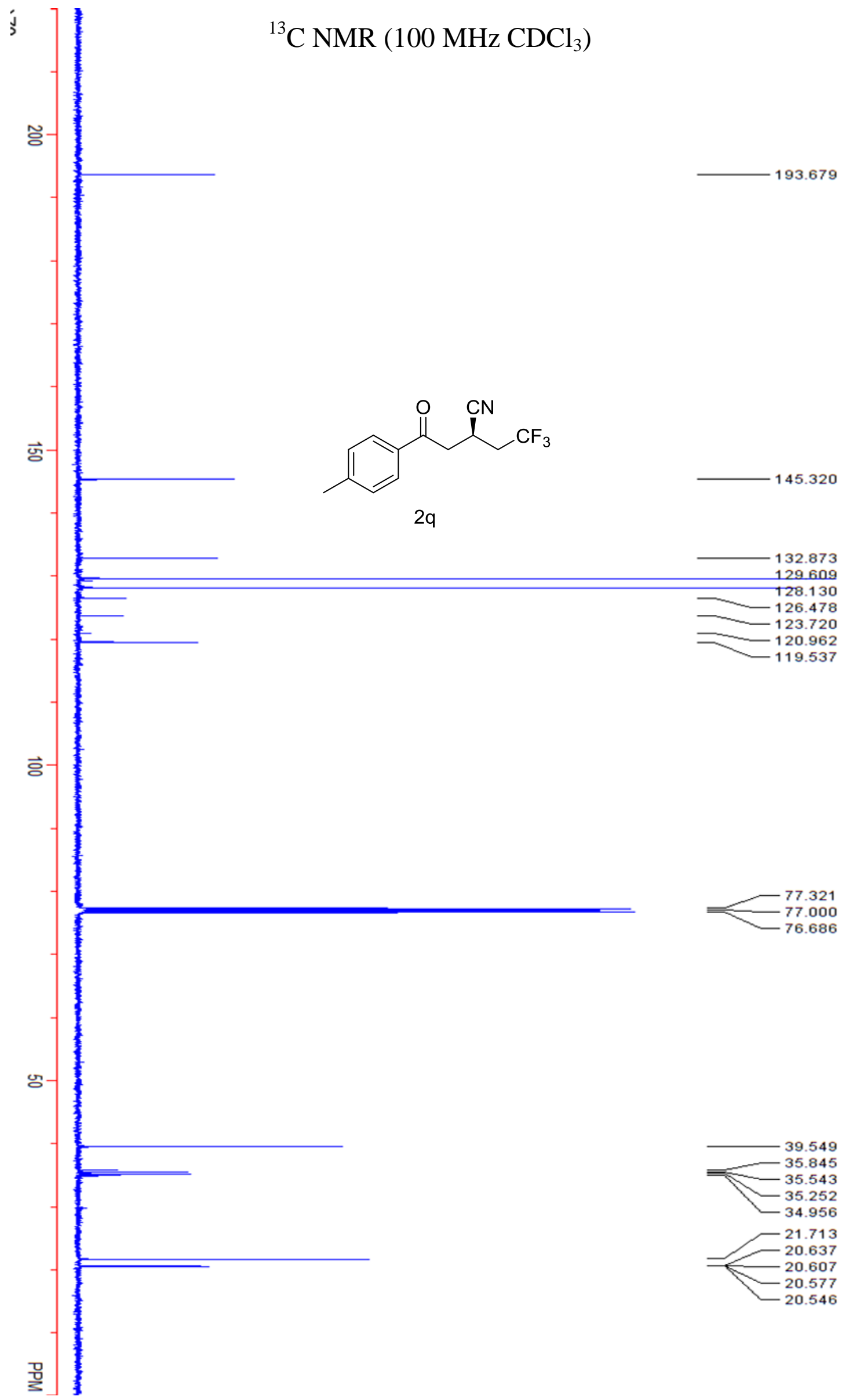




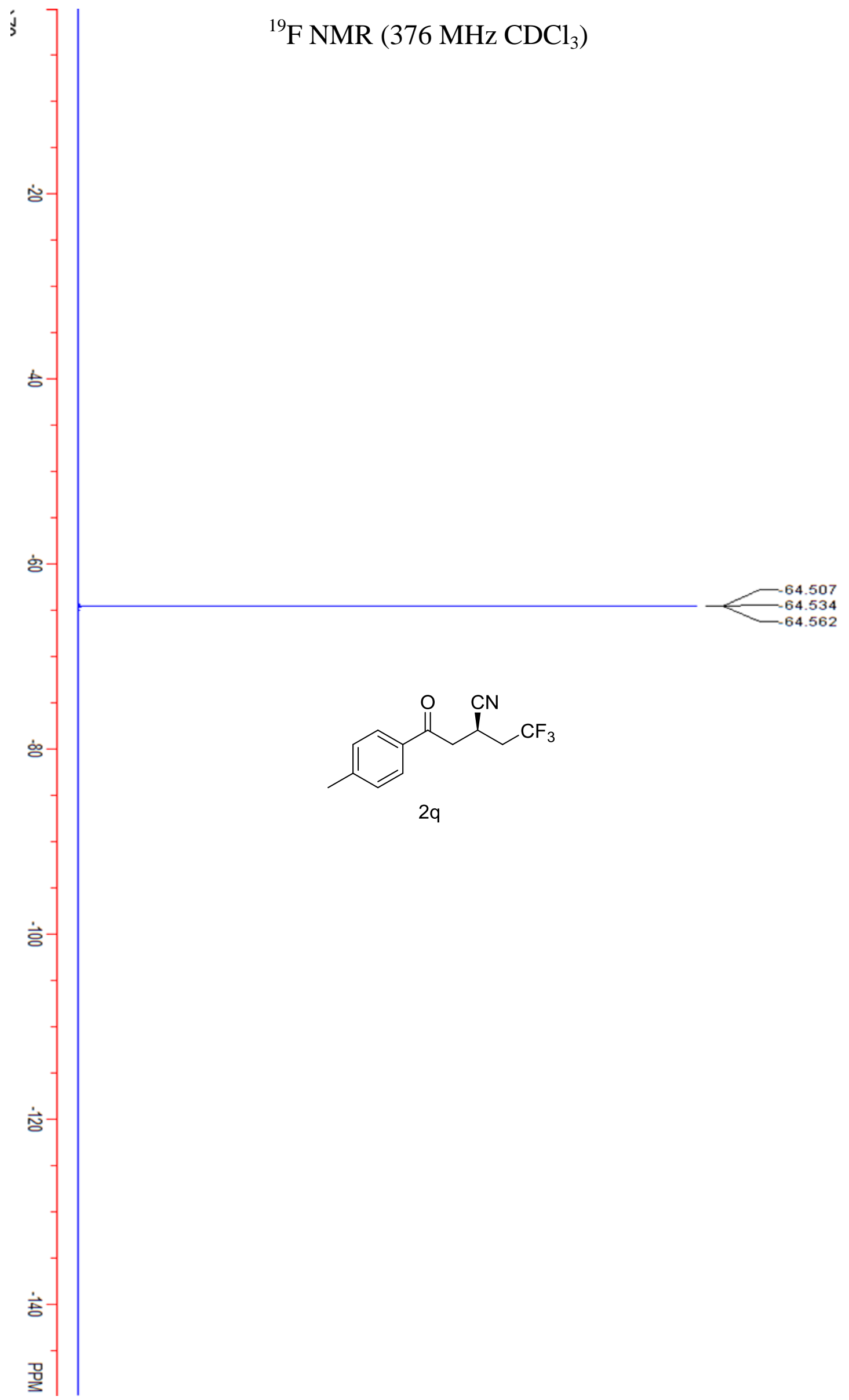



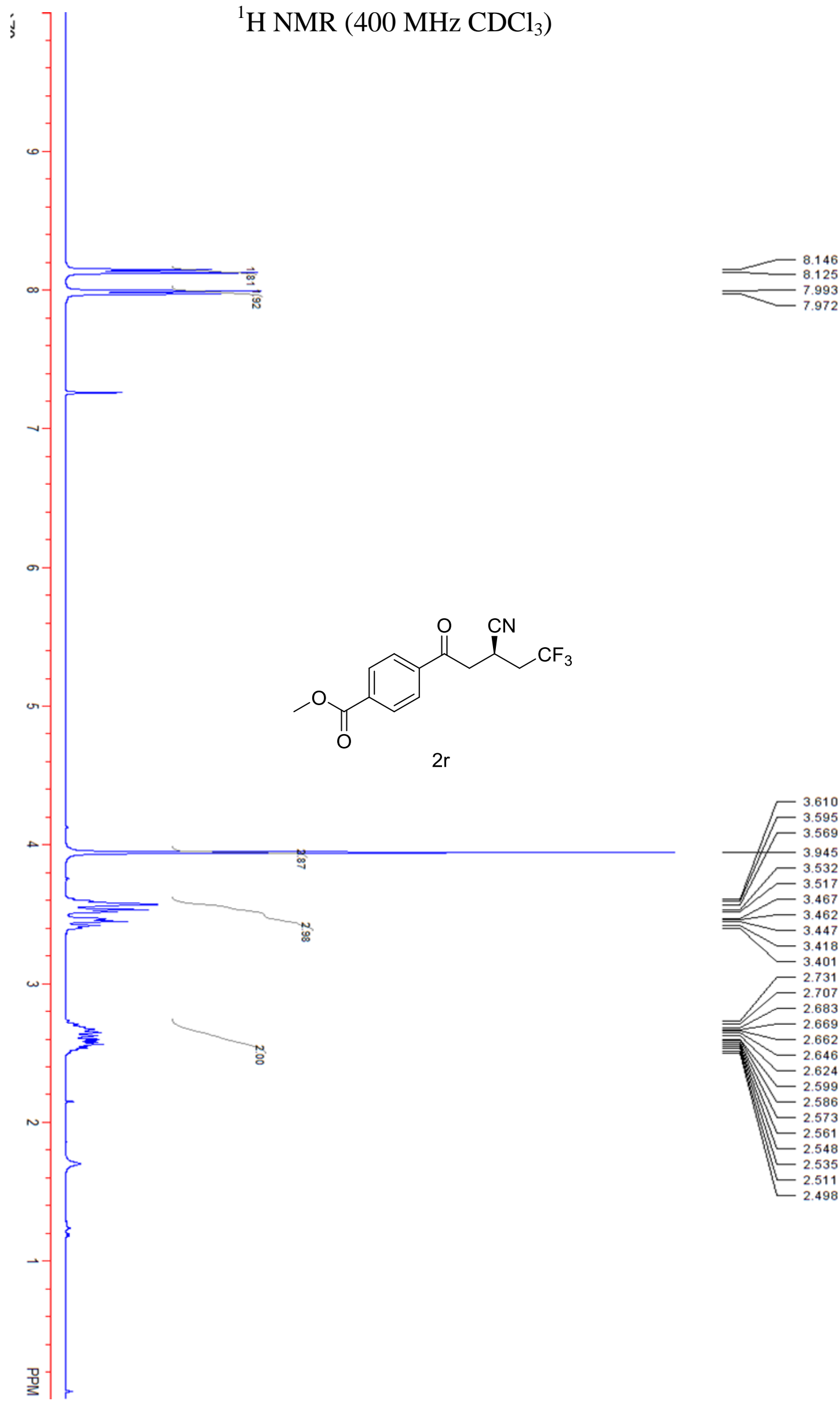


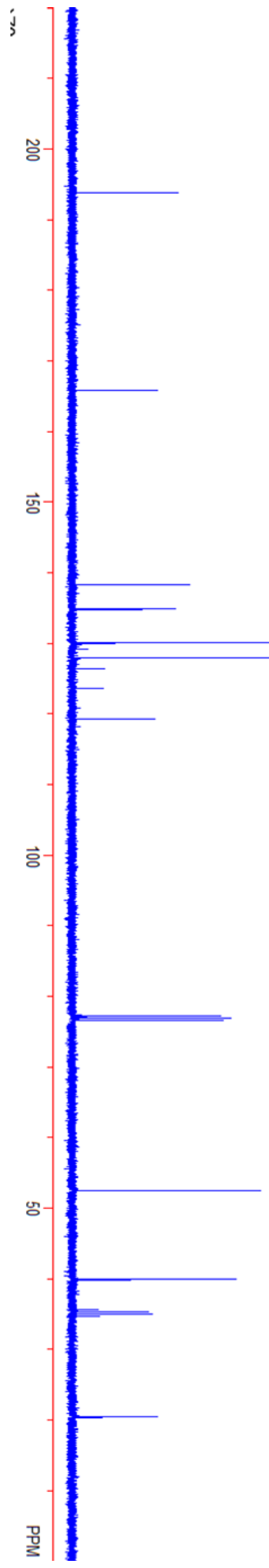

${ }^{13} \mathrm{C}$ NMR (100 MHz $\mathrm{CDCl}_{3}$ )
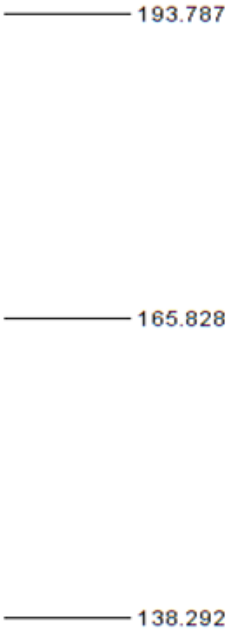

134.808

130.041

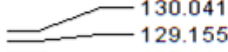

ב-127.941

$-127.883$

$-126.392$

$-123.638$

$-120.929$

$-119.239$<smiles>COC(=O)c1ccc(C(=O)C[C@H](C#N)CC(F)(F)F)cc1</smiles>
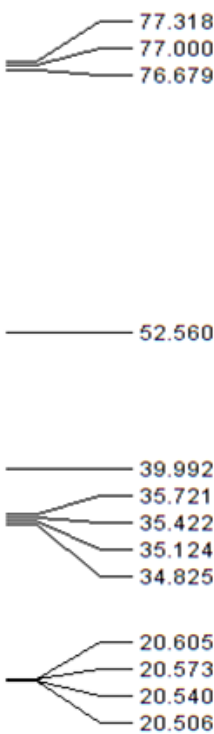

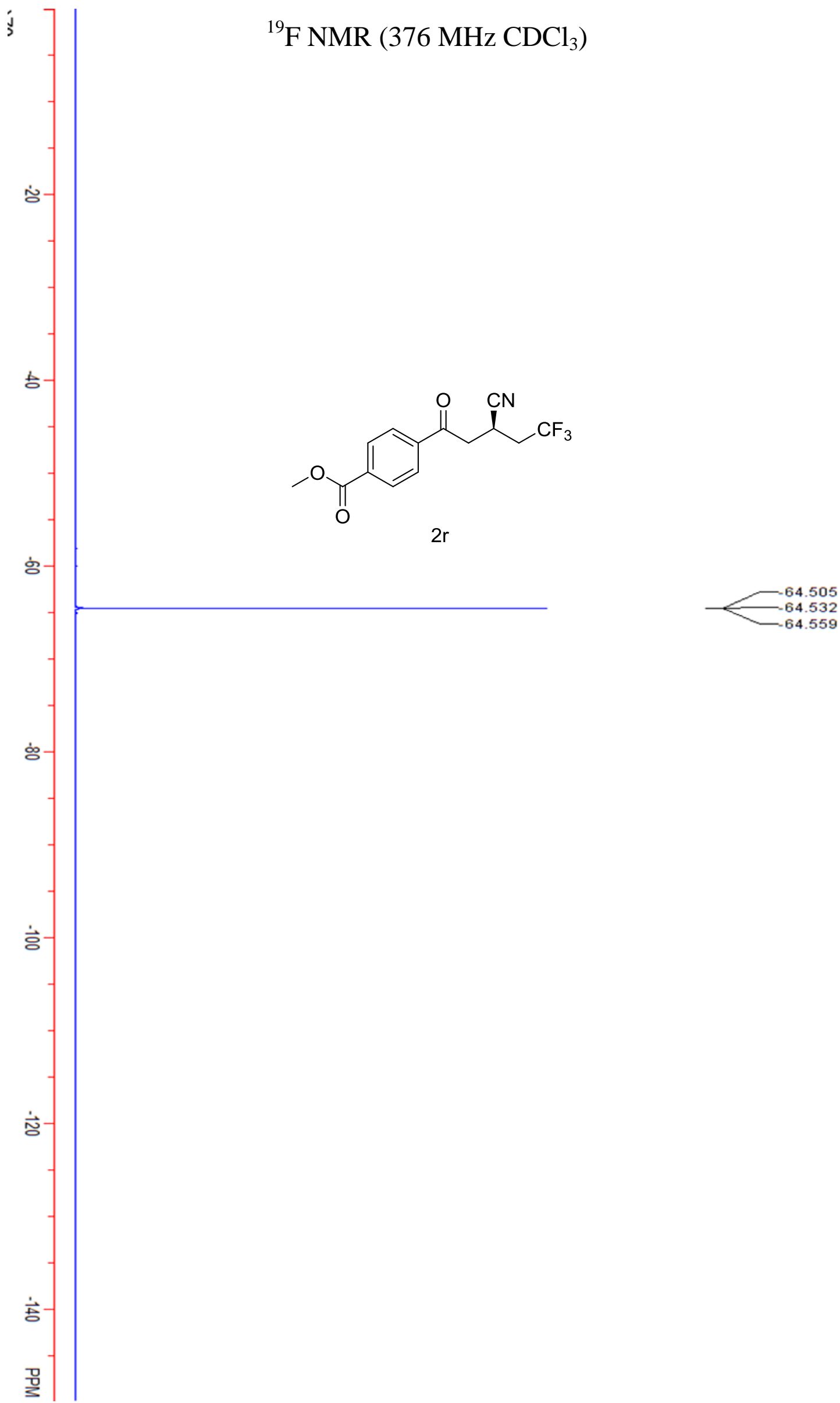


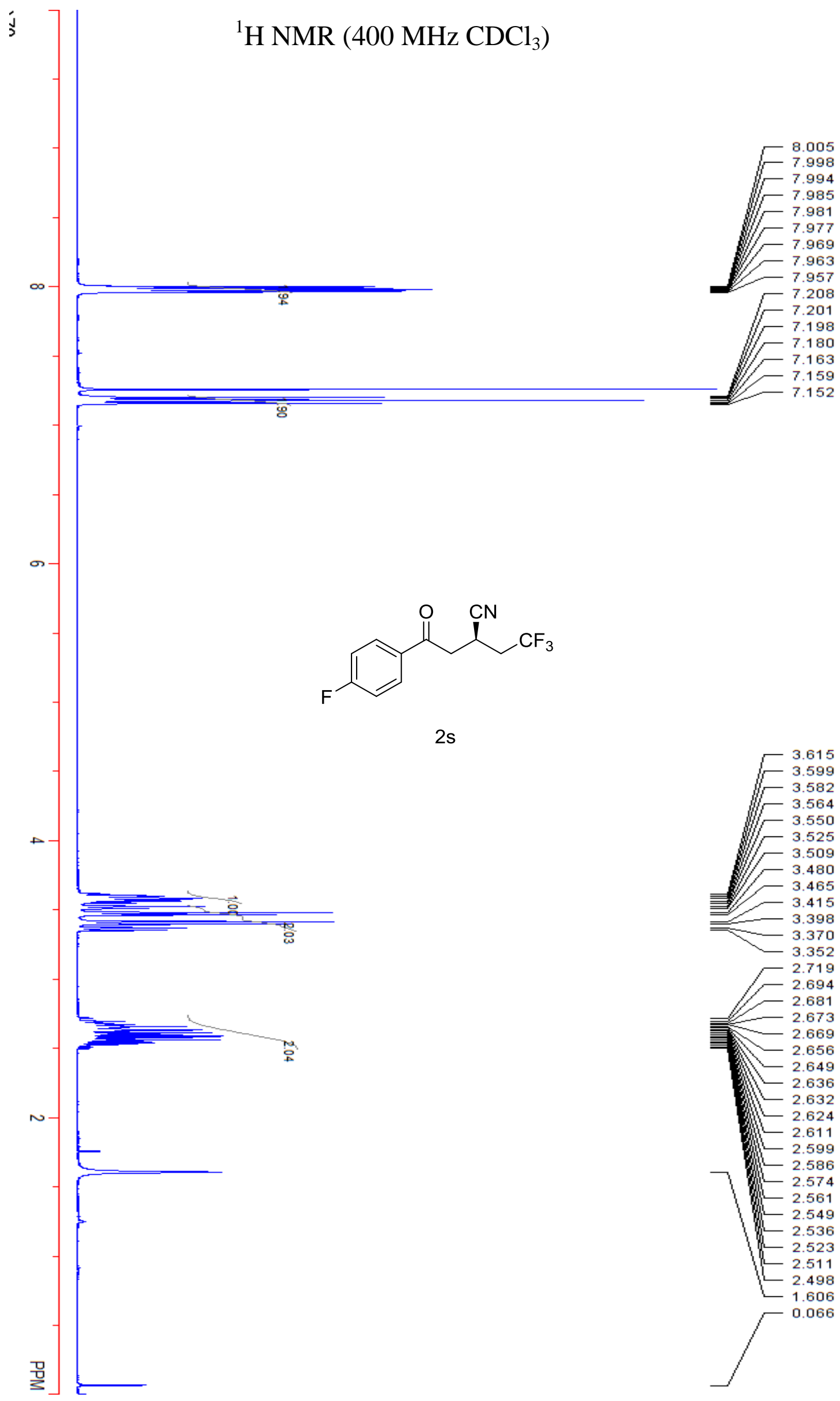




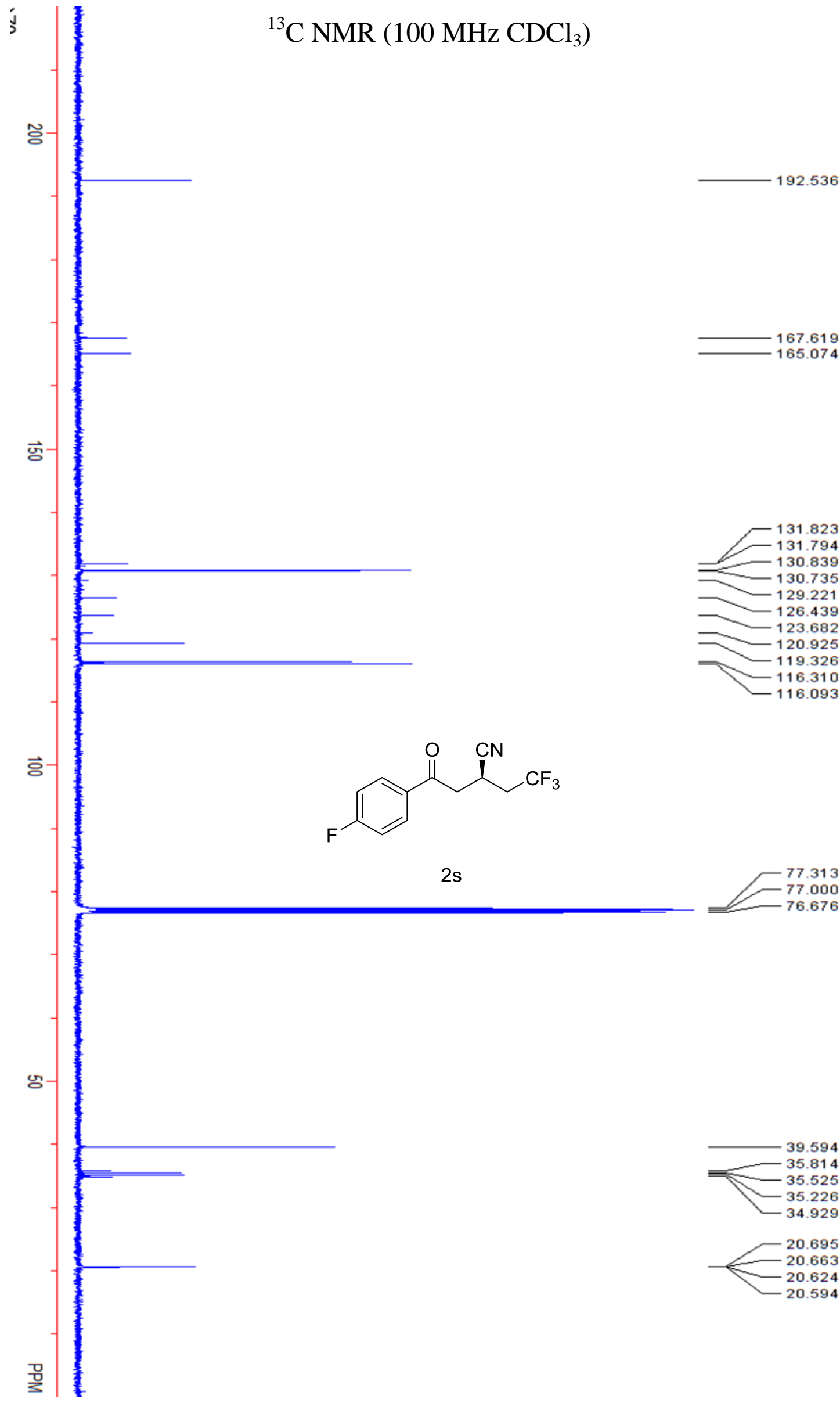




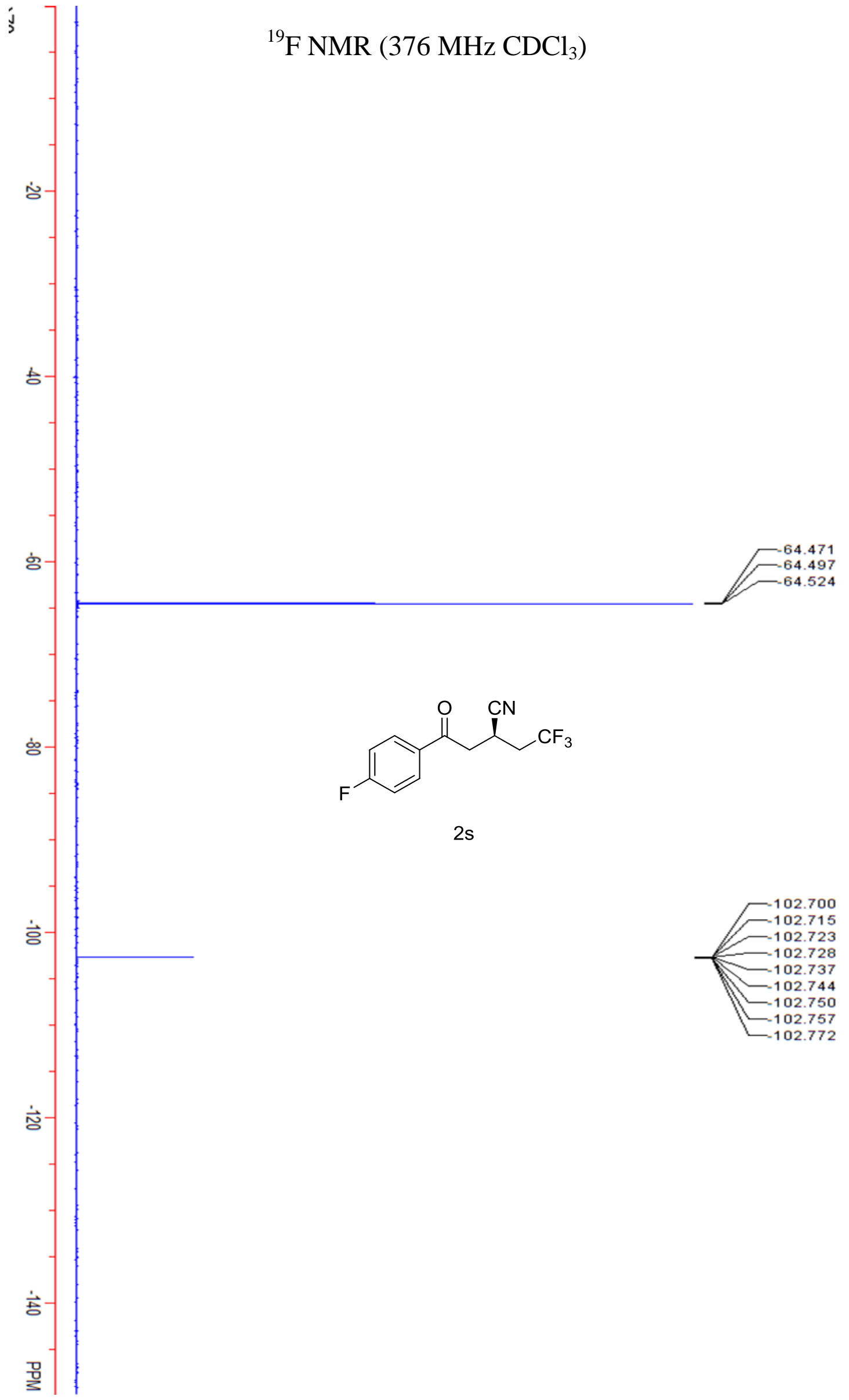



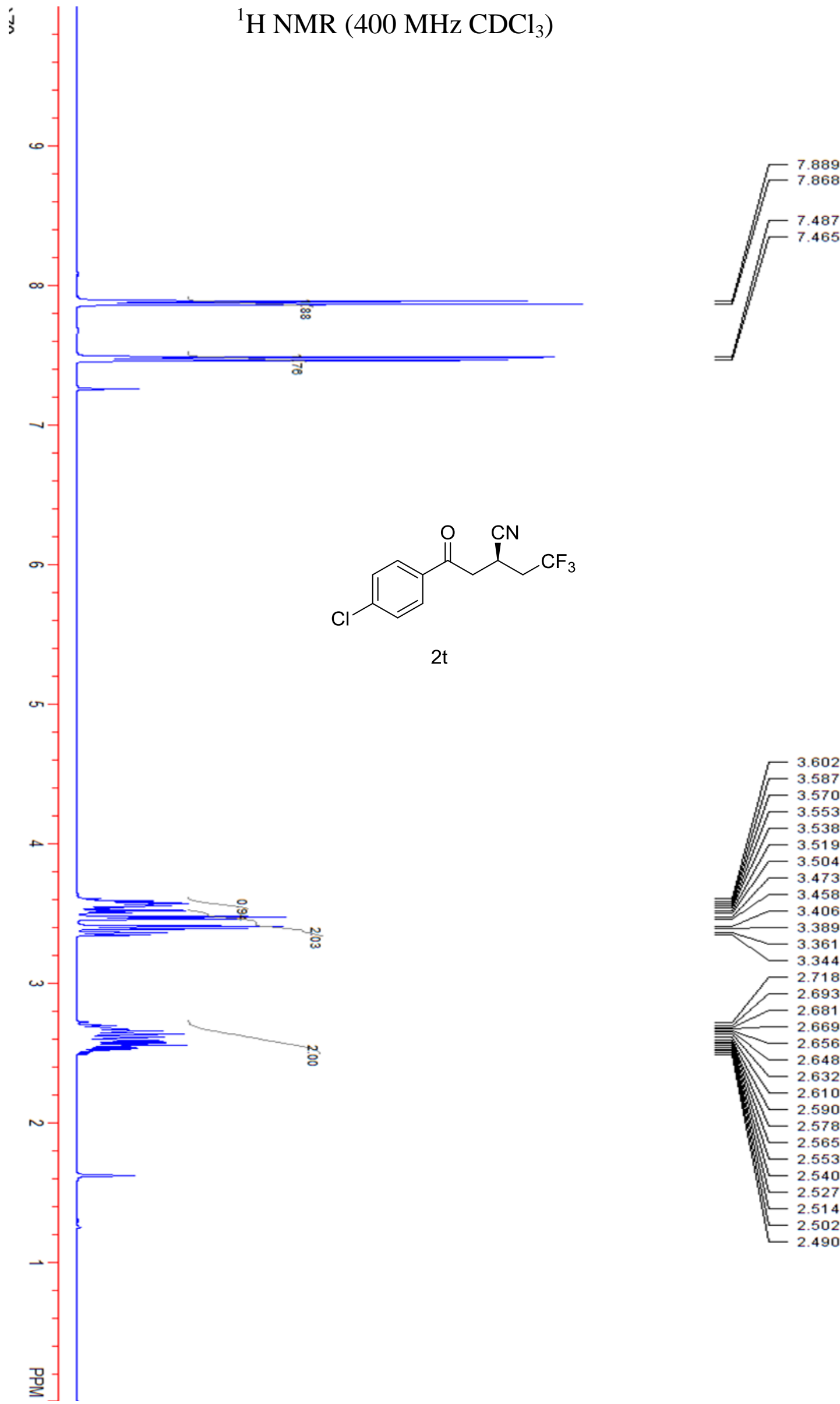

3.602 3.519 3.504 3.389 3.361

3.344 2.718 2.693 2.681 2.669 2.656 2.648 2.632 2.610 2.590
-2.578 2.565 - 2.553

$M-2.540$ 2.527
- 2.514 2.502 2.490 


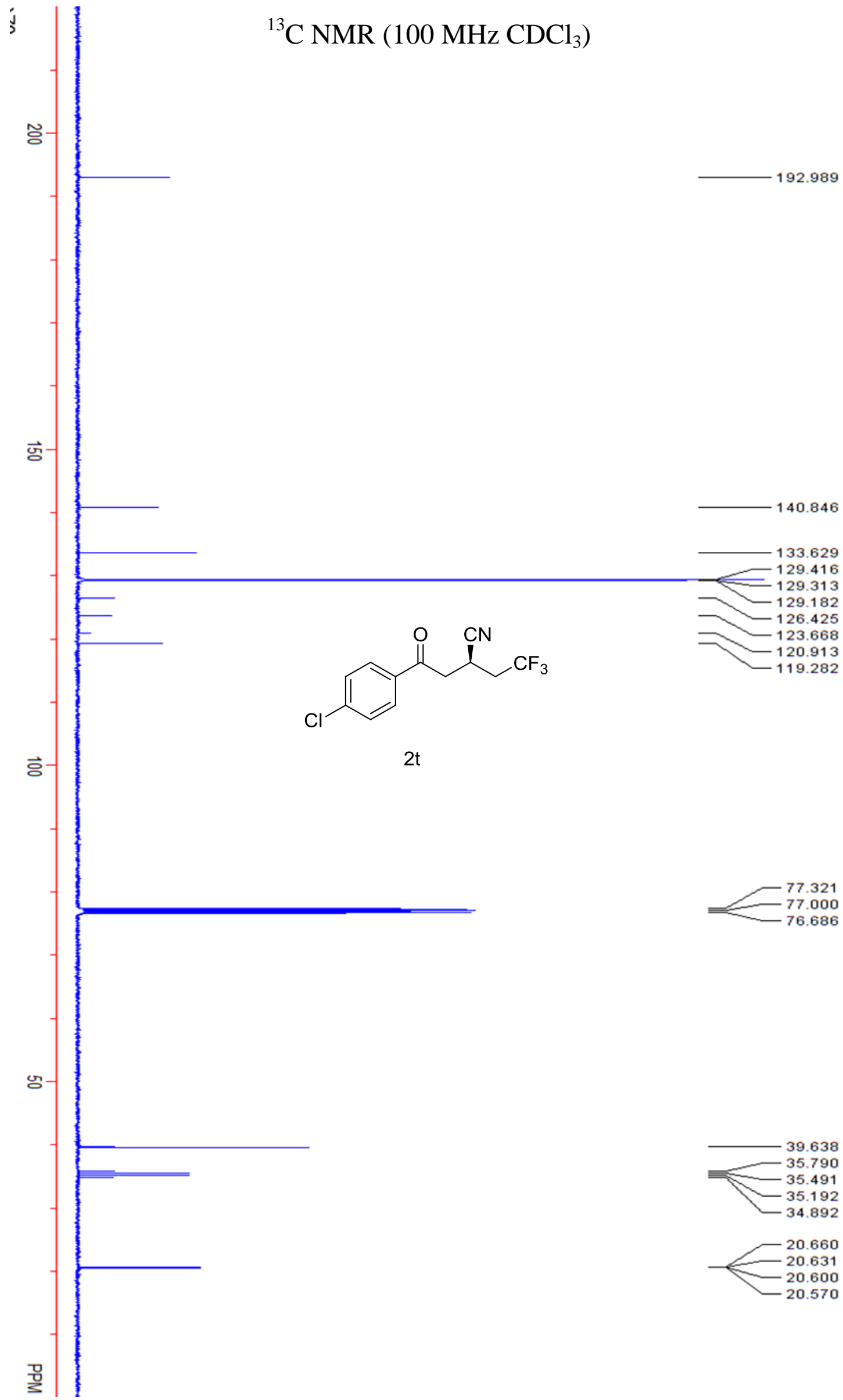




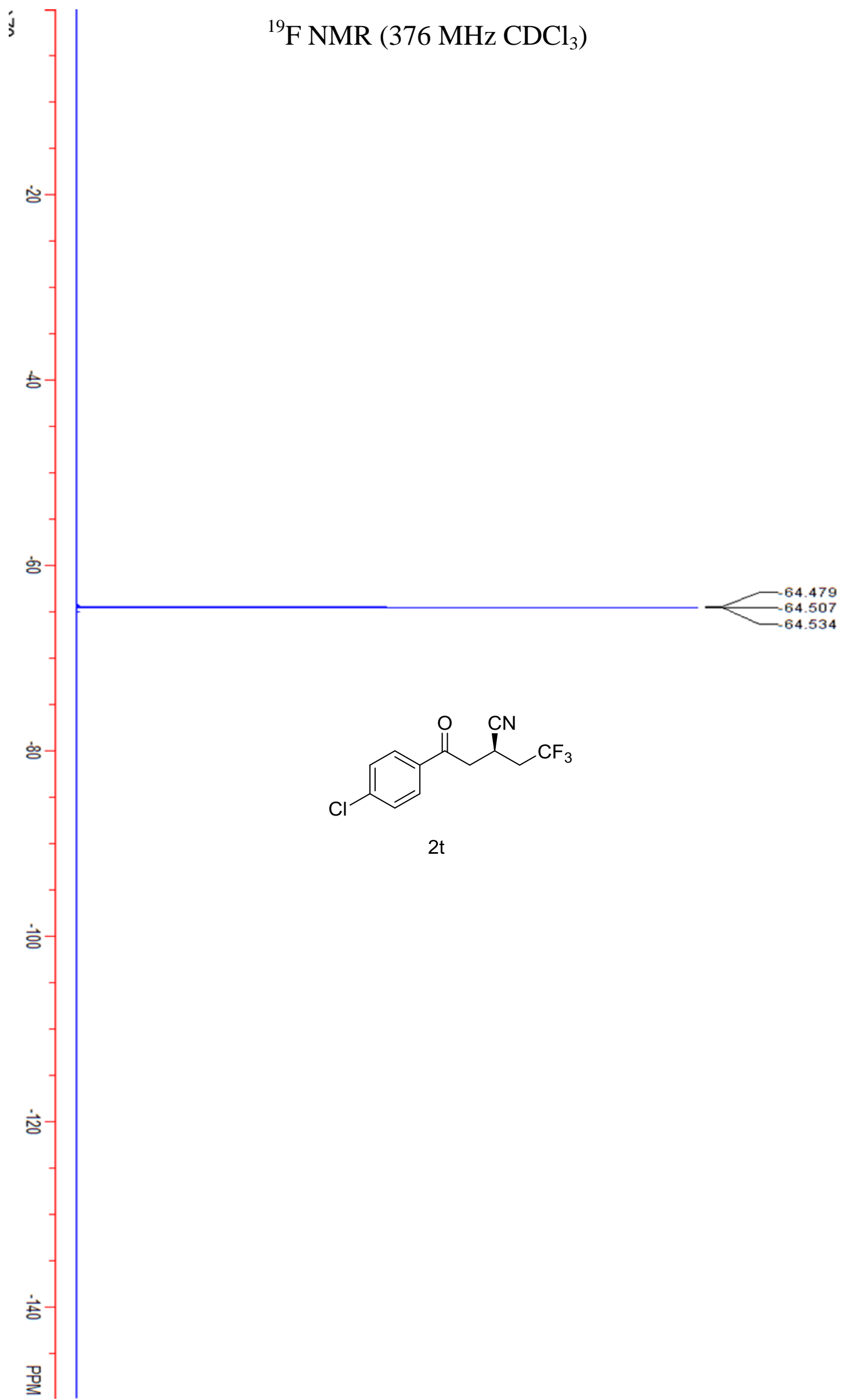




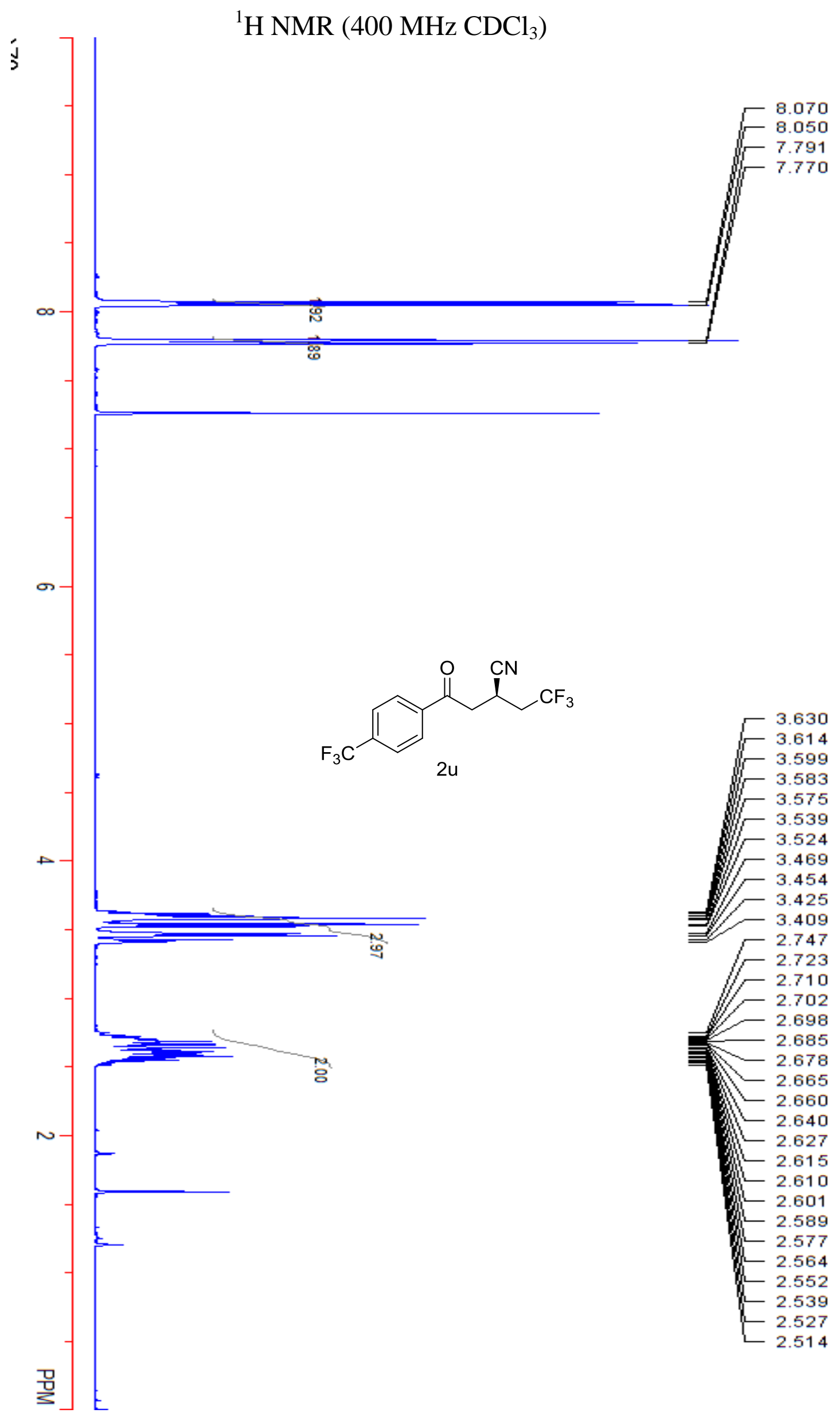




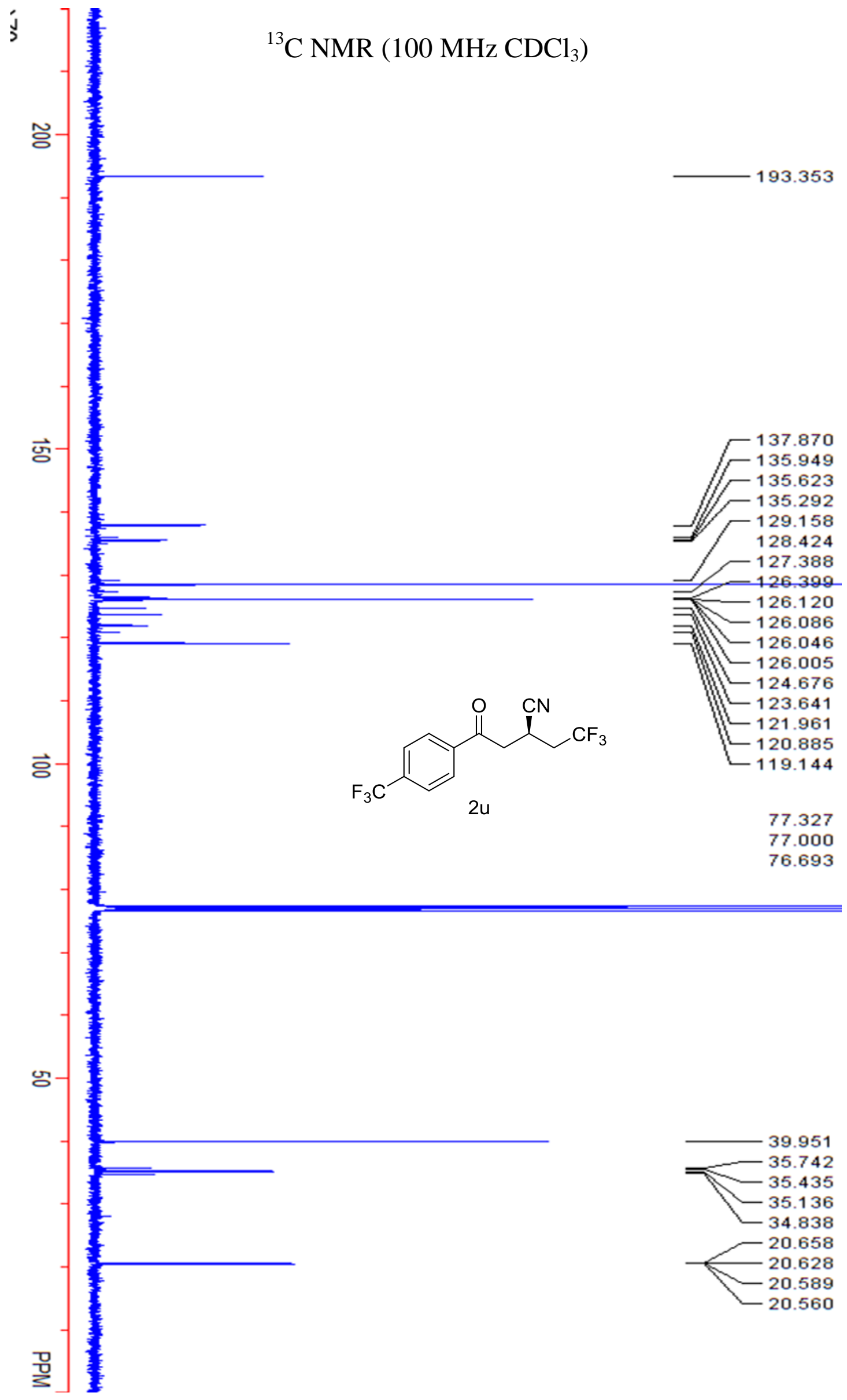




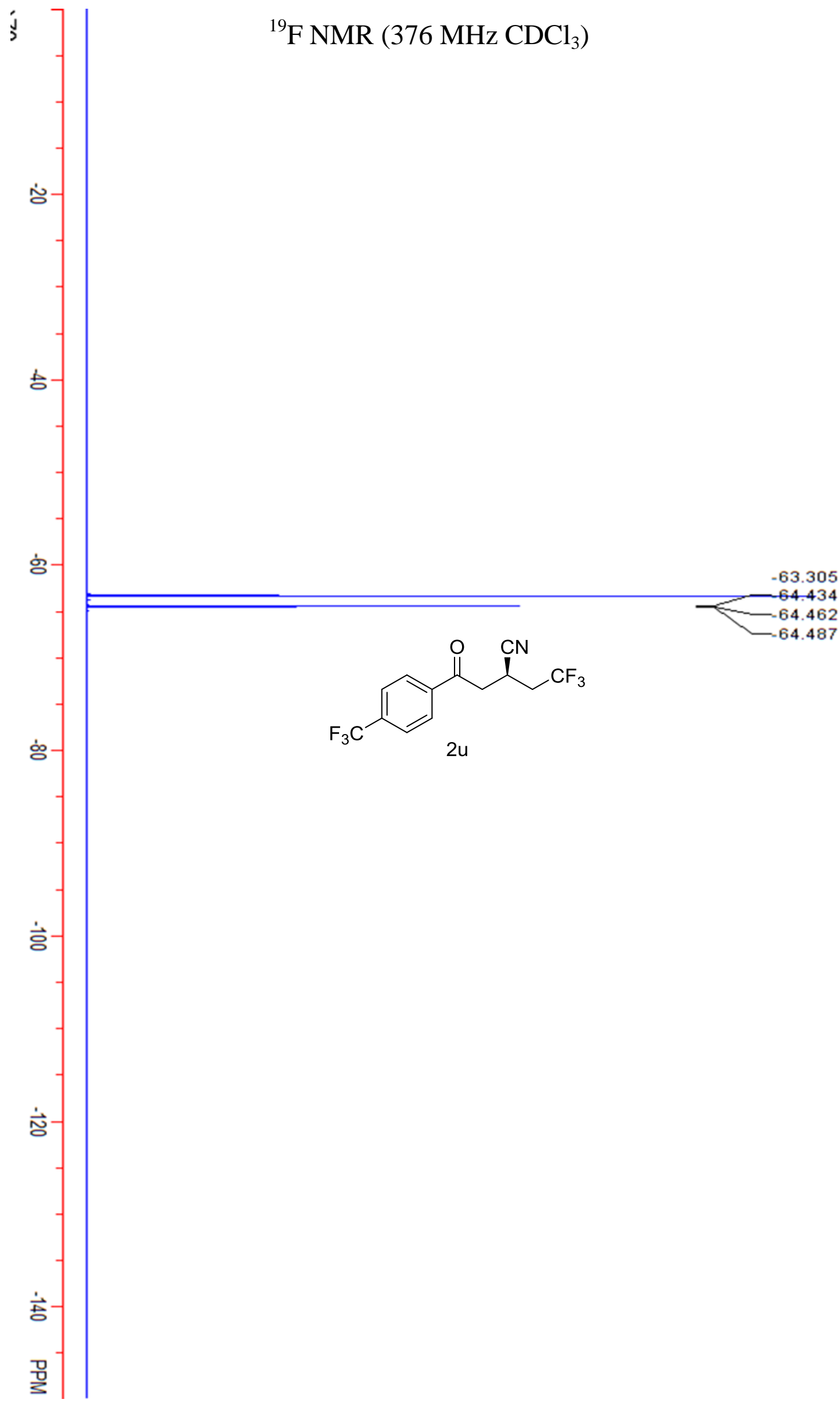



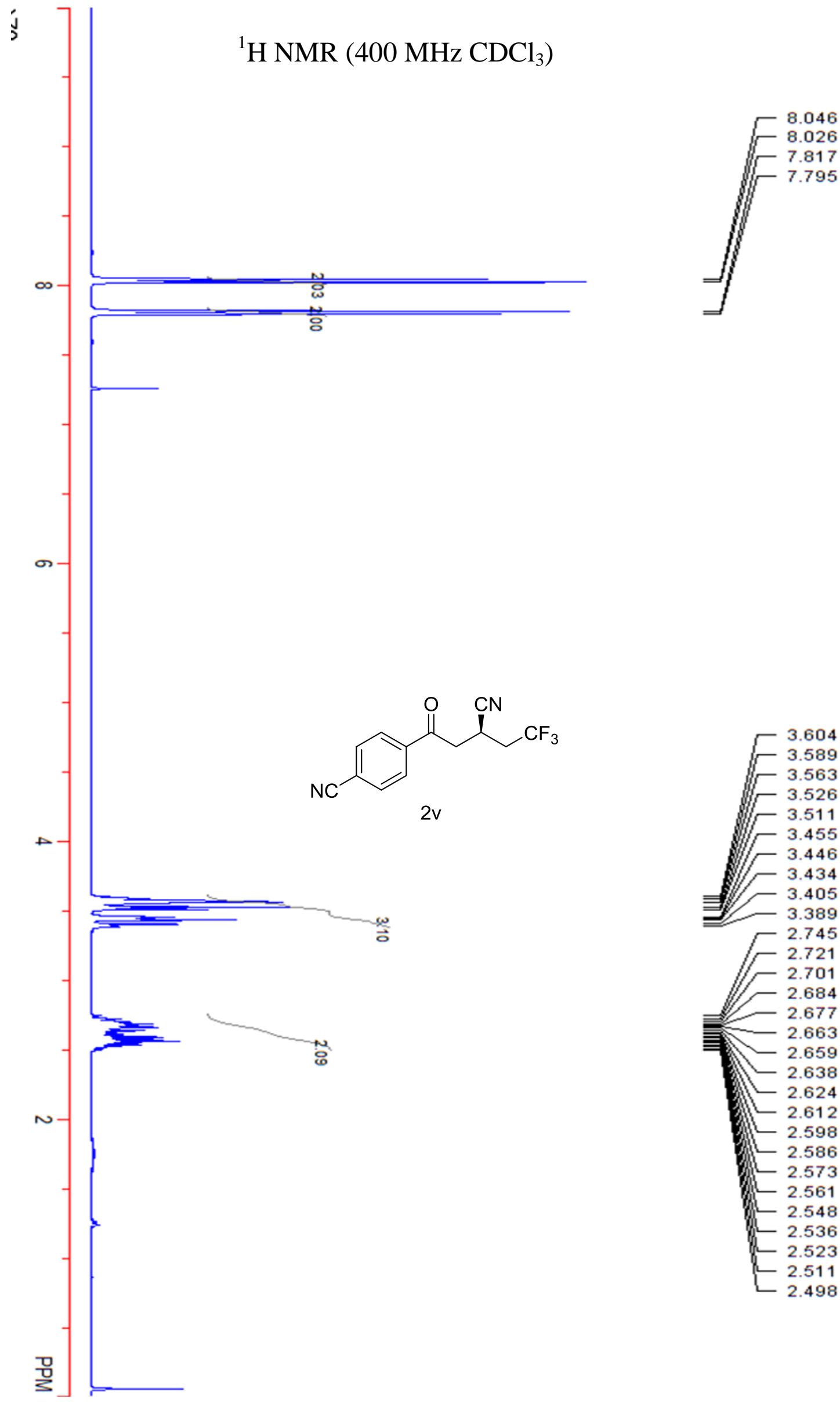


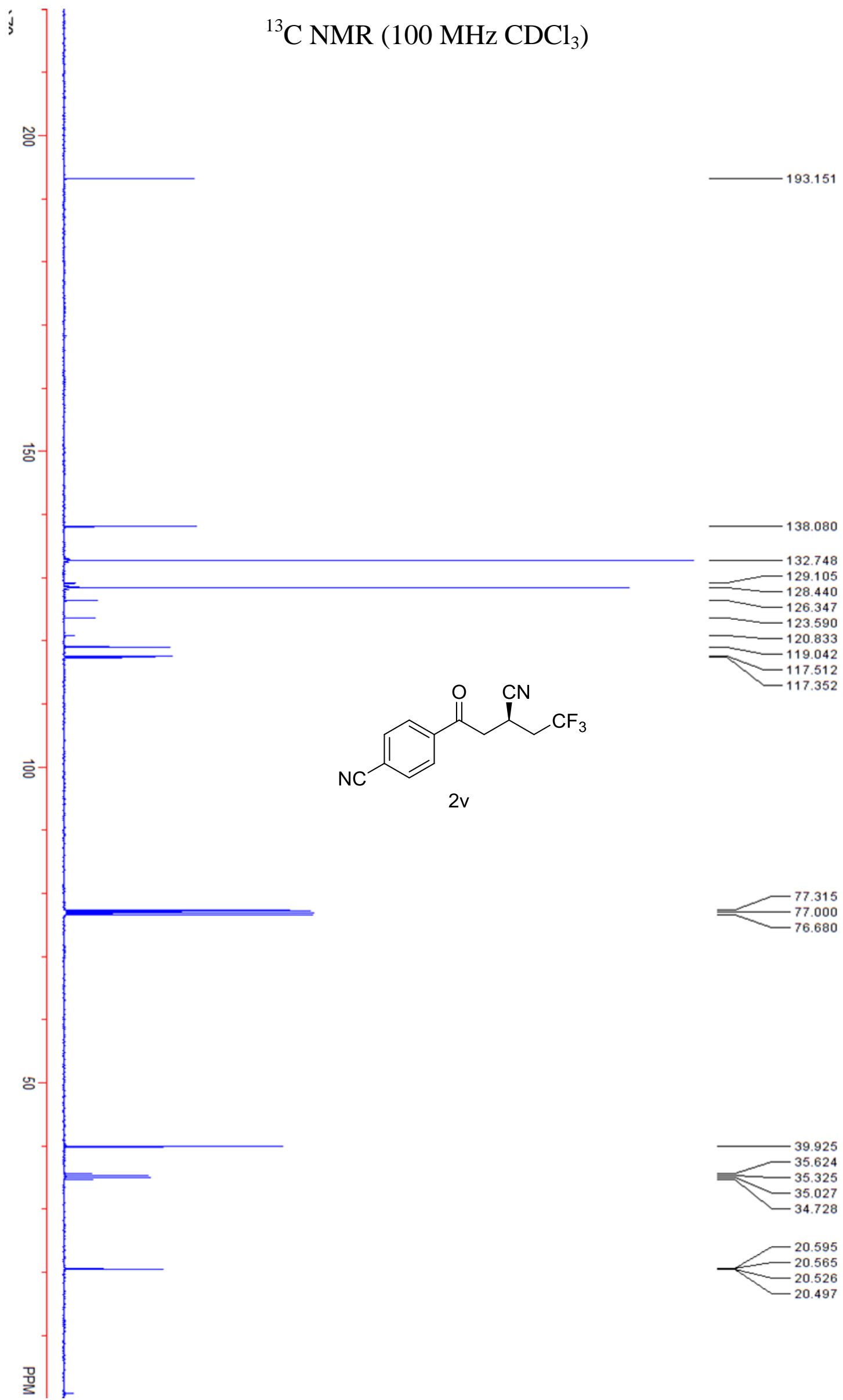




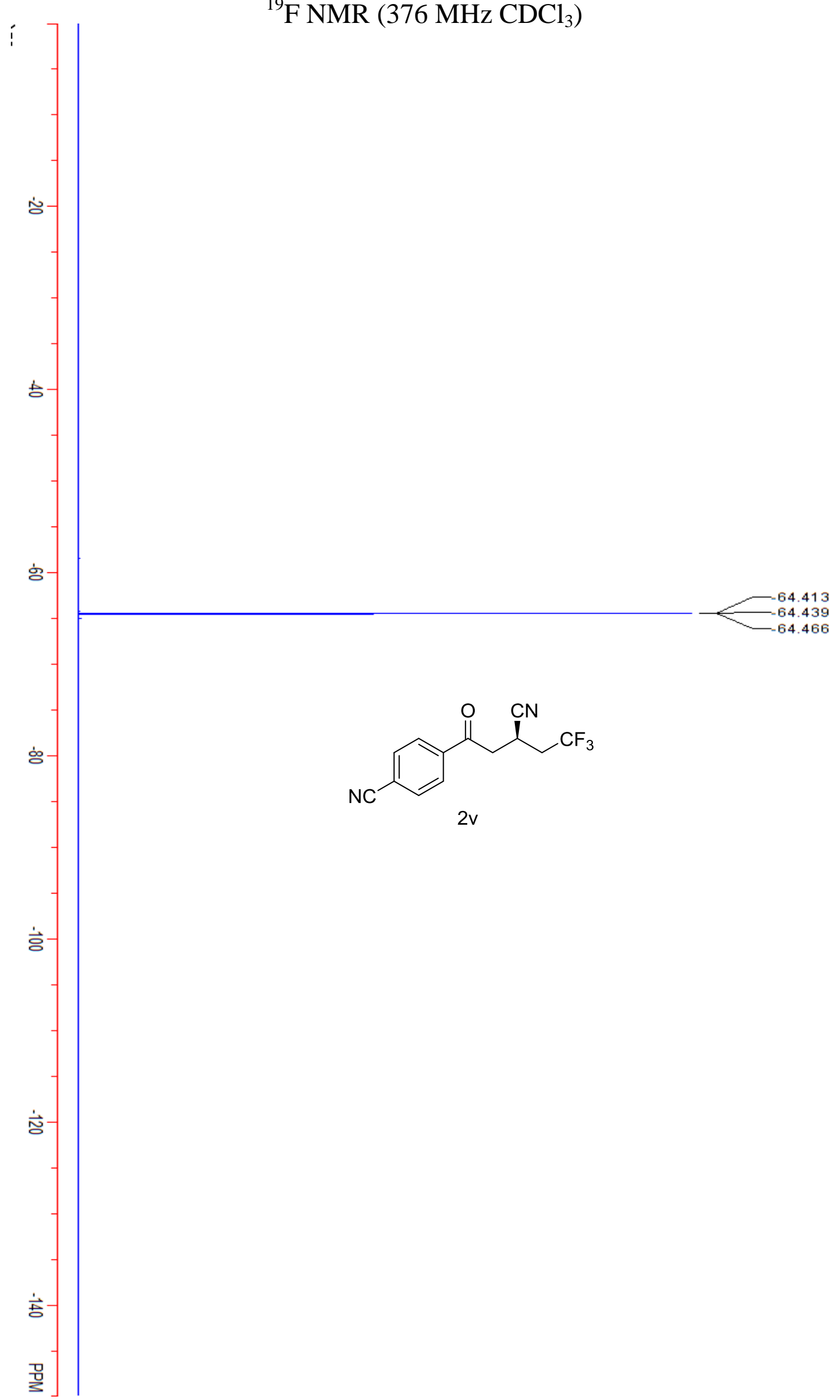




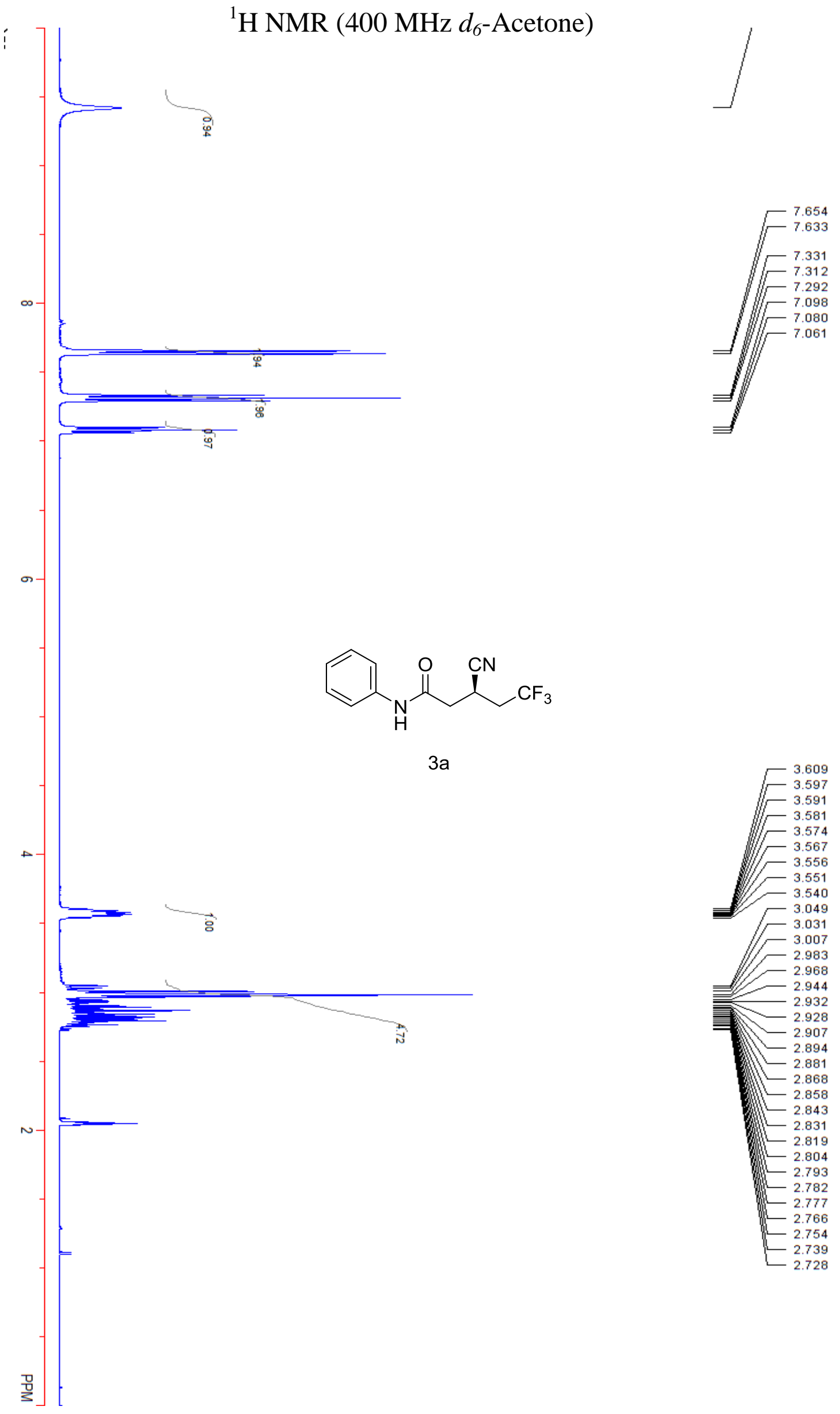




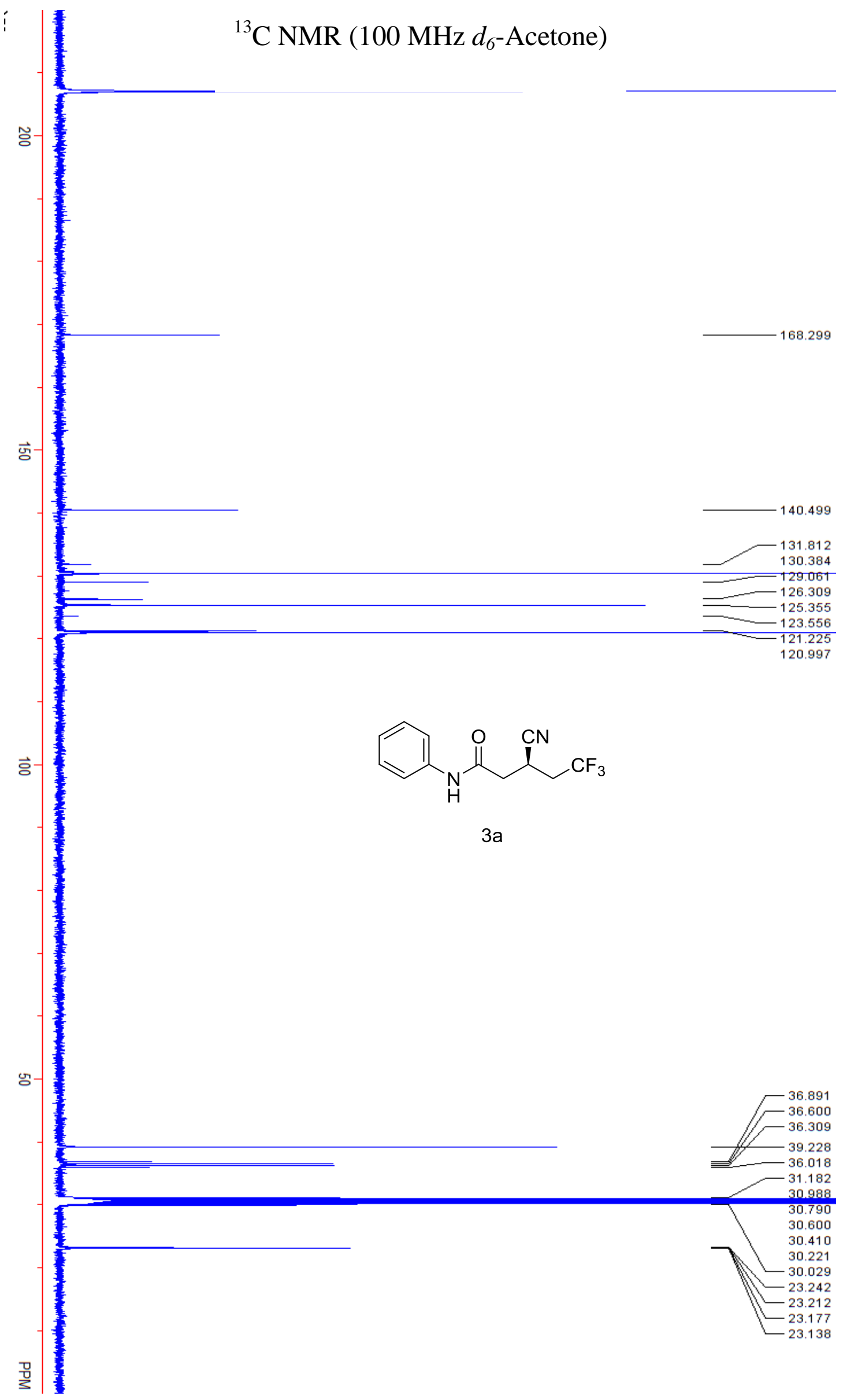




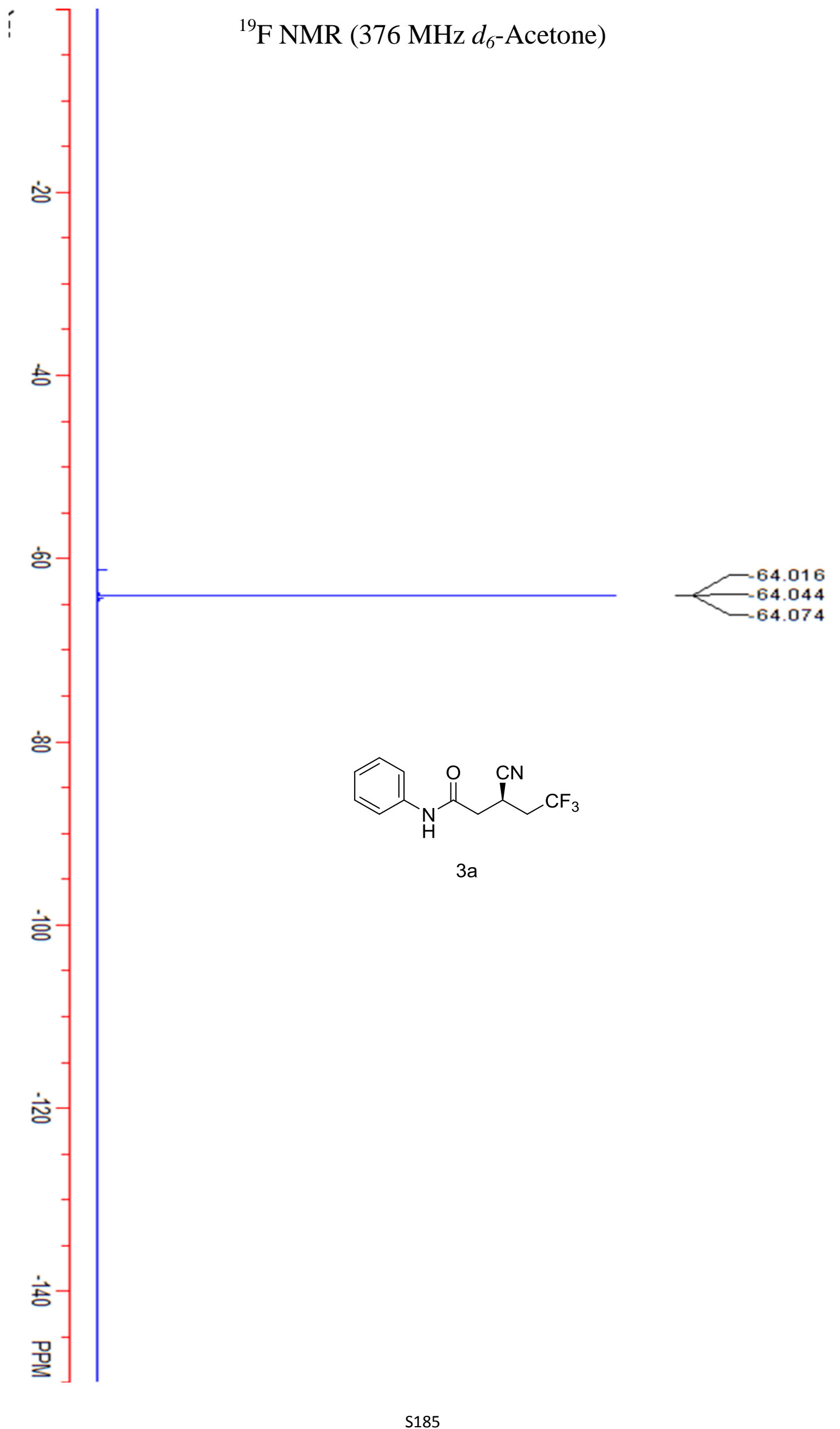




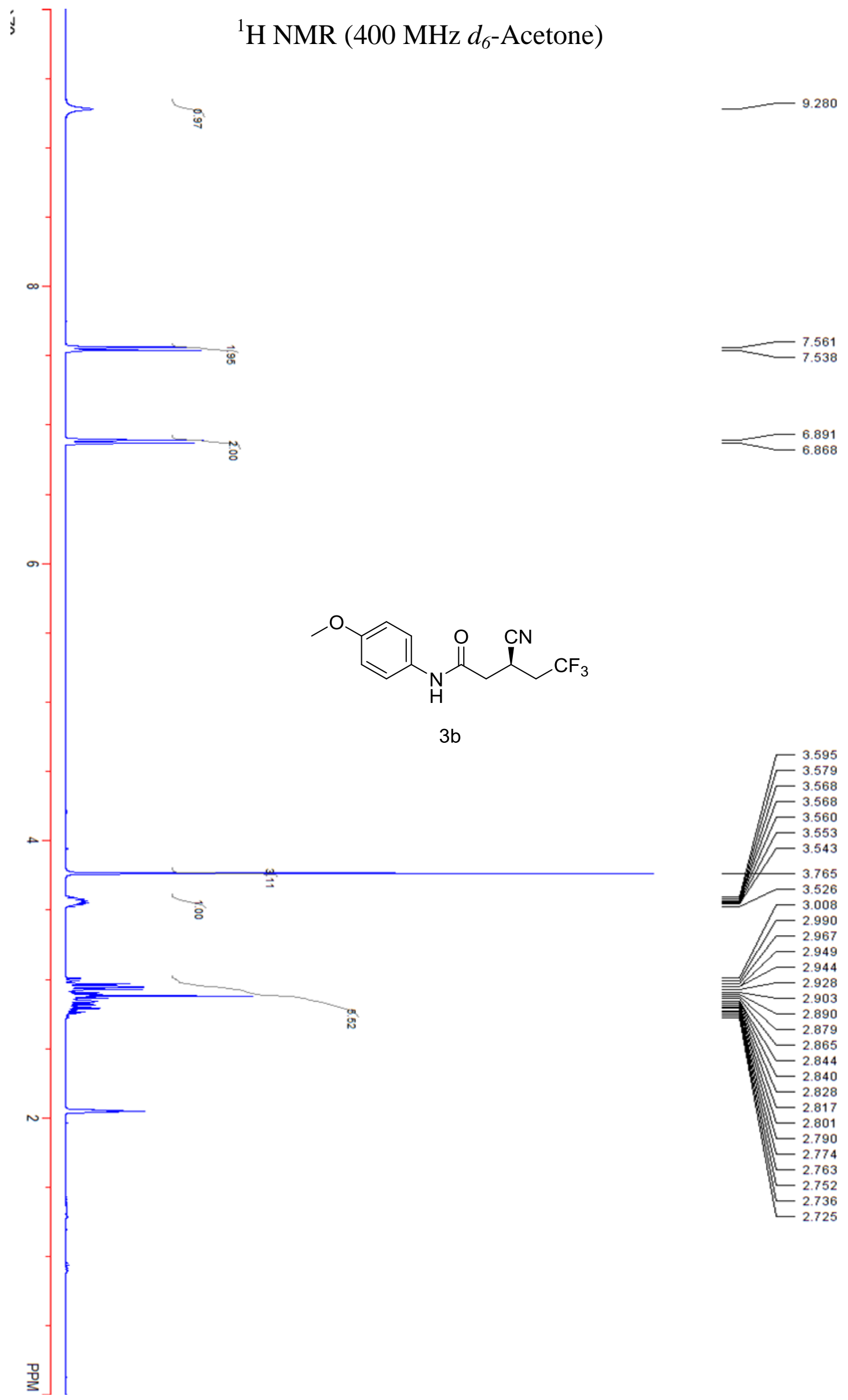




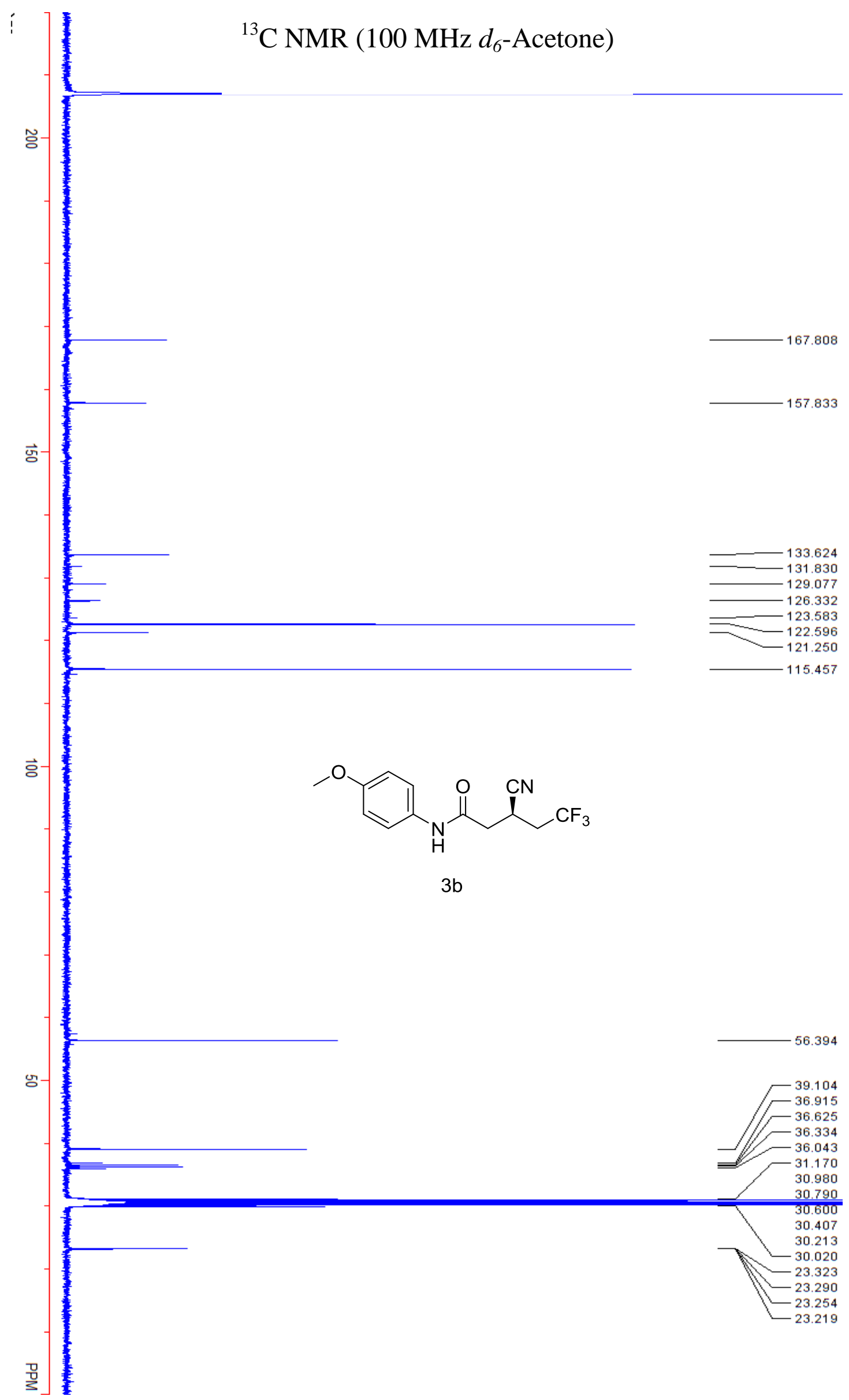




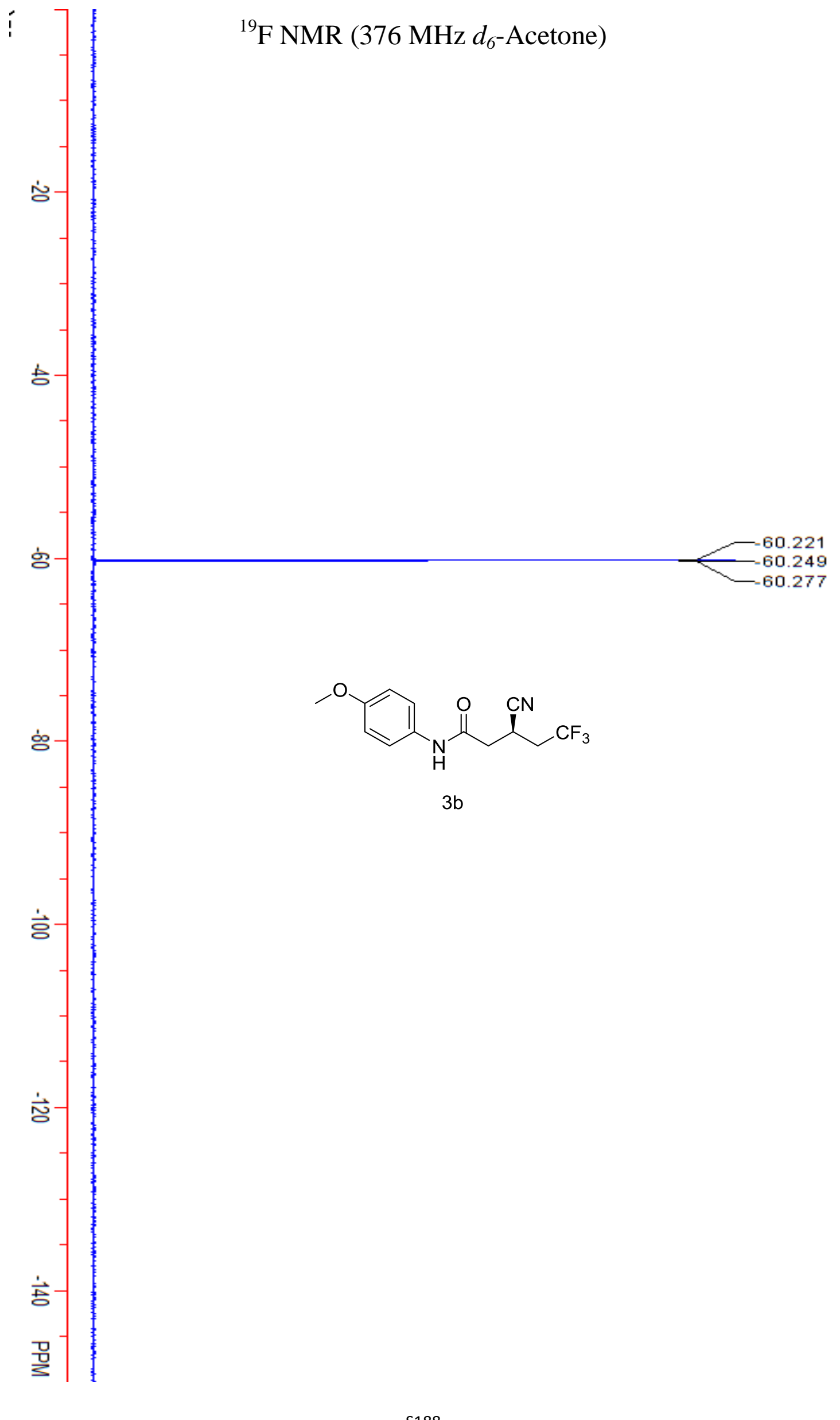




\section{${ }^{1} \mathrm{H}$ NMR (400 MHz $d_{6}$-Acetone)}

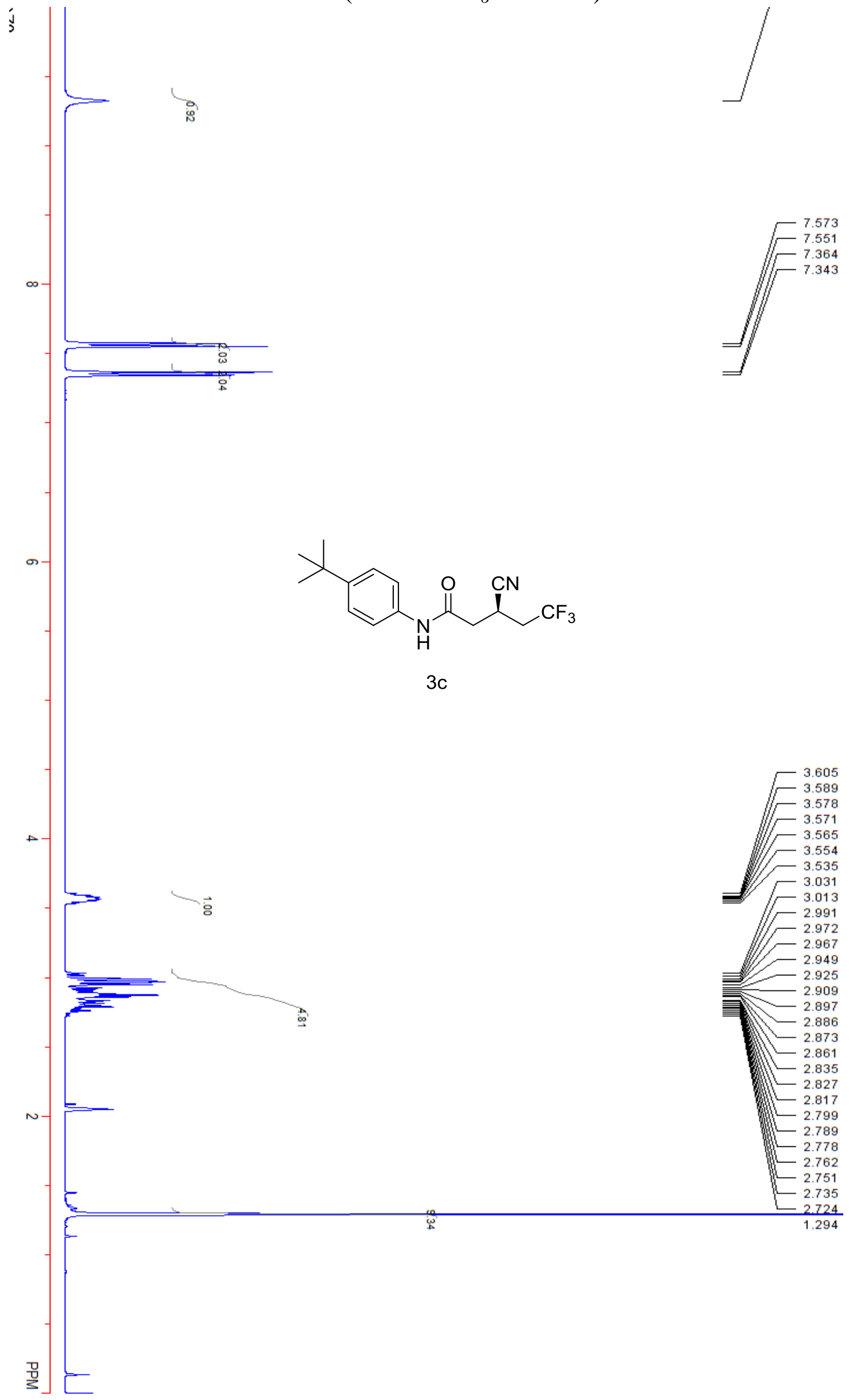




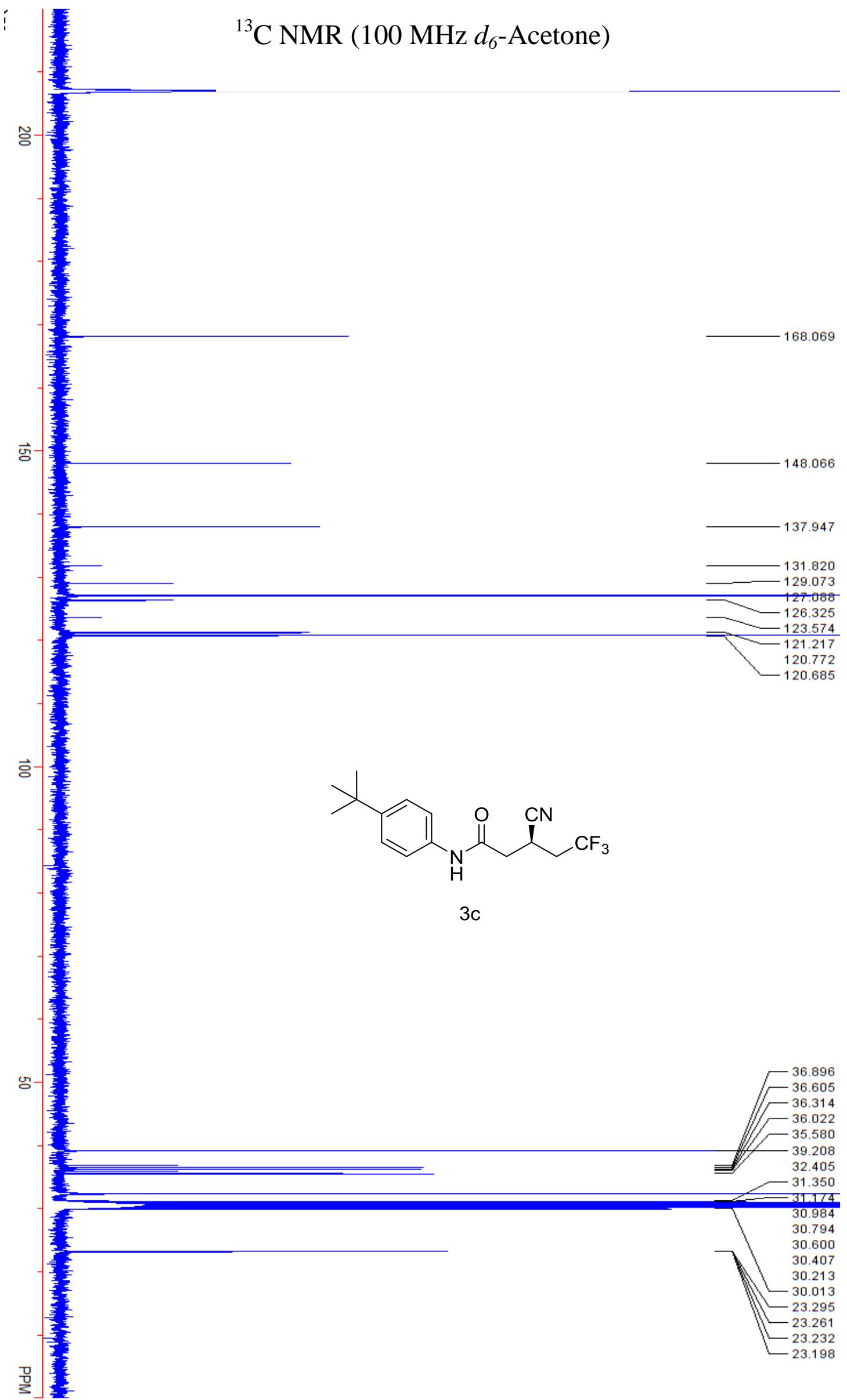



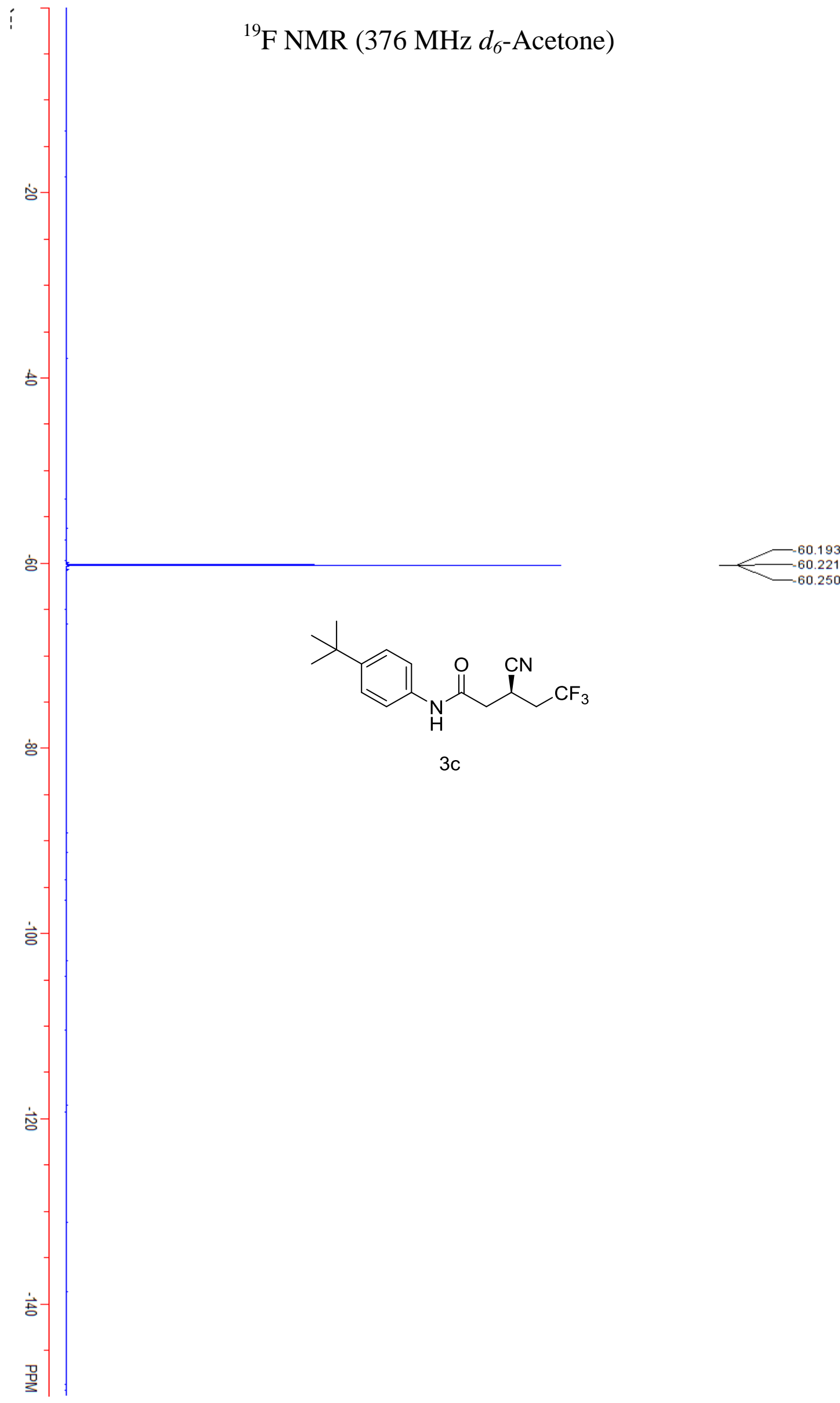

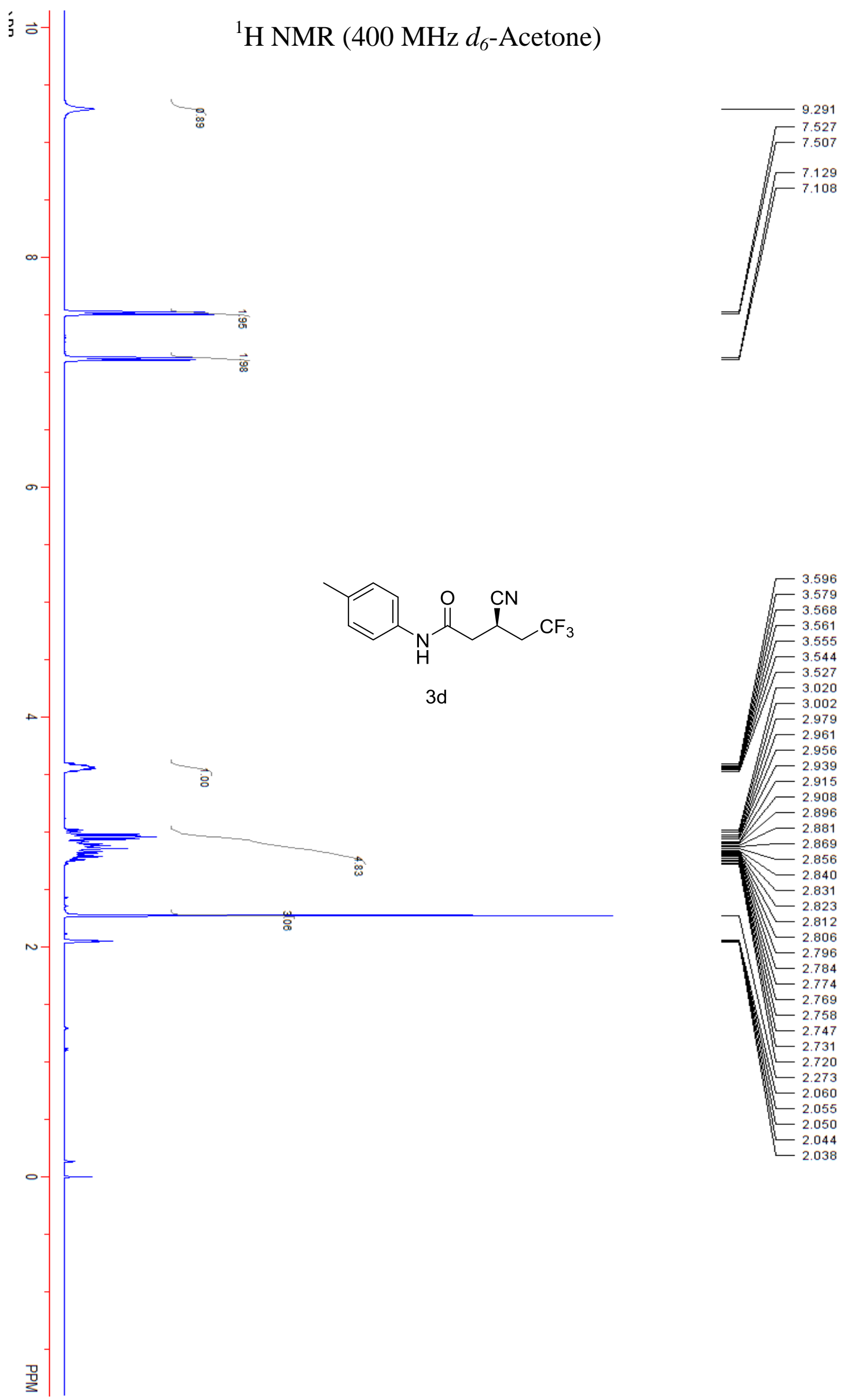


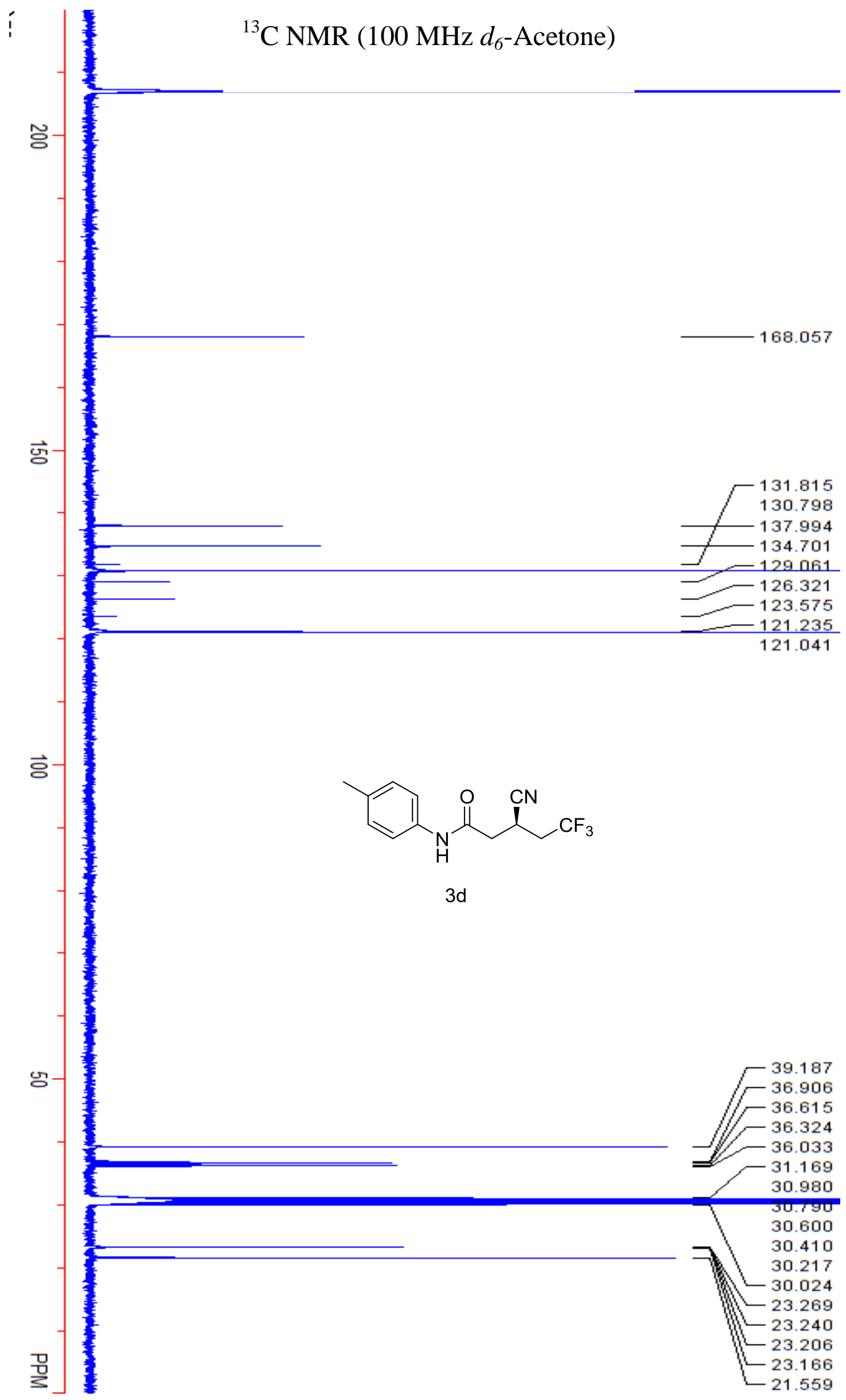




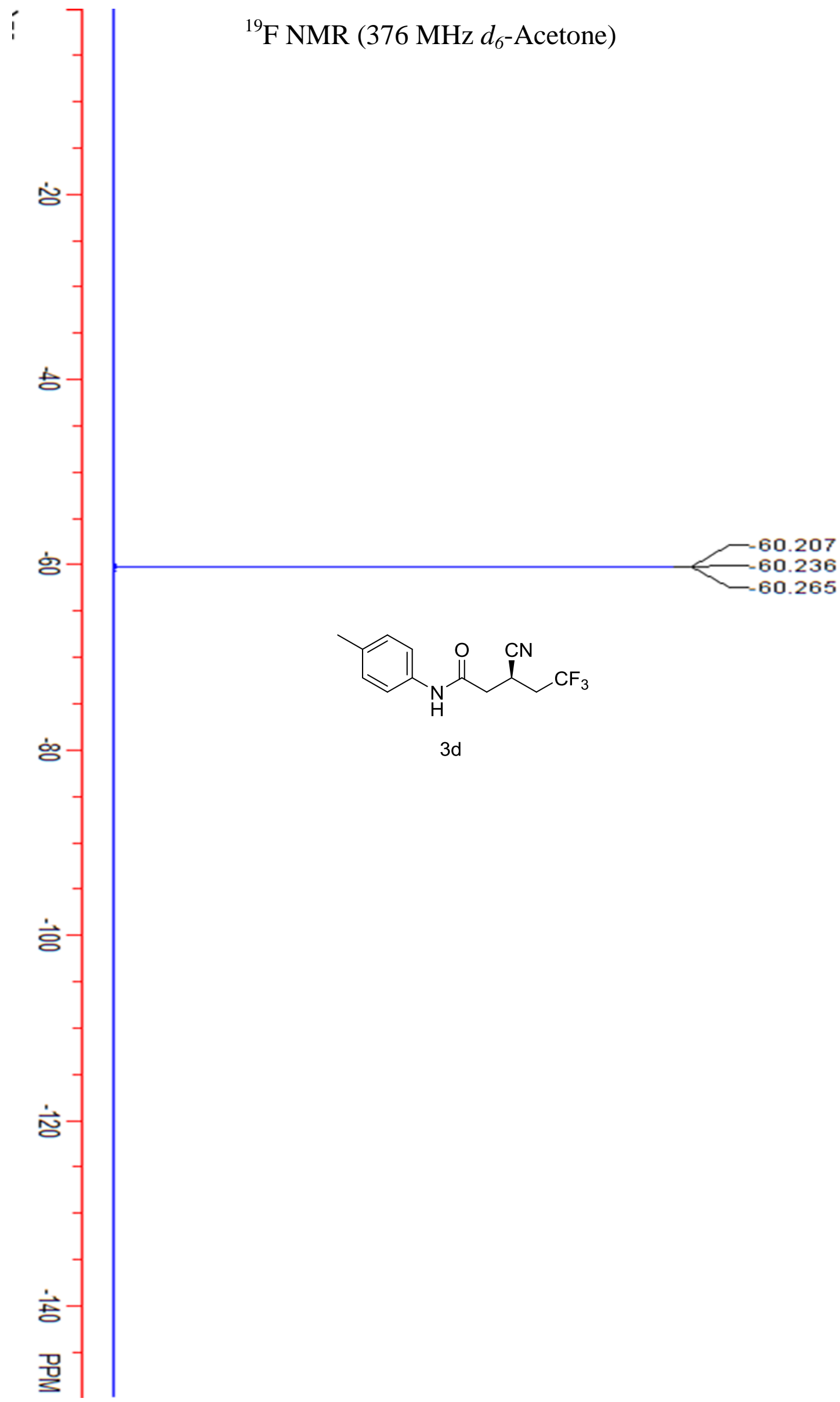




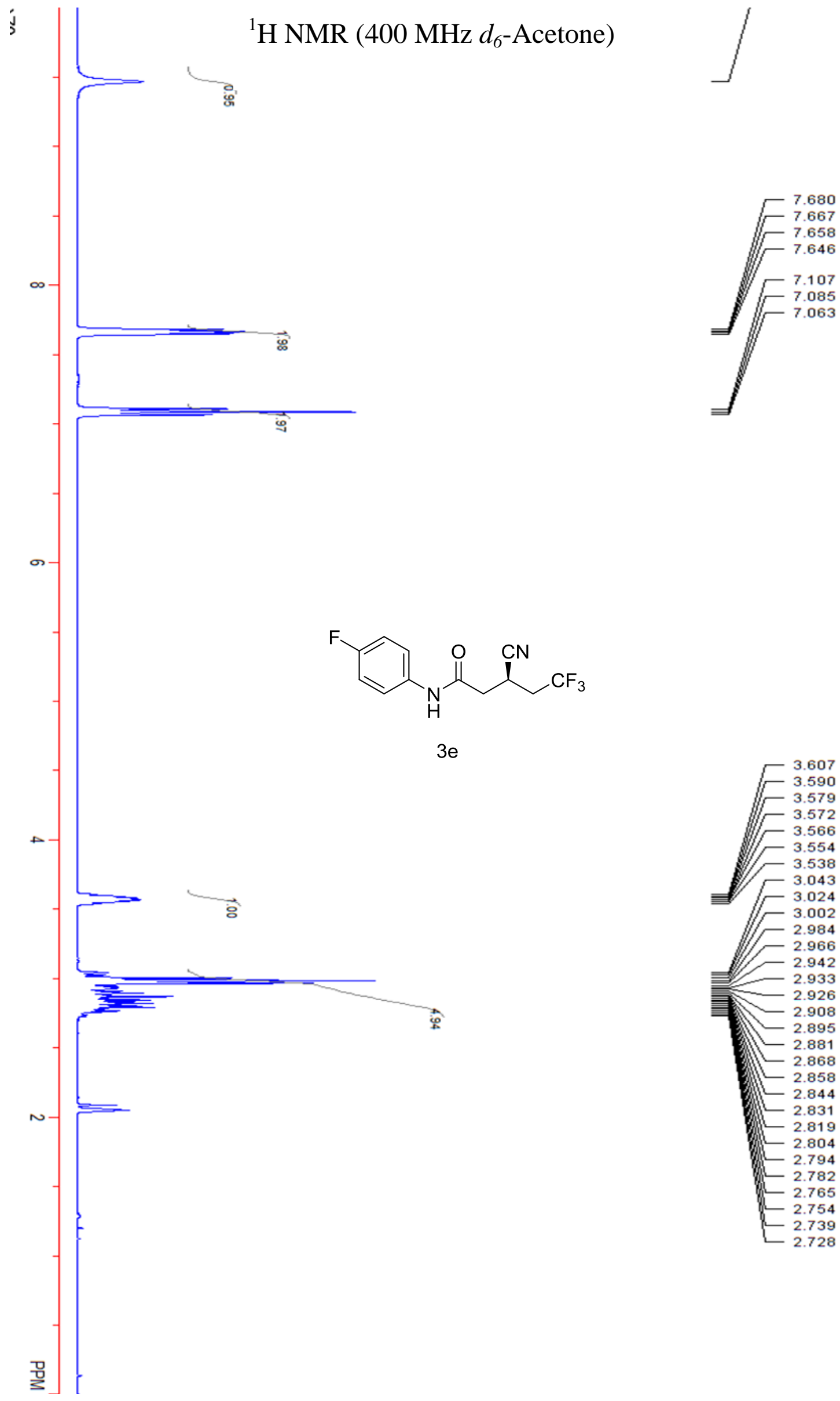




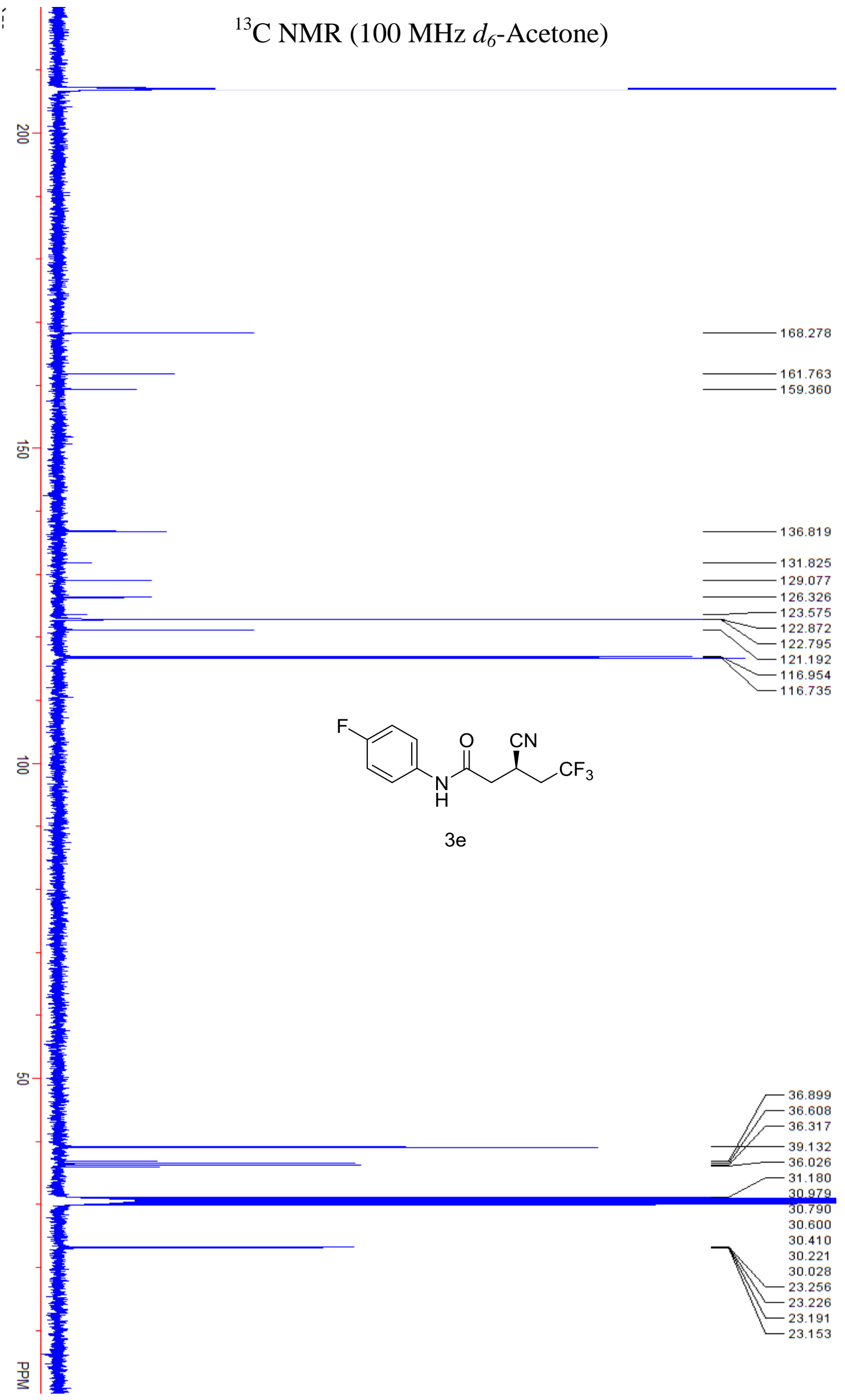




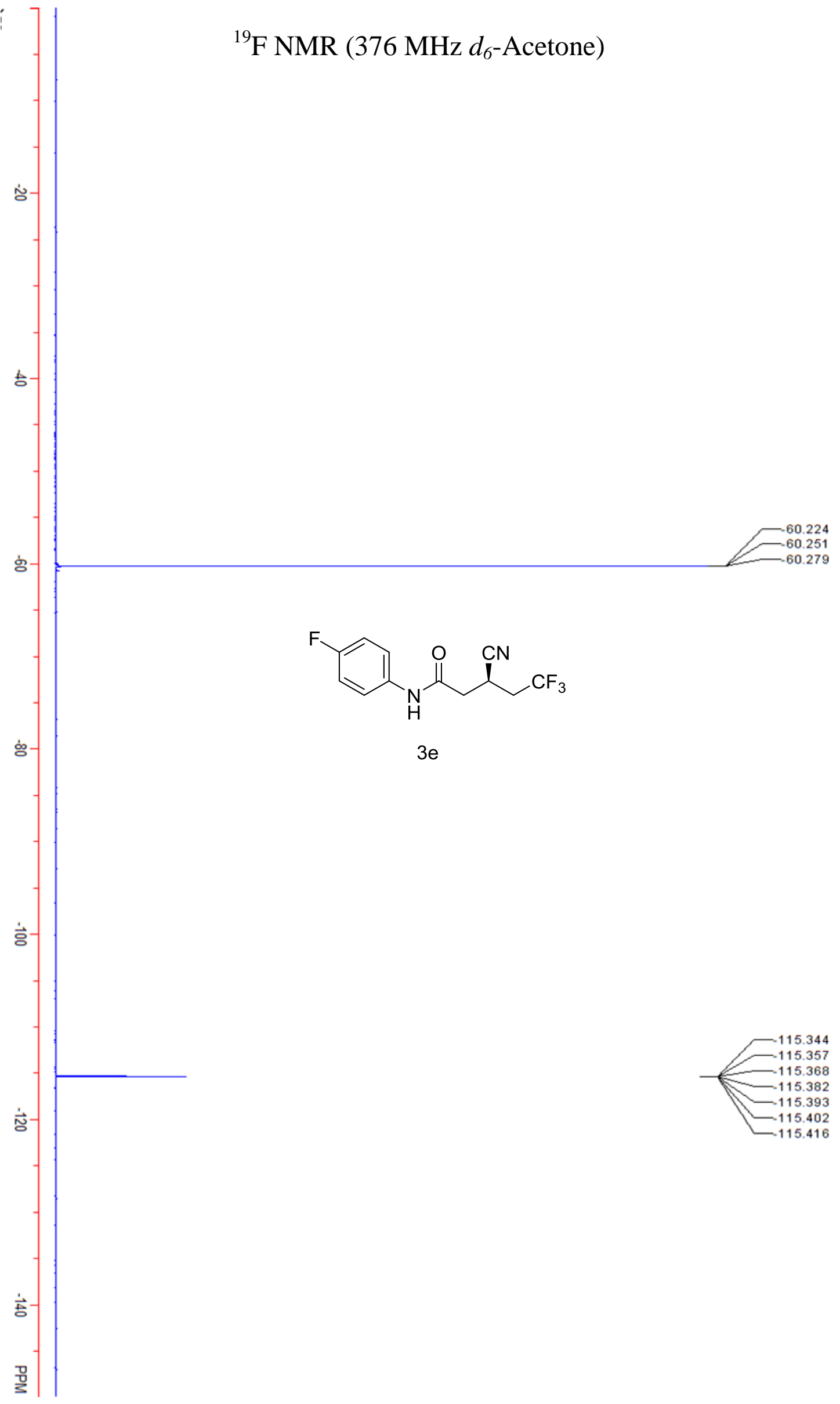




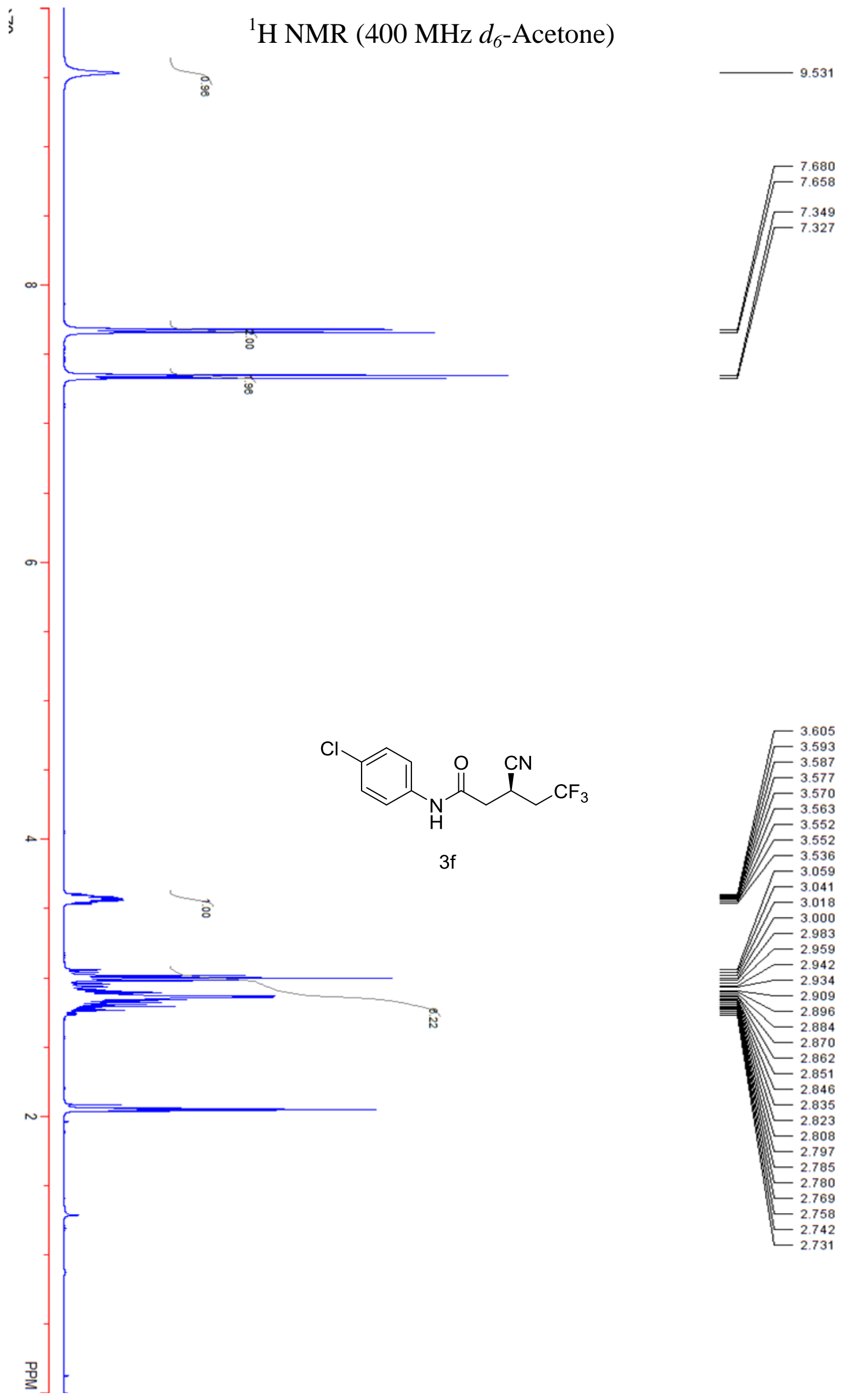




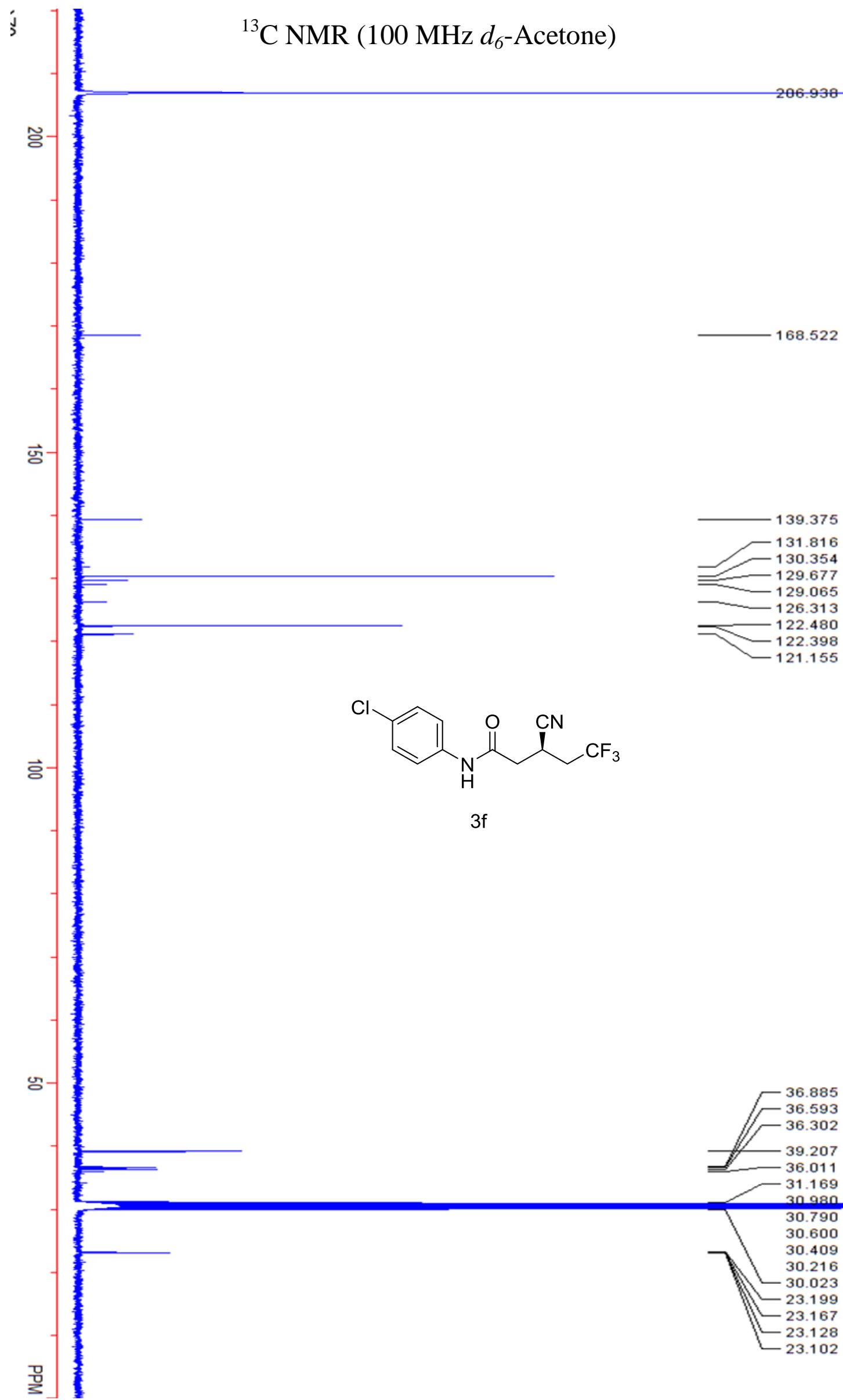




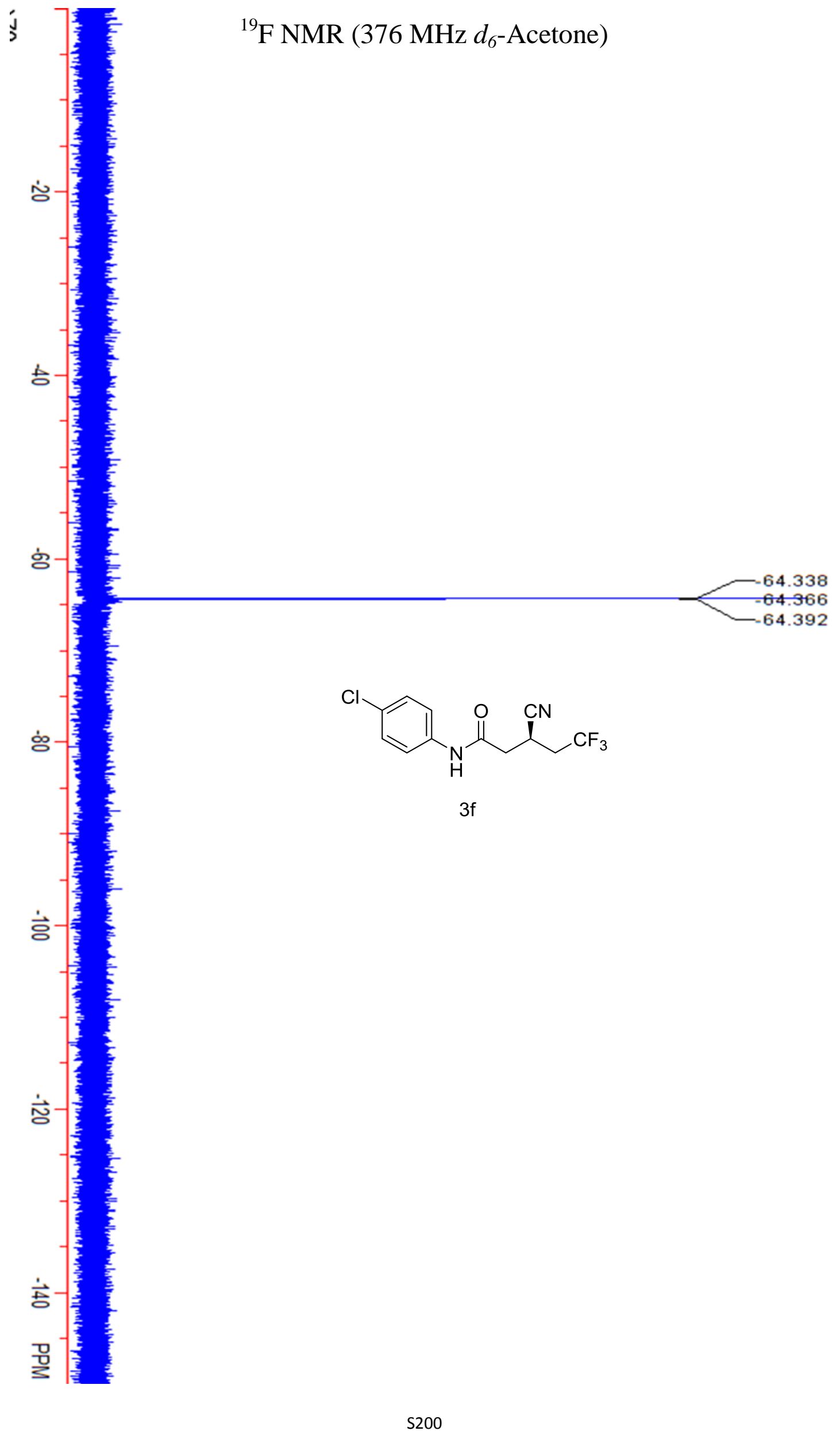




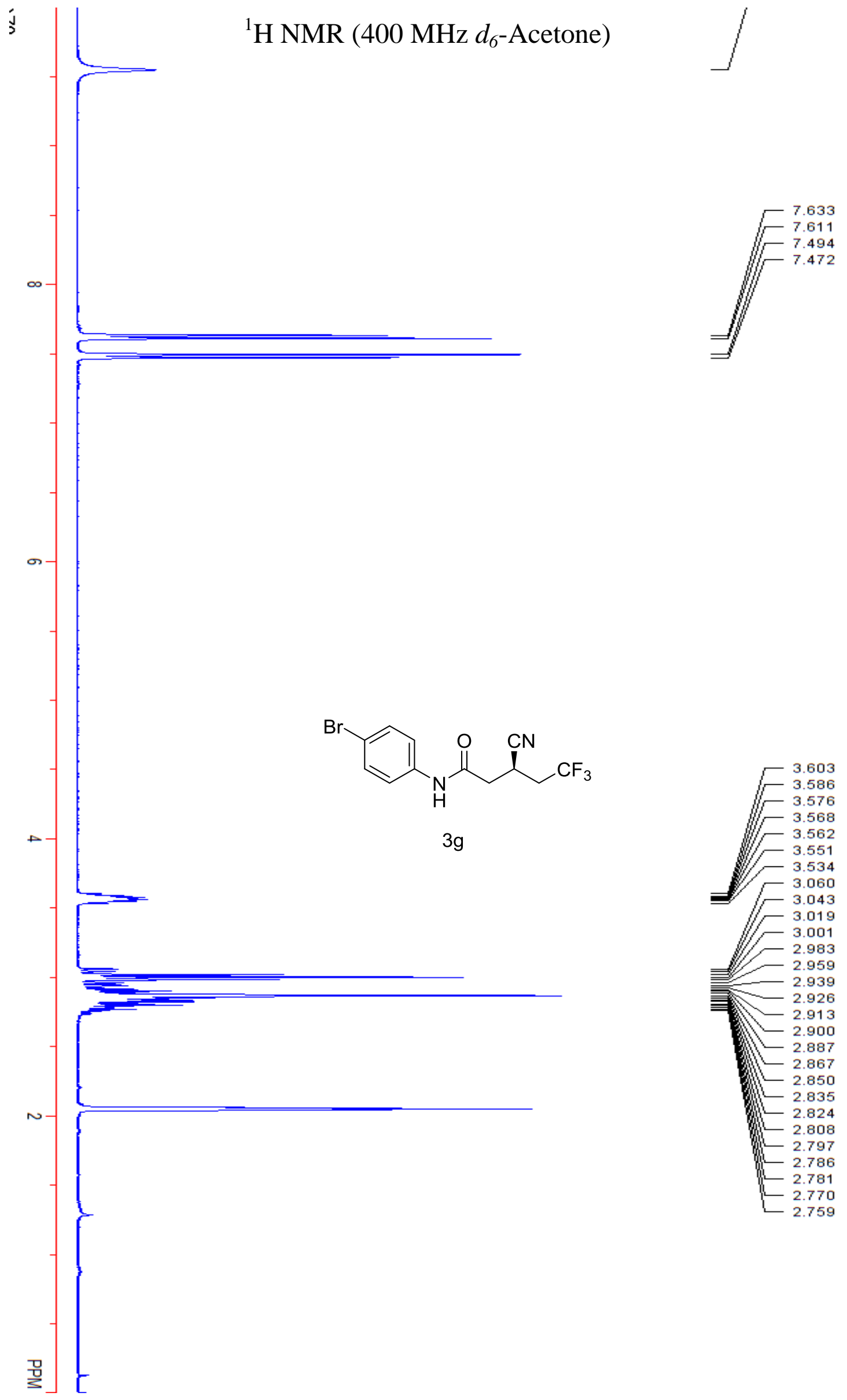




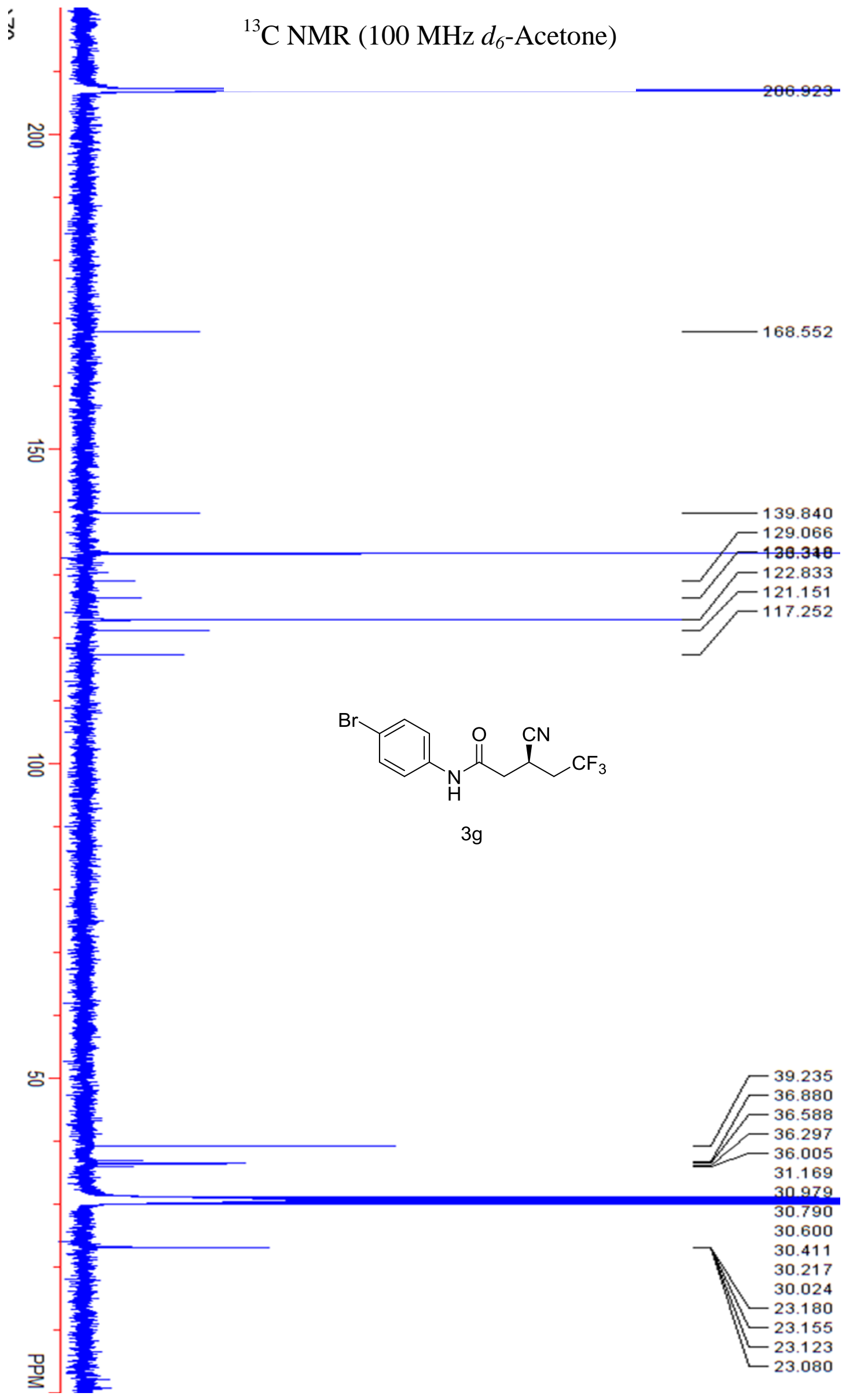




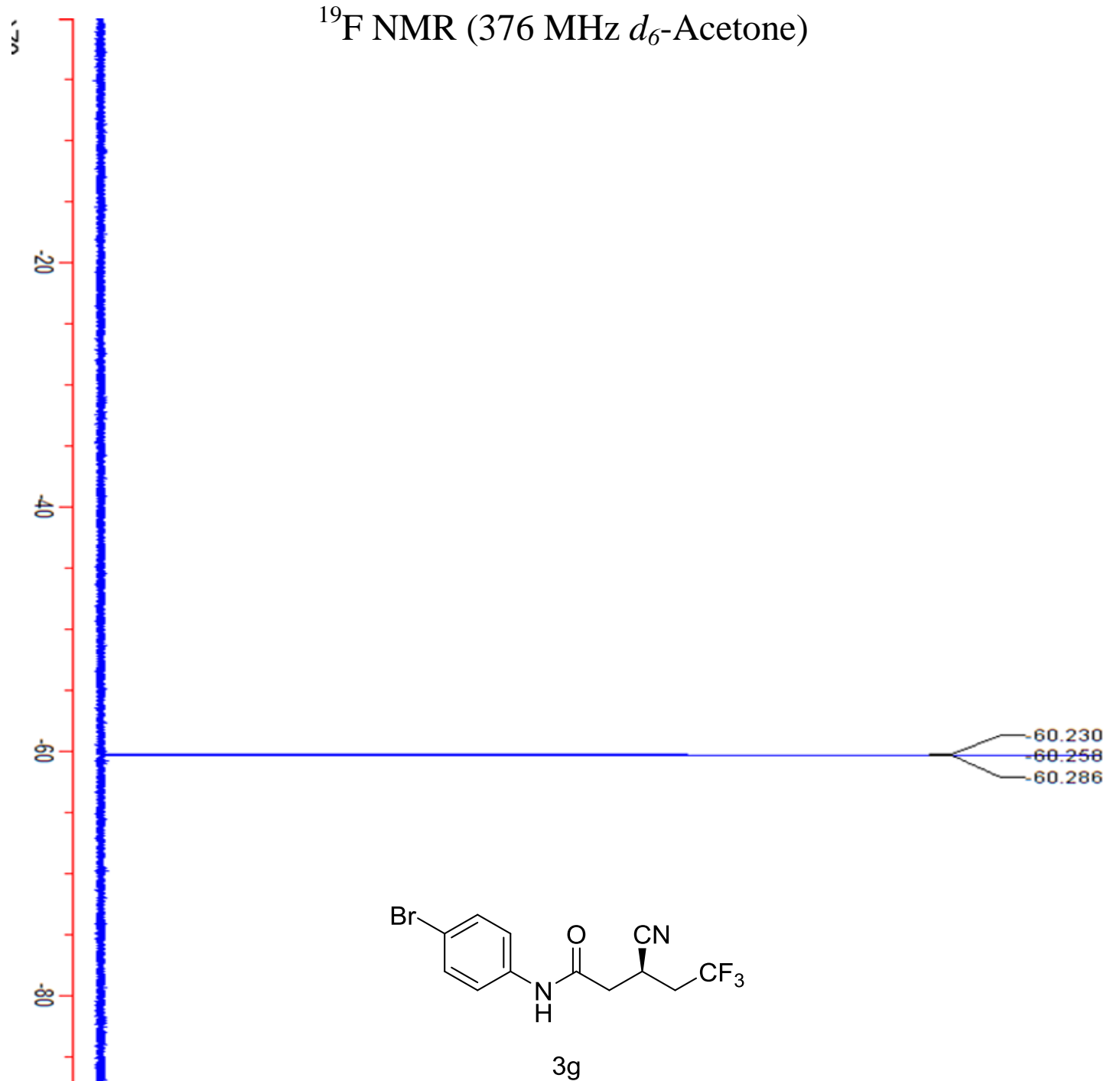

$3 g$ 
${ }^{1} \mathrm{H}$ NMR (400 MHz $\mathrm{CDCl}_{3}$ )

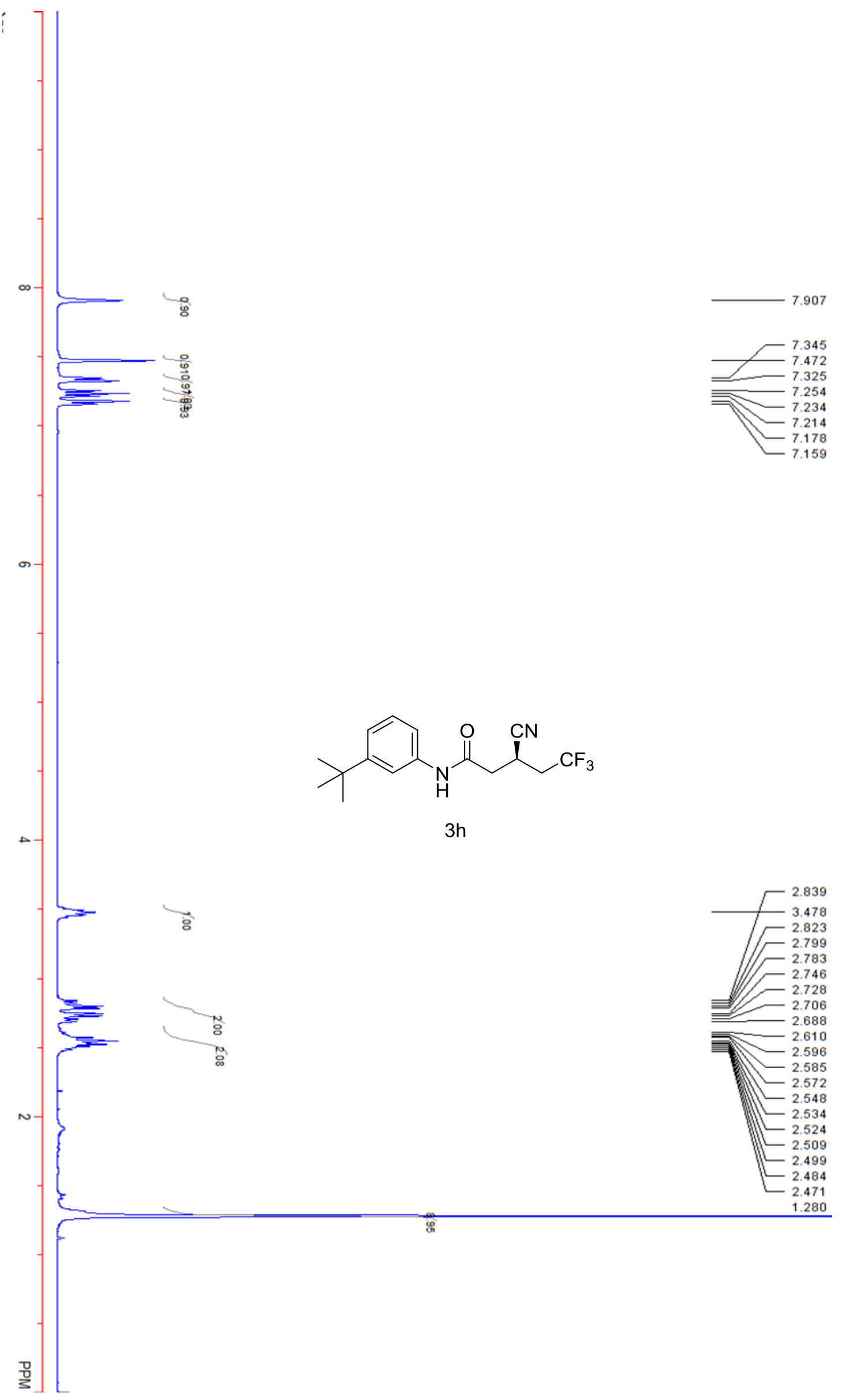




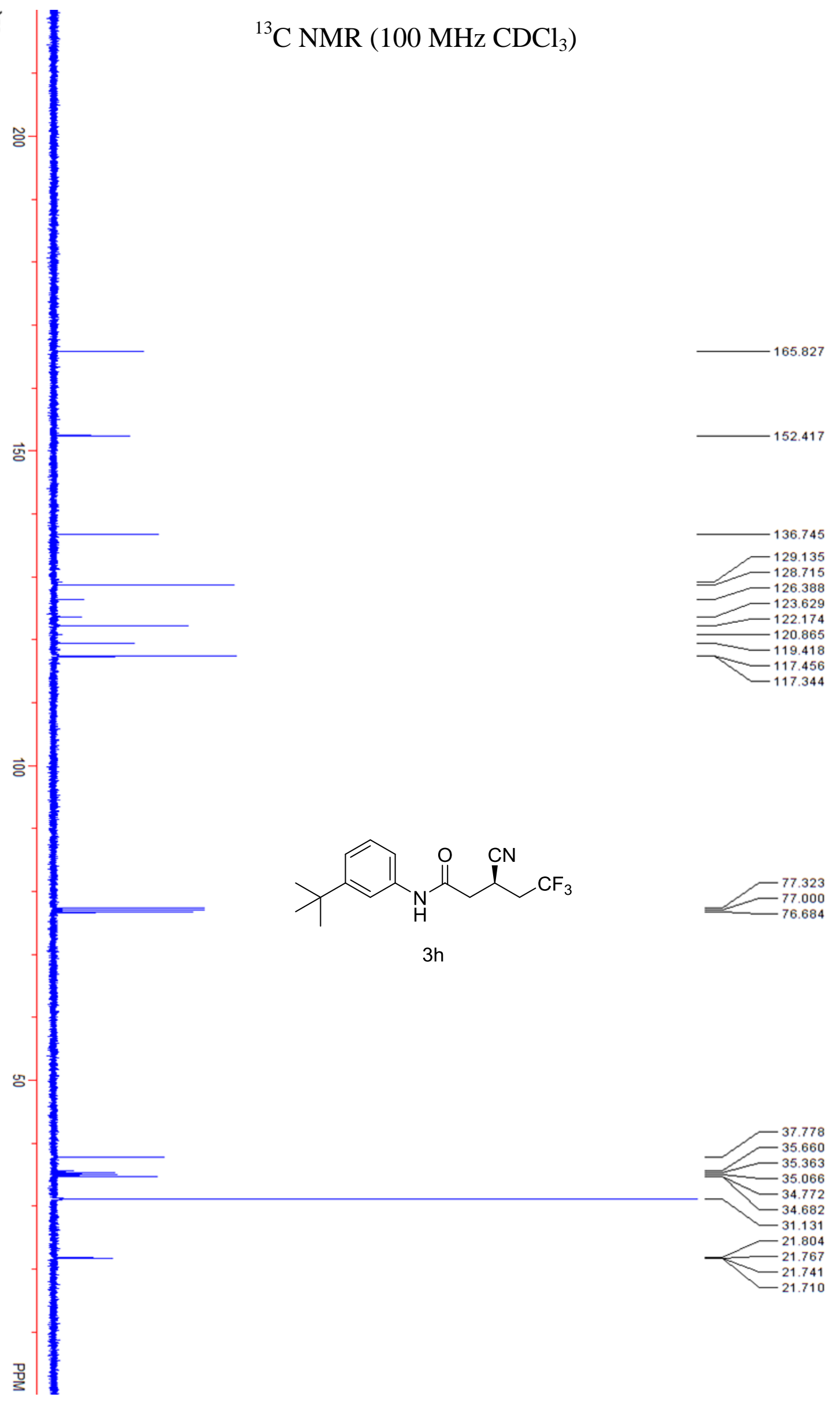



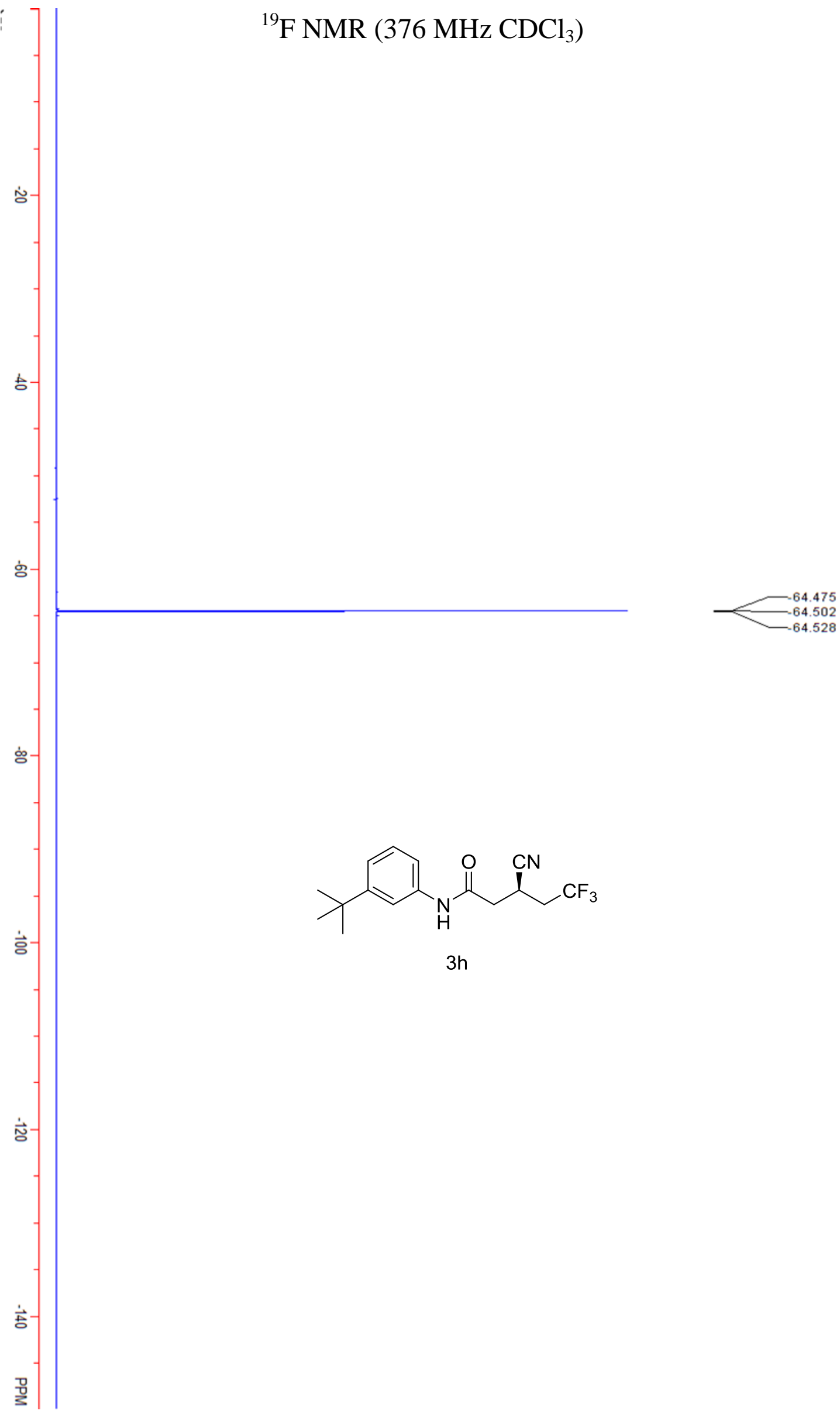

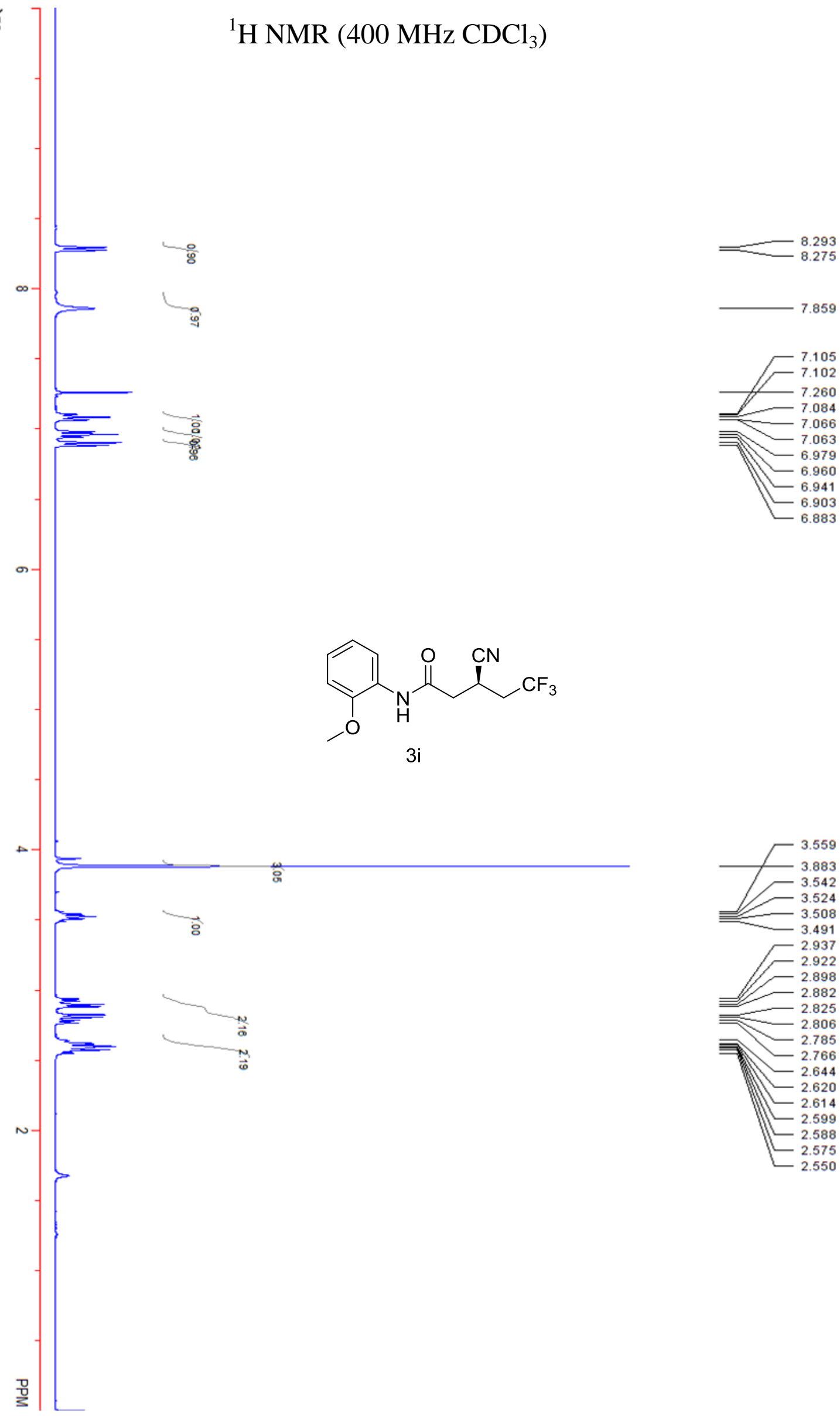


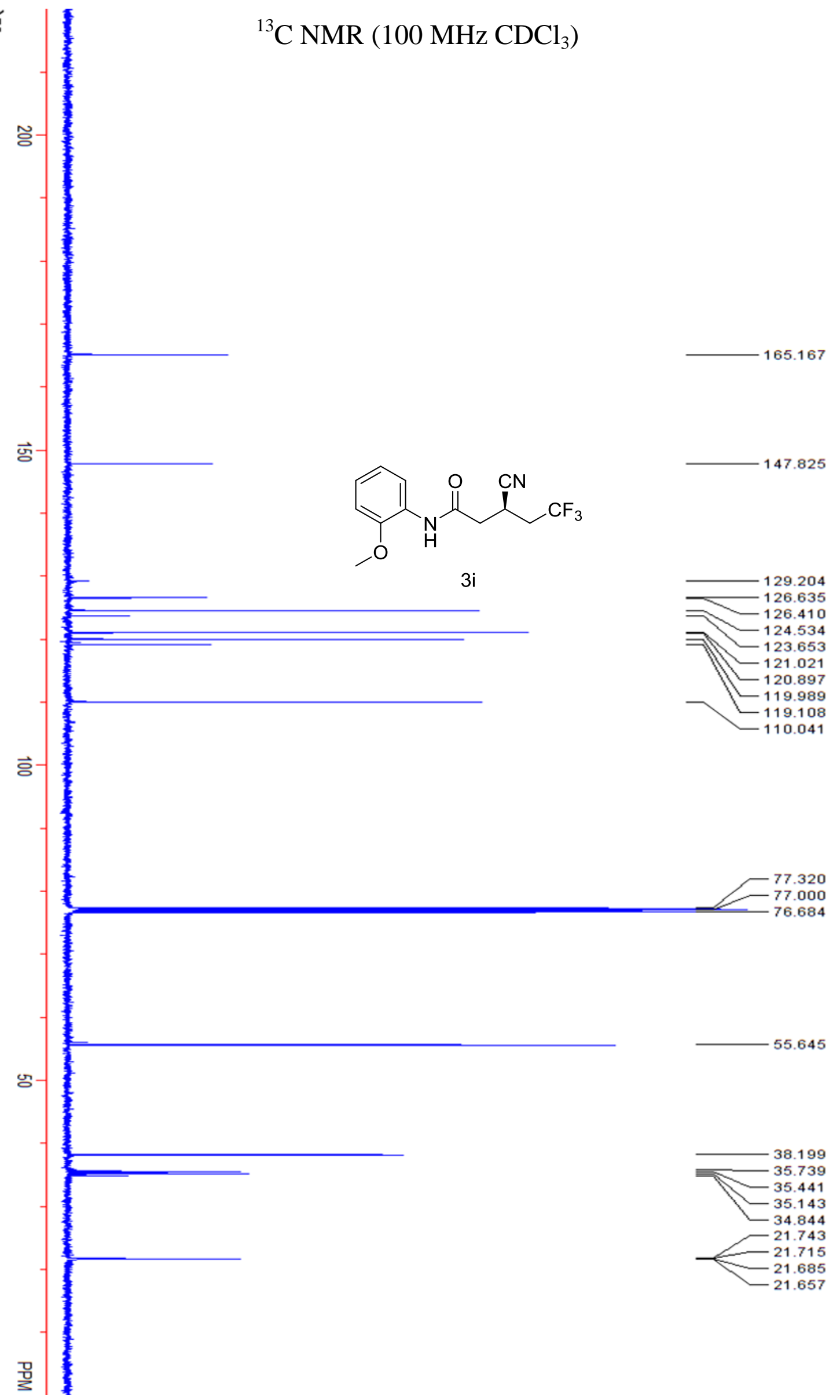



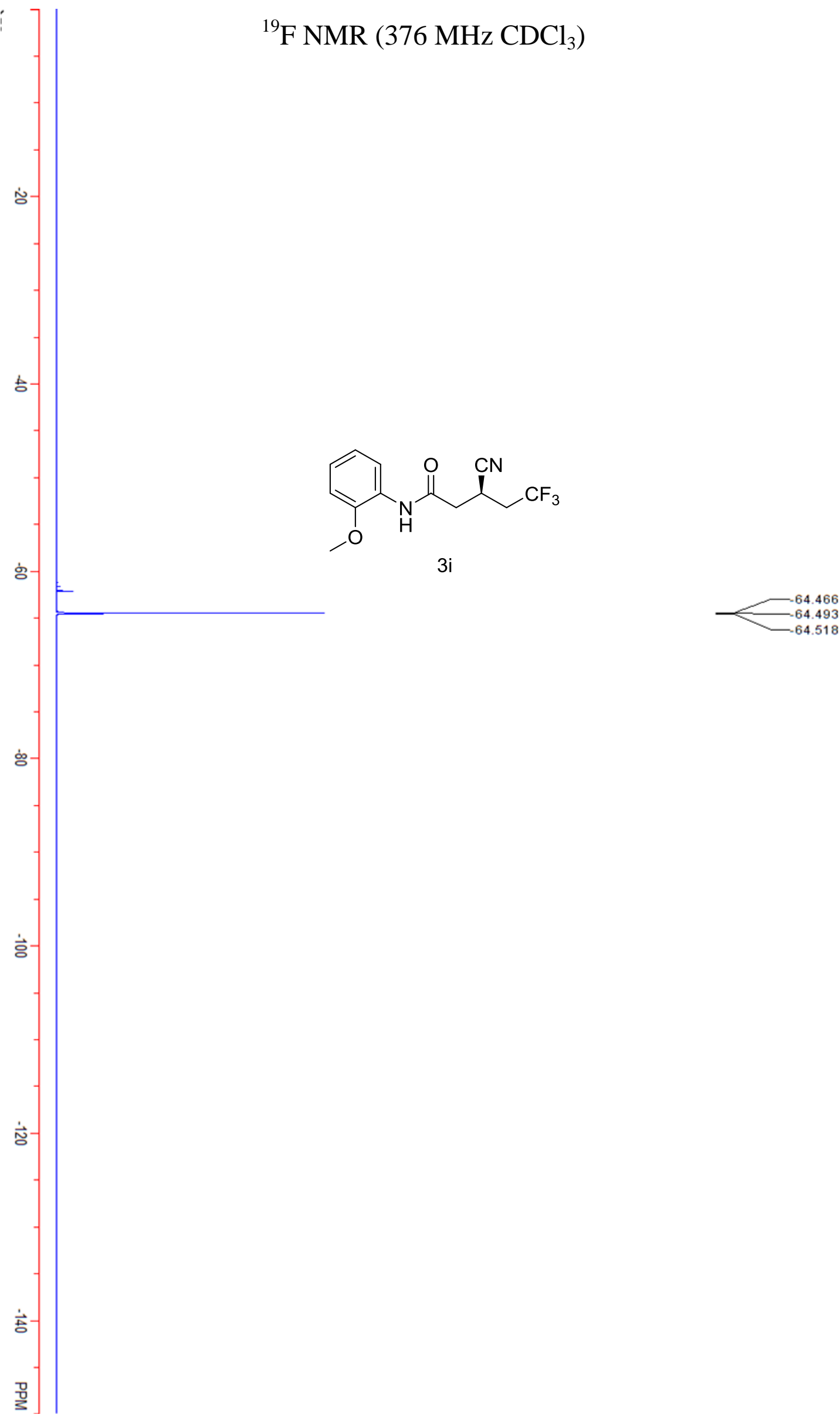


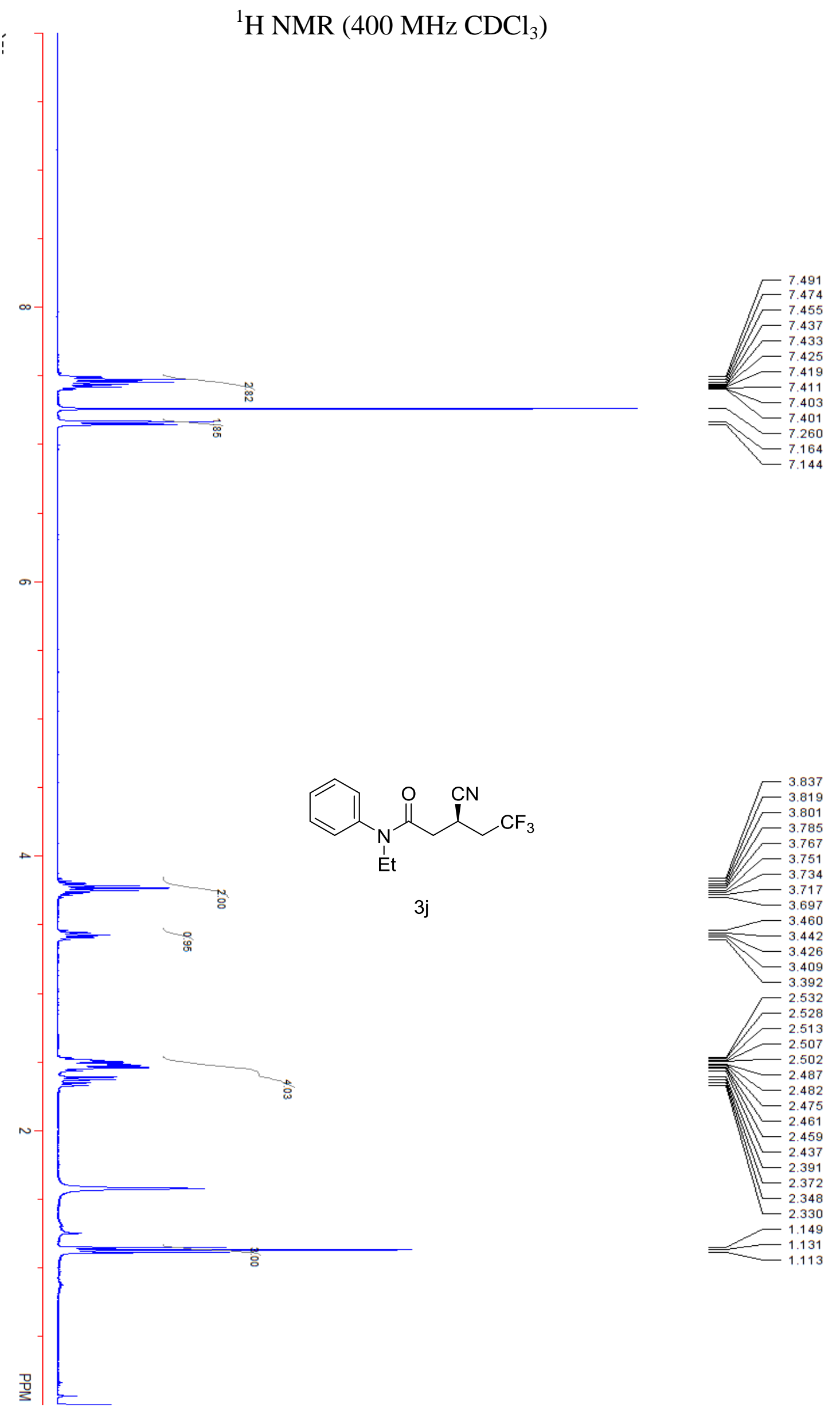



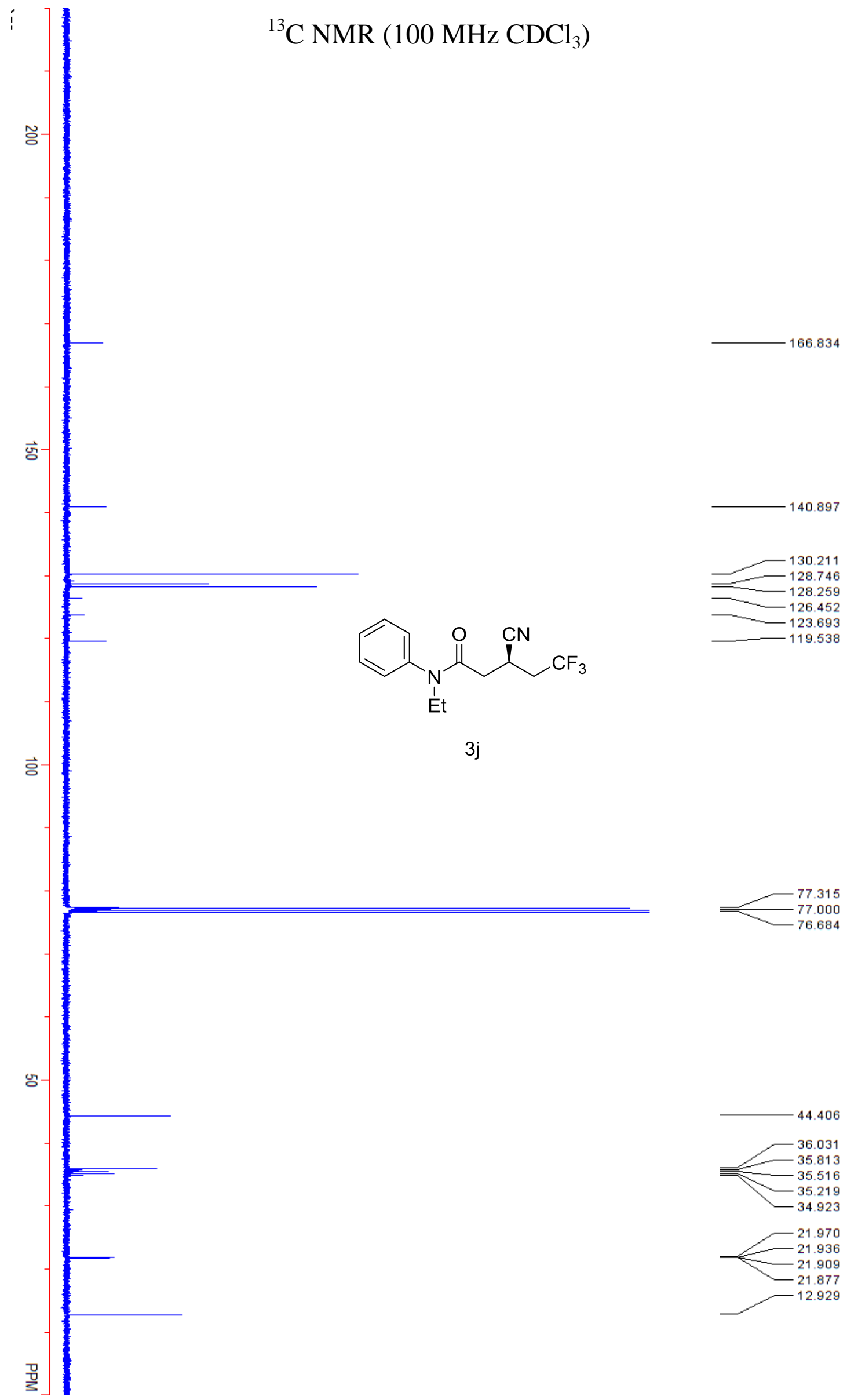


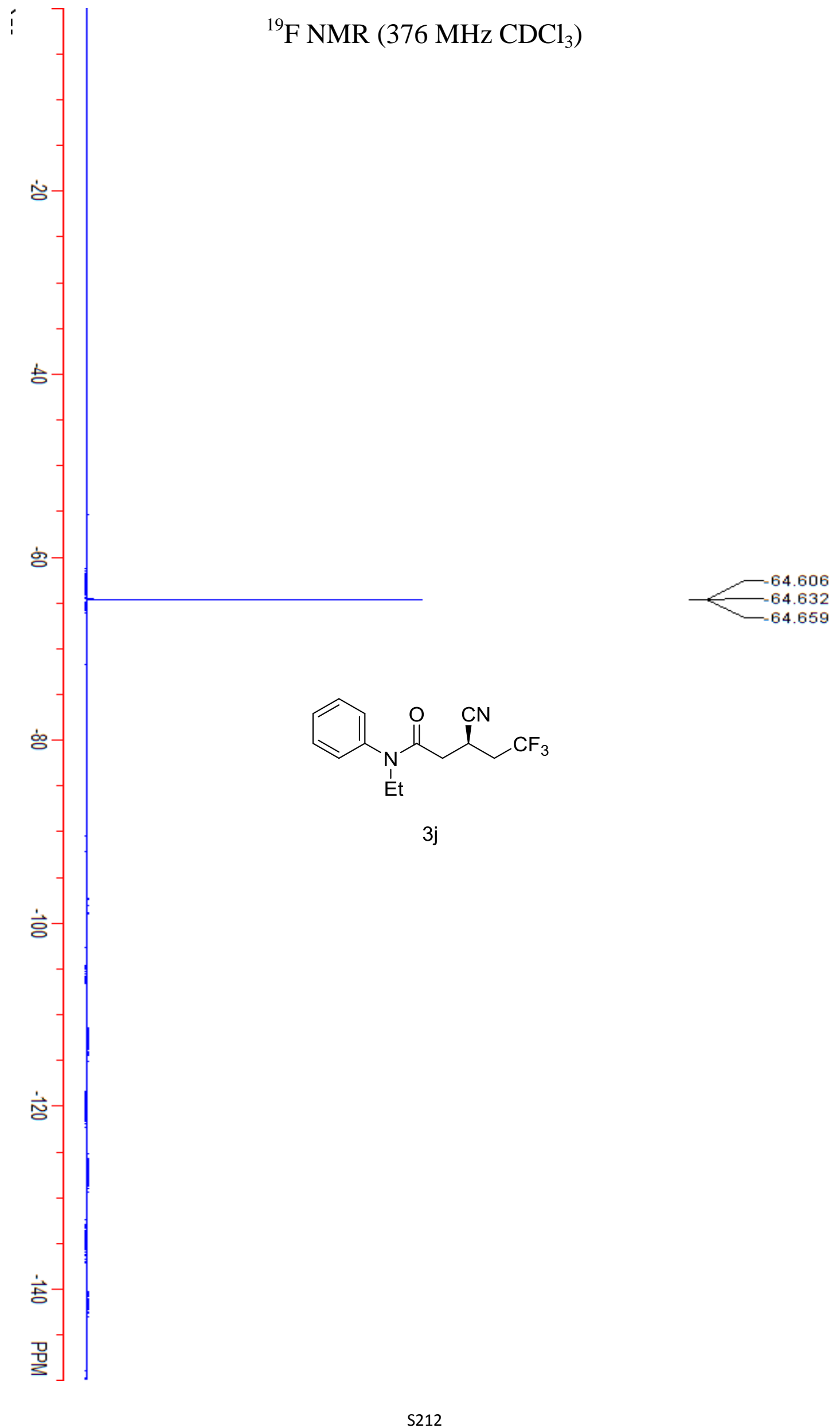




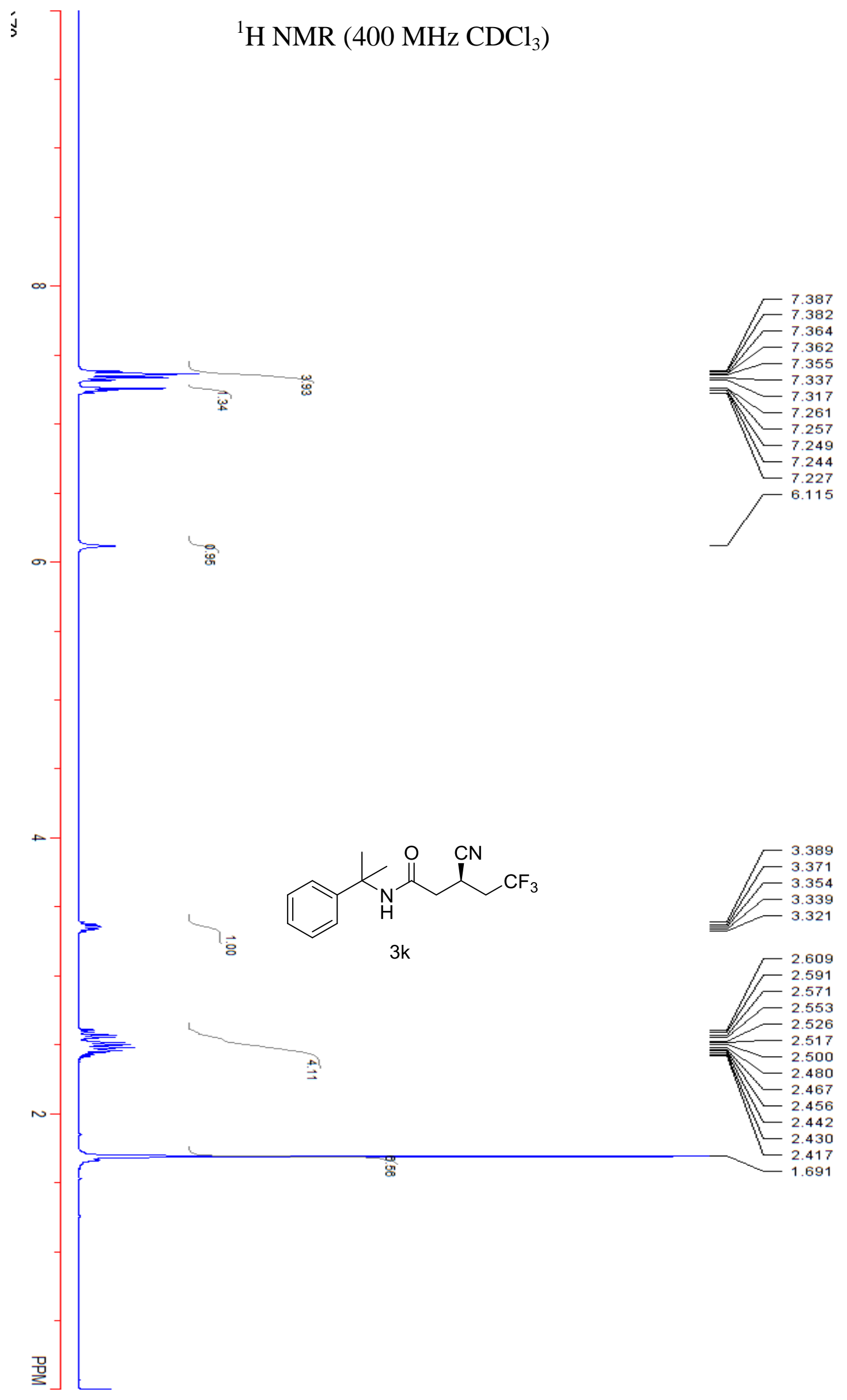




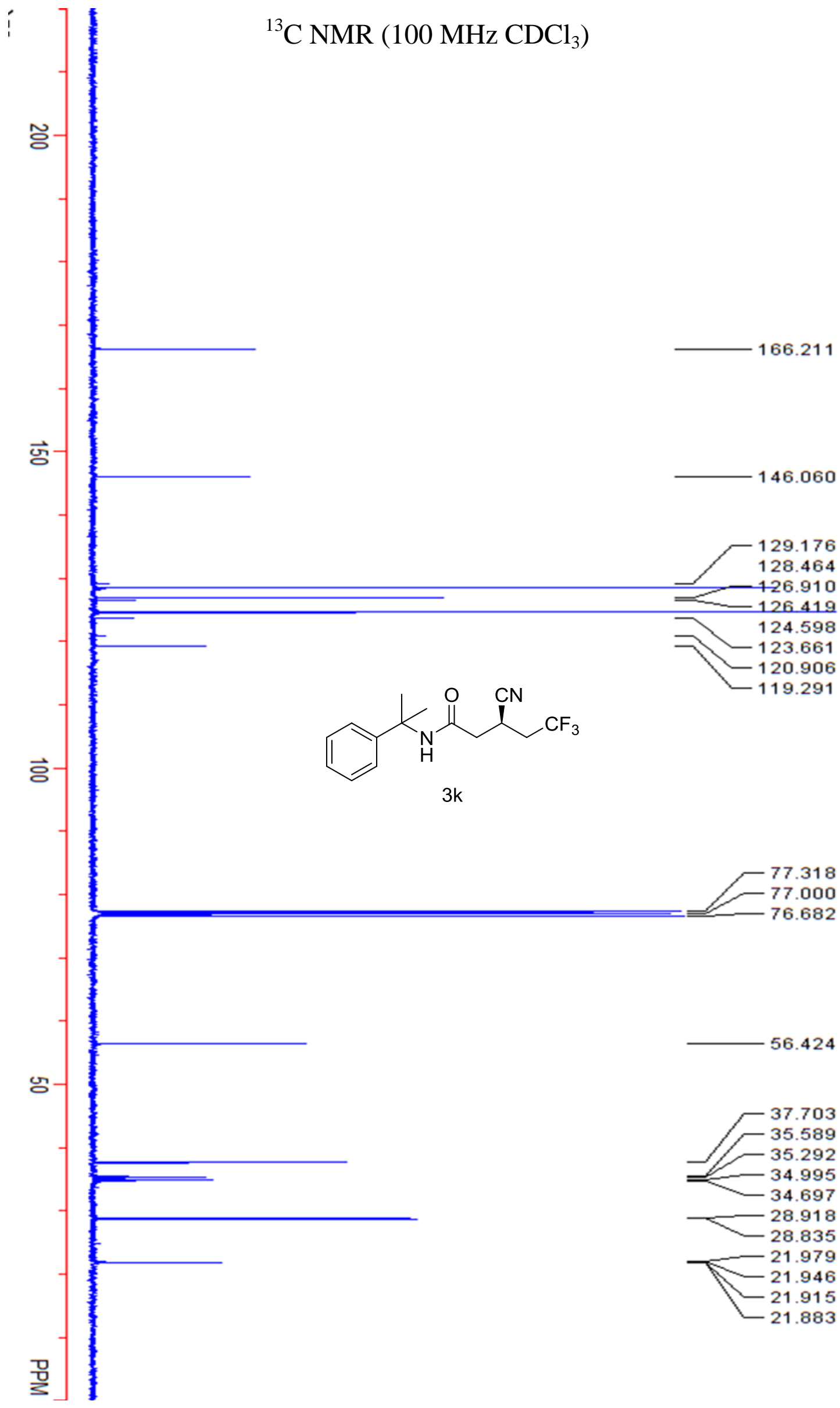




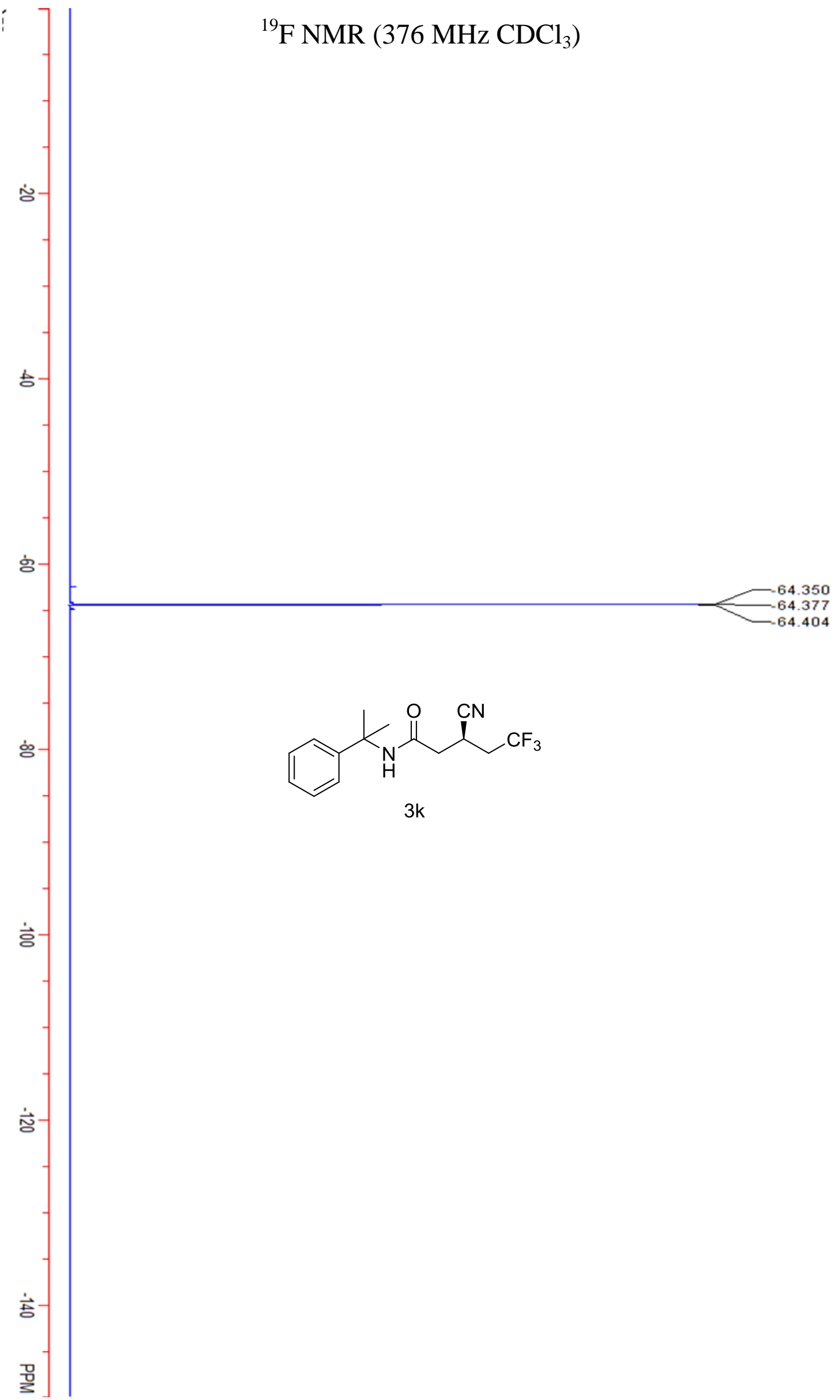



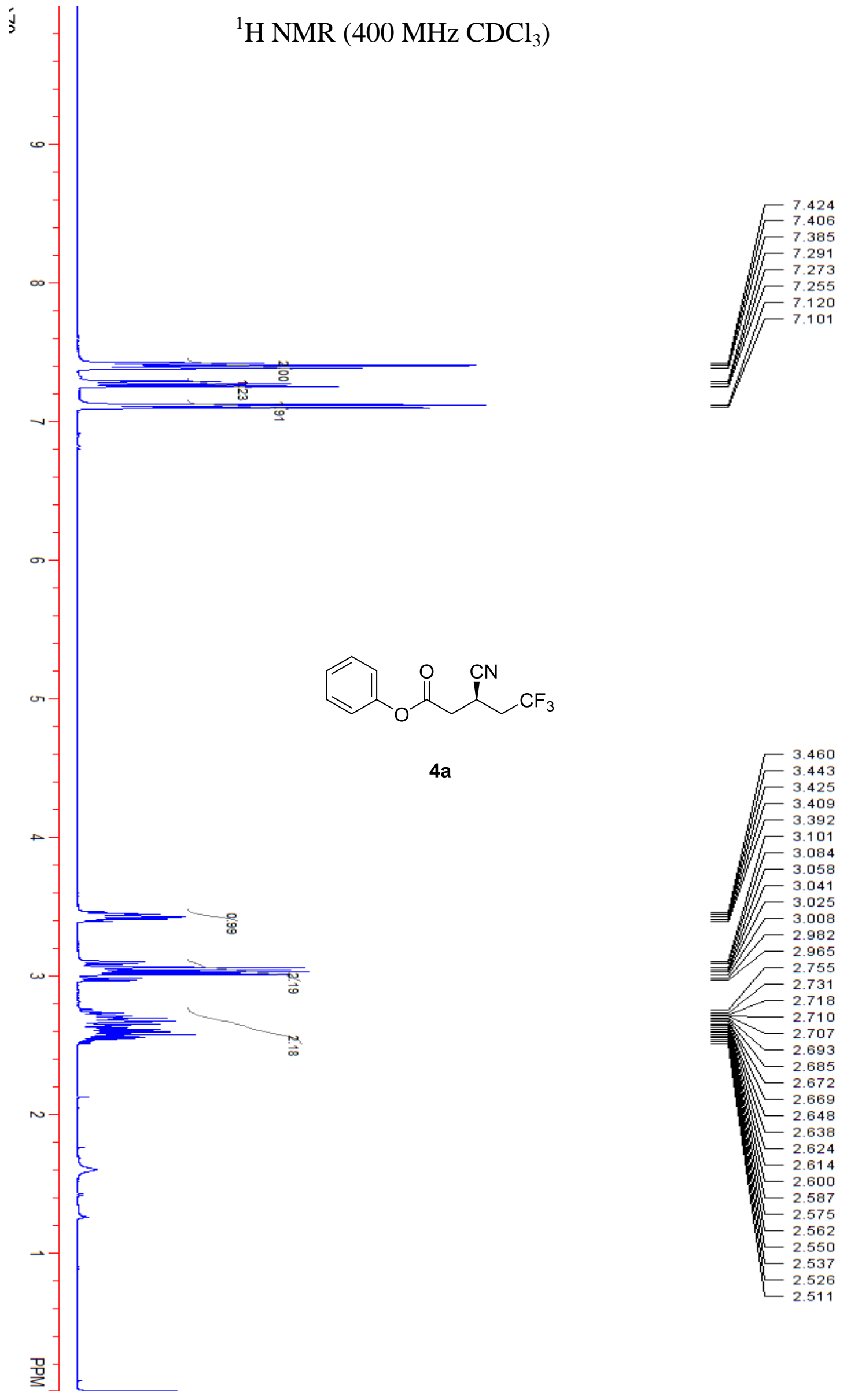

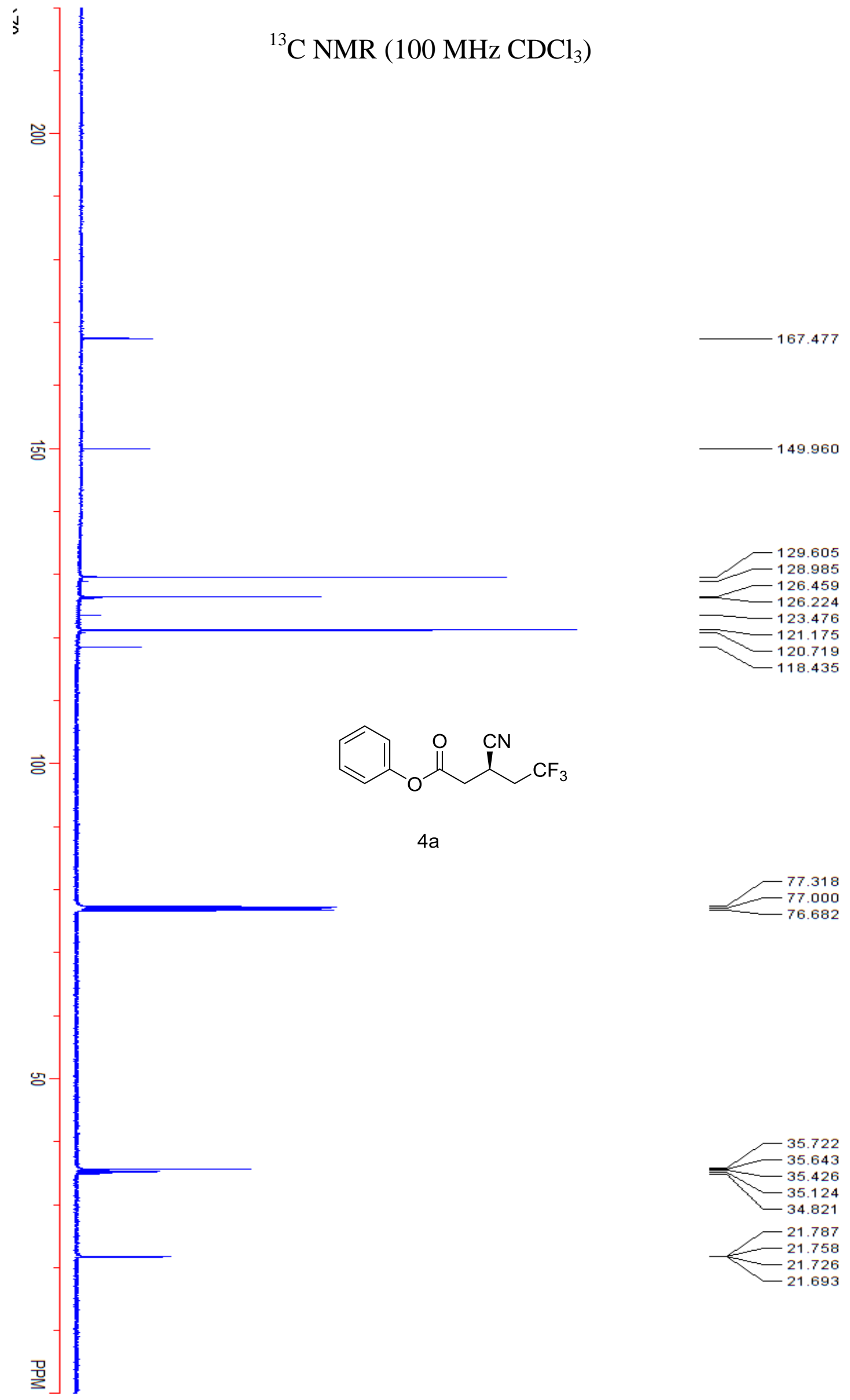

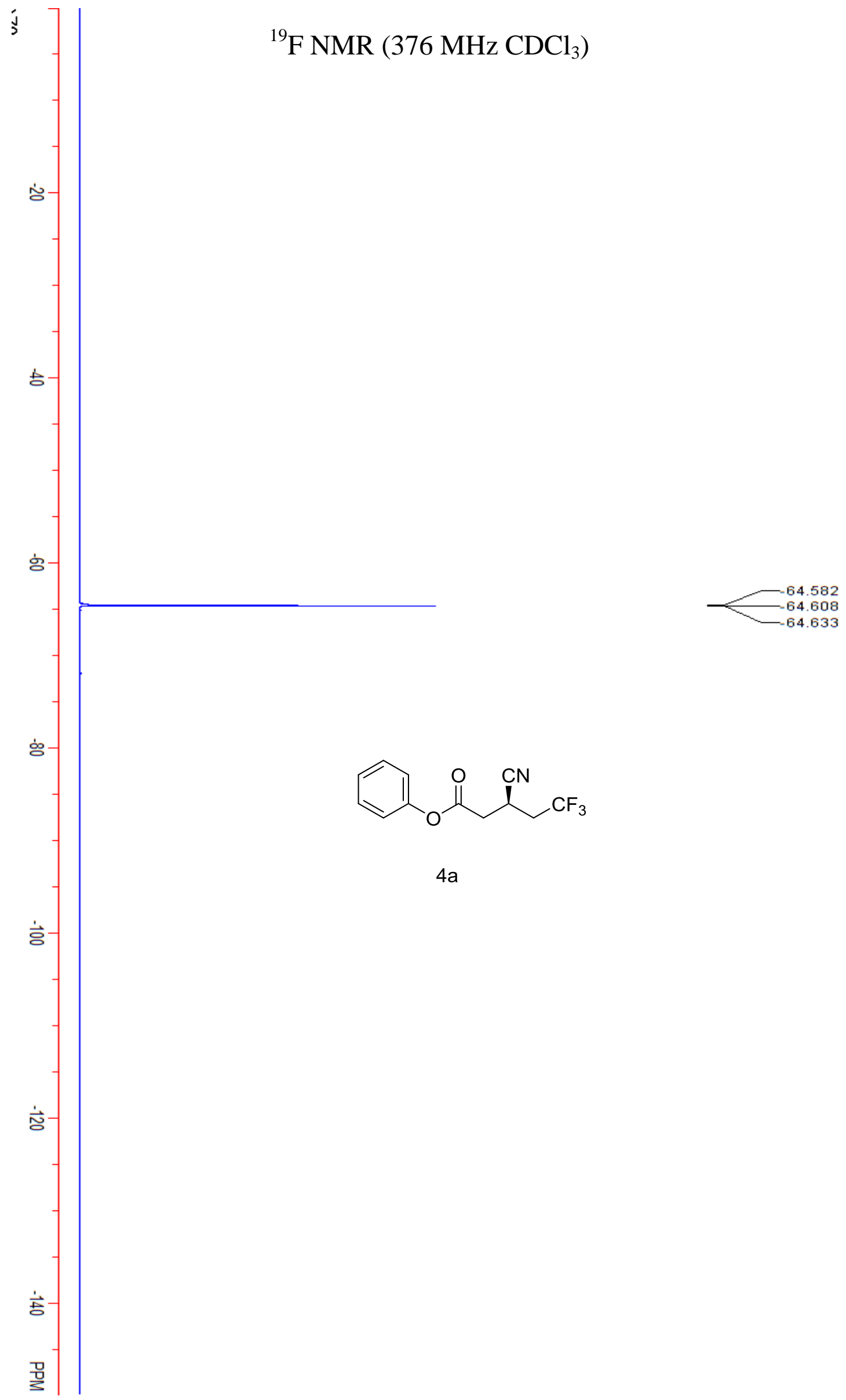


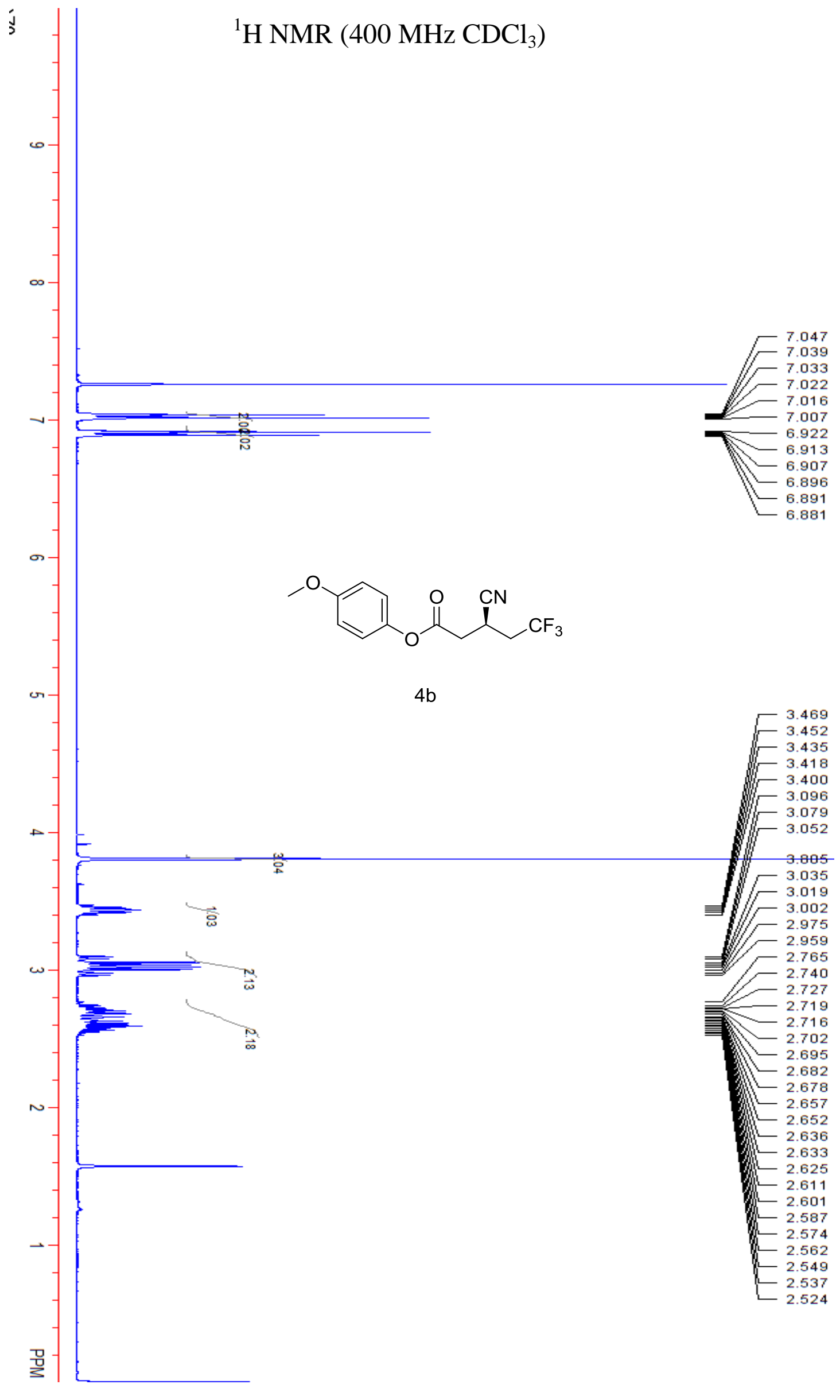



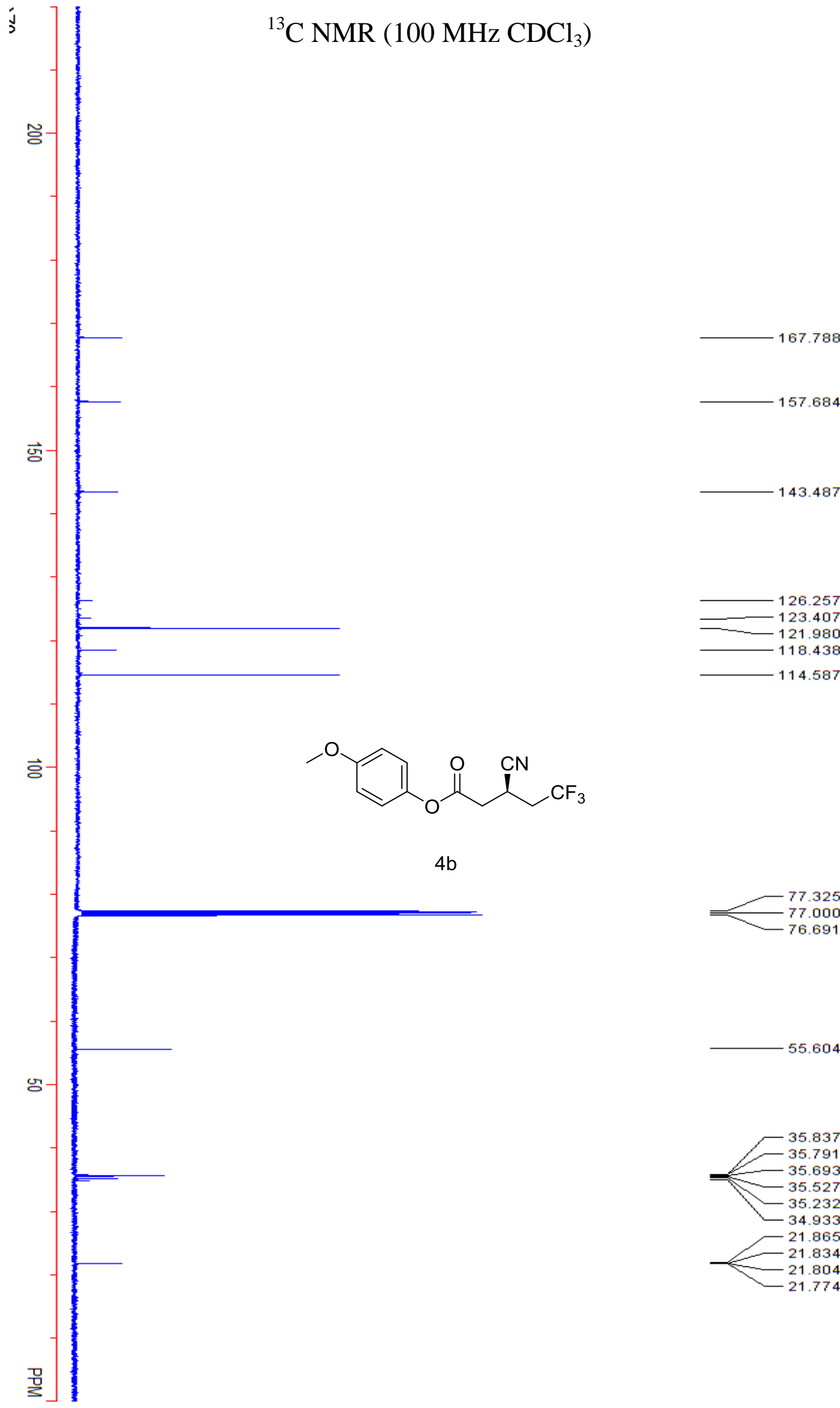

55.604 


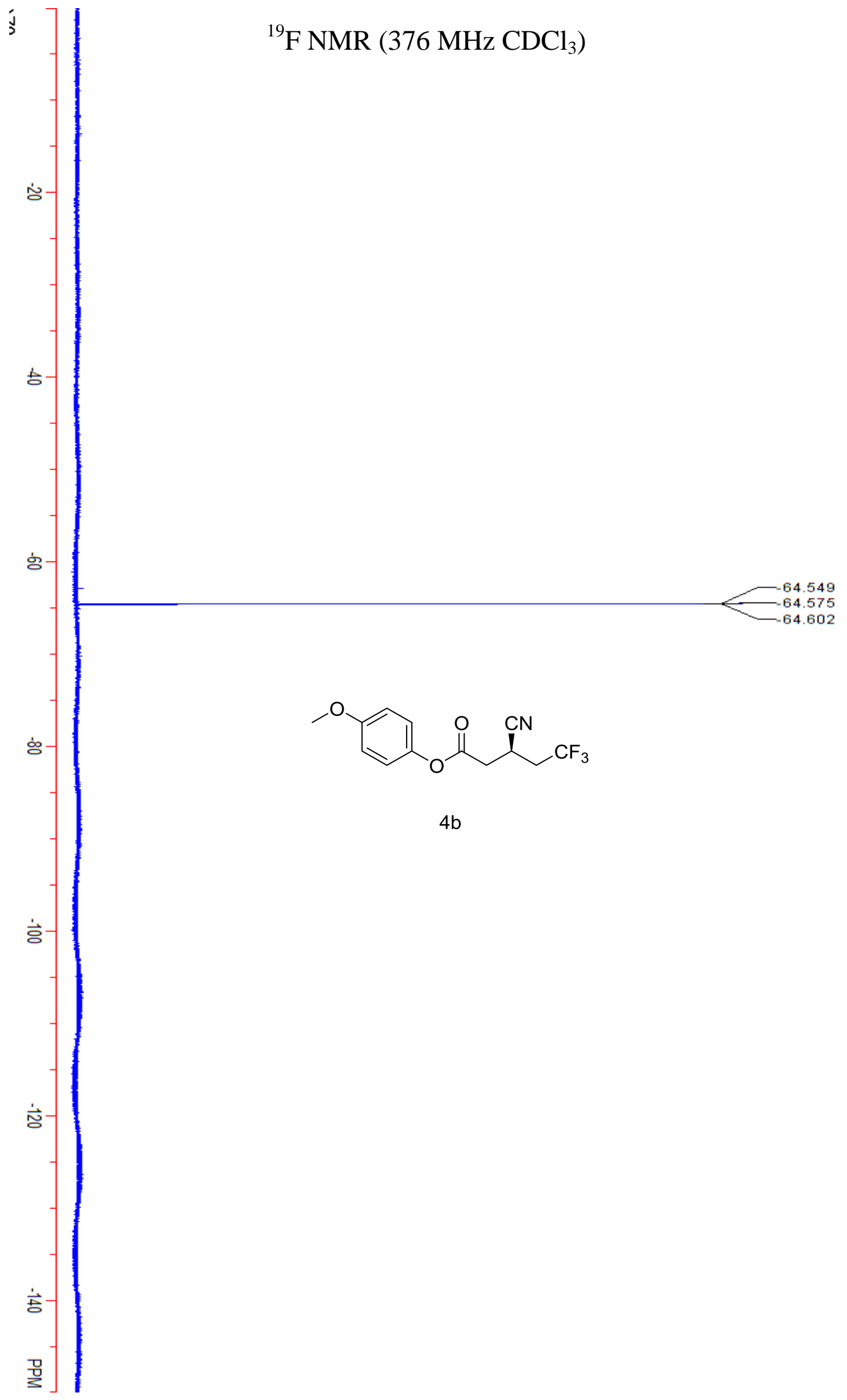




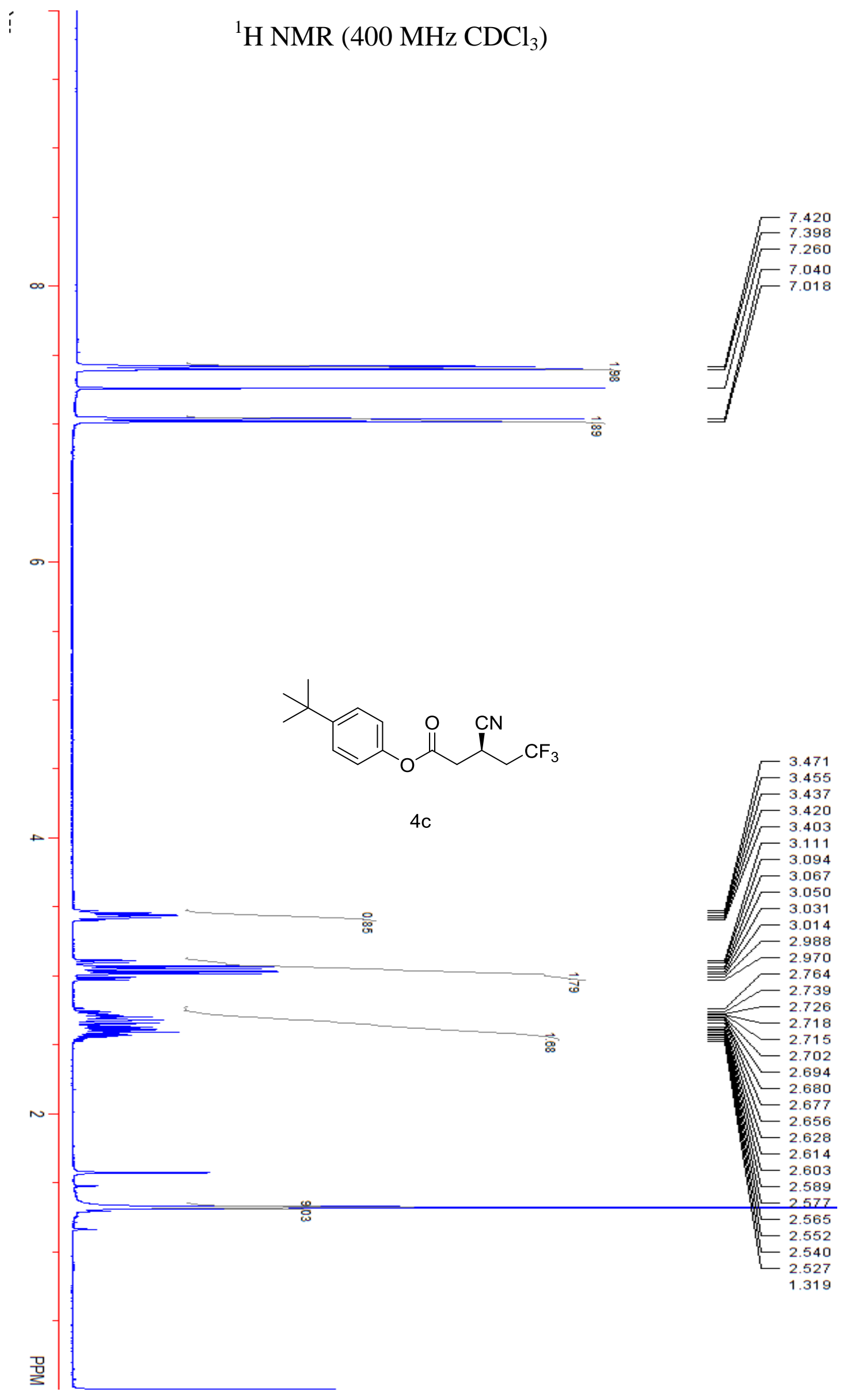




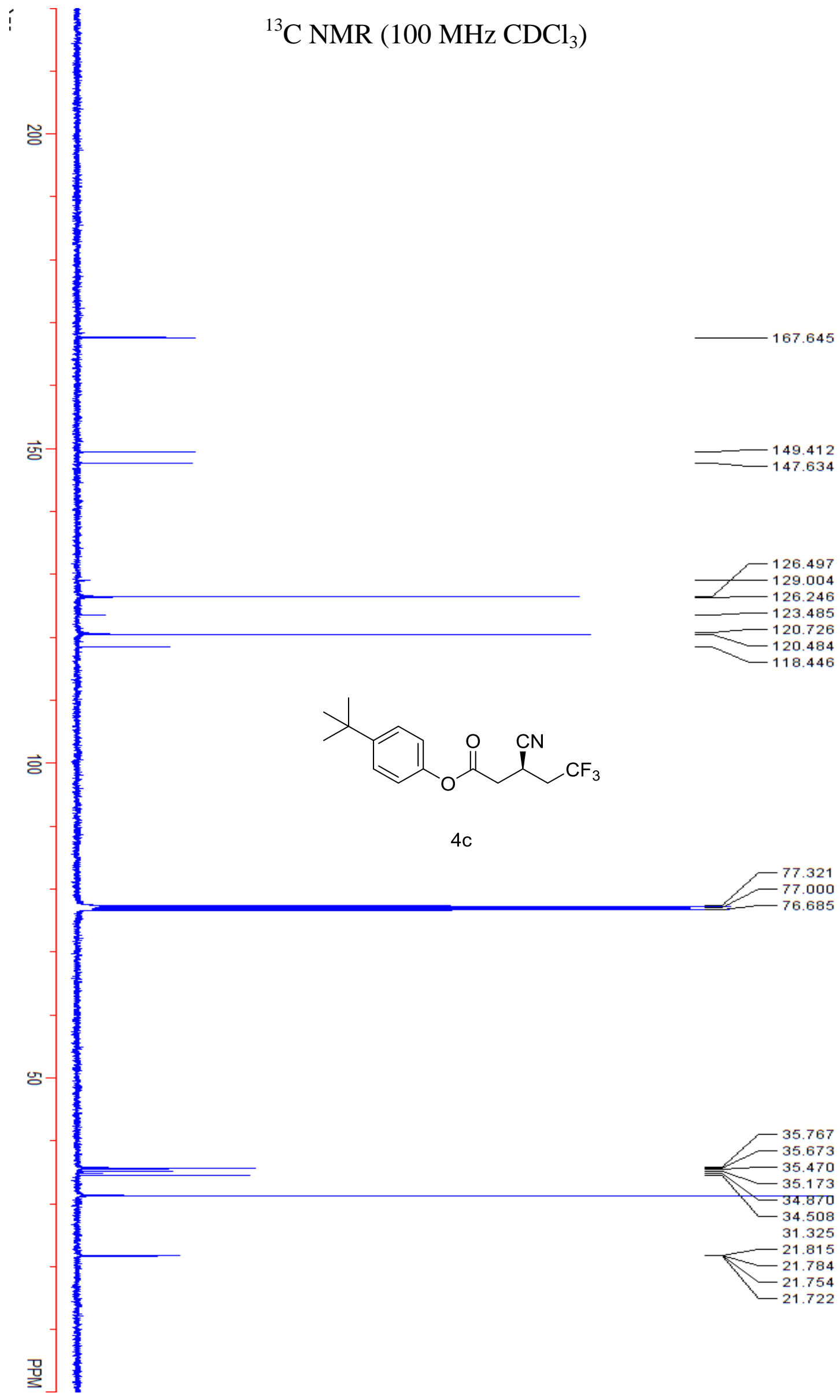




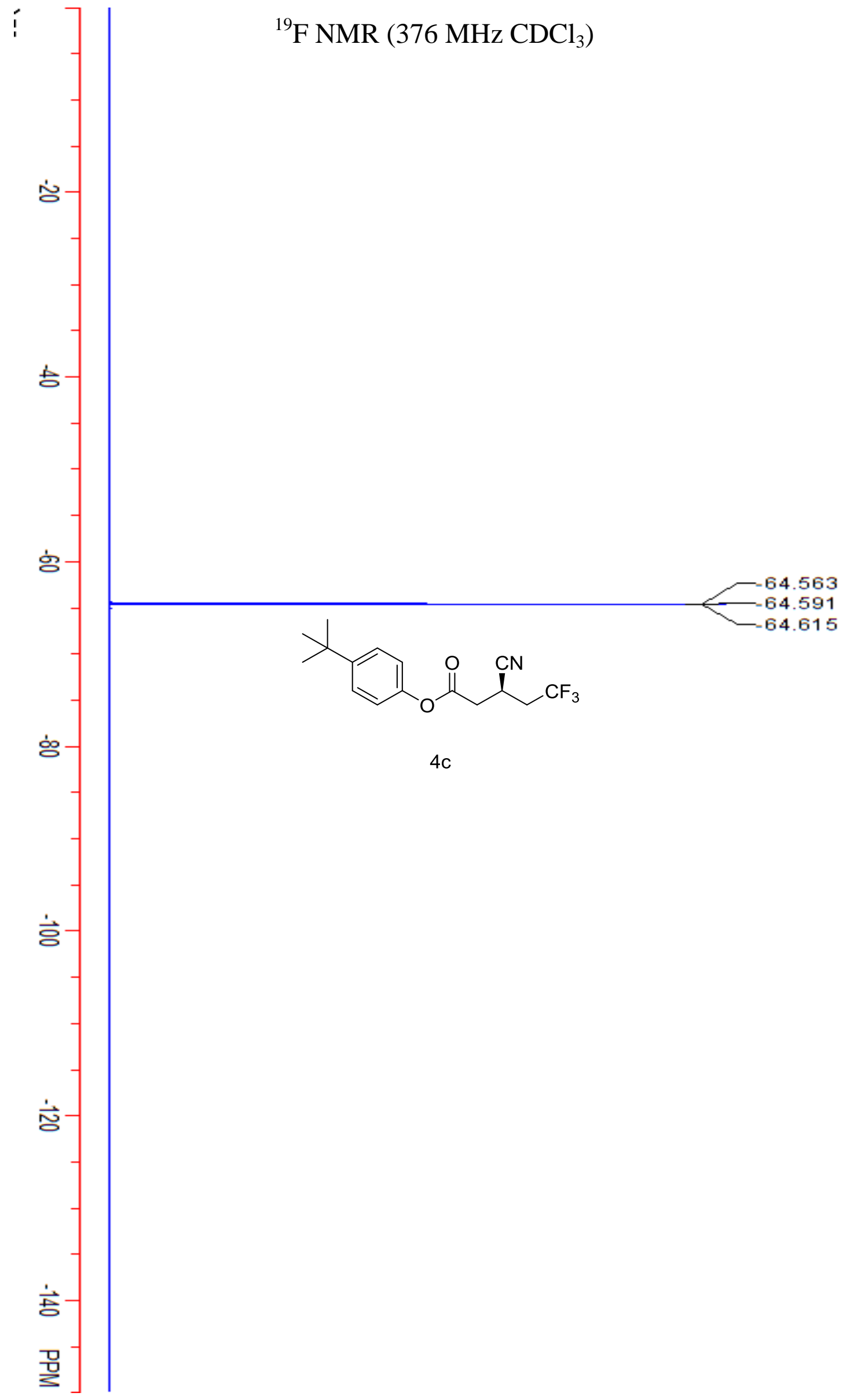




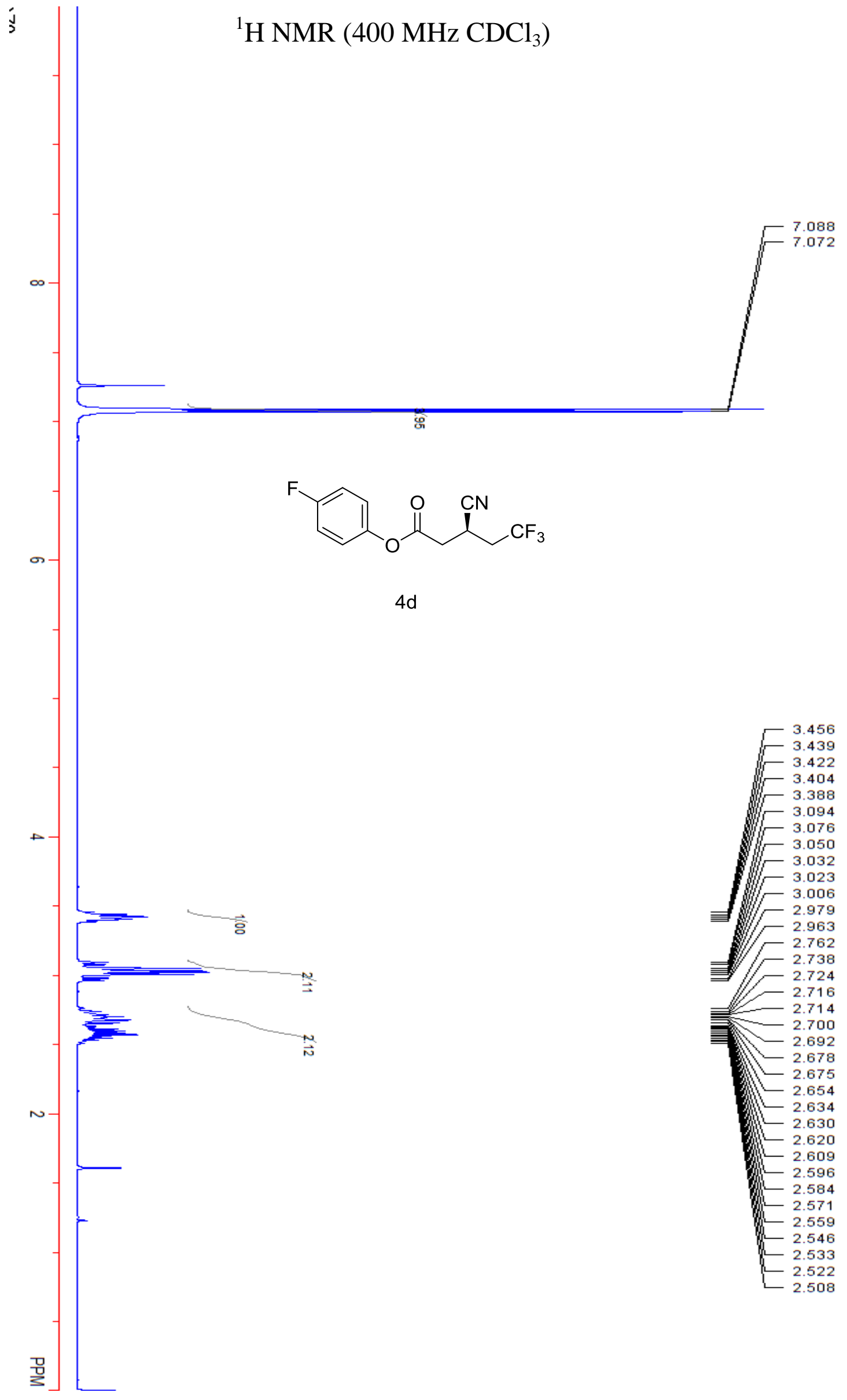




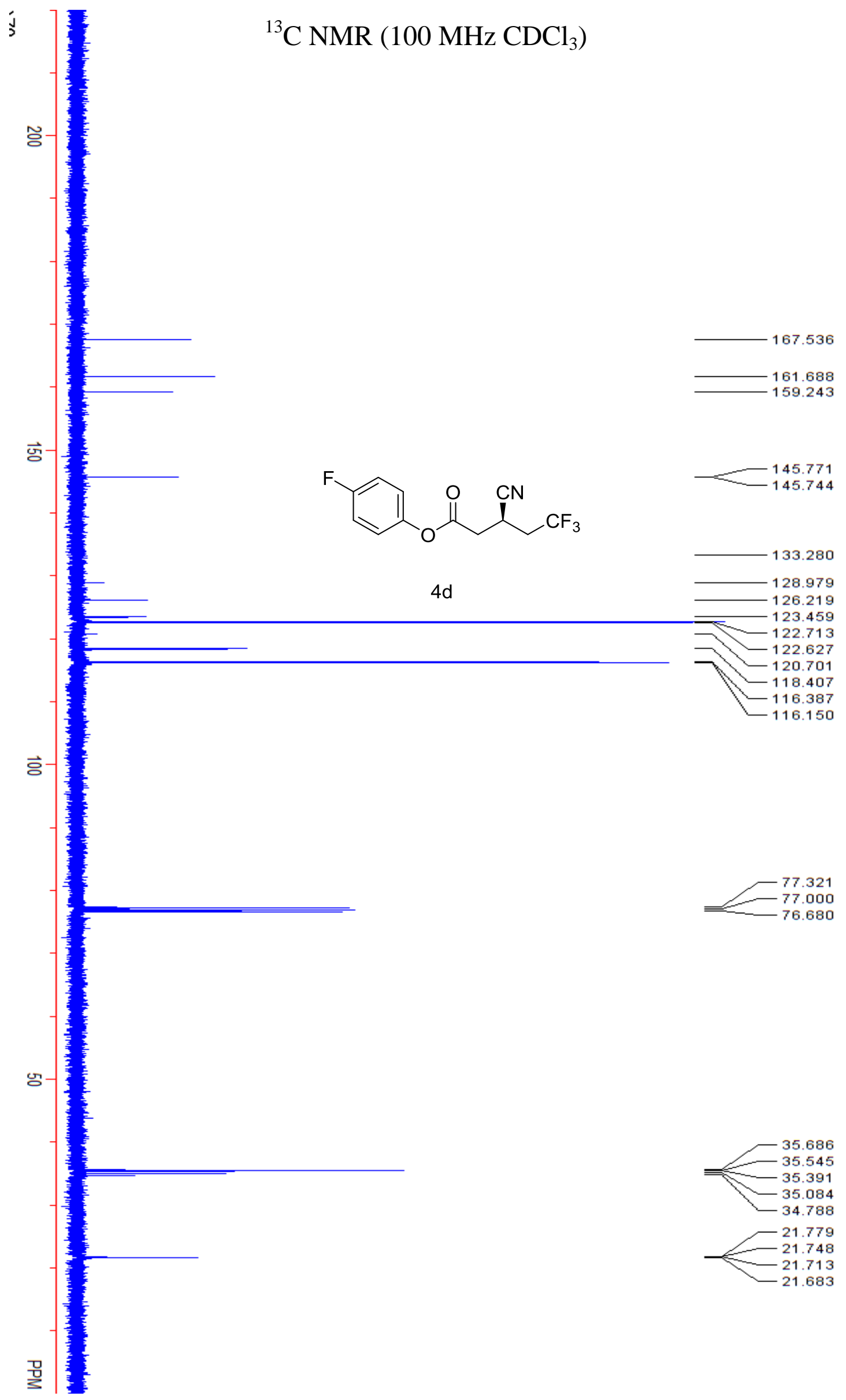




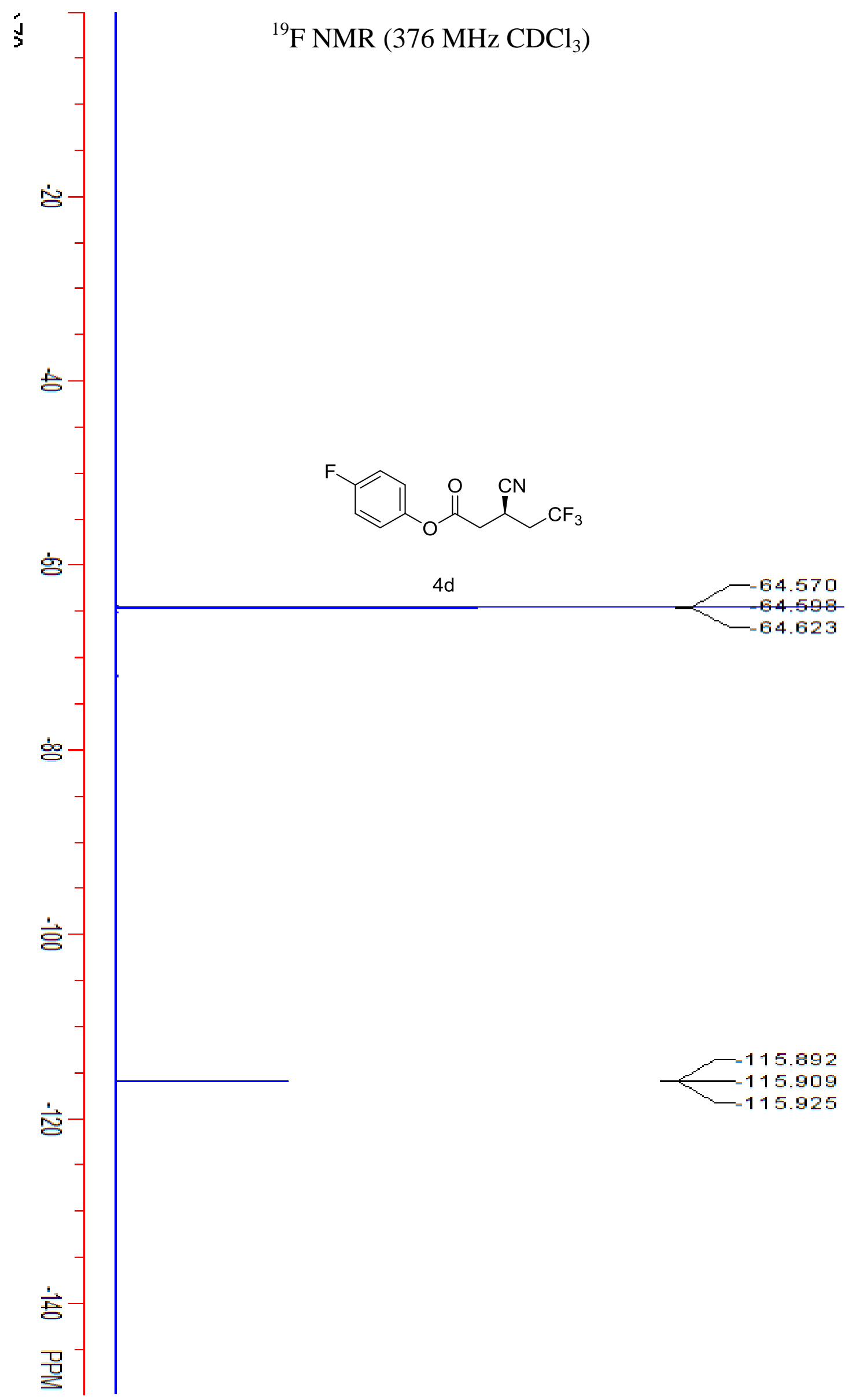




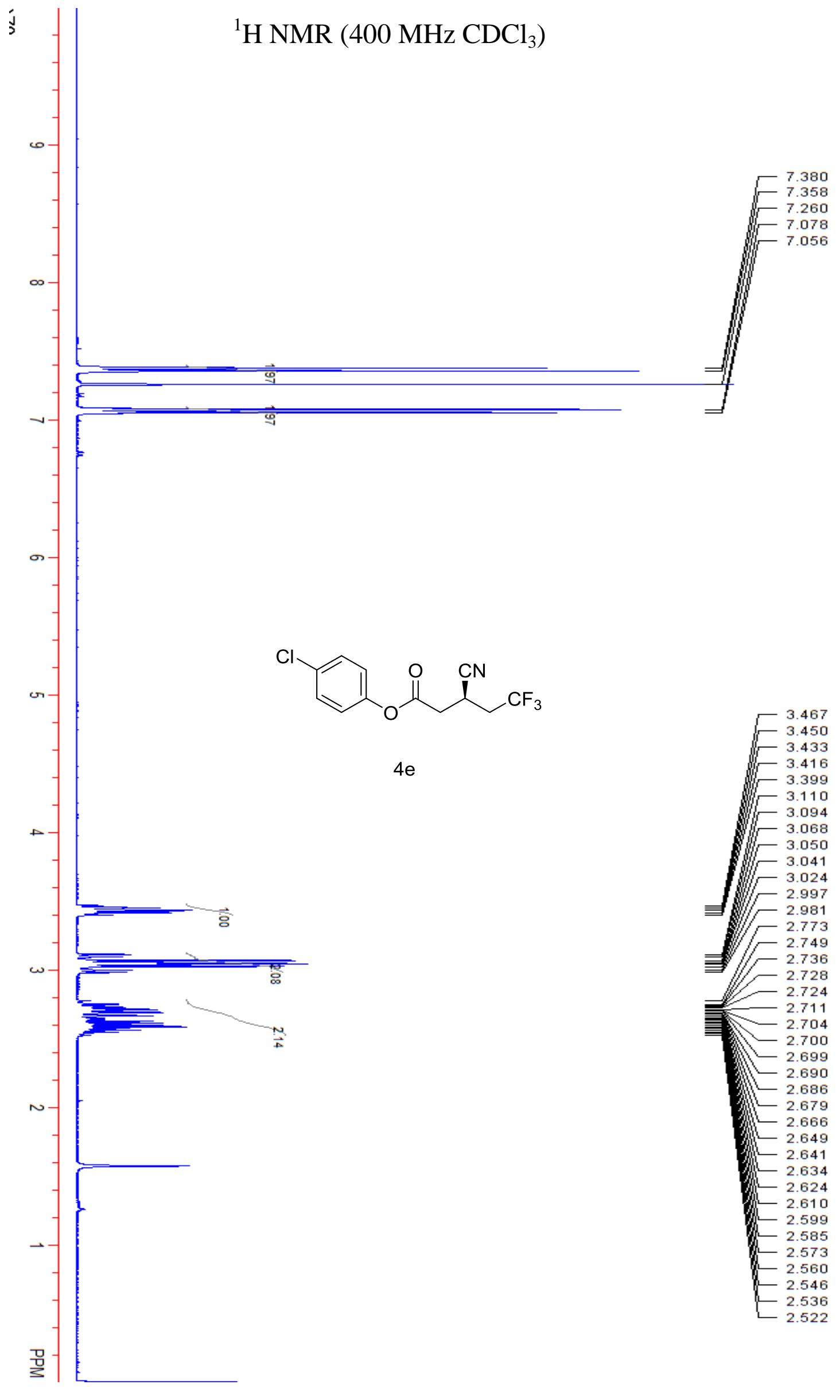


${ }^{13} \mathrm{C}$ NMR (100 MHz $\mathrm{CDCl}_{3}$ )

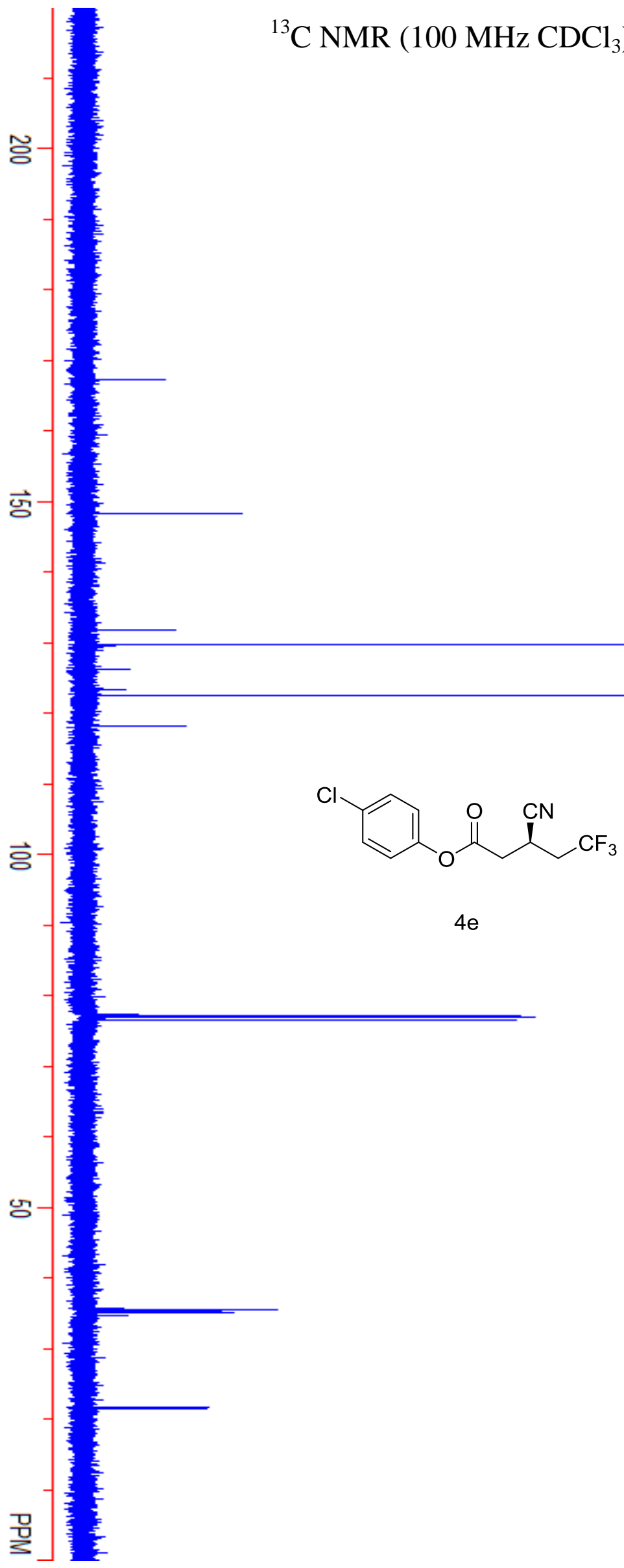

167.253

148.386

131.934 129.686 129.668 126.194 123.435 122.581 $-118.315$

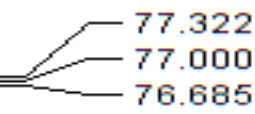




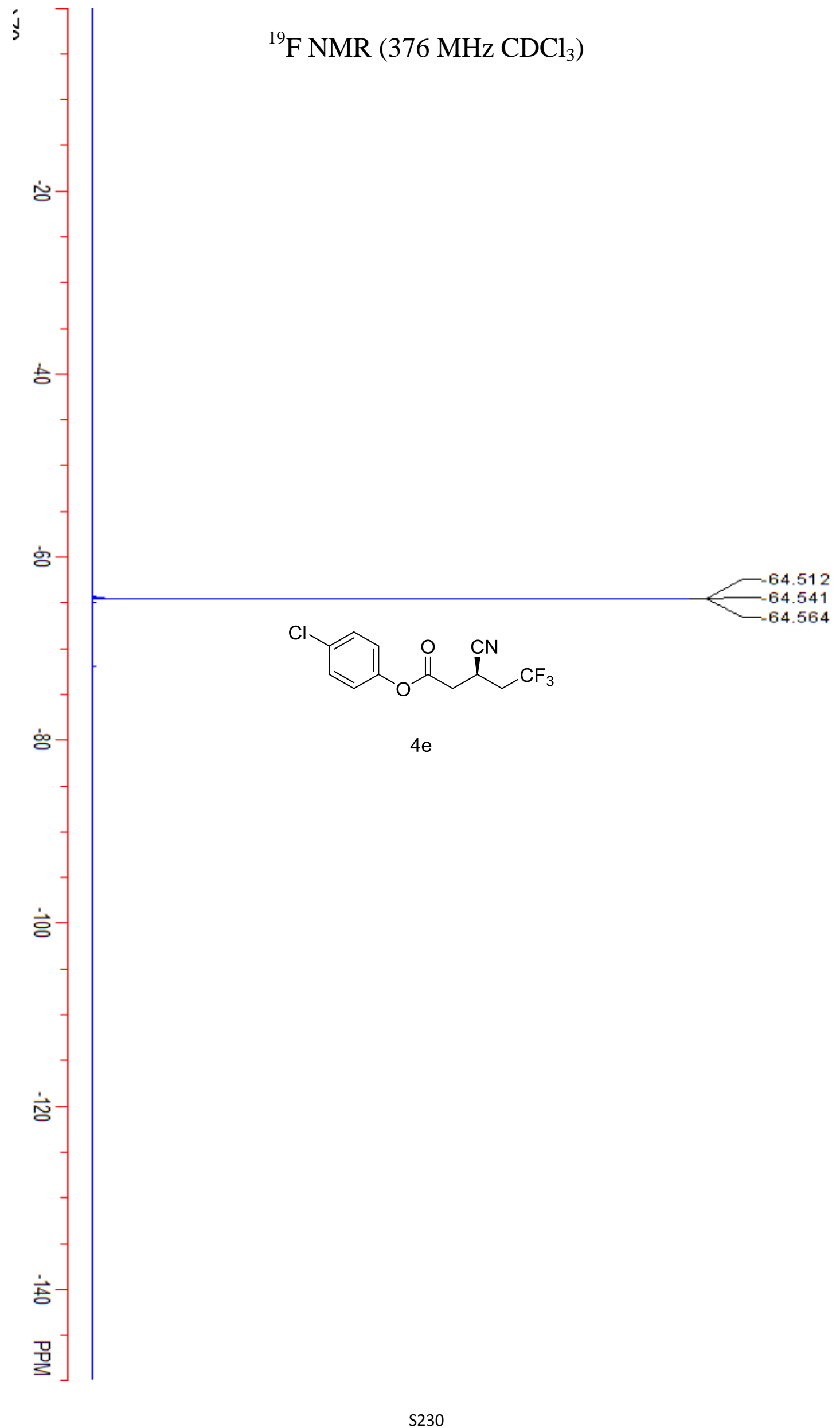




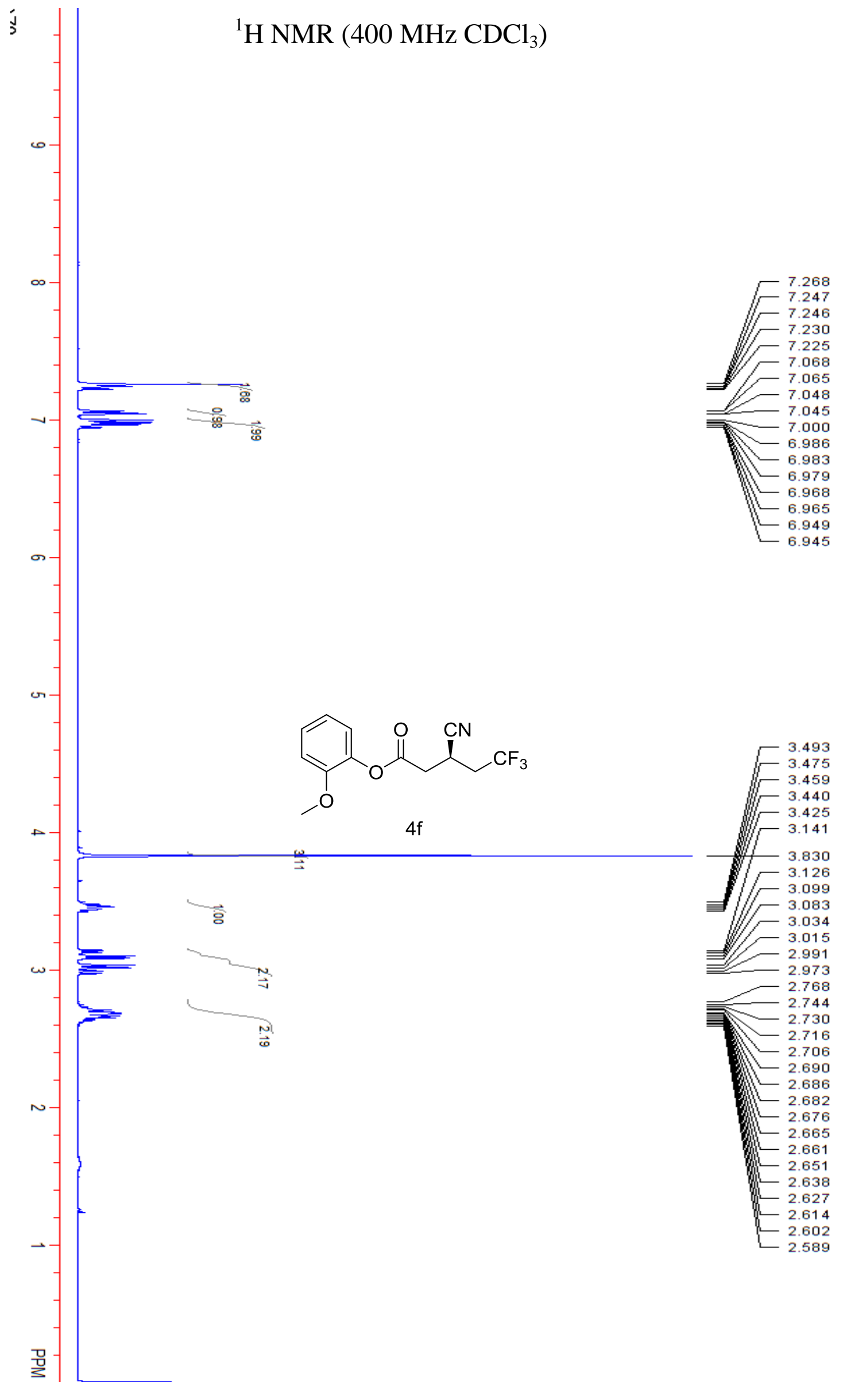




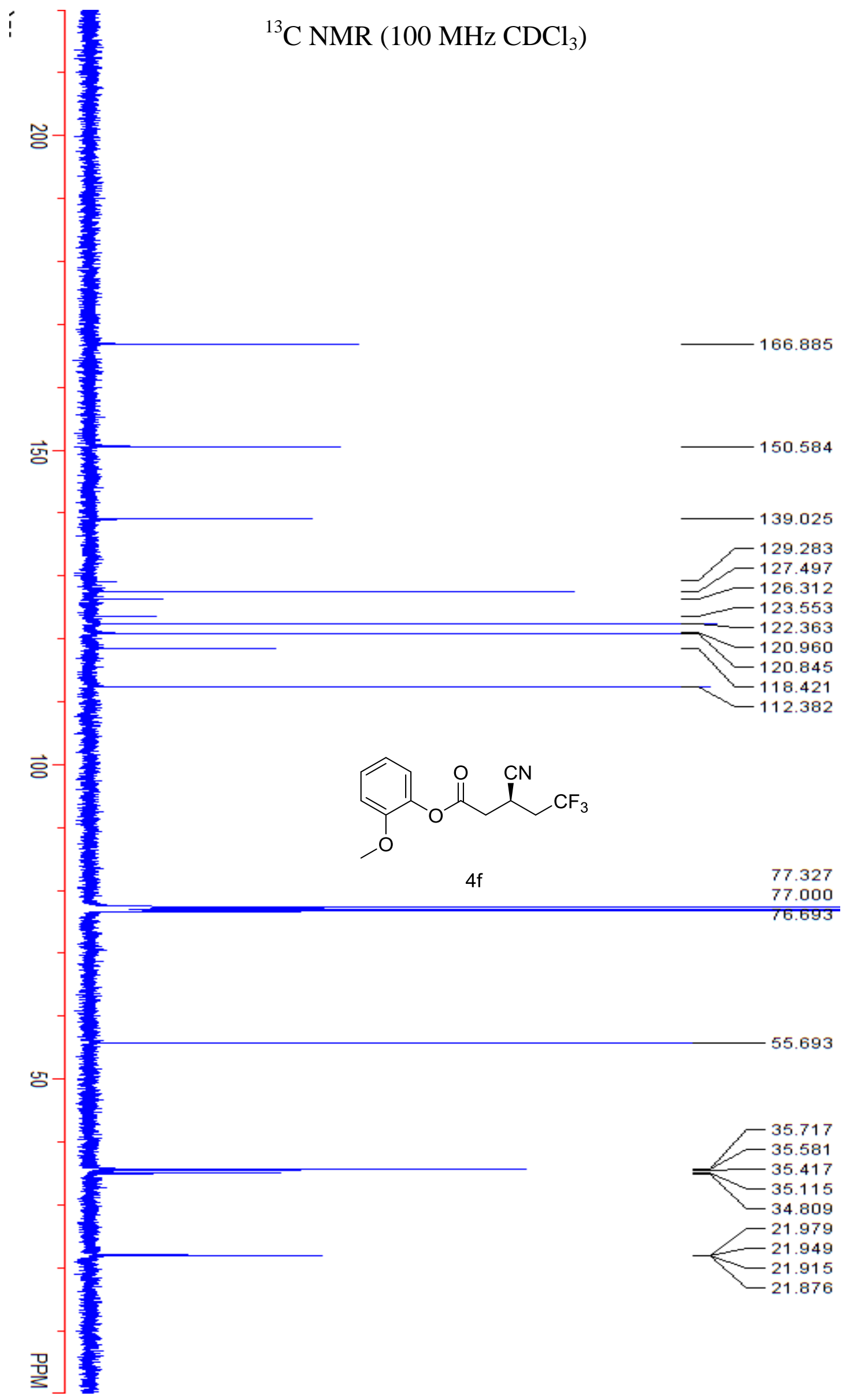




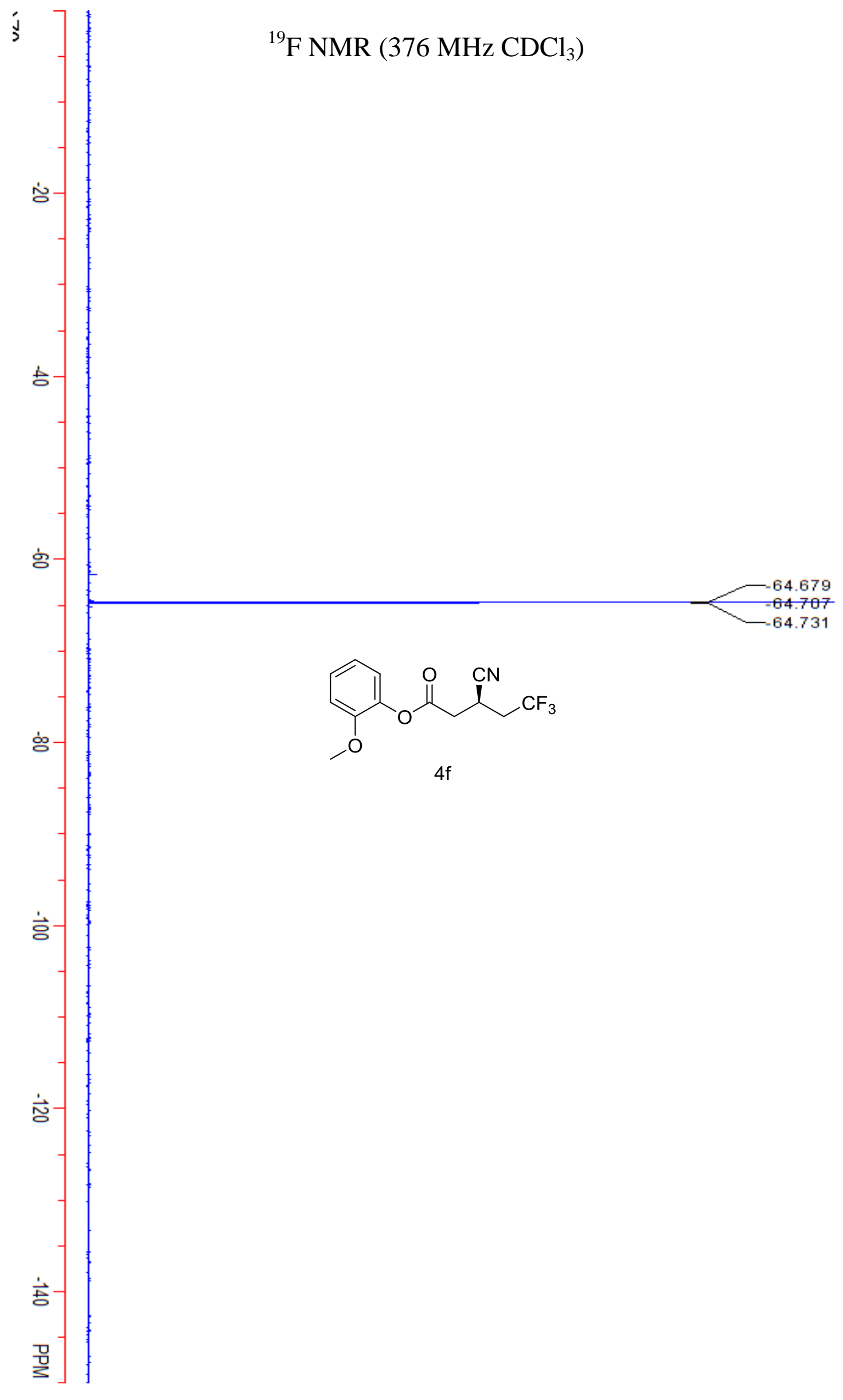




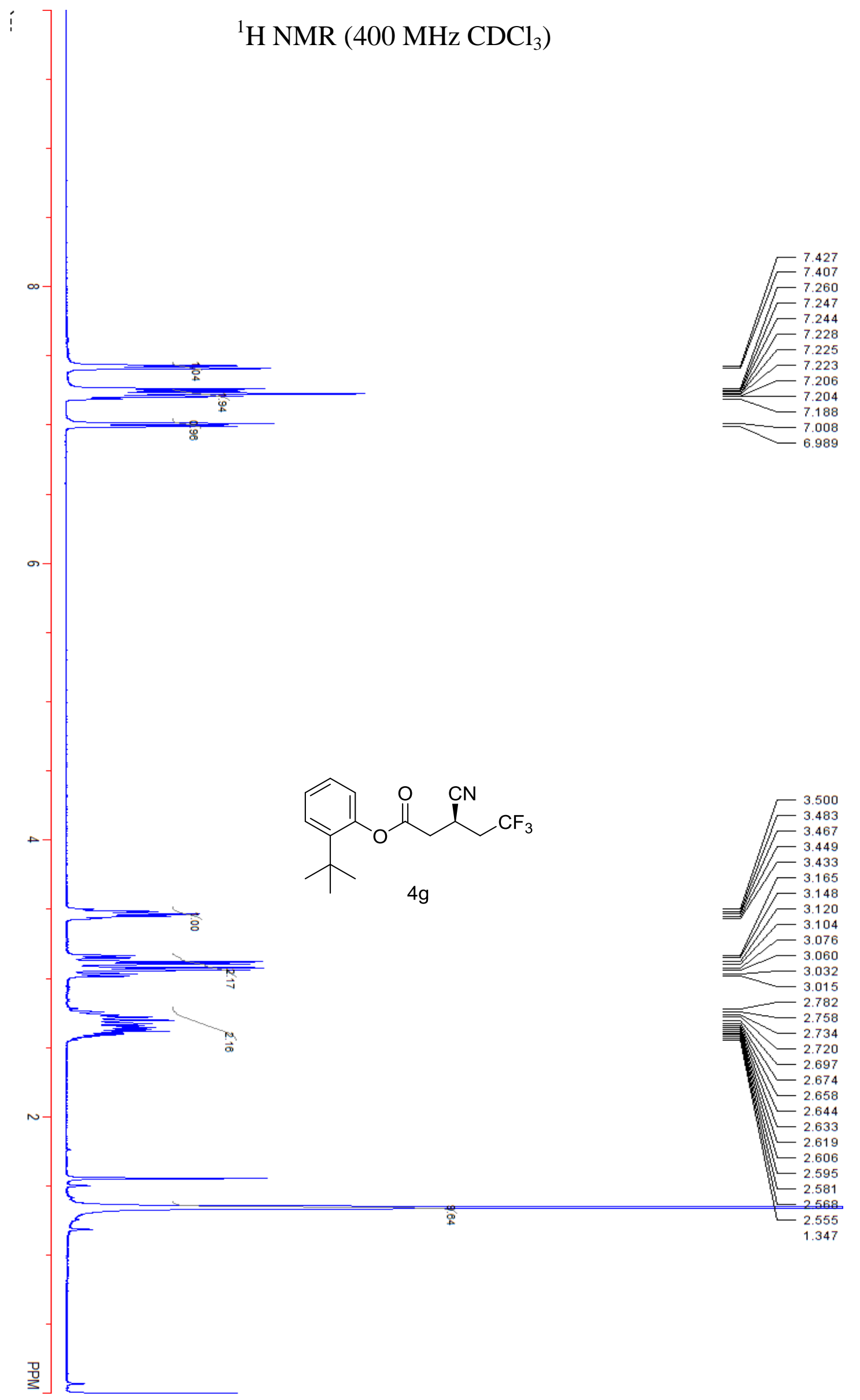




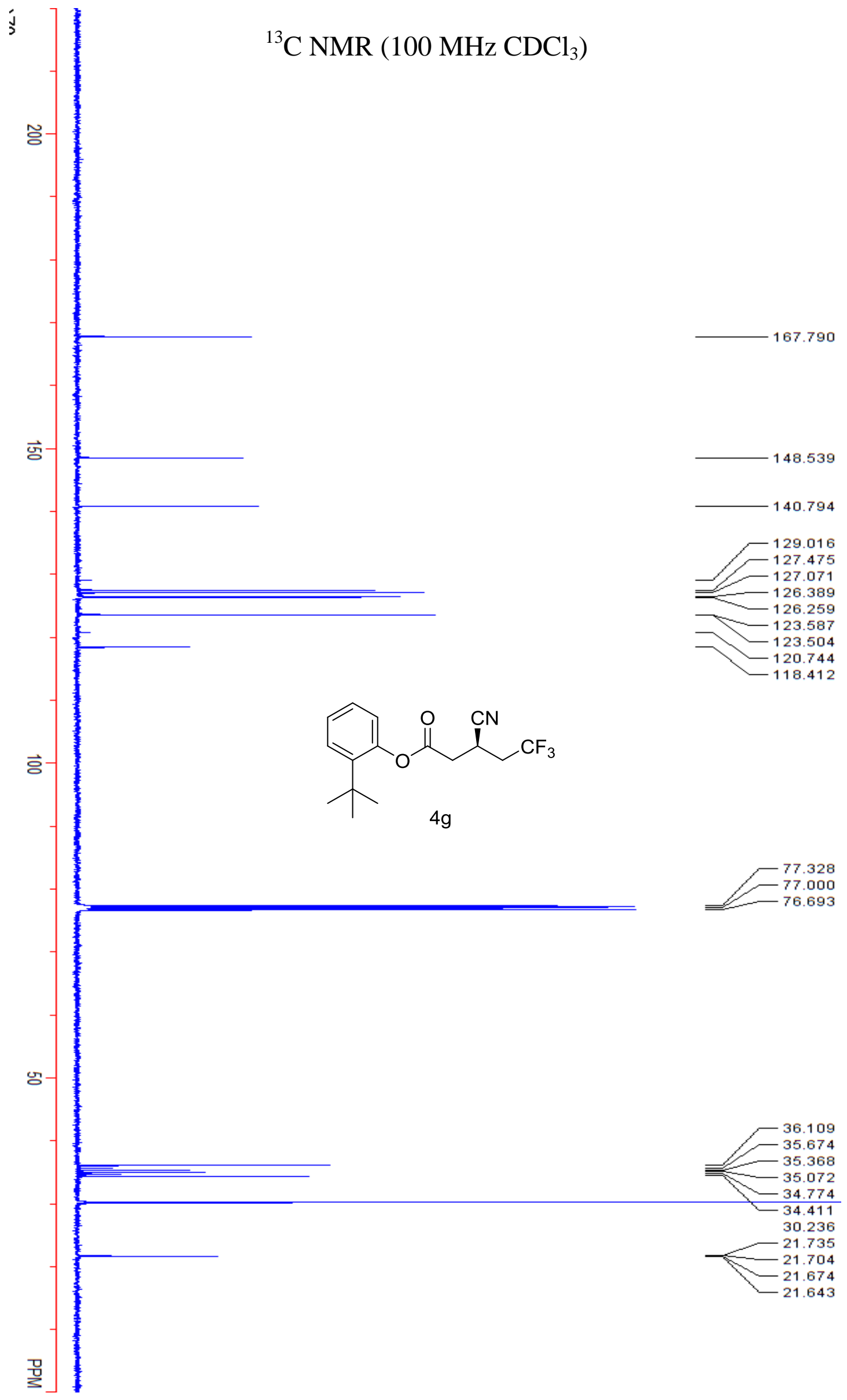



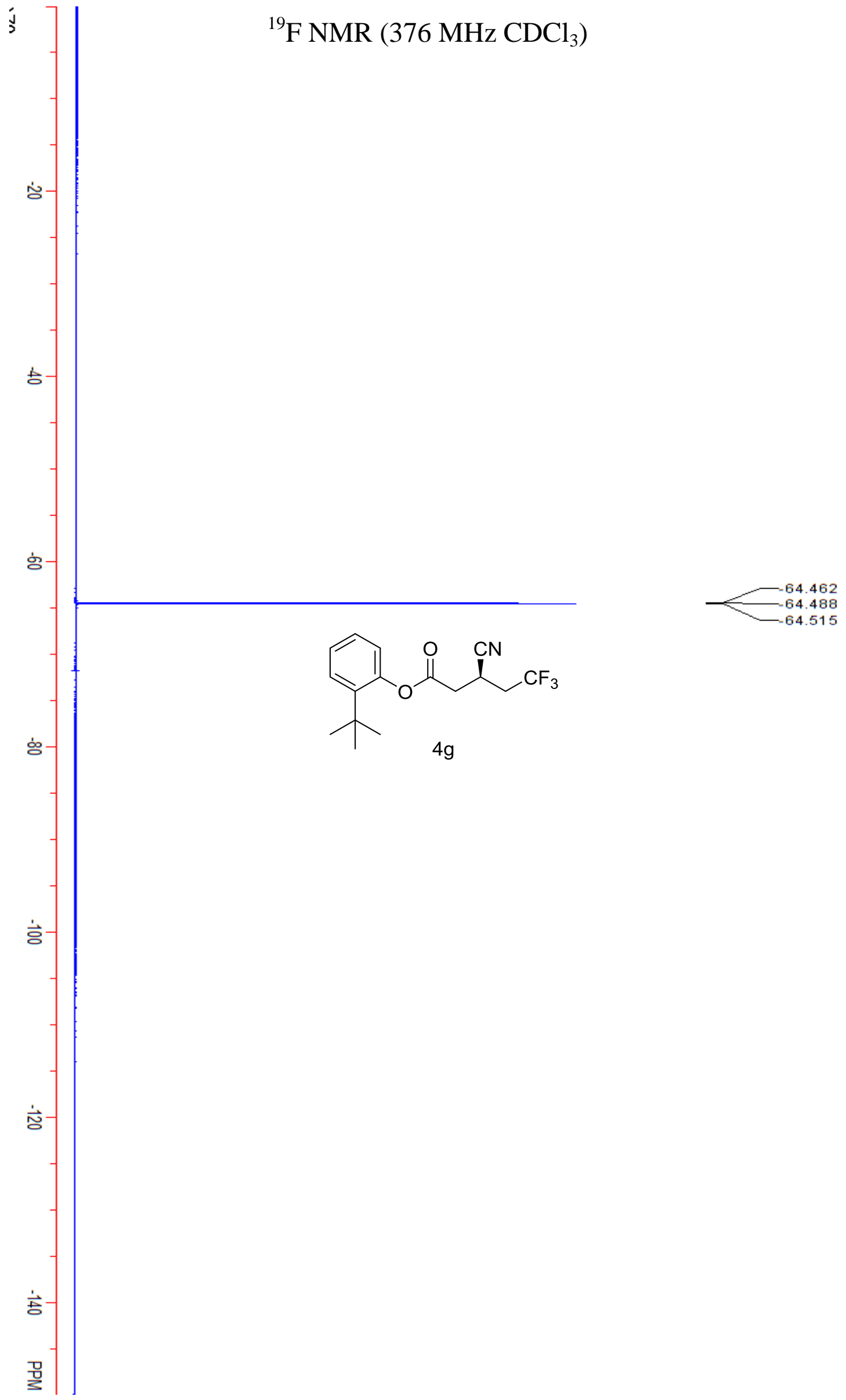

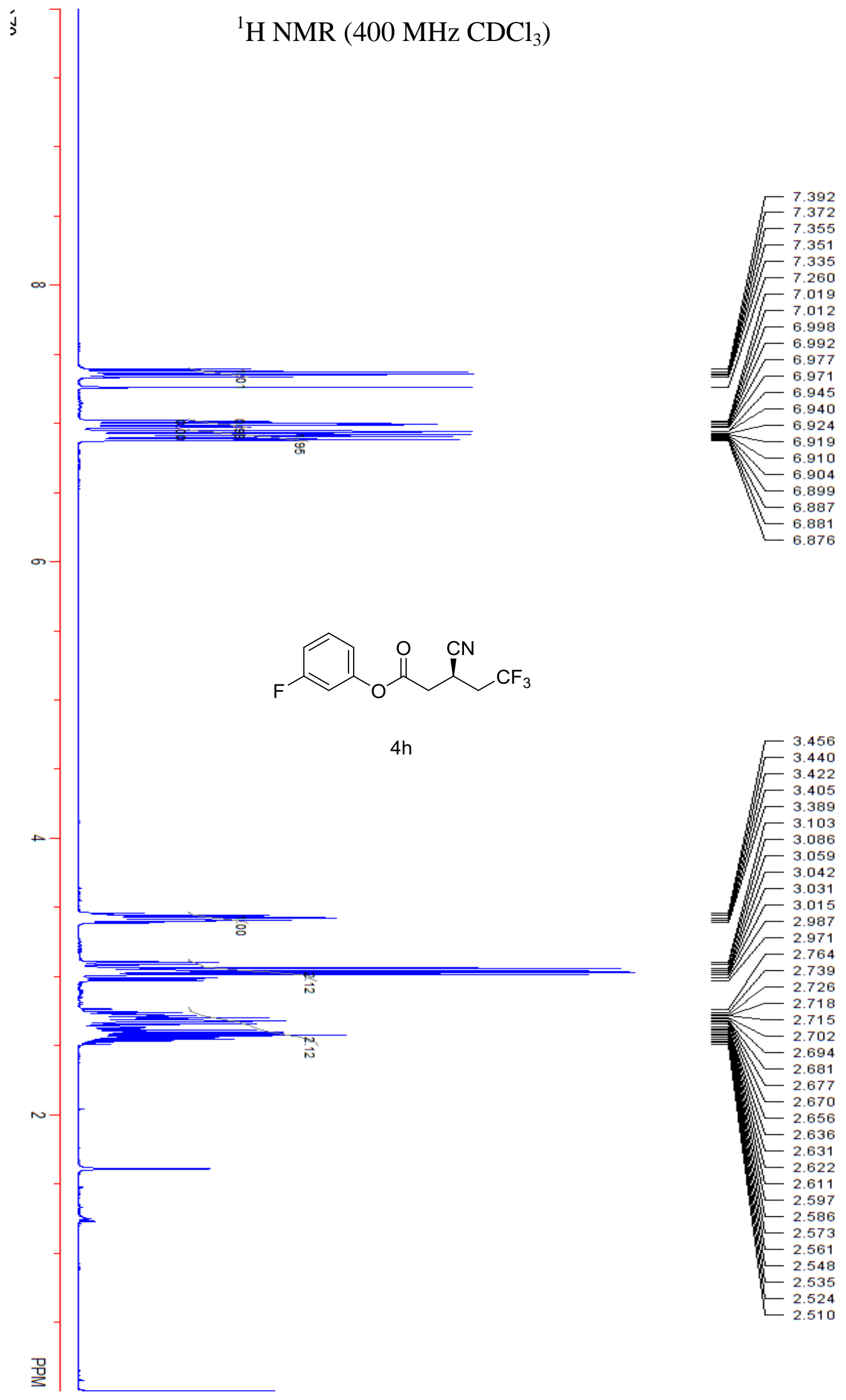


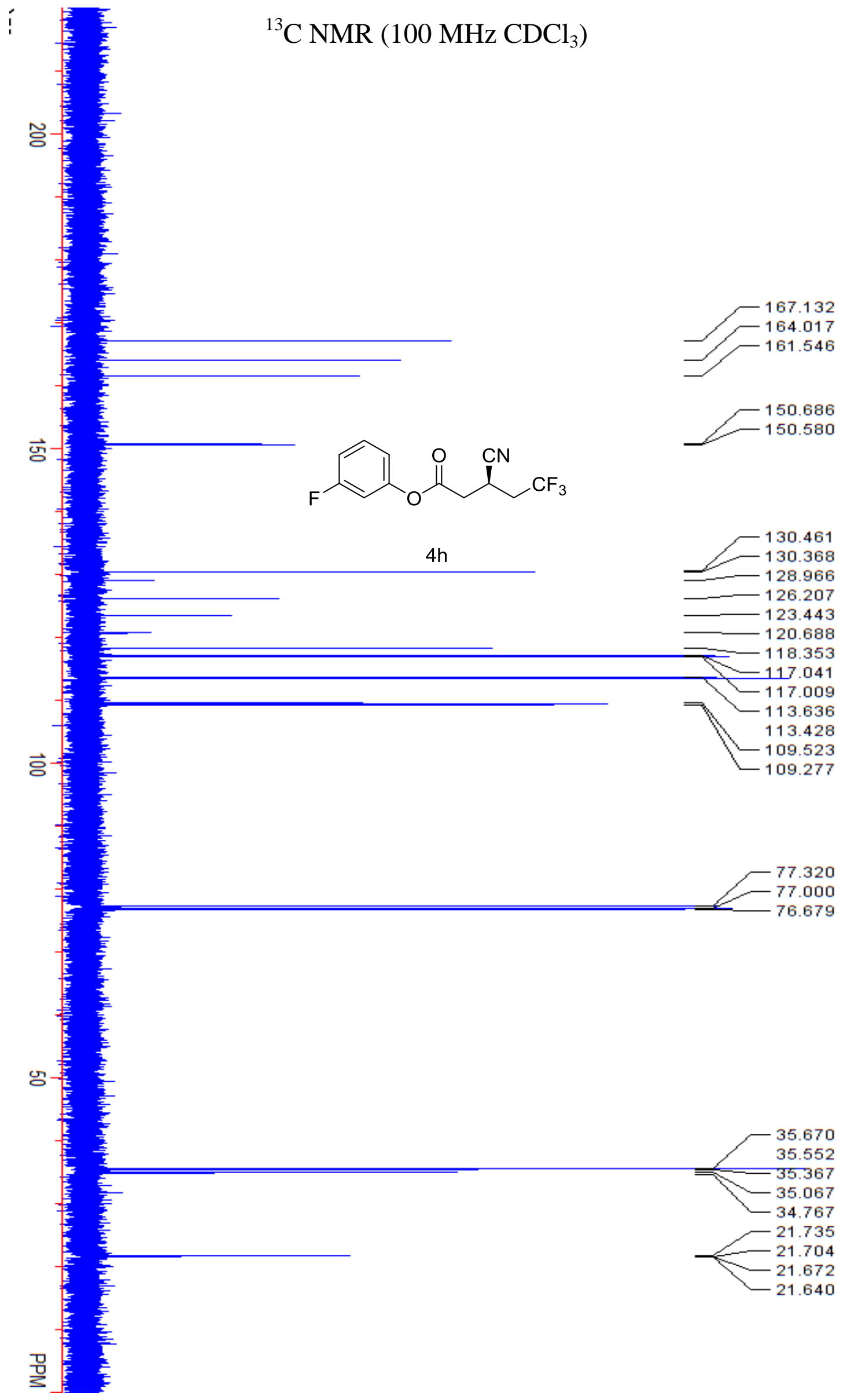




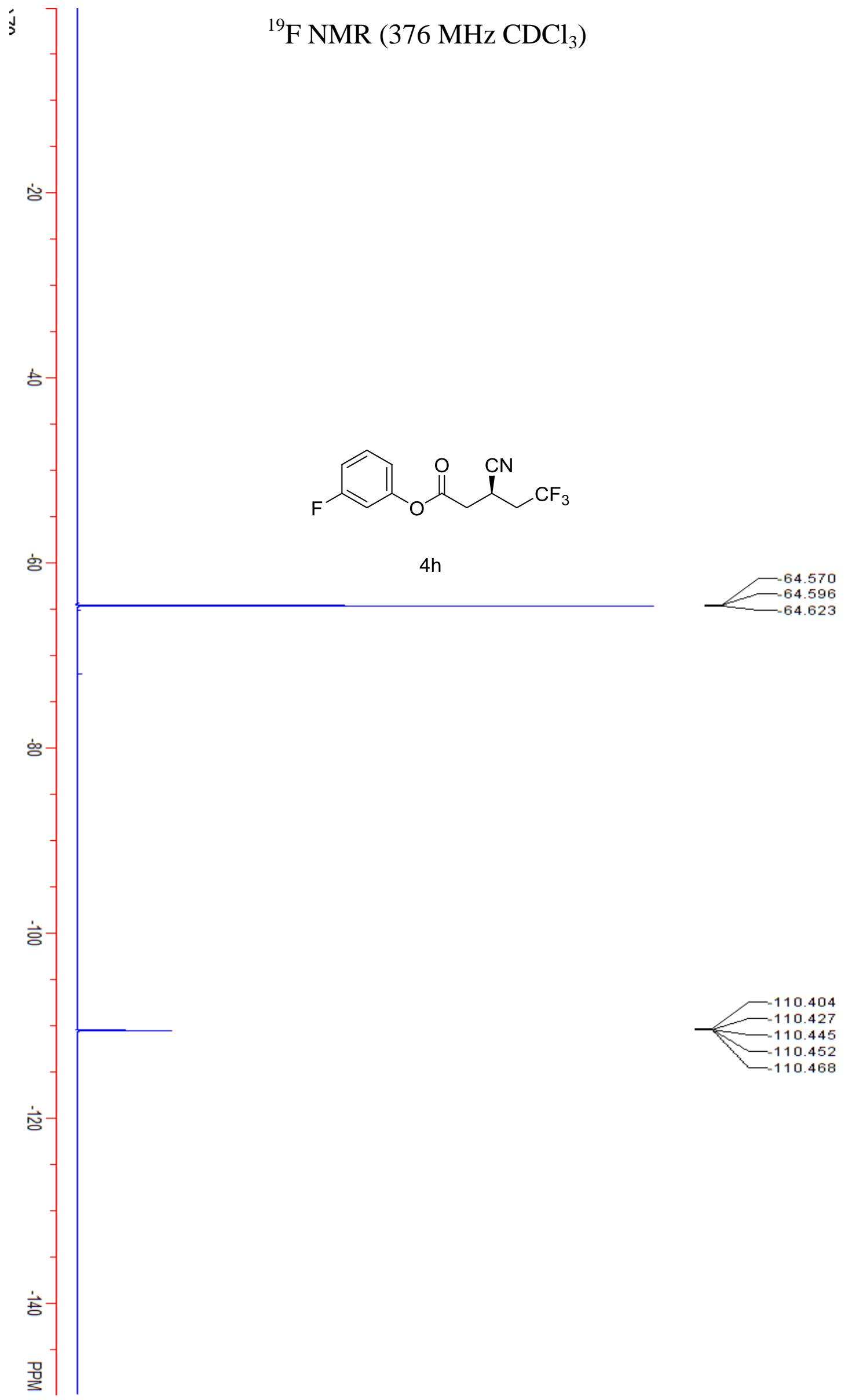




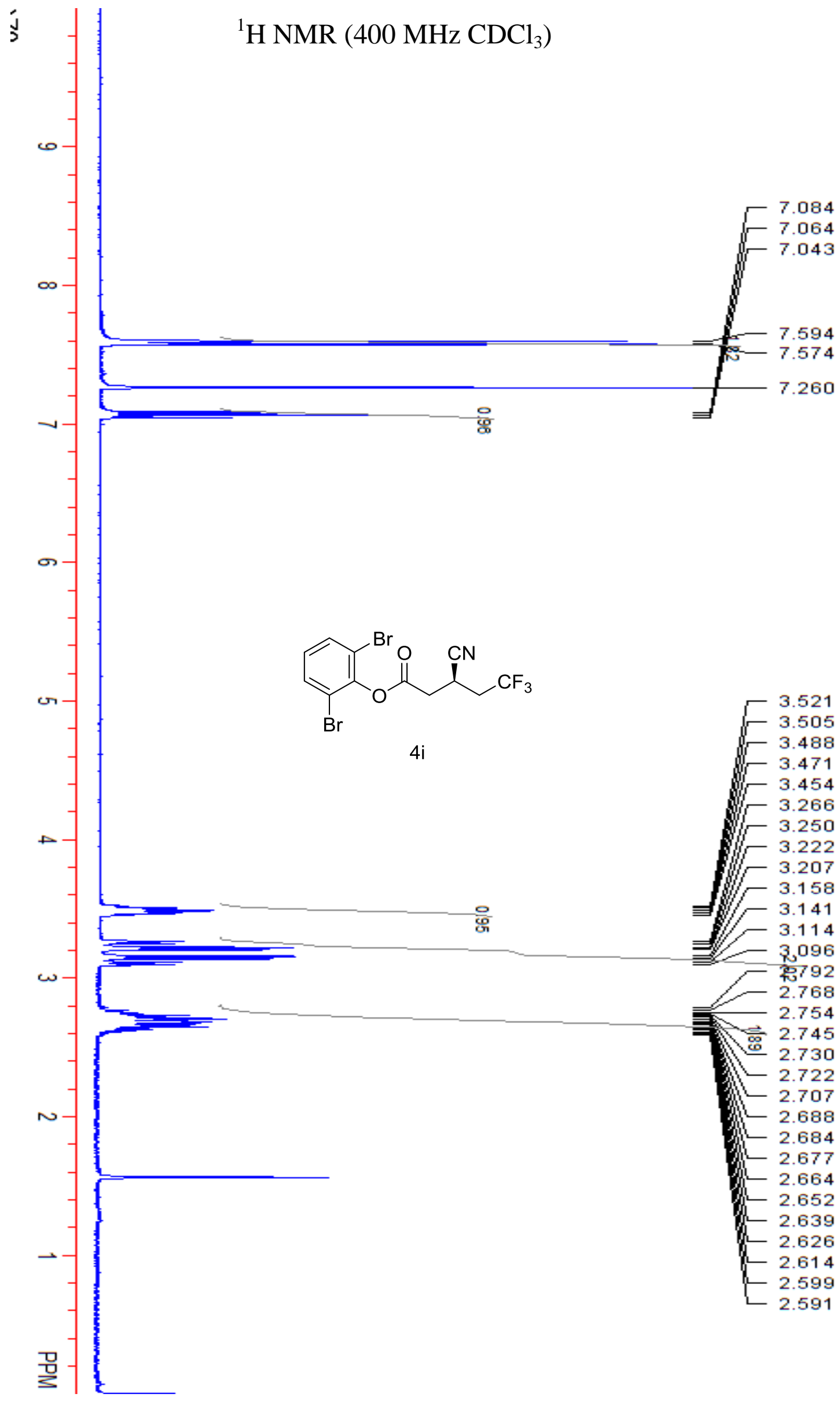




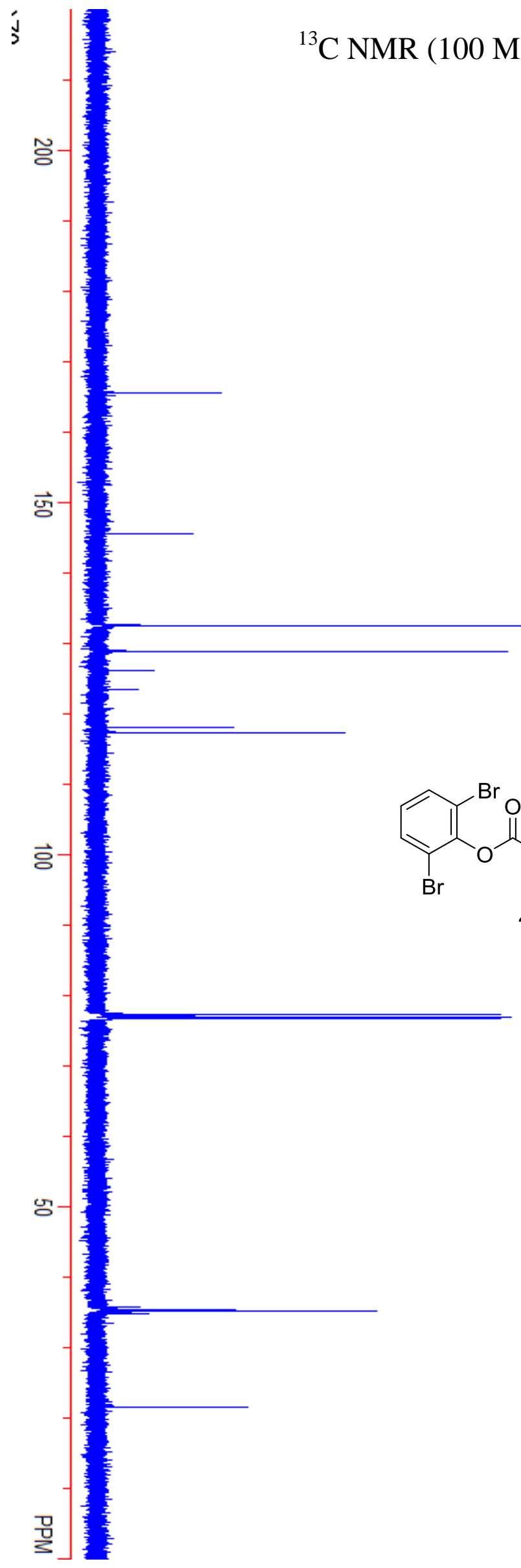

165.529

145.454

432.549

128.804

126.186

123.424

118.105

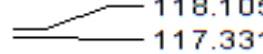

CN

$\mathrm{CF}_{3}$

$4 i$
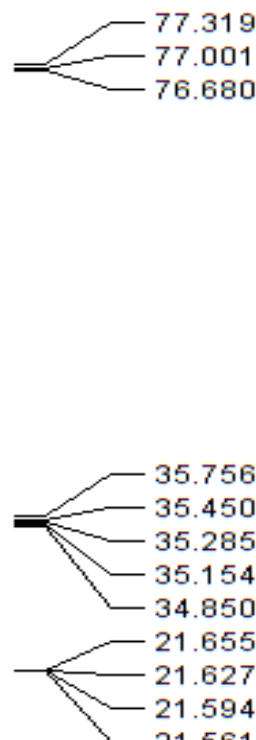


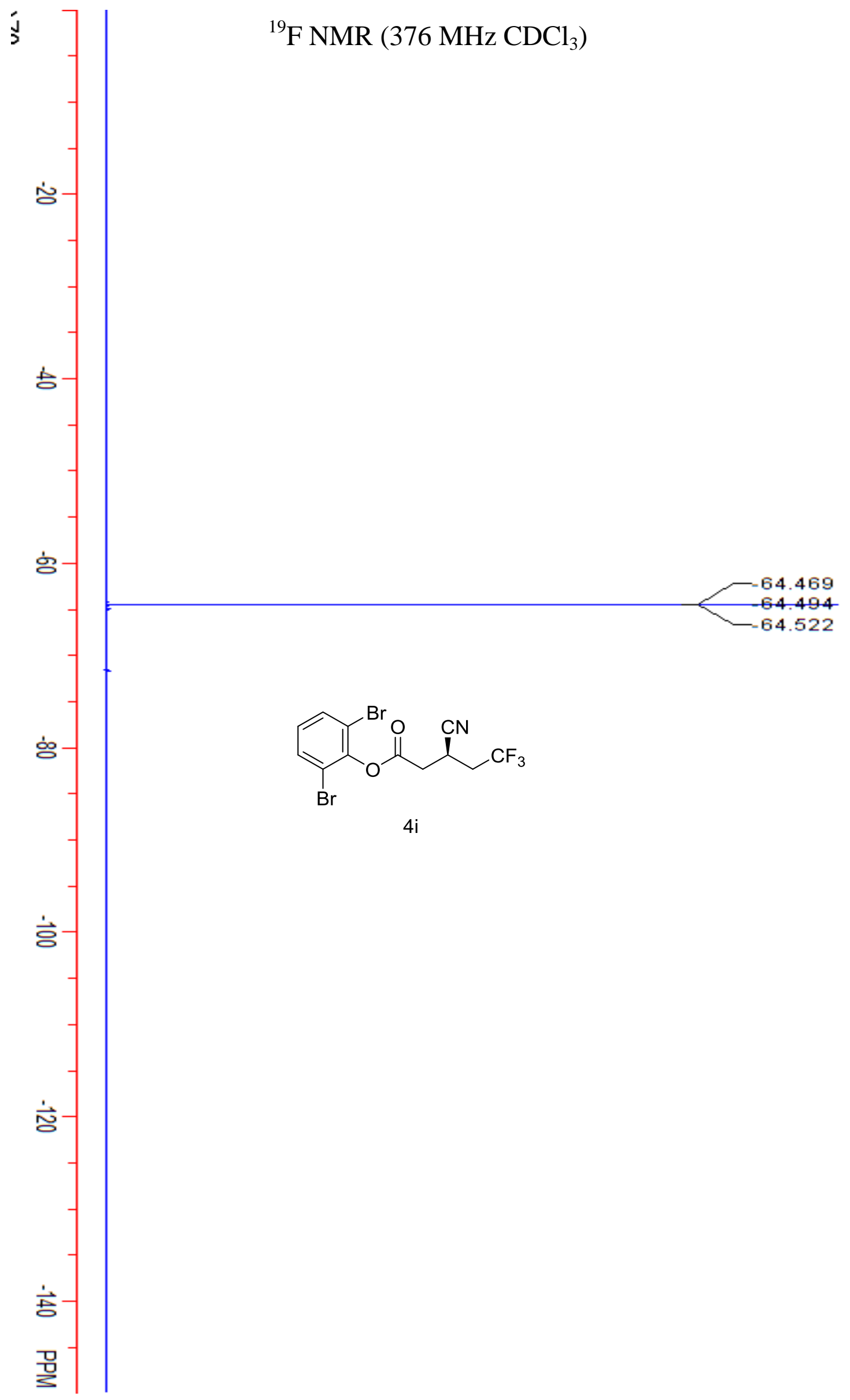




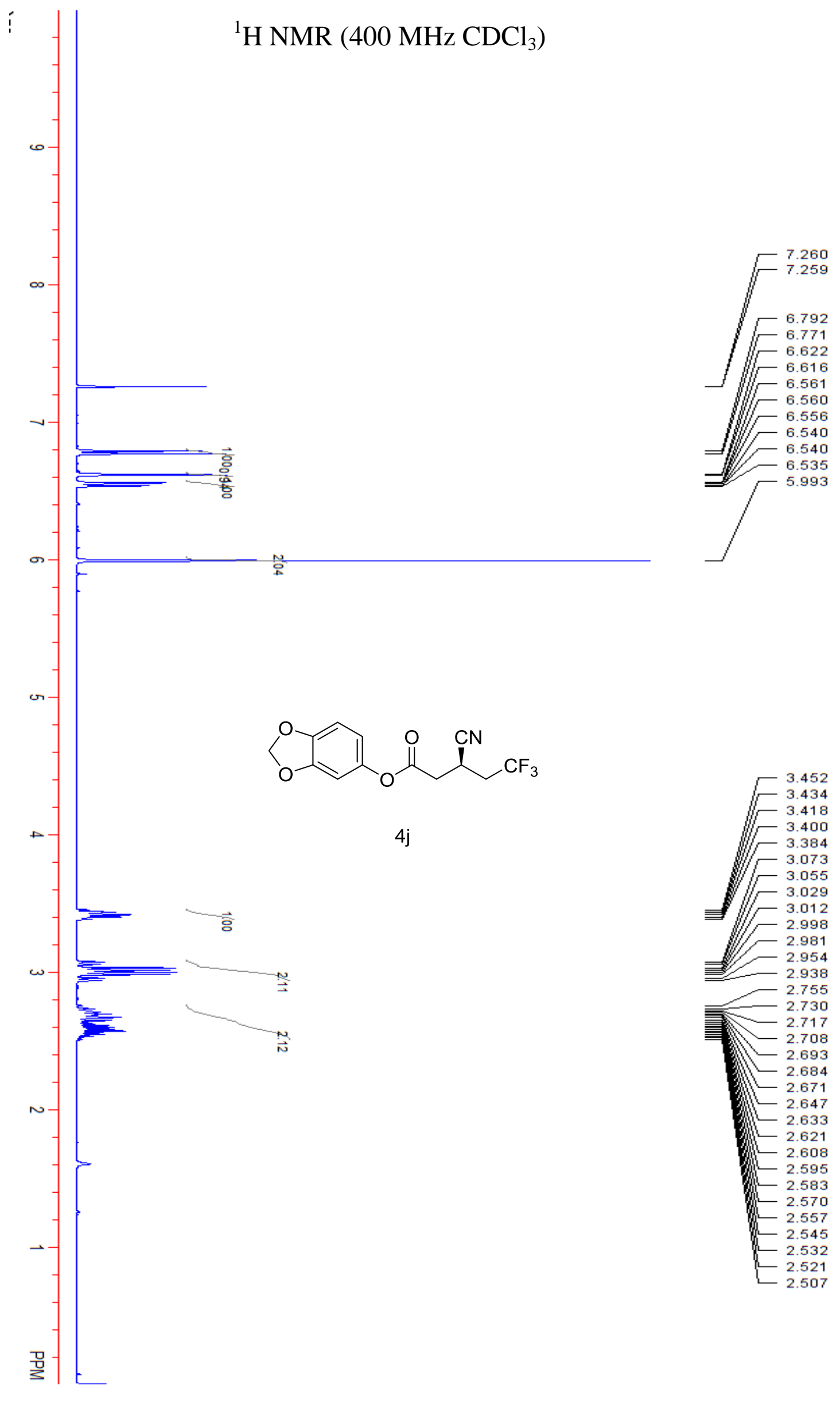




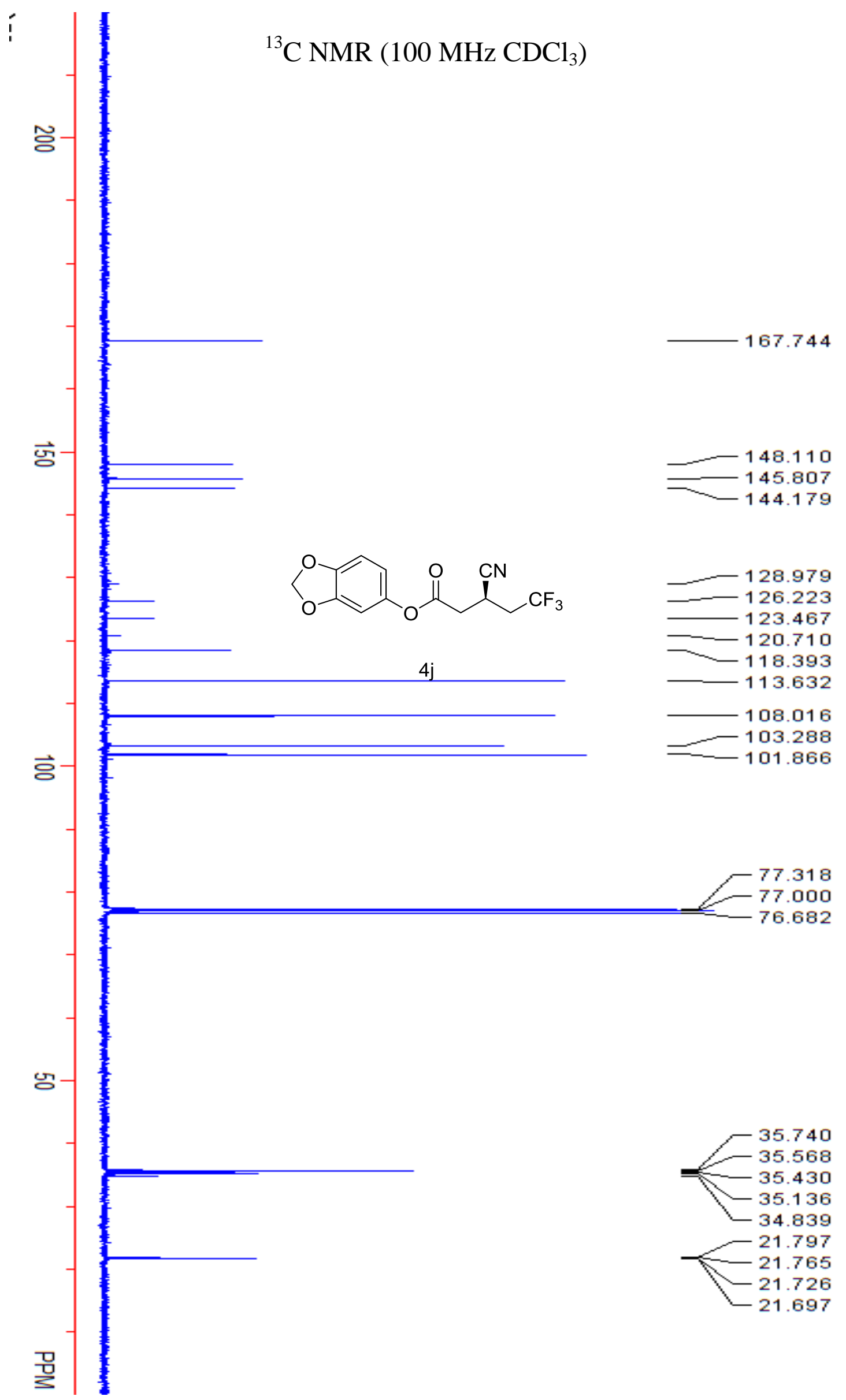



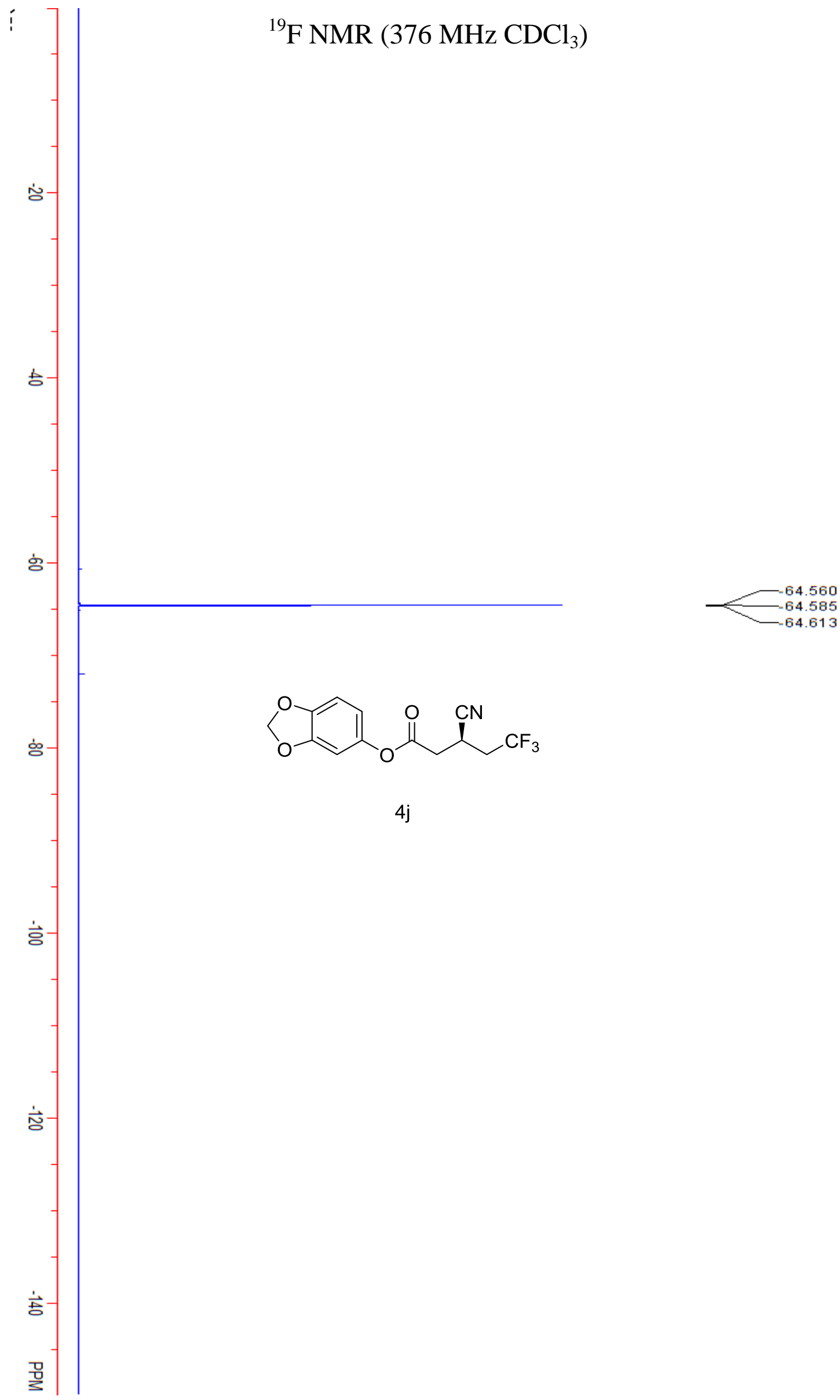

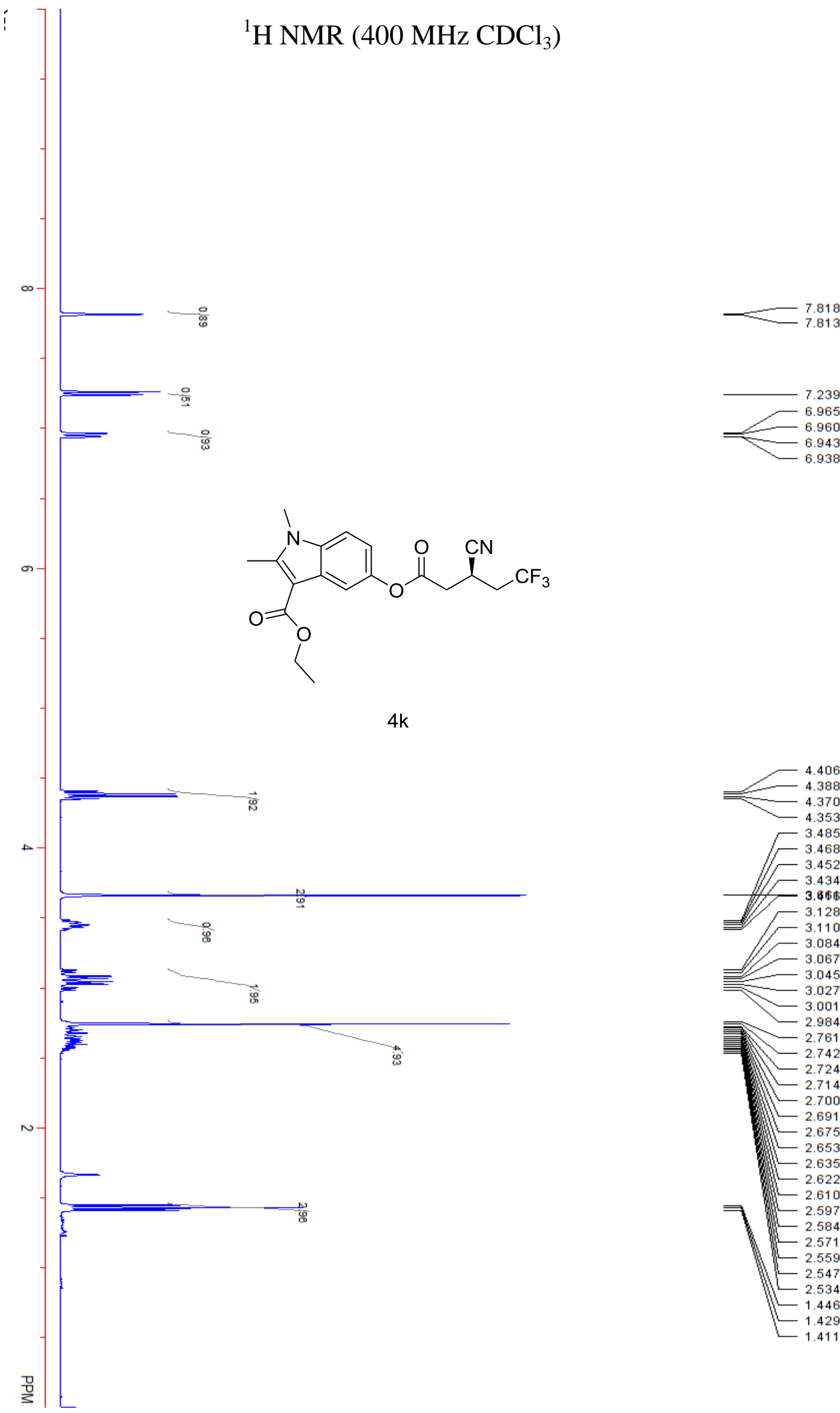


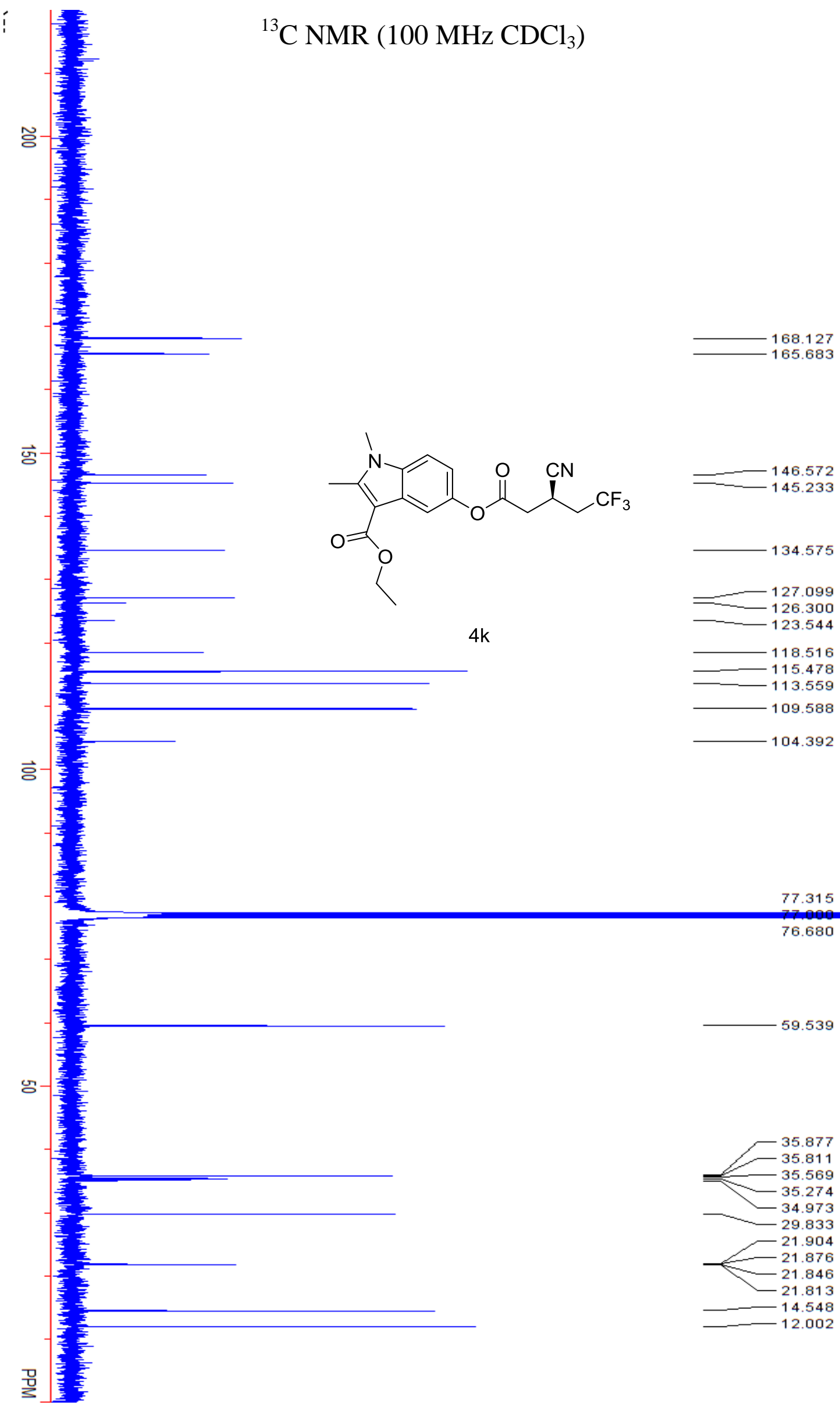




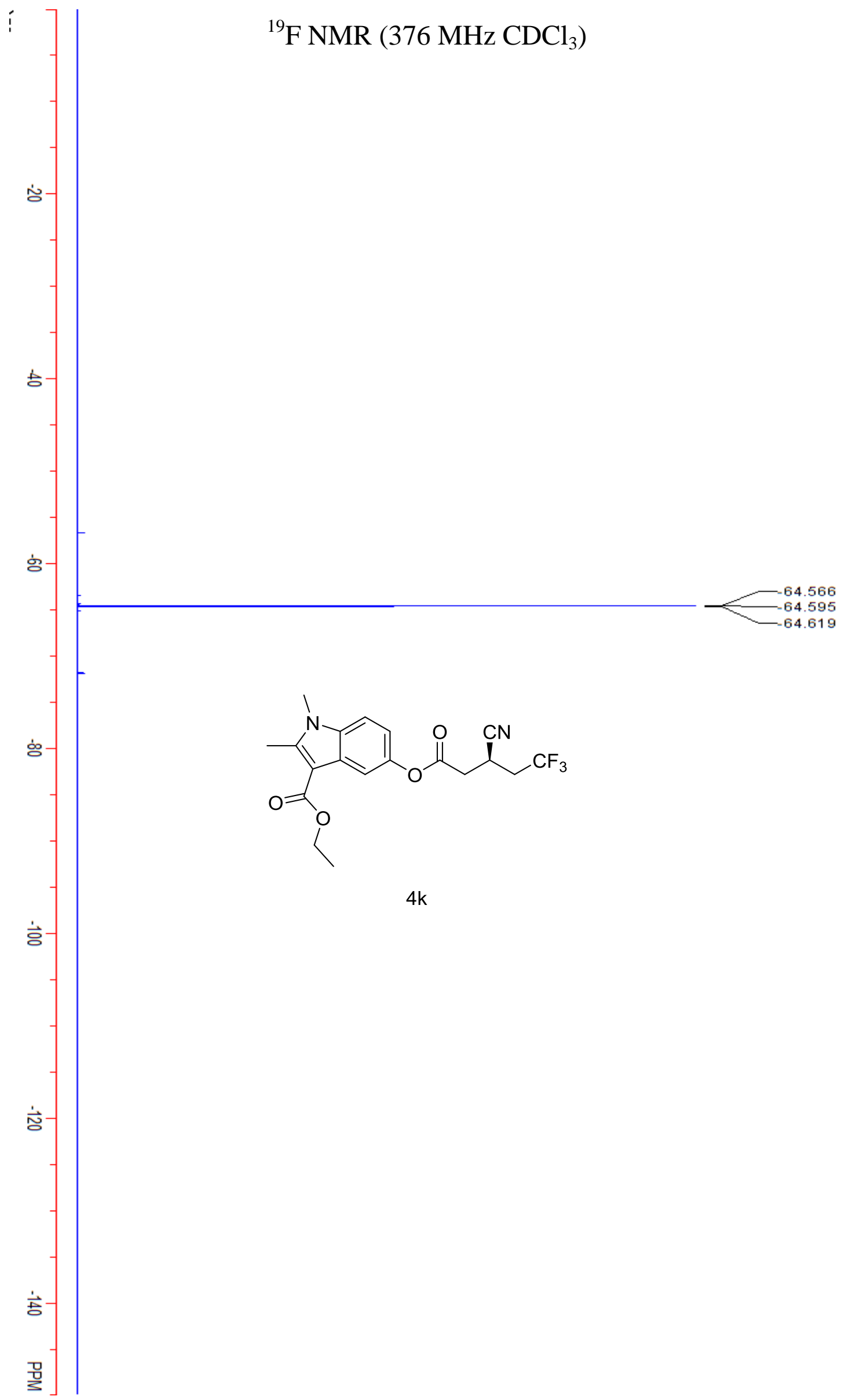




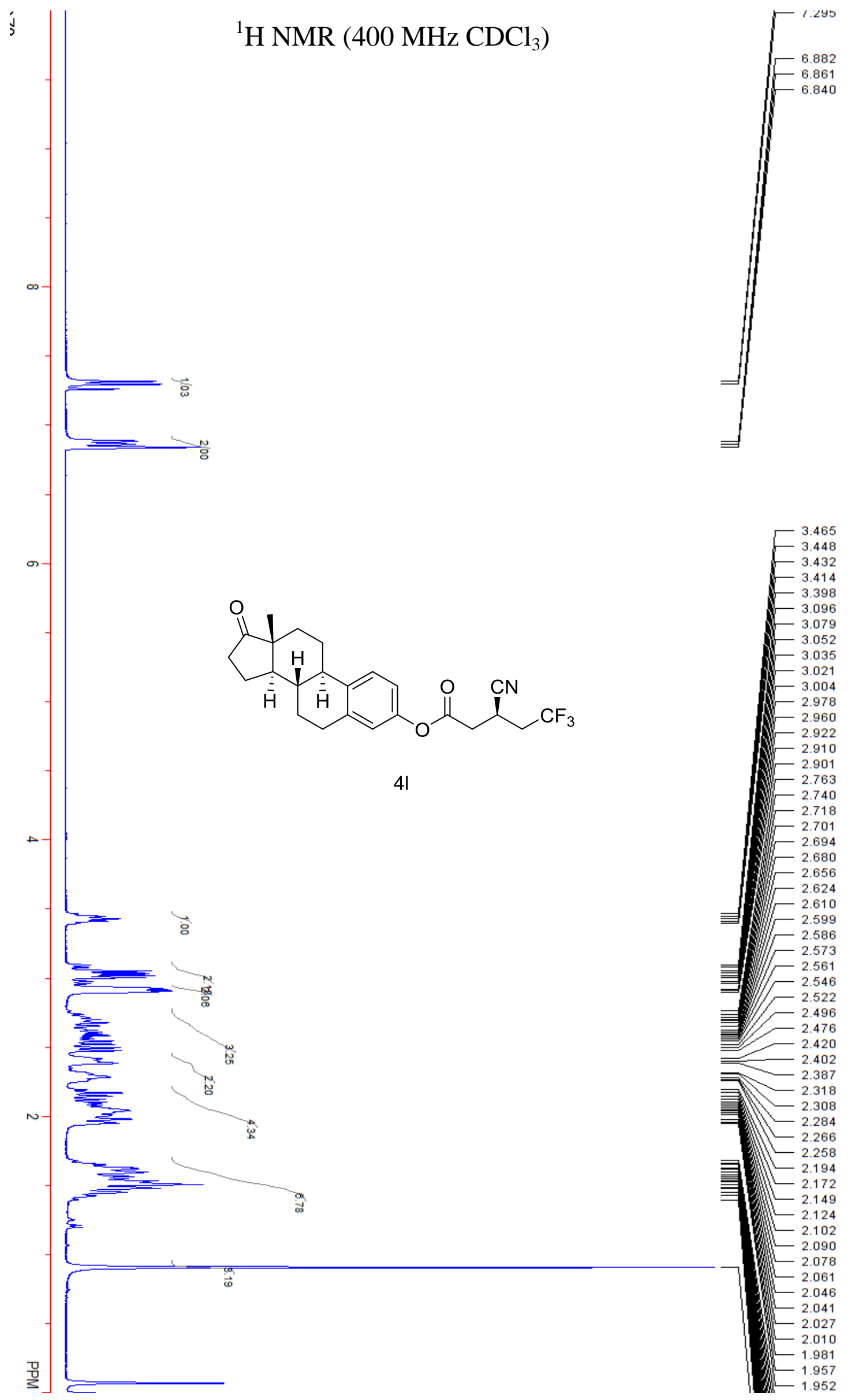



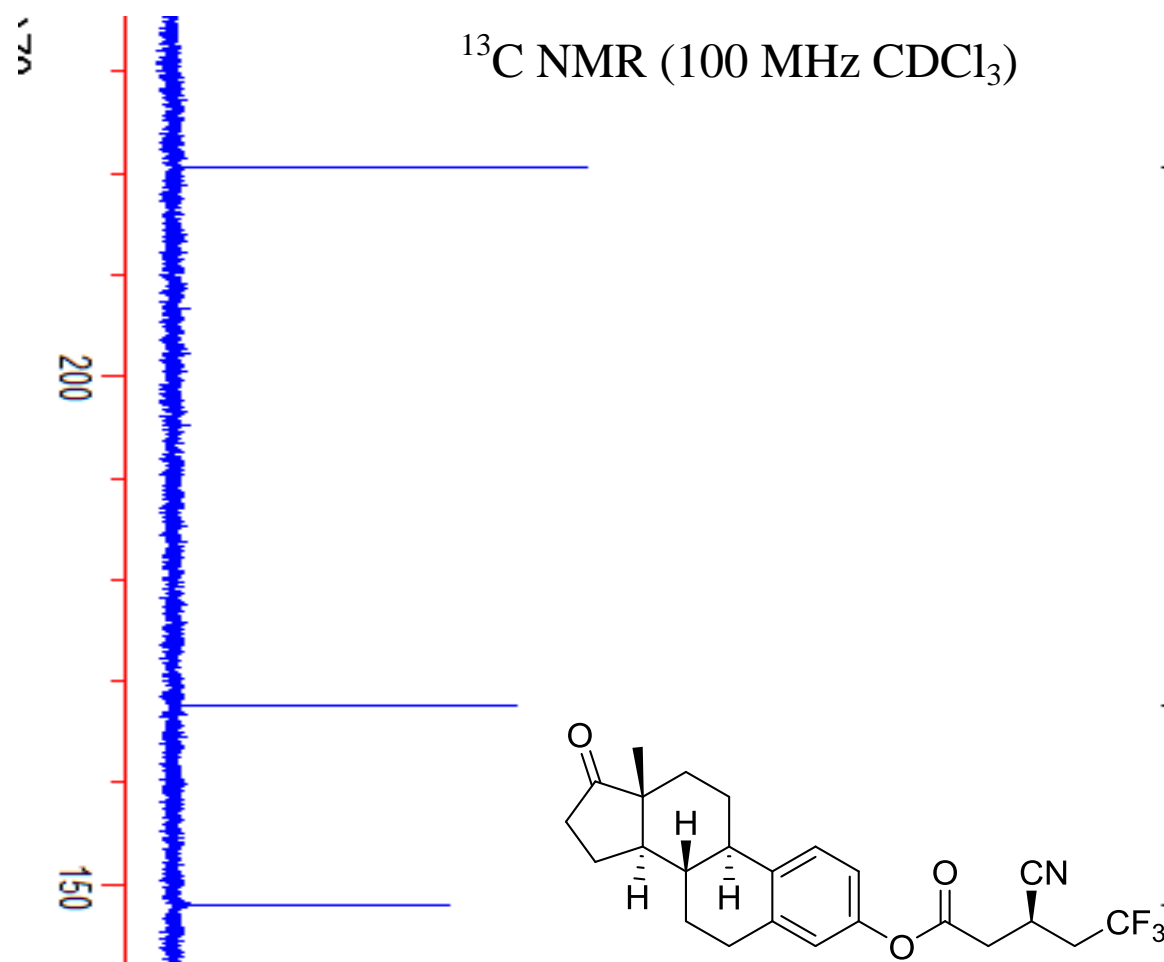

167.665

$\overrightarrow{\text { 횽 }}$

용

41

77.319

77.000

76.682

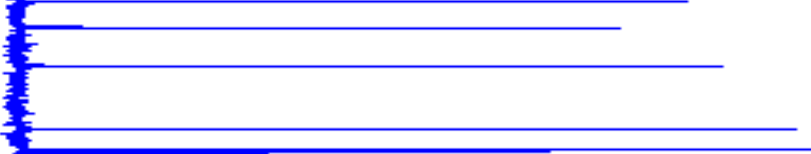

50.372

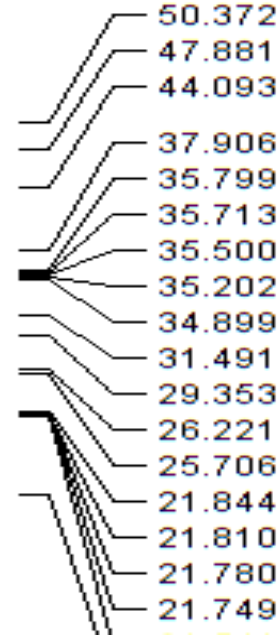


${ }^{19} \mathrm{~F}$ NMR (376 MHz $\left.\mathrm{CDCl}_{3}\right)$

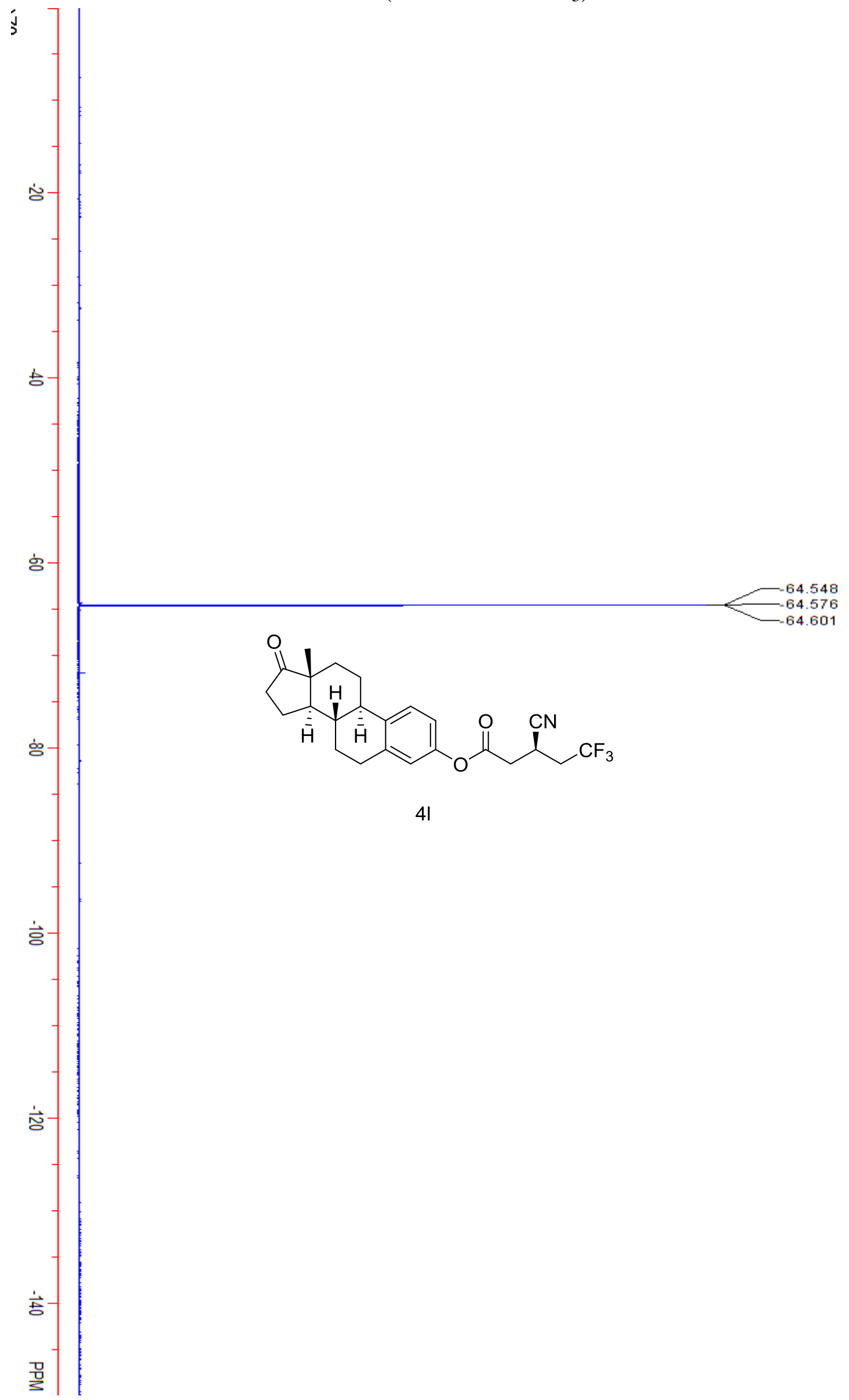




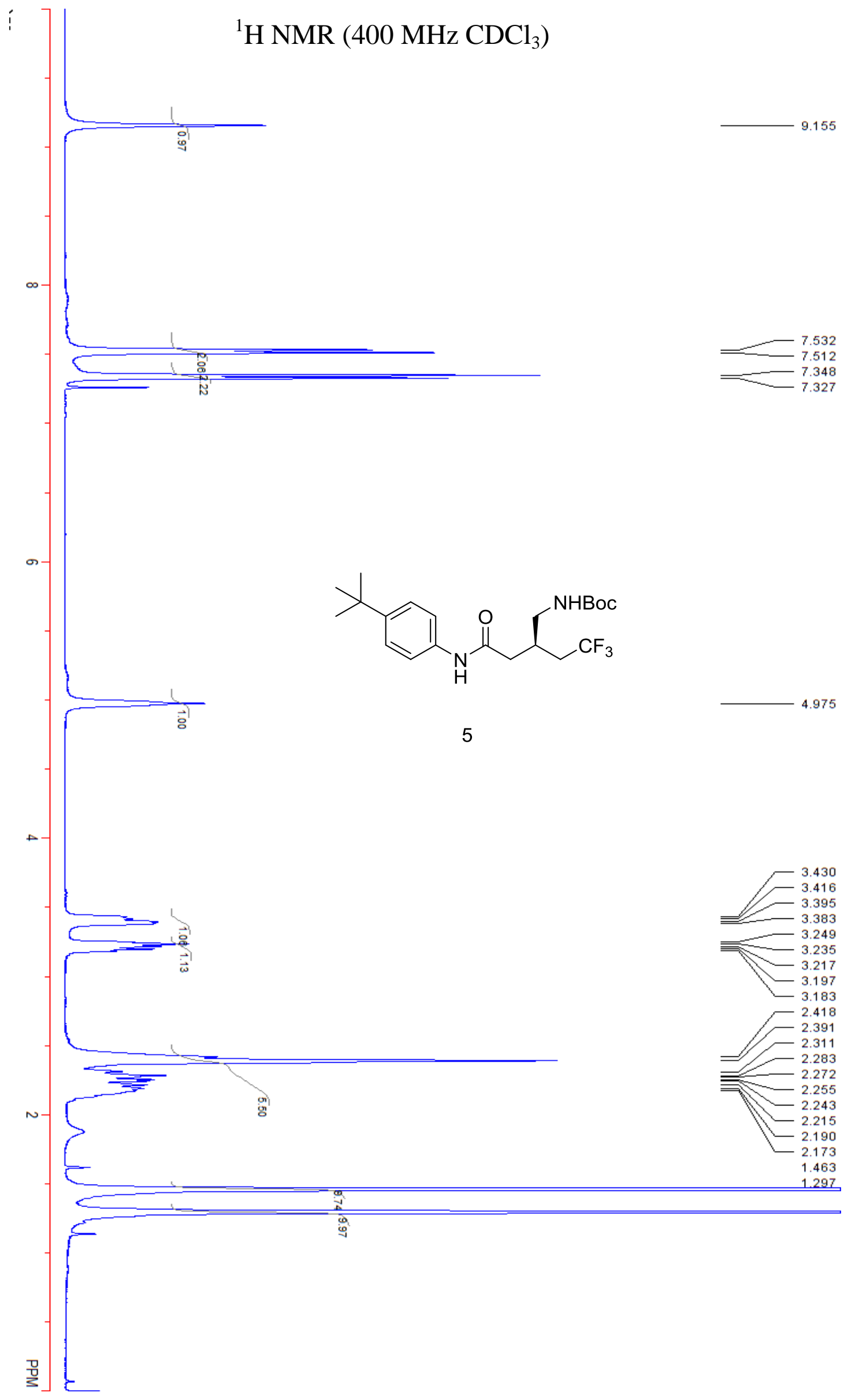




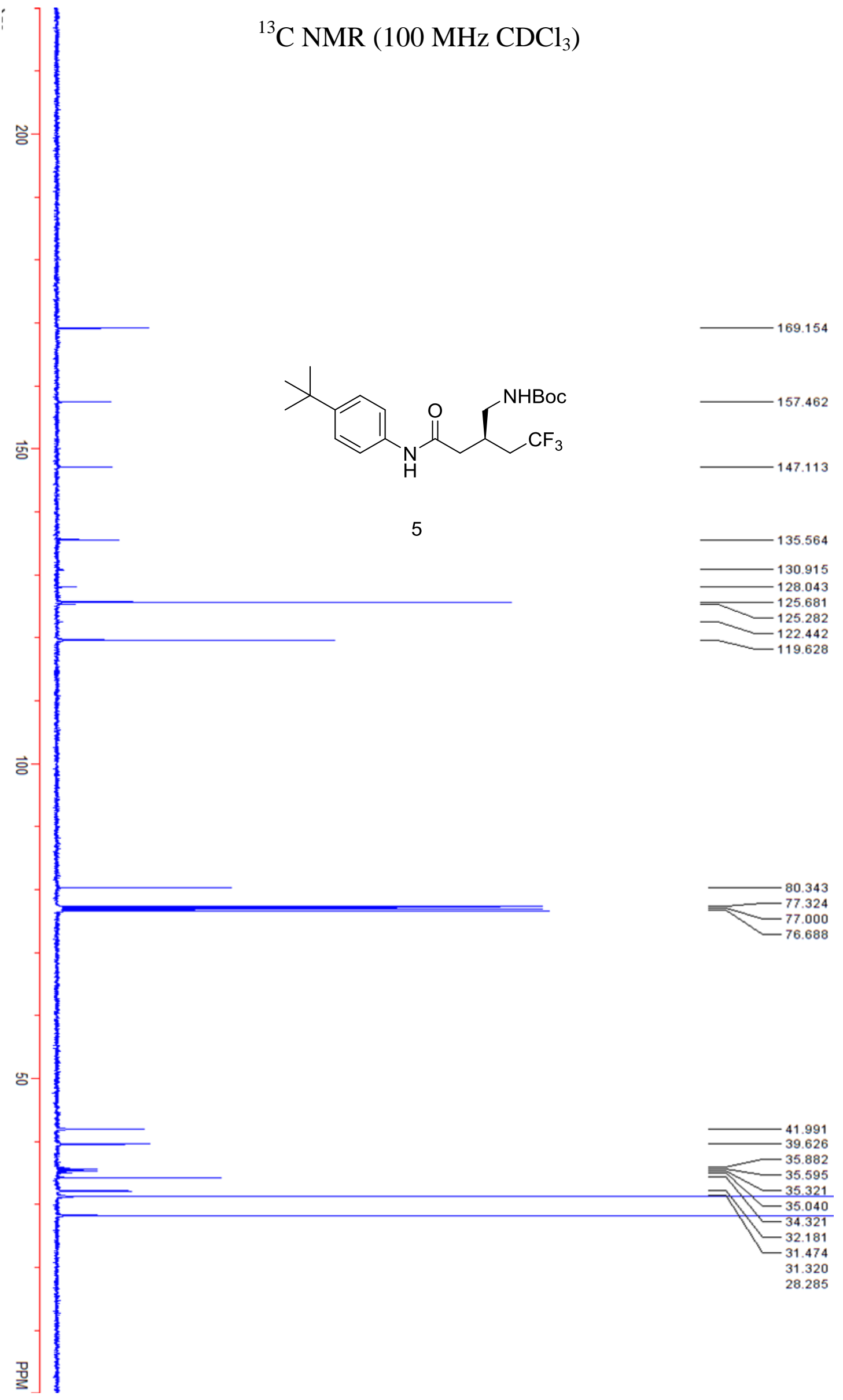




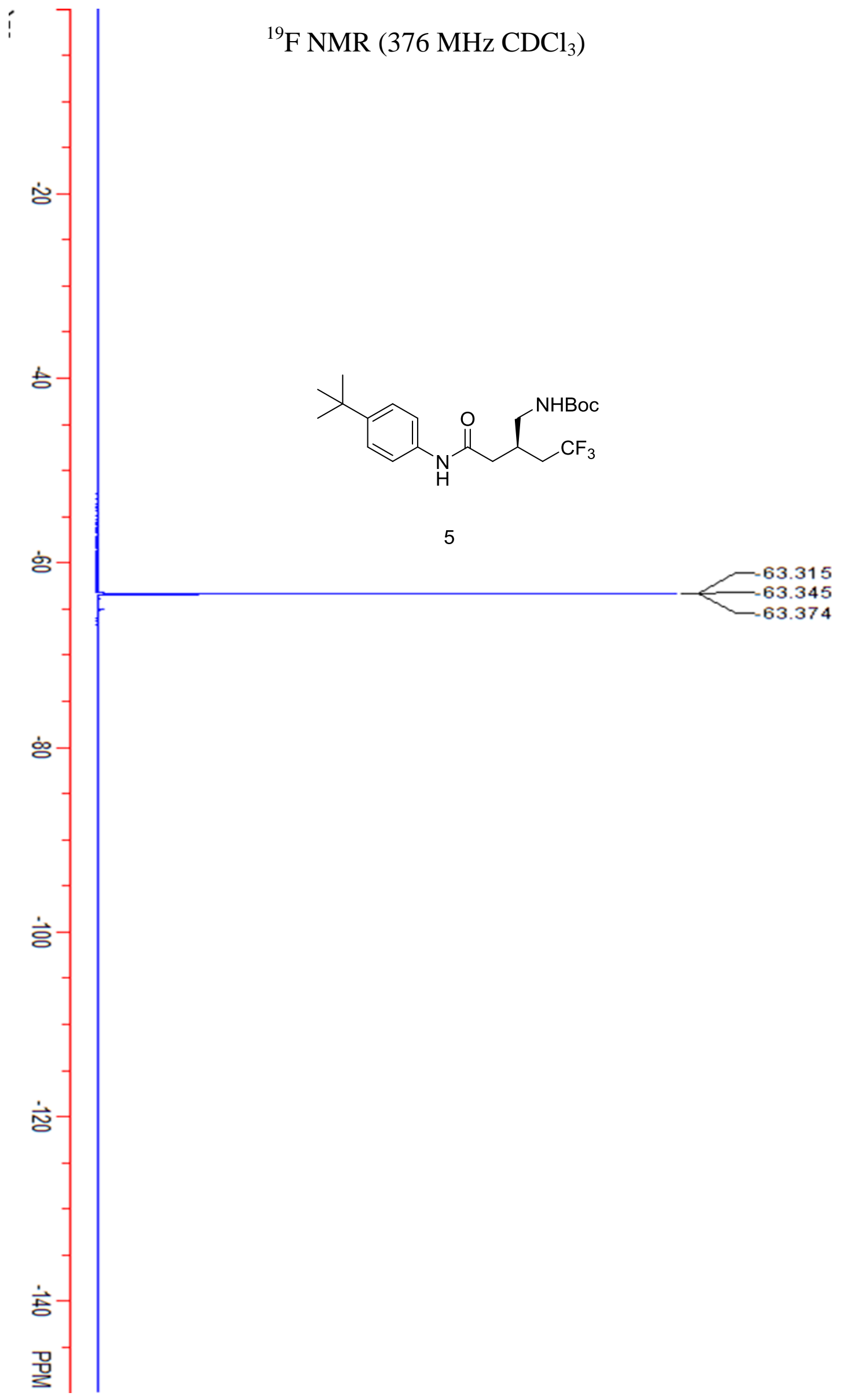



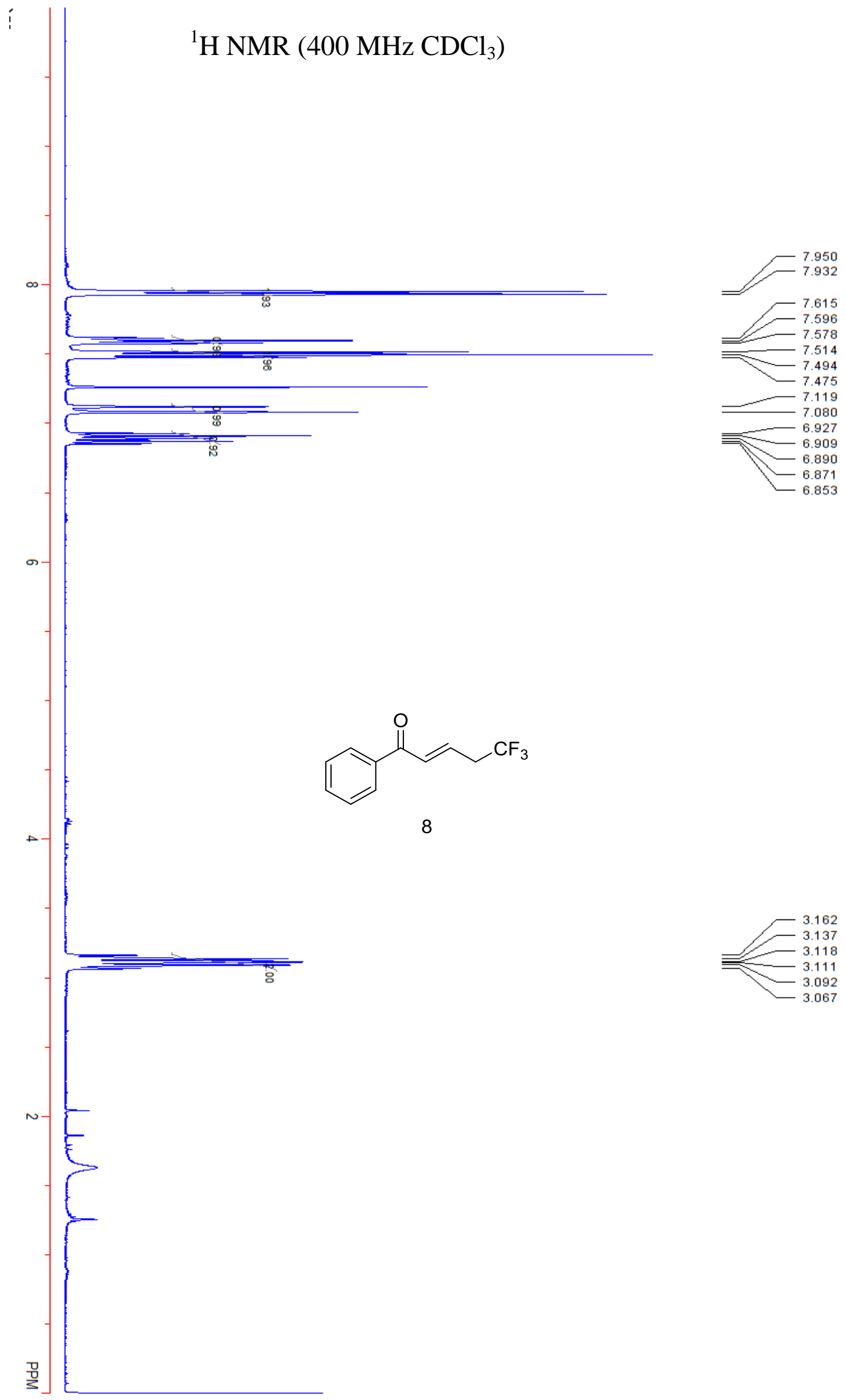


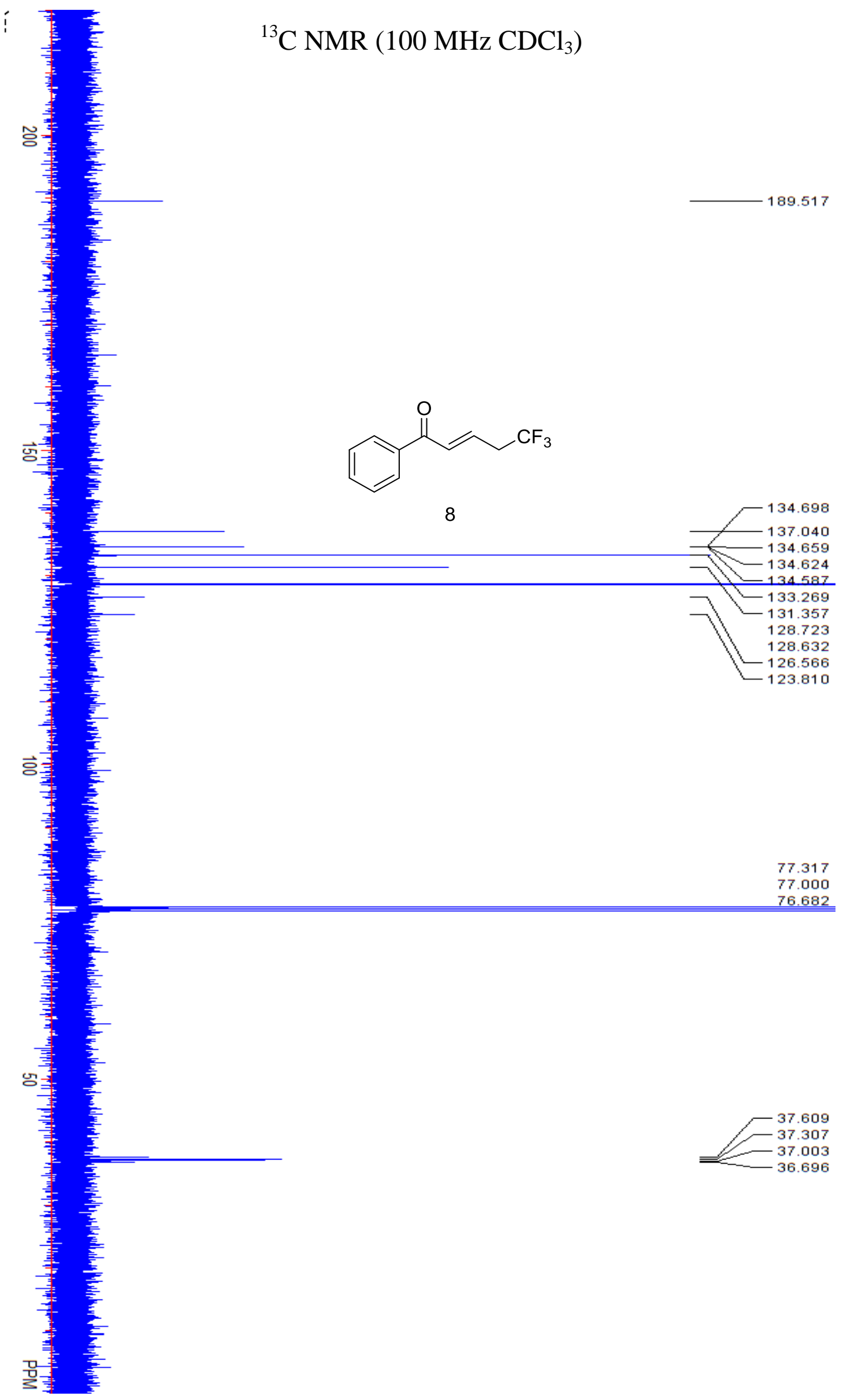



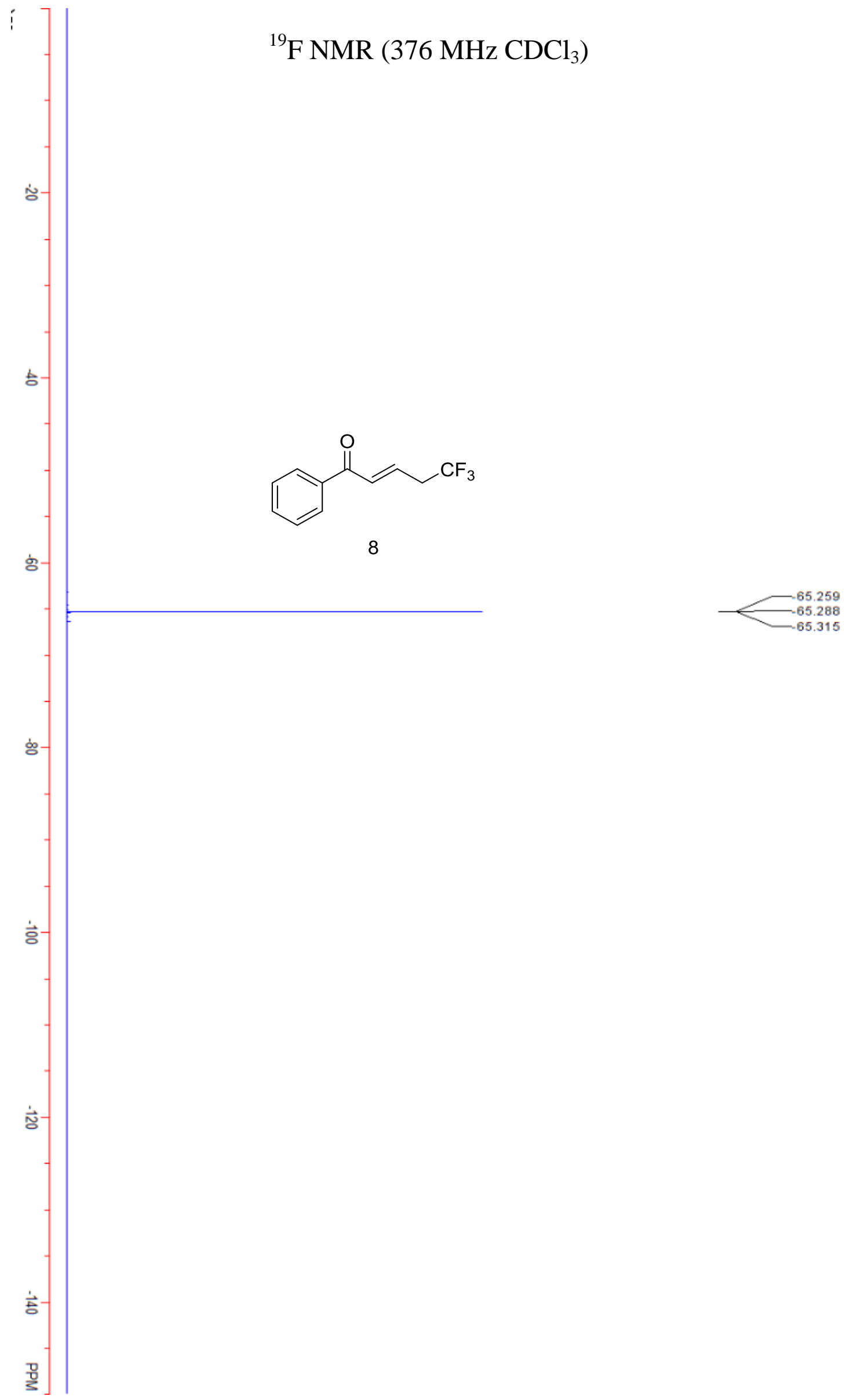


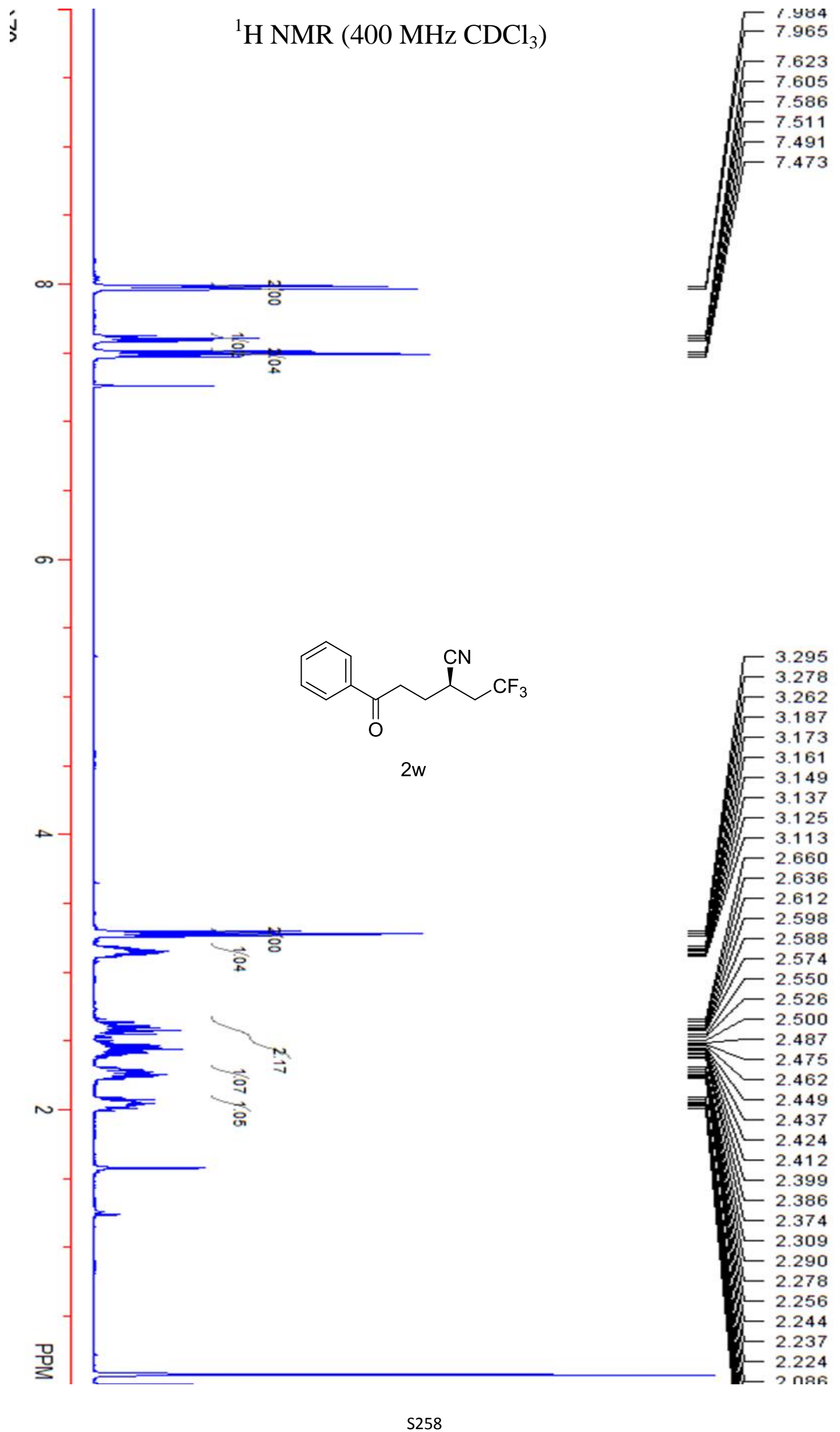




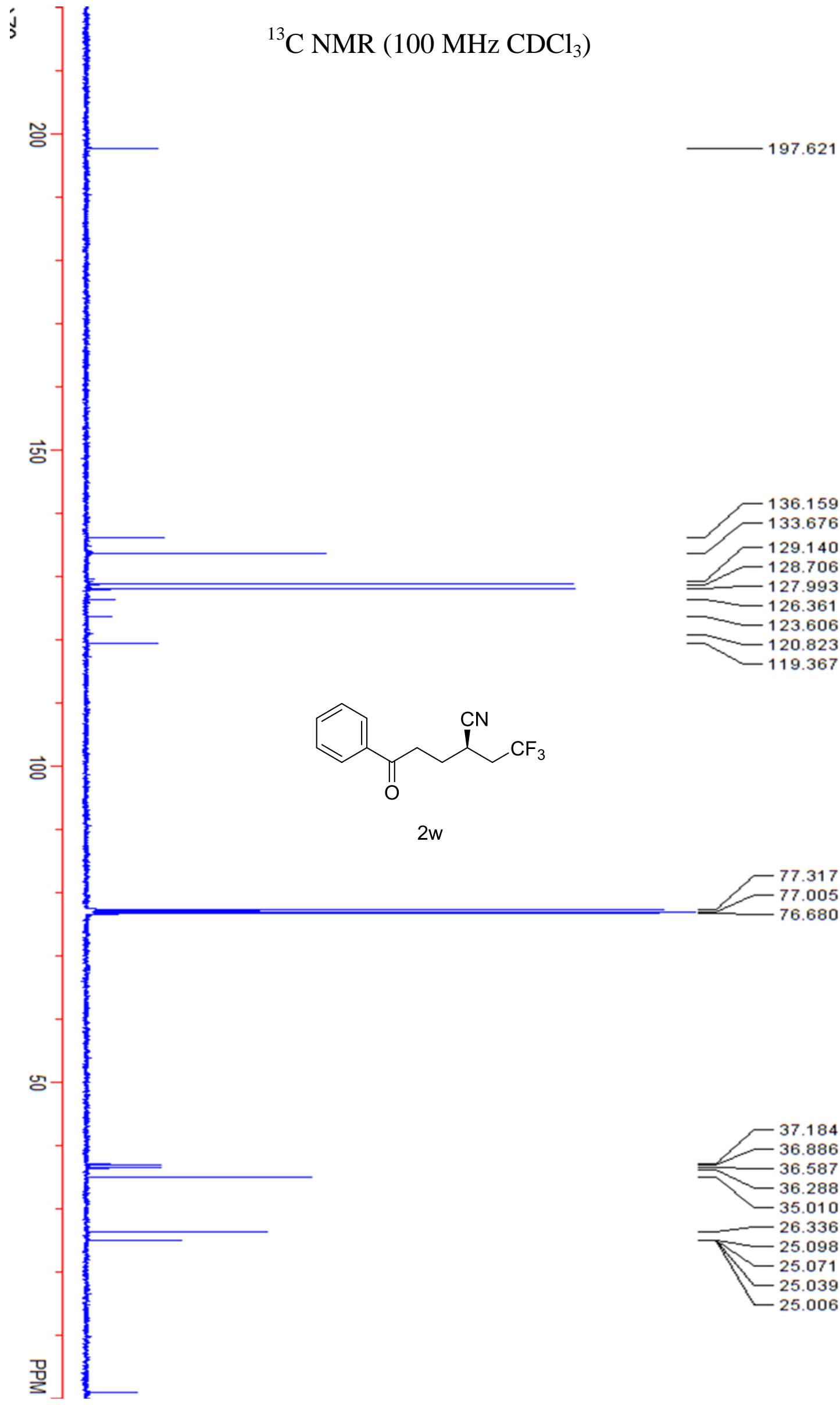



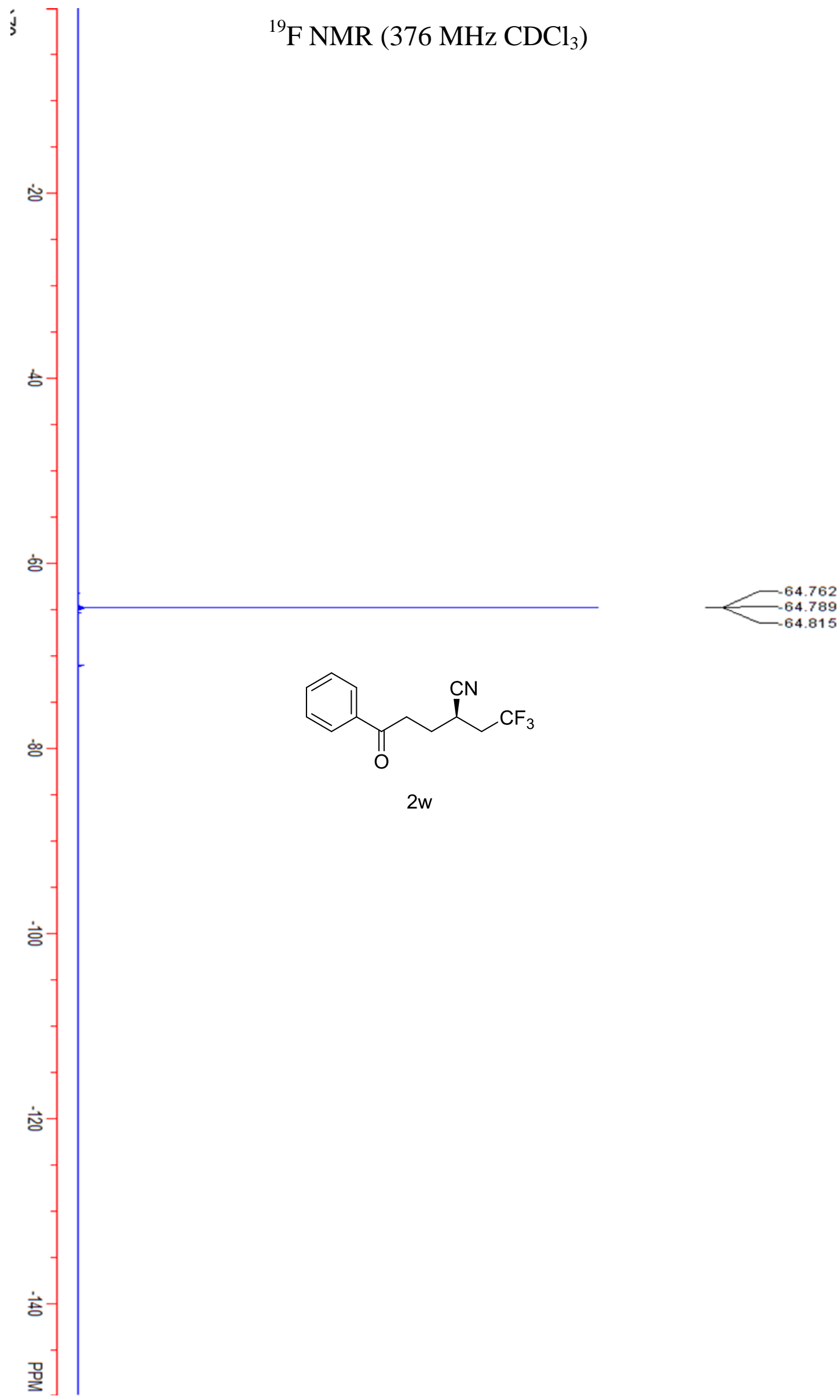

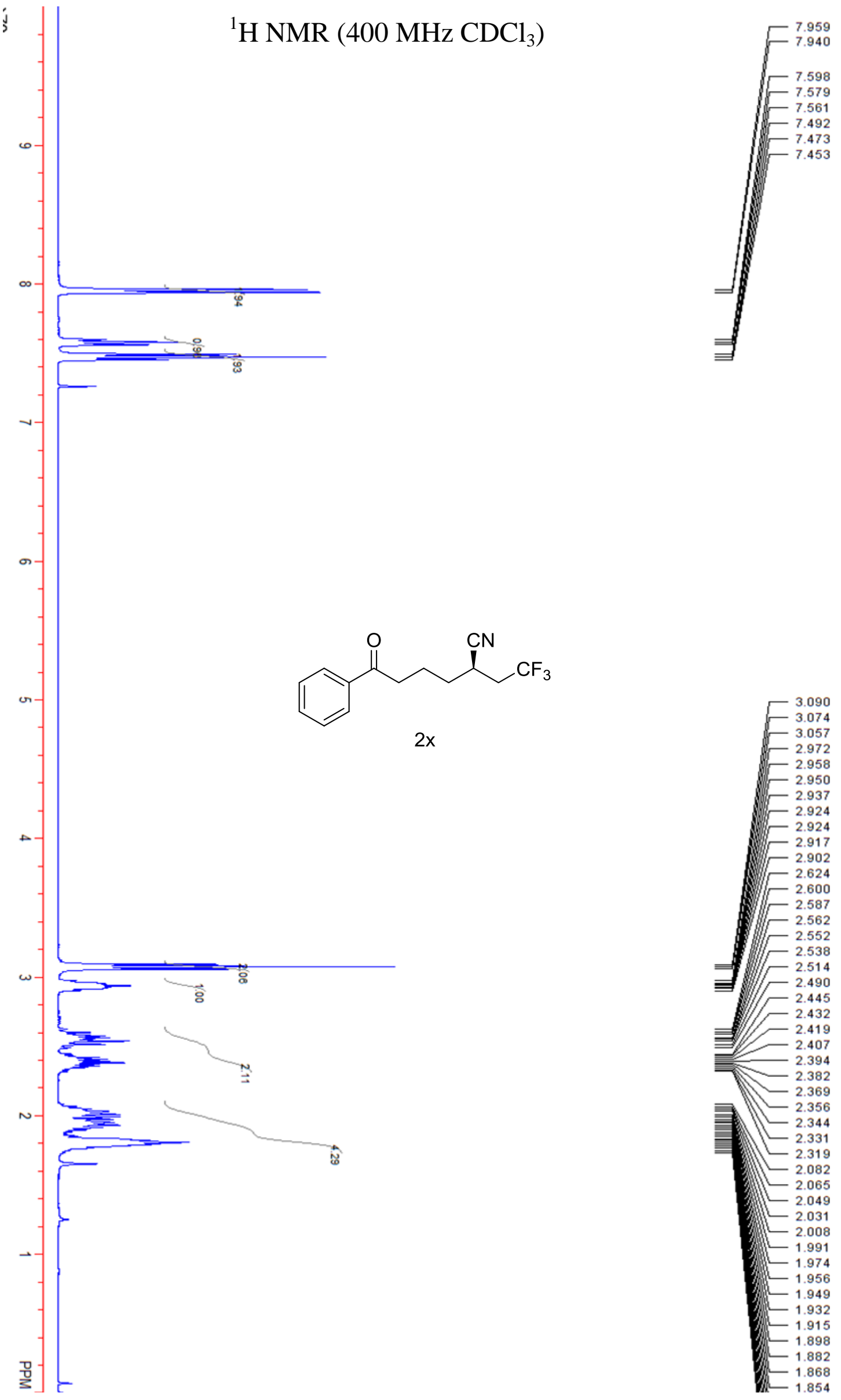


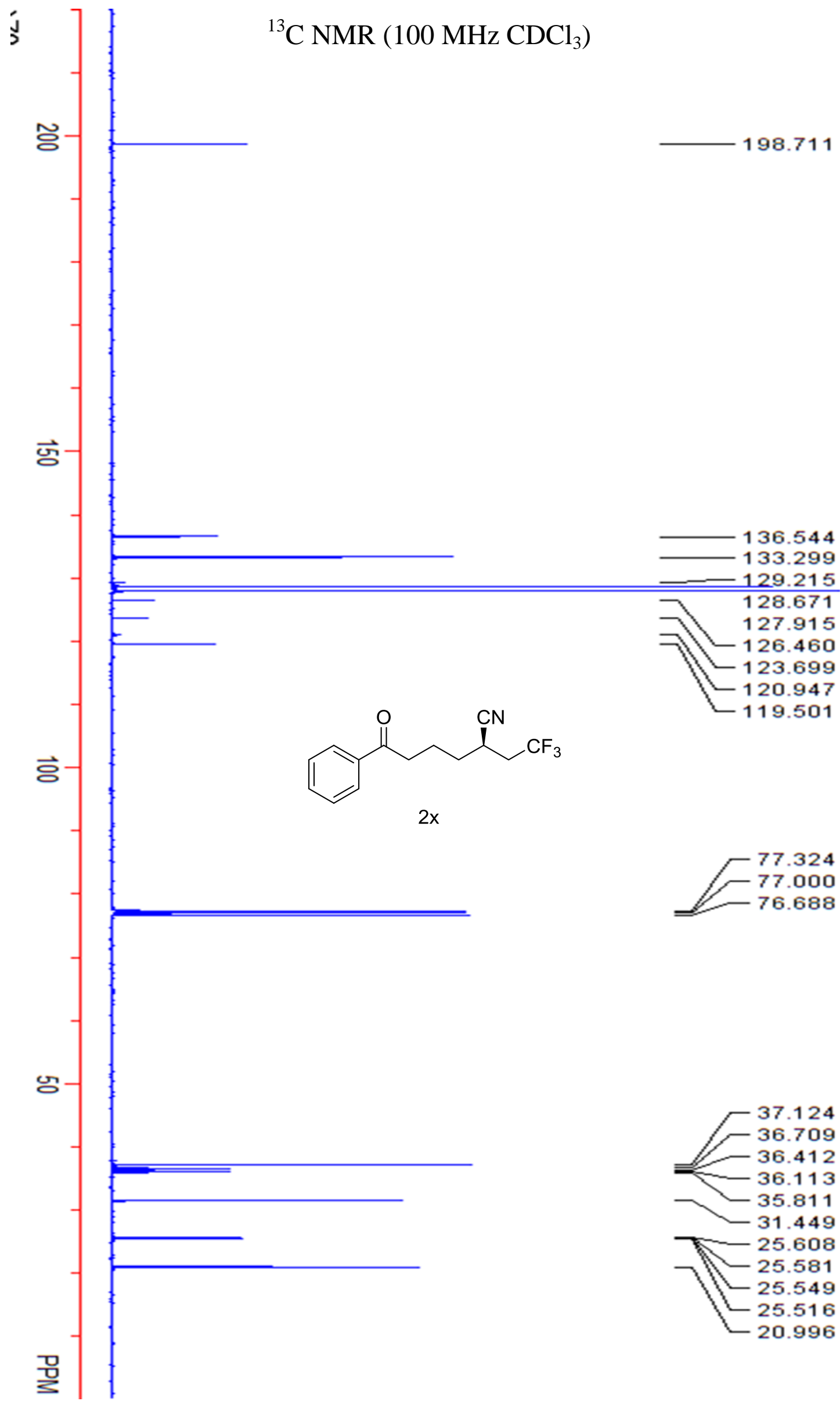



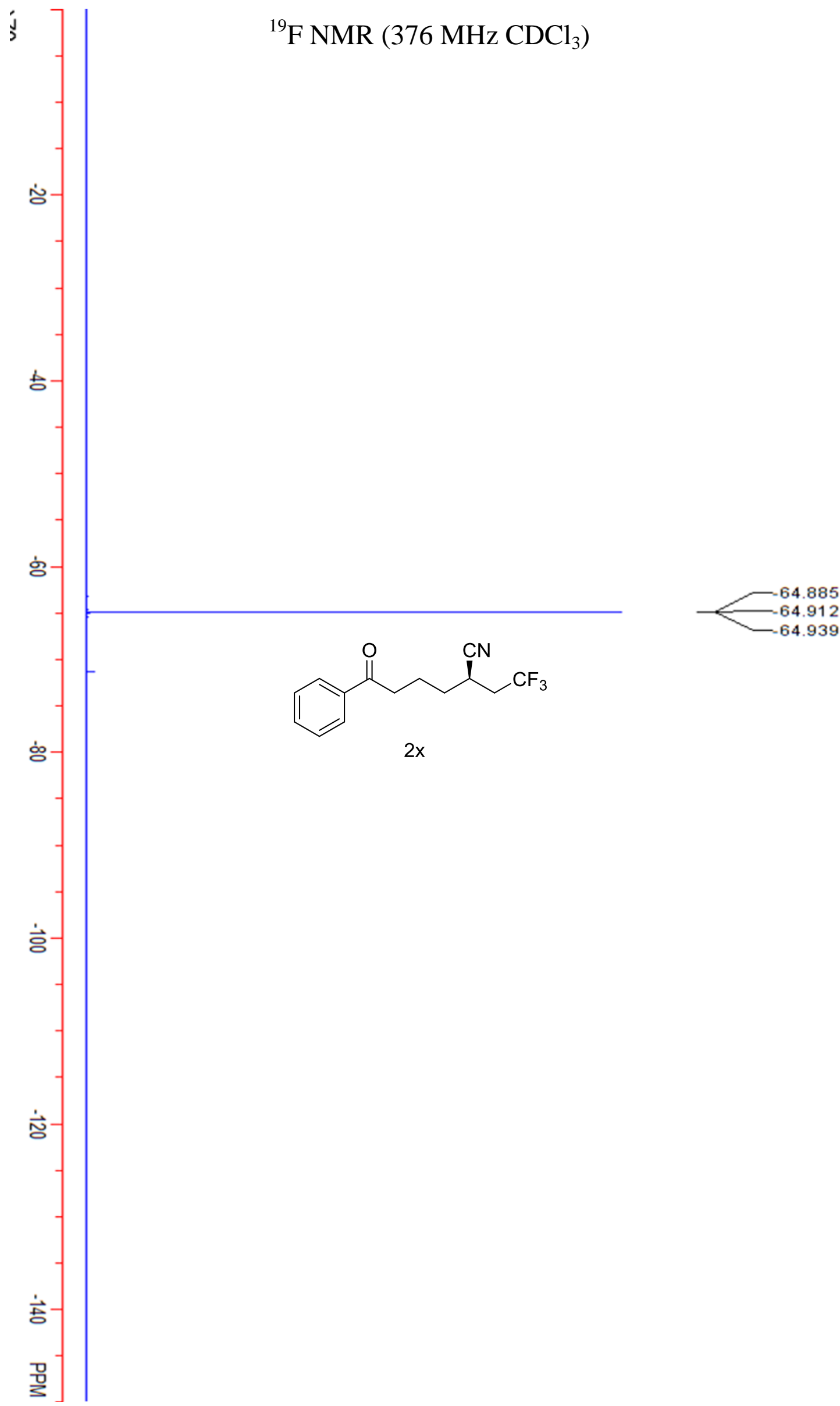

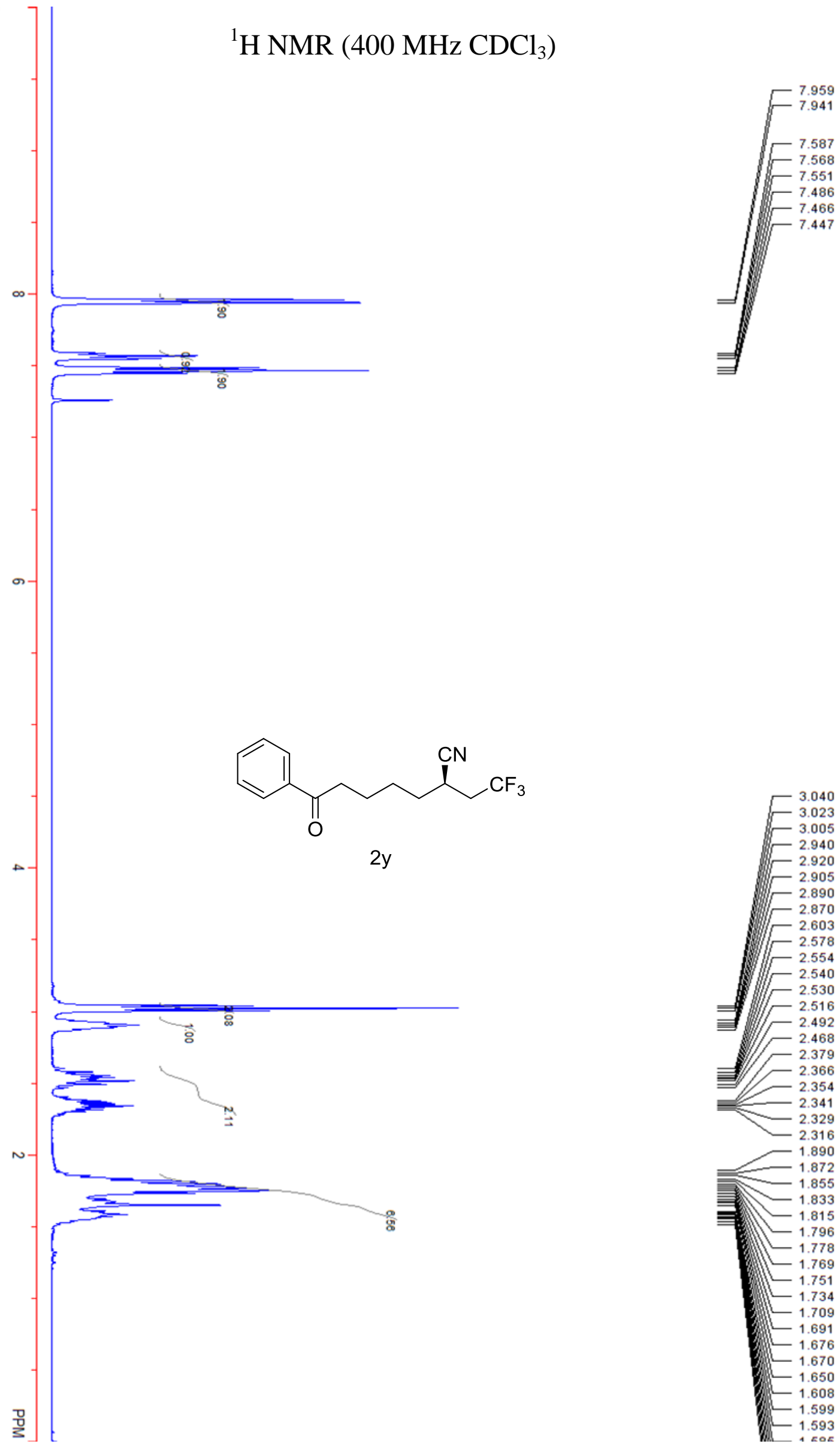

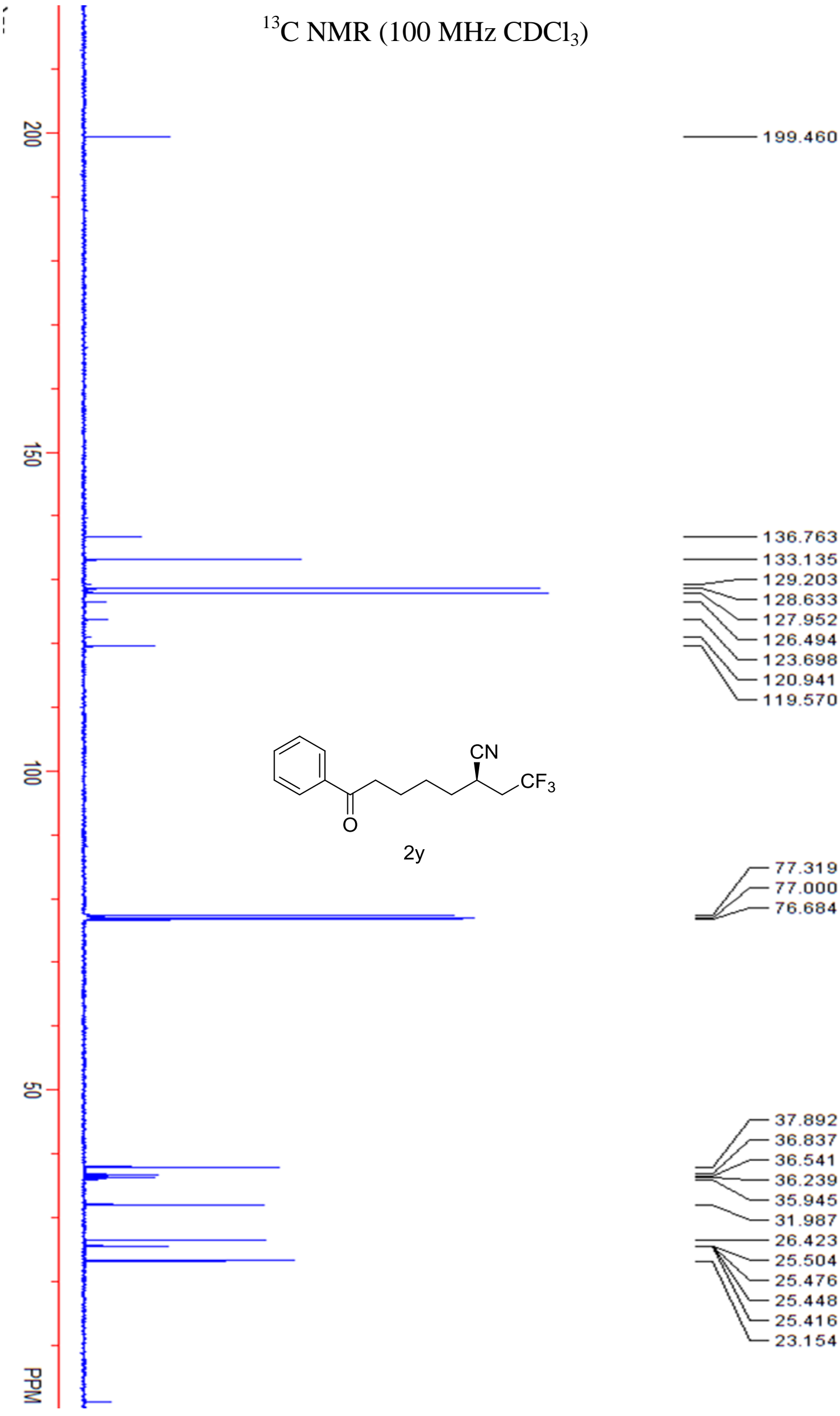


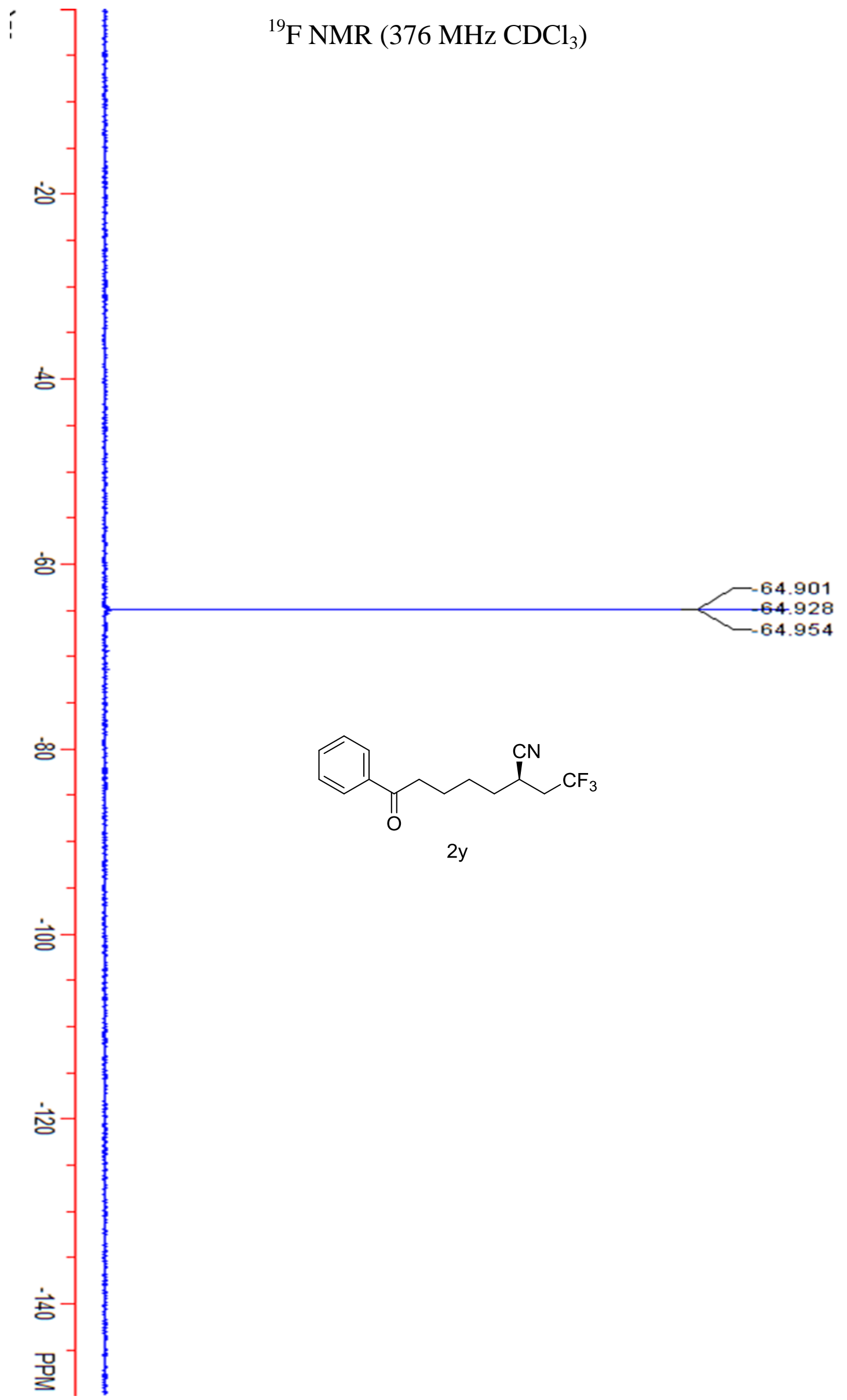



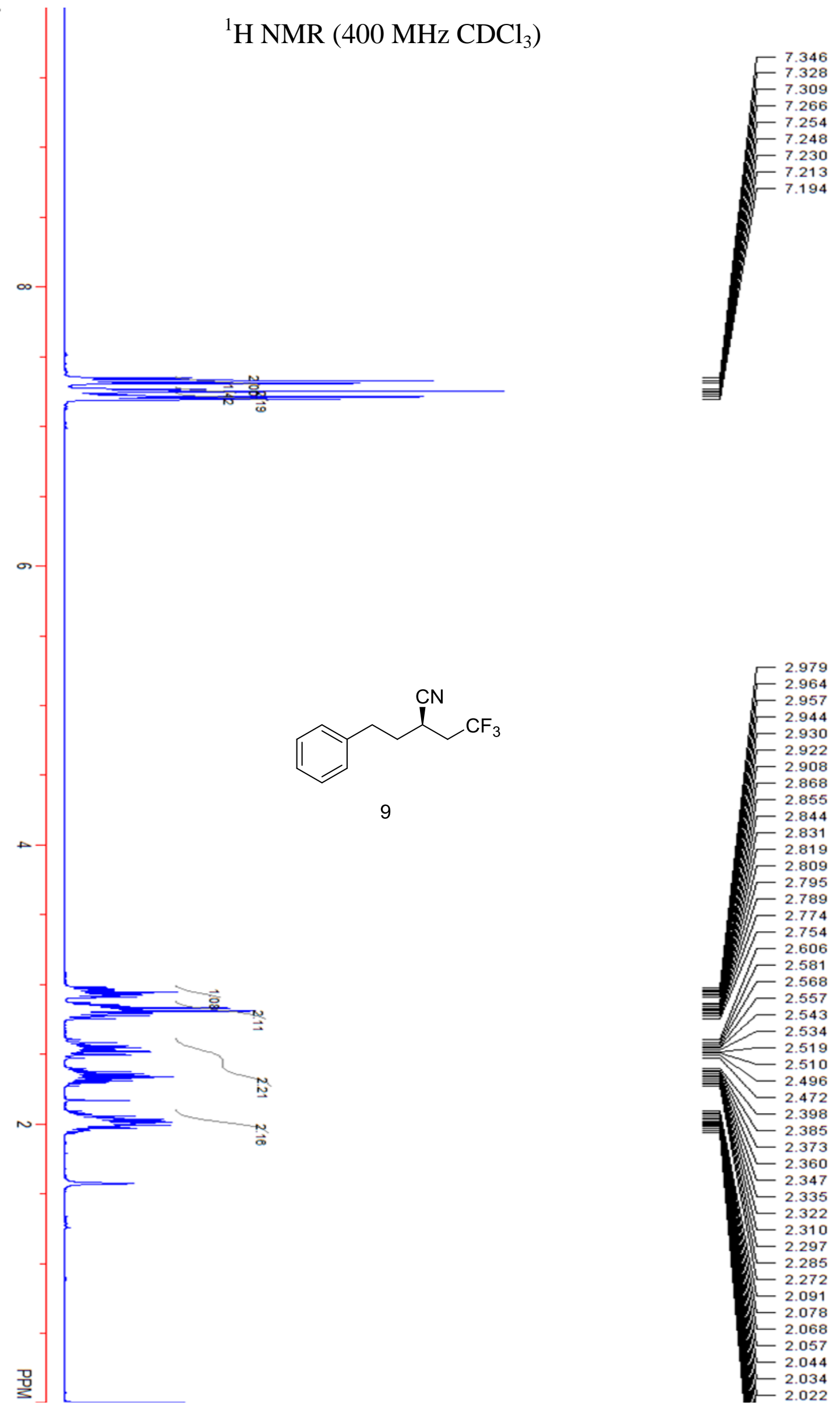


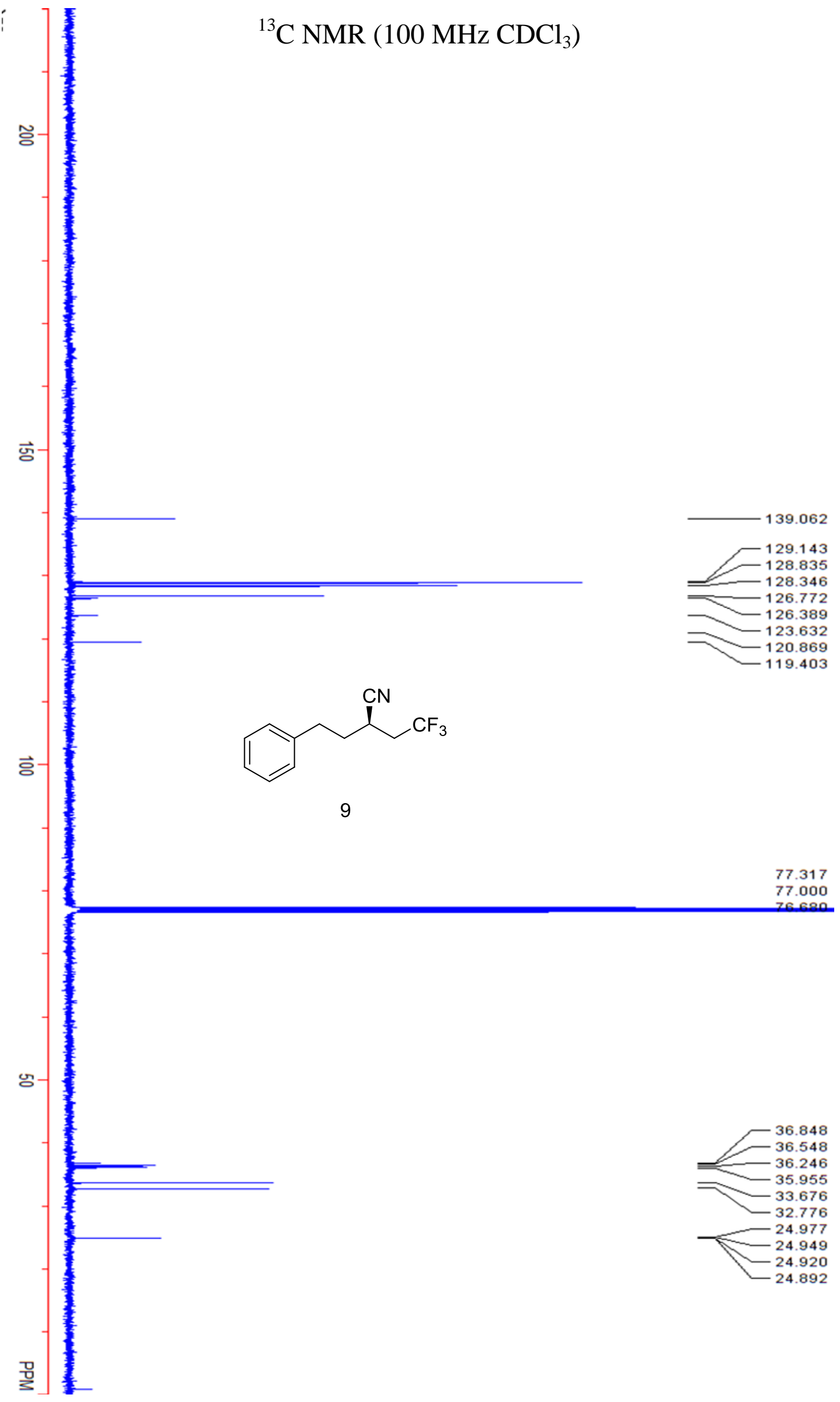




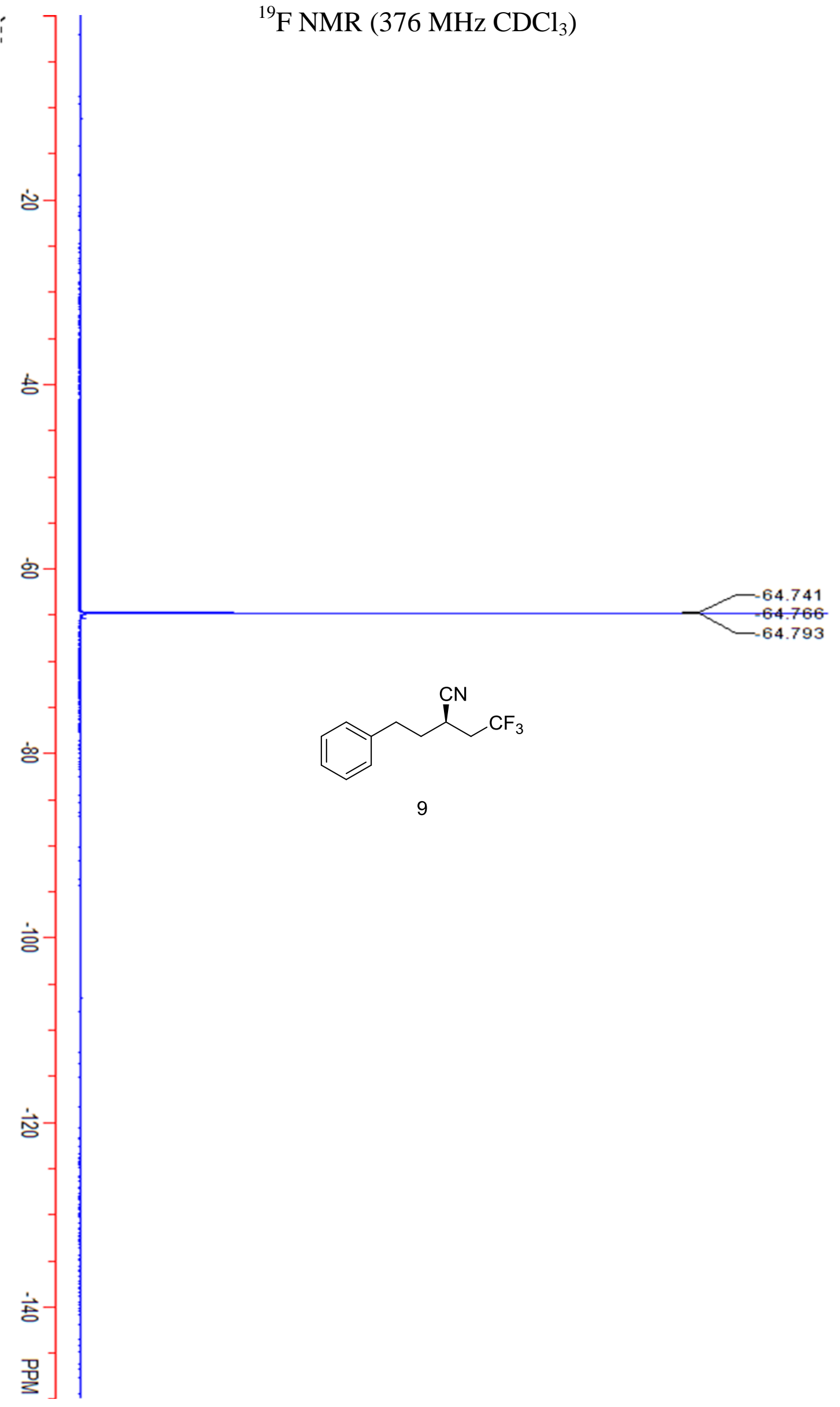



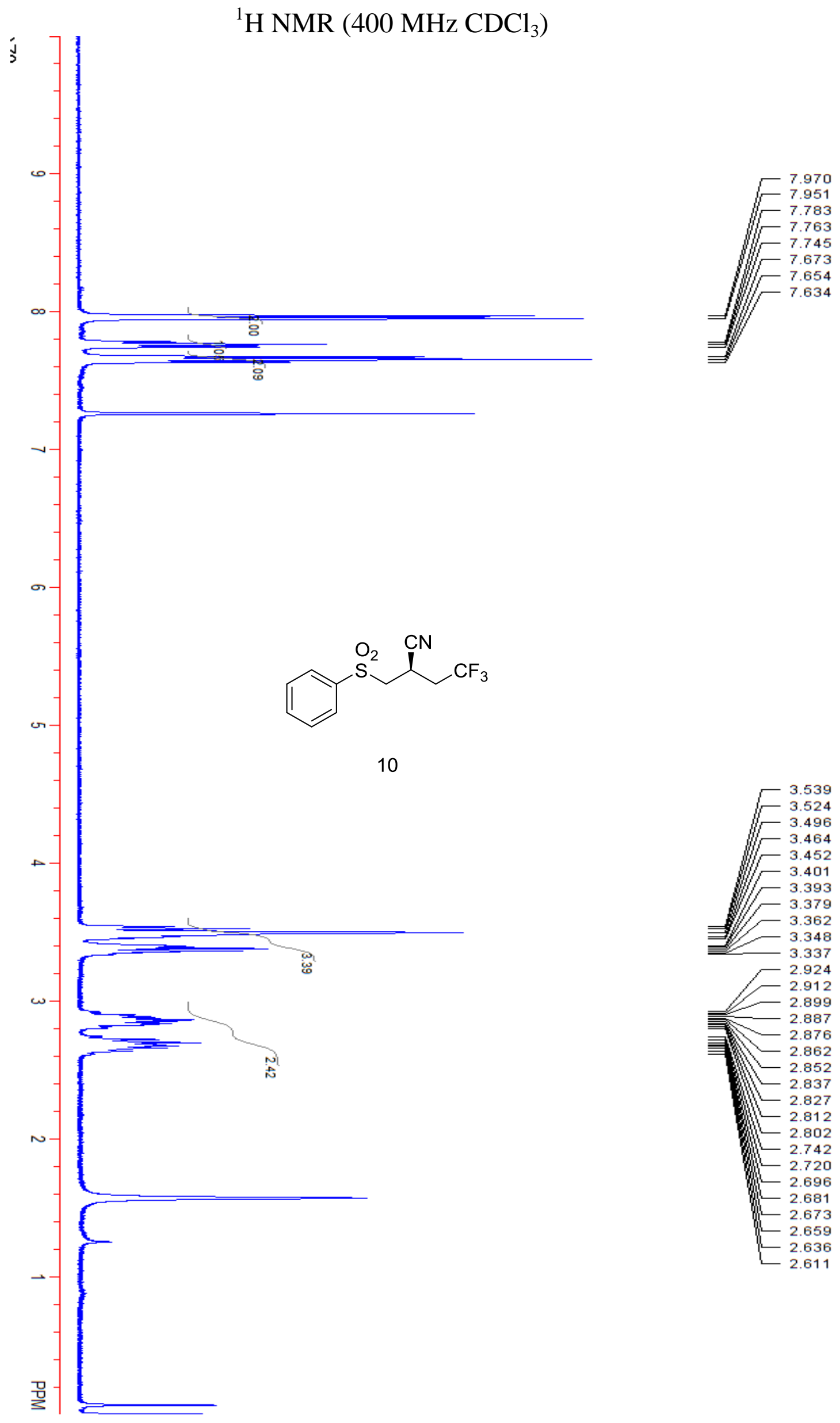

3.337

- 2.924

2.912

2.899

2.887

2.876

2.862

2.852

2.837

2.827

2.812

2.802

2.742

2.720

2.696

2.681

2.673

2.636

2.611 


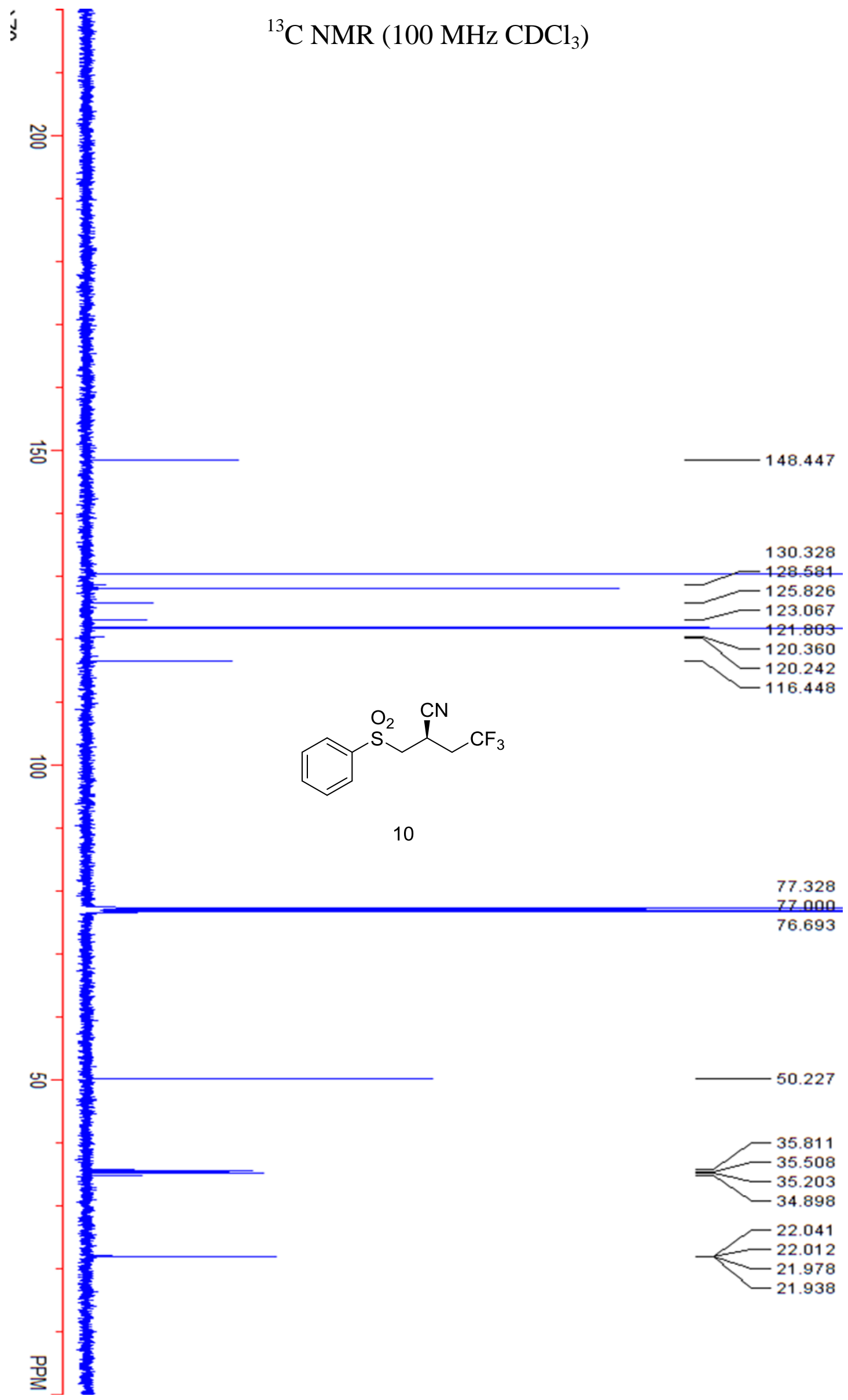




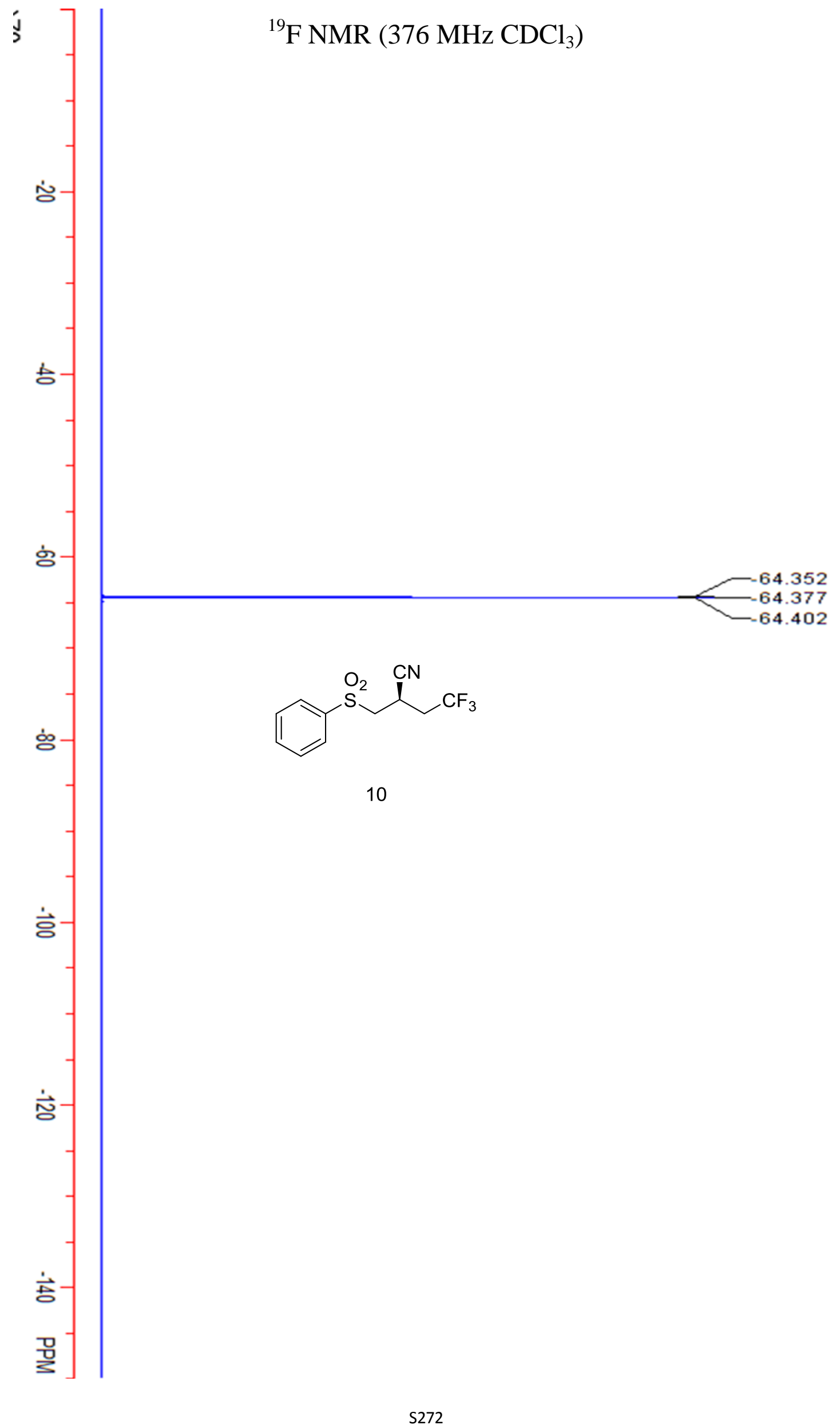



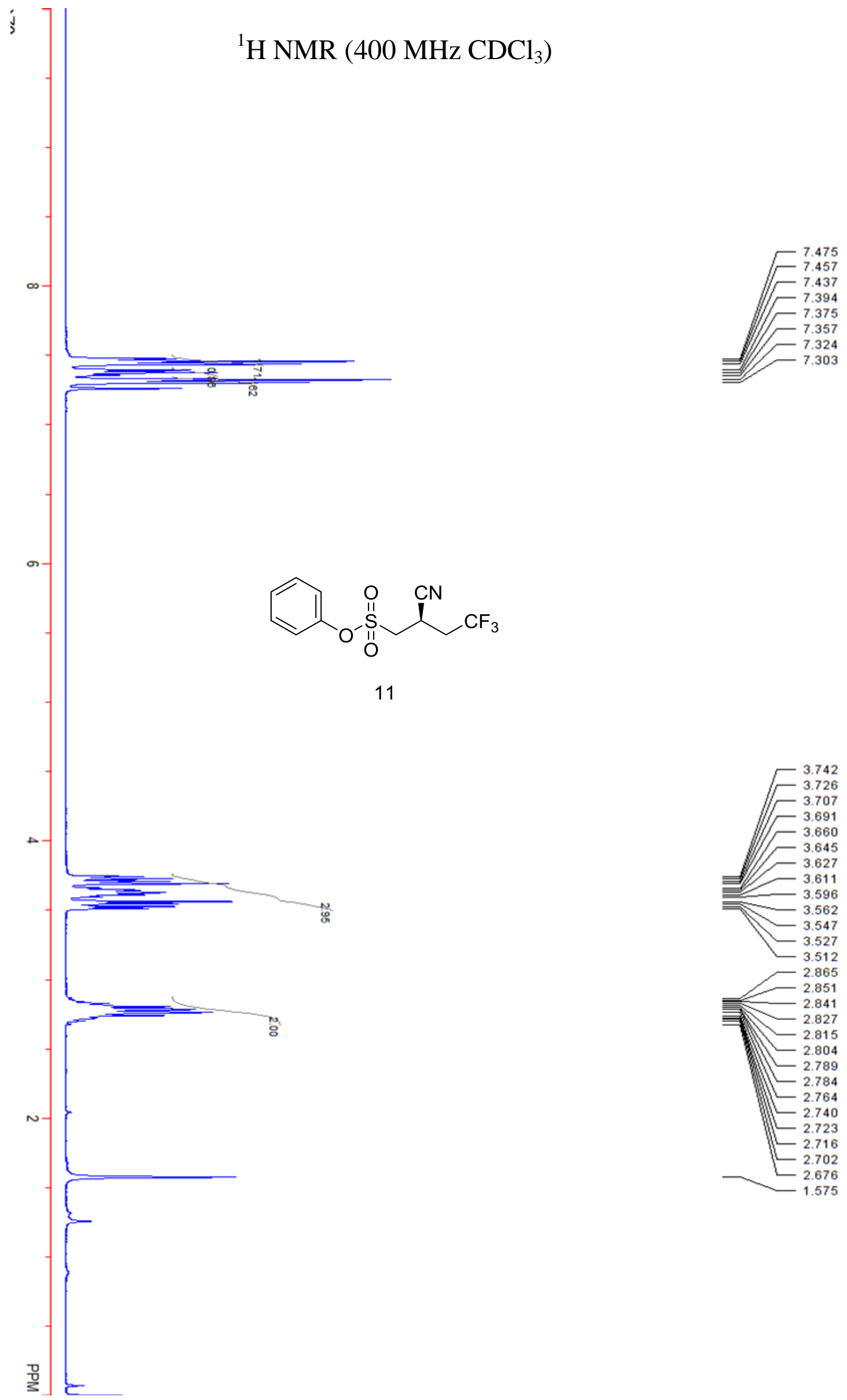


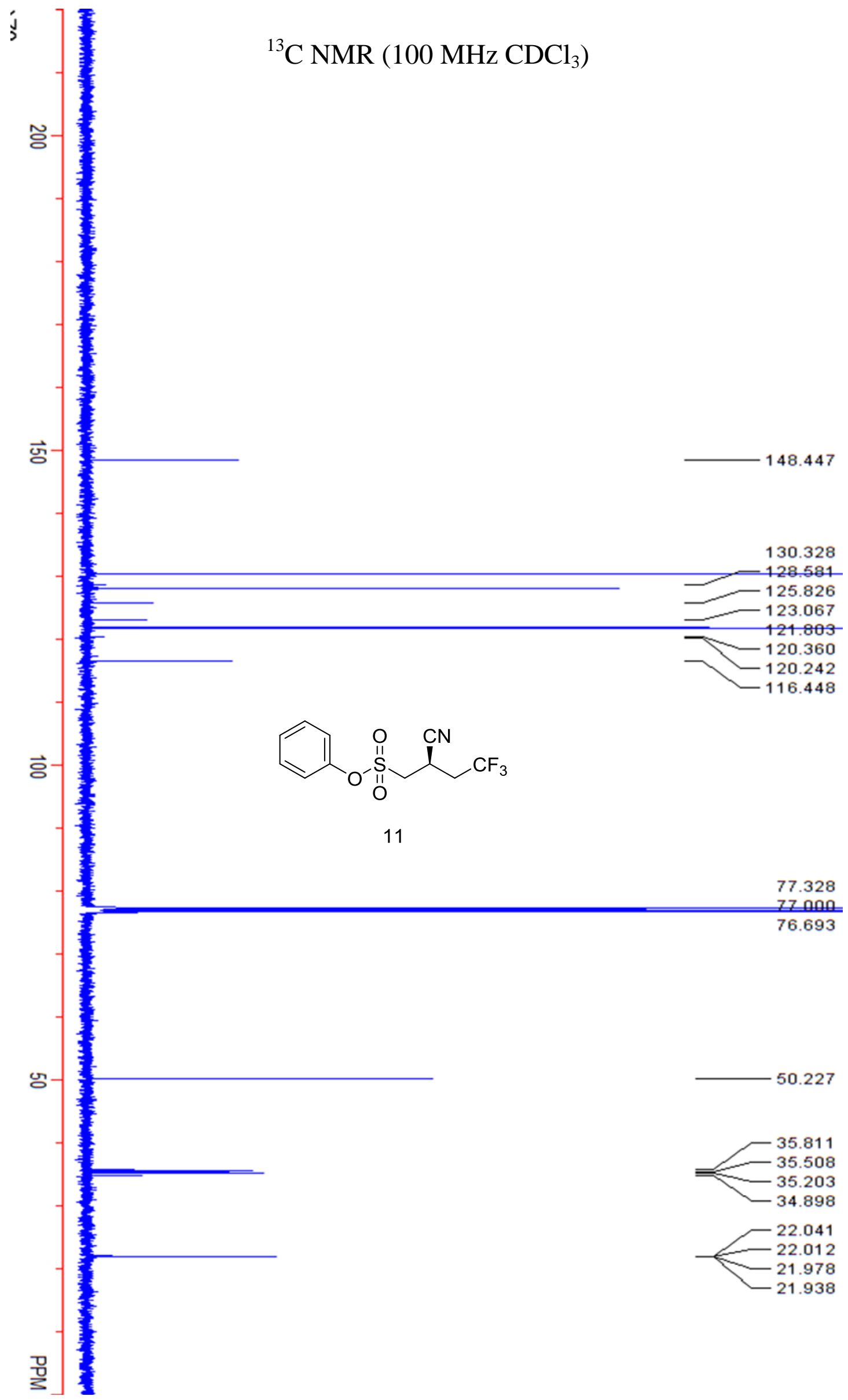




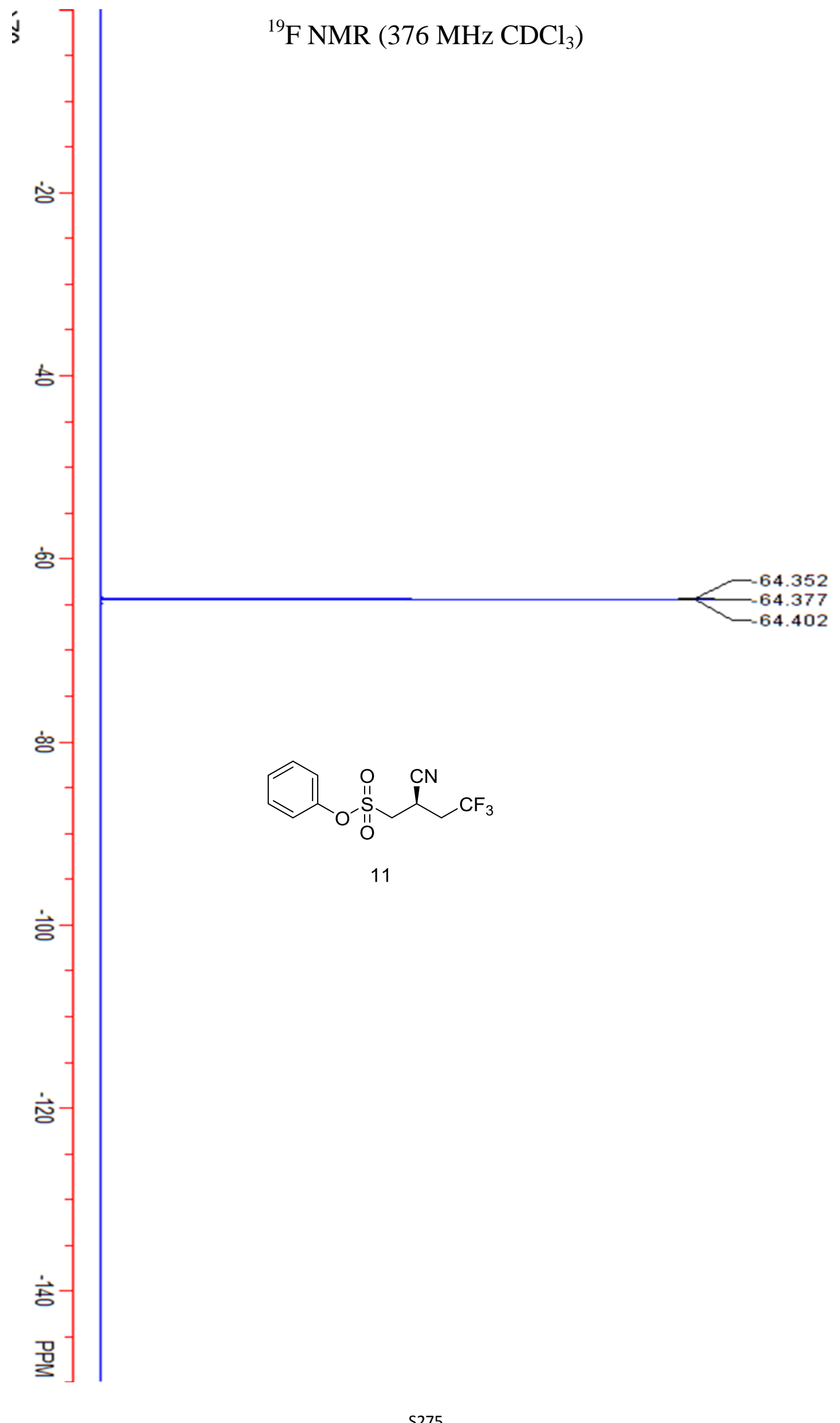




\section{HPLC Analysis of Products}
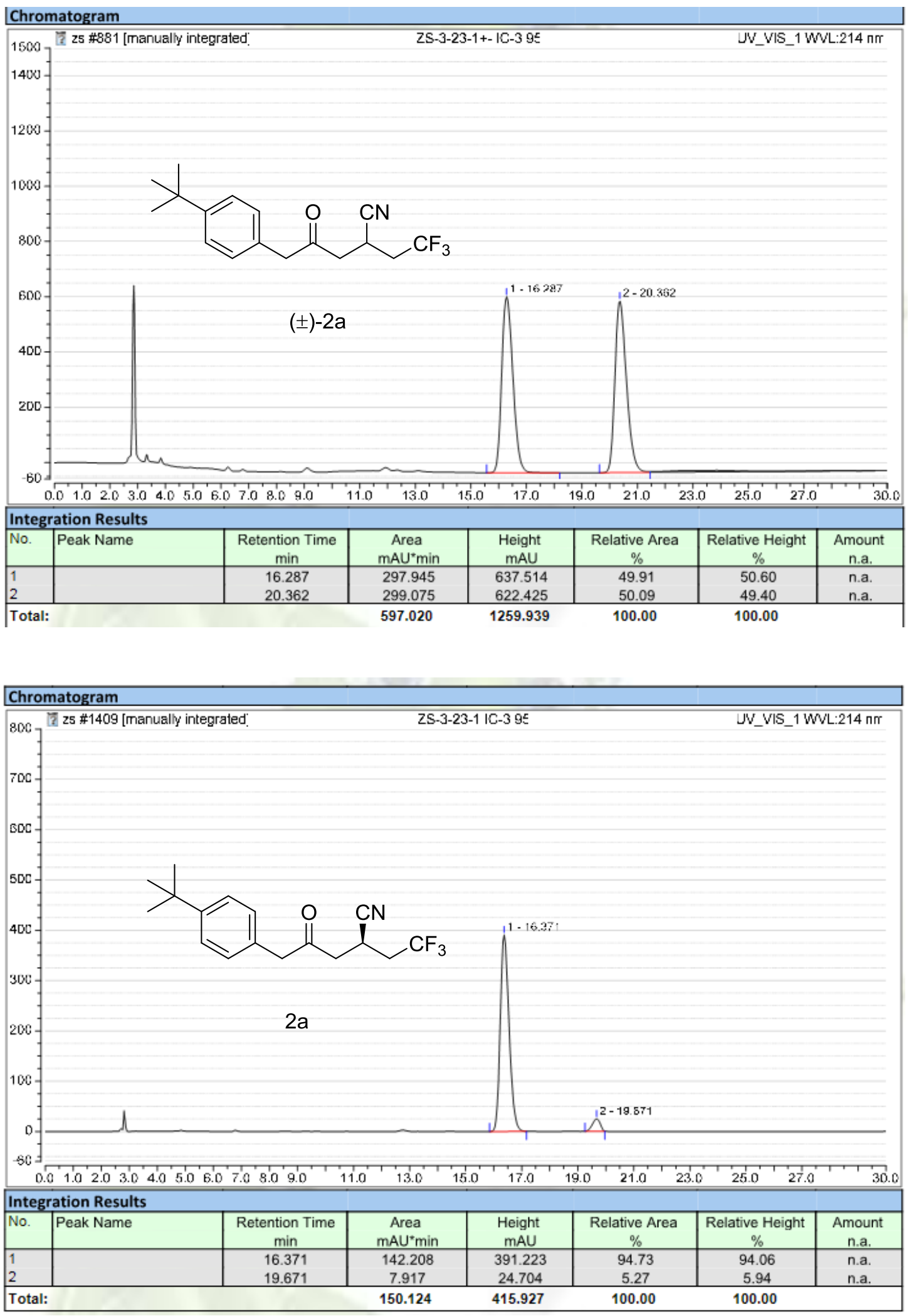

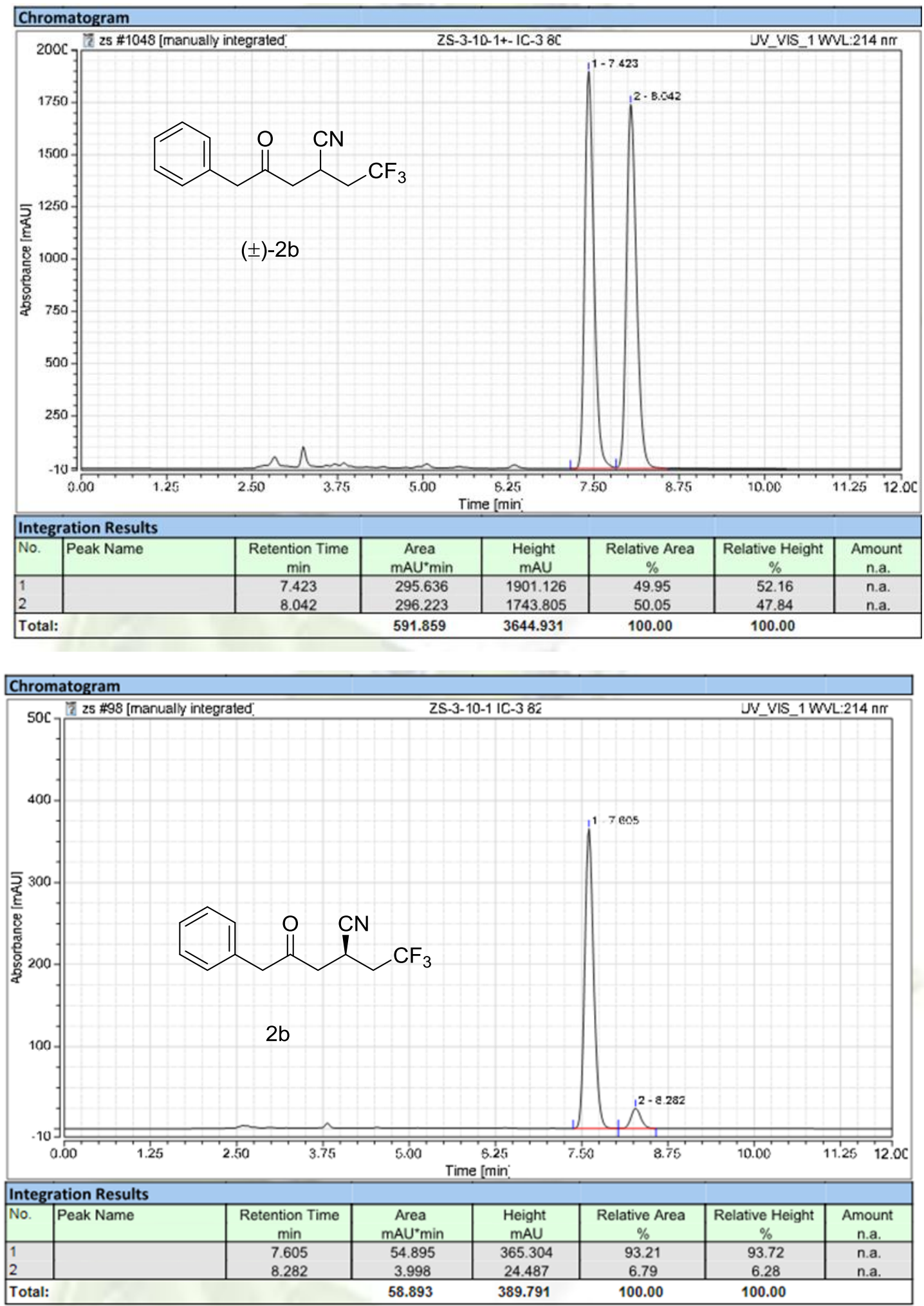


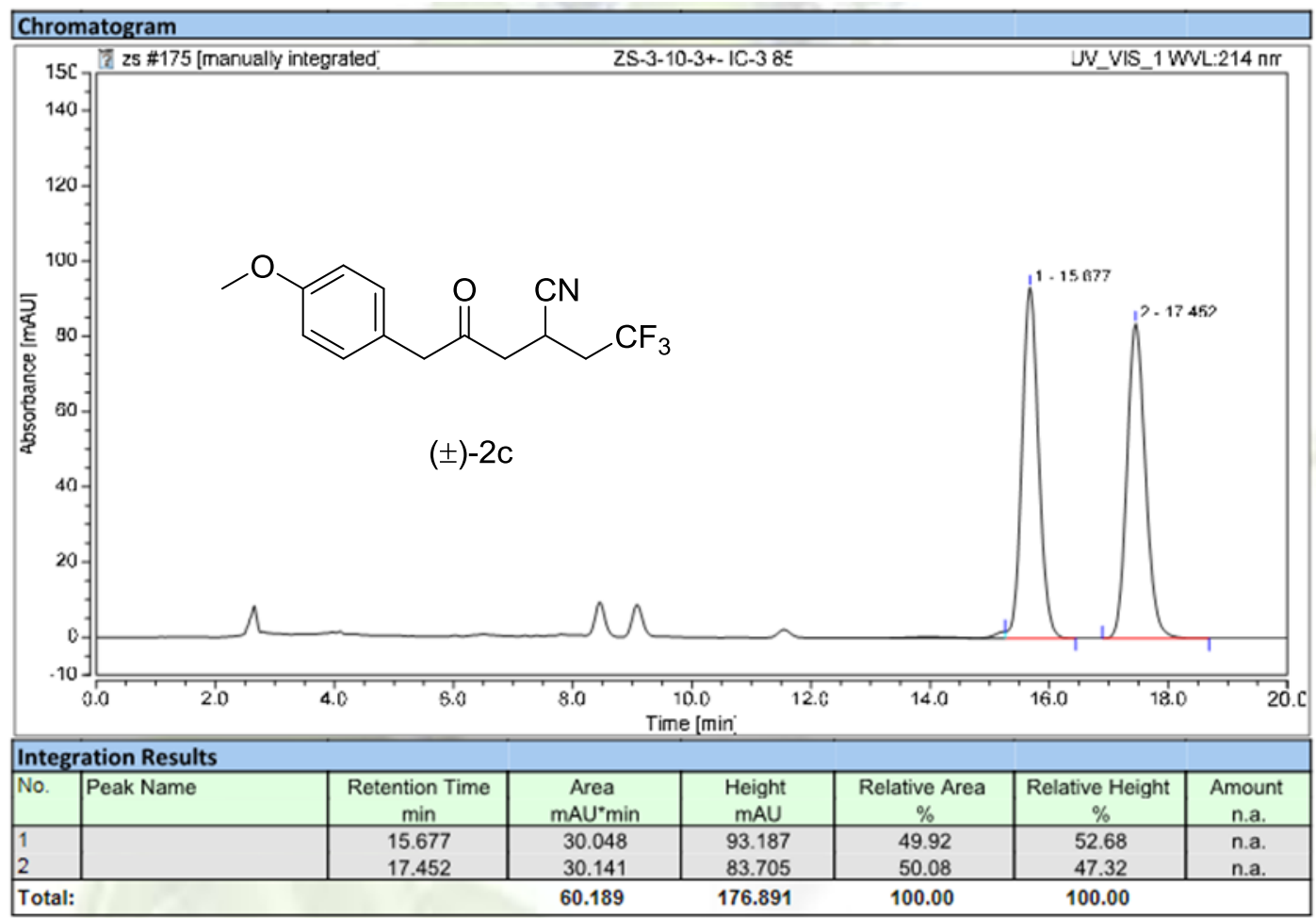

Chromatogram

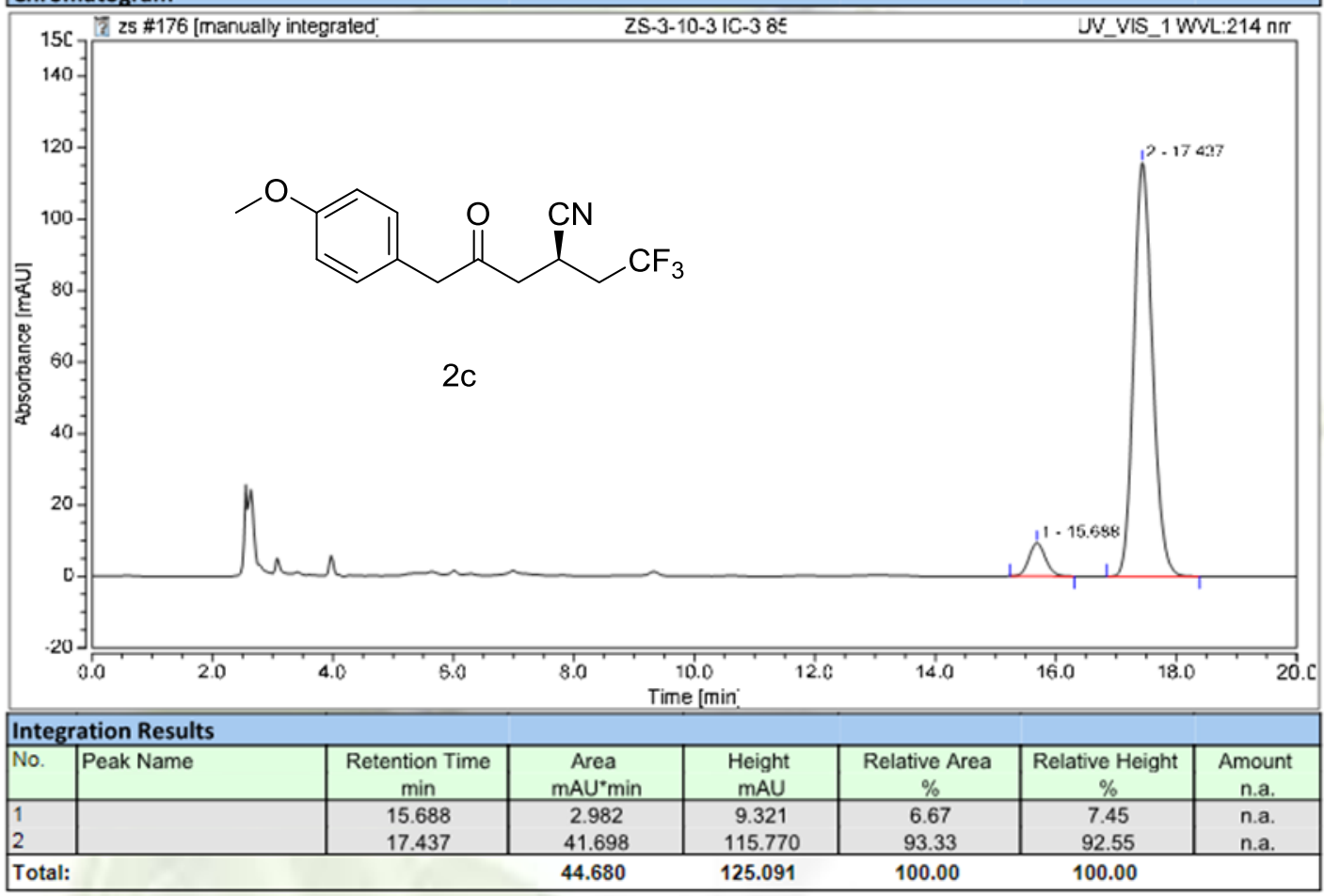



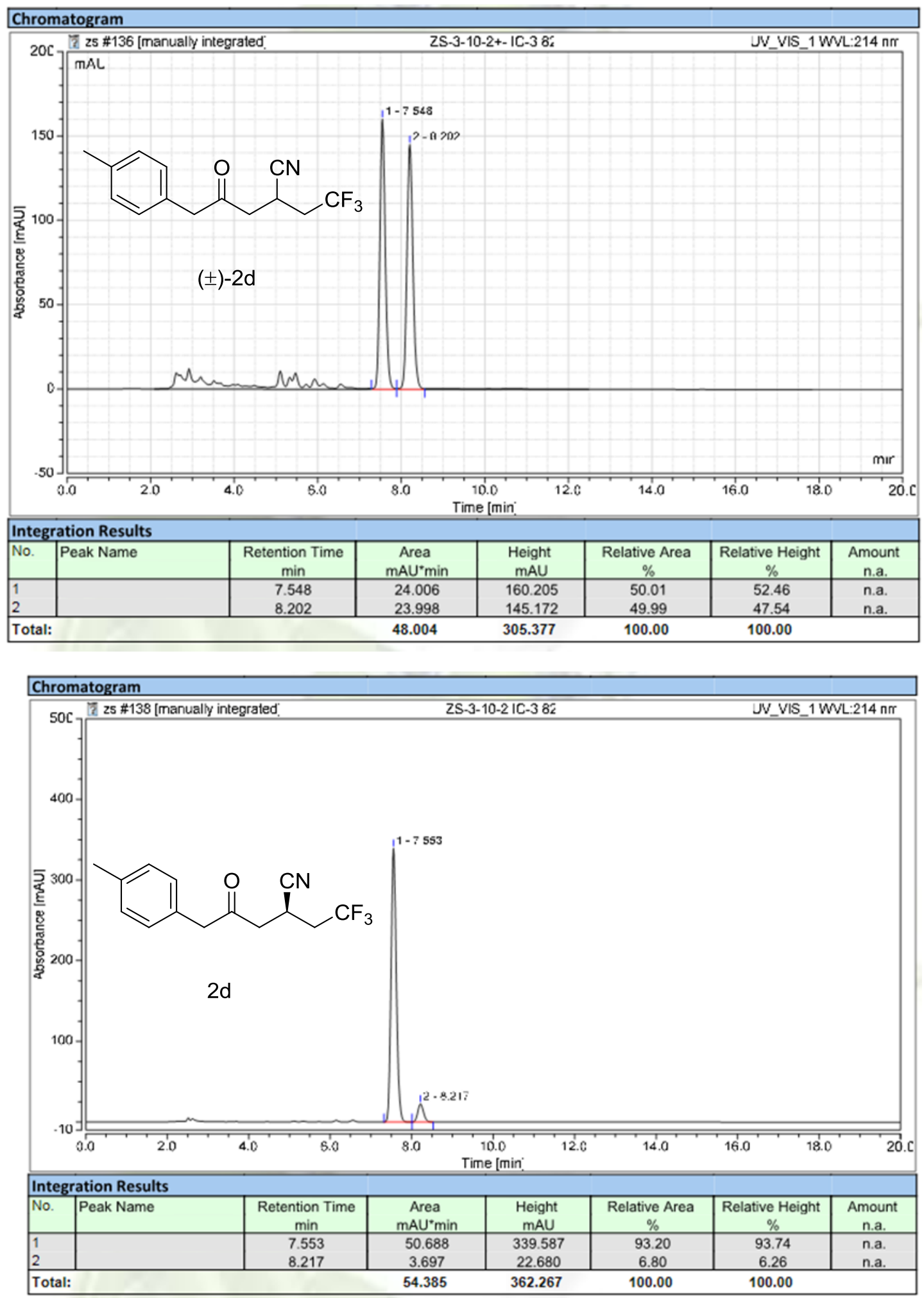

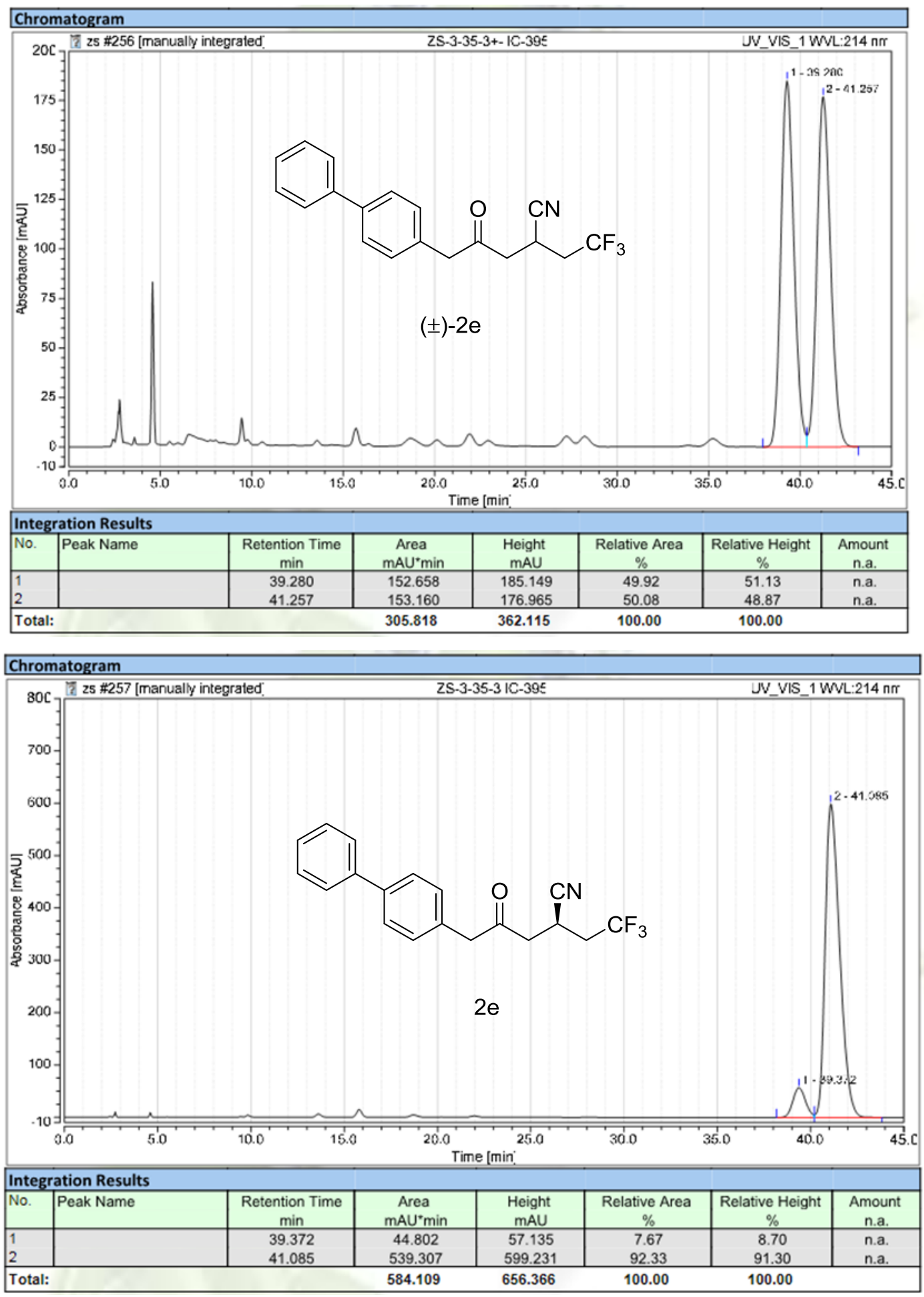

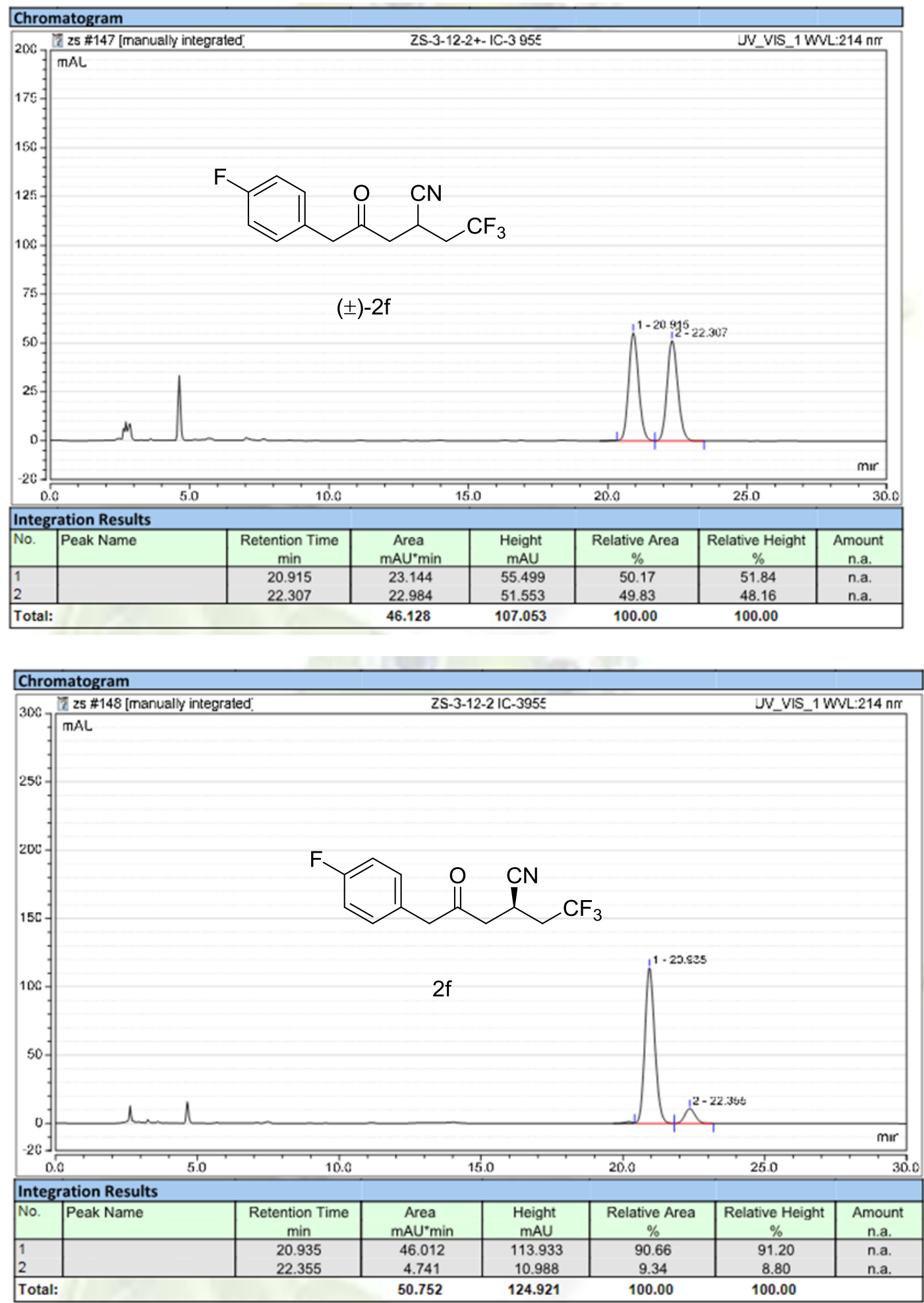

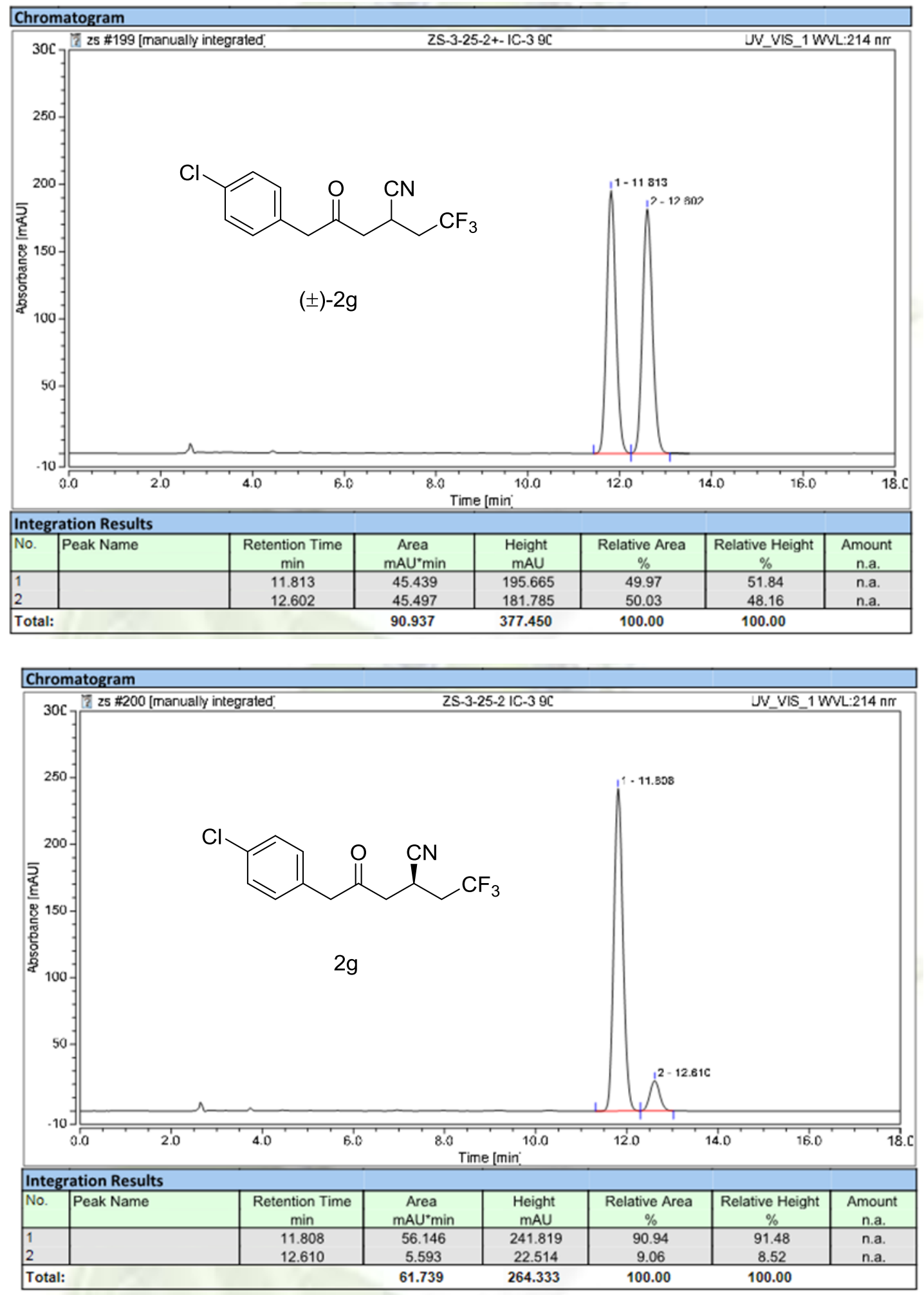

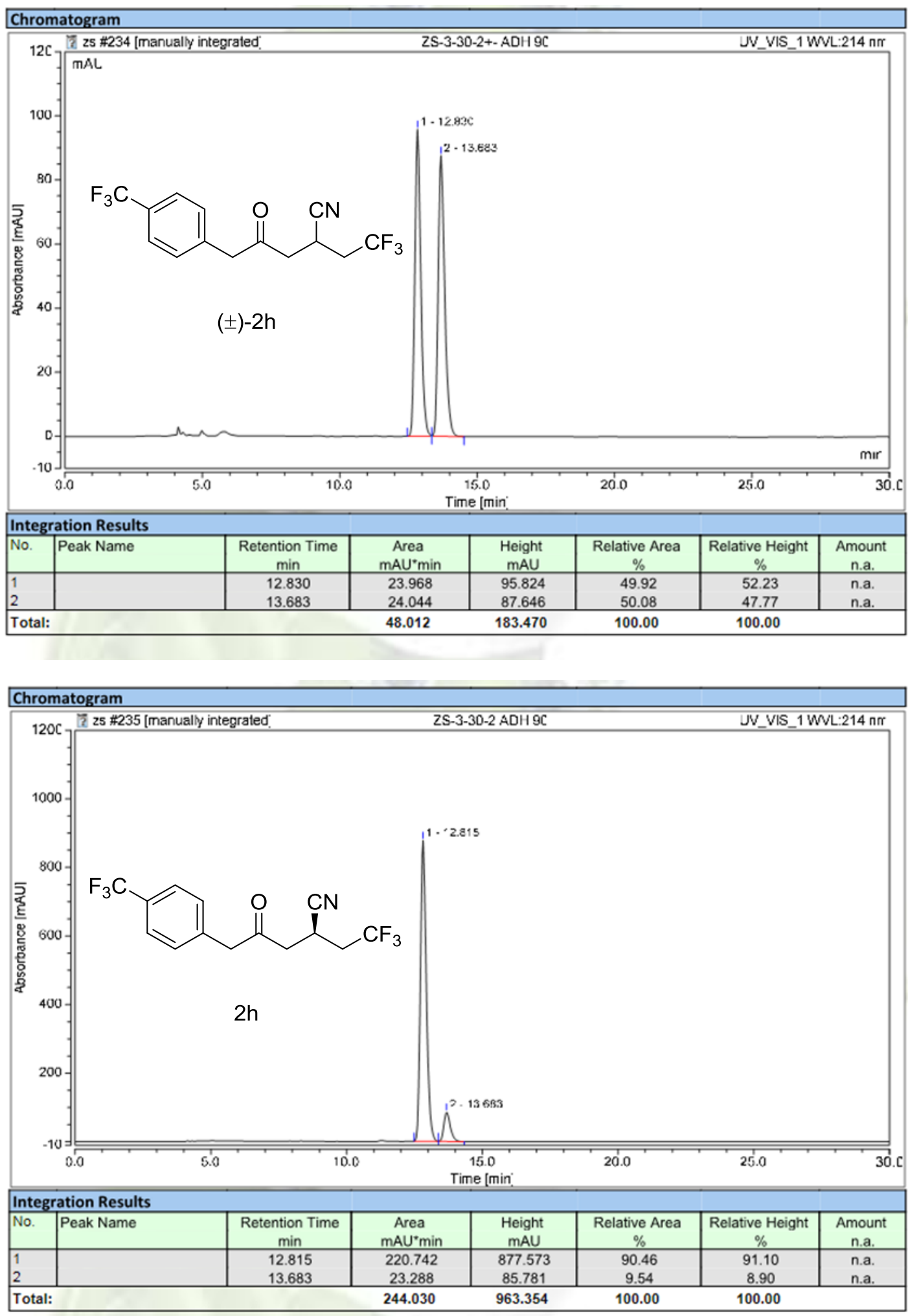

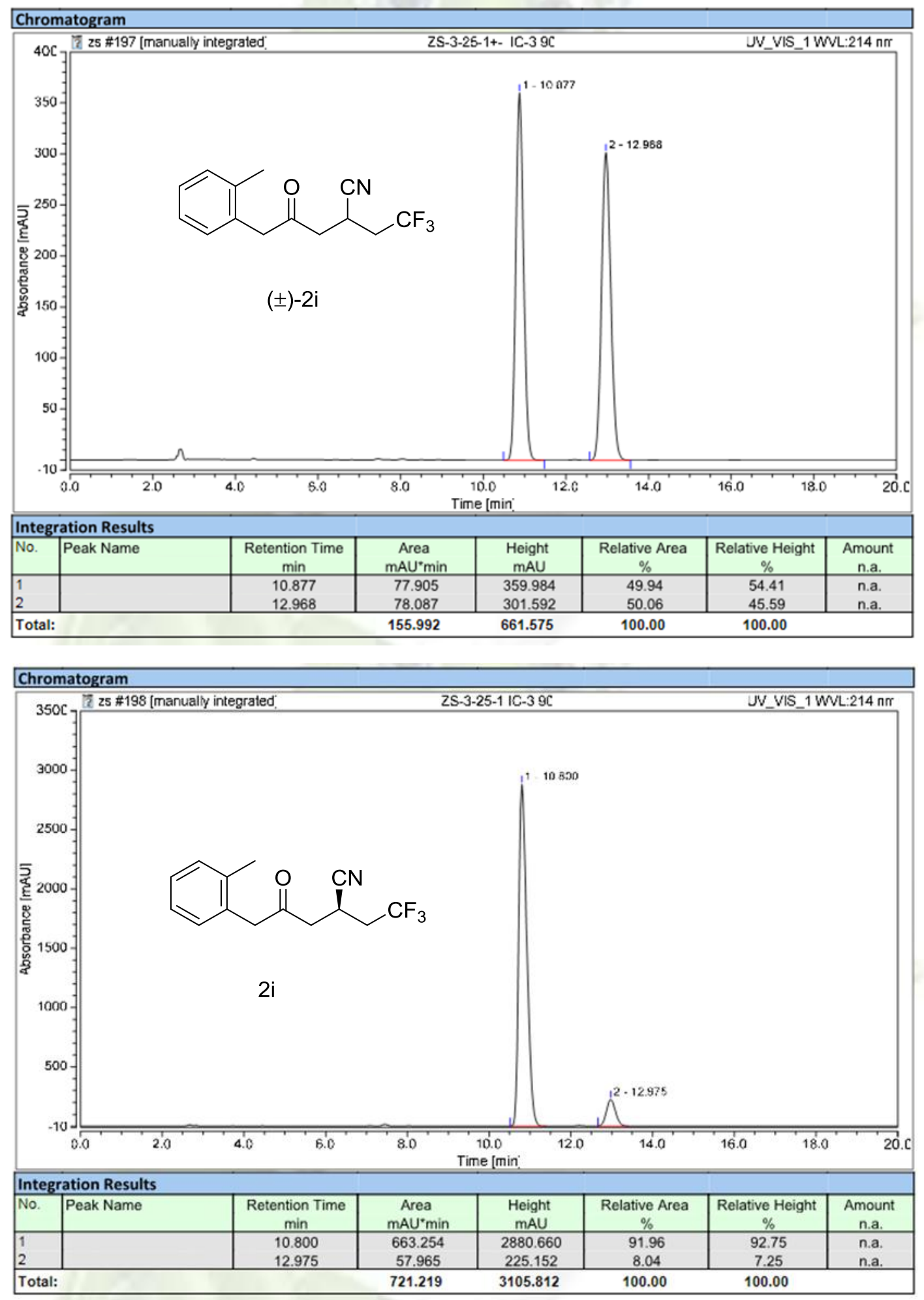

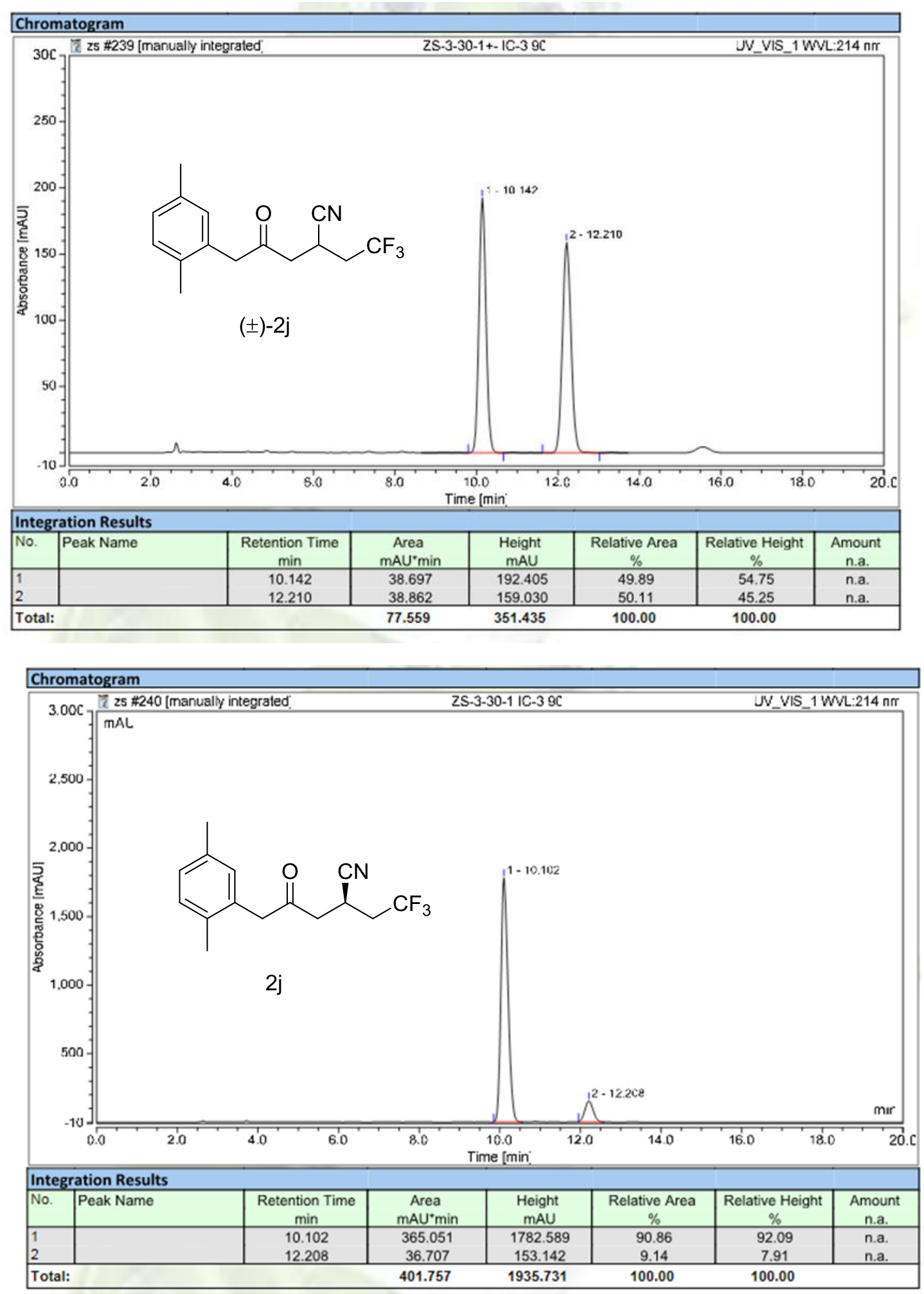


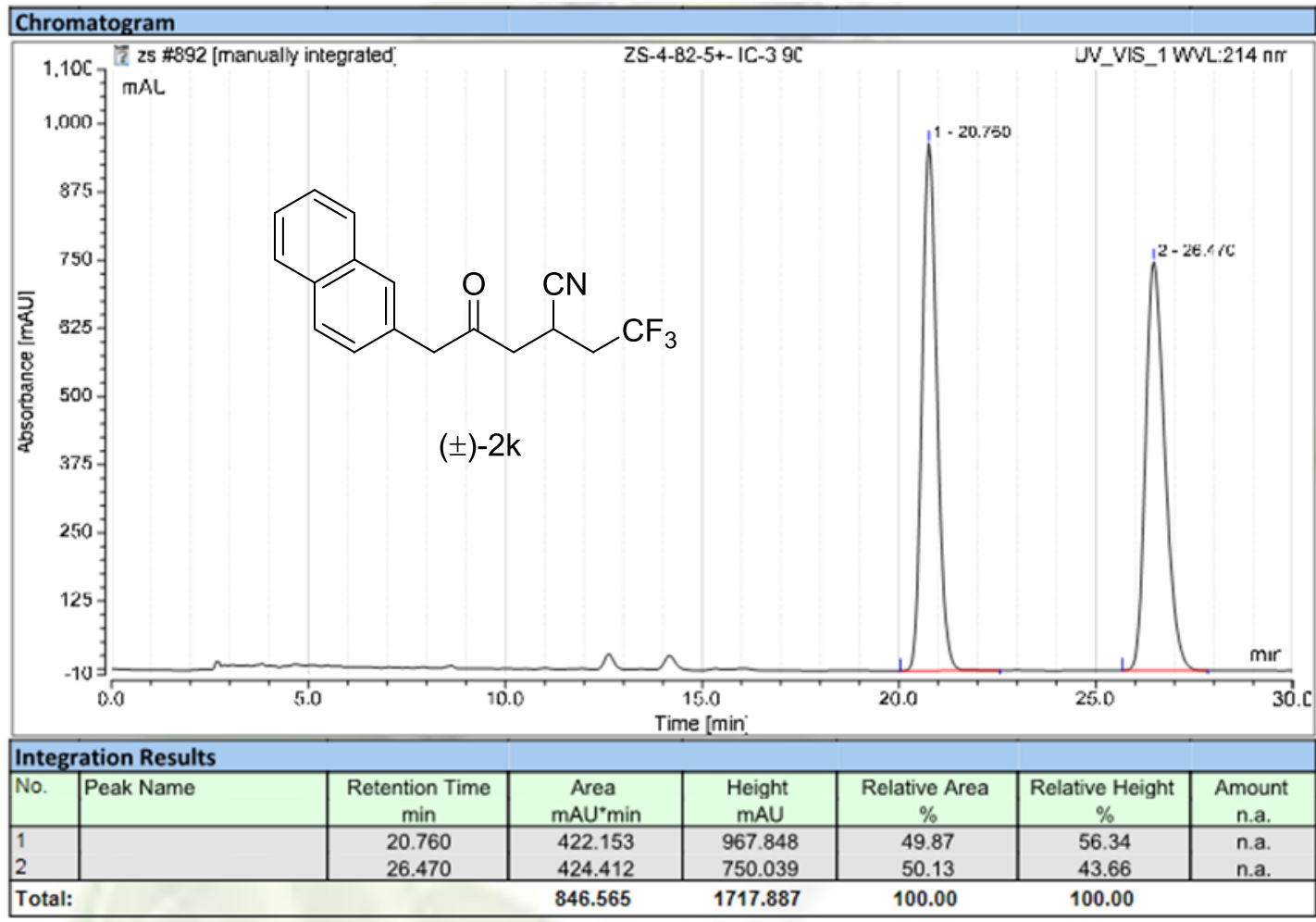

\section{Chromatogram}

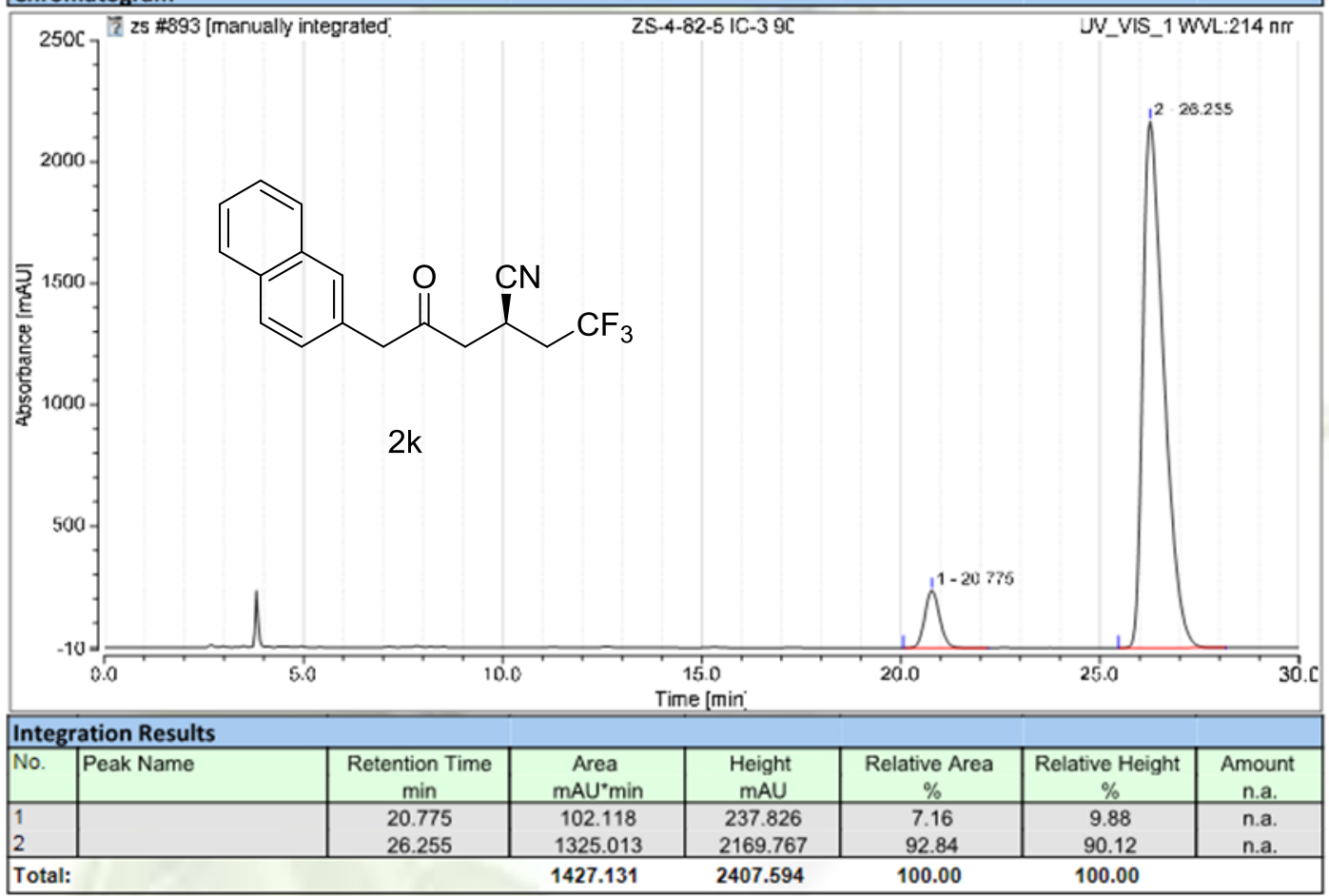



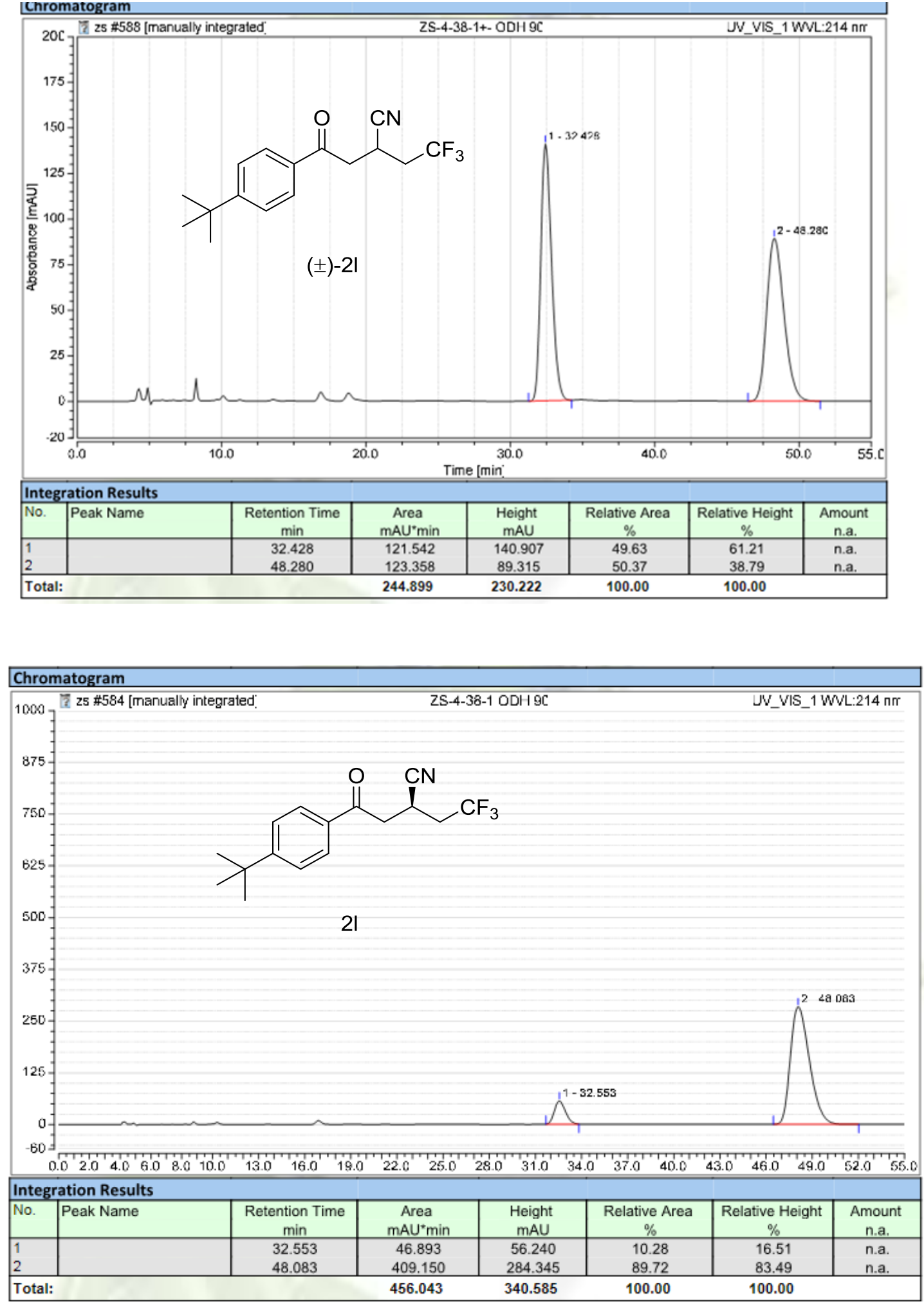

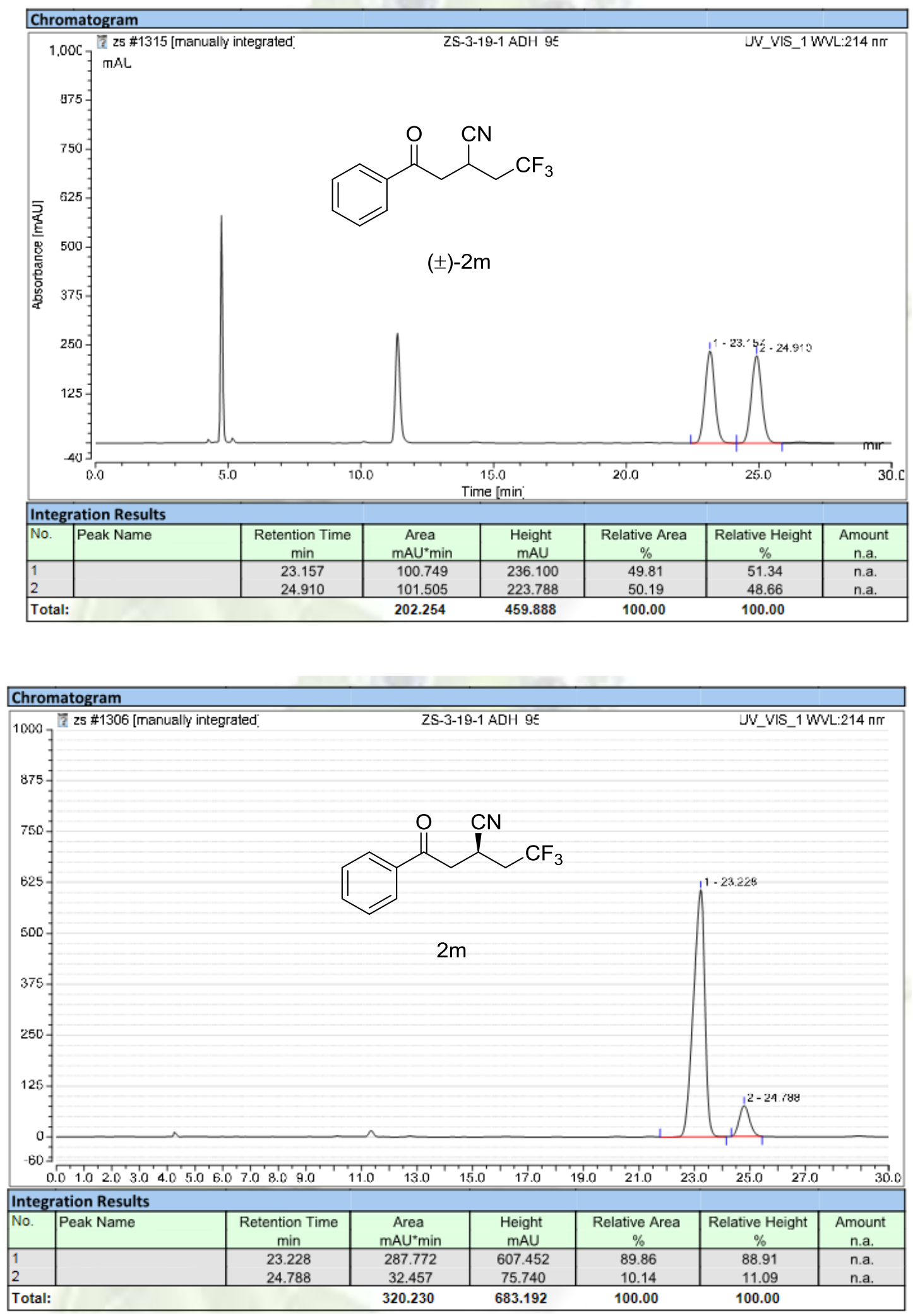

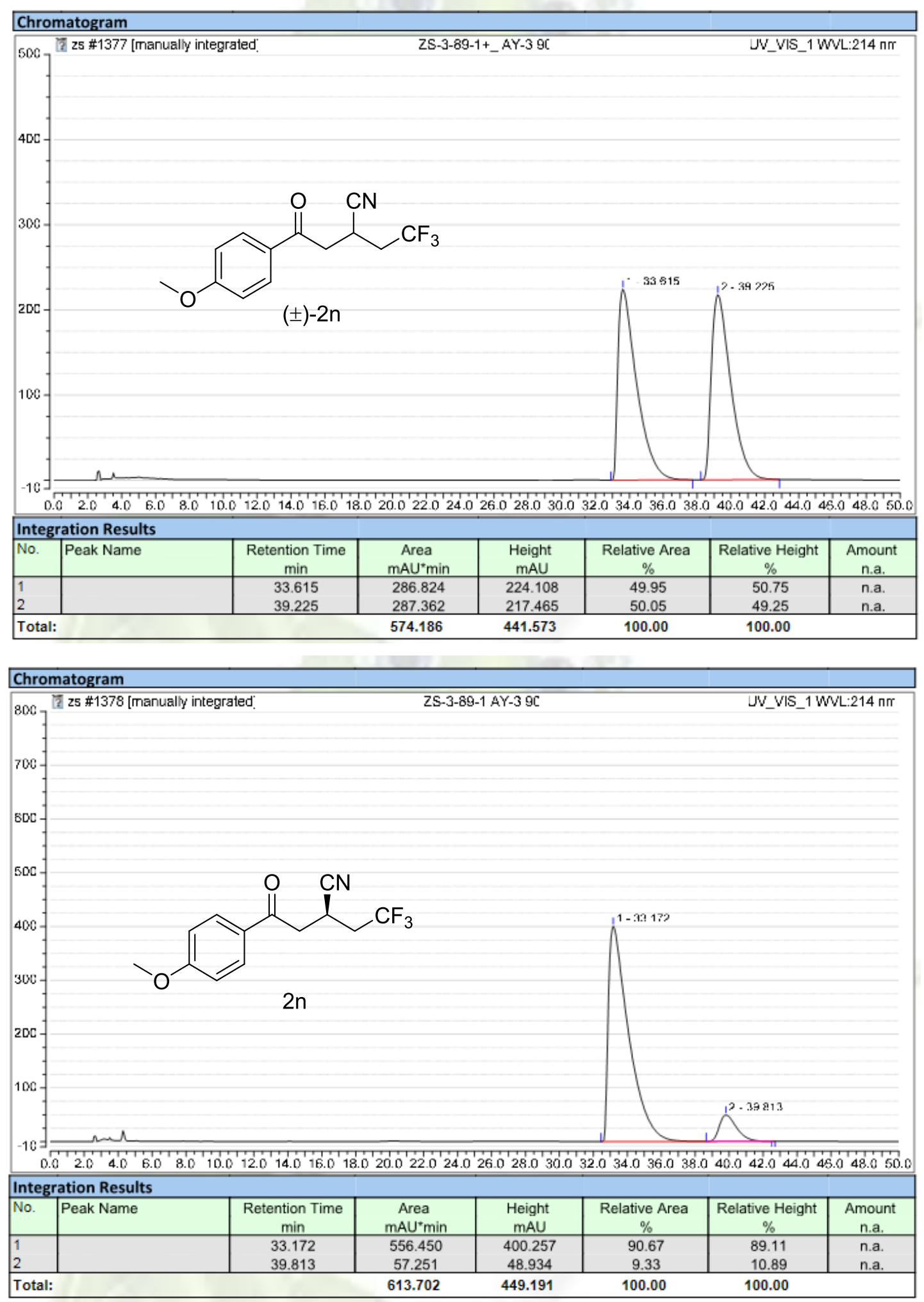

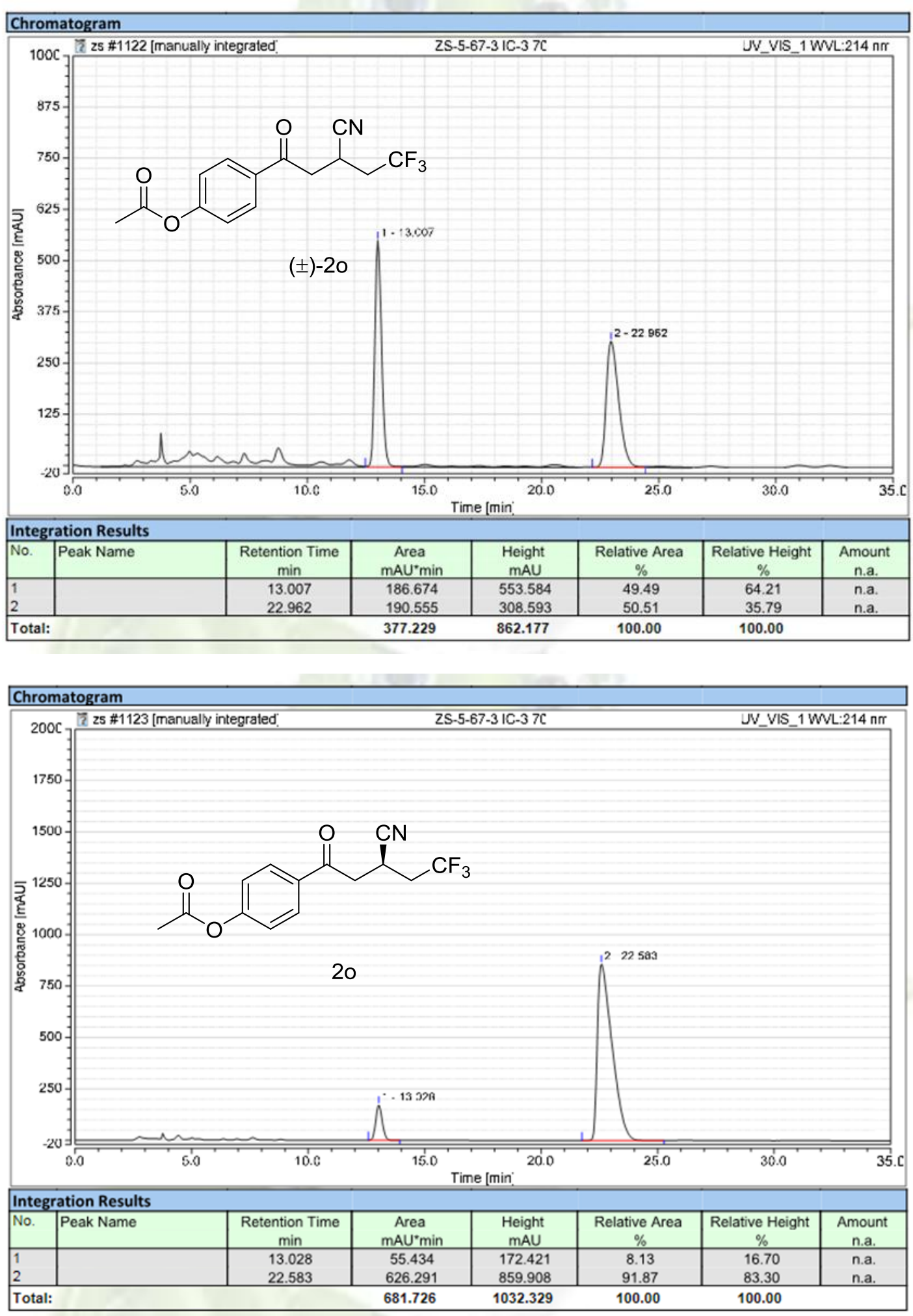

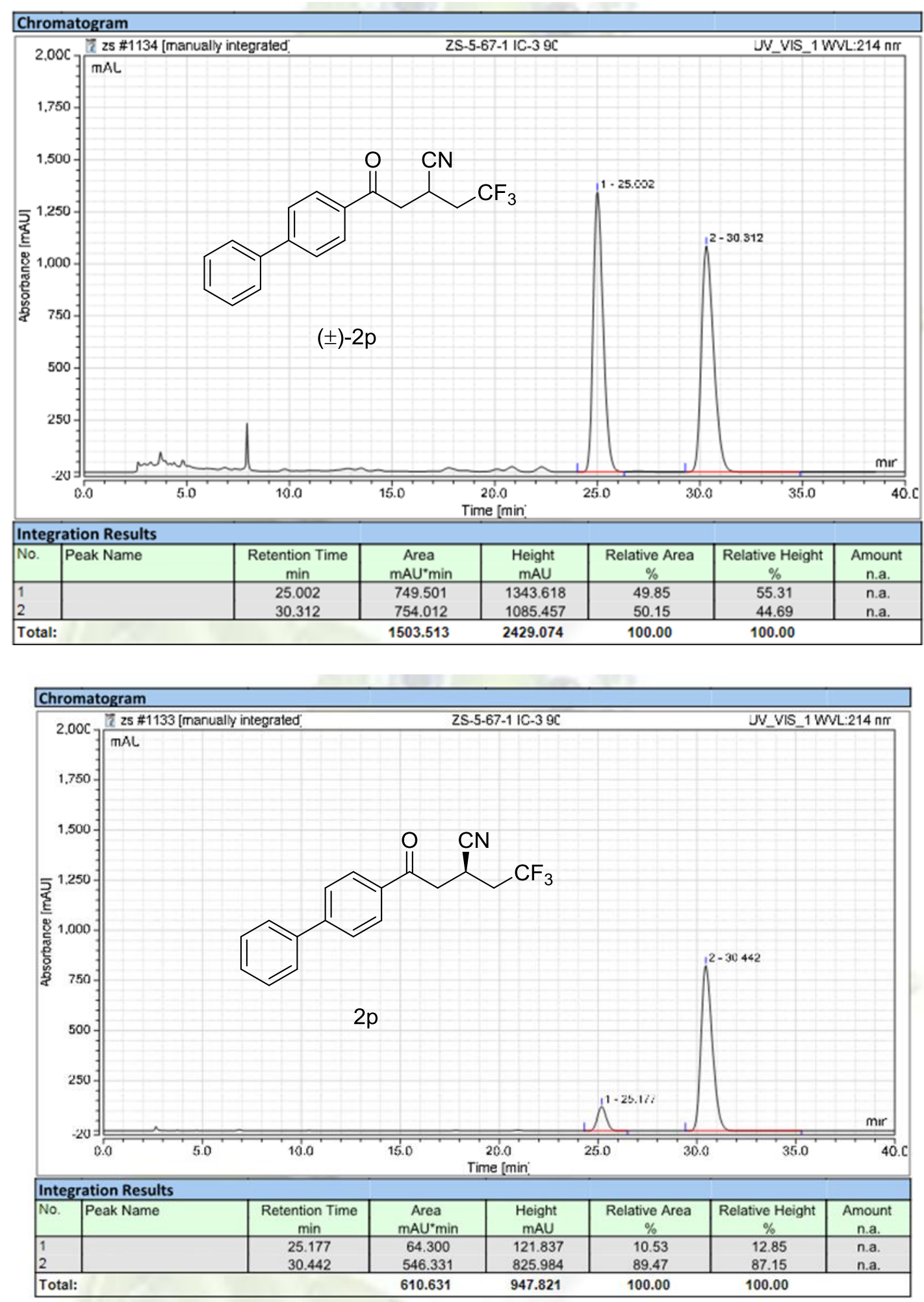

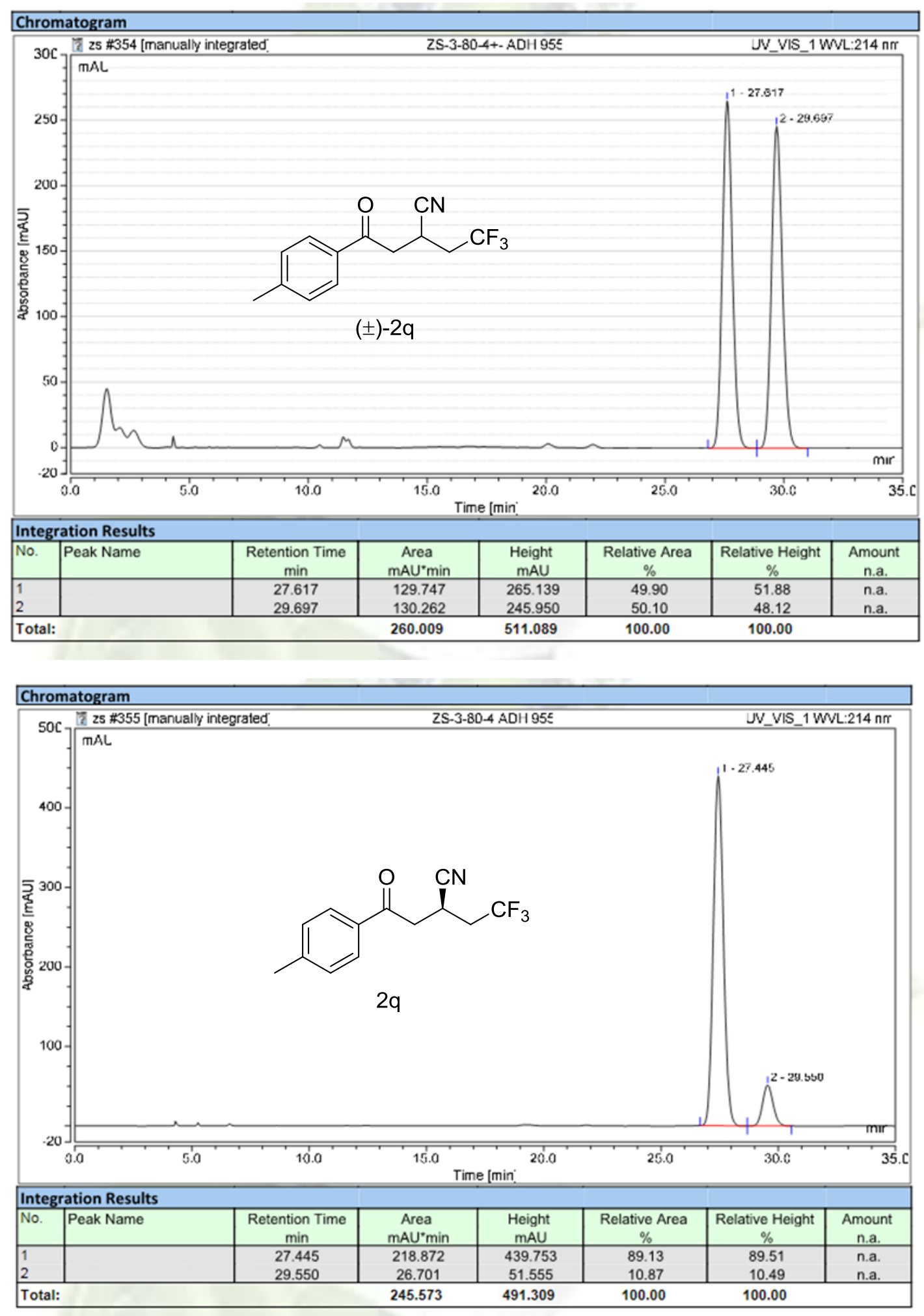

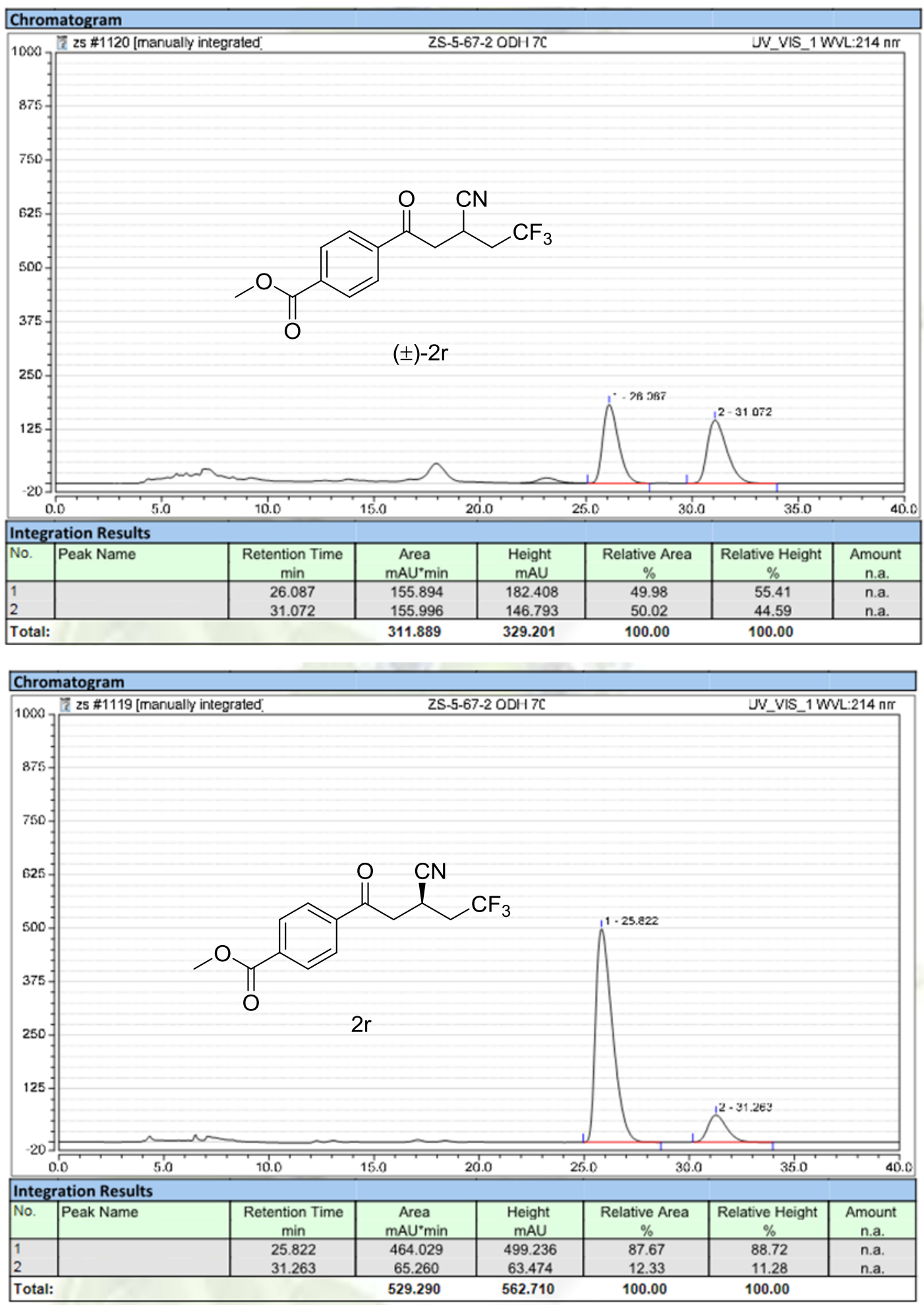

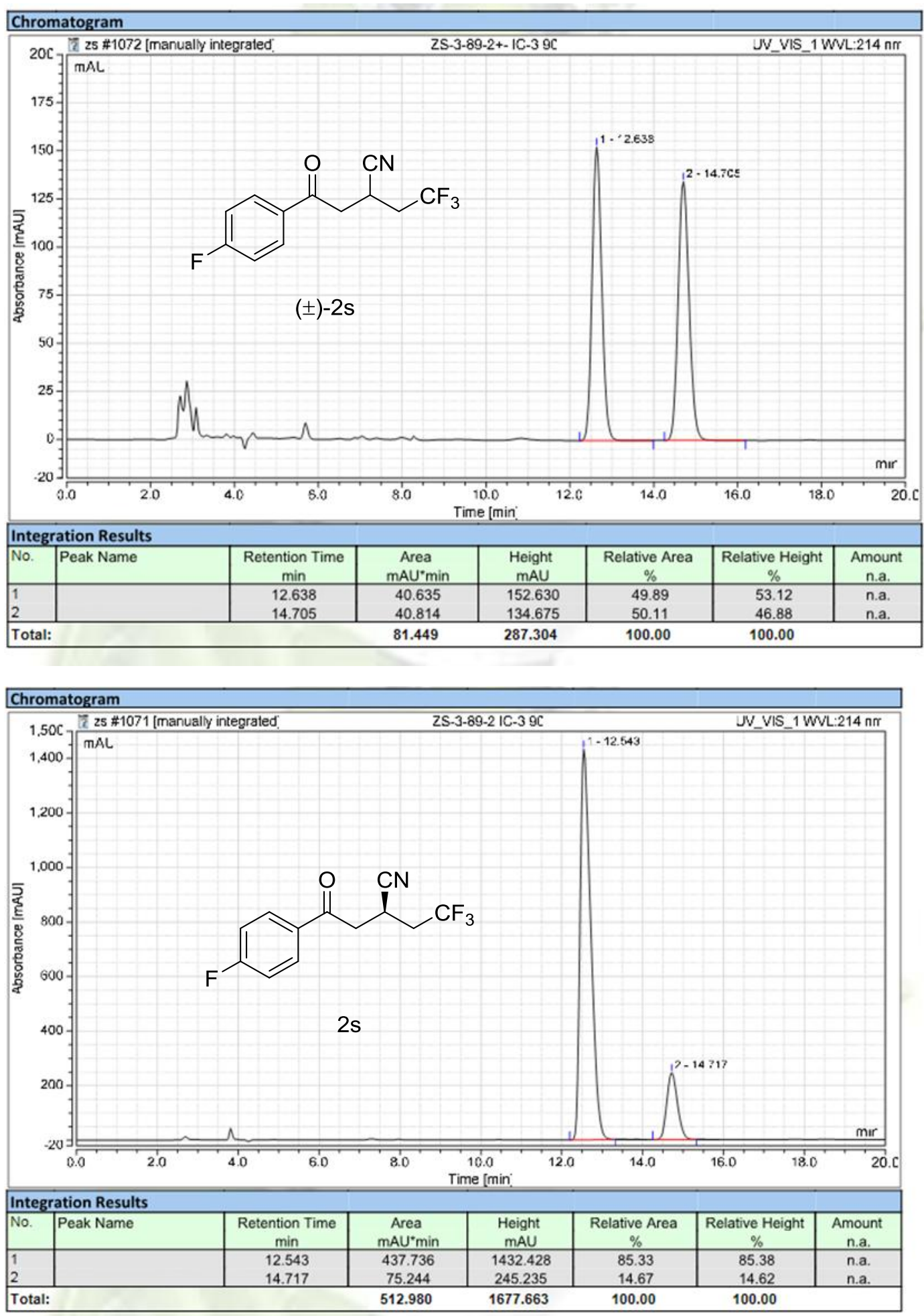

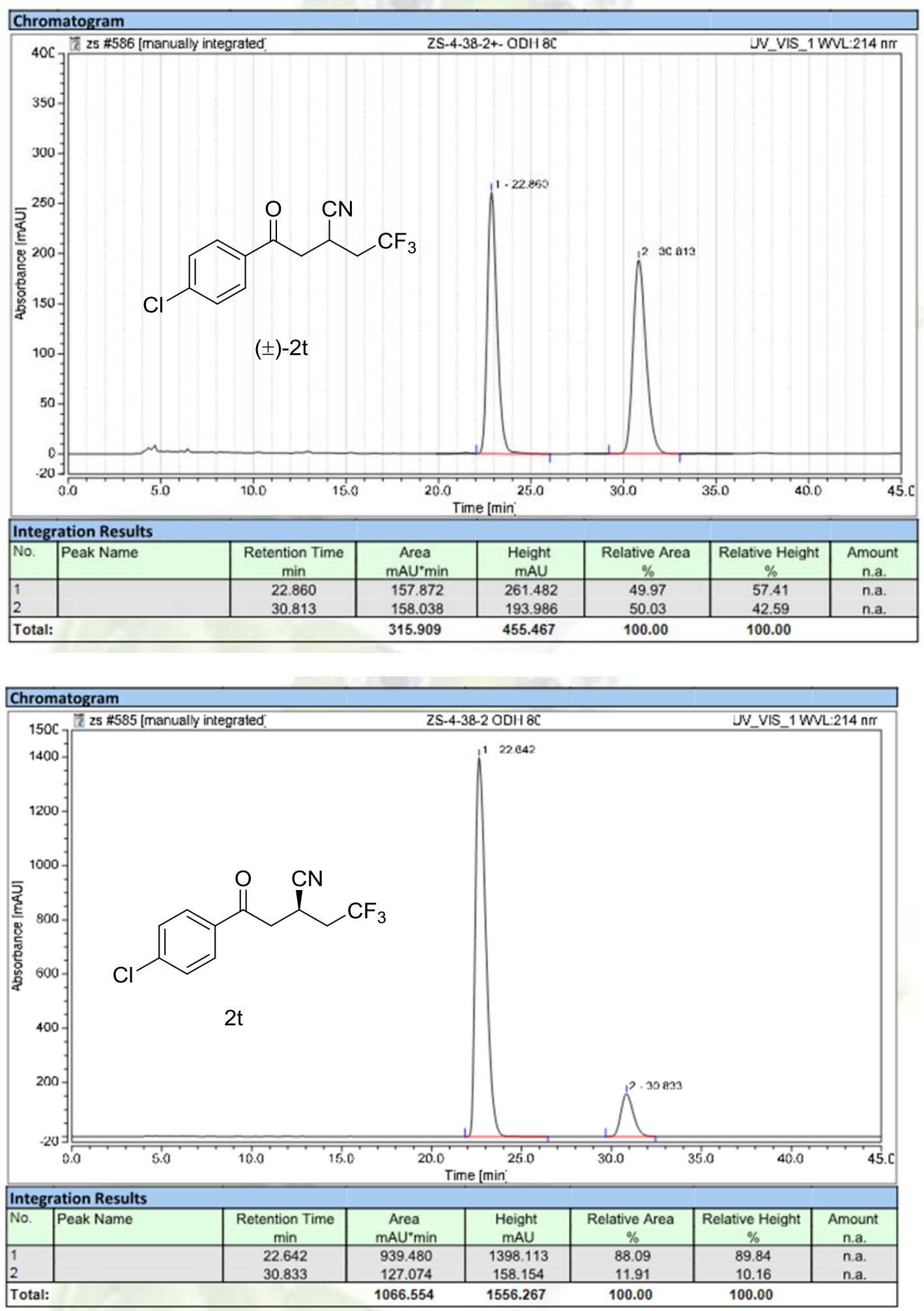

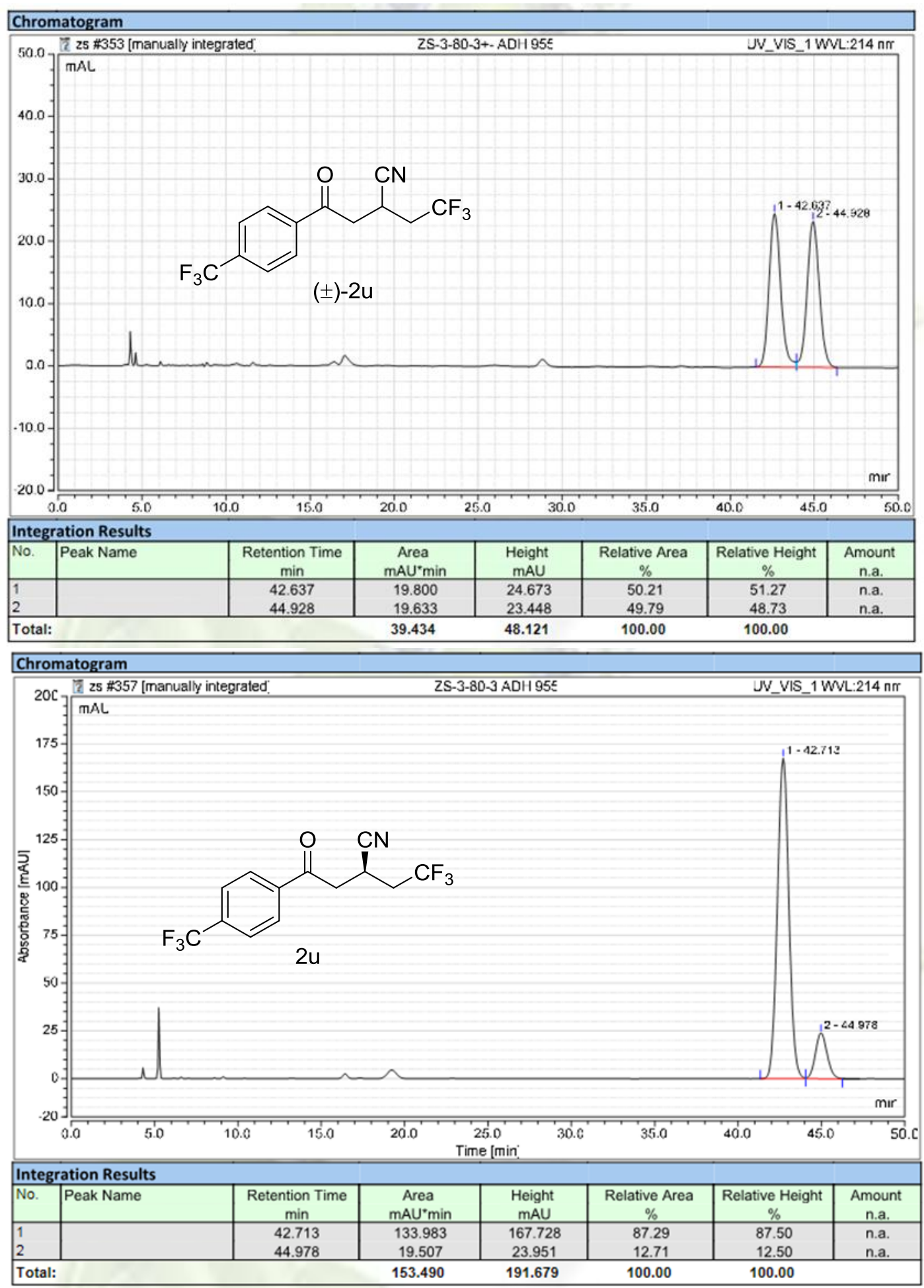

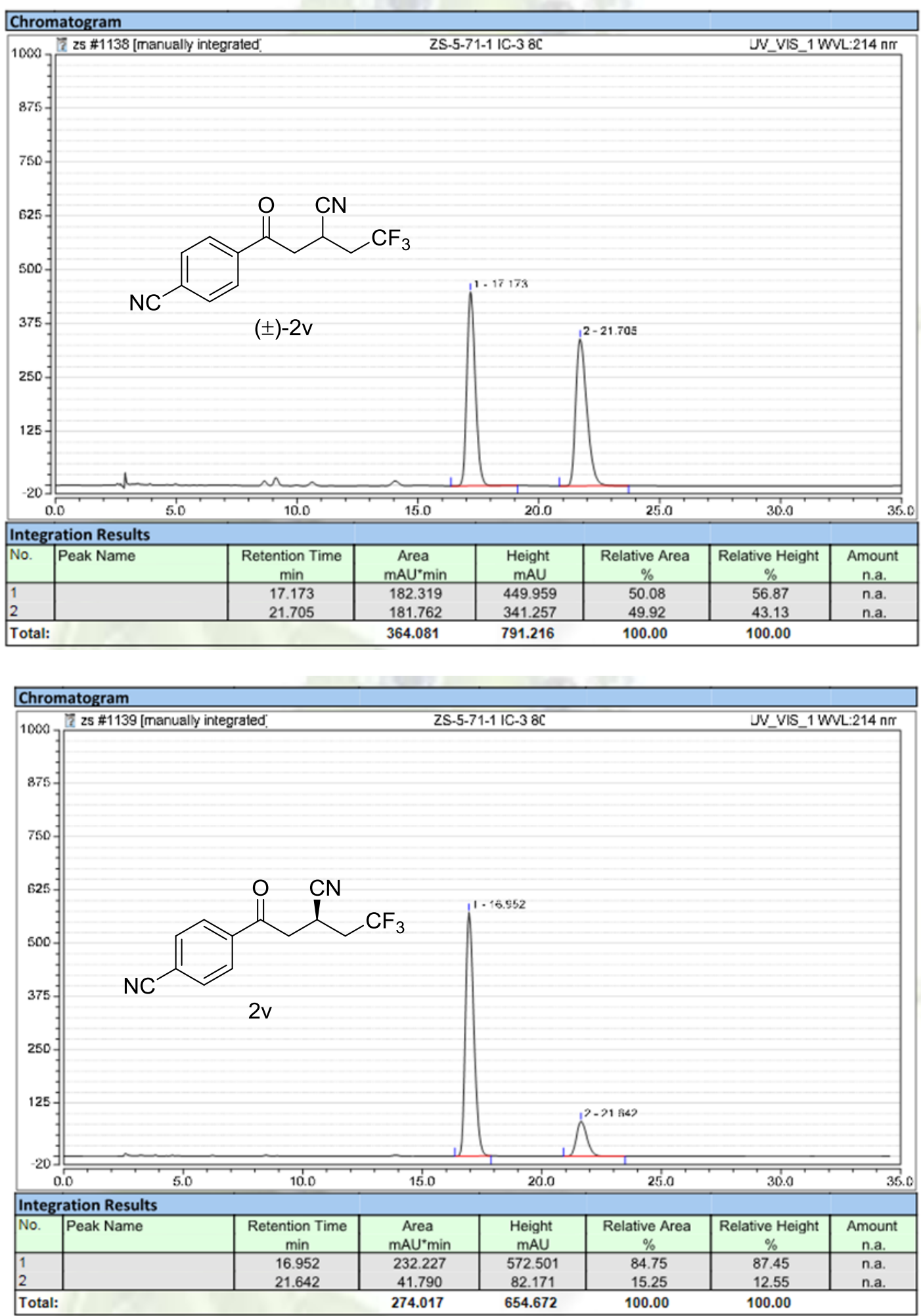

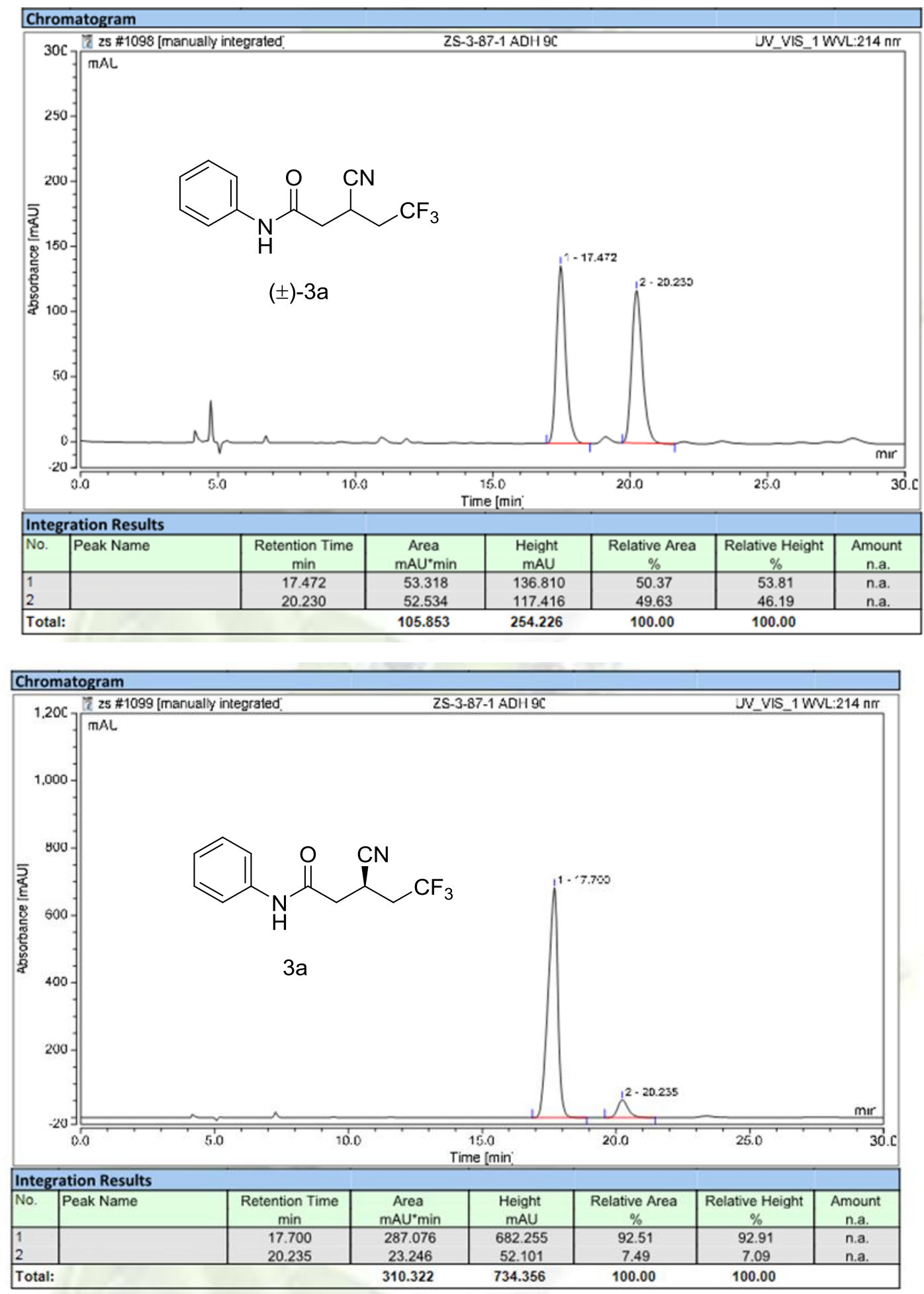

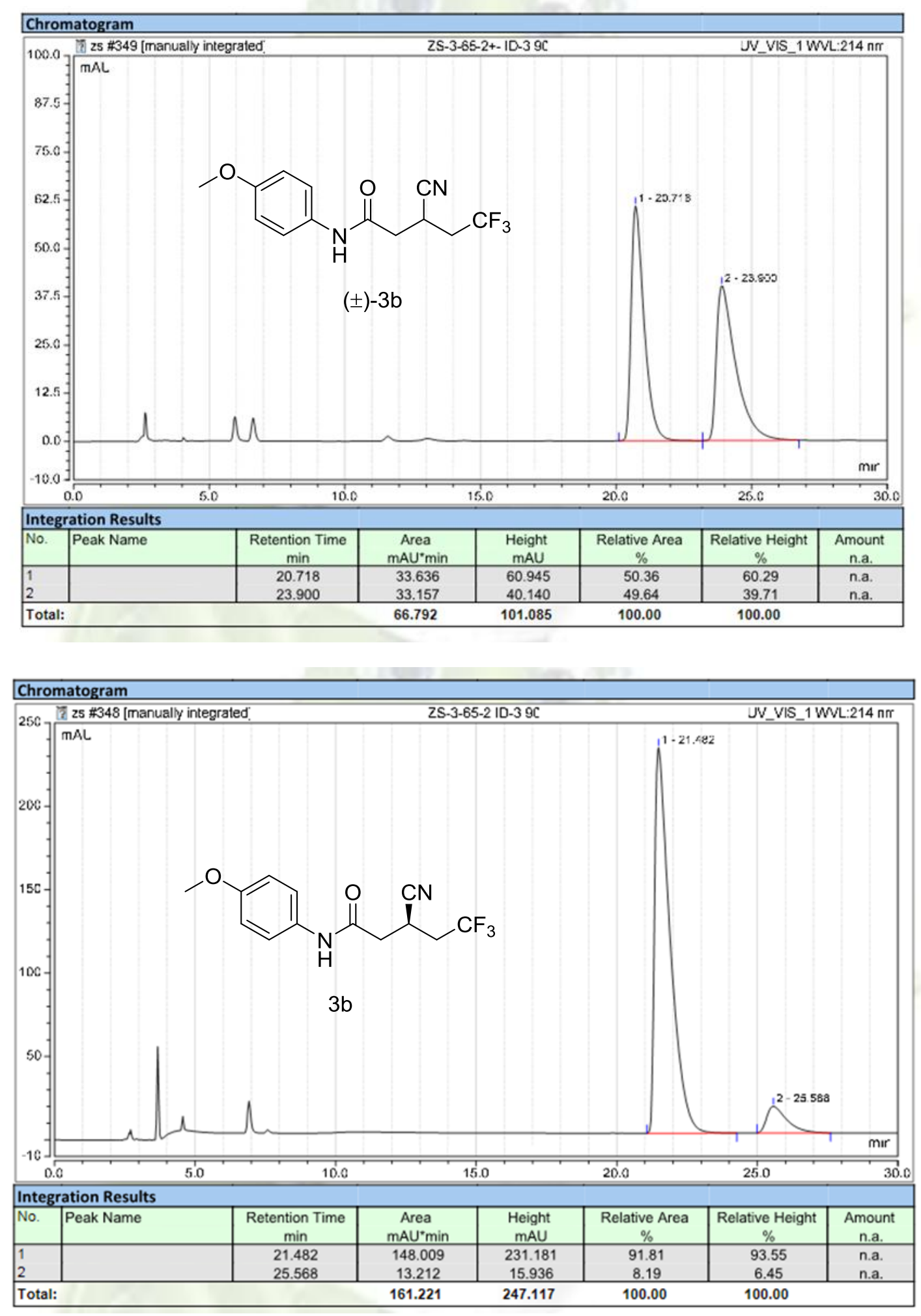

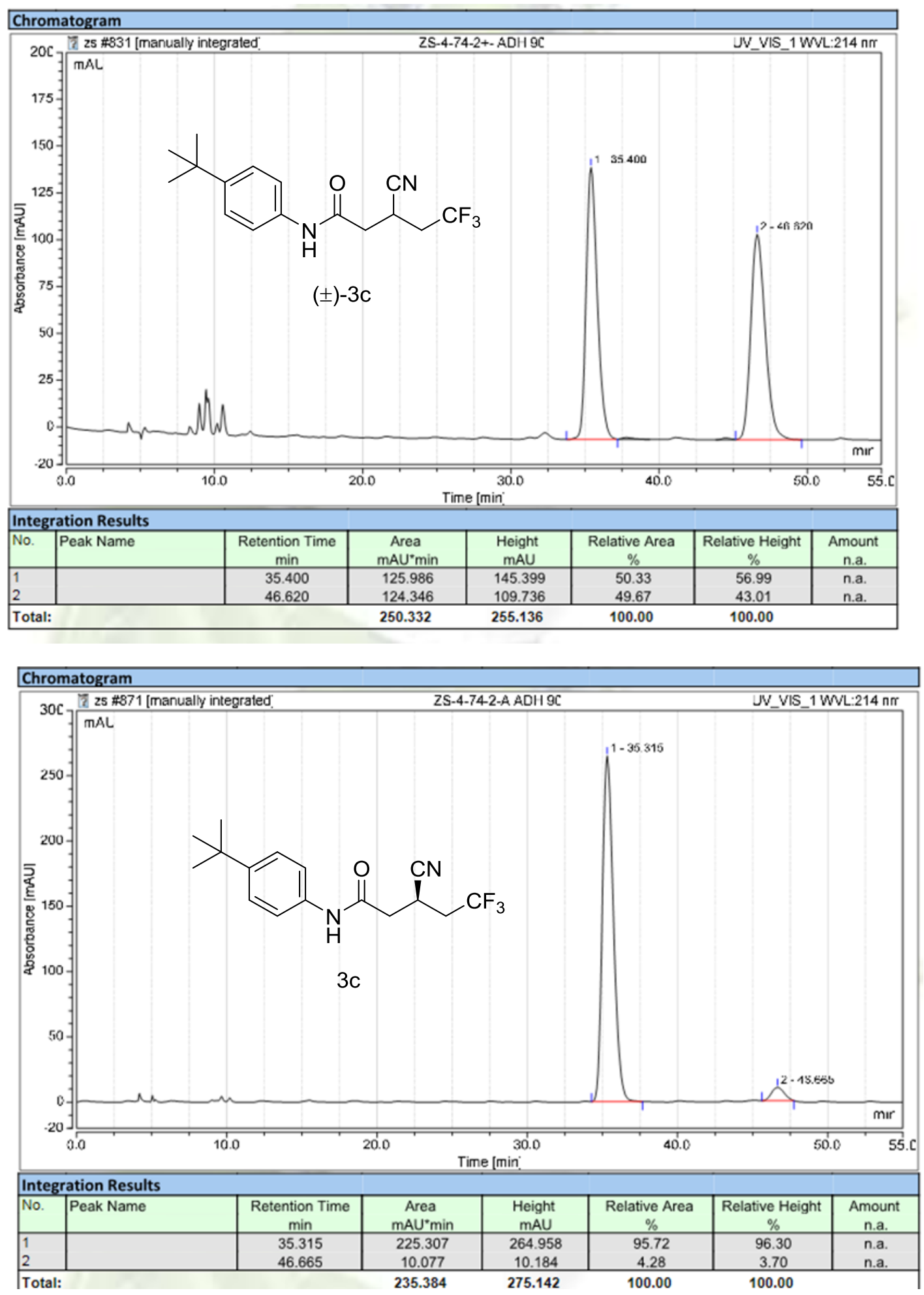

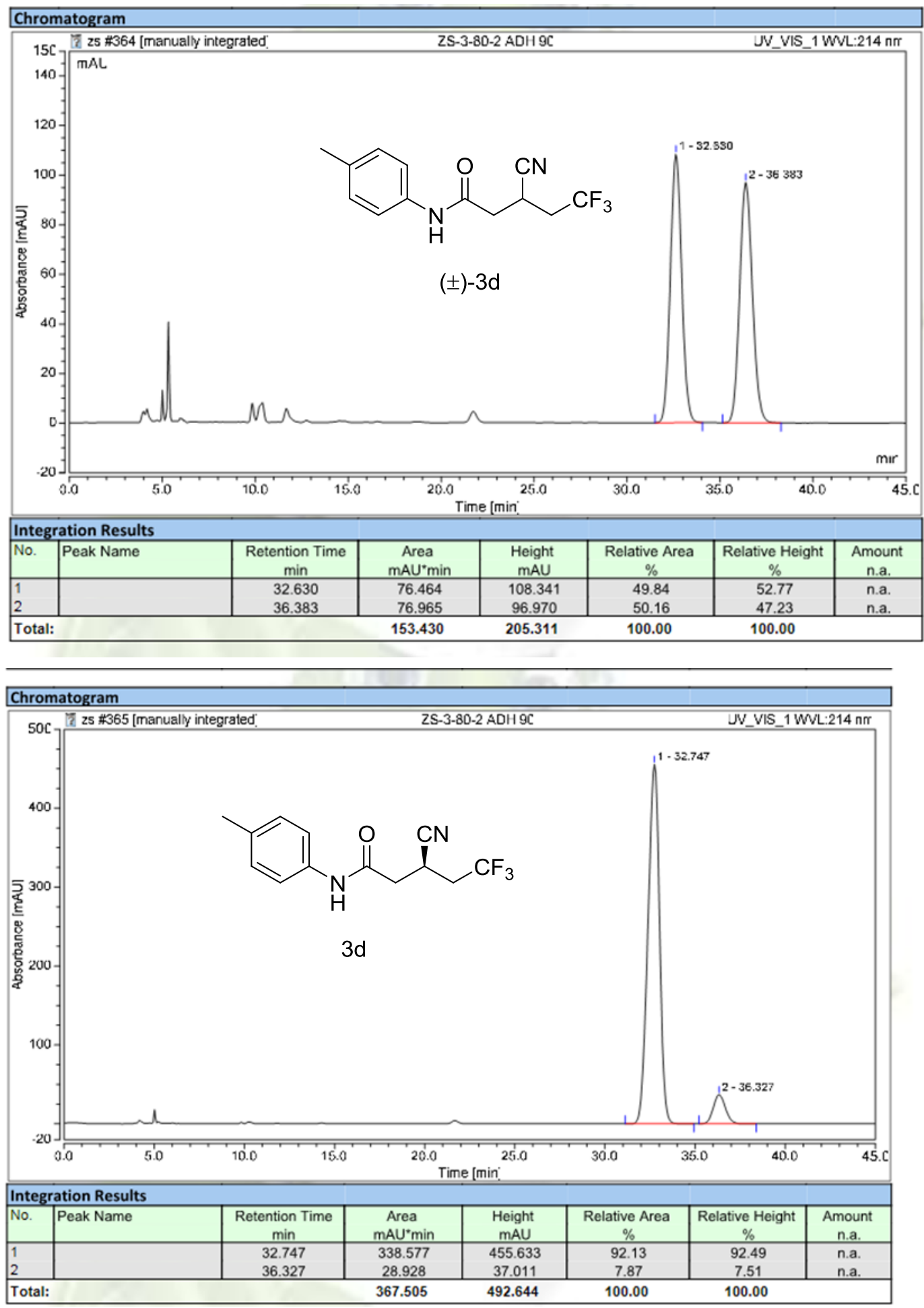

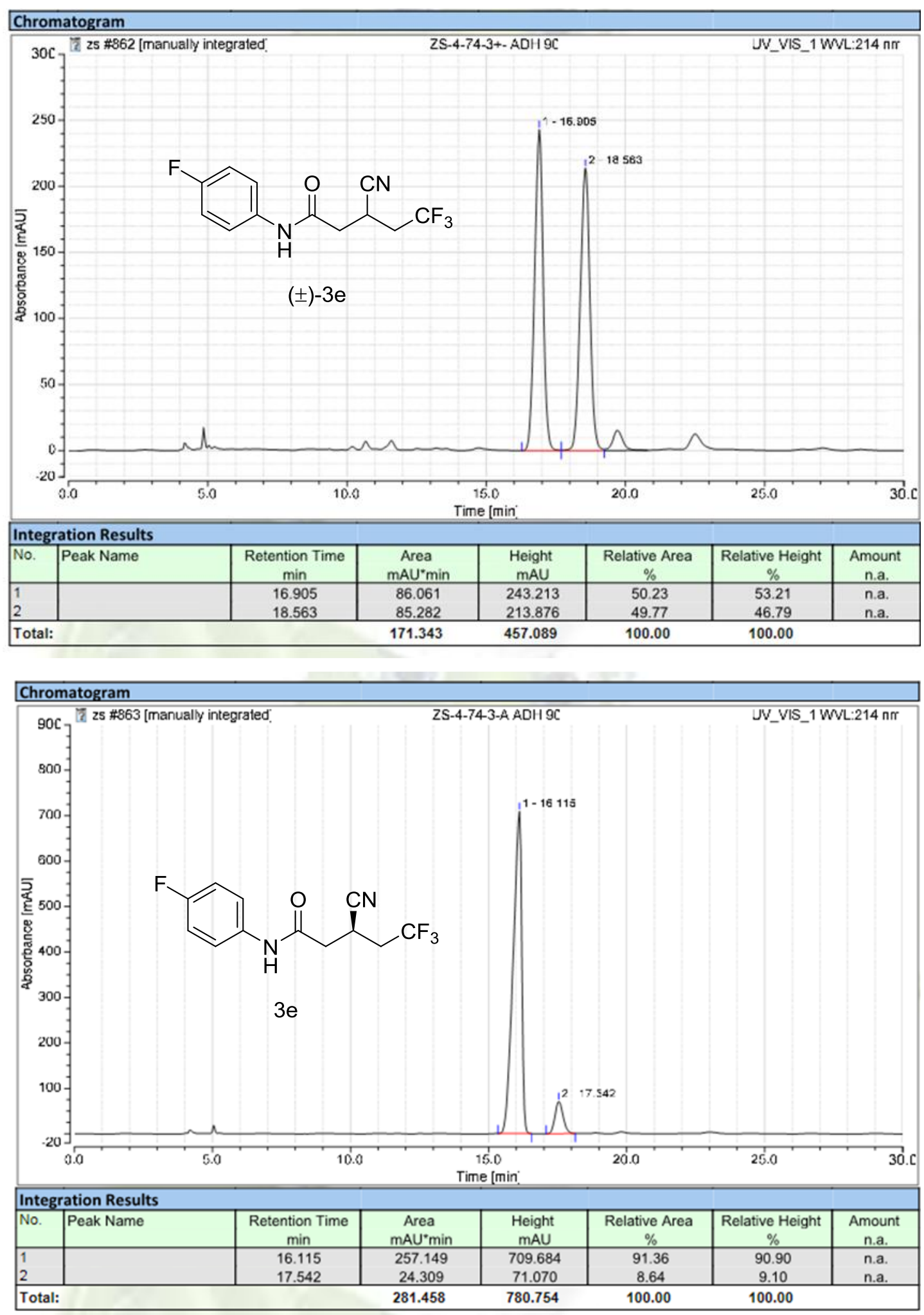

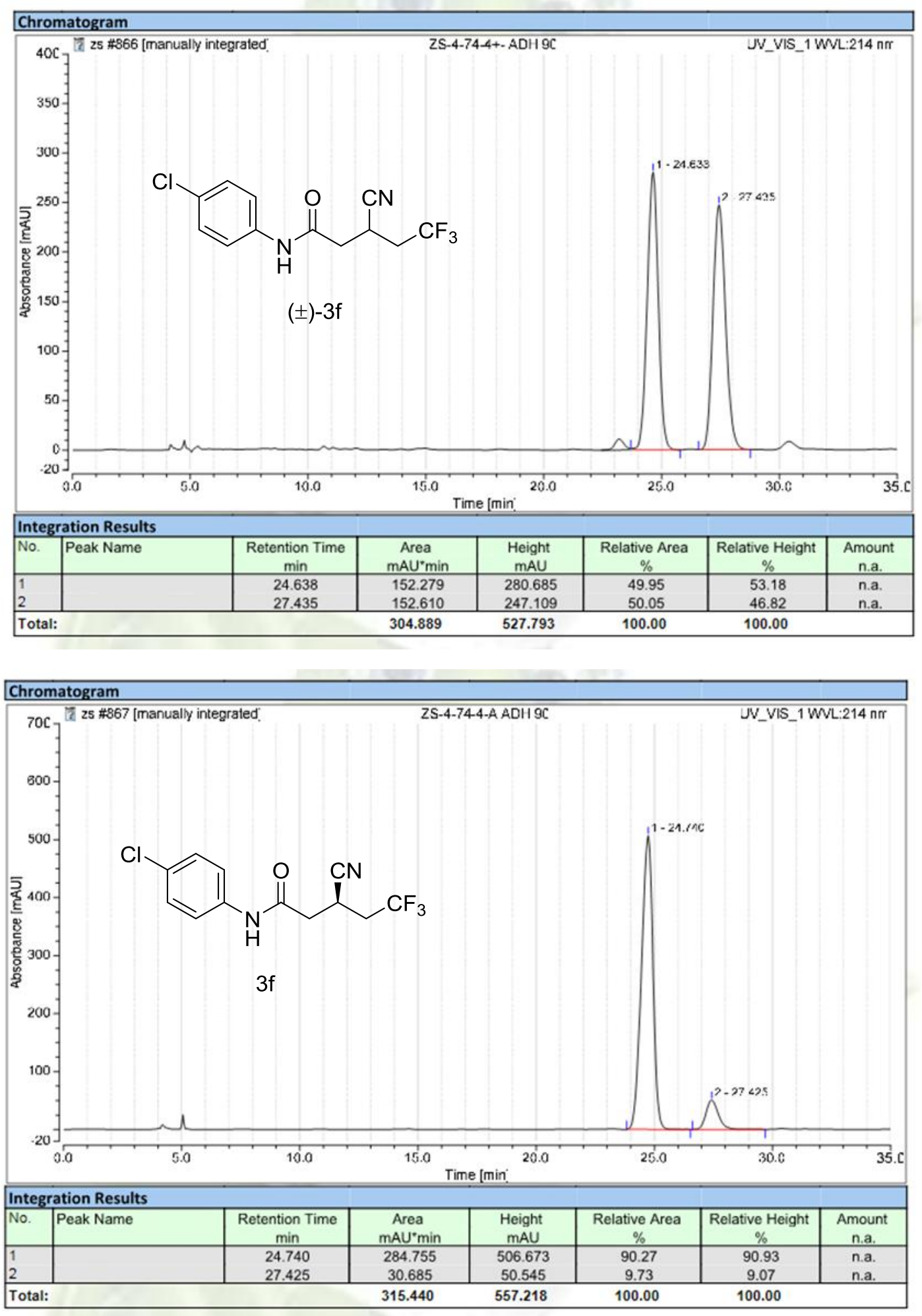

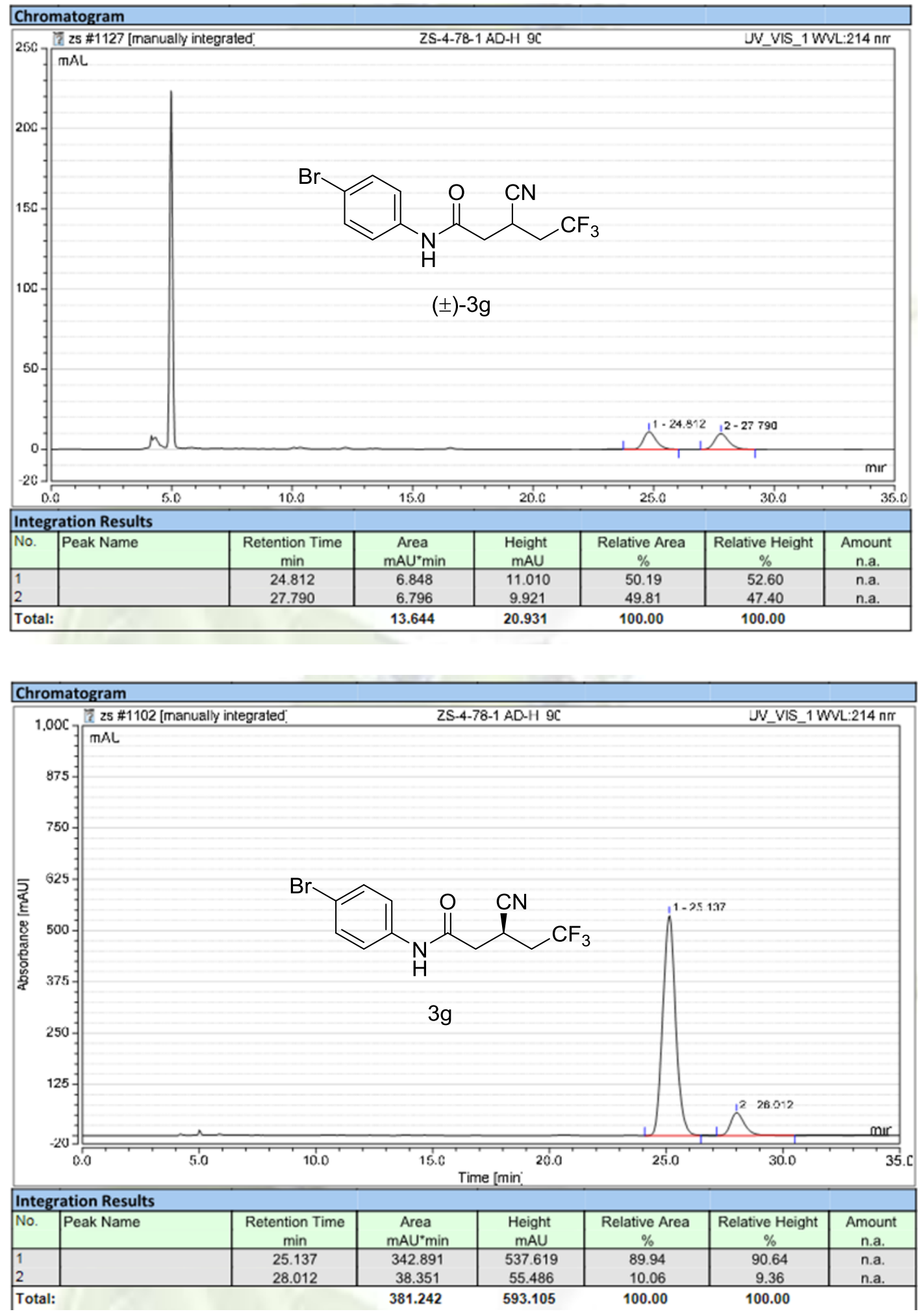


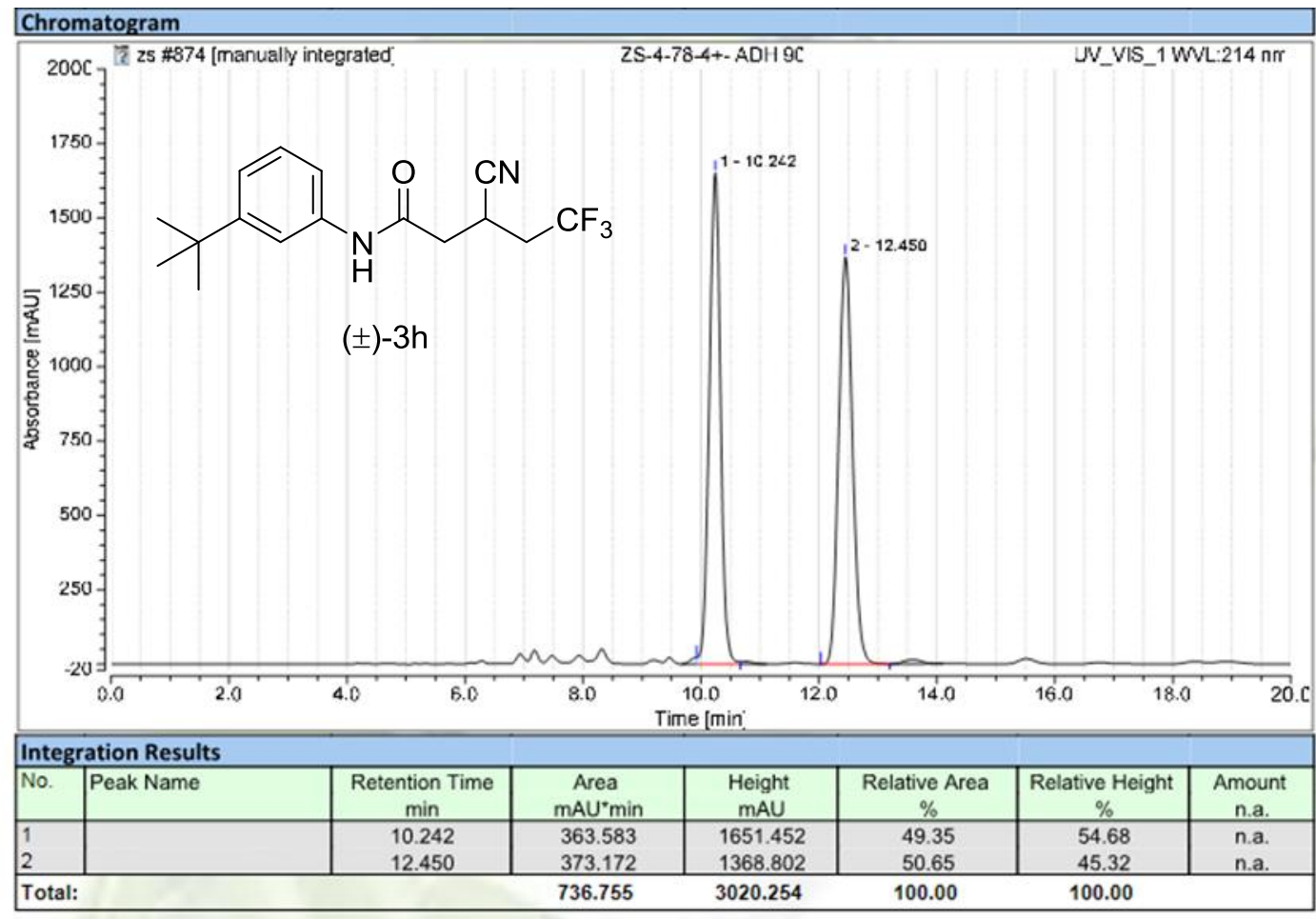

\section{Chromatogram}

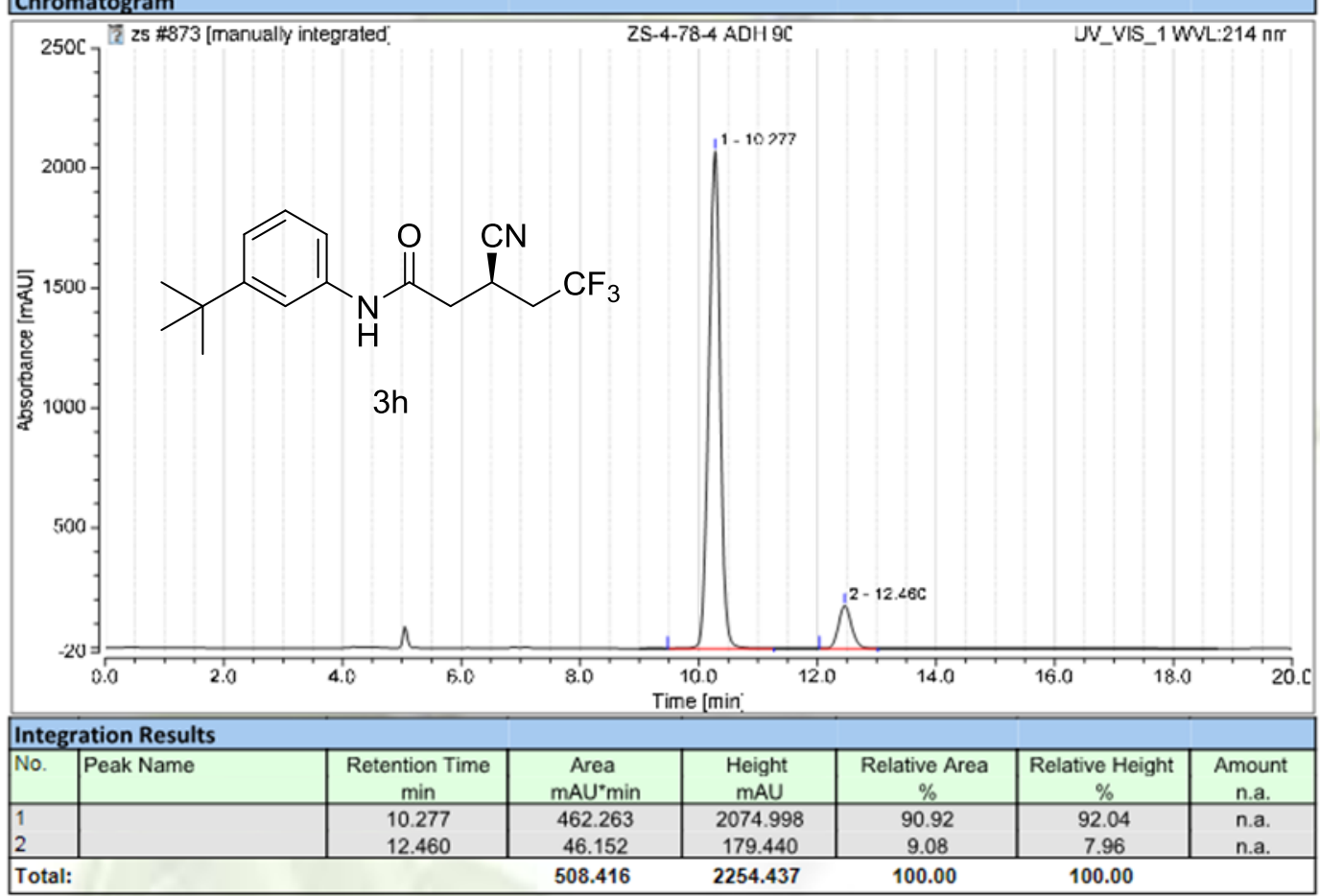



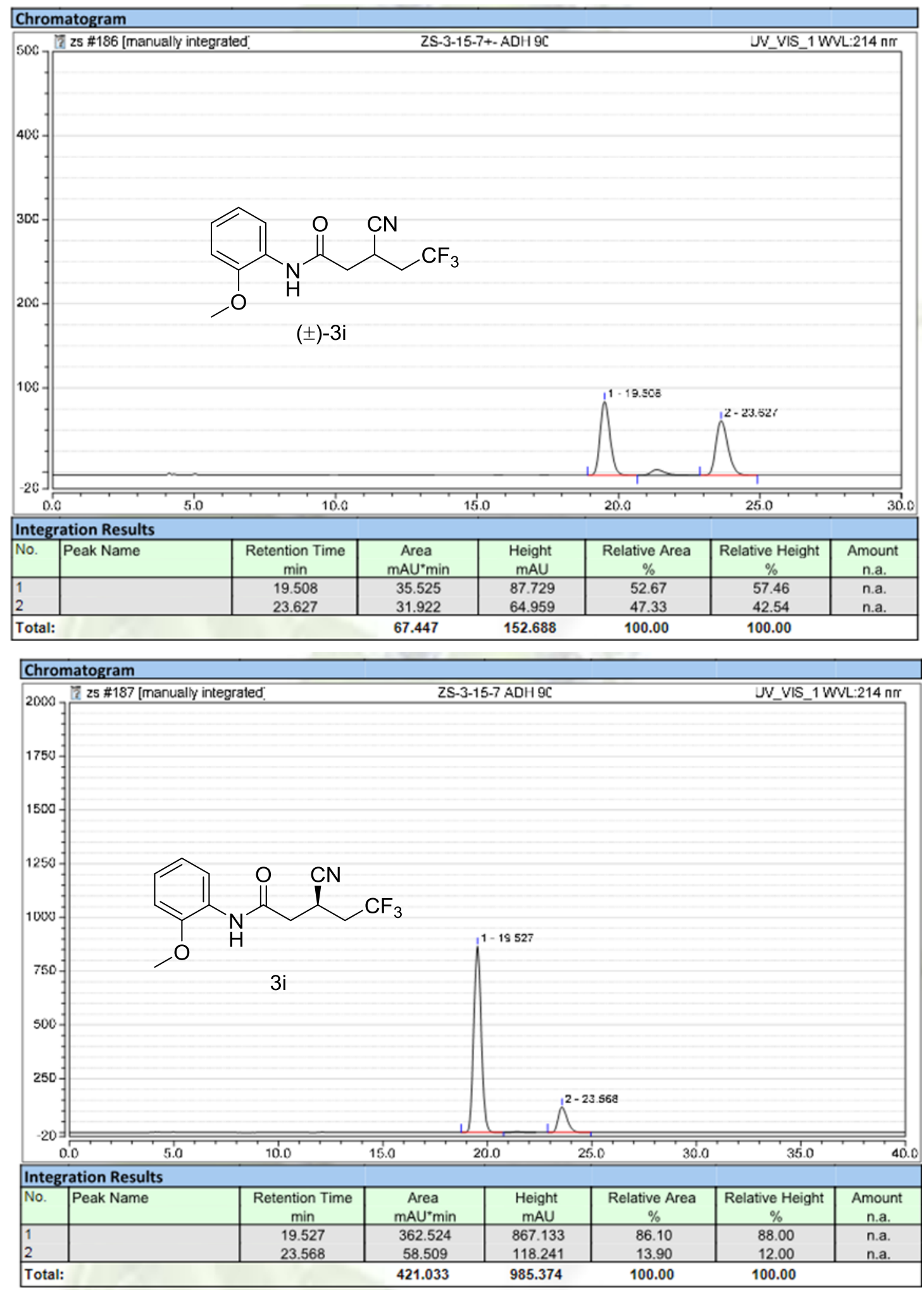

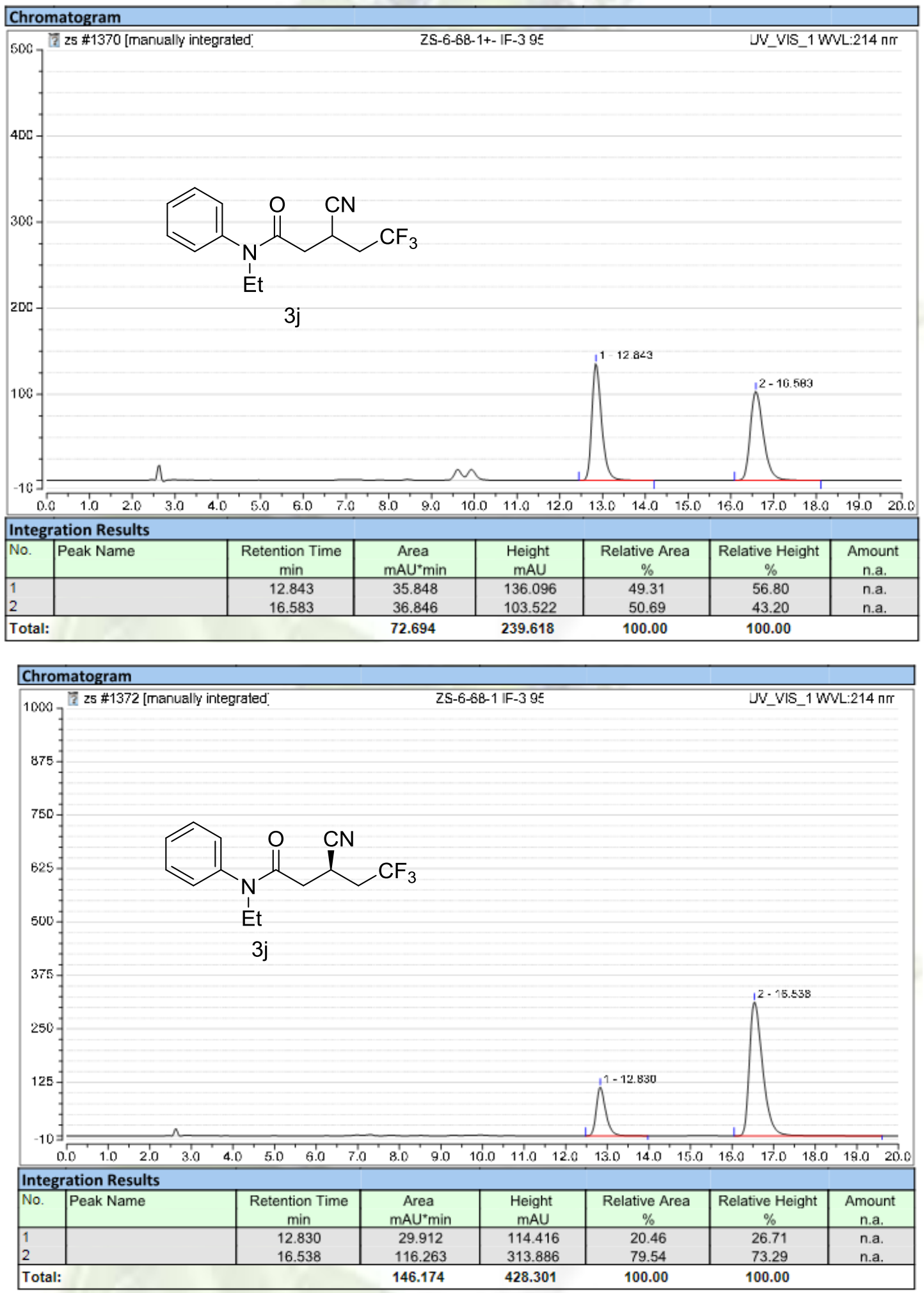

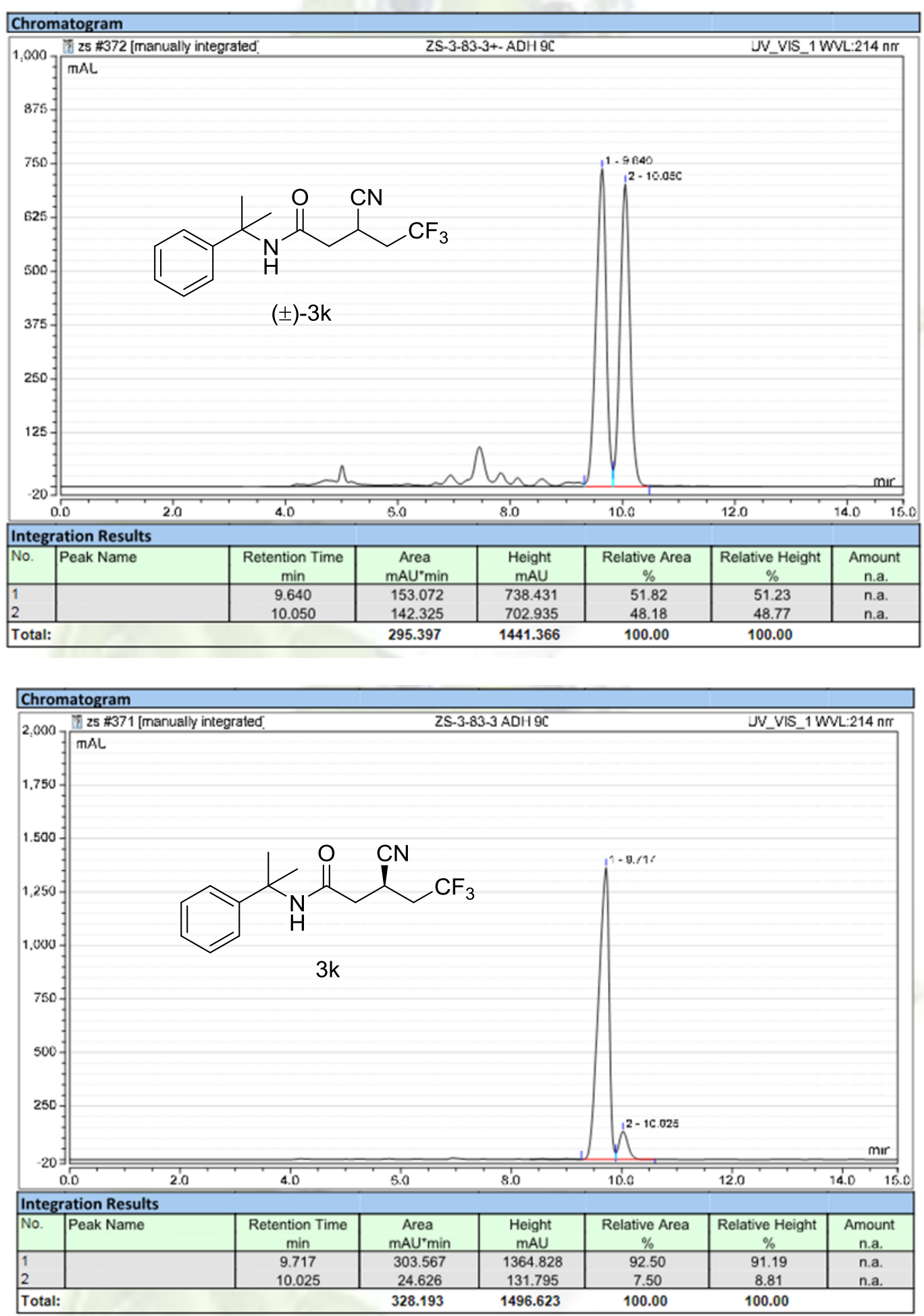

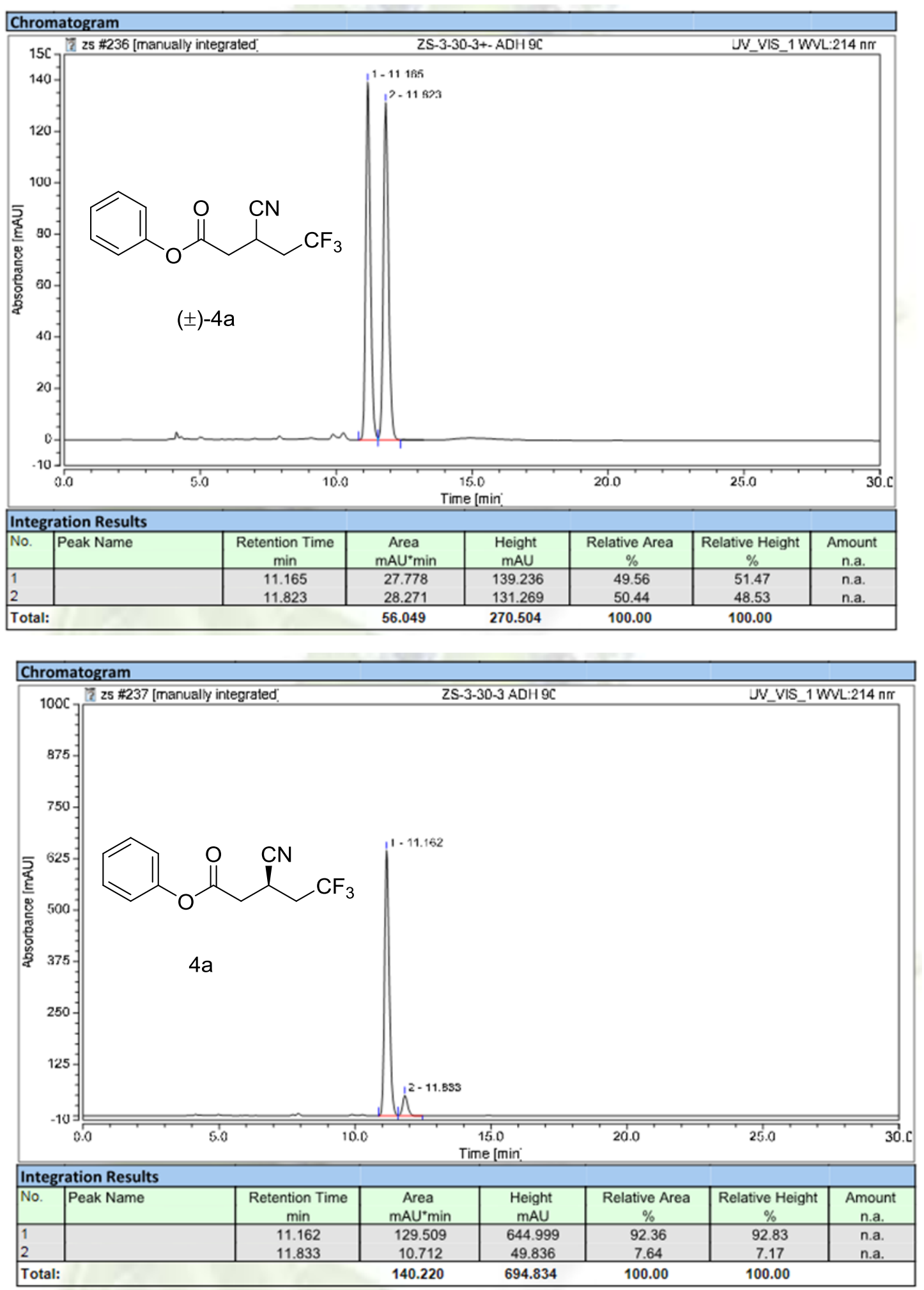

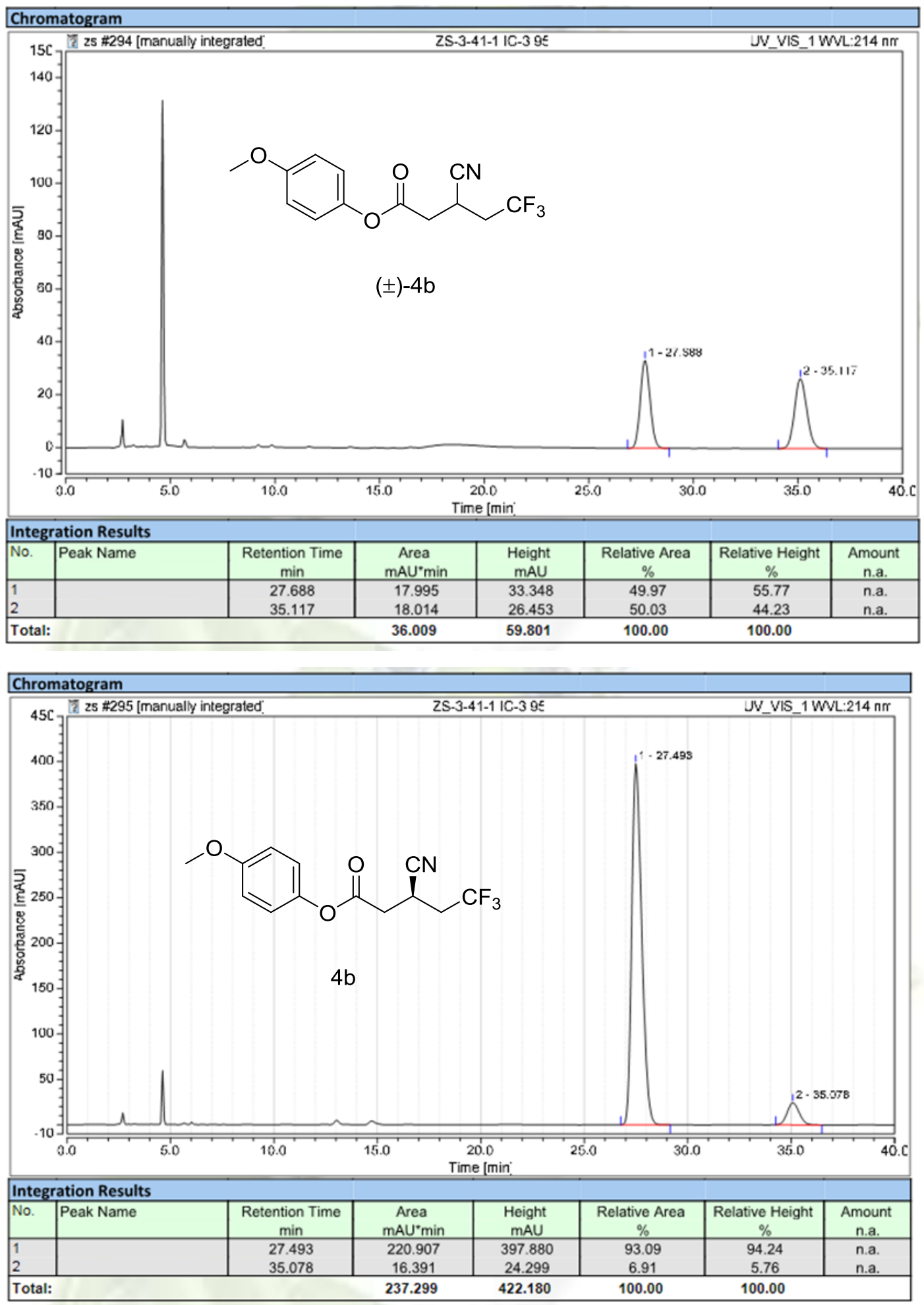

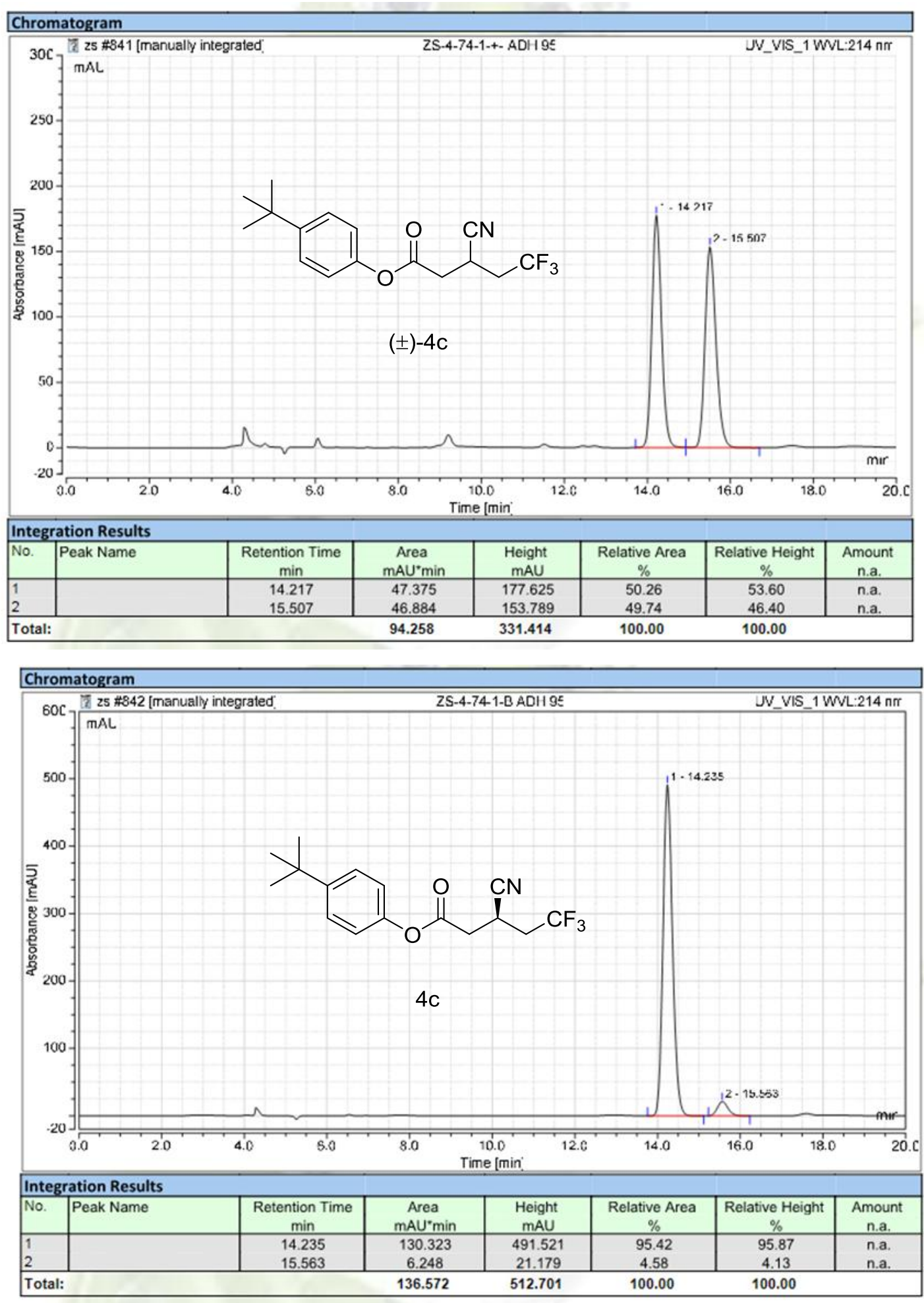

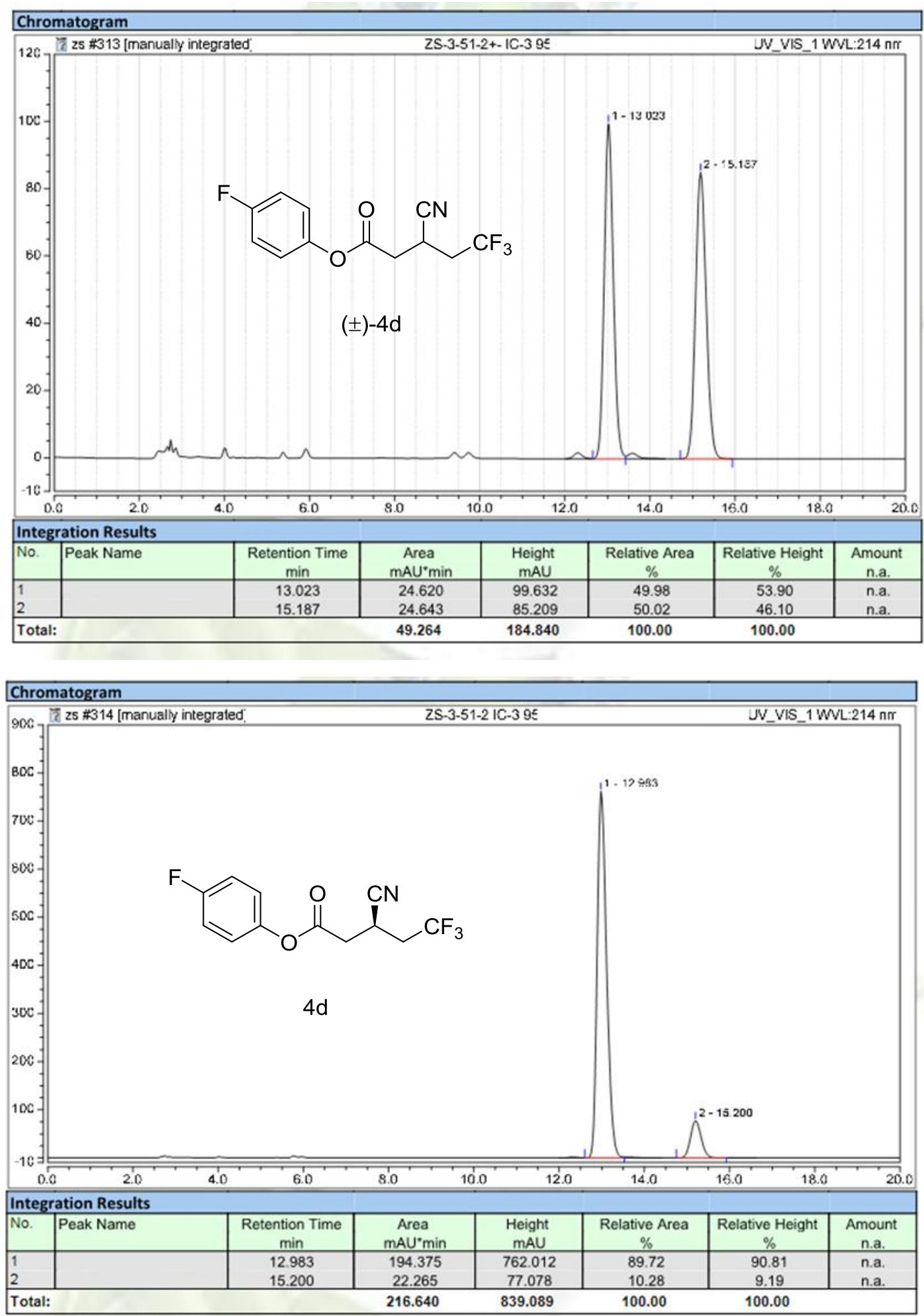

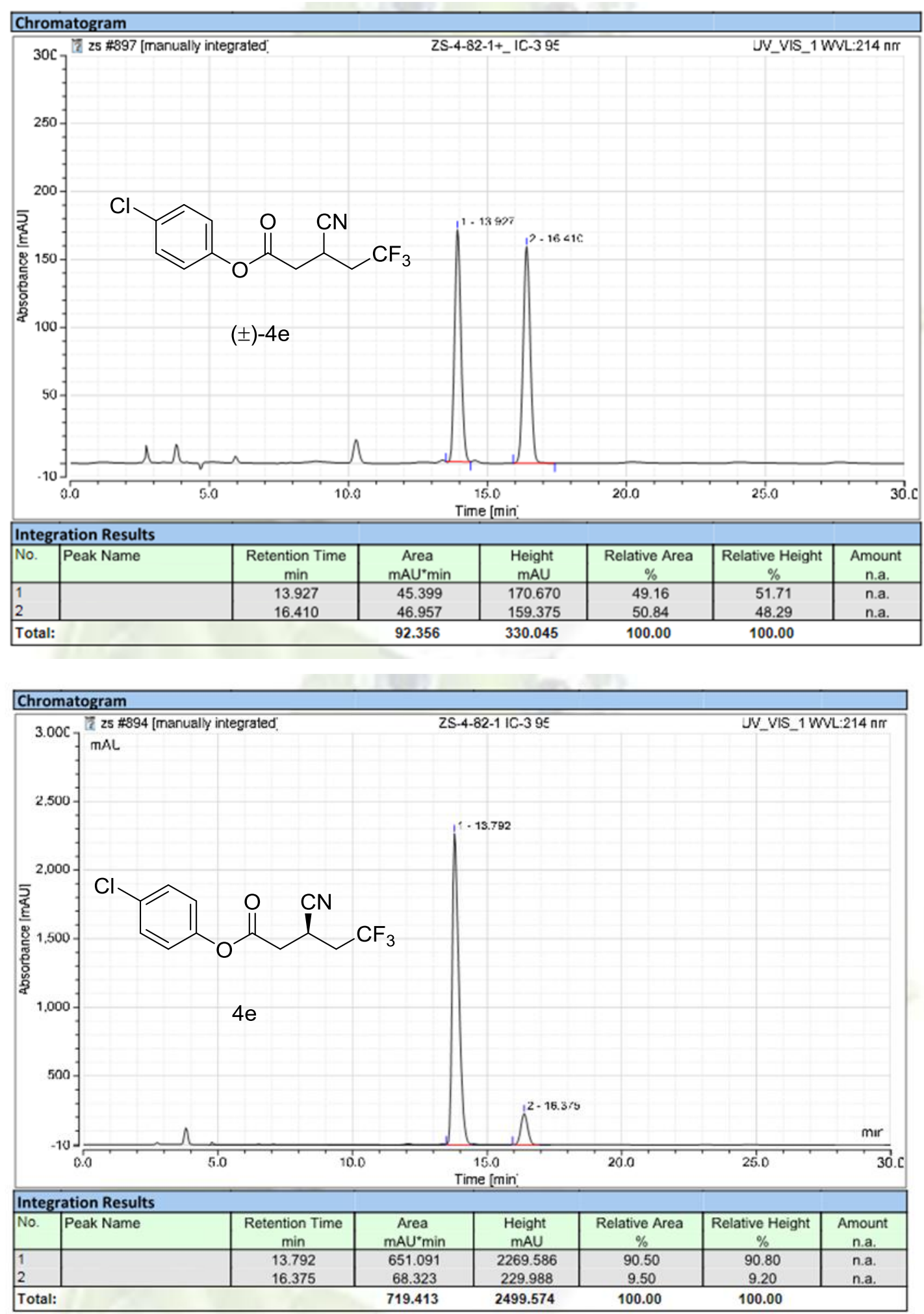

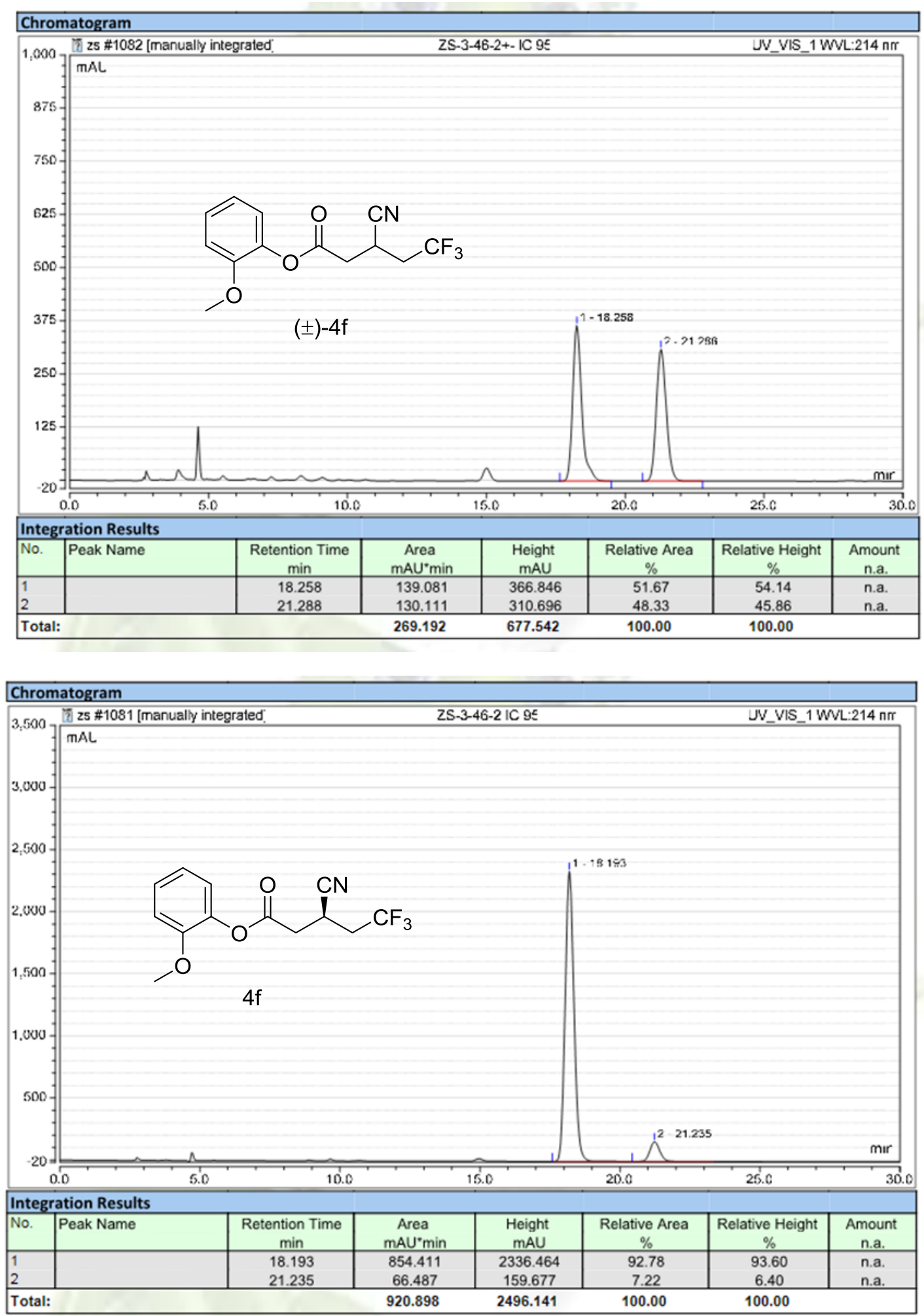

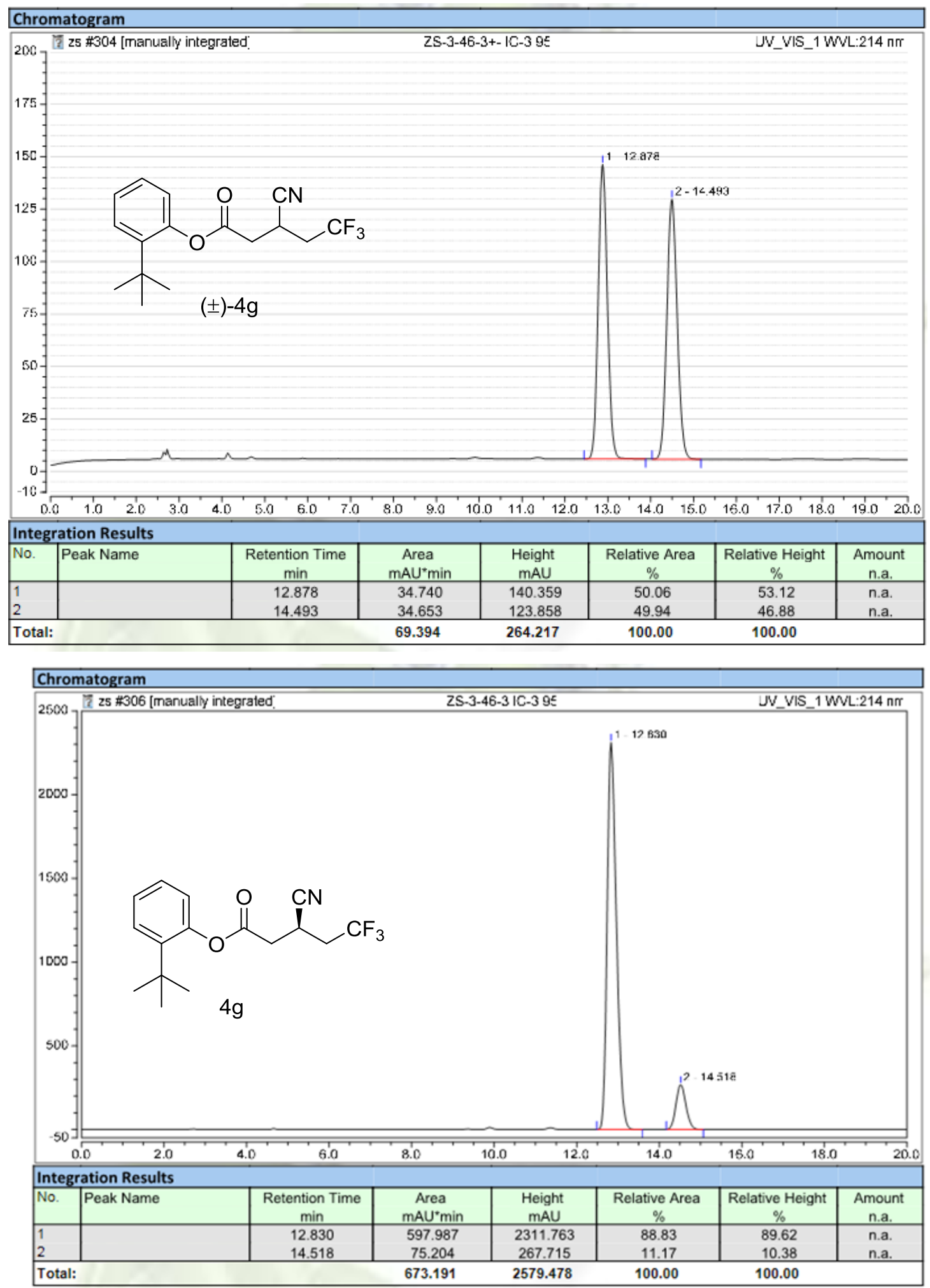

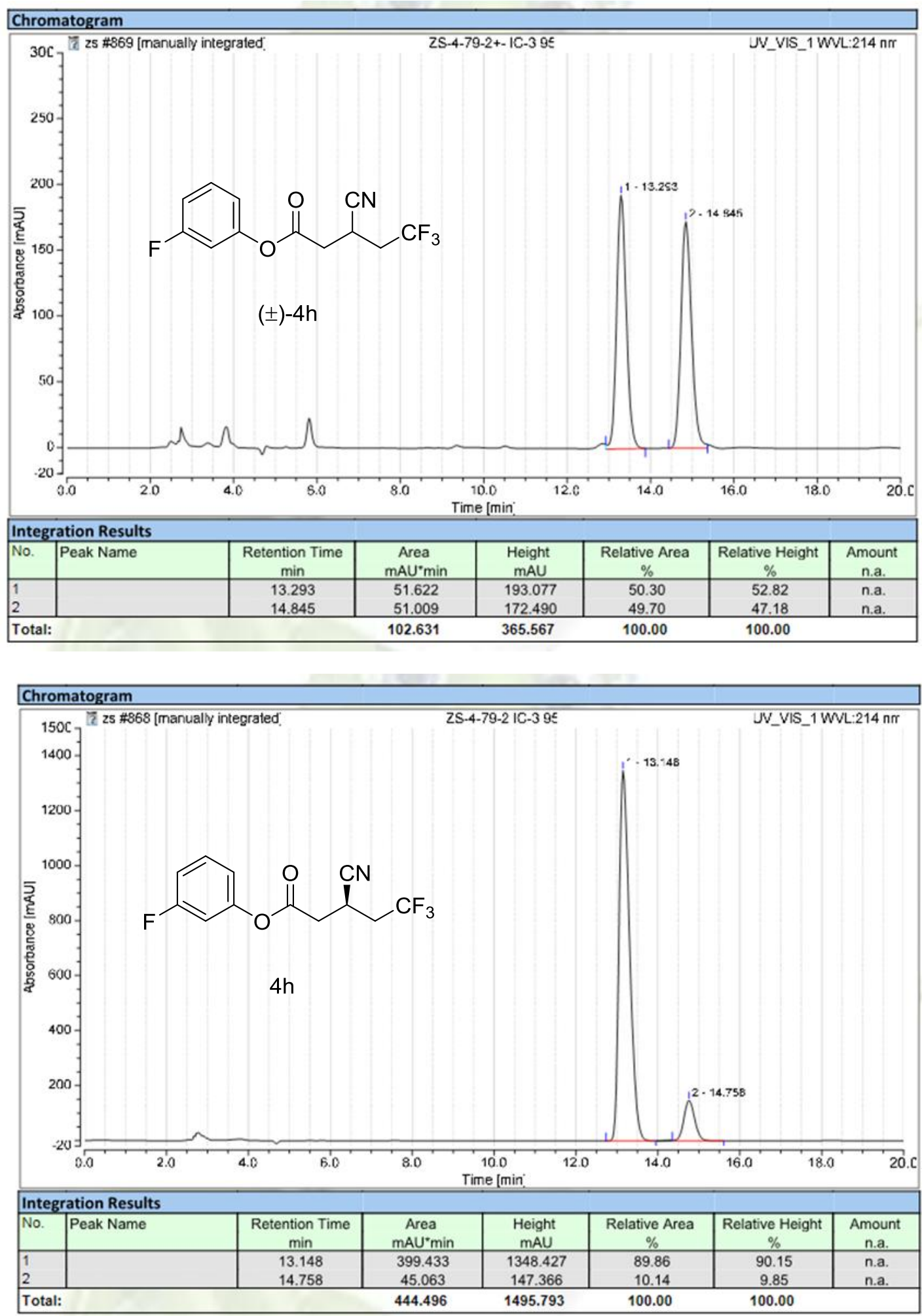

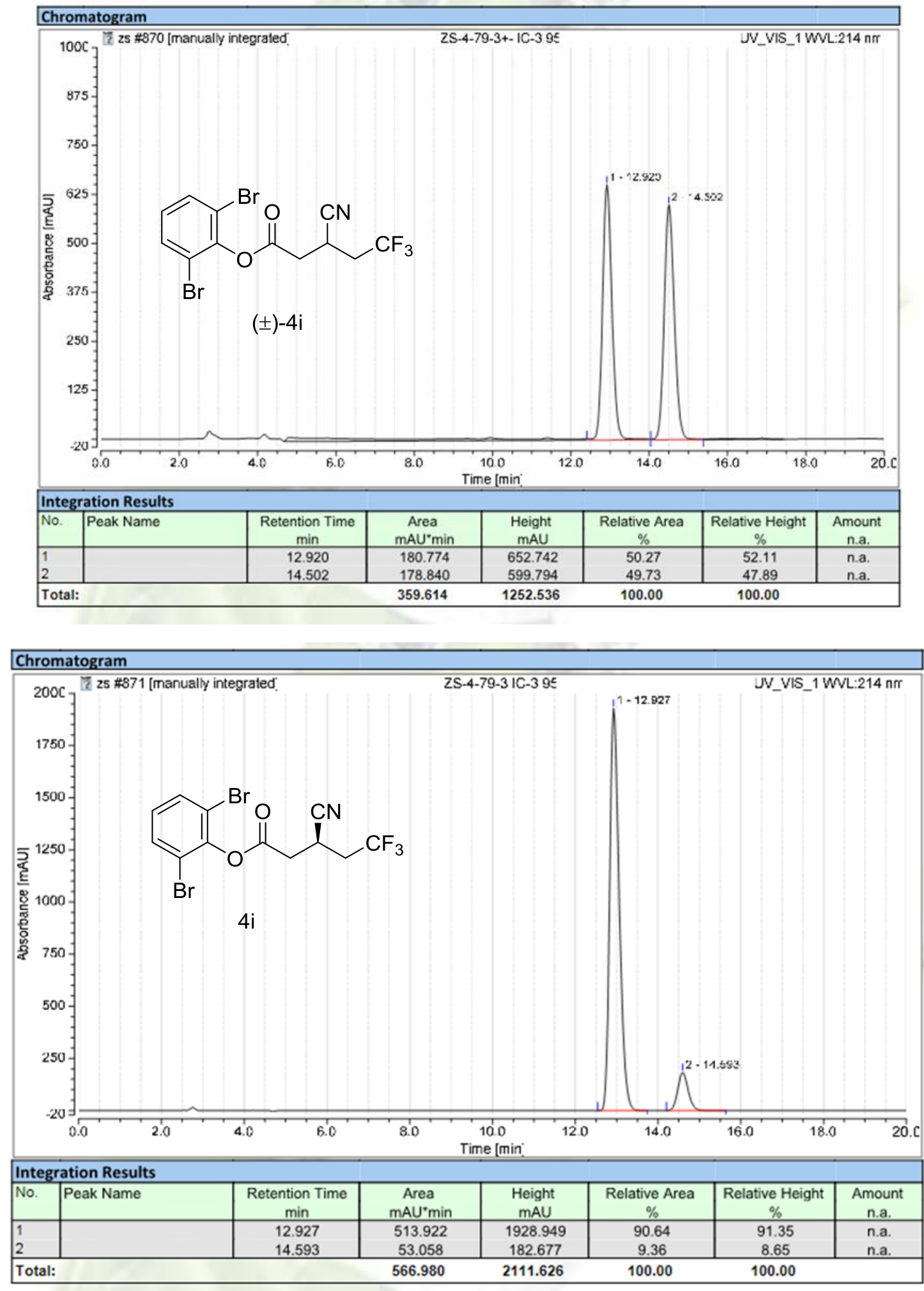

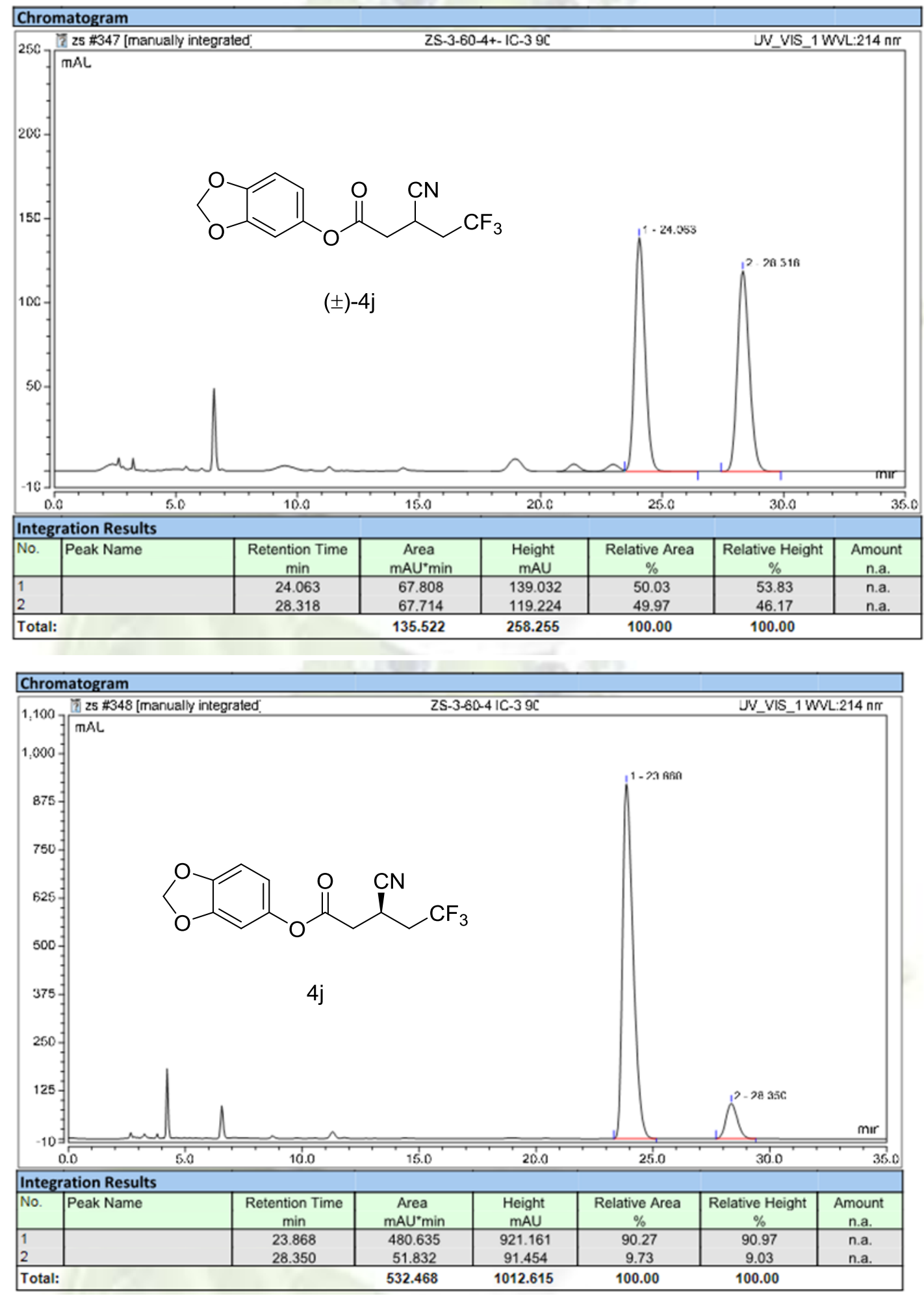

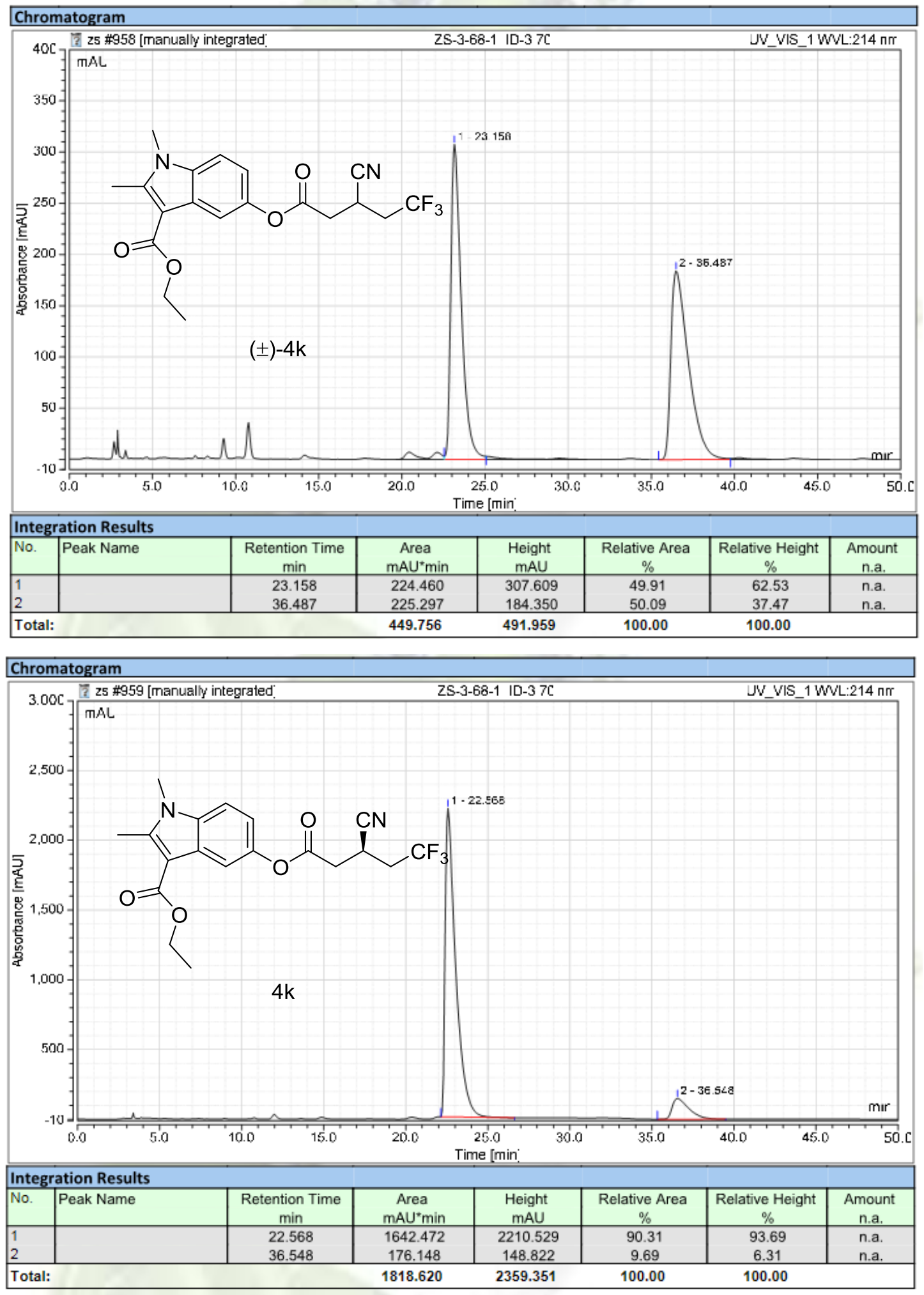


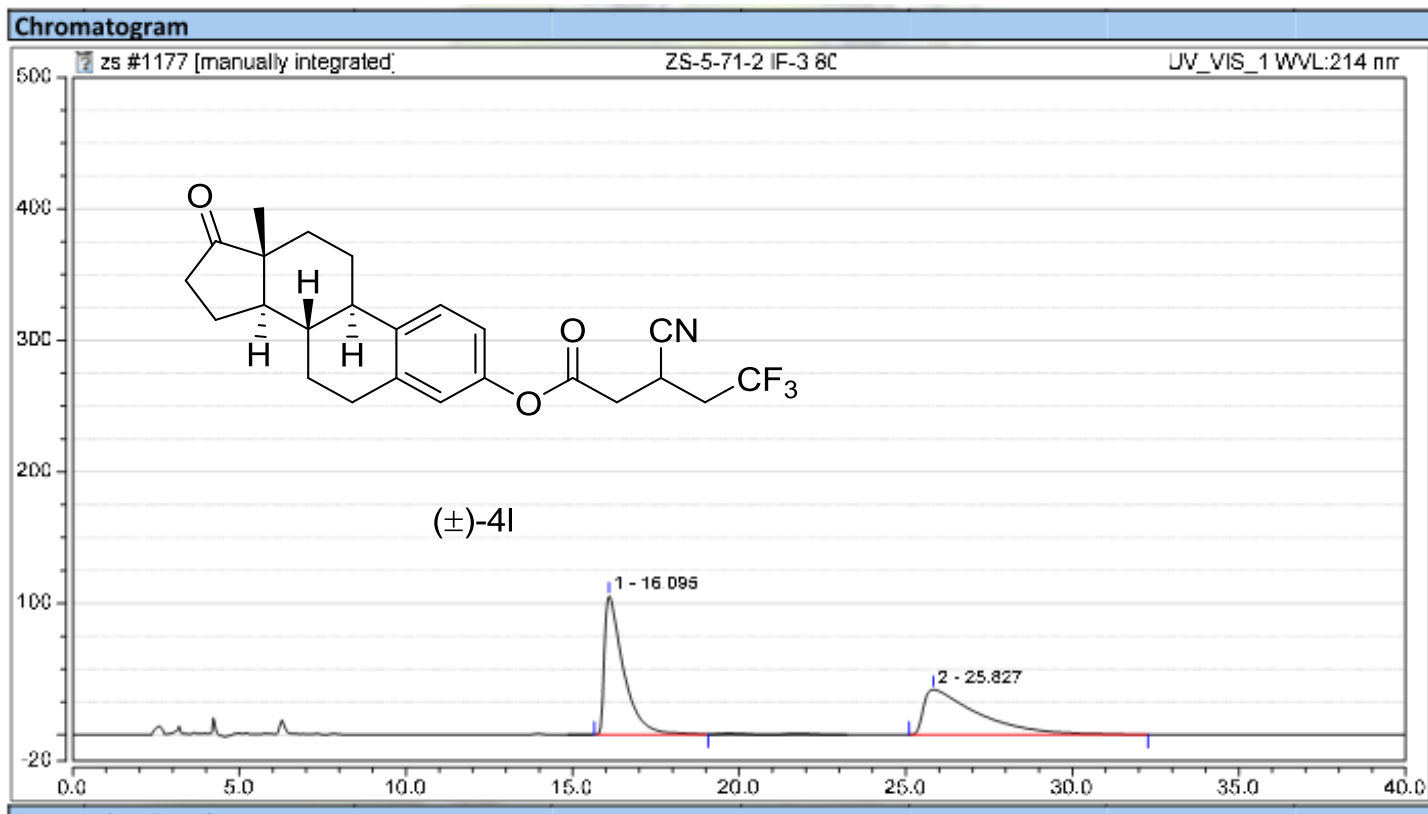

\begin{tabular}{|l|l|c|c|c|c|c|c|}
\hline \multicolumn{2}{|l|}{ Integration Results } \\
\hline No. & Peak Name & $\begin{array}{c}\text { Retention Time } \\
\text { min }\end{array}$ & $\begin{array}{c}\text { Area } \\
\text { mAU*min }\end{array}$ & $\begin{array}{c}\text { Height } \\
\text { mAU }\end{array}$ & $\begin{array}{c}\text { Relative Area } \\
\%\end{array}$ & $\begin{array}{c}\text { Relative Height } \\
\%\end{array}$ & $\begin{array}{c}\text { Amount } \\
\text { n.a. }\end{array}$ \\
\hline 1 & & 16.095 & 70.500 & 106.018 & 52.14 & 75.49 & n.a. \\
2 & 25.827 & 64.707 & 34.418 & 47.86 & 24.51 & n.a. \\
\hline
\end{tabular}

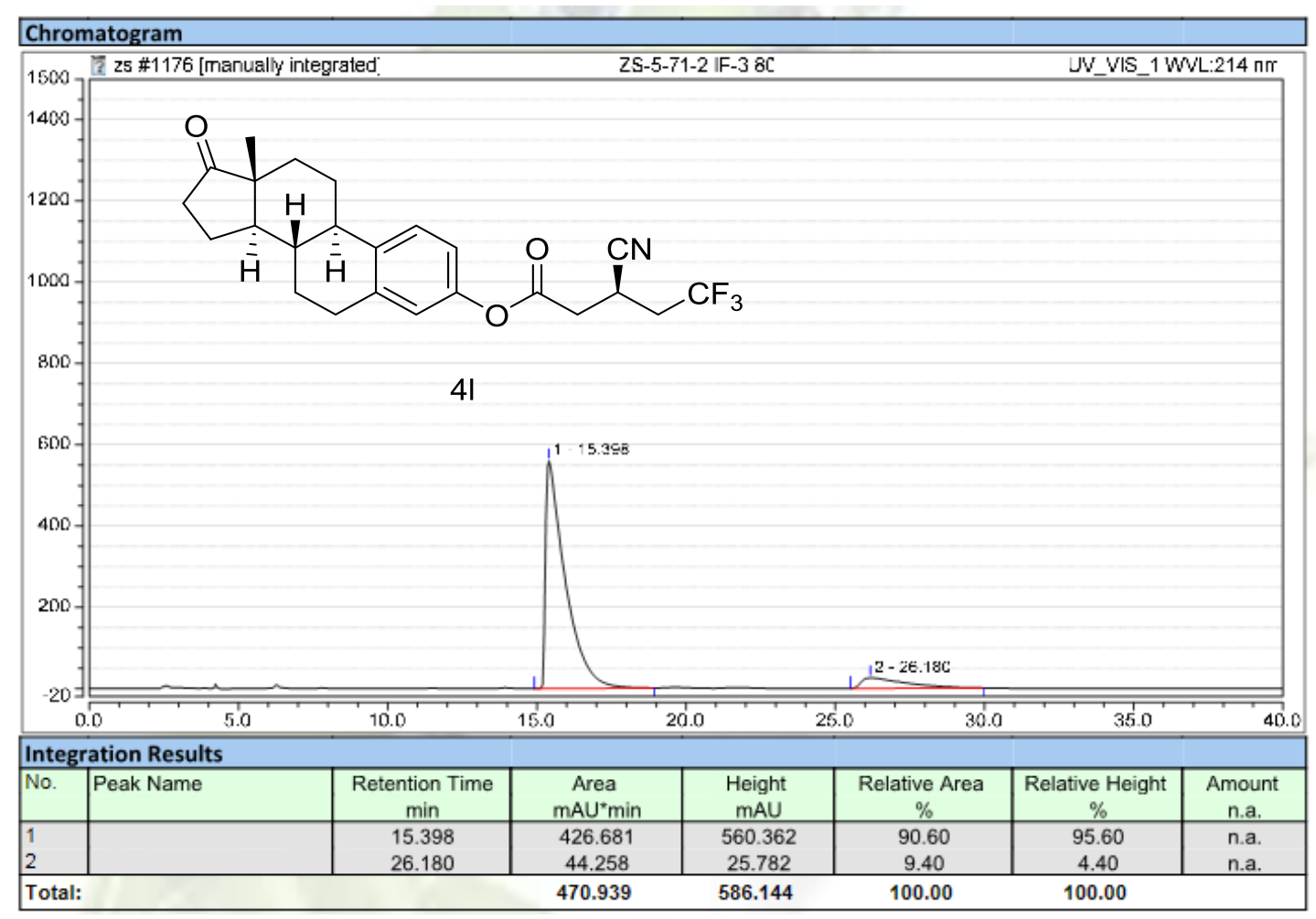



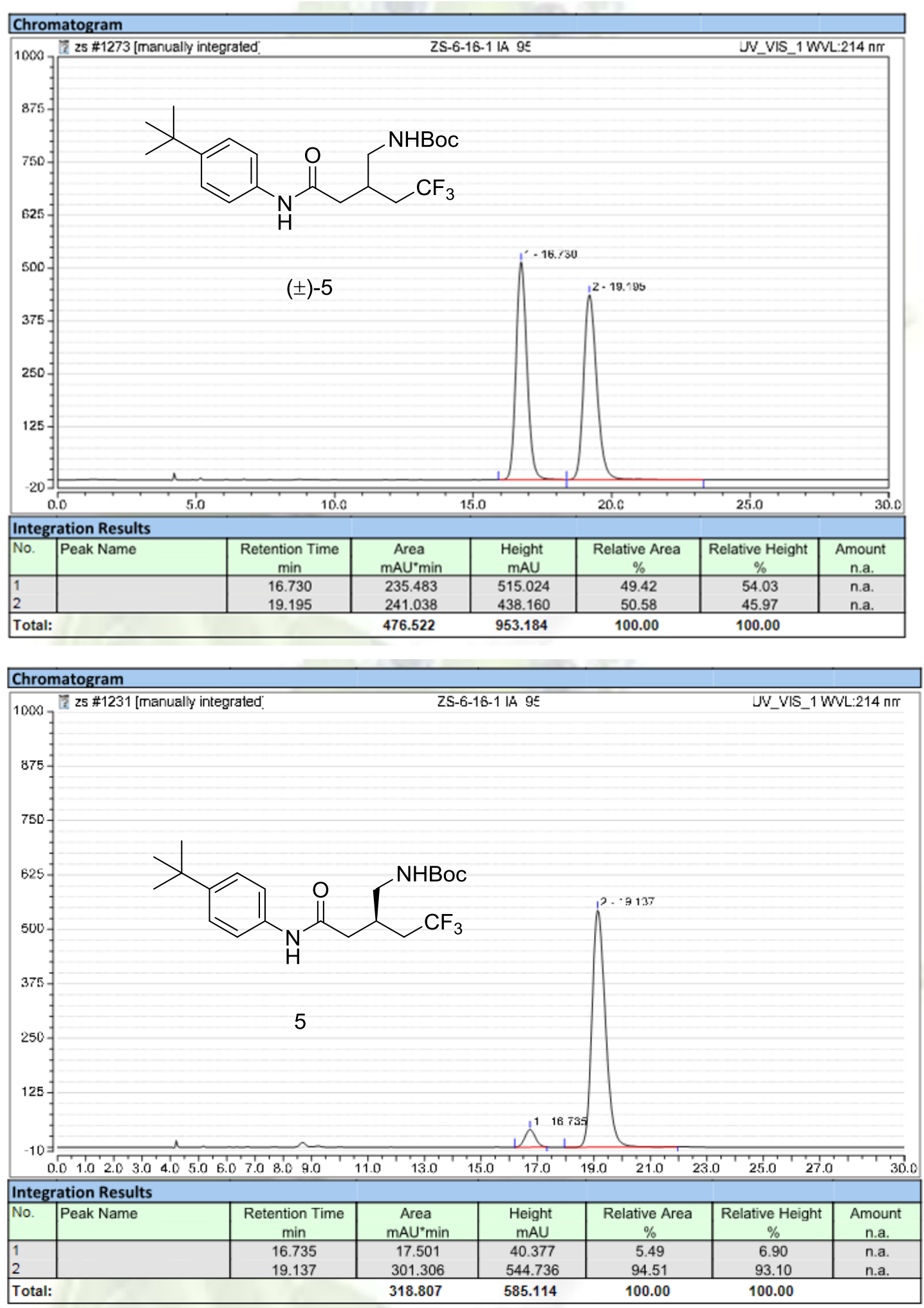


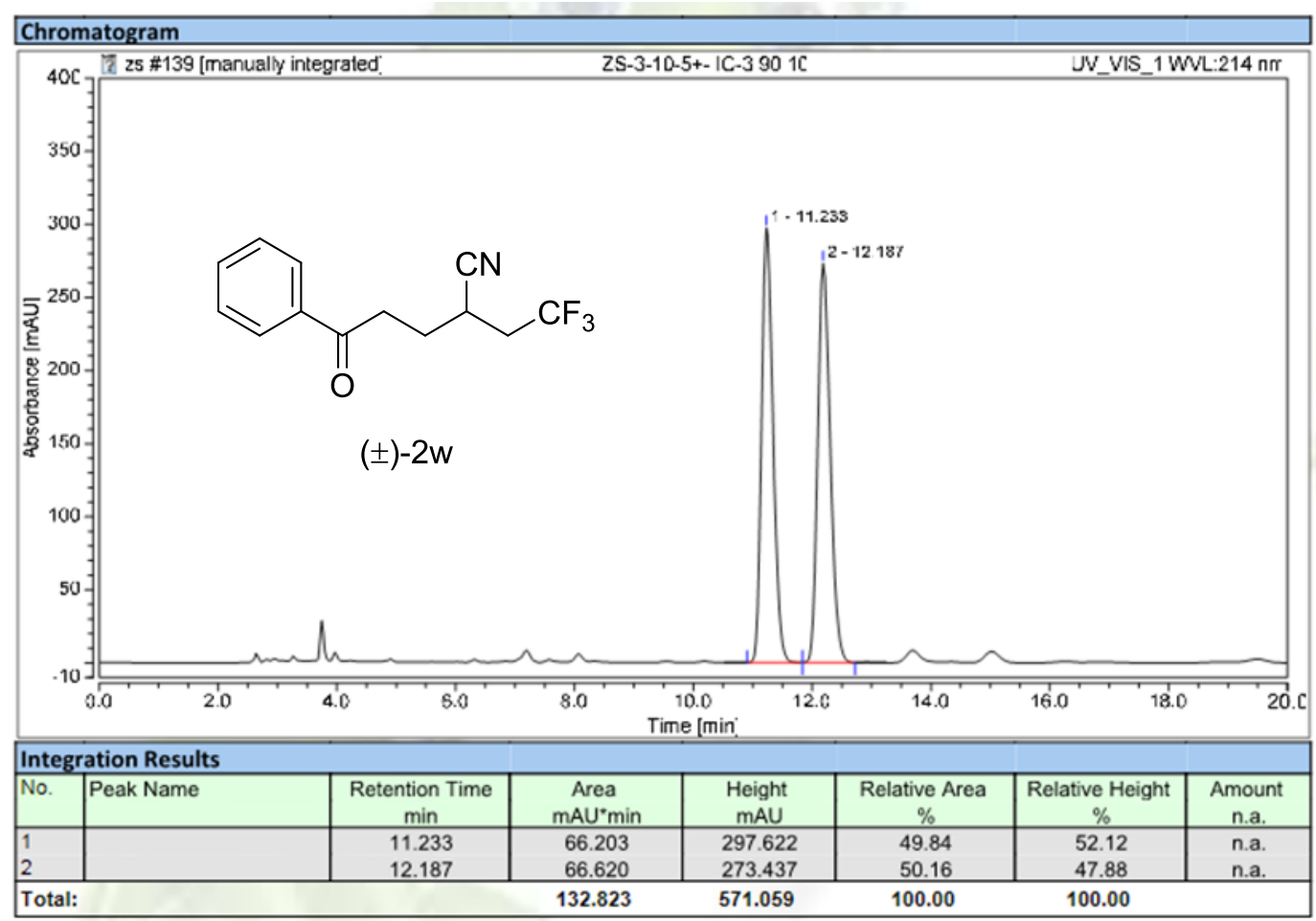

\section{Chromatogram}

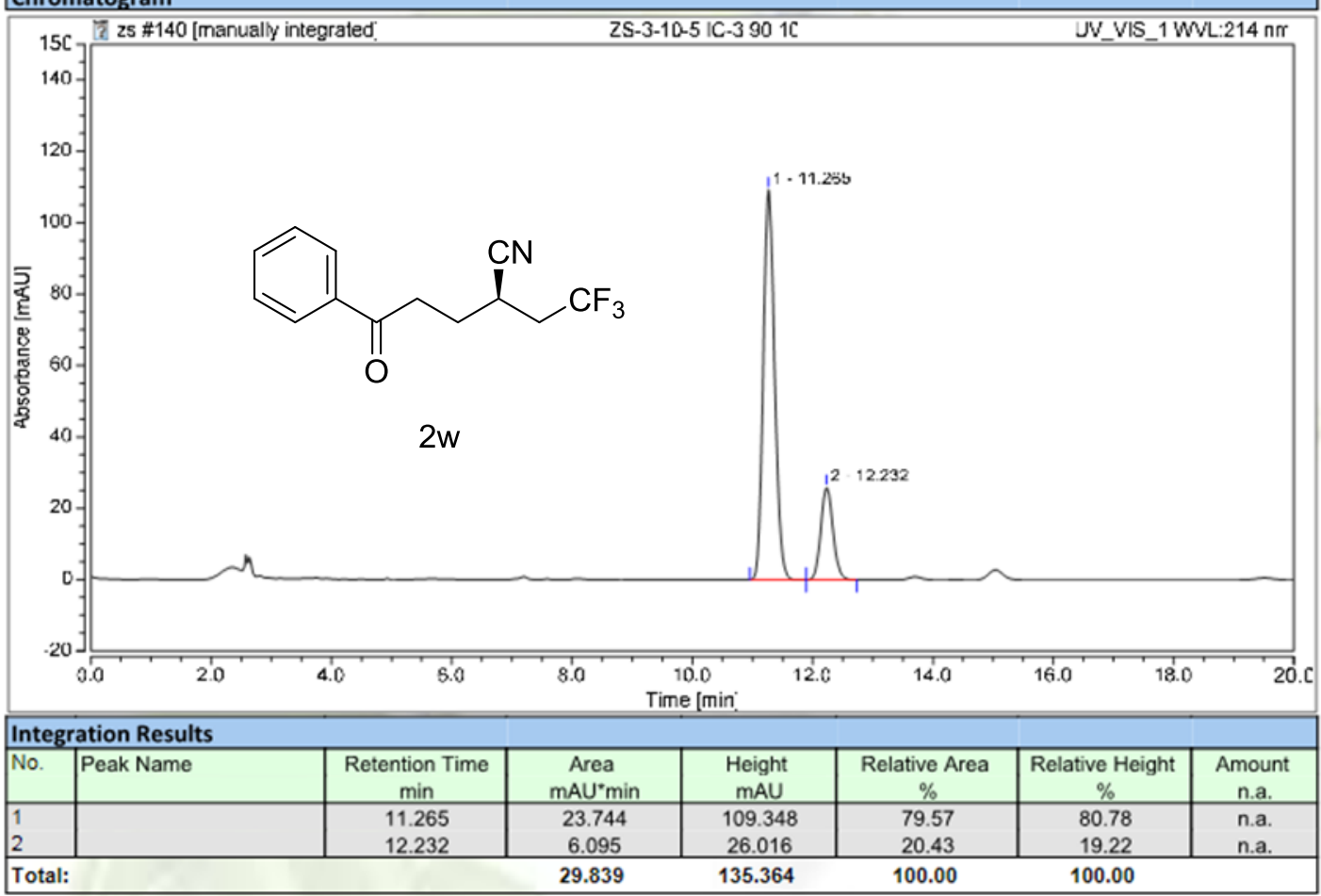



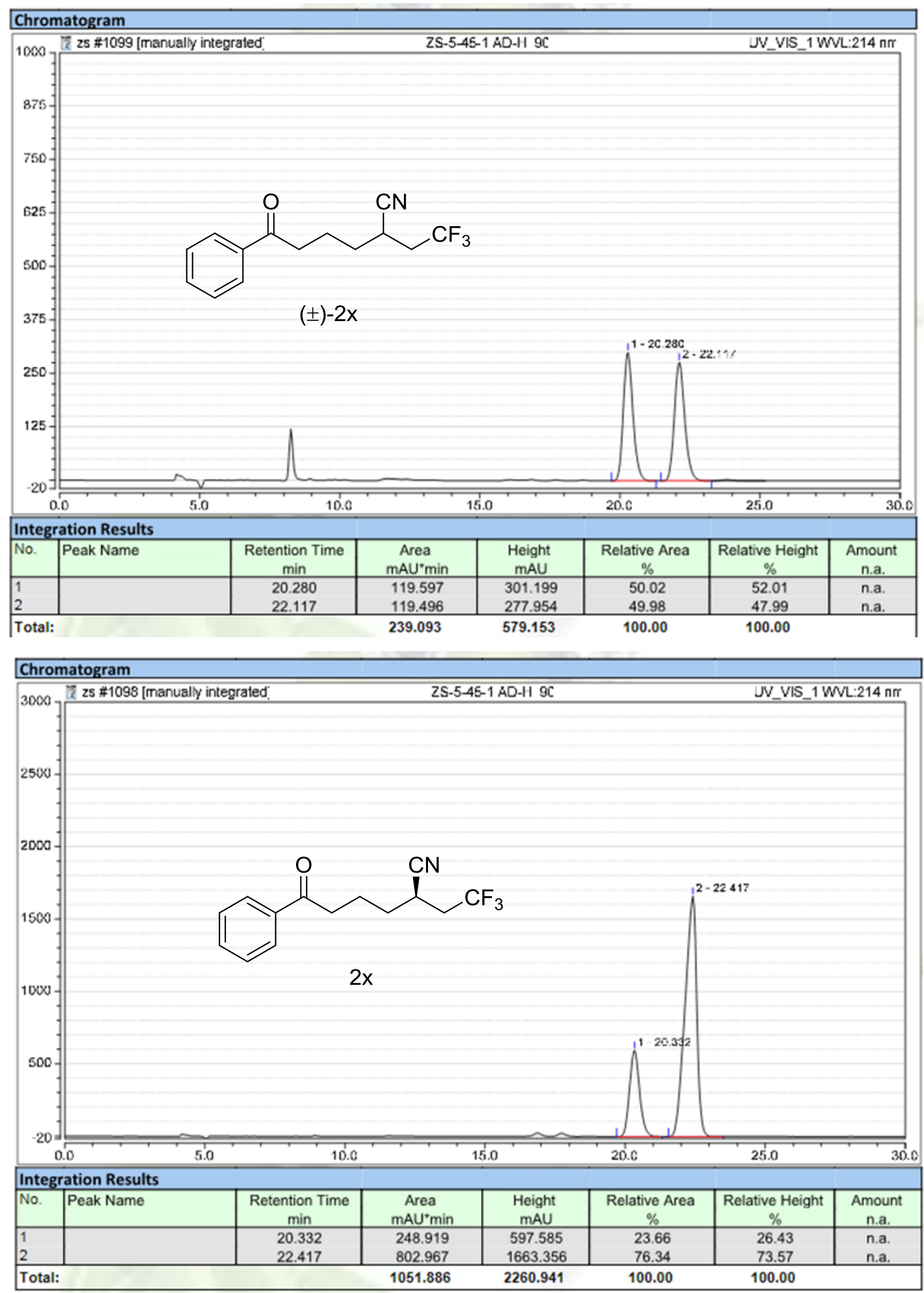

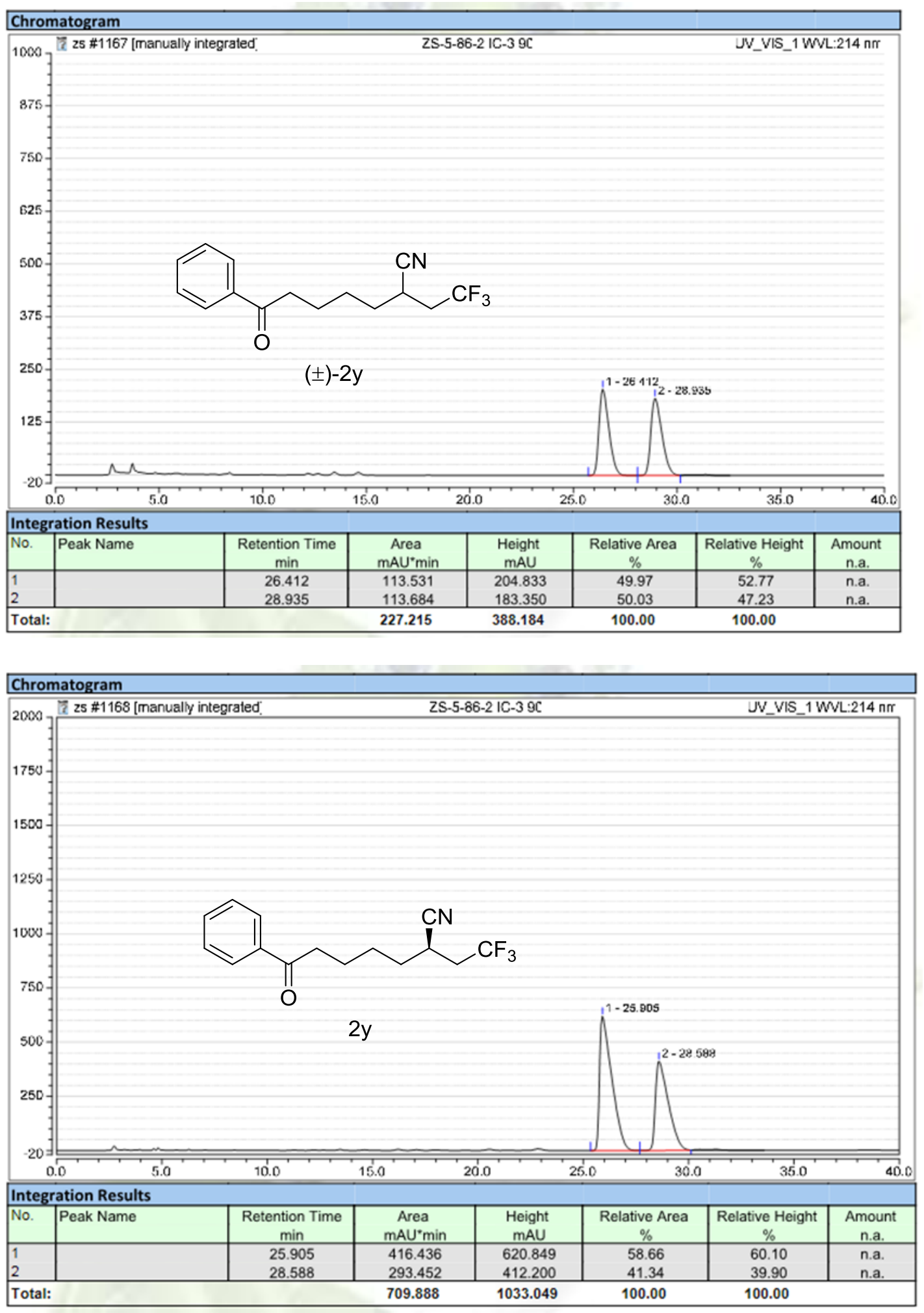

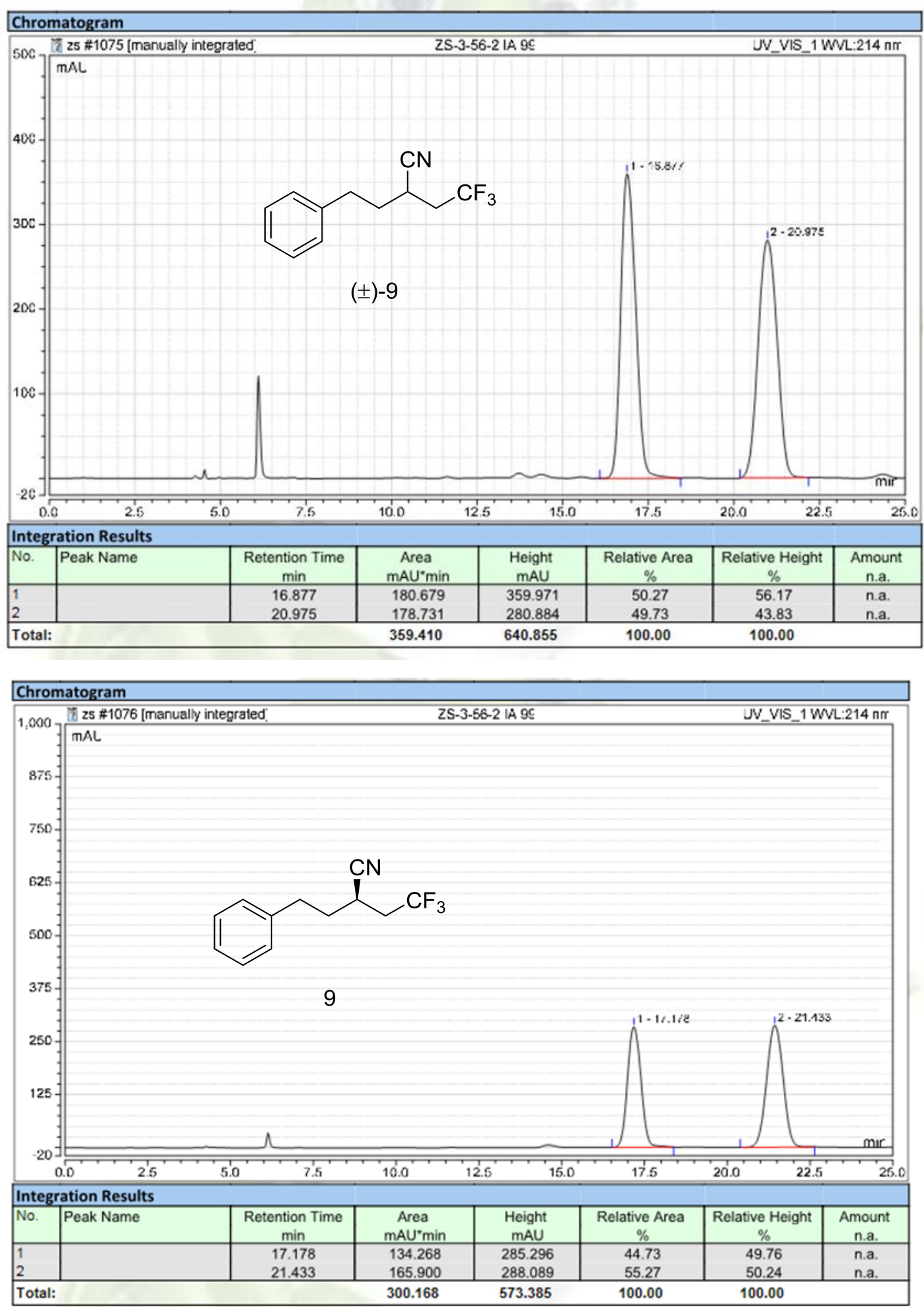

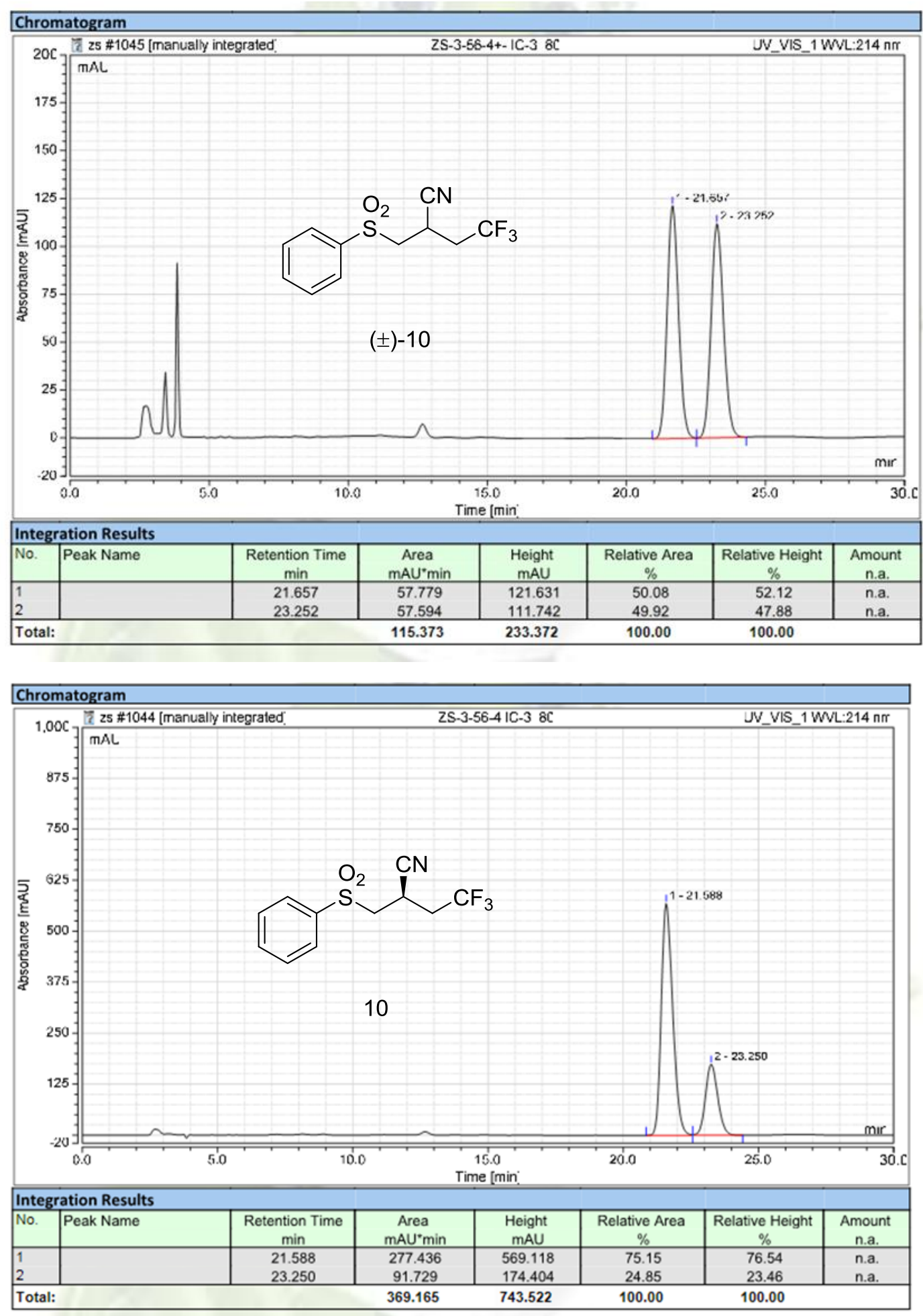

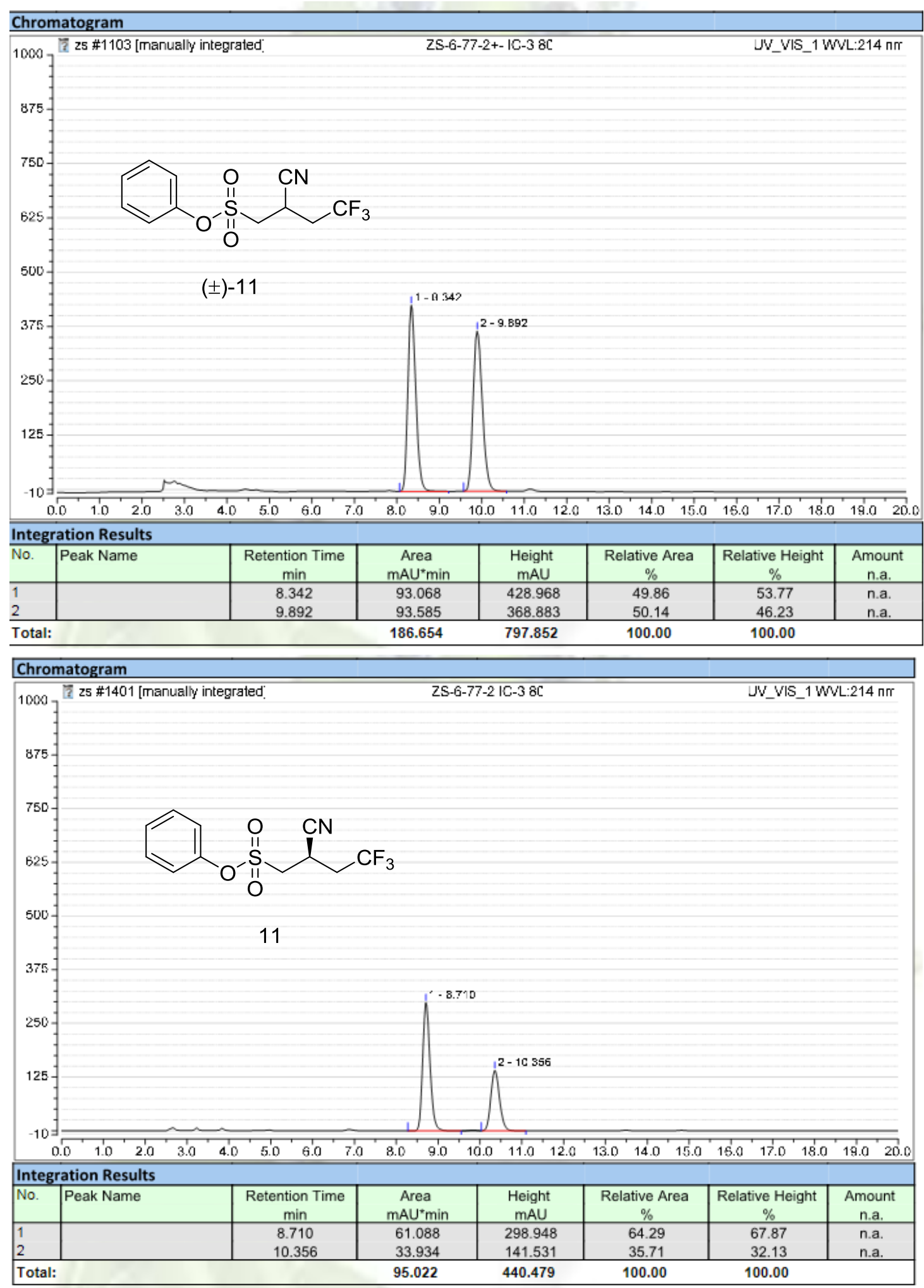


\section{References}

(S1) Lee, S-Y. A.; L, L-S. Tetrahedron Lett. 2000, 41, 8803-8806.

(S2) Peng, X-X.; Deng, Y-J.; Yang, X-L.; Zhang, L.; Yu, W.; Han, B. Org. Lett. 2014, $16,4650-4653$.

(S3) Yoo, B. W.; Choi, K. H.; Lee, S. J.; Nam, G. S.; Chang, K. Y.; Kim, S. H.; Kim, J. H.; Synthetic communications, 2002, 32, 839-846.

(S4) Zhuo, L. G.; Yao, Z. K.; Yu, Z. X. Org. Lett. 2013, 15, 4634-4637.

(S5) Guo, Y. Q.; Zhao, M. N.; Ren, Z. H.; Guan, Z. H. Org. Lett. 2018, 20, 3337-3340.

(S6) Toy, P. H.; Dhanabalasingam, B.; Newcomb, M.; Hanna, I. H.; Hollenberg, P. F. J.Org. Chem. 1997, 62, 9114-9122.

(S7) Ranaweera, R. A. A. U.; Scott, T.; Li, Q.; Rajam, S.; Duncan, A.; Li, R.; Evans, A.; Ault, B. S. Gudmundsdottir, A.D. J. Phys. Chem. A, 2014, 118,10433-10447.

(S8) Shi, P.; Wang, J.; Gan, Z. X.; Zhang, J. Y.; Zeng, R. S.; Zhao, Y. S. Chem.Commun. 2019, 55, 10523-10526.

(S9) Jean, M.; Weghe, P. van de. Tetrahedron Lett. 2011, 52, 3509-3513.

(S10) Wang, X.; Ye, Y.; Zhang, S.; Feng, J.; Xu, Y.; Zhang, Y.; Wang, J. J. Am. Chem. Soc. 2011, 133, 16410-16413.

(S11) Tan, X.; Song, T.; Wang, Z.; Chen, H.; Cui, L.; Li, C. Org. Lett. 2017, 19, 1634-1637. 